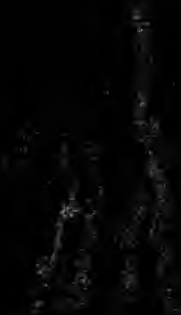

3

4
20

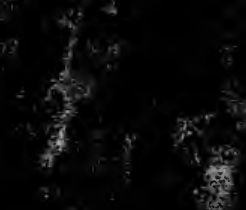

sy 

Adrlf. in Baeyen.

Gom his thantiful fufil

MPlPestim pis 
Digitized by the Internet Archive in 2007 with funding from Microsoft Corporation 

$4477 \mathrm{re}$

¿ Researches in organic chemistry

$$
\begin{gathered}
\text { by Henry Parkin } \\
\text { Vol. I } \\
1880-18893
\end{gathered}
$$


ON THE SYNTHETICAL FORMATION OF CLOSED CARBON-CHAINS.

By W. H. Perkin, Jun., Ph.D., Privatdocent at the University of Munich.

Part I. On Some Derivatives of Trimethylene.

INTRODUCTION.

OrGanIC chemistry is generally divided into two distinct sections, namely, the fatty series and the aromatic series.

The members of the first series are derivatives of methane, the simplest constituted hydrocarbon, and are characterised by their open or chain form, as for example in the case of normal hexane,

$$
\mathrm{CH}_{3} \cdot \mathrm{CH}_{2} \cdot \mathrm{CH}_{2} \cdot \mathrm{CH}_{2} \cdot \mathrm{CH}_{2} \cdot \mathrm{CH}_{3} \text {. }
$$

Aromatic compounds are, on the contrary, derivatives of a much more complicated basis, namely of benzene, $\mathrm{C}_{6} \mathrm{H}_{6}$, which, as was first shown by Kekulé in 1865, has the constitution

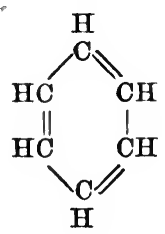

i.e., contains a ring consisting of six carbon-atoms joined together in such a way as to form a regular hexagon.

These two series differ in the most marked way from one another, the members of the aromatic series being particularly characterised by their extreme stability. Thus, whilst it is a matter of comparative ease to split up a more complicated fatty body into its simpler components, by chemical reactions, it is only with the greatest difficulty that the benzene-ring can in any way be decomposed.

In considering these great differences, one cannot but be surprised that no intermediate series should be known, the members of which would possess partly the character of fatty, and partly that of aromatic compounds.

It is not absolutely necessary that a carbon-ring should contain six carbon-atoms, as it is quite reasonable to suppose that rings constructed with $3,4,5,7$, \&c., carbon-atoms should be capable of existing. The few experiments which have up to the present time 
been tried to test this supposition, have not thrown much light on the subject.

The improbability of the existence of a three-carbon-ring

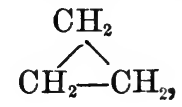

which would be isomeric with propylene, $\mathrm{CH}_{3} \cdot \mathrm{CH}: \mathrm{CH}_{2}$, is the subject of a long discussion in a paper by Victor Mejer (Annalen, 180, 196).

Reboul (Compt. rend., 22 Juin, 1874) attempted to obtain this hydrocarbon by treating trimethylene bromide with sodium at high temperatures, and found that a gas was formed, which he supposed to be ordinary propylene. Freund (Monatsh. Chem., 1882, 622), who has since very carefully studied this reaction, comes to very different conclusions. On heating trimethylene bromide, $\mathrm{CH}_{2} \mathrm{Br}^{\circ} \mathrm{CH}_{2} \mathrm{CH}_{2} \mathrm{Br}$, with sodium at temperatures not above the boiling point of the bromide $\left(165^{\circ}\right)$, Freund obtained a gas which when passed through bromine was only very slowly and with great difficulty attacked by it, trimethylene bromide being regenerated, whereas propylene, as is well known, is instantly absorbed by bromine, propylene bromide, $\mathrm{CH}_{3} \cdot \mathrm{CHBr} \cdot \mathrm{CH}_{2} \mathrm{Br}$ being formed.

Still more curious is the fact (also observed by Freund) that this gas when passed through fuming hydriodic acid is easily absorbed, normal propyl iodide, $\mathrm{CH}_{3} \cdot \mathrm{CH}_{2} \cdot \mathrm{CH}_{2} \mathrm{I}$, being produced.

Propylene, under the same circumstances, gives isopropyl iodide, $\mathrm{CH}_{3} \cdot \mathrm{CHI} \cdot \mathrm{CH}_{3}$.

There can therefore be no doubt that this gas is not propylene; it must therefore be trimethylene,

No attempts appear to have been made to synthesise the corresponding four-carbon-ring,

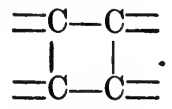

There is, however, one body in the aromatic series, namely,<smiles></smiles>

acenaphthene, which without the slightest doubt contains such a ring, 
and this being a very stable body, it is lawful to assume that the ring, $\mathrm{CH}_{2}-\mathrm{CH}_{2}$ $\underset{\mathrm{C} \mathrm{H}_{2}-\mathrm{CH}_{2}}{\mathrm{C}}$, should not be a body incapable of existence.

An example of a hydrocarbon in the aromatic series containing a five-carbon-ring is fuorene :-

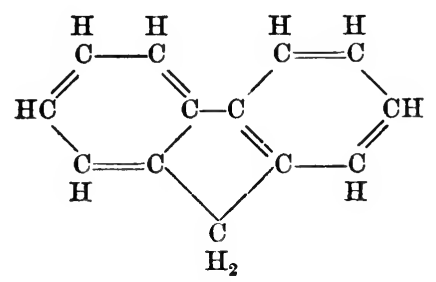

If we examine further the possibility of the existence o four- and five-carbon-rings alone, we find that several bodies in the fatty series are already well known, which are closely allied to these hypothetical hydrocarbons. Thus furfuran, $\underset{\mathrm{CH}=\mathrm{CH}}{\mathrm{C}=\mathrm{CH}}>\mathrm{O}$, is a fourcarbon-chain, which is closed by an oxygen-atom, a fact which should rather tend to weaken the compound than otherwise, and yet this body possesses a considerable degree of stability, forcing one to come to the conclusion that the simple ring, ${ }_{\mathrm{CH}}^{\mathrm{CH}}=\mathrm{CH}$ (that is, tetrene), should certainly be capable of existence. On pursuing the subject further, we find such bodies as-

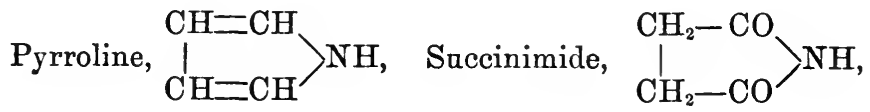

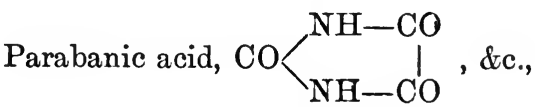

which all contain rings made up of five atoms of carbon or nitrogen, a fact which must lead one to suppose that the ring $\underset{\mathrm{C}-\mathrm{C}}{\mathrm{C}-\mathrm{C}}>\mathrm{C}$ cannot be an unstable combination. Such considerations as these made it appear interesting to pursue the subject further, and if possible either to fill up the gaps by synthesising the missing rings, or if that were not possible, at all events, to give some plausible reason why they should not be capable of existence.

In order to synthesise the four-carbon-ring, the first idea that would 
naturally occur would be to prepare a dibromide of the formula $\mathrm{CH}_{2} \mathrm{Br} \cdot \mathrm{CH}_{2} \cdot \mathrm{CH}_{2} \cdot \mathrm{CH}_{2} \mathrm{Br}$, and then treat this with solium,

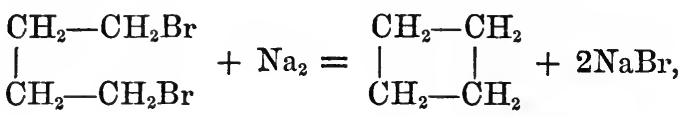

a reaction exactly similar to that by which Freund obtained trimethylene from trimethylene bromide, $\mathrm{CH}_{2} \mathrm{Br} \cdot \mathrm{CH}_{2} \cdot \mathrm{CH}_{2} \mathrm{Br}$, and sodium.

There is, however, curiously enough, no reaction in the fatty series by which dibromides can be prepared having the two bromineatoms situated at opposite ends of the carbon-chain; after a long series of experiments, no practicable method could be found for producing such compounds in sufficient quantities for further experiments.

Another method for forming these ring-compounds was, however, afterwards found, which did away in the meanwhile with the necessity of using bromides with longer chains than trimethylene bromide.

It is a well-known fact that if ethyl malonate is mixed with sodic ethylate, it forms a sodium compound, which when treated with the halogen-derivatives of the alcohol radicals gives the higher homologues of ethyl malonate; thus on treating it with propyl iodide, ethyl propylmalonate is formed-

$$
\begin{aligned}
& \mathrm{CHNa}_{\mathrm{COOC}_{2} \mathrm{H}_{5}}^{\mathrm{COOC}_{2} \mathrm{H}_{5}}+\mathrm{CH}_{3} \cdot \mathrm{CH}_{2} \cdot \mathrm{CH}_{2} \mathrm{I}=
\end{aligned}
$$

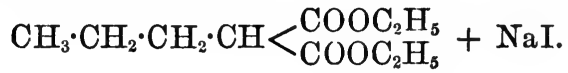

This ethyl propylmalonate again is itself capable of forming a sodium compound, which on further treatment with propyl iodide gives ethyl dipropylmalonate thus :-

$\mathrm{CH}_{3} \cdot \mathrm{CH}_{2} \cdot \mathrm{CH}_{2} \cdot \mathrm{CNa}<\mathrm{COOC}_{2} \mathrm{H}_{5}+\mathrm{CH}_{3} \cdot \mathrm{CH}_{2} \cdot \mathrm{CH}_{2} \mathrm{I}=$

$\underset{\mathrm{CH}_{3} \cdot \mathrm{CH}_{2} \cdot \mathrm{CH}_{2}}{\mathrm{CH}_{3} \cdot \mathrm{CH}_{2} \cdot \mathrm{CH}_{2}} \underset{\mathrm{COOOC}_{2} \mathrm{H}_{5}}{\mathrm{COOO}_{2} \mathrm{H}_{5}}+\mathrm{NaI}$.

Now if it were possible to brominate ethyl propylmalonate in such a way that the bromine would attack the methyl-group, and that group alone, the compound

$$
\mathrm{CH}_{2} \mathrm{Br}^{\circ} \mathrm{CH}_{2} \cdot \mathrm{CH}_{2} \cdot \mathrm{CH}<\mathrm{COOC}_{2} \mathrm{COOC}_{5} \mathrm{H}_{5}
$$

would be obtained, which when treated with sodic ethylate would from a sodium compound; this on heating should be decomposed in the following way :- 
$\mathrm{CH}_{2} \mathrm{Br} \cdot \mathrm{CH}_{2} \cdot \underset{\mathrm{Na}}{\mathrm{CH}_{2}}>\mathrm{C}<\underset{\mathrm{COOOC}_{2} \mathrm{H}_{5}}{\mathrm{COOC}_{2} \mathrm{H}_{5}}=\mathrm{CH}_{2}<\mathrm{CH}_{2}^{\mathrm{CH}_{2}}>\mathrm{C}<\mathrm{COOC}_{2} \mathrm{H}_{5}$

$+\mathrm{NaBr}$.

It is unfortunately a most difficult matter to prepare a brominated compound such as that represented above, and all experiments which were tried for this purpose failed. The bromine always enters the molecule in the $\mathrm{CH}$ group, forming a compound of the formula-

$$
\underset{\mathrm{Br}}{\mathrm{CH}_{3} \cdot \mathrm{CH}_{2} \cdot \mathrm{CH}_{2}}>\mathrm{C}<\mathrm{COOOC}_{2} \mathrm{H}_{5} \cdot
$$

This difficulty can, however, be got over, if one starts in the first place with a dibromide which already contains the two bromineatoms in $\mathrm{t}$ :e right position, and such a bromide is trimethylene bromide, $\mathrm{CH}_{2} \mathrm{Br} \cdot \mathrm{CH}_{2} \cdot \mathrm{CH}_{2} \mathrm{Br}$. If the sodium compound of ethyl malonate is treated with trimethylene bromide an ethereal salt is obtained, which, from the experiments already made, must without doubt contain a four-carbon-ring and have the following constitution:-

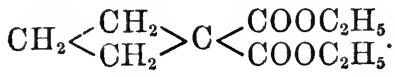

The proofs that this compound has this constitution are the following: If the action of trimethylene bromide on ethyl malonate is studied, it is seen that there are only two possible ways in which the reaction can take place :-I. That a body possessing the above constitution is formed. II. Or that the reaction is the following :-

$\mathrm{CHNa}<\mathrm{COOC}_{2} \mathrm{H}_{5}+\mathrm{CH}_{2} \mathrm{Br} \cdot \mathrm{CH}_{2} \cdot \mathrm{CH}_{2} \mathrm{Br}=$

$$
\mathrm{CH}_{2}: \mathrm{CH} \cdot \mathrm{CH}_{2} \cdot \mathrm{CH}<_{\mathrm{COOC}_{2} \mathrm{H}_{5}}^{\mathrm{COOC}_{2} \mathrm{H}_{5}}+\mathrm{NaBr}+\mathrm{HBr},
$$

i.e., that ethyl allylmalonate is formed.

That this is not the case I shall be able to show decisively when the substance in question is treated of in Part II of this paper. One is therefore forced to come to the conclusion that a derivative of a closed chain containing four carbon-atoms has been formed. This reaction is, moreover, not confined to trimethylene bromide. Ethylene bromide acts on ethyl malonate in exactly the same way, producing compounds which are periectly analogous to those ohtained by the action of trimethylene bromide.

'Thus the first product of the action of ethylene bromide on ethyl malonate is a three-1 ing-derivative, having the formula-

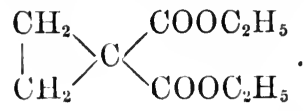


It was now necessary to find some suitable form of nomenclature for these bodies, and after some consideration it was thonght that the simplest method would be to regard the saturated hydrocarbons themselves as multiples of methylene, $\mathrm{CH}_{2}$, and thus name them di-, tri-, tetra-, penta-, \&c., methylene, as is easiest seen from the following table:-

\begin{tabular}{|c|c|c|c|c|c|}
\hline$=\mathrm{CH}_{2}$ & $\begin{array}{l}\mathrm{CH}_{2} \\
\mathrm{CH}_{2}\end{array}$ & $\mathrm{CH}_{2}-\mathrm{CH}_{2}$ & $\underset{\mid}{\mathrm{CH}_{2}}-\stackrel{\mathrm{CH}}{2}_{2}$ & $\overbrace{\mathrm{CH}_{2}-\overbrace{\mathrm{CH}}^{\mathrm{CH}}}^{\mathrm{CH}_{2}}$ & $\overbrace{\mathrm{CH}_{2}}^{\mathrm{CH}_{2}} \overbrace{\mathrm{CH}_{2}}^{\mathrm{CH}_{2}}$ \\
\hline Methylene. & $\begin{array}{c}D i- \\
\text { methylene } \\
\text { (Ethylene) }\end{array}$ & $\underset{\text { methylene }}{\text { Tri- }}$ & $\begin{array}{c}\text { Tetra- } \\
\text { methylene }\end{array}$ & $\begin{array}{c}\text { Penta- } \\
\text { methylene }\end{array}$ & $\begin{array}{c}\mathrm{Hexa}- \\
\text { methylene }\end{array}$ \\
\hline
\end{tabular}

This is not only very clear and easy to understand, but is also of general application to all hydrocarbons which are built up of $\mathrm{CH}_{2}$ groups.

Another question is how to distinguish the different possible isomeric derivatives of such hydrocarbons from each other, and I think that in order to meet this difficulty in the clearest and most satisfactory manner, it will be best to number the different carbonatoms in the ring as proposed by Baeyer (Ber., 17, 960); thus :-
$\mathrm{CH}_{2}$
(2) $\mathrm{CH}_{2}-\mathrm{CH}_{2}(3)$
Trimethylene.
(4) $\mathrm{CH}_{2}-\mathrm{CH}_{2}$ (1)
Tetramethylene.

The necessity for some such system of nomenclature will be seen directly on examining the following table of the carboxylic acids of trimethylene, which have all been obtained :-

I. $\overbrace{\mathrm{CH}_{2}-\mathrm{CH}_{2}}^{\mathrm{CH}} \cdot \mathrm{COOH}=$ Trimethylenecarboxylic acid.

II. $\overbrace{\mathrm{CH}_{2}-\mathrm{CH}_{2}}^{\mathrm{C}<\mathrm{COOH}}=$ Trimethylenedicarboxylic acid $(1,1)$.

III. $\overbrace{\mathrm{CH}_{2}-\mathrm{CH} \cdot \mathrm{COOH}}^{\mathrm{CH} \cdot \mathrm{COOH}}=$ Trimethylenedicarboxylic acid $(1,2)$.

IV. $\overbrace{\mathrm{CH}_{2}-\mathrm{CH} \cdot \mathrm{COOH}}^{\mathrm{C}<\mathrm{COOH}}=$ Trimethylenetricarboxylic acid $(1,1,2)$. 

$\overbrace{\mathrm{COOH} \cdot \mathrm{CH}-\mathrm{CH} \cdot \mathrm{COOH}}^{\mathrm{CH} \cdot \mathrm{COOH}}=\begin{gathered}\text { Trimethylenetricarboxylic acid } \\ (1,2,3) .\end{gathered}$

V.

VI. $\begin{gathered}\stackrel{\mathrm{C}<\mathrm{COOH}}{\mathrm{COOH}} \\ \mathrm{COOH} \cdot \mathrm{CH}-\mathrm{CH} \cdot \mathrm{COOH}\end{gathered}=$ Trimethylenetetracarboxylic acid

All other derivatives of such closed chains can be formulated in an exactly similar way, and thus directly recognised and identified by the name. Thus a compound of the following complicated structure-

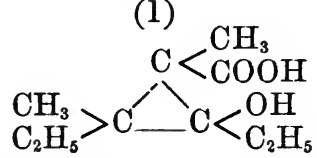

would be dimethyldiethylhydroxytrimethylenecarboxylic acid $(1,3,2$, $3,2,1)$.

Part I of this paper, I have devoted entirely to the derivatives of trimethylene. In Part II I hope to have the honour of bringing an account of some tetramethylene-derivatives before the Society.

Before entering into the details of this research, I must express my sincerest thanks to Professor Adolf Baeyer, in whose laboratory the experiments were carried out, for the great interest which he always took in the work, and for his valuable help in overcoming all difficulties, and also to Herr Carl Bernhart for his assistance in the experimental part.

\section{Action of Ethylene Bromide on Ethyl Malonate.}

\section{Ethylic Trimethylenedicarboxylate $(1,1)$.}

If the sodium compound of ethyl malonate is digested with ethylene bromide for some hours, sodic bromide is precipitated and the ethyl salt of trimethylenedicarboxylic acid $(1,1)$ is formed according to the following equation :-

$$
\begin{gathered}
\left.2 \mathrm{CHNa}<\mathrm{COOC}_{2} \mathrm{H}_{5}+\underset{\mathrm{CH}_{2} \mathrm{Br}}{\mathrm{COOC}_{2} \mathrm{H}_{5}}=\underset{\text { Ethyl trimethylenedicarboxylate. }}{\mathrm{CH}_{2}}\right\rangle_{\mathrm{COOC}_{2} \mathrm{H}_{5}}^{\mathrm{CH}_{2} \mathrm{Br}}+ \\
\mathrm{CH}_{2}<\mathrm{COOC}_{2} \mathrm{H}_{5} \\
\mathrm{COOH}_{2} \mathrm{H}_{5}
\end{gathered}
$$

In carrying out this synthesis, the following method, which I give 
in full, has, after a great many experiments, been found to give the best results.

10 grams of sodium are dissolved in 100 to 120 grams of absolute alcohol, and, after cooling well, a mixture of 70 grams of ethyl malonate and 42 grams of ethylene bromide are slowly poured in, care being taken that the mixture does not get hot. The milky liquid is then transferred to a soda-water bottle and heated in a water-bath for six hours at $100^{\circ}$, by which means the sodium compound is completely decomposed. The end of the reaction can easily be determined by: taking out a small portion, diluting it with water, and testing with. litmus-paper, when the liquid should have a neutral reaction.

In preparing this compound, it has been found convenient to work with five such portions, that is, with 350 grams of ethyl malonate. As soon as the reaction is completed the contents of the five bottles are mixed with twice the bulk of water and extracted three or four times with ether. The ethereal solution is then well washed with water, dried over calcic chloride, and the ether distilled off, when 385 grams of an almost colourless oil remain behind; on distilling this, it gives the following fractions :-

$$
\begin{aligned}
120-180^{\circ} & =16 \text { grams. } \\
130-2 \cdot 25^{\circ} & =330 \quad " \\
\text { Residue in retort } & =13 \quad,
\end{aligned}
$$

The fraction $180-225^{\circ}$, which contains all the ethyl trimethylenedicarboxylate, as well as the unchanged ethyl malonate, is next mixed with 70 grams of ethylene brumide, a solution of 17 grams of sodium in 180 to 200 grams of absolute alcohol is poured in, and the whole is again heated in soda-water bottles for six hours, by which means a great part of the ethyl malonate is transformed into ethyl trimethylenecarboxylate. Water is then added, and the product extracted with ether and treated exactly as described before. On distilling off the ether, 325 grams of oil remain behind, which on fractioning give the following results :-

$$
\begin{aligned}
& 120--130^{\circ}=24 \text { grams } \\
& 180-225=275 " \\
& \text { Residue }=12 ",
\end{aligned}
$$

The fraction $180-225^{\circ}$ still contains a considerable quantity of unchanged ethyl malonate which is removed by treating it with sodic ethylate and benzyl chloride. By this means all the ethyl malonate present is transformed into ethyl benzylmalonate boiling at $300^{\circ}$, which can easily be separated by fractional distillation. This fraction, $180-225^{\circ}$, is therefore mixed with 120 grams of benzyl chloride, and the whole heated with 20 grams of sodium dissolved in 
220 grams of absolute alcohol for two hours, at the end of which time the reaction is finished. After adding water and extracting with ether, 355 grams of oil are obtained which, on distillation, give the following fractions :-

$$
\begin{aligned}
& 120-170^{\circ}=4 \text { grams. } \\
& 170-260=202 \quad " \\
& \text { Residue }=132 \quad "
\end{aligned}
$$

The fraction $170-260^{\circ}$ contains all the ethyl trimethylenedicarboxylate, together with a certain quantity of benzyl ether,

$$
\mathrm{C}_{6} \mathrm{H}_{5} \cdot \mathrm{CH}_{2} \cdot \mathrm{O} \cdot \mathrm{C}_{2} \mathrm{H}_{5} \text {, }
$$

produced by the action of benzyl chloride on any excess of sodic ethylate. This can, however, be almost entirely removed by oftrepeated careful fractioning either with a column or with a Würtz flask with a long reck $(30-40 \mathrm{~cm}$.). In this way, 100 to 120 grams of nearly pure ethyl trimethylenedicarboxylate are obtained boiling at $208-210^{\circ}$ (at $720 \mathrm{~mm}$.).

Should this ether when tested be found to contain chlorine (due to traces of undecomposed benzyl chloride), it is mixed with its own volume of glacial acetic acid and warmed on a water-bath with a little zinc-dust. After diluting with water, the product is extracted several times with ether, the ethereal solution well washed with water and dilute sodic carbonate solution, and dried over potassic carbonate. After removing the ether, the residual oil is again purified by fractioning.

This substance gave the following numbers on analysis :-

I. $0 \cdot 2064$ gram substance gave $0 \cdot 1453$ gram $\mathrm{H}_{2} \mathrm{O}$ and $0 \cdot 4364$ gram $\mathrm{CO}_{2}$.

II. $0 \cdot 2018$ gram substance gave $0 \cdot 1420$ gram $\mathrm{H}_{2} \mathrm{O}$ and $0 \cdot 4284$ gram $\mathrm{CO}_{2}$.

III. 0.1977 gram substance gave 0.1320 gram $\mathrm{H}_{2} \mathrm{O}$ and 0.4201 gram $\mathrm{CO}_{2}$.

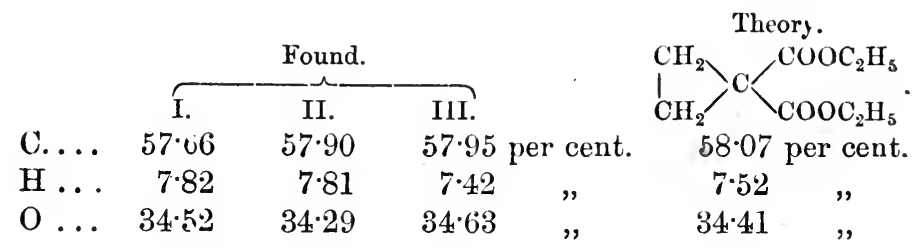

The vapour-density determined in aniline vapour by Hofmann's method gave-

$$
\mathrm{D}=6.32^{\circ} \text {. Theory, } \mathrm{C}_{9} \mathrm{H}_{14} \mathrm{O}_{4}, \mathrm{D}=6.43^{\circ} .
$$


The substance is therefore ethyl trimethylenedicarboxylate. It is a colourless, pleasant-smelling oil which boils at $210^{\circ}\left(720 \mathrm{~mm}\right.$., $213^{\circ}$ at $760 \mathrm{~mm}$.$) . Its density is :-$

$$
d \frac{15^{\circ}}{15^{\circ}}=1.06463 . \quad d \frac{25^{\circ}}{25^{\circ}}=1.05664 .
$$

The boiling point, 206-208 ${ }^{\circ}$, which I first gave in a short notice in the Berichte $(17,55)$, is slightly too low.

\section{Trimethylenedicarboxylic Acid $(1,1)$.}

In order to obtain this acid, pure ethyl trimethylenedicarboxylate was dissolved in a little alcohol and a slight excess of strong alcoholic potash slowly poured in. As soon as the violence of the reaction had subsided, the whole was heated in a flask connected with a reflux condenser for about four hours on a water-bath, by which means the ether was easily saponified. Water was then added, the whole heated on a water-bath until the alcohol had been driven off, and the residue filtered. The filtrate was then acidified with dilute sulphuric acid and repeatedly extracted with pure ether, until a drop of the ethereal solution on evaporation on a watch-glass left only a very slight residue. The ethereal solution was then dried over calcic chloride and the bulk of the ether distilled off. The concentrated ethereal solution, on evaporation, deposited an almost colourless semisolid acid, which solidified almost entirely after standing for some hours over sulphuric acid in a vacuum. In order to remove a small quantity of mother-liquor, the crystals were spread out on a porous plate, by which means they were obtained almost colourless. They were further purified by recrystallisation from chloroform or ether, and then analysed with the following result:-

I. 0.1983 gram substance gave 0.0870 gram $\mathrm{H}_{2} \mathrm{O}$ and 0.3335 gram $\mathrm{CO}_{2}$.

II. 0.1920 gram substance gave 0.0813 gram $\mathrm{H}_{2} \mathrm{O}$ and 0.3212 gram $\mathrm{CO}_{2}$.

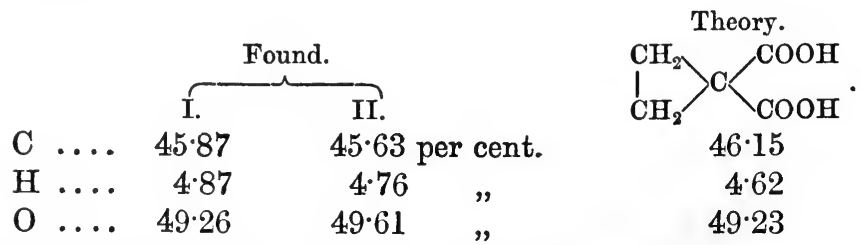

This substance is therefore trimethylenedicarboxylic acid $(1,1)$. It melts at $140-141^{\circ}$, and at $160^{\circ}$ begins to decompose slowly, much more rapidly at $200^{\circ}$, carbonic anhydride being driven off. It is easily soluble in water, much more so than in ether, and can be 
recrystallised from this solvent by allowing the concentrated solution to stand over sulphuric acid in a vacuum.

It is thus obtained in beautiful colourless prisms, which, however, contain water of crystallisation. Trimethylenedicarboxylic acid is only extremely slowly oxidised by potassic permanganate in alkaline solution, and it is but little affected by warming with chromic acid and dilute sulphuric acid. The acid is best purified by allowing the concentrated ethereal solution to evaporate slowly, when beautiful colourless prisms are formed. Professor Haushofer was kind enough to measure them for me, with the following results :-

$$
\begin{gathered}
\text { Crystalline System; Triclinic. } \\
\begin{array}{c}
\alpha=88^{\circ} 20^{\prime} \\
\beta=92 \quad 40 \\
\gamma=9238 \\
a: \iota: c=0.7712: 1: 0.8702 .
\end{array}
\end{gathered}
$$

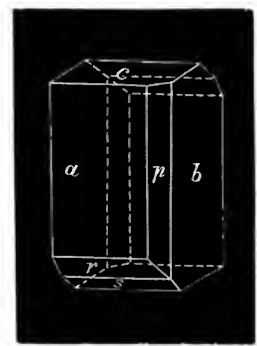

Apparently rectangular prismatic crystals, with the combinations, $\infty \overline{\mathrm{P}} \infty(a), \infty \breve{\mathrm{P}}_{\infty}(b)$, o $\mathrm{P}(c), \infty \mathrm{P}_{1}^{\prime}(p), \overline{\mathrm{P}}_{1} \infty(r), \frac{1}{2} \overline{\mathrm{P}}_{1} \infty(s)$. Usually, $r$ is in the direction of the vertical axis. The faces, with the exception of $b$ and $p$, not well developed. The face $s$ was only observed in one crystal. The cleavage is perfect in the direction $r$, the cleavage surface is fibrous as in gypsum in the direction P.

$\begin{array}{ll}c: a={ }^{*} 92^{\circ} 36^{\prime} & \text { Calculated. } \\ a: b={ }^{*} 9234 & - \\ c: b=* 8848 & - \\ b: p=* 12914 & - \\ r: a=* 13733 & - \\ s: a=11800 & 118^{\circ} 47^{\prime} \\ r: p=12647 & 126 \quad 59\end{array}$

The solution of this acid in chloroform is not affected by bromine at ordinary temperatures, and only very slowly on warming, hydro- 
bromic acid being evolved. Neither is it affected by treating it with sodium amalgam, even after remaining in contact with it for some days.

In order to characterise this acid better, the following salts were next examined.

Ammonium Salt.-This salt is easily prepared by neutralising the aqueous solution of the free acid with ammonia. On allowing the solution to evaporate over sulphuric acid in a vacuam, the salt crystallises out in beautiful four-sided plates /, which give a fine play of colours under the influence of polarised light.

Silver Salt.-If nitrate of silver is added to a solution of the am. monium salt, the silver salt is precipitated as a white voluminous mass, almost insoluble in cold water. It dissolves, although with difficulty, in hot water, and separates out on cooling as a white powder, which on examination with a microscope is seen to consist of a mass of small needles.

For analysis, the salt was dried over sulphuric acid in a vacuum.

I. 0.2440 gram substance gave 0.0273 gram $\mathrm{H}_{2} \mathrm{O}, 0.1516$ gram $\mathrm{CO}_{2}$, and 0.1539 gram Ag.

II. 0.3054 gram substance gave 0.0314 gram $\mathrm{H}_{2} \mathrm{O}, 0.1892$ gram $\mathrm{CO}_{2}$, and $0 \cdot 1920$ gram $\mathrm{Ag}$.

\begin{tabular}{|c|c|c|c|c|c|}
\hline \multicolumn{4}{|c|}{ Found. } & \multirow{3}{*}{\multicolumn{2}{|c|}{$\begin{array}{l}\text { Theory. } \\
\mathrm{CH}_{2} \mathrm{CH}_{2}<\mathrm{COOAg} . \\
17 \cdot 44 \text { per cent. }\end{array}$}} \\
\hline & I. & II. & & & \\
\hline C .... & 16.95 & 16.89 & cent. & & \\
\hline H.... & $1 \cdot 24$ & $1 \cdot 14$ & , & $1 \cdot 16$ & ", \\
\hline Ag.... & $63 \cdot 07$ & $62 \cdot 87$ & $"$ & $62 \cdot 79$ & $"$ \\
\hline $\mathrm{O}$. & $18 \cdot 74$ & $19 \cdot 10$ & ," & $18 \cdot 61$ & \\
\hline
\end{tabular}

Copper Salt.-If a solution of cupric sulphate is added to the ammonium salt, a beautiful deep blue solution is obtained, but no precipitate is formed. On boiling, however, or on partial evaporation, the copper salt crystallises out in a mass of magnificent deep blue crystals, which when seen under the microscope have the following forms.

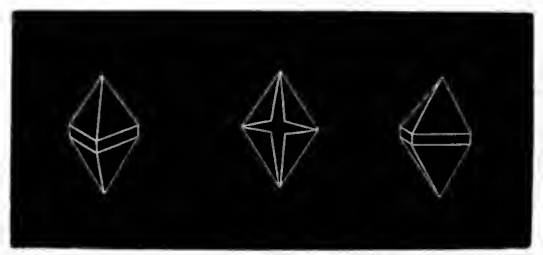


The salt was collected, well washed with hot water, in which it is sparingly soluble, and dried over sulphuric acid in a vacunm. It then contains $1 \mathrm{~mol} . \mathrm{H}_{2} \mathrm{O}$, which, however, cannot be directly determined, as the whole is nct driven off below $170-180^{\circ}$, and at that temperature the salt nndergoes slight decomposition.

I. 0.354 .3 gram substance gave $0 \cdot 1318$ gram $\mathrm{CuO}$.

II. $0 \cdot 3432$

$0 \cdot 1330$,

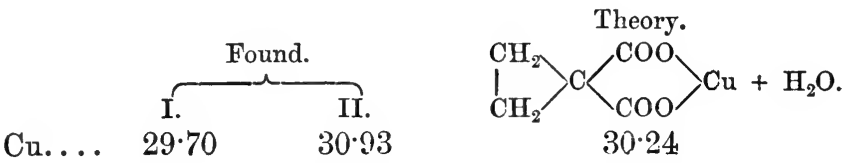

When heated to $160^{\circ}$, this beautiful deep blue salt is transformed into a light green powder.

Barium Salt.-This salt was prepared by adding a solution of barium chloride to the ammonium salt, when it is obtained as a heavy white precipitate, which under the microscope is seen to consist of a mass of fine crystals. This salt is free from water of crystallisation. Analysis gave the following numbers:-

0.2695 gram substance gave 0.2350 gram $\mathrm{BaSO}_{4}$.

Found.

Ba...... 51.27 per cent.
Theory. $\mathrm{C}_{5} \mathrm{H}_{4} \mathrm{BaO}_{4}$.

$51 \cdot 69$

Lead Salt.-This salt is precipitated on adding lead acetate to a solution of the ammonium salt as a heary white amorphous mass, practically insoluble in cold water. In hot water it is slightly soluble, and separates out on cooling in an amorphous condition. It appears to contain no water of crystallisation. For analysis, the salt was dried at $100^{\circ}$.

I. 0.3028 gram substance gave 0.2716 gram $\mathrm{PbSO}_{4}$.

II. $0.2381 \quad, \quad, \quad 0.2154 \quad$,

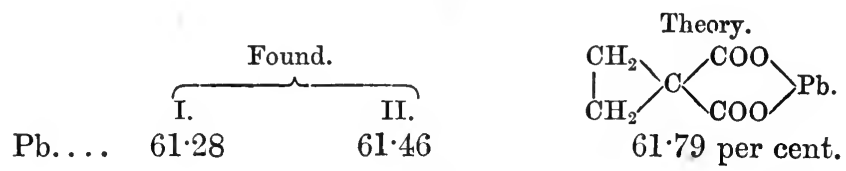

Besides the above-mentioned salts, two others have been examined by Roeder (Annalen, 227, 16), namely :-

The acid barium salt, $\left(\mathrm{C}_{5} \mathrm{H}_{5} \mathrm{O}_{4}\right)_{2} \mathrm{Ba}+4 \mathrm{H}_{2} \mathrm{O}$, which crystallises from water in prismatic crystals.

The acid silver salt, $\mathrm{C}_{5} \mathrm{H}_{5} \mathrm{O}_{4} \mathrm{Ag}$, which crystallises from water in long colourless needles. 
Action of Hydrobromic Acid on Trimethylenedicarboxylic Acid.

\section{$\gamma$-Bromethylmalonic Acid.}

It has already been mentioned that trimethylenedicarboxylic acid is not only unaffected by nascent hydrogen, but that its solution in chloroform can be left for a long time in contact with bromine, without any appreciable change taking place. As soon, however, as it is treated with hydrobromic acid (sp. gr. 1.83), it undergoes a remarkable change, taking up the elements of $\mathrm{HBr}$, and being instantly converted into bromethylmalonic acid according to the equation-

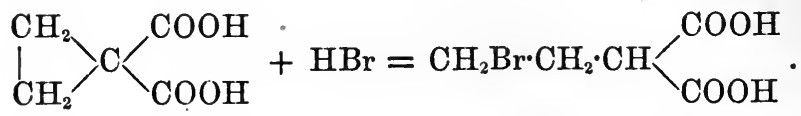

This decomposition was first noticed by Fittig and Roeder (Ber., 16, 2592), but as it was of considerable importance I repeated the experiment, and found their observations to be correct. If trimethylenedicarboxylic acid is mixed in a test tube with a concentrated solution of hydrobromic acid (sp. gr. 1.83) it dissolves, forming a clear solution, which, however, on shaking, or if the sides of the vessel be scratched with a glass rod, begins to deposit crystals, the crystallisation going on till the whole becomes solid. The crystals are next roughly separated from the mother-liquor by spreading the mass out on a porous plate and then drying it over sulphuric acid in a vacuum. It is easily obtained pure by one crystallisation from chloroform. Analysis :-

0.3313 gram substance gave 0.2941 gram AgBr.

Found. $\quad \mathrm{C}_{5} \mathrm{H}_{7} \mathrm{BrO}_{4}$.

Br.... 37.78 per cent. $\quad 37 \cdot 91$ per cent.
(Fittig and Roeder). $37 \cdot 79$ per cent.

This acid melts at $116-117^{\circ}$. Its constitution is further proved by its transformation into carbobutyrolactonic acid by boiling with water. (Fittig-Roeder, loc. cit.)

$\mathrm{CH}_{2} \mathrm{Br} \cdot \mathrm{CH}_{2} \cdot \mathrm{CH} \cdot \mathrm{COOH}$<smiles>C#CCCC(=O)O</smiles>

$\mathrm{CH}_{2} \cdot \mathrm{CH}_{2} \cdot \mathrm{CH} \cdot \mathrm{COOH}$<smiles></smiles>

Carbobutyrolactonic acid. 


\section{Trimethylenemonocarboxylic Acid, $\mathrm{CH}_{2}-\mathrm{CH} \cdot \mathrm{COOH}$.}

If trimethylenedicarboxylic acid is heated in an oil-bath at $210^{\circ}$, large quantities of carbonic anhydride are given off, and a colourless acid-smelling oil distils over, leaving a considerable residue in the retort; this, however, can be driven over by raising the temperature of the bath at the last to $250^{\circ}$. The distillate is next roughly purified by dissolving it in sodic carbonate solution, heating for some time in a water-bath with animal charcoal, and filtering; the filtrate is then acidified with dilute sulphuric acid, when an oily acid is precipitated, which is extracted once or twice with pure ether. The ethereal solution, after drying over calcic chloride and distilling off the ether, deposits a colourless oil, which distils almost completely between $180^{\circ}$ and $195^{\circ}$.

This was further purified by repeated fractional distillation, and at last obtained pure as a colourless oil boiling constantly between $182^{\circ}$ and $184^{\circ}$.* This fraction gave the following numbers on analysis :-

I. $0 \cdot 1653$ gram substance gave $0 \cdot 1076$ gram $\mathrm{H}_{2} \mathrm{O}$ and $0 \cdot 3373$ gram $\mathrm{CO}_{2}$.

II. $0 \cdot 1843$ gram substance gave $0 \cdot 1221$ gram $\mathrm{H}_{2} \mathrm{O}$ and 0.3750 gram $\mathrm{CO}_{2}$.

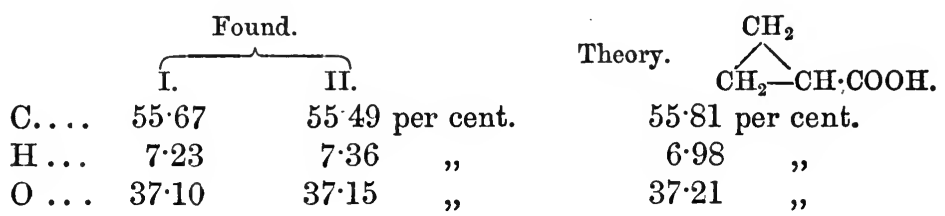

The substance is therefore trimethylenemonocarboxylic acid, and is formed from the dicarboxylic acid according to the equation-<smiles>CCC(C(=O)O)C(=O)O</smiles>

When cooled in a freezing mixture it solidifies to a white crystalline mass, which, however, melts again at the ordinary temperature. $\dagger$ This acid has the same formula as the three crotonic acids, but in all its properties, both physical and chemical, it is entirely different from them.

* Not $188-190^{\circ}$, which was first given in the Berichte $(17,57)$.

+ Roeder (Annalen, 227,25$)$ gives the melting point of this acid as $18-19^{\circ}$, and the boiling point $180-181^{\circ}$. 
$\alpha$-Crotonic acid, $\mathrm{CH}_{3} \cdot \mathrm{CH}: \mathrm{CH} \cdot \mathrm{COOH}$, boils at $180-181^{\circ}$, and melts at $72^{\circ}$.

$\beta$-Crotonic acid, $\mathrm{CH}_{2}: \mathrm{CH} \cdot \mathrm{CH}_{2} \cdot \mathrm{COOH}$, boils at $171 \cdot 9^{\circ}$, and does not solidify at $-15^{\circ}$.

Methacrylic acid, $\mathrm{CH}_{2}: \mathrm{C}\left(\mathrm{CH}_{3}\right)^{\circ} \mathrm{COOH}$, boils at $160 \cdot 5^{\circ}$, and melts at $16^{\circ}$.

These acids differ most from trimethylenecarboxylic acid in their behaviour towards bromine. The three crotonic acids, being unsaturated acids combine directly with $1 \mathrm{~mol}$. of bromine. Trimethylenedicarboxylic acid, on the contrary, is not affected by bromine at ordinary temperatures, and only very slowly on warming, hydrobromic acid being evolved even when a large excess of the acid is present.

Trimethylenemonocarboxylic acid is partly soluble in water, forming a strongly acid solution. It has a powerful odour, recalling somewhat that of butyric acid, and a burning taste. It is heavier than water.

The ammonium salt was obtained by dissolving the acid in ammonia, and then allowing the solution to stand over sulphuric acid in a vacuum until all excess of ammonia had evaporated. On adding nitrate of silver to this solution, the silver salt is precipitated as a white voluminous mass sparingly soluble in cold water. After well washing with water and drying over sulphuric acid in a vacuum, it gave the following analytical results :-

0.2030 gram substance gave 0.04 .97 gram $\mathrm{H}_{2} \mathrm{O}$ and 0.1838 gram $\mathrm{CO}_{2}$.

Found.

C.... 24.69 per cent.

H ... 2.72 ,

$\mathrm{Ag}$.. 55.83 ,

O.... $16 \cdot 76 \quad$,

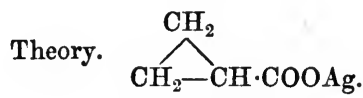

24.87 per cent.

$2 \cdot 59$

$55 \cdot 96$

$16 \cdot 58$

The silver salt dissolves sparingly in hot water, and crystallises out in small needles, on cooling.

On distilling the dry salt, a low-boiling oil is given off which appears to be the anhydride of the acid.

The calcium salt, $\left(\mathrm{C}_{4} \mathrm{H}_{5} \mathrm{O}_{2}\right)_{2} \mathrm{Ca}+6 \mathrm{H}_{2} \mathrm{O}$, and the barium salt, $\left(\mathrm{C}_{4} \mathrm{H}_{5} \mathrm{O}_{2}\right)_{2} \mathrm{Ba}+2 \mathrm{H}_{2} \mathrm{O}$, have been examined and analysed by Roeder (loc. cit.). They are both very easily soluble in water, and crystallise in colourless needles on evaporating their solutions. 


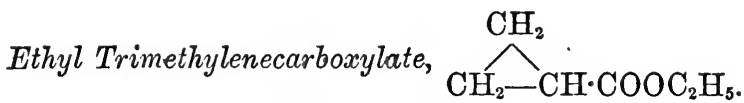

In order to obtain this, the silver salt of the pure acid was digested with a slight excess of ethyl iodide and a little pure ether for two hours on a water-bath; in this way, the salt was easily decomposed, iodide of silver being precipitated; this was separated by filtration, and washed once or twice with small quantities of pure ether. The filtrate was then carefully distilled, particnlarly at first, so that none of the ethereal salt, which is very volatile, should be mechanically carried over with the ether. As soon as all the ether had distilled over, the thermometer rose rapidly to $125^{\circ}$, between which temperature and $14.0^{\circ}$ almost all went over. This portion was then repeatedly refractioned until an oil was obtained which distilled constantly between $133^{\circ}$ and $134^{\circ}$, and gave the following numbers on analysis :-

0.1232 gram substance gave 0.0988 gram $\mathrm{H}_{2} \mathrm{O}$ and 0.2853 gram $\mathrm{CO}_{2}$.

Found.

C..... $63 \cdot 16$ per cent.

H $\ldots . .8 .91$,

O..... 27.93 ,
Theory.

$63 \cdot 16$ per cent.

$8 \cdot 77$,

$28 \cdot 07 \quad$,

The substance was therefore ethyl trimethylenecarboxylate. It is a volatile oil of pleasant ethereal odour. It is not affected by treatment with bromine at ordinary temperatures, and curiously enough only very slowly so when boiled with it, hydrobromic acid being given off.

\section{Theoretical Considerations.}

In the foregoing paper it has been taken for granted that the substances produced by the action of ethylene bromide on ethyl malonate are trimethylene-derivatives. This does not, however, necessarily follow, as there are three kinds of compounds which could be formed by this reaction, namely-

I.<smiles>CCOC(=O)C1(C(=O)OCC)CC1</smiles>

Ethyl trimethylenedicarboxylate $(1,1)$.
II.<smiles>CC=C(C(=O)OCC)C(=O)OCC</smiles>

Ethyl ethylidenemalonate.
III.<smiles>C=CC(C(=O)OCC)C(=O)OCC</smiles>

Ethyl vinylmalonate. 
It would be very strange if a substance having the constitution expressed in formula II were formed, as this would involve an intermolecular change from $=\mathrm{CH} \cdot \mathrm{CH}: \mathrm{CH}_{2}$ to $=\mathrm{C}: \mathrm{CH} \cdot \mathrm{CH}_{3}$. The ethyl ethylidenemalonate has moreover been fully examined by Komnenos (Annalen, 218, 145), and in comparing this with ethyl trimethylenedicarboxylate it is easy to see that the two substances are entirely different. On heating with alcoholic potash, ethyl ethylidenemalonate is entirely decomposed into its constituents, aldehyde and malonic acid, whereas ethyl trimethylenedicarboxylate on hydrolysis gives the corresponding acid, as has been shown in this paper. That ethyl trimethylenedicarboxylate is not merely ethyl vinylmalonate (formula III) is much more difficult to prove, as it is not possible, by treating ethyl malonate with vinyl bromide, to obtain ethyl vinylmalonate synthetically, and thus enable a direct comparison to be made. As ethyl vinylmalonate is unsaturated, it would be expected to give a bromide when treated with bromine at ordinary temperatures, but this is not the case. Further, it should give on distillation an acid of the formula $\mathrm{CH}_{2}: \mathrm{CH} \cdot \mathrm{CH}_{2} \cdot \mathrm{COOH}$, which ought to be identical with one of the three known crotonic acids.

Trimethylenemonocarbonic acid, however, possesses properties entirely different from any of these acids. This difference is shown most decidedly in their behaviour towards bromine. The crotonic acids being unsaturated acids take up with the greatest ease $1 \mathrm{~mol}$. of bromine, but trimethylenecarboxylic acid may be left for hours in contact with bromine without any appreciable change. All these considerations tend therefore to show that trimethylenedicarboxylic acid cannot have the constitution of vinylmalonic acid.

The readiness with which trimethylenedicarboxylic acid combines with hydrobromic acid, forming bromethylmalonic acid, appears at first sight strange, but when it is considered that trimethylene itself undergoes a decomposition exactly similar, the difficulty disappears. Freund (Monatsh. Chem., 1882, 625) states that when trimethylene is made to pass through highly concentrated hydriodic acid, the ring is split, and normal propyl iodide is formed according to the equation-

$$
\overbrace{\mathrm{CH}_{2}-\mathrm{CH}_{2}}^{\mathrm{CH}_{2}}+\mathrm{HI}=\mathrm{CH}_{3} \cdot \mathrm{CH}_{2} \cdot \mathrm{CH}_{2} \mathrm{I} \text {. }
$$

Fittig and Roeder (loc. cit.) noticed, however, other decompositions of this acid which are difficult to explain on the assumption of the existence of a trimethylene-ring in it. Trimethylenedicarboxylic acid is decomposed on warming with dilute sulphuric acid into the isomeric carbobutyılactonic acid, $\mathrm{O}_{\mathrm{O}}^{\mathrm{CH} \cdot \mathrm{CH}_{2} \cdot \mathrm{CH} \cdot \mathrm{COOH}}$ and what is 
still more strange is the fact that this same trimethylenedicarboxylic acid on simple distillation (as Fittig has shown) gives as much as 30 per cent. of butyrolactone,

$\mathrm{CH}_{2} \cdot \mathrm{CH}_{2} \cdot \mathrm{CH}_{2}$

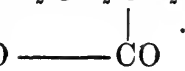

These reactions are easy to explain, on the assumption that the acid is a vinylmalonic acid, such unsaturated acids having a strong tendency to undergo molecular change, and form the isomeric lactones. Otherwise it is necessary to suppose that in all these reactions the trimethylene-ring is first split, as in the above-mentioned case, with hydrobromic acid. If, however, the two formulæ are examined-<smiles>C=CC(C(=O)O)C(=O)O</smiles>

Vinylmalonic acid.<smiles>O=C(O)C1(C(=O)O)CCC1</smiles>

Trimethylenedicarboxylic acid.

more closely, it is directly apparent that vinylmalonic acid is nothing more than a mono-substituted malonic acid corresponding with ethylmalonic acid, $\mathrm{CH}_{3} \cdot \mathrm{CH}_{2}>\mathrm{C}<\mathrm{COOH}$, whereas trimethylenedicarboxylic acid is a di-substituted malonic acid corresponding with dimethylmalonic acid, ${\stackrel{\mathrm{CH}}{\mathrm{CH}_{3}}>\mathrm{C}<\mathrm{COOH}}_{\mathrm{COOH}}^{\mathrm{CH}^{2}}$

Now it is well known from the beautiful researches of Conrad and Bischoff, that on treating mono-substituted malonic ethers with sodic ethylate, they form sodium compounds which, when further treated with the iodides of the alcohol radicals, benzyl chloride, \&c., give di-substituted malonic ethers, thus :-

$\underset{\mathrm{Na}}{\mathrm{CH}_{3} \cdot \mathrm{CH}_{2}}>\mathrm{C}<\underset{\mathrm{COOC}_{2} \mathrm{H}_{5}}{\mathrm{COOC}_{2} \mathrm{H}_{5}}+\mathrm{CH}_{3} \cdot \mathrm{CH}_{2} \mathrm{I}=$

Sodium compound of ethylic ethylmalonate.

$$
\underset{\mathrm{CH}_{3} \cdot \mathrm{CH}_{2}}{\mathrm{CH}_{3} \cdot \mathrm{CH}_{2}}>\mathrm{C}<\mathrm{COOOC}_{2} \mathrm{H}_{5}+\mathrm{NaI} \text {. }
$$

Ethylic diethylmalonate.

It is therefore clear that if the trimethylenedicarboxylic acid be a vinylmalonic acid, that its ether when treated with sodic ethylate and benzyl chloride (for instance) would give benzylvinglmalonic acid, $\mathrm{CH}_{6}: \mathrm{H}_{5} \cdot \mathrm{CH}_{2}>\mathrm{C}<\mathrm{COOH}^{\mathrm{COOH}}$. Should the ether, on the contrary, remain unacted on, this would be the best proof that the acid is a disubstituted malonic acid-that is, really has the constitution-

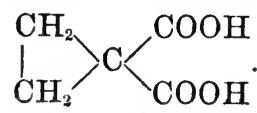


For the purpose of testing this, 77 grams of ethyl trimethylenecarboxylate were mixed with 50 grams of benzyl chloride, and a solution of 9 grams of sodium in 100 grams of absolute alcohol slowly poured in, the whole being well cooled during the operation. The mixture was then heated on a water-bath in a flask connected with a reflux condenser. The reaction set in directly, and was finished as soon as a sample, when mixed with water, was neutral to test-paper.

The product was then diluted with water and several times extracted with ether. The ethereal solution after drying over calcic chloride and evaporating, deposited 120 grams of a slightly yellowish-coloured oil which on distillation gave the following fractions :-

$$
\begin{aligned}
& \text { Under } 180^{\circ} \ldots \ldots \ldots \ldots=7 \text { grams. } \\
& \text { From } 180^{\circ} \text { to } 220^{\circ} \ldots \ldots .=95, \\
& \text { Residue left in the re- } \\
& \text { tort above } 220^{\circ} \ldots \ldots .=7,
\end{aligned}
$$

The residue above $220^{\circ}$ was then transferred to a small retort, first roughly distilled over and then fractioned. Almost all went over below $220^{\circ}$, leaving a trace of a dark-brown oil behind which was too small for further examination.

The fractions below $220^{\circ}$ were mixed and saponified by boiling with a slight excess of alcoholic potash for about four hours. On adding water a quantity of oil was precipitated which was extracted with ether and fractioned; the whole distilled over between 180 and $190^{\circ}$, and was found to consist of almost pure benzyl ether, $\mathrm{C}_{6} \mathrm{H}_{5} \cdot \mathrm{CH}_{2} \cdot \mathrm{O}^{\cdot} \mathrm{C}_{2} \mathrm{H}_{5}$. This fraction weighed 49 grams, which, allowing for loss during fractioning, \&c., is almost equivalent to the amount ( 54 grams) which should have been produced had the whole of the benzyl chloride used in the above experiment reacted with the sodic ethylate, and its presence in such quantity is the best proof that the ethyl trimethylenedicarboxylate had not been affected.

The solution of the potash salts produced by the hydrolysis were then evaporated on a water-bath to drive off alcohol and ether, acidulated with dilute sulphuric acid, and extracted several times with pure ether. The ethereal solution was carefully dried over calcic chloride, filtered, and the ether distilled off, when a quantity of a beautifully crystalline acid remained; this after recrystallisation from ether melted at $140^{\circ}$ and possessed all the properties of trimethylenedicarboxylic acid. In order to be certain, a part was converted into the silver salt and analysed with the following result:-

0.2107 gram substance gave $0 \cdot 1316$ gram silver.

Found.

$\mathrm{Ag} \ldots \quad 62 \cdot 4.5$ per cent.
Theory. $\mathrm{C}_{5} \mathrm{H}_{4} \mathrm{O}_{4} \mathrm{Ag}_{2}$.

$62 \cdot 79$ per cent.

The acid, therefore, had been quite unacted on by the benzyl chloride. 
In order to confirm this, a second experiment was made with perfectly pure ethyl trimethylenedicarboxylate which had been prepared by treating the silver salt of the pure acid with ethyl iodide. This time, 15 grams of the pure ether were treated with a large excess of sodic ethylate and benzyl chloride (namely, 4 grams $\mathrm{Na}$ and 20 grams benzyl chloride) in the manner described above. On fractioning the product, it all distilled over below $215^{\circ}$, leaving a mere trace of residue which could not be driven over without superheating the vapour and thus causing the thermometer to rise. The distillate was then saponified, but, as in the previous case, nothing but trimethylenedicarboxylic acid was obtained; no trace of a benzyl-derivative had been formed. In order to be still more certain, a large quantity of high-boiling residues, produced in purifying crude ethyl trimethylenedicarboxylate, as described at the beginning of this paper, were also examined, as it seemed likely that if any benzylvinylmalonic acid had been produced that it would probably be present in considerable quantity in these oils.

They were first distilled under diminished pressure $(50 \mathrm{~mm}$.) and then saponified. In this way a large quantity of a beautifully crystalline product was obtained consisting mostly of benzylmalonic acid. Traces of another acid were also present which on analysis proved to be dibenzylmalonic acid. As I have not been able to find a description of this acid, I give a short account of its properties. Dibenzylmalonic acid is sparingly soluble in cold water, more easily so in hot, and crystallises, on cooling slowly, in thick prisms; on rapid cooling, it is precipitated in fine colourless needles.

The analysis gave the following numbers:-

$0 \cdot 1011$ gram substance gave 0.2653 gram $\mathrm{CO}_{2}$ and 0.0545 gram $\mathrm{H}_{2} \mathrm{O}$.

\begin{tabular}{|c|c|c|}
\hline C .... & $\begin{array}{l}\text { Found. } \\
71: 56 \text { per cent. }\end{array}$ & 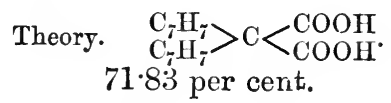 \\
\hline & $5 \cdot 99 \quad$, & $5 \cdot 63 \quad$ \\
\hline & $22 \cdot 45$ & $22 \cdot 54$ \\
\hline
\end{tabular}

Dibenzylmalonic acid melts at $170-172^{\circ}$, and is decomposed at a higher temperature into carbonic anhydride and a solid acid, probably dibenzylacetic acid.

No trace of any other acid besides this and benzylmalonic acid could be found. There is, therefore, no doubt that trimethylenedicarboxylic acid is a di-substituted malonic acid, and has the constitution $\stackrel{\mathrm{CH}_{2}}{\mathrm{CH}_{2}}>\mathrm{C}\left\langle\begin{array}{l}\mathrm{COOH} \\ \mathrm{COOH}\end{array}\right.$

Ethyl trimethylenedicarboxylate is produced by the action of 
ethyl bromide on the mono-sodium compound of ethyl malonate, according to the equation given on p. 817 , and not on a $d i$-sodinm compound, as might be expected, thus :-

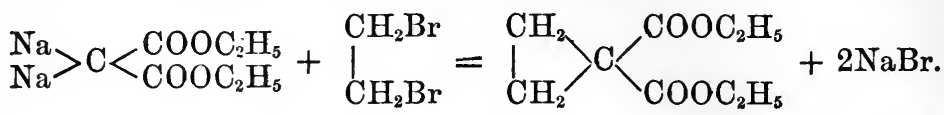

This is proved by the fact that large quantities of ethyl malonate always remain nnchanged in this reaction, which would not be the case if the above equation were correct.

The fact that di-substituted acetoacetic ethers are formed from the mono-sodium compound of the latter has often been noticed. Allen and Kölliker (Annalen, 227, 113) found that when perfectly pure ethyl sodacetoacetate was treated with triphenylmethane bromide, that a di-substituted derivative was formed and half the ethyl acetoacetate regenerated according to the equation-

$$
\begin{aligned}
& 2 \mathrm{CHNa}<\mathrm{COOC}_{2} \mathrm{H}_{5}+2 \mathrm{BrC}\left(\mathrm{C}_{6} \mathrm{H}_{5}\right)_{3}=2 \mathrm{NaBr}+
\end{aligned}
$$

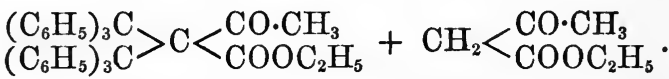

Similar results were obtained by Emil Fischer and Hermann Koch (Ber., 16, 651) on treating ethyl sodacetoacetate with phthalyl chloride, and also by Wislicenus who followed up this reaction, using ethyl malonate in place of ethyl acetoacetate.

\section{Action of Ethis Dibromosuccinate on Ethyl Malonate.}

Ethyl Trimethylenetetracarboxylate $(1,1,2,3)$.

In order to get further knowledge as to the properties of the trimethylene-ring, it was important to prepare some of the other possible carboxylic acids, more especially the tricarboxylic acid $(1,2,3)$, in which the three carboxyl-groups are attached to three different carbon-atoms, an acid which would therefore correspond with the hydromellitic acid of the benzene series-

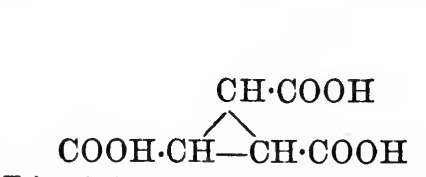

Trimethylenetricarboxylic acid $(1,2,3)$.
$\mathrm{CH} \cdot \mathrm{COOH}$

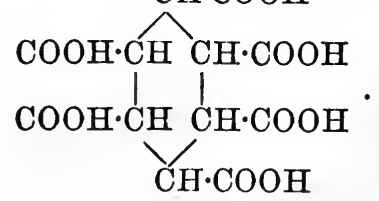

Hydromellitic acid.

The synthesis of this acid may be accomplished in the following way : 
When the sodium compound of malonic ether is heated with dibromosuccinic ether, a violent reaction sets in, sodic bromide is precipitated, and the ethyl salt of trimethylenetetracarboxylic acid $(1,1,2,3)$ is formed according to the equation-

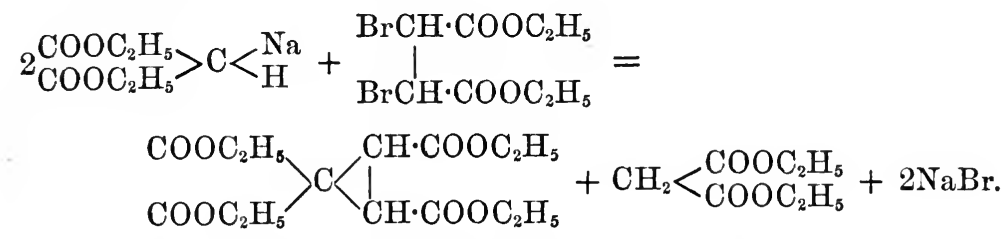

On hydrolysis, the free tetracarboxylic acid is obtained, which on prolonged heating at $200^{\circ}$ gives off carbonic anhydride, forming trimethylenetricarboxylic acid $(1,2,3)$ thus-

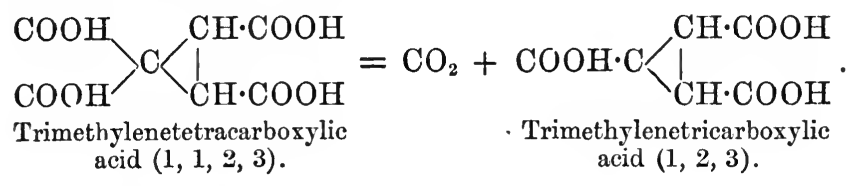

This synthesis is best carried out as follows: 4.5 grams of sodium are dissolved in 50 grams of absolute alcohol, and when cold mixed with 30 grams of ethyl malonate, 35 grams of ethyl dibromosuccinate (prepared by treating ethyl fumarate with bromine) are now slowly added. A violent reaction sets in after each addition of the bromosuccinate, so that it is necessary to add it in small quantities at a time and cool well during the operation. As soon as it is all added, the product, which has a deep yellow colour, is heated in a flask connected with a reflux condenser for about an hour on a waterbath, in order to ensure the reaction being complete. Water is then added, and the oil which is precipitated is several times extracted with ether. The ethereal solution is well washed with water, dried over calcic chloride, and the ether distilled off, when a quantity of an almost colourless oil remains behind, which is next roughly fractioned under diminished pressure (at $85 \mathrm{~mm}$.). A large quantity distills below $150^{\circ}$ (consisting principally of unchanged ethyl malonate), the thermometer then rises rapidly to $235^{\circ}$, between which temperature and $255^{\circ}$ about half the weight of the original oil comes over. This is then several times very carefully fractioned under the same pressure, and at last obtained pure as a thick, colourless oil boiling constantly at $245-247^{\circ}$ ( $85 \mathrm{~mm}$.). 'The analysis gave the following numbers :-

$0 \cdot 2377$ gram substance gave $0 \cdot 1532$ gram $\mathrm{H}_{2} \mathrm{O}$ and $0 \cdot 4710$ gram $\mathrm{CO}_{2}$. 
Found.

C .... 54.04 per cent.

H .... $7 \cdot 16 \quad$,

O .... 38.80 ,
Theory.

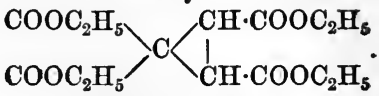

$54 \cdot 54$ per cent.

$6 \cdot 67$

38.79 ,

The substance is therefore ethyl trimethylenetetracarboxylate $(1,1,2,3)$. It has an agreeable odour, and does not solidify at-10 . It is isomeric with ethyl dicarboxyglutaconate-*

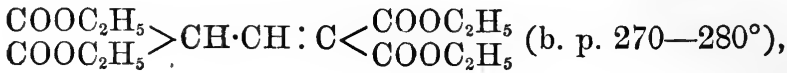

and with ethyl propargylenetetracarboxylate- $\uparrow$

$\underset{\mathrm{COOC}_{2} \mathrm{H}_{5}}{\mathrm{COOC}_{2} \mathrm{H}_{5}}>\mathrm{CH} \cdot \mathrm{CH}<\underset{\mathrm{C} \cdot \mathrm{OOC}_{2} \mathrm{H}_{5}}{\mathrm{COOC}_{2} \mathrm{H}_{5}}$ (b. p. $220-230^{\circ}$ at $40 \mathrm{~mm}$.).

\section{Trimethylenetetracarboxylic Acid $(1,1,2,3)$.}

In order to obtain this acid, the pure ethyl-derivative was boiled for about five hours in a flask connected with a condenser, with a fairly concentrated solution of sodie hydrate, by which the oil was entirely saponified. The solution, which was slightly brownish, was then evaporated almost to dryness, acidified with dilnte sulphuric acid ( 1 to 1 ), and extracted with pure ether. This extraction had to be repeated at least twenty times, as the acid is exceedingly soluble in water, in fact much more so than in ether. For this reason also any excess of water should be avoided. The ethereal solution was then carefully dried over calcic chloride, filtered, and the ether distilled off, great care being taken that the residue was not heated above $50^{\circ}$. By this means a very thick almost colourless oil was obtained, which after standing for some days over sulpharic acid in a vacuum, solidified almost entirely, forming curious circular masses very much resembling the mineral wavellite. In order to remove traces of oily matter, the crystals were first spread out on a porous plate, then repeatedly washed with benzene and light petroleum, and finally dried over sulphuric acid in a vacuum.

The following numbers were obtained on analysis :-

0.2643 gram substance gave 0.0816 gram $\mathrm{H}_{2} \mathrm{O}$ and 0.3805 gram $\mathrm{CO}_{2}$. 


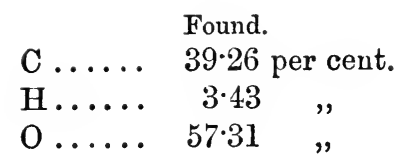

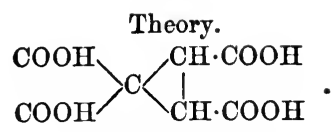

38.53 per cent.

$2 \cdot 75 \quad$ "

58.72,

The acid was therefore trimethylenetetracarboxylic acid $(1,1,2,3)$. This acid, obtained in the way described above, is a hard colourless crystalline mass, which melts at $95-100^{\circ}$, being at the same time decomposed into trimethylenetricarboxylic acid and carbonic anhydride. It is easily soluble in water, ether, alcohol, and acetone, sparingly so in light petroleum, benzene, and tolnene. The aqueous solution has a strongly acid reaction.

The ammonium salt is easily prepared by neutralising the aqueous solution of the acid with ammonia; it is extremely soluble in water. On adding argentic nitrate, the silver salt is obtained as a heavy white precipitate, which after collecting and washing with water, was dried over sulphuric acid in a vacuum and analysed with the following result :-

0.2773 gram substance gave 0.0196 gram $\mathrm{H}_{2} \mathrm{O}$ and 0.1354 gram $\mathrm{CO}_{2}$.

0.2038 gram substance gave 0.1350 gram Ag.

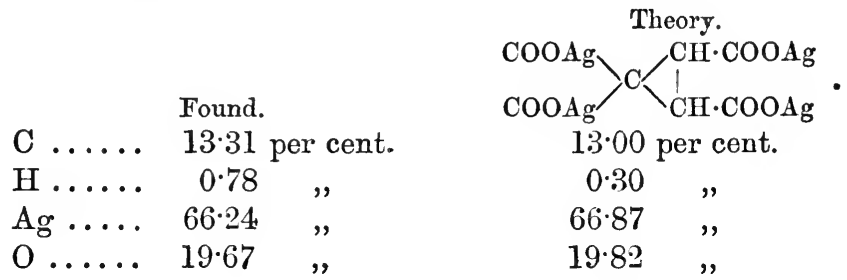

This salt is difficult to analyse. On warming, it decomposes suddenly, giving off a quantity of carbonic anhydride, and leaving a mass of silver threads. It is necessary, therefore, in order to get good numbers, to mix the salt thoroughly with finely divided oxide of copper, and conduct the combustion very siowly. The silver salt is, however, otherwise stable, and only slowly affected by exposure to light.

Barium chloride gives with the solution of the ammonium salt a white gelatinous precipitate of the barium salt, almost insoluble in water. The same salt is produced on adding an excess of barytawater to an aqueous solution of the free acid.

The copper salt appears to be easily soluble in water. The calcium salt of this acid is the most characteristic. If a solution of 
calcic chloride is added to the ammonium salt, no precipitate is produced; on warming, however, the calcium salt crystallises out in micróscopic needles, which are much more soluble in cold than in hot water. The analyses show that this salt has the formula $\mathrm{C}_{7} \mathrm{H}_{2} \mathrm{O}_{8} \mathrm{Ca}_{2}+\mathrm{H}_{2} \mathrm{O}$.

I. 0.2670 gram substance lost, when heated at $130^{\circ}$ for four hours, 0.0158 gram and gave 0.2276 gram $\mathrm{CaSO}_{4}$.

II. 0.2474 gram substance lost, when heated at $120^{\circ}$ for five hours, 0.0146 gram and gave 0.2113 gram $\mathrm{CaSO}_{4}$.

Found.

\begin{tabular}{|c|c|c|c|}
\hline & $\overbrace{25 \cdot 07}$ & $\begin{array}{l}\text { II. } \\
25 \cdot 12 \text { per cent. }\end{array}$ & $\begin{array}{c}\text { Theory. } \mathrm{C}_{7} \mathrm{H}_{2} \mathrm{O}_{8} \mathrm{Ca}_{2}+\mathrm{H}_{2} \mathrm{O} \text {. } \\
25 \cdot 64 \text { per cent. }\end{array}$ \\
\hline & $5 \cdot 91$ & $5 \cdot 91$ & $5 \cdot 71$ \\
\hline
\end{tabular}

\section{Trimethylenetricarboxylic Acid $(1,2,3)$.}

As has already been mentioned, trimethylenetetracarboxylic acid, when heated to $100^{\circ}$, melts and is decomposed, giving off carbonic anhydride, so that it is doubtful whether this is really the melting point or simply the decomposing point of the acid.

In order to obtain the tricarboxylic acid, it was found best to heat the tetracarboxylic acid to a temperature not exceeding $200^{\circ}$, otherwise a dark-coloured mass was obtained, which was difficult to purify. As soon as the evolution of carbonic anhydride had ceased, the whole was allowed to cool; a pale brown thick oily mass was obtained, which could not be made to crystallise. It was therefore dissolved in sodic hydrate, boiled for some time with freshly ignited animal charcoal, and filtered. The filtrate, which was almost colourless, was then acidified with dilute sulphuric acid, repeatedly extracted with ether, and the ethereal solution dried with calcic chloride. On distilling off the ether, an almost colourless oil remained behind, which, after standing for some days over sulphuric acid in a racuum, almost entirely solidified. In order to purify these crystals as much as possible, they were first spread out on a porous plate, and lastly well washed with benzene and light petroleum. On account of the small quantity at my disposal, I was not able to obtain this acid in a more definitely crystalline condition.

The analyses gave the following results :-

I. 0.1694 gram substarice gave 0.0621 gram $\mathrm{H}_{2} \mathrm{O}$ and 0.2583 gram $\mathrm{CO}_{2}$.

II. 0.1459 gram substance gave 0.0525 gram $\mathrm{H}_{2} \mathrm{O}$ and 0.2220 gram $\mathrm{CO}_{2}$. 

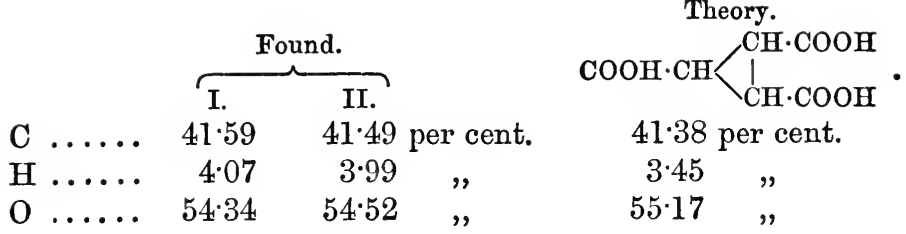

Trimethylenetricarboxylic acid $(1,2,3)$ had, therefore, been formed from the tetracarboxylic acid, by the simple splitting off of $\mathrm{CO}_{2}$, as shown on p. 823 .

This acid melts at $150-153^{\circ}$; it is easily soluble in water, alcohol, and acetone, sparingly so in light petroleum, benzene, carbon bisulphide, and chloroform, and can be obtained in definite crystals by mixing the alcoholic solution with benzene, and allowing the whole to evaporate slowly at ordinary temperatures.

The free acid, when heated, first gives off water and then chars, a small quantity of a colourless oil distilling over, which solidifies on cooling. This sublimate is sparingly soluble in ether, and appears to be an anhydride of the acid.

The ammonium salt was next prepared by neutralising the aqueous solution with ammonia. On adding silver nitrate, a white amorphous precipitate was obtained, which, after well washing and drying, first over sulphuric acid and then at $130^{\circ}$, gave the following numbers on analysis :-

I. 0.2890 gram substance gave 0.0266 gram $\mathrm{H}_{2} \mathrm{O}, 0.1582$ gram $\mathrm{CO}_{2}$, and 0.1880 gram $\mathrm{Ag}$.

II. 0.2418 gram substance gave 0.1575 gram Ag.

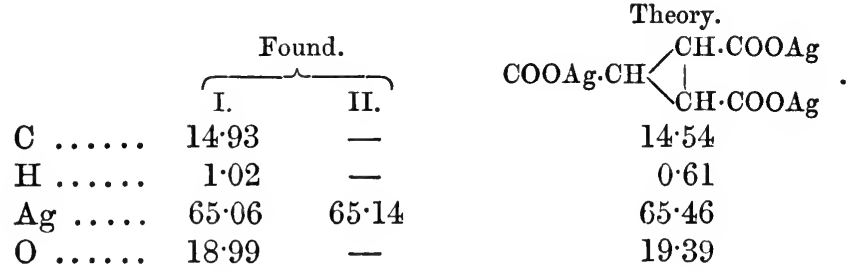

Cupric sulphate gives, with the ammonium salt, a beautiful light green precipitate, which is sparingly soluble in water. The barium and lead salts are white precipitates, almost insoluble in water. On adding calcic chloride to a dilute solution of the ammonium salt, no precipitate is formed. On warming, however, the calcium salt is precipitated in fine crystals ; it is, as in the case of the calcium salt of the tetracarboxylic acid, more soluble in cold water than in hot.

It is remarkable that hydromellitic acid, which is the acid corre- 
sponding with this acid in the benzene series, should also give a calcium salt, which is more easily soluble in cold than in hot water. Trimethylenetricarboxylic acid $(1,2,3)$ is isomeric with the trimethylenetricarboxylic acid $(1,1,2)$, obtained by Conrad and Guthzeit (Ber., 17, 1185) by the action of $\alpha$ - $\beta$-dibromopropionic acid on ethyl malonate, as is easily seen from the following formulæ:-

\section{$\mathrm{CH} \cdot \mathrm{COOH}$ \\ $\mathrm{COOH} \cdot \mathrm{CH}-\mathrm{CH} \cdot \mathrm{COOH}$}

Trimethylenetricarboxylic acid $(1,2,3)$. Melting point, $150--153^{\circ}$.

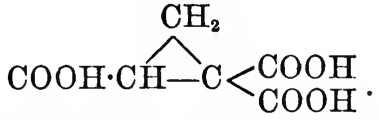

Trimethylenetricarboxylic acid $(1,1,2)$. Melting point, $184^{\circ}$.

The chief difference in these two acids is seen on their behaviour when heated. As already noticed, the acid $(1,2,3)$ chars, giving only a trace of anhydride. The acid $(1,1,2)$, on the contrary, containing two carboxyl-groups attached to one carbon-atom, is easily decomposed into the trimethylenedicarboxylic acid $(1,2)$,

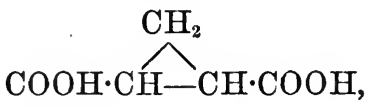

and carbonic anhydride.

The trimethylenetricarboxylic acids are also isomeric with aconitic acid, carboxyglutaconic acid, \&c. The acid $(1,2,3)$ is, perhaps, identical with aceconitic acid obtained by Baeyer (Annalen, 135, $206)$, by the action of sodium on ethyl monobromacetate.

\section{Action of Ethylene Bromide on Ethrl Acetoacetate.}

In the preceding sections, I have described some derivatives of trimethylene which are produced by the action of ethylene bromide and ethyl dibromosuccinate on ethyl malonate. As, however, ethyl acetoacetate and ethyl benzoylacetate generally enter into the reactions in which ethyl malonate takes part, it seemed probable that by the study of the action of ethylene bromide on these compounds interesting trimethylene-derivatives might also be produced.

It was also thought that such derivatives prepared from ethyl benzoylacetate would probably be crystalline, and therefore easy to characterise.

In the following I shall, however, first describe the substances obtained by the action of ethylene bromide on ethyl acetoacetate, leaving the benzoyl-derivatives to a later chapter. 


\section{Ethyl Acetyltrimethylenecarboxylate $(1,1)$,

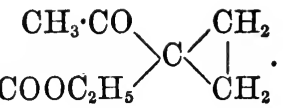

This ether, which is the principal product of the action of ethylene bromide on ethyl sodacetoacetate, is best prepared as follows:-To a solution of 5 grams of sodium in 60 grams of absolute alcohol, 26 grams of ethyl acetoacetate are slowly added, and then the whole heated with 20 grams of ethylene bromide in a soda-water bottle at $100^{\circ}$ for eight hours. In carrying out this operation five such quantities, that is, 130 grams of ethyl acetoacetate, were generally worked with at once. At the end of this time a quantity of sodic bromide is precipitated, and the reaction is generally finished, as is proved by the product having a neutral reaction when diluted with water. After adding water, the oily layer which sinks to the bottom is separated from the aqueous liquid by means of a separating funnel, and dried over calcic chloride. The aqueous solution is then extracted once or twice with ether, well washed with water, dried over calcic chloride, and the ether distilled off. The oil which remains behind is then mixed with the principal product and the whole carefully fractioned with a column. Between $120-150^{\circ}$, a small quantity of unchanged ethylene bromide comes over, then the temperature rises rapidly to $180^{\circ}$, between which and $220^{\circ}$ by far the greater part distils over, weighing about 120 grams. A considerable residue, however, always remains behind, with the examination of which $I$ am at the present time engaged.

The fraction $180-220^{\circ}$ still contained about 40 per cent. of unchanged ethyl acetoacetate; it was therefore further heated with 10 grams of sodium (dissolved in 120 grams of absolute alcohol) and 40 grams of ethyl bromide for 10 hours at $100^{\circ}$. The oil, after separating in the manner described above, was then refractioned, when the principal quantity again distilled over between $180^{\circ}$ and $220^{\circ}$. This was now most carefully refractioned with a column until an oil was obtained which distilled orer almost constantly between $195^{\circ}$ and $200^{\circ}$. As analysis showed that this product still contained traces of ethyl acetoacetate, the whole was well shaken with a fairly concentrated aqueous potash solution for some hours, and then allowed to stand over night. By this means the ethyl acetoacetate dissolves, and is saponified, whereas most of the ethyl acetyltrimethylenecarboxylate remains unchanged. After extracting with ether and repeated fractioning, the product was probably almost pure, and boiled constantly between $197^{\circ}$ and $200^{\circ}$. The analyses gave the following numbers:-

I. 0.1488 gram substance gave $0 \cdot 1070$ gram $\mathrm{H}_{2} \mathrm{O}$ and 0.3337 gram $\mathrm{CO}_{2}$. 
II. 0.2113 gram substance gave 0.1532 gram $\mathrm{H}_{2} \mathrm{O}$ and 0.4735 gram $\mathrm{CO}_{2}$.

III. 0.2512 gram substance gave 0.1833 gram $\mathrm{H}_{2} \mathrm{O}$ and 0.5620 gram $\mathrm{CO}_{2}$.

IV. 0.1773 gram substance gave 0.1276 gram $\mathrm{H}_{2} \mathrm{O}$ and 0.3973 gram $\mathrm{CO}_{2}$.

Found.

$\overbrace{\text { I. II. }}^{\text {III. }}$ IV.

C ... $61 \cdot 16 \quad 61 \cdot 12 \quad 61 \cdot 02 \quad 61 \cdot 12$ p.c.

H... $\quad 7.99 \quad 8.05 \quad 8.10 \quad 7.99$,

$\begin{array}{llllll}0 & \ldots & 30.85 & 30.83 & 30.88 & 30.89\end{array}$

Theory.

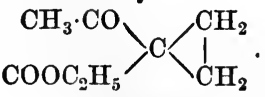

61.54 p. c.

$7 \cdot 69$,

$30 \cdot 76$,

This compound is therefore ethyl acetyltrimethylenecarboxylate $(1,1)$ and is produced by the action of ethylene bromide on the sodium compound of ethylacetoacetate, according to the following equation:-

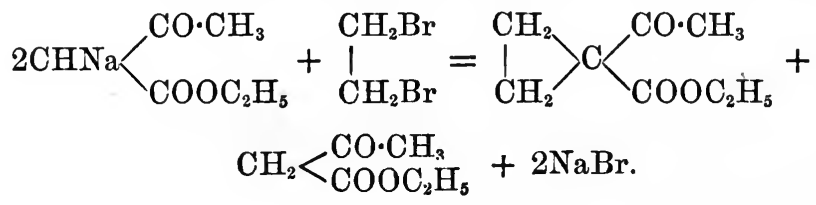

A vapour-density determination which was made by Hofmann's method, by vaporising the substance in a vacuum at $184^{\circ}$ (aniline vapour), gave the following result :-

$$
\begin{array}{cc}
\text { Found. } & \text { Theory. } \mathrm{C}_{8} \mathrm{H}_{12} \mathrm{O}_{3} \text {. } \\
\text { D...... } & 4 \cdot 94
\end{array}
$$

Ethyl acetyltrimethylenecarboxylate is a peculiarly smelling colourless oil, boiling between $197^{\circ}$ and $200^{\circ}$. It does not solidify at $0^{\circ}$. The sp. gr. and magnetic rotation determinations, which were performed by my father, gave the following numbers :-

Density :-

$$
\begin{array}{ll}
d \frac{4^{\circ}}{4^{\circ}}=1.03436 . & d \frac{6.5^{\circ}}{6.5^{\circ}}=1.03256 . \\
d \frac{15^{\circ}}{15^{\circ}}=1.02549 . & d \frac{25^{\circ}}{25^{\circ}}=1.01834 .
\end{array}
$$




\begin{tabular}{c|c|c}
\multicolumn{1}{c|}{$t}$. & Sp. rotation. & Mol. rotation. \\
\hline $12 \cdot 5$ & $1 \cdot 1696$ & $9 \cdot 865$ \\
$12 \cdot 5$ & $1 \cdot 1596$ & $9 \cdot 780$ \\
$12 \cdot 5$ & $1 \cdot 1643$ & $9 \cdot 820$ \\
$12 \cdot 5$ & $1 \cdot 1623$ & $9 \cdot 802$ \\
\hline $12 \cdot 16$ & $9 \cdot 817$
\end{tabular}

Dr. Gladstone was so kind as to determine the refractive equivalent, and gave me the following account of his results.

\begin{tabular}{c|c|c|c|c|c|c}
\hline Temp. & $\mu_{\text {A. }}$ & $\mu_{\mathrm{D} .}$ & $\mu_{\mathrm{H} .}$ & $\begin{array}{c}\text { Specific } \\
\text { refraction A. }\end{array}$ & $\begin{array}{c}\text { Specific } \\
\text { dispersion. }\end{array}$ & $\begin{array}{c}\text { Refractive } \\
\text { equivalent A. }\end{array}$ \\
\hline $22^{\circ}$ & $1 \cdot 4600$ & 1.4667 & 1.4910 & 0.4517, & 0.0305 & $70 \cdot 46$ \\
\hline
\end{tabular}

"If the ethyl acetyltrimethylenecarboxylate were a member of the series below the tetramethylene compound* I examined before, it ought to give a refractive equivalent of 68 : the equivalent found, 70.46 , indicates an additional pair of double linked carbon-atoms; this seems perplexing, especially as the dispersion does not indicate any increase in that direction."

The yield of ether obtained by the above method is not good, a quantity of high-boiling oil being produced at the same time; this appears to be the product of the action of $1 \mathrm{~mol}$. of ethylene bromide on 2 mols. of ethyl acetoacetate.

When treated with phosphorous pentachloride, ethyl acetyltrimethylenecarboxylate is violently attacked, chlorinated bodies being formed which are at present under examination.

On warming with alcoholic potash, the ether is easily saponified. It appears to combine with lyydroxylamine and phenylhydrazine, forming thick oils, which as they could not be obtained crystalline were not further examined.

\section{Acetyltrimethylenecarboxylic Acid $(1,1)$.}

In order to obtain this acid, the pure ether was mixed with an excess of a fairly concentrated solution of alcoholic potash, and then allowed to stand at ordinary temperatures to saponify. In a short

* I hope to be able to have the honour of laying an account of this substance before the Society in a short time. 
time, crystals of a potassic salt separated, the liquid becoming slightly brownish. At the end of about five days water was added, and any unchanged ether carefully separated from the potassic salt by repeated treatment with ether. The aqueous solution was then acidified with dilute sulphuric acid, and several times extracted with small quantities of ether. The ethereal solution after being well washed and dried over calcic chloride was evaporated on a water-bath, when the crude acid was left as a thick slightly brownish oil, which did not solidify after standing for some days over sulphuric acid in a vacuum.

In order to purify this crude product, it was dissolved in dilute sodic carbonate, and after warming on a water-bath, well shaken with animal charcoal and filtered; by this means an almost colourless solution of the sodic salt was produced, which was then acidified with sulphuric acid, and extracted repeatedly with pure ether. The ethereal solution, after carefully drying over calcic chloride and evaporating, deposited an almost colourless oil ; this after standing for some days over sulphuric acid in a vacuum gave the following numbers on analysis :-

0.1624 gram substance gave 0.0975 gram $\mathrm{H}_{2} \mathrm{O}$ and 0.3375 gram $\mathrm{CO}_{2}$.

Found.

C ..... 56.68 per cent.

H..... 6.67 ,

O $\ldots \ldots 36 \cdot 65 \quad$

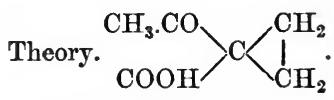

56.25 per cent.

$6 \cdot 25$

$37 \cdot 50 \quad$,

The substance was therefore acetyltrimethylenecarboxylic acid $(1,1)$. This acid is fairly easily soluble in water, the solution being strongly acid to test-paper. It does not solidify when kept at $-10^{\circ}$ for some hours, but becomes so thick that the vessel containing it may be turned over without any of the substance falling out. The solution in alkalis is easily decomposed on adding iodine, iodoform being formed in abundance. Sodic hypochlorite also produces chloroform when added to a solntion of the acid in sodic carbonate. On warming in a test-tube above $100^{\circ}$, the acid is decomposed, carbonic anhydride being driven off, and neutral oils formed, which will be more fully described later on.

The salts of acetyltrimethylenecarboxylic acid are very characteristic.

The ammonium salt is easily prepared by dissolving the acid in a slight excess of ammonia and allowing the solution to evaporate over sulphuric acid in a vacuum. It crystallises in colourless needles, which are very soluble in water. 
The silver salt is obtained by adding nitrate of silver to the hot solution of the ammonium salt. On cooling, it crystallises out in nodular masses, which consist of a mass of fine needles. These were first dried between filter-paper and then over sulphuric acid in a vacuum. The analysis gave the following numbers:-

I. 0.2693 gram substance gave 0.0791 gram $\mathrm{H}_{2} \mathrm{O}, 0.3053$ gram $\mathrm{CO}_{2}$, and 0.1223 gram Ag.

II. 0.4212 gram substance gave 0.1938 gram $\mathrm{Ag}$.

III. $0 \cdot 3224$

" $0 \cdot 1470$,

Theory.

Found.

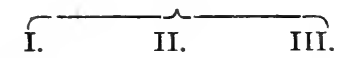

C...... 30.92

III.

H. ...... $3 \cdot 26$

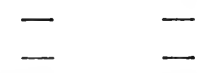

Ag $\ldots . . .45 \cdot 41$

$46 \cdot 01$

$45 \cdot 60$

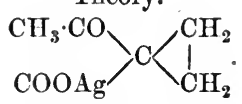

O ...... 20.41

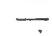
$30 \cdot 64$ per cent. $2 \cdot 98$

4595

$20 \cdot 42$

This salt is very stable, and only slowly darkened when exposed to the influence of diffused light.

If a solution of ferric chloride is added to the ammonium salt, a dark brown precipitate is formed, which on boiling dissolves, producing a dark red solution. Ferrous sulphate gives no precipitate. Mercuric chloride, with a solution of the ammonium salt, gives a white curdy precipitate, which on boiling dissolves, and crystallises out again in needles on cooling. Mercurous chloride gives a black precipitate. Baric chloride, calcic chloride, magnesic sulphate, stannous chloride, and nickelous sulphate, do not give any precipitate with the ammonium salt. The lead salt is a white precipitate soluble in hot water.

Acetyltrimethylenecarboxylic acid in alkaline solutions is a very stable compound; it may be boiled with alcoholic potash for a considerable time without being decomposed.

When boiled with dilute sulphuric acid, it is easily split up, abun. dance of carbonic anhydride being given off, and a dark brownish oil remaining behind; on extracting this with ether and fractioning, it could not be obtained pure, and appeared to be a mixture of several bodies. The same decomposition occurs on boiling the acid with water alone, the residual oil, however, appears to be purer.

From the stability not only of the acid itself, but more especially of its salts, as will have been noticed from the above description, it is clear that this acid, or rather its ethereal salt, is something quite different from ordinary substituted ethyl acetoacetates. From the method of its formation, it must, however, be either ethyl acetyltrimethylenecarboxylate or an ethyl vinylacetoacetate of the formula 


\section{$\mathrm{CH}_{2}: \mathrm{CH} \cdot \mathrm{CH}<{\mathrm{CO} \cdot \mathrm{CH}_{3}}_{3}^{\mathrm{COOC}_{2} \mathrm{H}_{5}}$.}

All acetoacetic ethers, as is well known; are easily, split up on boiling with potash into acids and ketones. Thus ethylic dimethylacetoacetate (which only differs from ethylic acetyltrimethylenecarboxylate by two hydrogen-atoms) gives on hydrolysis isopropyl methyl ketone, dimethylacetic acid, acetic acid, and carbonic anhydride, thus :-

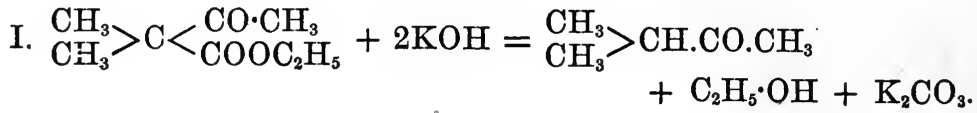

II. $\underset{\mathrm{CH}_{3}}{\mathrm{CH}_{3}}>\mathrm{C}<\underset{\mathrm{COOC}_{2} \mathrm{H}_{5}}{\mathrm{CO} \cdot \mathrm{CH}_{3}}+2 \mathrm{KOH}=\underset{\mathrm{CH}_{3}}{\mathrm{CH}_{3}}>\mathrm{CH} \cdot \mathrm{COOK}+\mathrm{C}_{2} \mathrm{H}_{5} \cdot \mathrm{OH}$ $+\mathrm{CH}_{3} \cdot \mathrm{COOK}$.

It was only by using great precautions that Ceresole (Ber., 15, 1874) succeeded in obtaining diméthylacetoacetic acid, and found that this acid was extremely unstable, being slowly and completely decomposed, even at ordinary temperatures, into isopropyl methyl ketone and carbonic anhydride-a behaviour very different to that of acetyltrimethylenecarboxylic acid, which can be kept for weeks withont the slightest decomposition setting in. The salts of dimethylacetoacetic acid are, moreover, almost as unstable as the acid itself : thus the silver salt is decomposed directly on warming, silver being precipitated, whereas the silver salt of acetyltrimethylenecarboxylic acid can be recrystallised from boiling water without decomposition. It is evident that a very great difference exists between the latter acid and all the substituted acetoacetic acids which have been prepared, and which behave in a way perfectly analogons to dimethylacetoacetic acid. There can, therefore, be no doubt that the acid is not a vinylacetoacetic acid, but that it is really acetyltrimethylenecarboxylic acid $(1,1)$.

\section{Acetyltrimethylene, $\mathrm{CH}_{3} \mathrm{CO} \cdot \mathrm{CH} \backslash \underset{\mathrm{CH}_{2}}{\mathrm{CH}_{2}}$.}

As has already been mentioned, acetyltrimethylenecarboxylic acid is easily decomposed on boiling with water or dilute acids into neutral oils and carbonic anhydride, and, as it was thought that it would be interesting to follow up this reaction, several such decomposition experiments were tried, and the oils prodaced carefully examined without any definite result. It was then found that the acid itself underwent a similar decomposition when distilled, carbonic anhydride and low-boiling oils being given off, and, as it appeared likely that the latter might be more easy to purify than those produced by boiling 
with dilute acids, the careful examination of the product was undertaken with the following results:-

A considerable quantity of acetyltrimethylenecarboxylic acid was, in portions of 5 grams at a time, heated at $200^{\circ}$ in a retort connected with a condenser until the evolution of carbonic anhydride ceased. During the operation, a colourless oil distilled over leaving a considerable residue in the retort, which $I$ have not as yet been able to examine.

The distillate was next carefully fractioned in a Würtz flask with a long neck, and the fraction $100-125^{\circ}$ collected separately. This was then repeatedly refractioned until at last a beautiful colourless oil was obtained boiling constantly at $112-113^{\circ}$ (at $720 \mathrm{~mm}$.). It gave the following numbers on analysis:-

$0 \cdot 1915$ gram substance gave $0 \cdot 1663$ gram $\mathrm{H}_{2} \mathrm{O}$ and $0 \cdot 4994$ gram $\mathrm{CO}_{2}$.

Found.

C .... $71 \cdot 12$ per cent.

H $\ldots . .964$,

O .... 19.24,
Theory. $\mathrm{CH}_{8} \cdot \mathrm{CO} \cdot \mathrm{CH}\left\langle\mathrm{CH}_{2} \mathrm{CH}_{2}\right.$

$71 \cdot 42$ per cent.

9.52,

$19 \cdot 05 \quad "$

This substance was therefore acetyltrimethylene or trimethylene methyl ketone. It is produced from acetyltrimethylenecarboxylic acid by the splitting off of carbonic anhydride according to the equation-<smiles>COC1(C(=O)O)CC1</smiles>

Acetyltrimethylene is a colourless, mobile oil, of powerful odour, recalling somewhat that of camphor. It appears to be easily polymerised, particularly when treated with acids, high-boiling oils, which at present have not been further examined, being formed.

The yield of acetyltrimethylene from acetyltrimethylenedicarboxylic acid is comparatively small, owing to the production of these more complicated condensation-products.

Acetyltrimethylene unites with hydroxylamine, producing a compound which crystallises in needles; it is probably the oxime-

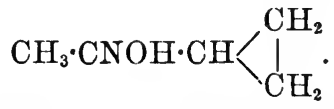

Unfortunately the quantity at my disposal was too small to admit of analysis. 


\section{Action of Ethylene Bromide on Ethyl Benzoylacetate.}

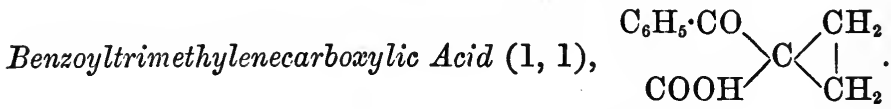

In previous researches on ethyl benzoylacetate (Trans., 1884, 170) I have shown that it is not only capable of entering into all the reactions in which ethyl acetoacetate takes part, but that, on account of the power of crystallising possessed by its derivatives, it is a much more convenient substance for use in synthetical researches than the acetoacetate. On studying the action of ethylene bromide on ethyl benzoylacetate, it was found, as was to be expected, that compounds exactly analogous to those produced by the action of ethylene bromide on ethyl acetoacetate were formed, the acetjl-group in the latter derivatives being replaced by the benzoyl-group. In carrying out this reaction the following method was found to give the best results.

5 grams of sodium are dissolved in 60 grams of absolute alcohol, and then a mixture of 40 grams of ethyl benzoylacetate and 20 grams of ethylene bromide slowly added, care being taken that the mixture does not get hot. The whole is then heated in a soda-water bottle to $100^{\circ}$ for six hours, at the end of which time the reaction is complete. The dark brownish-coloured mass is now mixed with about three times its volume of water and several times extracted with ether, the ethereal solution being well washed with water and carefully dried over calcic chloride. On distilling off the ether, a dark-brown oil remains behind, consisting of ethyl benzoyltrimethylenecarboxylate and unchanged ethyl benzoylacetate. This oil is further digested with 1.5 grams of sodium (dissolved in absolute alcohol) and 8 grams of ethylene bromide at $100^{\circ}$ for six hours, and then treated as described above. A dark-brown oil is thus obtained which, besides ethyl benzoyltrimethylenecarboxylate, still contains some unchanged ethyl benzoylacetate which is very difficult to completely remove by fractional distillation. It is best, therefore, to convert it into the free acid in the following way:-The crude product is mixed with an excess of a fairly concentrated solution of alcoholic potash and allowed to stand at ordinary temperatures for about seven days, in order that the ether may be completely saponified. On adding water, a considerable quantity of oil is precipitated (consisting principally of ethylene bromide, acetophenone, and a little unchanged ether) and must be removed by repeated treatment with ether.

The alkaline solution is then acidified with dilute sulphuric acid, when a thick brownish-coloured oil is precipitated, which is several times extracted with ether. The ethereal solution, after well washing 
and drying over calcic chloride, deposits the crude acid on evaporation, as a thick reddish-brown oil, which, on standing over sulphuric acid in a vacuum, deposits a quantity of crystals.

The whole mass is, however, best purified by redissolving it in hot sodic carbonate, agitating well with animal charcoal for about half an hour, and filtering.

A much lighter coloured solution of sodic salt is thus obtained, which is again acidified with dilute sulphuric acid and extracted with ether.

On evaporation, the ethereal solution deposits an oil, which, although still dark-coloured, usually solidifies almost completely on standing over sulphuric acid in a vacuum for a few days. The crystals are next roughly purified from the oily mother-liquor by. spreading out on a porous plate, when they become almost colourless. The mass of crystals is dissolved in ether, and the solution concentrated on a water-bath till crystallisation sets in. If this solution be now allowed to concentrate further by evaporation at ordinary temperatures, the benzoyltrimethylenecarboxylic acid crystallises out in magnificent colourless prisms, which are pure when once recrystallised.

Besides this acid, the mother-liquors contain benzoic acid, which, however, on account of its greater solubility in ether, is easy to get. rid of by repeated crystallisation from this solvent. The analysis of the pure acid gave the following results :-

I. 0.1867 gram substance gave 0.0888 gram $\mathrm{H}_{2} \mathrm{O}$ and 0.4737 gram $\mathrm{CO}_{2}$.

II. 0.214 .3 gram substance gave 0.1048 gram $\mathrm{H}_{2} \mathrm{O}$ and 0.5482 gram $\mathrm{CO}_{2}$.

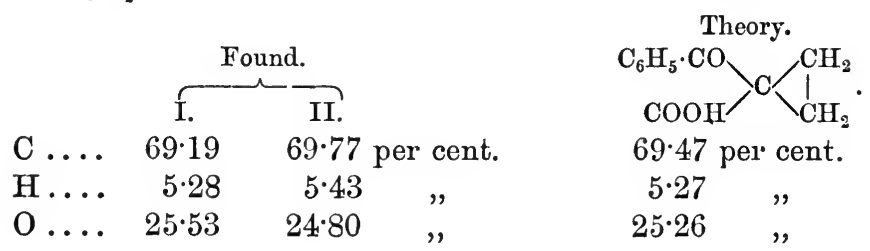

This substance was, therefore, benzoyltrimethylenecarboxylic acid $(1,1)$, its ethereal salt being formed by the action of ethylene bromide on ethyl benzoylacetate thus :-

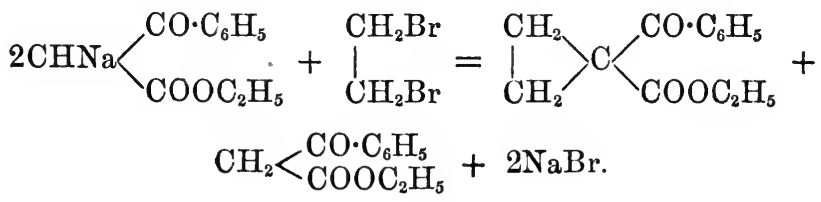

Benzoyltrimethylenecarboxylic acid melts at $148-149^{\circ}$, being decom- 
posed at the same time into carbonic anhydride and benzoyltrimethylene, a compound which will be fully described later on.

It is fairly easily soluble in ether, chloroform, benzene, and bisulphide of carbon; sparingly in water. If, however, the acid is dissolved in ammonia, it is precipitated in a very finely divided state on adding dilute sulphuric acid. Thus prepared it dissolves, although with difficulty, in boiling water, and erystallises out on cooling in long colourless needles, which appear to have a slightly higher melting point than the acid crystallised from other solvents. The acid crystallises best, however, from ether. If the concentrated ethereal solution be allowed to evaporate slowly, most magnificent colourless prisms are obtained, which Professor Haushofer was kind enough to examine. He gave me the following description of this body:-

Crystalline System, Monoclinic.

$$
\begin{gathered}
a: b: c=2 \cdot 3298: 1: 2 \cdot 1319 . \\
\beta=83^{\circ} 4^{\prime} .
\end{gathered}
$$

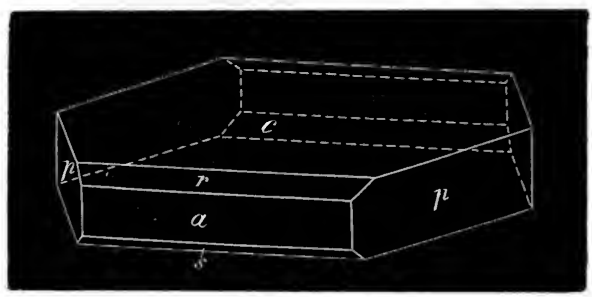

Pale yellow transparent crystals with the combinations $\mathrm{OP}(c)$. $\infty \mathrm{P}(p), \infty \mathrm{P} \infty(a),-\mathrm{P} \infty(r), \mathrm{P} \infty(s)$; tabular in the direction of the face $c$, usually elongated in the direction of the orthodiagonal. The face $c$ is usually bent somewhat concave; cleavage imperfect in the

\begin{tabular}{|c|c|c|c|c|}
\hline \multicolumn{3}{|c|}{ Measured. } & \multirow{2}{*}{\multicolumn{2}{|c|}{ Calculated }} \\
\hline$a: c=$ & $* 96^{\circ}$ & $56^{\prime}$ & & \\
\hline$a: r=$ & *136 & 13 & - & \\
\hline$p: p=$ & *133 & 14 & - & \\
\hline$s: a=$ & 128 & 42 & $128^{\circ}$ & 40 \\
\hline$r: c=$ & 140 & 38 & 140 & 43 \\
\hline$p: c=$ & 92 & 50 & 92 & 4.5 \\
\hline
\end{tabular}
direction $p$.

Although a certain general morphological resemblance between this compound and the carboxylic acid of benzoyltetramethylene can be observed, as for example in the relation of the angles $c: r$, in the 
basal angles $\left(23^{\circ} 14^{\prime}\right.$ and $\left.20^{\circ} 46^{\prime}\right)$, or in the relation of the axes $a: b$, no inference can be safely drawn from these circumstances.

The ammonium salt of this acid is easily prepared by dissolring the pure acid in an excess of ammonia and allowing the solution to evaporate over sulphuric acid in a vacuum. It is thus obtained as a colourless, crystalline mass, very easily soluble in water. On adding dilnte acids to a solution of the ammonium salt, the acid is precipitated in a pure condition as a mass of minute colourless needles. If nitrate of silver is added to the solution of the ammonium salt, the silver salt is obtained as a white curdy precipitate insoluble in water. After well washing and drying over sulphuric acid in a vacuum, the following numbers were obtained on analysis :-

0.2352 gram substance gave 0.0677 gram $\mathrm{H}_{2} \mathrm{O}, 0.3817$ gram $\mathrm{CO}_{2}$, and 0.0854 gram Ag.

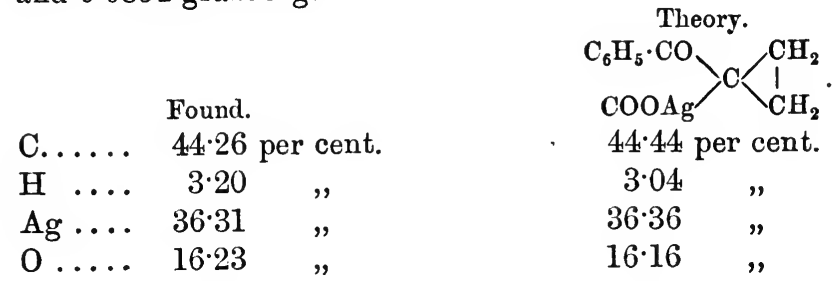

This salt is very stable, and is only slowly darkened when exposed to diffused light.

Ferric chloride gives with a solution of the ammonium salt a reddish-white precipitate, which, on warming, becomes slightly darker coloured. Ferrous sulphate, baric chloride, and calcic chloride give no precipitates. The copper salt is obtained on adding cupric sulphate to the ammonium salt, as a light green precipitate, wnich is sparingly soluble in water. Plumbic acetate gives with the ammonium salt a heavy white precipitate, which dissolves, although witn diffculty, in boiling water, and on cooling crystallises out in small colourless needles. All these salts are very stable.

Benzoyltrimethylenecarboxylic acid itself may be boiled for a long time with strong solutions of alcoholic potash without any appreciable change taking place. On preparing this acid, however, from the crude ethereal salt, it is better to saponify at ordinary temperatures, and not to boil, as the impurities present, such as ethyl benzoylacetate, acetophenone, \&c., are liable to be changed into resinous bodies, which render the further purification of the acid a matter of great difficulty. The stability of this acid towards alkalis is quite as remarkable, if not more so, than that of acetyltrimethylenecarboxylic acid, and it is quite out of the question to suppose that it has the constitution of a substituted vinylbenzoylacetic acid of the formula- 


\section{$\mathrm{CH}_{2}: \mathrm{CH} \cdot \mathrm{CH}<\mathrm{COO} \cdot \mathrm{C}_{6} \mathrm{H}_{5}$.}

As I have shown in previous researches, it is practically impossible to obtain the substituted benzoylacetic acids by the hydrolysis of their ethers at ordinary temperatures. On warming with potash, they are naturally completely split up, not a trace of the corresponding acids being left.

$$
\text { Ethyl Benzoyltrimethylenecarboxylate }(1,1),{ }_{\mathrm{COOC}_{2} \mathrm{H}_{5}}^{\mathrm{C}_{6} \mathrm{H}_{5} \cdot \mathrm{CO}}>\mathrm{C} /\left.\right|_{\mathrm{CH}_{2}} ^{\mathrm{CH}_{2}}
$$

In order to obtain this in a pure state, the silver salt of benzoyltrimethylenecarboxylic acid was mixed with a slight excess of ethyl iodide and a little pure ether, and heated in a flask connected with a reflux condenser for about two hours on a water-bath. The silver salt was thus easily decomposed. After filtering from the precipitated iodide of silver, the ethereal solution was evaporated on a water-bath, when a colourless oil remained behind; this on distillation went over completely between $280^{\circ}$ and $290^{\circ}$. On refractioning, by far the greater part distilled over between $280^{\circ}$ and $283^{\circ}(720 \mathrm{~mm}$.), as an almost colourless oil, which gave the following numbers on analysis :-

I. $0 \cdot 1359$ gram substance gave 0.0793 gram $\mathrm{H}_{2} \mathrm{O}$ and 0.3540 gram $\mathrm{CO}_{2}$.

II. 0.1312 gram substance gave 0.0765 gram $\mathrm{H}_{2} \mathrm{O}$ and 0.3446 gram

\begin{tabular}{|c|c|c|c|}
\hline \multicolumn{3}{|c|}{ Found. } & $\begin{array}{l}\text { Theory. } \\
\mathrm{C}_{6} \mathrm{H}_{5} \cdot \mathrm{CO}\end{array}$ \\
\hline & I. & II. & $\mathrm{COOC}_{2} \mathrm{H}_{5}$ \\
\hline C .... & $71 \cdot 04$ & $71 \cdot 63$ per cent. & 71.56 per cent. \\
\hline H.... & $6 \cdot 48$ & $6 \cdot 47$ & $6 \cdot 42$ \\
\hline $0 \ldots$ & $22 \cdot 48$ & $21 \cdot 90$ & $22 \cdot 02$ \\
\hline
\end{tabular}
$\mathrm{CO}_{2}$.

The substance was therefore ethyl benzoyltrimethylenecarboxylate $(1,1)$. It is a thick colourless oil, possessing a faintly aromatic odour and burning taste. It does not appear to solidify when exposed to a temperature of $-10^{\circ}$.

It distils without the least decomposition at $280-283^{\circ}(720 \mathrm{~mm}$.), and is easily saponified by warming with alcoholic potash, benzyltrimethylenecarboxylic acid being regenerated.

$$
\text { Benzoyltrimethylene, } \mathrm{C}_{6} \mathrm{H}_{5} \cdot \mathrm{CO} \cdot \mathrm{CH}\left\langle\left.\right|_{\mathrm{CH}_{2}} ^{\mathrm{CH}_{2}} \cdot\right.
$$

As already mentioned, benzoyltrimethylenecarboxylic acid, when 
heated a few degrees above its melting point, is completely decomposed, carbonic anhydride being given off, and a yellowish oil remaining behind, which on cooling does not again solidify. As it was thought that interesting results might be obtained by following up this decomposition, 9 grams of the pure acid were heated in an oilbath at $200^{\circ}$, until no more carbonic anhydride was evolved. The residue, which was a thick brownish-coloured oil, was then carefully fractioned, almost the whole (about 6.1 grams) distilling over between $235^{\circ}$ and $242^{\circ}$. This distillate was then once or twice refractioned, and thus easily obtained perfectly pure as an almost colourless oil boiling constantly at $239-239.5^{\circ}(720 \mathrm{~mm}$.).

The analyses gave the following results :-

I. $0 \cdot 1730$ gram substance gave $0 \cdot 1114$ gram $\mathrm{H}_{2} \mathrm{O}$ and 0.5205 gram $\mathrm{CO}_{2}$.

II. 0.1382 gram substance gave 0.0920 gram $\mathrm{H}_{2} \mathrm{O}$ and 0.4150 gram $\mathrm{CO}_{2}$.

III. $0 \cdot 1597$ gram substance gave $0 \cdot 1014$ gram $\mathrm{H}_{2} \mathrm{O}$ and 0.4791 gram $\mathrm{CO}_{2}$.

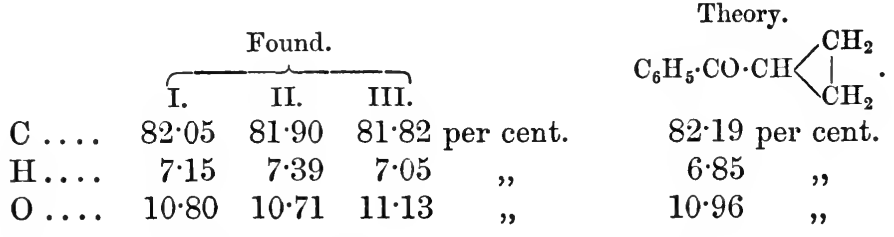

The vapour-density of this compound was determined by Hofmann's method by volatilising the substance in a vacuum in aniline vapour. The following numbers were obtained:-

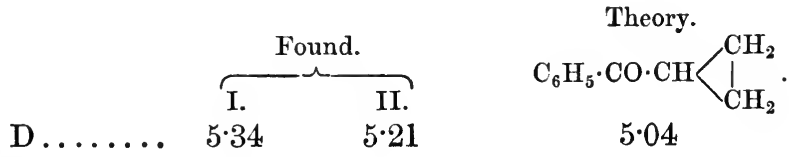

This substance was, therefore, without doubt benzoyltrimethylene. It is formed from benzoyltrimethylenecarboxylic acid by the simple splitting off of carbonic anhydride, according to the equation-

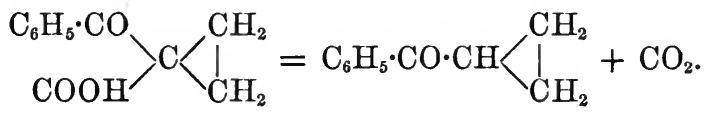

Benzoyltrimethylene is a colourless oil, of aromatic odour, which does not solidify when cooled down to $-10^{\circ}$. It is formed almost quantitatively according to the above equation. 9 grams of benzoyltrimethylenecarboxylic acid should give 6.9 grams of benzoyltri- 
methylene, and in the experiment 6.1 grams were obtained boiling between $235^{\circ}$ and $242^{\circ}$, which, allowing for loss by distillation, is as much as could be expected when such small quantities are operated with. It is rather curious that such a good yield should be obtained, when it is remembered that the amount of acetyltrimethylene obtained by the decomposition of acetyltrimethylenecarboxylic acid is so small. The acetyl-derivative appears, therefore, to be less stable than the benzoyl one.

Benzoyltrimethylene is not affected when treated with bromine at ordinary temperatures; on heating, however, substitution takes place, abundance of hydrobromic acid being evolved. The product, after washing with sodic carbonate solution, is a colourless, very heavy oil, which, however, owing to the small amount at my disposal, could not be further examined. The solution of benzoyltrimethylene in chloroform can be boiled for a considerable time with bromine without any appreciable change taking place, a proof that it cannot be unsaturated.

\section{Action of Hydrobromic Acid on Benzoyltrimethylenecarboxylic $\operatorname{AClD}(1,1)$.}

w-Bromopropyl Phenyl Ketone, $\mathrm{C}_{6} \mathrm{H}_{5} \cdot \bullet \mathrm{O} \cdot \mathrm{CH}_{2} \cdot \mathrm{CH}_{2} \cdot \mathrm{CH}_{2} \mathrm{Br}$.

It has already been shown (p. 814) that trimethylenedicarboxylic acid $(1,1)$, when treated with fuming hydrobromic acid, is converted into bromethylmalonic acid, the trimethylene-ring being split. This result confirms the remarkable observations of Freund (loc. cit., p. 818), that the trimethylene-ring, although very stable when treated with bromine, is easily decomposed when acted on by fuming hydrobromic or hydriodic acid. It was thought advisable to further prove this point, if possible, by subjecting other differently constituted trimethylene-derivatives to the same treatment. It appeared probable that benzoyltrimethylenecarboxylic acid, being such a beautifully crystalline substance, might be expected to give well-defined decomposition products, which could easily be purified and identified; on this account it seemed to be especially adapted for further examination.

The pure acid was first obtained in a finely divided condition by dissolving it in ammonia and precipitating with dilute sulphuric acid; the sandy mass was then well washed and dried over sulphuric acid in a vacuum.

On mixing the dry substance with fuming hydrobromic acid (sp. gr. 1.83) it did not dissolve, and no reaction was at first perceptible. After standing, however, for about an hour, at ordinary temperatures, 
small bubbles began to appear, and on agitating a quantity of gas was evolved which proved to be carbonic anhydride. This evolution of carbonic anhydride lasted for about a week, at the end of which time the decomposition was complete, the liquid dividing into two distinct layers, the upper one, which was a brownish oil, being the product of the reaction. This was separated, dissolved in a little ether, and well shaken with a dilute solution of sodic carbonate in order to remove any excess of hydrobromic acid.

After drying over potassic carbonate and distilling off the ether, a slightly brownish-coloured oil remained behind, which, however, soon solidified to a hard crystalline mass. The substance, which is practically pure, can be freed from minute traces of mother-liquor by spreading it out on a porous plate. It then gave the following numbers on analysis :-

I. 0.1924 gram substance gave 0.0849 gram $\mathrm{H}_{2} \mathrm{O}$ and 0.3703 gram $\mathrm{CO}_{2}$.

II. 0.307u gram substance gave 0.2510 gram $\mathrm{AgBr}$.

Found.

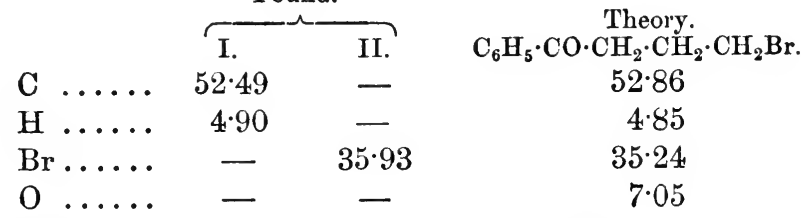

It is, therefore, $\omega$-bromopropyl phenyl ketone.* It is formed from benzoyltrimethylenecarboxylic acid $(1,1)$ according to the equation-

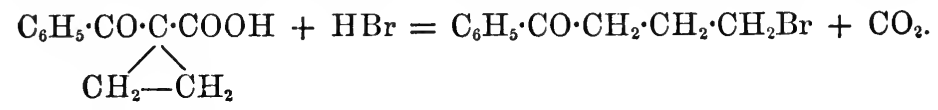

This reaction is perfectly analogous to the formation of $\gamma$-bromethylmalonic acid from trimethylenecarboxylic acid $(1,1)$.

$\boldsymbol{\omega}$-Bromopropyl phenyl ketone melts at $37-39^{\circ}$. It is easily soluble in light petroleum, methyl alcohol, ethyl alcohol, chloroform, ether, carbon bisulphide, benzene, acetone, and ethyl acetate, but is insoluble in water. It is precipitated from its solutions on evaporation always as an oil, and cannot, therefore, be purified by recrystallisation. The oily drops when touched with a glass rod directly solidify.

When boiled with a dilute solution of silver nitrate, this compound is easily decomposed, quantities of silver bromide being precipitated, and an oily body formed; owing to the small quantity at my disposal, it could not be further examined.

* With regard to the non enclature of this body, sce Baejer (Ber., 17, 960). 
Bromethylmalonic acid behaves in exactly the same way, being converted into carbobutyrolactone; it is probable that this decomposition product of $\omega$-bromopropyl phenyl ketone is $\omega$-hydroxypropyl phenyl ketone, formed according to the following equation:-

$$
\begin{aligned}
& \mathrm{C}_{6} \mathrm{H}_{5} \cdot \mathrm{CO} \cdot \mathrm{CH}_{2} \cdot \mathrm{CH}_{2} \cdot \mathrm{CH}_{2} \mathrm{Br}+\mathrm{H}_{2} \mathrm{O} \\
&=\mathrm{C}_{6} \mathrm{H}_{5} \cdot \mathrm{CO} \cdot \mathrm{CH}_{2} \cdot \mathrm{CH}_{2} \cdot \mathrm{CH}_{2} \cdot \mathrm{OH}+\mathrm{HBr} .
\end{aligned}
$$

Alcoholic potash appears to have the same effect. $\omega$-Bromopropyl phenyl ketone is formed with still greater ease when benzoyltrimethylene is allowed to stand for some time with fuming hydrobromic acid. From these experiments, it would appear that the remarkable splitting of the trimethylene-ring under the influence of the halogen acid takes place, with even greater ease in the case of the derivatives than of the hydrocarbon itself.

\section{Action of Hydroxylamine on Benzoyltrimethylene.}

In the action of ethylene bromide on ethyl malonate, there are only two interpretations of the reaction, namely, (1) that a trimethylene-derivative or (2) that a vinyl-derivative is formed.

As soon as ethyl malonate is replaced by ethyl benzoylacetate, however, a third possibility comes into account, namely, that the reaction could take place according to one of the following equations:-

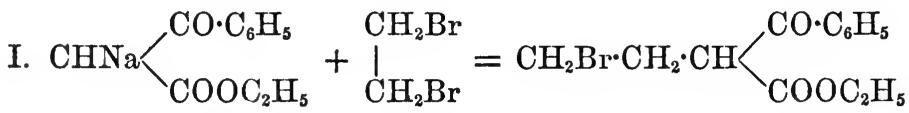
$+\mathrm{NaBr}$.

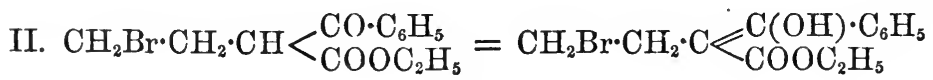<smiles>C=COC(=O)OCC</smiles>

Such a reaction, although improbable, is still by no means impossible, and it appeared necessary in this case also to bring some decided experimental proof to bear on the question. According to the above series of equations, benzoyltrimethylene would have the formula $\mathrm{CH}_{2}{ }_{\mathrm{CH}_{2} \cdot \mathrm{CH}}^{\mathrm{O}-\mathrm{C}_{6} \cdot \mathrm{C}_{6} \mathrm{H}_{5}}$, that is, be a furfurane-derivative and not a ketone. In order to decide this, it was only necessary to treat benzoyltrimethylene with hydroxylamine and see whether it formed an oxime 
or not. The experiment showed that it combined with hydroxylamine like all other ketones, forming benzoyltrimethyleneoxime,

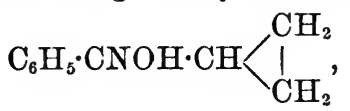

a definite proof that it contained the ketone-group $\mathrm{CO}$, and, therefore, could not be a furfurane-derivative. In preparing the oxime, the following method was found to give the best results : -3 grams of benzoyltrimethylene are dissolved in a little alcohol, and then a mixture of 4 grams of hydroxylamine hydrochloride and 3 grams of potassic carbonate dissolved in a small quantity of water are added, the quantities of alcohol and water being so regulated that the benzoyltrimethylene remains completely dissolved. The whole is allowed to stand for about a week at ordinary temperatures to ensure the completion of the reaction, then acidified with a little dilute sulphuric acid and extracted once or twice with ether. The ethereal solution, after well washing with water to remove the alcohol as much as possible, is dried over calcic chloride and evaporated, when a thick colourless oil remains behind; this almost completely solidifies on standing over sulphuric acid in a vacuum for a short time.

The crystals, after being spread out on a porous plate to roughly remove traces of mother-liquor and then recrystallised from ether, are easily obtained pure. The analysis gave the following results :-

I. 0.2107 gram substance gave 0.1330 gram $\mathrm{H}_{2} \mathrm{O}$ and 0.5741 gram $\mathrm{CO}_{2}$.

II. 0.2748 gram substance gave $21 \cdot 5$ c.c. $\mathrm{N} . \quad t=17^{\circ}$. Bar. $=$ $718 \mathrm{~mm}$.

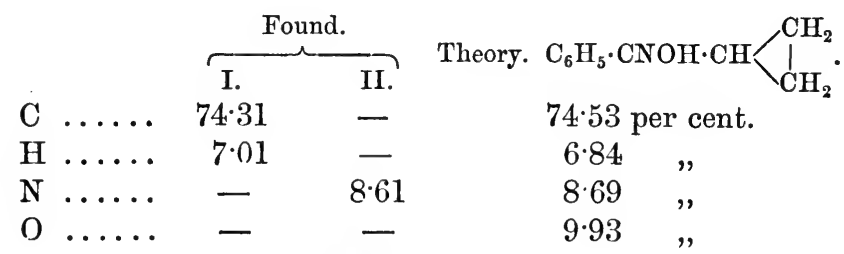

Benzoyltrimethyleneoxime had therefore been formed according to the equation-

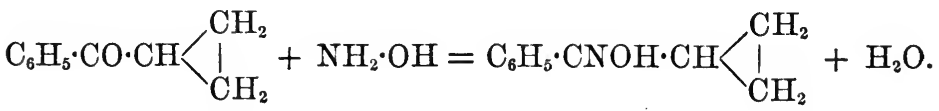

Benzoyltrimethyleneoxime melts at $86-87^{\circ}$. It crystallises from light petroleum, boiling at $50-70^{\circ}$, in curiously striped leafy masses which are colourless when pure. It can be obtained in beautiful 
prisms by allowing the ethereal solution to evaporate slowly. It is easily soluble in ether, benzene, chloroform, alcohol, and carbon bisulphide, sparingly so in cold light petroleum. It appears to be completely decomposed on long keeping, the crystals disappearing and an uninviting brownish oil being formed.

A result totally different from the preceding is obtained when benzoyltrimethylene is heated with hydroxylamine and hydrochloric acid in a sealed tube, the reaction being much more complicated. If a mixture of 4 grams of benzoyltrimethylene, 4 grams of hydroxylamine hydrochloride, 30 grams of absolute alcohol, and a few drops of hydrochloric acid be digested in a sealed tube at $130-140^{\circ}$, the mixture becomes darker and darker coloured till at the end of six hours the tube appears to be perfectly black. If, however, a small quantity of the contents be taken out and diluted with alcohol the whole dissolves, producing a beautiful deep-blue solution which in reflected light shows an intense brick-red fluorescence. The colour being so beautiful it was thought that it would be interesting to further examine the same in order if possible to see what relation existed between it and benzoyltrimethyleneoxime. All attempts to obtain the product, which proved to be a mixture of the hydrochlorides of two bases, in a crystalline condition being unsuccessful, it was decomposed with alkali and the free bases themselves worked on. To obtain the bases, the contents of several such tubes as those described above were dissolved in alcohol, gently warmed on a waterbath, and then agitated with a dilute solution of sodic carbonate. The beautiful blue colour instantly disappeared and a brownish-red precipitate was formed which, after standing for a short time, was collected, thoroughly washed with water until free from inorganic matter, and then dried over sulphuric acid in a vacuum. The blackish-red amorphous mass thus obtained was next treated with various solvents in the hope of obtaining something crystalline for analysis, but without result. It was, however, soon discovered that the product was not homogeneous, but consisted of two distinct bases, one of which was fairly easily soluble, the other almost insoluble in ether. In order to separate these, the crude substance was first obtained in as finely-divided condition as possible by powdering under water and then repeatedly extracted with pure ether, until a residue was left which did not further dissolve, and which will be described in detail further on.

The dark brownish-red ethereal extract was next washed with water, carefully dried over potassic carbonate, and the ether distilled off. By this means a blackish, semi-solid mass remained behind which, on standing over sulphuric acid, soon became hard and brittle, but could not by any means be obtained in a crystalline condition. 
It was powdered, carefully dried over sulphuric acid in a vacuum, and analysed with the following results :-

I. 0.1780 gram substance gave 0.1009 gram $\mathrm{H}_{2} \mathrm{O}$ and 0.5244 gram $\mathrm{CO}_{2}$.

II. 0.1737 gram substance gave 0.0984 gram $\mathrm{H}_{2} \mathrm{O}$ and 0.5113 gram $\mathrm{CO}_{2}$.

III. $0 \cdot 1824$ gram substance gave $0 \cdot 1049$ gram $\mathrm{H}_{2} \mathrm{O}$ and 0.5384 gram $\mathrm{CO}_{2}$.

IV. 0.2188 gram substance gave 12.4 c.c. $\mathrm{N} . t=21^{\circ}$. Bar. $=$ $718 \mathrm{~mm}$.

V. 0.3055 gram substance gave $18 \cdot 1$ c.c. $\mathrm{N} . \quad t=24^{\circ}$. Bar. $=$ $718 \mathrm{~mm}$.

VI. 0.2699 gram substance gave $16 \cdot 1$ c.c. $\mathrm{N} .=22^{\circ}$. Bar. $=$ $718 \mathrm{~mm}$.

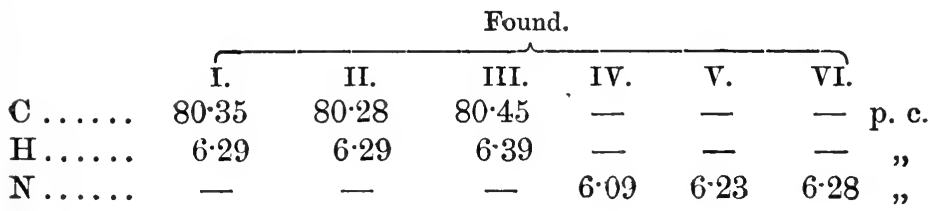

Theory. $\mathrm{C}_{30} \mathrm{H}_{28} \mathrm{~N}_{2} \mathrm{O}_{2}$.

C........... 86.36 per cent.

H............ 6.25 ,

N............ $6.25 \quad$,

$0 \ldots \ldots \ldots \ldots \ldots, 7 \cdot 14 \quad$,

Analyses I, II, IV, and V were done with the sample first prepared, III and VI from a later preparation; it would, therefore, seem as though the body were homogeneous.

This base has, therefore, the formula $\mathrm{C}_{30} \mathrm{H}_{28} \mathrm{~N}_{2} \mathrm{O}_{2}$, and is possibly formed from 3 mols. of benzoyltrimethyleneoxime according to the equation:

$$
3 \mathrm{C}_{6} \mathrm{H}_{5} \cdot \mathrm{CNOH} \cdot \mathrm{CH}\left\langle\mathrm{CH}_{2}^{\mathrm{CH}_{2}}=\mathrm{C}_{30} \mathrm{H}_{28} \mathrm{~N}_{2} \mathrm{O}_{2}+\mathrm{NH}_{3}+\mathrm{H}_{2} \mathrm{O}\right. \text {. }
$$

It is easily soluble in acetone, aniline, and nitrobenzene, forming brownish-red solutions; it is more sparingly soluble in ether, alcohol, benzene, and toluene. On allowing the solution in acetone to evaporate at ordinary temperatures, the base is precipitated as a blackishred, semi-solid mass which, on standing, soon becomes hard and brittle. When broken up, it has a shining surface somewhat resem. bling that of ordinary pitch.

It dissolves in acetic acid forming a greyish-blue solution which 
contains the acetate of the base. If the brownish-red alcoholic solution is treated with zinc-dust and ammonia it is reduced and becomes colourless; on shaking with air it is reoxidised, the colour again appearing. Distilled with zinc-dust, the base is entirely broken up, oily bodies being given off which have a peculiar odour recalling somewhat that of aniline. When distilled with soda-lime, a heavy oil comes over in considerable quantity which possesses in a marked way the characteristic smell of benzonitrile.

The salts of this base are easily obtained by adding dilute acids to its alcoholic solution. They are deep blue, and the solutions possess a most magnificent brick-red fluorescence. The hydrochloride which appeared to be the best characterised salt, was prepared in the following way :-A concentrated solution of the base in ether was carefully dried over potassic carbonate and, after filtering, saturated with gaseous hydrogen chloride. In this way, the pure hydrochloride was precipitated as a deep-blue amorphous mass. This was rapidly collected, well washed with an ethereal solution of hydrogen chloride, and then allowed to stand in a desiccator partly filled with sticks of potash and partly with sulphuric acid in a vacuum until the excess of hydrochloric acid had been removed. A beantiful dark-bluish mass was thus obtained which had a magnificent coppery lustre, and outwardly resembled indigo in every respect.

The analyses gave the following result:-

I. 0.2120 gram substance gave 0.1100 gram $\mathrm{H}_{2} \mathrm{O}$ and 0.5390 gram $\mathrm{CO}_{2}$.

II. $0 \cdot 2012$ gram substance gave $0 \cdot 1057$ gram $\mathrm{H}_{2} \mathrm{O}$ and 0.5130 gram $\mathrm{CO}_{2}$.

III. 0.2203 gram substance gave 11.5 c.c. $\mathrm{N} . \quad t=21^{\circ} . \quad$ Bar. $=$ $717 \mathrm{~mm}$.

IV. 0.2078 gram substance gave 0.1141 gram AgCl.

V. 0.3795 gram substance gave 0.2160 gram AgCl.

Found.

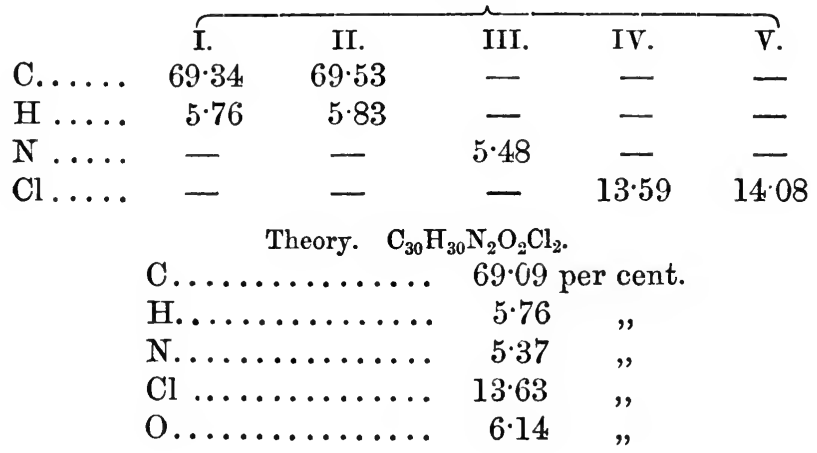


This salt has, therefore, the formula $\mathrm{C}_{30} \mathrm{H}_{30} \mathrm{~N}_{2} \mathrm{O}_{2} \mathrm{Cl}_{2}$. It dissolves in warm water, more easily in alcohol, forming beautiful indigo-blue solutions, which possess the magnificent brick-red fluorescence already described. It is remarkable that the solutions of this hydrochloride should show the same absorption-spectrum as indigo. If the solution in alcohol is warmed with zinc-dust and a little hydrochloric acid, the colour changes first to pink, and then entirely disappears. On filtering and shaking with air a beautiful crimson solution is produced which possesses a red fluorescence even more marked than that of the original substance, and reminds one strongly of magdala red.

If platinum chloride is added to the solution of the hydrochloride in alcohol, the platinochloride is precipitated as a bluish-black amorphous mass which, after being collected, well washed with water, and dried over sulphuric acid in a vacuum, gave the following numbers on analysis :-

0.2536 gram of substance gave 0.0559 gram of platinum.

$$
\begin{aligned}
& \text { Found. Theory. }\left(\mathrm{C}_{30} \mathrm{H}_{30} \mathrm{~N}_{2} \mathrm{O}_{2} \mathrm{Cl}_{2}\right) \mathrm{PtCl}_{4} \text {. } \\
& \text { Pt..... 22.04 per cent. } \quad 22 \cdot 76 \text { per cent. }
\end{aligned}
$$

This platinochloride is sparingly soluble in water and alcohol. From the above results there can be but little doubt that the base of this colouring matter has the empirical formula $\mathrm{C}_{30} \mathrm{H}_{28} \mathrm{~N}_{2} \mathrm{O}_{2}$, and is capable of forming salts such as $\left(\mathrm{C}_{30} \mathrm{H}_{28} \mathrm{~N}_{2} \mathrm{O}_{2}\right), 2 \mathrm{HCl}$ with 2 mols. of the acid.

No clue as to the arrangement of the atoms in this complicated molecule has as yet been obtained, and the further examination has for the meantime been postponed until our knowledge of the trimethylene compounds is more developed.

The second base, produced by the action of hydroxylamine on benzoyltrimethylene, which is left behind after extracting the base $\mathrm{C}_{30} \mathrm{H}_{28} \mathrm{~N}_{2} \mathrm{O}_{2}$ with ether, was then examined.

After washing well with water and drying over sulphuric acid in a vacuum, it presented exactly the appearance of amorphous phosphorus, and gave the following numbers on analysis :-

I. 0.1453 gram substance gave 0.0707 gram $\mathrm{H}_{2} \mathrm{O}$ and 0.4107 gram $\mathrm{CO}_{2}$.

II. $0 \cdot 1167$ gram substance gave 0.0576 gram $\mathrm{H}_{2} \mathrm{O}$ and 0.3302 gram $\mathrm{CO}_{2}$.

III. 0.1321 gram substance gave 0.0630 gram $\mathrm{H}_{2} \mathrm{O}$ and 0.3738 gram $\mathrm{CO}_{2}$.

IV. $0 \cdot 1470$ gram substance gave 10.6 c.c. $\mathrm{N} . \quad t=22^{\circ}$. Bar. $=$ $716 \mathrm{~mm}$.

V. 0.2025 gram substance gave 14.5 c.c. $\mathrm{N} . \quad t=20^{\circ}$. Bar. $=$ $716 \mathrm{~mm}$. 


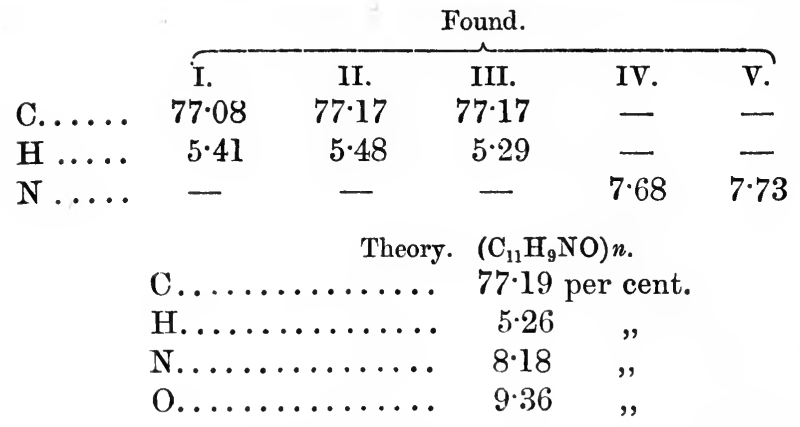

Analyses I, II, and IV were done for the first sample, III and V from a preparation made later on. This base appears, therefore, to have the formula $\left(\mathrm{C}_{11} \mathrm{H}_{9} \mathrm{NO}\right) n$, perhaps $\mathrm{C}_{33} \mathrm{H}_{27} \mathrm{~N}_{3} \mathrm{O}_{3}$.

It is sparingly soluble in boiling alcohol, benzene, and toluene, but easily so in acetone, aniline, and nitrobenzene, forming reddishbrown solutions, from which it is precipitated in an amorphous condition on evaporation. All attempts to obtain it in a crystalline condition were unsuccessful.

It dissolves in alcoholic hydrochloric acid, producing a greenishviolet solution, which fluoresces in the same way as, although to a much less extent than, the salts of the base $\mathrm{C}_{30} \mathrm{H}_{28} \mathrm{~N}_{2} \mathrm{O}_{2}$.

It dissolves in concentrated sulphuric acid with an intense olivegreen colour; on diluting with water, a green amorphous mass is precipitated, which is perhaps the sulphate of the base.

\section{Action of Propylene Bromide on Ethyl Acetoacetate.}

Ethyl Acetylmethyltrimethylenecarboxylate $(1,2,1)$,<smiles>CCOC1(CC)CC1C</smiles>

It was next thought necessary, in order to prove whether the above method for the formation of trimethylene-derivatives was of general application, to try the action on ethy] acetoacetate of some other dibromide which was constituted similarly to ethylene bromide. For this purpose propylene bromide, $\mathrm{CH}_{3} \cdot \mathrm{CHBr} \cdot \mathrm{CH}_{2} \mathrm{Br}$, was selected, and the reaction carried out in the following way:-

4.6 grams of sodium were dissolved in 60 grams of alssolute alcohol, and then a mixture of 26 grams of ethyl acetoacetate and 21 grams of propylene bromide were slowly poured in, the whole being well cooled during the operation.

This mixture, after being heated at $100^{\circ}$ for 10 hours in a soda- 
water bottle, had a neutral reaction, a sign that the reaction was finished. Water was then added, and the oily product several times extracted with ether, the ethereal solution dried over calcic chloride, and the ether distilled off. The residual oil was again heated at $100^{\circ}$ for 10 hours with 2.3 grams of sodium (dissolved in 35 grams of absolute alcohol) and 12 grams of propylene bromide.

The product was isolated, as described before, and carefully fractioned, when, after a little unchanged propylene bromide had gone over, by far the greater quantity distilled between $180^{\circ}$ and $230^{\circ}$, a thick blackish oil remaining in the retort. On repeatedly fractioning the portion $180-230^{\circ}$, it was at last obtained pure, as a colourless oil boiling at $215-217^{\circ}(720 \mathrm{~mm}$.) ; it gave the following numbers on analysis :-

0.1813 gram substance gave 0.1375 gram $\mathrm{H}_{2} \mathrm{O}$ and 0.4225 gram $\mathrm{CO}_{2}$.

Found.

C ..... 63.55 per cent.

H ..... 8.42 ,

O .... 28.03 ,<smiles>CCOC(=O)OCC</smiles>
63.53 per cent.

$8 \cdot 24$,

$28 \cdot 23$,

This substance was therefore ethyl acetylmethyltrimethylenecarboxylate $(1,2,1)$, and is formed by the action of propylene bromide on ethyl acetoacetate, according to the following equation :-

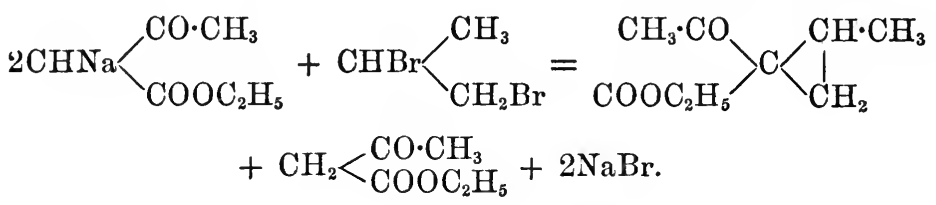

It is a colourless faintly smelling oil, which boils at $215-217^{\circ}$ (720 mm.). On cooling down below $-10^{\circ}$, it becomes very thick and appears inclined to crystallise. The yield is not good, a considerable quantity of high-boiling oils being formed at the same time.

Acetylmethyltrimethylenecarboxylic Acid $(1,2,1)$,

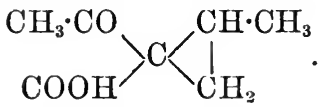

In order to obtain this acid, the pure ether was mixed with excess of a moderately concentrated solution of alcoholic potash, and allowed to stand for about seven days at the ordinary temperature, at the end of which time the hydrolysis was nearly complete. The product was 
then diluted with water and a trace of unchanged oil carefully removed by extraction with ether. On acidifying the solution of the potassic salt of the acid with dilute sulphuric acid, it became only slightly turbid, and no crystalline acid separated out. The whole was, therefore, several times extracted with pure ether, the ethereal solution well washed with water, in order to remove as much alcohol as possible, and then dried over calcic chloride. On distilling off the ether an almost colourless oily acid remained behind, which, on standing for some days over sulphuric acid in a vacaum, did not crystallise. In order to purify it, it was dissolved in a little dilute ammonia, well shaken with pure animal charcoal and filtered, a trace of colouring matter being thus almost entirely remored. On acidifying with dilute sulphuric acid and extracting with ether as before, a very thick almost colourless oil was obtained, which, after standing for some days over sulphuric acid in a vacuum in order to remove the last traces of ether, was analysed with the following result :-

0.2021 gram substance gave 0.1369 gram $\mathrm{H}_{2} \mathrm{O}$ and 0.4347 gram $\mathrm{CO}_{2}$.

\begin{tabular}{lrl}
\multicolumn{4}{c}{ Found. } \\
C .... & $58 \cdot 66$ & per cent. \\
H.... & $7 \cdot 52 \quad "$ \\
O ... & $33.82 \quad "$
\end{tabular}

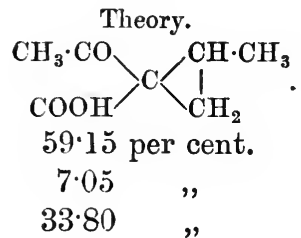

It was therefore acetylmethyltrimethylenecarboxylic acid $(1,2,1)$. This acid does not appear to be decomposed by boiling with alcoholic potash. When heated in a test-tube, it is split up into carbonic anhydride and a low-boiling oil, which has the same peculiar odour as acetyltrimethylene. Unfortunately, owing to the small amount of material at my disposal, I was not able to purify and analyse it. It is probably acetylmethyltrimethylene $(1,2), \mathrm{CH}_{3} \cdot \mathrm{CO} \cdot \mathrm{CH}\left\langle_{\mathrm{CH}_{2}}^{\mathrm{CH} \cdot \mathrm{CH}_{3}}{ }^{\prime}\right.$.

Acetylmethyltrimethylenecarboxylic acid is fairly easily soluble in water, but less so than acetyltrimethylenecarboxylic acid.

The ammonium salt was prepared by dissolving the free acid in ammonia and allowing the excess of the latter to evaporate over sulphuric acid in a vacuum. On adding silver nitrate to the solution of this salt, the silver salt of the acid was precipitated as a white curdy mass slightly soluble in water. After collecting, washing well with water, and drying over sulphuric acid in a vacuum, the following results were obtained on analysis (see p. 855):- 
OF CLOSED CARBON-CHAINS.

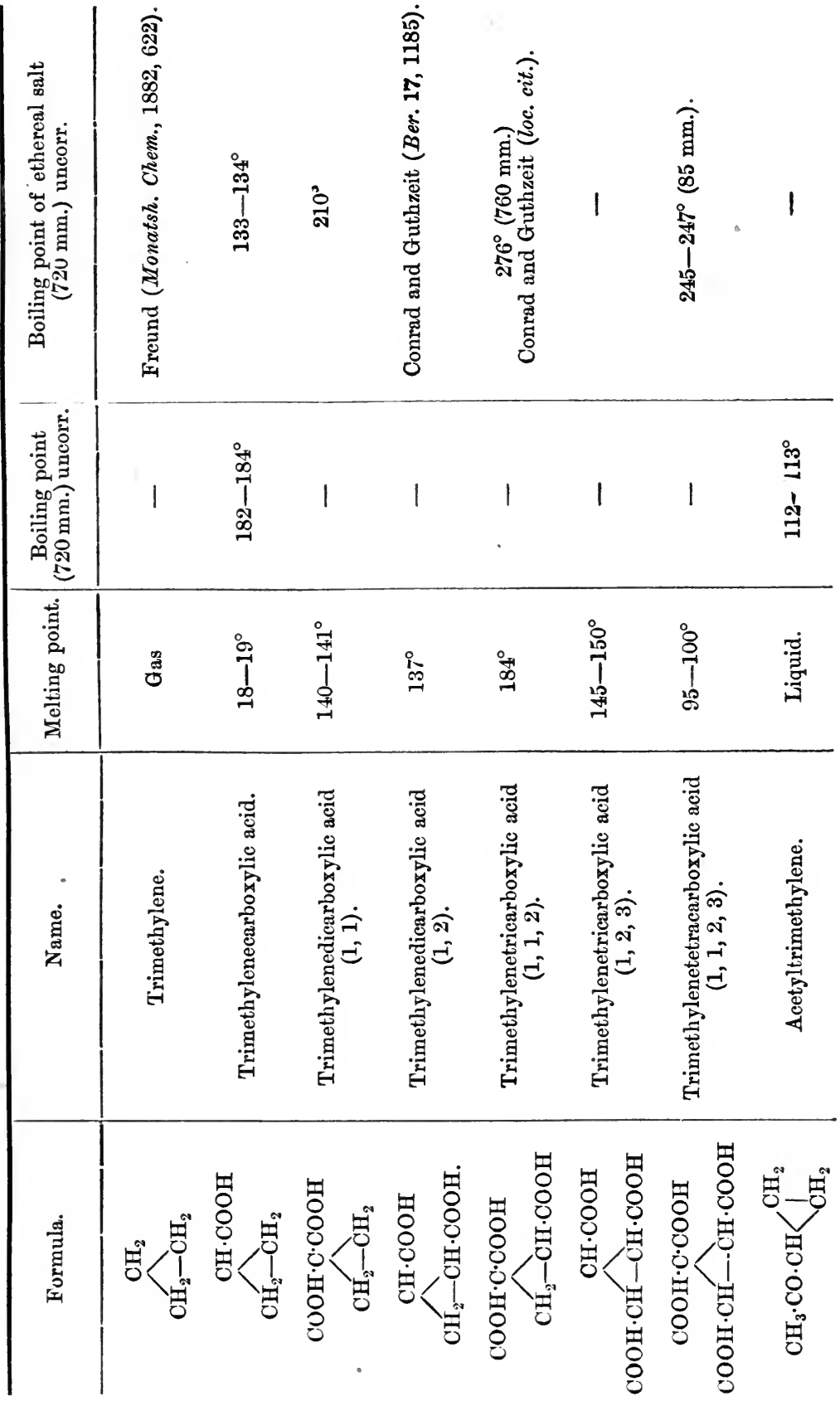




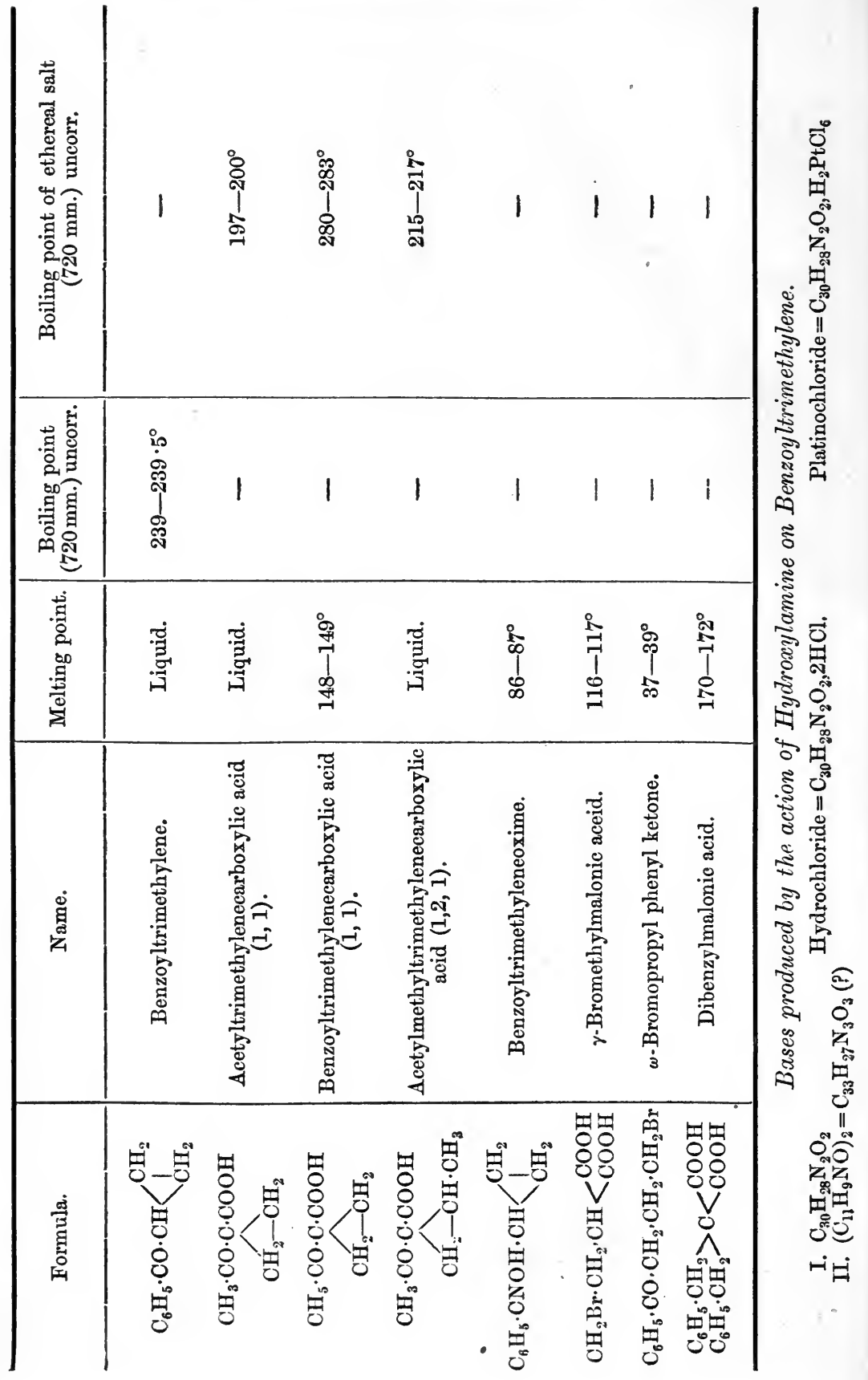


$0 \cdot 3112$ gram substance gave $0 \cdot 1339$ gram silver.

Found.

Ag...... 43.02 per cent.
Theory. $\mathrm{C}_{7} \mathrm{H}_{2} \mathrm{O}_{3}$ Ag. 43.38 per cent.

It is evident, therefore, that the action of propylene bromide on ethyl acetoacetate is exactly similar to that of ethylene bromide, so that it is probable that this reaction for forming trimethylenederivatives is a general one.

In conclusion, I append a table (pp. 853 and 854) of the compounds mentioned in this paper. 

[Reprinted from the Journal of the Chemical Society, November, 1887. Vol. LI.]

THE SYNTHETICAL FORMATION OF CLOSED CARBONCHAINS. PART I (continued). TRIMETHYLENEDICARBOXYLIC ACID.

$$
\text { By W. H. Perkin, Jun., Ph.D. }
$$

Is the previous paper on trimethylene-derivatives (Trans., 47, 818), among other substances an acid was described under the name of trimethylenedicarboxylic acid, which for several reasons was supposed to have the constitution $\prod_{\mathrm{CH}_{2}}^{\mathrm{CH}_{2}}>\mathrm{C}\left\langle{ }_{\mathrm{COOH}}^{\mathrm{COOH}}\right.$, and to be a true trimethylenederivative.

As was shown in this paper, it was not necessary that this acid should have this formula; it is perfectly possible to assume that in this reaction an unsaturated compound had been formed, such as that represented by the formula-

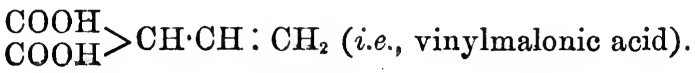


One of the grounds given as being greatly in favour of the trimethylene formula was that the ethereal salt of this acid when treated with benzyl chloride and sodium ethylate did not give the ethereal salt of benzyl vinylmalonic acid-

$$
\stackrel{\mathrm{COOH}}{\mathrm{COOH}}>\mathrm{C}\left(\mathrm{C}_{7} \mathrm{H}_{7}\right) \cdot \mathrm{CH}: \mathrm{CH}_{2},
$$

which it should have done had it been simply a mono-substituted ethyl malonate.

Fittig and Marbarg (Ber., 18, 3414) have since doubted the value of this proof, because in carrying out the experiment I mixed the ethylic trimethylenedicarboxylate first with the benzyl chloride, and then added the sodium ethylate, which of course admits of the possibility of the sodium ethylate acting on the benzyl chloride at once, before any sodium compound of the ethylic trimethylenedicarboxylate had been formed.

In order to clear up this point, I have repeated this experiment in the following way:-14 grams of ethylic trimethylenedicarboxylate was first carefully added to a cold solution of 1.8 grams of sodium dissolved in 20 grams of absolute alcohol, and after the mixture had stood for about two minutes it was treated with 9.5 grams of benzyl chloride and heated to boiling on a water-bath for about an hour, at the end of which time the prodnct showed neutral reaction. Water was then added, the precipitated oil extracted twice or thrice with ether, the ethereal solution washed with water, dried over calcic chloride, and the ether distilled off.

The residue on fractioning distilled orer almost entirely between $175^{\circ}$ and $220^{\circ}$, leaving only a very small residue behind. There had evidently been no ethylic benzyl vinylmalonate formed, as a substance of that constitution would have a much higher boiling point. On submitting this fraction to hydrolysis, it split up into trimethylenedicarboxylic acid and benzyl ethyl ether, $\mathrm{C}_{6} \mathrm{H}_{5} \cdot \mathrm{CH}_{2} \cdot \mathrm{O} \cdot \mathrm{C}_{2} \mathrm{H}_{5}$, the amount of the latter which was present being almost that which should have been formed had all the benzyl chloride reacted simply with the sodic ethylate. It is therefore clear that ethylic trimethylenedicarboxylate, as before stated, does not form a sodium compound when treated with sodium ethylate. Fittig and Marburg also state that I, in my previous publication, laid great stress on the fact that vinaconic acid (trimethylenedicarboxylic acid) did not combine with bromine as supporting the view that the acid was a trimethylene-derivative. This, however, is not so. Freund (Monatshefte, 1882, 625) has shown in his experiments on trimethylene that this gas is acted on by bromine, though very slowly, trimethylene bromide being formed- 
$\overbrace{\mathrm{CH}_{2}-\mathrm{CH}_{2}}^{\mathrm{CH}_{2}}+\mathrm{Br}_{2}=\mathrm{CH}_{2} \mathrm{Br} \cdot \mathrm{CH}_{2} \cdot \mathrm{CH}_{2} \mathrm{Br}$.

It always appeared to me remarkable, in considering this result, that trimethylenedicarboxylic acid if it really were a trimethylenederivative should not combine with bromine at all. In order to test this, I dissolved some of the acid in chloroform, added an excess of bromine, and let the mixture stand 14 days in the dark. At the end of this time, no apparent change had taken place, and on allowing the mixture to evaporate in an open dish a white crystalline acid was obtained, which proved to be unaltered trimethylenedicarboxylic acid. The same results were also obtained by Roeder (Annalen, 227, 18), and Fittig, in his theoretical paper on the constitntion of vinaconic acid (ibid., 227, 29), comes to the conclusion that this acid is either not at all, or at all events only very slightly, acted on by bromine at ordinary temperatures.

Fittig and Marburg at a later date (Ber., 18, 3413) repeated the experiment in sunlight, and found that under these conditions a change did slowly take place, a brominated acid of the formula $\mathrm{C}_{3} \mathrm{H}_{4} \mathrm{Br}_{2}(\mathrm{COOH})_{2}$ being formed, a result which shows now clearly the perfect analogy existing between the reactions of trimethylene itself and those of its dicarboxylic acid.

The further examination of this dibromo-acid must prove conclusively whether trimethylenedicarboxylic acid really is a trimethylene-derivative or not. If this is so, the dibromo-acid must have one of the two following formulæ:-

I.

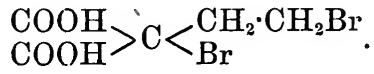

II.

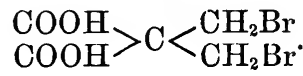

Whereas if it be vinylmalonic acid, the dibromo-acid must have the formula-

\section{$(\mathrm{COOH})_{2} \cdot \mathrm{CH} \cdot \mathrm{CHBr} \cdot \mathrm{CH}_{2} \mathrm{Br}$.}

Some very interesting experiments, which tend strongly to support the trimethylene formula for the acid in question, have lately been instituted by Franchimont (Rec. Trav. Chim., 1885, 398 and 399). This chemist has, during the course of his experiments on the action of pure nitric acid on organic compounds in the fatty series, made the discovery that mono-substituted malonic acids when treated with pure nitric acid are completely and rapidly decomposed with evolution of 2 mols. of carbonic anhydride, whereas the di-substituted malonic acids under similar treatment remain perfectly unaltered.

Franchimont was kind enough to include trimethylene- and tetra- 
methylene-dicarboxylic acid in his investigation, and as a result he found that these two acids behaved exactly as di-substituted malonic acids. Even after standing for 10 days with the nitric acid, not a single bubble of carbonic anhydride was evolved in either case, and on extracting with ether he was able to recover almost the entire quantity of the acids unchanged.

Turning from the chemical to the physical properties of trimethylenedicarboxylic acid, it is interesting to see that here also all the experiments which have been made on the refractive index and magnetic rotation of the ethylic salt of this acid fully bear out the view that it is really a trimethylene-derivative and not vinylmalonic acid.

Dr. Gladstone was kind enough to determine the refractive index of ethylic trimethylenedicarboxylate, and has given me the following account of his experiments; the measurements were made at a temperature of $7^{\circ}:-$

$$
\begin{aligned}
\text { Specific gravity .......... } & =1.0708 \\
\text { Refraction for A.......... } & =1.4351 \\
, \quad \text { D......... } & =1.4399 \\
\text { H......... } & =1.4554 \\
\text { Specific refraction for A ... } & =0.4063 \\
\text { "dispersion . . ... } & =0.0190 \\
\text { Refractive equivalent A ... } & =75.57
\end{aligned}
$$

The conclusion which Dr. Gladstone draws from these experiments is that ethylic trimethylenedicarboxylate is a saturated compound. The calculated refractive equivalent of a substance of the formula $\mathrm{C}_{9} \mathrm{H}_{14} \mathrm{O}_{4}$ is $75 \cdot 6$, a number which agrees closely with that found.

Again, the dispersion of a saturated substance of this formula should be about 0.0190 , which was actually found. If, on the other hand, this ethereal salt bad contained a double band, the dispersion would have been from 0.0240 to 0.0250 .

The experiments on the magnetic rotation of ethylic trimethylenedicarboxylate, which were conducted by my father, show also very clearly that this substance must be a saturated body. The following are the results of his experiments :-

Specific gravity :

$$
\begin{aligned}
& d \frac{15^{\circ}}{15^{\circ}}=1 \cdot 06455, \\
& d \frac{25^{\circ}}{25^{\circ}}=1 \cdot 05657 .
\end{aligned}
$$




\begin{tabular}{c|c|c}
\multicolumn{1}{c|}{$t}$. & Sp. rotation. & Mol. rotation. \\
\hline $17 \cdot 0^{\circ}$ & $0 \cdot 9454$ & $9 \cdot 191$ \\
$17 \cdot 5$ & $0 \cdot 9439$ & $9 \cdot 180$ \\
$18 \cdot 0$ & $0 \cdot 9436$ & $9 \cdot 181$ \\
$18 \cdot 5$ & $0 \cdot 9389$ & $9 \cdot 138$ \\
$14 \cdot 4$ & $0 \cdot 9416$ & $9 \cdot 136$ \\
$14 \cdot 4$ & $0 \cdot 9443$ & $9 \cdot 162$ \\
$14 \cdot 4$ & $0 \cdot 9465$ & $9 \cdot 183$ \\
$14 \cdot 8$ & $0 \cdot 9435$ & $9 \cdot 157$ \\
\hline $16 \cdot 1$ & $0 \cdot 9434$ & $9 \cdot 166$
\end{tabular}

The calculated molecular rotation of a saturated substance of the formula-<smiles>CCOC(=O)OCC1(CC)CC1</smiles>

would be 8.842 , whereas the calculated magnetic rotation of ethylic vinylmalonate is $10 \cdot 258$. The numbers found show, therefore, clearly that the substance is not ethylic vinylmalonate.

From the results of all these experiments, it is extremely probable that trimethylenedicarboxylic acid $(1,1)$ really has the formula-

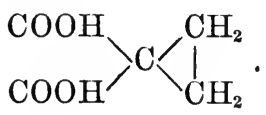

I hope at some later date to be able to prepare vinylmalonic acid, in order that it may be possible by direct comparison to decide between these two formulø. 

THE SYNTHETICAL FORMATION OF CLOSED CARBONCHAINS. Pari I (continued). THE ACTION OF ETHYLENE BROMIDE ON THE SODIUM-DERIVATIVES OF ETHYLIC ACETOACETATE, BENZOYLACETATE, AND ACETONEDICARBOXYLATE.

$$
\text { By P. C. Freer, Ph.D., and W. H. Perkin, Jun., Ph.D. }
$$

IN two papers bronght before the notice of the Society by one of as a short time ago (this vol., pp. 1 and 702), the remarkable difference in the behaviour of the sodium-derivatives of ethylic malonate on the one hand and those of ethylic acetoacetate, benzoylacetate, and acetonedicarboxylate on the other hand, towards trimethylene bromide was discussed.

It was shown that in the case of ethylic malonate, a tetramethylenederivative, that is, ethylic tetramethylenedicarboxylate,

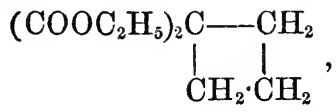

was formed, whereas in the case of ethylic sodacetoacetate, for example, no tetramethylene-derivative was produced, but a reaction took place which is represented by the following equation:-

$$
\begin{aligned}
& .{ }^{2} \mathrm{COOC}_{2} \mathrm{HH}_{5}>\mathrm{CHNa}+\mathrm{BrCH}_{2} \cdot \mathrm{CH}_{2} \cdot \mathrm{CH}_{2} \mathrm{Br}= \\
& \mathrm{COOC}_{2} \mathrm{H}_{5} \cdot \stackrel{\mathrm{CH} \cdot \mathrm{CH}_{2} \cdot \mathrm{CH}_{2}}{\mathrm{CH}_{3} \cdot \mathrm{C}}-\mathrm{O} \mathrm{CH}_{2}+\underset{\mathrm{COOC}_{2} \mathrm{H}_{5}}{\mathrm{CH}_{3} \mathrm{CO}}>\mathrm{CH}_{2}+2 \mathrm{NaBr} .
\end{aligned}
$$

with formation of ethylic methyldehydrohexonecarboxylate.

In a paper published some time since, the results of a series of experiments on the action of ethylene bromide on the sodium compounds of ethylic acetoacetate and benzoylacetate were communicated, and the substances formed in the reaction were assumed to be trimethylene-derivatives. Thus, for example, the reaction between ethylic sodacetoacetate and ethylene bromide was represented as taking place according to the following equation :-

$$
{ }_{2}^{\underset{\mathrm{COOC}_{2} \mathrm{H}_{5}}{\mathrm{CH}_{3} \cdot \mathrm{CO}}>\mathrm{CHNa}+\mathrm{BrCH}_{2} \cdot \mathrm{CH}_{2} \mathrm{Br}} \underset{\underset{\mathrm{COOC}_{2} \mathrm{H}_{5}}{\mathrm{COO}_{\mathrm{CH}_{2}}}>\stackrel{\mathrm{CH}}{\mathrm{CH}_{3} \cdot \mathrm{CO}} !_{\mathrm{CH}_{2}}^{\mathrm{CH}_{3} \cdot \mathrm{CO}_{2}}>\mathrm{CH}_{2}+2 \mathrm{NaBr} .}{\mathrm{COOC}_{2} \mathrm{H}_{5}}+
$$

From the results of the experiments on the action of trimethylene 
bromide on ethylic sodacetoacetate, it was, however, much more likely that the above decomposition would be more correctly represented thus :-

$$
{ }_{2} \underset{\mathrm{COOC}_{2} \mathrm{H}_{5}}{\mathrm{CH}_{3} \cdot \mathrm{CO}}>\mathrm{CHNa}+\mathrm{BrCH}_{2} \cdot \mathrm{CH}_{2} \mathrm{Br}=
$$

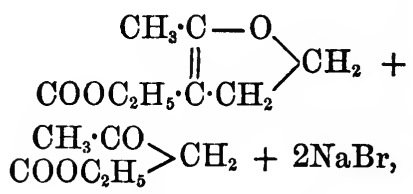

in which case the product would be ethylic methyldehydropentonecarboxylate, and not a derivative of trimethylene as shown in the first equation. This possibility made it necessary to again study this reaction, in the hope of being able to decide whether these compounds really were pentone-derivatives or trimethylene-derivatives.

In experimenting in this direction, a large number of reactions have been studied, most of which can be equally well explained on the assumption of either the trimethylene or the pentone formula, and the only one which has thrown any decisive light on the question is that which takes place when the acid, which has already been described (loc. cit., p. 831) as acetyltrimethylenecarboxylic acid, is distilled. This reaction was mentioned in the previous paper (p. 834), and it is there stated that the acid on distillation is split up into carbonic anhydride, and a colourless oil boiling at $113^{\circ}$ (acetyltrimethylene).

As in these experiments only very small quantities of the acid were worked with, and it now appeared that the further study of this substance might throw considerable light on the question of the constitution of the original acid, this decomposition has been restudied, using much larger quantities of the acid. The result has shown that the oil produced in this reaction is a mixture of two substances, the one which is present in by far the larger quantity being the oil boiling at $113^{\circ}$, which was previously noticed, the other, which we have only found in very small quantities, being an oil of ethereal odour boiling at about $90^{\circ}$. The first of these is acetyltrimethylene, the second methyldehydropentone or the anhydride of acetopropyl alcohol. The constitutional formulæ of these two isomeric substances is therefore-

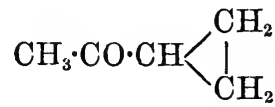

Acetyltrimethylene (b. p. $113^{\circ}$ ).

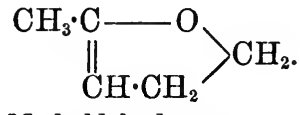

Methyldehydropentone

(b. p. $90^{\circ}($ ?))

The remarkable difference between these two compounds is seen in their behaviour towards water at ordinary temperatures. Acetyltrimethylene dissolves but remains unchanged, whereas methyldehydropentone is converted into acetopropyl alcohol. 


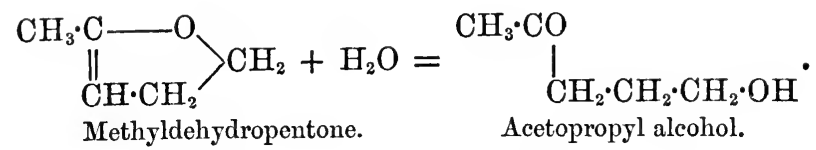

The further examination of the properties of acetyltrimethylene has shown that it is a methyl ketone, and is a saturated compound; the only formula therefore which can be assigned to it (at all events according to the system of nomenclature now in use) is the one given above.

From these results, it follows that the product of the action of ethylene bromide on ethylic sodacetoacetate is a mixture of two substances-ethylic acetyltrimethylenecarboxylate and ethylic methyldehydropentonecarboxylate, which have respectively the following formulæ:-<smiles>CCOC1(CC)CC1</smiles>

and<smiles>CCOC(=O)OCC</smiles>

As the second substance is present in such very small quantities* we propose, for simplicity sake, to treat the product of this reaction in the following paper, as consisting only of ethylic acetyltrimethylenecarboxylate.

We have unfortunately not yet been able to isolate ethylic methyldehydropentonecarboxylate in a state of purity, but we hope, in experimenting further on this subject, to find some means of separating the very small quantity of this substance which is formed in the reaction from the large quantities of ethylic acetyltrimethylenecarboxylate and unchanged ethylic acetoacetate.

The constitution of ethylic acetyltrimethylenecarboxylate, as deduced from the above experiments, is very remarkably supported by the study of its magnetic rotation, and also that of its decomposition products, which has been instituted by my father.

The only constitutional formula possible for ethylic acetyltrimethylenecarboxylate, other than that given above, is that of ethylic vinglacetoacetate, $\mathrm{COOC}_{2} \mathrm{CH}_{5} \cdot \mathrm{CO}>\mathrm{CH} \cdot \mathrm{CH}: \mathrm{CH}_{2}$.

Now this differs from ethylic allylacetoacetate in having one $\mathrm{CH}_{2}$ less than the latter, it therefore follows that the magnetic rotation of ethylic vinylacetoacetate should be that of ethylic allylacetoacetate $(10.383)$ - the value of a $\mathrm{CH}_{2}$-group $(1 \cdot 023)$, that is, $9 \cdot 360$. Now the magnetic rotation of the purest sample of ethylic acetyltrimethylene-

* Under some conditions, which we have as yet been unable to determine, it does not appear to be formed at all, a fact which is borne out by the study of the numbers obtained as the magnetic rotation of different samples. 
carboxylate yet examined was found to be $8 \cdot 198$, which shows at once that it cannot be ethylic vinylacetoacetate.

Against the conclusions deduced above from the results of the chemical examination of the products of the distillation of acetyltrimethylenecarboxylic acid, it may be urged that during this reaction some intermolecular change has taken place; that this is not the case, however, the following considerations will show. The magnetic rotation of acetyltrimethylene is $5 \cdot 245$. Now if acetyltrimethylene is really the basis of the original product of the action of ethylene bromide on ethylic sodacetoacetate, it follows that the magnetic rotation of this substance should differ from that of ethylic acetyltrimethylenecarboxylate by the value of $\left(\mathrm{COOC}_{2} \mathrm{H}_{5}-\mathrm{H}\right)$.

The value of this group, as has previously been mentioned (this vol., p. 708), is easily deduced by subtracting the magnetic rotation of acetone from that of ethylic acetoacetate, and is thus found to be 2.987 .

If we now add this to the magnetic rotation of acetyltrimethylene, we get as the calculated magnetic rotation of ethylic acetyltrimethylenecarboxylate $8 \cdot 232$, a number which agrees sufficiently with that actually found $(8 \cdot 198)$, so that at present both the chemical reactions and the physical measurements are in favour of the assumption that these compounds are trimethylene-derivatives.

Several other reactions of acetyltrimethylenecarboxylic acid and its ethereal salt have been studied, among which the more important are the following :-

Acetyltrimethylenecarboxylic acid, when boiled with water, is slowly converted into carbonic anhydride and acetopropyl alcohol, thus :

$$
\left.\underset{\mathrm{COOH}}{\mathrm{CH}_{3} \cdot \mathrm{CO}}\right\rangle_{\mathrm{CH}}\left\langle\underset{\mathrm{CH}_{2}}{\mathrm{CH}_{2}}+\mathrm{H}_{2} \mathrm{O}=\underset{\text { Acetopropyl alcohol. }}{\mathrm{CH}_{3} \cdot \mathrm{CO} \cdot \mathrm{CH}_{2} \cdot \mathrm{CH}_{2} \cdot \mathrm{CH}_{2} \cdot \mathrm{OH}}+\mathrm{CO}_{2} .\right.
$$

Ethylic acetyltrimethylenecarboxylate dissolves easily in a concentrated aqueous solution of hydrogen bromide, being thereby converted into ethylic $\omega$-bromethylacetoacetate :-

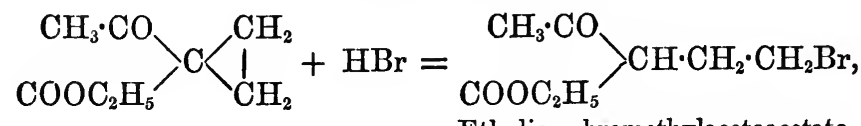

Ethylic $\omega$-bromethylacetoacetate.

a reaction which is a counterpart of the formation of bromethylmalonic acid by the action of hydrogen bromide on trimethylenedicarboxylic acid.

These reactions are undoubtedly difficult to explain on the 
assumption that these substances are trimethylene-derivatives, as in that case we are bound to assume that by the action of hydrogen bromide, or, more curiously still, by simply boiling with water, the trimethylene-ring is split. At the same time, it must be remembered that the properties of such a ring as the trimethylene-ring are at present totally unknown, and therefore that no definite argument can be drawn from these peculiarities.

While carrying out this research, experiments were also made on the action of ethylene bromide on the disodium-derivative of ethylic acetonedicarboxylate, originally in the hope of obtaining pentamethylene-derivatives, thus :-

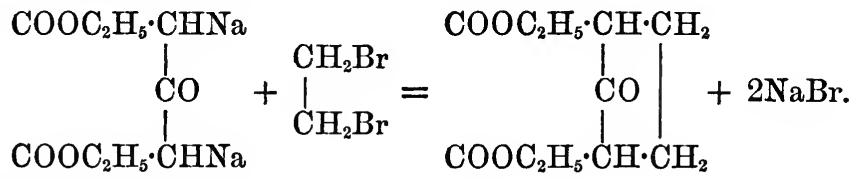

It was, however, soon discovered that in this case also trimethylenederivatives were formed, the reaction apparently taking place in the following way :-

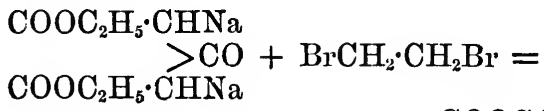

$\mathrm{COOC}_{2} \mathrm{H}_{5} \cdot \mathrm{CHNa}$

$\mathrm{COOC}_{2} \mathrm{H}_{5} \cdot \mathrm{CH} \cdot \mathrm{CH}_{2} \cdot \mathrm{CH}_{2} \mathrm{Br}+\mathrm{NaBr}$.

$\mathrm{COOC}_{2} \mathrm{H}_{5} \cdot \mathrm{CH}_{2} \cdot \mathrm{CO}$ $\mathrm{COOC}_{2} \mathrm{H}_{5}>\mathrm{CNa} \cdot \mathrm{CH}_{2} \cdot \mathrm{CH}_{2} \mathrm{Br}=$

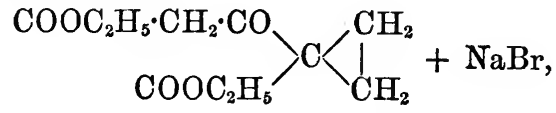

the product of the reaction being ethylic acetyltrimethylenedicarboxylate.

The proof of this fact is that on boiling the acid produced by the hydrolysis of this ethereal salt with water, it is decomposed into carbonic anhydride and acetopropyl alcohol, a reaction which is expressed by the following equation :-

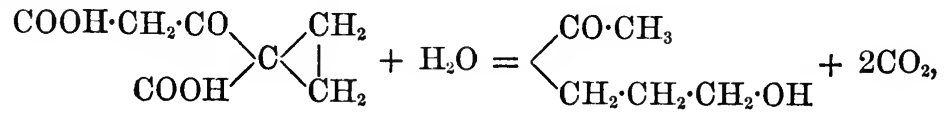

a reaction resembling in every respect the formation of acetopropyl alcohol and $1 \mathrm{~mol}$. of carbonic anhydride, from acetyltrimethylenecarboxylic acid, by the action of boiling water. 
It is obvious that there are still several points of interest in this research which require more satisfactory explanation, and it is hoped that further experiments which are being carried on will throw some light on these difficulties.

Ethylic Acetyltrimethylenecarboxylate,

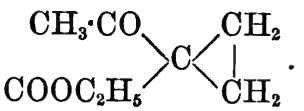

In commencing this research, it was necessary in the first place to prepare pure ethylic acetyltrimethylenecarboxylate.

For this purpose, a large quantity of the crude product of the action of ethylene bromide on ethylic sodacetoacetate (prepared as described in a previous paper, Trans., 1885, 829), using, however, 300 grams of ethylic acetoacetate in each experiment, was carefully fractioned, and the fraction $180-210^{\circ}$ first separated. This portion was then submitted to most careful fractioning with a long colonna. After repeating this operation about 20 times, an oil was obtained boiling constantly at $195-196^{\circ}$, which gave the following numbers on analysis :-

I. 0.2031 gram substance gave 0.1465 gram $\mathrm{H}_{2} \mathrm{O}$ and 0.4565 gram $\mathrm{CO}_{2}$.

II. $0 \cdot 1631$ gram substance gave $0 \cdot 1189$ gram $\mathrm{H}_{2} \mathrm{O}$ and $0 \cdot 3682$ gram $\mathrm{CO}_{2}$.

\begin{tabular}{|c|c|c|c|}
\hline \multirow[b]{3}{*}{ C .. } & \multirow[b]{2}{*}{$\begin{array}{l}\text { Theory. } \\
\mathrm{C}_{8} \mathrm{H}_{12} \mathrm{O}_{3} \text {. }\end{array}$} & \multicolumn{2}{|c|}{ Found. } \\
\hline & & I. & II. \\
\hline & 61.54 p. c. & $61 \cdot 30$ & $61 \cdot 54 \mathrm{p}$ \\
\hline $\mathrm{H} \ldots .$. & $7 \cdot 69 \leadsto$ & $8 \cdot 01$ & 8.04, \\
\hline $0 \ldots \ldots$ & 30.77 & $30 \cdot 69$ & $30 \cdot 42$ \\
\hline
\end{tabular}

The determination of the magnetic rotation gave the following numbers :-

$$
\begin{aligned}
& d_{\overline{15^{\circ}}}^{15^{\circ}}=1 \cdot 05174 \\
& d_{\overline{20^{\circ}}}^{20^{\circ}}=1 \cdot 04810 \\
& d_{\overline{25^{\circ}}}^{25^{\circ}}=1 \cdot 04390
\end{aligned}
$$


Magnetic Rotations.

\begin{tabular}{c|c|c}
$t$. & Sp. rotation. & Mol. rotation. \\
\hline $19 \cdot 7^{\circ}$ & $1 \cdot 0245$ & $8 \cdot 471$ \\
$19 \cdot 7$ & $1 \cdot 0175$ & $8 \cdot 417$ \\
$19 \cdot 4$ & $1 \cdot 0221$ & $8 \cdot 451$ \\
$19 \cdot 4$ & $1 \cdot 0189$ & $8 \cdot 425$ \\
$19 \cdot 4$ & $1 \cdot 0201$ & $8 \cdot 433$ \\
$18 \cdot 0$ & $1 \cdot 0219$ & $8 \cdot 439$ \\
$18 \cdot 0$ & $1 \cdot 0165$ & $8 \cdot 395$ \\
$17 \cdot 6$ & $1 \cdot 0233$ & $8 \cdot 448$ \\
$17 \cdot 6$ & $1 \cdot 0203$ & $8 \cdot 423$ \\
\hline Average $18 \cdot 7$ & $1 \cdot 0205$ & $8 \cdot 434$ \\
\hline
\end{tabular}

On still further fractioning this preparation, a product was obtained which boiled at $195-195 \cdot 5^{\circ}$, and which gave the following numbers :-

Sp. gr.

$$
\begin{aligned}
& d \frac{15^{\circ}}{15^{\circ}}=1 \cdot 05152, \\
& d \frac{25^{\circ}}{25^{\circ}}=1.0439 .
\end{aligned}
$$

Magnetic Rotations.

\begin{tabular}{c|c|c}
$t$. & Sp. rotation. & Mol. rotation. \\
\hline $15 \cdot 5^{\circ}$ & $\begin{array}{c}1 \cdot 0080 \\
1 \cdot 0120\end{array}$ & $\begin{array}{r}8 \cdot 310 \\
8 \cdot 346\end{array}$ \\
\hline $16 \cdot 5$ & $8 \cdot 328$
\end{tabular}

As these numbers were still not so good as might be desired, a specimen of ethylic acetyltrimethylenecarboxylate was prepared from the silver salt of acetyltrimethylenecarboxylic acid by treatment with ethyl iodide. In order to do this, 60 grams of carefully purified acetyltrimethylenecarboxylic acid were dissolved in about five times its weight of water, carefully neutralised with dilute ammonia, and then mixed with a slight excess of a solution of nitrate of silver. This caused the precipitation of a very small quantity of silver salt, which was filtered off and discarded.

The clear solution was now evaporated in an open porcelain dish over a free flame as rapidly as possible to about one-third of its bulk, quickly filtered from a small quantity of a black precipitate which always forms, and allowed to stand in a cool place for 12 hours. At the 
end of this time, it was found that a considerable quantity of silver salt had crystallised out on the bottom of the dish, in the hard greyishwhite nodular masses which are so characteristic of this acid. These were collected, well washed with water, and allowed to dry on a porous plate over sulphuric acid in a vacuum. On further evaporation of the mother-liquor, another crop of crystals was obtained, and this operation was continued until no more salt separated.

The dry silver salt thus prepared was then powdered and digested with an excess of ethyl iodide and some pure ether for four hours, at the end of which time the decomposition was complete. The ethereal solution was now filtered from the precipitated iodide of silver, the latter well washed with pure ether, and the ethereal solution evaporated.

In this way, a colourless oil was obtained, which on fractioning boiled constantly at $195 \cdot 6-196^{\circ} 6^{\circ}$.

\section{Analysis.}

0.1793 gram substance gave 0.1304 gram $\mathrm{H}_{2} \mathrm{O}$ and 0.4067 gram $\mathrm{CO}_{2}$.

\begin{tabular}{|c|c|c|}
\hline & $\begin{array}{l}\text { Theory. } \\
\mathrm{C}_{8} \mathrm{H}_{12} \mathrm{O}_{3} \text {. } \\
61 \cdot 54 \text { per cent. }\end{array}$ & $\begin{array}{l}\text { Found. } \\
61 \cdot 76 \text { per cent. }\end{array}$ \\
\hline & $7 \cdot 69$ & 8.02 \\
\hline$\ldots$ & $30 \cdot 77$ & $30 \cdot 22$ \\
\hline
\end{tabular}

The sp. gr. and magnetic rotation of this preparation gave the following results :-

$$
\begin{aligned}
& d \frac{15^{\circ}}{15^{\circ}}=1 \cdot 04703, \\
& d \frac{25^{\circ}}{25^{\circ}}=1 \cdot 03930 .
\end{aligned}
$$

Magnetic Rotations.

\begin{tabular}{c|c|c}
$t$. & Sp. rotation. & Mol. rotation. \\
\hline $14 \cdot 0^{\circ}$ & $0 \cdot 9960$ & $8 \cdot 238$ \\
$14 \cdot 25$ & $0 \cdot 9975$ & $8 \cdot 252$ \\
$16 \cdot 5$ & $0 \cdot 9945$ & $8 \cdot 241$ \\
\hline Arerage $14 \cdot 76$ & $0 \cdot 9960$ & $8 \cdot 245$
\end{tabular}

This product was then again carefully fractioned and re-examined with the following results :-

$$
d \frac{15^{\circ}}{15^{\circ}}=1 \cdot 04753
$$


Magnetic Rotations.

\begin{tabular}{c|c|c}
\multicolumn{1}{c|}{$t}$. & Sp. rotation. & Mol. rotation. \\
\hline $15^{\circ}$ & $0 \cdot 9873$ & $8 \cdot 171$ \\
15 & $0 \cdot 9943$ & $8 \cdot 229$ \\
15 & $0 \cdot 9903$ & $8 \cdot 195$ \\
\hline Average 15 & $0 \cdot 9906$ & $8 \cdot 198$
\end{tabular}

The value $8 \cdot 198$, which is believed to most nearly represent the true magnetic rotation of ethylic acetyltrimethylenecarboxylate, differs very widely from the number previonsly published for that substance, namely, 9.817 (Trans., loc. cit., p. 831). The preparation from which this latter value was deduced was specially prepared for the determination of its physical properties by treating a large quantity of the crude product of the action of ethylene bromide on ethylic acetoacetate, boiling at $180-220^{\circ}$, with potash until about three-fourths had been hydrolysed. The remaining oil was then fractioned and found to boil between $197^{\circ}$ and $210^{\circ}$. By repeated refractioning a portion was separated boiling between $204^{\circ}$ and $206^{\circ}$, which on examination gave the numbers already pablished. As it was possible that some mistake had been made, the product was re-examined with the following results :-

Magnetic Rotation.

\begin{tabular}{c|c|c}
$t$. & Sp. rotation. & Mol. rotation. \\
\hline $12 \cdot 5^{\circ}$ & $1 \cdot 1696$ & $9 \cdot 865$ \\
$12 \cdot 5$ & $1 \cdot 1596$ & $9 \cdot 780$ \\
$12 \cdot 5$ & $1 \cdot 1643$ & $9 \cdot 820$ \\
$12 \cdot 5$ & $1 \cdot 1623$ & $9 \cdot 802$ \\
\hline Average $12 \cdot 5$ & $1 \cdot 1636$ & $9 \cdot 817$
\end{tabular}

The number 9.817 is the same as that obtained in the original measurements.

On refractioning the product, it was found to boil, with the exception of one or two drops at the end, between $204^{\circ}$ and $206^{\circ}$. It is at present impossible to understand what this substance was, as even if it had consisted entirely of ethylic methyldehydropentonecarboxylate, the rotation would not have been so high. 


\section{Action of Water on Acetyltrimethylenecarboxylic Acid.}

Acetyltrimethylenecarboxylic acid when boiled with water is slowly split up into acetopropyl alcohol and carbonic anhydride as mentioned in the introduction.

In preparing acetyltrimethylenecarboxylic acid, it is not necessary to start from the pure ethereal salt or to condnct the hydrolysis at ordinary temperatures. Considerable quantities of this acid have been obtained in the following way. The fraction $180-210^{\circ}$ of the crude product of the action of etbylene bromide on ethylic acetoacetate is mixed with twice the calculated quantity of potash, dissolved in methyl alcohol (or pure ethyl alcohol), and boiled for five hours on a water-bath. The alcohol is then distilled off, the product dissolved in water, and evaporated in an open basin on a water-bath till the last traces of alcohol have been expelled. When cool, dilute sulphuric acid is added in excess, and the acid extracted about 10 times with pure ether. The ethereal solution, after drying over calcic chloride and evaporating, deposits the acetyltrimethylenecarboxylic acid as a slightly brownish-coloured oil, which is pure enough for ordinary purposes. If required in a pure state, it is treated as described previously (loc. cit., 832).

To prepare acetopropyl alcohol, the crude acid is dissolved in five times its weight of water, and boiled with reflux condenser until the evolution of carbonic anhydride has ceased, which is usually the case in about 14 hours. If the resulting clear solution, after cooling, is saturated with anhydrous potassic carbonate, the acetopropyl alcohol separates on the surface of the liquid as a brownish-coloured oily layer, which should be extracted at least 20 times with pure ether. The ethereal solution is then dried over carbonate of potash, filtered, and distilled, when the crude alcohol remains behind as a slightly brownish-coloured syrup.

This crude product cannot be purified by distillation under ordinary pressures, as it is to a considerable extent decomposed into water and its anhydride. When rapidly heated under a pressure of $100 \mathrm{~mm}$., however, it distils over between $140^{\circ}$ and $150^{\circ}$ almost without decomposition. After two distillations, a product was obtained boiling constantly at $144-145^{\circ}$, which gave the following numbers on analysis :-

0.2396 gram substance gave 0.2095 gram $\mathrm{H}_{2} \mathrm{O}$ and 0.5174 gram $\mathrm{CO}_{2}$.

\begin{tabular}{|c|c|c|}
\hline $\mathrm{CH}_{3} \cdot \mathrm{CO}$ & $\begin{array}{l}\text { Theory. } \\
\cdot \mathrm{CH}_{2} \cdot \mathrm{CH}_{2} \cdot \mathrm{CH}_{2} \cdot \mathrm{OH} \text {. }\end{array}$ & Found. \\
\hline C ..... & 58.82 per cent. & 58.88 per cent. \\
\hline & $9 \cdot 81 \quad$ & $9 \cdot 71$ \\
\hline $0 \ldots \ldots$ & $31 \cdot 37$ & $31 \cdot 41$ \\
\hline
\end{tabular}


The determinations of its sp. gr. and magnetic rotation gave the following numbers :-

$$
\begin{aligned}
& d \frac{15^{\circ}}{15^{\circ}}=1.00514, \\
& d \frac{20^{\circ}}{20^{\circ}}=1.00197, \\
& d \frac{25^{\circ}}{25^{\circ}}=0.99896 .
\end{aligned}
$$

\begin{tabular}{|c|c|c|c|}
\hline & $t$. & Sp. rotation. & Mol. rotation. \\
\hline & $\begin{array}{l}25^{\circ} \\
25 \\
25 \\
25\end{array}$ & $\begin{array}{l}0.9787 \\
0.9773 \\
0.9802 \\
0.9735\end{array}$ & $\begin{array}{l}5 \cdot 552 \\
5 \cdot 543 \\
5 \cdot 560 \\
5 \cdot 522\end{array}$ \\
\hline Average & 25 & 0.9774 & $5 \cdot 544$ \\
\hline
\end{tabular}

Magnetic Rotations.

On comparing this value, $5 \cdot 544$, with that previously obtained (Trans., 1887, 719) for acetobutyl alcohol,

$$
\mathrm{CH}_{3} \cdot \mathrm{CO} \cdot \mathrm{CH}_{2} \cdot \mathrm{CH}_{2} \cdot \mathrm{CH}_{2} \cdot \mathrm{CH}_{2} \cdot \mathrm{OH}=6 \cdot 502,
$$

it will be seen that the difference for the $\mathrm{CH}_{2}$-group $=0.958$, a value closely corresponding with the true value $=1.023$; this clearly shows that the constitution of acetopropyl alcohol must be similar to that of acetobutyl alcohol, which is the next member higher in the series. The reason why the numbers do not agree exactly is that it was found impossible to obtain acetopropyl alcohol free from its anhydride.

Experiments are in progress with the object of further proving the constitution of acetopropyl alcohol to be that assigned to it above.

Acetopropyl alcohol is a thick, colourless syrup which mixes with water in all proportions. When slowly heated, it is completely decomposed into its anhydride and water, so that the distillate from such an operation is found to consist of two layers, which, however, in a few hours completely amalgamate again with regeneration of acetopropyl alcohol.

Acetopropyl alcohol does not appear to have any action on an ammoniacal solution of silver at the ordinary temperature; on warming the mixture in a water-bath, however, reduction rapidly sets in. It does not reduce Fehling's solution in the cold, and only very slowly on boiling. 
If the aqueous solution of acetopropyl alcohol is mixed with a solution of phenylhydrazine hydrochloride in sodic acetate, the phenylhydrazine compound is precipitated as a yellowish oil which was not further examined as it did not crystallise. When boiled with an excess of concentrated nitric acid, acetopropyl alcohol is violently attacked with formation of red fumes. If, however, five drops of the alcohol be mixed with two drops of concentrated nitric acid and gently warmed, an intense pinkish-brown solution is obtained, which is changed to yellow on the addition of alkali. When treated with iodine and potash, acetopropyl alcohol yields iodoform.

\section{Decomposition of Acetyltrimethylenecarboxylic Acid by Heat.}

In studying this decomposition, 15 grams of carefully purified acid contained in a small retort connected with a well-cooled condenser was heated gradually to about $200^{\circ}$ in a metal bath. At about $120^{\circ}$, rapid decomposition set in, carbonic anhydride was evolved, and a small quantity of a colourless oil together with a little water passed over. On raising the temperature to $200^{\circ}$, the principal product of the reaction distilled, leaving a considerable residue in the retort, which has not been examined, but probably consists of complicated condensation products. From 75 grams of acid treated in this way, 35 grams of crude distillate were obtained. This was carefully fractioned in a Würtz flask with a long neck, when about 14 grams of an oil mixed with a little water distilled over between $65^{\circ}$ and $95^{\circ}$. The thermometer then rose rapidly to $135^{\circ}$, a further quantity of 15 grams coming over below this temperature. Above $135^{\circ}$, there was a small residue consisting of high boiling condensation products mixed with a little acetopropyl alcohol.

The fraction $65-95^{\circ}$, after drying over anliydrous potassic carbonate, was refractioned, when it was found that the boiling point had undergone a most remarkable alteration, for now only 4 grams passed over between $85^{\circ}$ and $95^{\circ}$, the remainder boiling almost constantly between $112^{\circ}$ and $114^{\circ}$.

The fraction $85-95^{\circ}$ consists for the most part of the anhydride of acetopropyl alcohol, in a more or less pure state, and in order to prove this, the oil was mixed with twice its weight of water, and allowed to stand at the ordinary temperature; at the end of two days it was found that the oily layer had almost entirely disappeared, it having in fact been converted into acetopropyl alcohol. This was isolated by saturating the liquid, which had previously been carefully freed from oily drops, with potassic carbonate, and extracting several times with ether. On distilling the ethereal solution, after drying it over potassic carbonate, a small quantity of a colourless syrup was 
obtained, which on standing for a short time over sulphuric acid gave the following numbers on analysis :-

0.1405 gram substance gave 0.1241 gram $\mathrm{H}_{2} \mathrm{O}$ and 0.3068 gram $\mathrm{CO}_{2}$.

\begin{tabular}{|c|c|c|c|c|}
\hline $\mathrm{CH}_{3} \cdot \mathrm{CO}$ & Theory. & $\mathrm{I}_{2} \cdot \mathrm{OH}$. & Found. & \\
\hline $\mathrm{C} \ldots \ldots$ & $58 \cdot 82$ & cent. & 59.55 & cent \\
\hline$\ldots$ & $9 \cdot 81$ & " & $9 \cdot 72$ & $川$ \\
\hline O. & $31 \cdot 37$ & ", & $30 \cdot 73$ & \\
\hline
\end{tabular}

These numbers agree sufficiently well to show that acetopropyl alcohol had been formed, and a further examination of the behaviour of this oil with phenylhydrazine hydrochloride and oxidising agents left no doubt as to its identity.

The portion of the original oil boiling at $95-135^{\circ}$ was now refractioned; the principal fraction obtained boiled at $110-120^{\circ}$, and weighed 8 grams. This was mixed with the other sample of oil boiling at $112-114^{\circ}$, and the two submitted to three or four careful distillations. In this way, an oil was obtained boiling constantly at $113-114^{\circ}$, which is identical with that of the acetyltrimethylene described by one of us in a previous paper (loc. cit., 835). It was not again analysed.

This substance was also subjected to a physical examination by my father with the following results :-

Sp. gr.

$$
\begin{aligned}
& d \frac{15^{\circ}}{15^{\circ}}=0.90471 \\
& d \frac{20^{\circ}}{20^{\circ}}=0.90083 \\
& d_{\overline{25^{\circ}}}^{25^{\circ}}=0.89706 .
\end{aligned}
$$

Magnetic Rotations.

\begin{tabular}{c|c|c}
$t$. & Sp. rotation. & Mol. rotation. \\
\hline $23 \cdot 7^{\circ}$ & $1 \cdot 0055$ & $5 \cdot 225$ \\
$22 \cdot 0$ & $1 \cdot 0138$ & $5 \cdot 261$ \\
$22 \cdot 0$ & $1 \cdot 0088$ & $5 \cdot 235$ \\
$22 \cdot 0$ & $1 \cdot 0115$ & $5 \cdot 249$ \\
$22 \cdot 0$ & $1 \cdot 0133$ & $5 \cdot 251$ \\
$22 \cdot 0$ & $1 \cdot 0100$ & $5 \cdot 241$ \\
\hline Average $22 \cdot 3$ & $1 \cdot 0105$ & $5 \cdot 245$
\end{tabular}

Acetyltrimethylene possesses in every respect the characteristics 
of a ketone. It dissolves slowly in sodic bisulphite, forming an easily soluble bisulphite compound which, on the addition of alkalis, is decomposed with separation of the original substance. When mixed with phenylhydrazine, a violent reaction sets in, water separates, and an oily phenylhydrazine compound is formed which has not been further examined.

In order to prove that acetyltrimethylene differs very widely from the anhydride of acetopropyl alcohol, 5 grams of the former substance were mixed with 20 grams of water and allowed to stand for six weeks, the whole being shaken up every day. A considerable quantity of the oil dissolved in the water, and it was thought at first that some decomposition had taken place. The whole was therefore extracted with very pure ether, dried over potassic carbonate, and the ether very carefully distilled off. On fractioning the residue, almost the whole quantity passed over between $110^{\circ}$ and $120^{\circ}$, only a very small residue remaining which could not be driven over without superheating. Acetyltrimethylene is therefore not the anhydride of a ketone alcohol.

On heating with water for 10 hours at $100-120^{\circ}$, acetyltrimethylene appears to be converted into acetopropyl alcohol, but as this is still doubtful no weight can be laid on the result, and the experiment must be repeated.

Action of Hydrogen Bromide on Ethylic Acetyltrimethylenecarboxylate.

\section{Ethylic w-Bromethylacetoacetate, $\underset{\mathrm{COOC}_{2} \mathrm{H}_{5}}{\mathrm{CH}_{3} \cdot \mathrm{CO}}>\mathrm{CH} \cdot \mathrm{CH}_{2} \cdot \mathrm{CH}_{2} \mathrm{Br}$.}

Ethylic acetyltrimethylenecarboxylate combines readily with hydrogen bromide, with evolution of heat and formation of ethylic w-bromethylacetoacetate, as shown in the introduction, the reaction. being parallel to the formation of ethylic w-bromethyl malonate from ethylic trimethylenedicarboxylate.

In order to prepare ethylic $\omega$-bromethylacetoacetate, pure ethylic acetyltrimethylenecarboxylate is dissolved in three times its volume of concentrated hydrogen bromide solution (sp. gr. 1.85), the mixture being kept well cooled. After being allowed to stand for about 10 minutes at the ordinary temperature, it is poured into ice-water. This causes the separation of a heavy oil which is extracted from the aqueous solution by means of ether. After washing with water, and drying over calcic chloride, the ether is distilled off, when nearly pure ethylic $\omega$-bromethylacetoacetate remains behind in the form of a heavy oil, of slightly yellowish colour. In order to obtain it in a state fit for analysis, it was allowed to stand for some days over sticks of caustic potash in a vacuum. 
A bromine determination gave the following result, which approximately agrees with the formula $\mathrm{C}_{8} \mathrm{H}_{13} \mathrm{BrO}_{3}$.

Theory.

Br........ 33.47 per cent.
Found.

$32 \cdot 89$ per cent.

Ethylic w-bromethylacetoacetate is a heavy oil, with an odour closely resembling that of camphor. When exposed to the air for any length of time, it becomes discoloured, small quantities of hydrogen bromide being at the same time evolved. It cannot be distilled. When treated with zinc-dust and acetic acid, an oil is obtained which on distillation boils constantly at $195^{\circ}$ and appears to be ethylic ethylacetoacetate. It was not analysed.

Ethylic $\omega$-bromethylacetoacetate is completely decomposed when heated with alcoholic potash, uninviting tarry products being formed which could not be purified. Hydrolysis with dilute hydrochloric acid was found however to give interesting results, the bromide splitting up in this way into carbonic anhydride, hydrogen bromide, and acetopropyl alcohol, thus :-

$$
\begin{aligned}
& \underset{\mathrm{COOC}}{\mathrm{CH}_{2} \cdot \mathrm{HO}}>\mathrm{CH} \cdot \mathrm{CH}_{2} \cdot \mathrm{CH}_{2} \mathrm{Br}+2 \mathrm{H}_{2} \mathrm{O}= \\
& \underset{\mathrm{H}}{\mathrm{CH}_{3} \cdot \mathrm{CO}}>\mathrm{CH} \cdot \mathrm{CH}_{2} \cdot \mathrm{CH}_{2} \cdot \mathrm{OH}+\mathrm{C}_{2} \mathrm{H}_{5} \cdot \mathrm{OH}+\mathrm{HBr}+\mathrm{CO}_{2} \text {. }
\end{aligned}
$$

\section{Acetopropyl Alcohol, $\mathrm{CH}_{3} \cdot \mathrm{CO} \cdot \mathrm{CH}_{2} \cdot \mathrm{CH}_{2} \cdot \mathrm{CH}_{2} \cdot \mathrm{OH}$.}

If a mixture of 20 grams of ethylic $\omega$-bromethylacetoacetate, 5 grams of concentrated hydrogen chloride solution, and 20 grams of water are boiled for about two hours in a flask fitted with a reflux condenser, the oil gradually disappears, forming a greenish solution, which now contains acetopropyl alcohol.

In order to isolate this, the solution is saturated with anhydrous potassic carbonate, when the acetopropyl alcohol rises to the top as a dark-coloured layer, which is repeatedly extracted with ether. After drying over potassium carbonate and distilling off the ether, the crade product remains behind as a dark-brown oil. This method of preparation was the first used to obtain acetopropyl alcohol, and on trying to purify the crude product for analysis, as in the case of acetobutyl alcohol, by fractioning under the ordinary pressure, and afterwards under reduced pressure $(300 \mathrm{~mm}$.), it was found that the decomposition which set in was considerable, and it was not till later that we succeeded in purifying acetopropyl alcohol, by fractioning under $100 \mathrm{~mm}$. pressure as described on page 829 . 
The method first used for preparing acetopropyl alcohol for analysis was the following:-

The crude alcohol was mixed with about five times its weight of water, filtered, and the solution extracted once or twice with pure ether, which dissolved out all the impurities, while the greater part of the acetopropyl alcohol remained behind.

In order to isolate this, it is necessary to saturate the solution with anhydrous potassic carbonate, and to extract at least 10 times with pure ether. After drying over potassic carbonate and distilling off the ether, nearly pure acetopropyl alcohol remains behind in the flask as an almost colourless oil. By repeating this process, a product was obtained, which on analysis gave the following numbers:-

0.1909 gram substance gave $0 \cdot 1670$ gram $\mathrm{H}_{2} \mathrm{O}$ and 0.4172 gram $\mathrm{CO}_{2}$.

\begin{tabular}{|c|c|c|}
\hline & $\begin{array}{l}\text { Theory. } \\
\mathrm{C}_{5} \mathrm{H}_{10} \mathrm{O}_{2} \text {. } \\
58.82 \text { per cent. }\end{array}$ & $\begin{array}{l}\text { Found. } \\
59.57 \text { per cent. }\end{array}$ \\
\hline U & $9 \cdot 81$ & $9 \cdot 83$ \\
\hline & $31 \cdot 37$ & $30 \cdot 60$ \\
\hline
\end{tabular}

A similar process of purification was also first applied to the acetopropyl alcohol obtained from acetyltrimethylenecarboxylic acid by boiling it with water, as described on p. 829 .

The analytical results obtained in this case were the following :-

I. $0 \cdot 1963$ gram substance gave 0.1714 gram $\mathrm{H}_{2} \mathrm{O}$ and 0.4249 gram $\mathrm{CO}_{2}$.

II. 0.0736 gram substance gave 0.0643 gram $\mathrm{H}_{2} \mathrm{O}$ and 0.1590 gram $\mathrm{CO}_{2}$.

\begin{tabular}{|c|c|c|c|}
\hline \multirow{2}{*}{\multicolumn{2}{|c|}{ Theory. }} & \multicolumn{2}{|c|}{ Found. } \\
\hline & & I. & II. \\
\hline$C \ldots \ldots$ & 58.82 per cent. & $59 \cdot 04$ & 58.92 per cent. \\
\hline$H \ldots \ldots$. & $9 \cdot 81$ & $9 \cdot 70$ & $9 \cdot 71$ \\
\hline $0 \ldots \ldots$ & $31 \cdot 37$ & $31 \cdot 26$ & $31 \cdot 37$ \\
\hline
\end{tabular}

Acetopropyl alcohol obtained from ethylic $\omega$-bromethylacetoacetate shows all the reactions of that obtained from acetyltrimethylenecarboxylic acid by boiling with water.

Unfortunately, we neglected determining the boiling point of the former, so as to prove the identity of the two products; but as the preparation of acetopropyl alcohol from acetyltrimethylenecarboxylic acid is so much more satisfactory, we have not had occasion to repeat the experiment with ethylic $\omega$-bromethylacetoacetate. 


\section{\%-Pentylene Glycol, $\mathrm{CH}_{3} \cdot \mathrm{CH}(\mathrm{OH}) \cdot \mathrm{CH}_{2} \cdot \mathrm{CH}_{2} \cdot \mathrm{CH}_{2} \cdot \mathrm{OH}$.}

Acetobutyl alcohol, when treated with sodium amalgam, is easily reduced to the corresponding $\delta$-hexylene glycol, as Lipp has shown (Ber., 18, 3282) It therefore seemed probable that acetopropy 1 alcohol, when subjected to similar treatment, would be converted into $\gamma$-pentylene glycol, a substance which would be of great value for the further synthesis of ring derivatives.

If acetopropyl alcohol (10 grams) be diluted with about 5 parts of water, and the theoretical quantity of sodium amalgam ( 3 per cent. Na) be slowly added to this solution, reduction sets in immediately without evolution of gas, the liquid becoming quite warm. A small quantity of resinous matter, which usually separates during the reduction, is removed by filtration, the colourless filtrate saturated with an excess of anhydrous carbonate of potash, and then repeatedly extracted with ether. After drying over potassium carbonate and distilling off the ether, \%-pentylene glycol remains behind in a nearly pure state. On rapid fractional distillation, the greater part distils over between $210-220^{\circ}$ as a colourless oil. This operation must be rapidly accomplished, otherwise a considerable quantity of the glycol is decomposed into water and its anhydride; owing to this fact, it was very difficult to obtain a specimen of $\gamma$-pentylene glycol sufficiently pure for analysis.

The best analytical numbers were obtained from a portion which was very rapidly distilled, and then as nearly as possible freed from anbydride and water by passing a stream of dry hydrogen through the liquid for some hours. The following results were then obtained :-

0.1167 gram substance gave 0.1179 gram $\mathrm{H}_{2} \mathrm{O}$ and 0.2485 gram $\mathrm{CO}_{2}$.

\begin{tabular}{|c|c|c|c|}
\hline $\mathrm{C}$ & & $\begin{array}{l}\text { Theory. } \\
\mathrm{C}_{5} \mathrm{H}_{12} \mathrm{O}_{2} \text {. } \\
57 \cdot 69 \text { per cent. }\end{array}$ & $\begin{array}{l}\text { Found. } \\
58.07 \text { per cent. }\end{array}$ \\
\hline $\mathrm{H}$ & & $11 \cdot 54$ & $11 \cdot 21$ \\
\hline $\mathrm{O}$ & .... & $30 \cdot 77$ & $30 \cdot 72$ \\
\hline
\end{tabular}

At some future date, should we be again in possession of this glycol, it is intended to purify it by rapid distillation in a vacuum.

$\gamma$-Pentylene glycol is a thick colourless inodorous syrup, which mixes with water in all proportions. If it be heated in a small retort for some time to its boiling point, water and a very volatile oil distil over, the glycol being in course of time apparently completely decomposed.

The decomposition into anhydride and water is much more readily accomplished by means of sulphuric acid (compare Lipp, Ber., 18, 
3282). If $\gamma$-pentylene glycol is heated with 50 per cent. sulphuric acid on a water-bath for some time, the anhydride distils over as a very volatile oil of ethereal smell. This when purified was found to boil at $78-83^{\circ}$.

An analysis gave the following result :-

$0 \cdot 1001$ gram substance gave 0.1040 gram $\mathrm{H}_{2} \mathrm{O}$ and 0.2547 gram $\mathrm{CO}_{2}$.

\begin{tabular}{|c|c|c|}
\hline $\mathrm{C}$ & $\begin{array}{l}\text { Theory. } \\
\mathrm{C}_{5} \mathrm{H}_{10} \mathrm{O} \text {. } \\
69 \cdot 77 \text { per cent. }\end{array}$ & $\begin{array}{l}\text { Found. } \\
69 \cdot 42 \text { per cent. }\end{array}$ \\
\hline $\mathrm{H} \ldots \ldots$. & $11 \cdot 62$ & $11.55 \quad "$ \\
\hline $0 \ldots \ldots$ & $18 \cdot 61$ & $19 \cdot 03$ \\
\hline
\end{tabular}

This anhydride is formed from $\gamma$-pentylene glycol by the abstraction of the elements of water, thus :-

$$
\mathrm{CH}_{3} \cdot \mathrm{CH}(\mathrm{OH}) \cdot \mathrm{CH}_{2} \cdot \mathrm{CH}_{2} \cdot \mathrm{CH}_{2} \cdot \mathrm{OH}=\mathrm{CH}_{2}\left\langle\underset{\mathrm{CH}\left(\mathrm{CH}_{3}\right)}{\mathrm{CH}_{2} \cdot \mathrm{CH}_{2}}>\mathrm{O}\right. \text {. }
$$

Action of Water on Benzoyltrimethylenecarboxylic Acid.

After the results obtained in studying the action of water on acetyltrimethylenecarboxylic acid, when, as was shown in a previous chapter, decomposition into carbonic anhydride and acetylpropyl alcohol takes place, it was thought that it would be interesting to experiment on the behaviour of benzoyltrimethylenecarboxylic acid under similar conditions.

In order to do this, it was necessary in the first place to obtain the acid in as finely divided a condition as possible. This was done by dissolving it ( 1 gram was used) in dilute ammonia (vide this vol., p. 733), and then carefully reprecipitating with dilute sulphuric acid. The fine powder thus obtained was well washed and heated in a sealed tube with 40 c.c. of water at $100-110^{\circ}$ for four or five hours, during which time (but more especially at first) the tube was frequently taken out and well agitated to ensure the acid dissolving in the water. On opening the tube, a small amount of carbonic anhydride escaped, and the contents were found to consist of an almost colourless liquid, in which a few small drops of a slightly brownish oil were swimming about. In order to get rid of these, the liquid was filtered several times through wet filter-paper, and the perfectly clear filtrate, after mixing with a little potassic carbonate, was then extracted five times with perfectly pure ether. The ethereal solution, after drying over potassic carbonate and filtering, was evaporated, first on a water-bath to a small bulk, and lastly on a watch-glass. In this way, a colourless oil of aromatic odour was obtained, which, however, was now almost insoluble even in boiling water. After standing in a 
vacuum over sulphuric acid for four days, an analysis was made with the following result :-

$0.12 \mathrm{I} 1$ gram substance gave 0.0771 gram $\mathrm{H}_{2} \mathrm{O}$ and 0.3620 gram $\mathrm{CO}_{2}$.

\begin{tabular}{|c|c|c|}
\hline $\mathrm{C}$ & $\begin{array}{l}\text { Theory. } \\
\mathrm{C}_{10} \mathrm{H}_{10} \mathrm{O} \text {. } \\
82 \cdot 19 \text { per cent. }\end{array}$ & $\begin{array}{l}\text { Found. } \\
81.52 \text { per cent. }\end{array}$ \\
\hline $\mathrm{H}$ & $6.85 \quad$ & $7 \cdot 06$ \\
\hline$\ldots$ & 10.95 & $11 \cdot 42$ \\
\hline
\end{tabular}

This substance is therefore not benzoylpropyl alcohol, $\mathrm{C}_{10} \mathrm{H}_{12} \mathrm{O}_{2}$, as was expected, but its anhydride.

In this decomposition, it is evident that the first reaction which takes place is the following:-

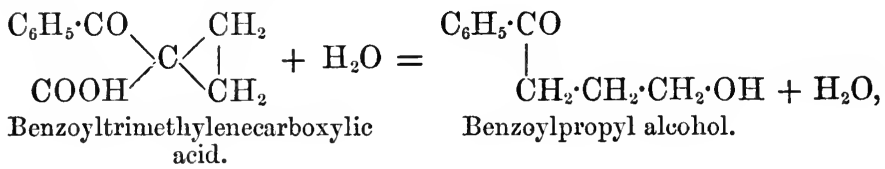

a. solution of benzoylpropjl alcohol resulting.

That a ketone alcohol is present is easily shown by adding to the clear aqueous solution a solution of phenylhydrazine acetate, when an oily phenylhydrazine compound is at once precipitated. On extracting the solution of this ketone alcohol with ether, drying over potassic carbonate, and distilling off the ether, this unstable substance is apparently at once split up into its anhydride and water, thus :-

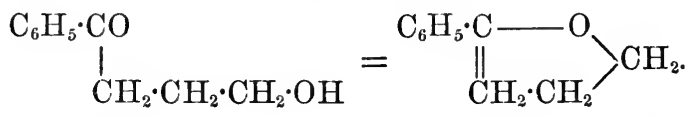

The instability of these aromatic ketone alcohols is very remarkable.

In a previous paper (this vol., p. 718), it was shown that acetobutyl alcohol, when rapidly heated, can be distilled with hardly any appreciable decomposition, whereas the corresponding aromatic alcohol, benzoylbutyl alcohol, (ibid., p. 733) on simply standing over sulphuric acid in a vacuum for some days, is converted into its anhydride and water. The present case is however still more remarkable. Acetopropyl alcohol, although not so stable as acetobutyl alcohol, can still be distilled without appreciable decomposition under a pressure of $100 \mathrm{~mm}$., whereas benzoylpropyl alcohol only appears to exist in solution, and on isolation is at once converted into its anhydride.

It is intended to further examine these interesting alcohols in order to be certain of these points, using larger quantities of material. One 
interesting point will be to determine whether the anhydride of benzoylpropyl alcohol is or is not identical with benzoyltrimethylene (Trans., 47, 840).

It seems almost probable that if these anhydride rings are so easily formed in the aromatic series, that the derivatives obtained from ethylic benzyolacetate and ethylene bromide are pentone- and not trimethylene-derivatives, in which case the acid described as benzoyltrimethylenecarboxylic acid would be benzoyldehydropentonecarboxylic acid, $\mathrm{COOH} \cdot \stackrel{\mathrm{C} \cdot \mathrm{CH}_{2} \mathrm{H}_{5} \cdot \mathrm{C}-\mathrm{O}}{\|} \mathrm{CH}_{2}$.

Further experiments must decide this point.

\section{Action of Phenylhydrazine on Ethylic Acetyltrimethylenecarboxylate.}

In order to prove the presence of a carbonyl-group in ethylic acetyltrimethylenecarboxylate, several experiments on the action of phenylhydrazine on this substance were made. If the pure ethereal salt is mixed with phenylhydrazine and warmed on a water-bath, a violent reaction sets in with separation of water and formation of a thick yellowish syrup, which even on long standing does not crystallise. On modifying the method slightly in the following way, better results were afterwards obtained. 5 grams of pure ethylic acetyltrimethylenecarboxylate was mixed with a little less than the theoretical quantity of phenylhydrazine ( $3 \cdot 3$ grams), the mixture heated on a water-bath until all action had ceased, and then submitted to distillation with steam in order to drive over any unchanged ethereal salt. If this product is allowed to stand for some weeks under water, crystals begin to radiate from various points on the bottom of the flask, and at last the oily mass completely solidifies, the whole presenting an appearance somewhat resembling that of cinnamic acid which has been fused and then allowed to cool slowly.

The phenylhydrazine compound thus obtained was broken away from the glass, dried over sulphuric acid in a vacuum, and then treated with various solvents, in the hope of purifying it by recrystallisation. The substance is very easily soluble in nearly all the usual solvents, and all attempts to obtain anything crystalline from these various solutions were unfortunately fruitless. An analysis of the crude substance was therefore made with the following result:-

$0 \cdot 2460$ gram substance gave $26 \cdot 0$ c.c. $N . \quad t=19^{\circ}$. Bar. $=760 \mathrm{~mm}$.

$$
\begin{array}{ll}
\multicolumn{3}{c}{\begin{array}{c}
\text { Theory. } \\
\mathrm{C}_{14} \mathrm{H}_{18} \mathrm{~N}_{2} \mathrm{O}_{2} .
\end{array}} & \text { Found. } \\
\mathrm{N} . \ldots \ldots \ldots \quad 11.38 \text { per cent. } & 12 \cdot 38 \text { per cent. }
\end{array}
$$

Although these numbers of course are only approximate, still they 
would appear to show that ethylic acetyltrimethylenecarboxylate reacts with phenylhydrazine in the same way as all substances containing the carbonyl-group, and that the above substance is formed according to the equation-

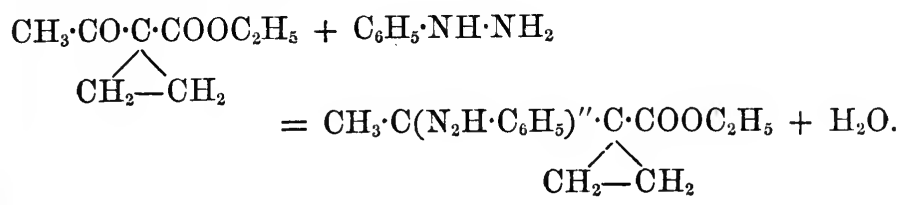

This would then be an additional proof that ethylic acetyltrimethylenecarboxylate is really an acetyltrimethylene-derivative, and does not contain an oxygen carbon-chain, as it would if its constitution were represented by the formula-

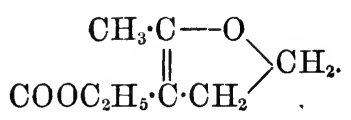

From the study of the action of phenglhydrazine on ethylic methyldehydrohexonecarboxylate, it is very improbable that a pentone ring would react with phenylhydrazine.

\section{Oxidation of Ethylic Acetyltrimethylenecarboxylate.}

During the course of these experiments, it appeared interesting to experiment on the action of oxidising agents on acetyltrimethylenecarboxylic acid or its ethereal salt, as it seemed possible that in this way trimethylenedicarboxylic acid $(1,1)$ might be produced, thus:-

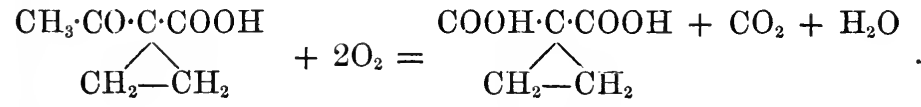

In the experiments to be described, ethylic acetyltrimethylenecarboxylate was usually employed.

1. Oxidation with Chromic Acid.-The pure ethereal salt was treated with an excess of chromic acid and dilute sulphuric acid. The oxidation sets in rapidly and was hastened at the last by gently warming the mixture. The product was then separated from chromium in the usual way, by precipitating with caustic soda, the alkaline filtrate boiled to hydrolyse any ethereal salt that might be present, acidified and several times extracted with ether. On distilling off the ether, a slight residue was obtained which, however, did not contain any trimethylenedicarboxylic acid. These experiments were then repeated with only sufficient chromic acid to oxidise the methyl-group in the 
ethylic acetyltrimethylenecarboxylate to the carboxyl-group, but it was found that in this case part of the ethereal salt had been completely destroyed, leaving the greater part quite unchanged.

2. Oxidation with Potassic Permanganate.-In these experiments the ethereal salt was first strongly agitated with dilute sodium carbonate solution, until a fine emulsion was formed, and then the calculated quantity of permanganate of potassium solution was slowly run in. The colour of the permanganate disappears instantly at first, but more slowly towards the end of the operation. After filtering from the precipitated manganese oxide, a little caustic soda was added, the solution boiled for some time, evaporated, acidified and repeatedly extracted with ether. On distilling off the ether, an uninviting brown substance remained behind, from which nothing but acetic acid could be extracted. There was no trimethylenedicarboxylic acid present.

3. Oxidation with Iodine in Alkaline Solution.-As it was thought possible that weaker oxidising agents than chromic acid and permanganate might yield better results, several experiments were made with iodine in alkaline solution. As in the previous experiment, an emulsion of the ethereal salt with dilute sodic carbonate solution was first prepared, and then treated with a little less than the calculated quantity of iodine. As soon as all the iodine had been added, any excess of ethereal salt was removed by extracting with ether, the aqueous solution was then acidified, and again several times extracted with ether. On distilling off the ether, a very small residue was obtained which evidently did not contain any trimethylenedicarboxylic acid. The principal products of the reaction were iodoform and considerable quantities of oxalic acid.

These oxidation experiments were repeated several times, not only with the ethereal salt but also with the free acid, but in no case could trimethylenedicarboxylic acid be obtained.

\section{Action of Phosphorous Pentachloride on Ethylic Acetyltrimethylene- carboxylate.}

In this experiment, 50 grams of the crude ethereal salt, boiling at $190-200^{\circ}$, were dissolved in twice the volume of chloroform, and to this 67 grams of phosphorus pentachloride were slowly added. The action was very violent, so that it was found necessary to cool the mixture with ice-water after each addition of the pentachloride, otherwise resinous substances are apt to be formed. After standing 24 hours to ensure the completion of the reaction, the mass was gradually poured inco ice and water, and then as soon as all the oxychloride and excess of pentachloride of phosphorus had been decomposed, the oil was extracted with ether. The ethereal solution 
was well washed, first with water and then with dilute carbonate of soda, dried over calcic chloride, filtered, and the ether distilled off; the yellowish oil which remained behind was purified by fractioning under diminished pressure $(150 \mathrm{~mm}$.). After repeated fractioning, an oil was obtained boiling constantly at $171-173^{\circ}(150 \mathrm{~mm}$.); this was analysed with the following result:-

$$
\begin{aligned}
& \text { Theory. } \\
& \mathrm{C}_{8} \mathrm{H}_{12} \mathrm{O}_{2} \mathrm{Cl}_{2} \text {. Found. } \\
& \text { Cl..... } \quad 33.65 \text { per cent. } \quad 28.90 \text { per cent. }
\end{aligned}
$$

On each fractioning, it was noticed that traces of gaseous hydrogen chloride were always given off; the experiments were therefore repeated, and the resulting oil now fractioned under a pressure of only $35 \mathrm{~mm}$. The portion boiling at $135-140^{\circ}$ gave the following analytical numbers :-

I. $0 \cdot 2180$ gram substance gave $0 \cdot 1194$ gram $\mathrm{H}_{2} \mathrm{O}$ and $0 \cdot 3629$ gram $\mathrm{CO}_{2} ; 0.4405$ gram substance gave 0.5430 gram $\mathrm{AgCl}$.

II. 0.2174 gram substance gave 0.1132 gram $\mathrm{H}_{2} \mathrm{O}$ and $0.3598 \mathrm{gram}$

\begin{tabular}{|c|c|c|c|c|c|}
\hline \multirow{2}{*}{\multicolumn{2}{|c|}{$\begin{array}{l}\text { Theory. } \\
\mathrm{C}_{8} \mathrm{H}_{12} \mathrm{O}_{2} \mathrm{Cl}_{2} .\end{array}$}} & \multicolumn{3}{|c|}{ Found. } & \\
\hline & & I. & II. & III. & \\
\hline $\mathrm{C}$ & 4540 p. c. & $45 \cdot 40$ & $45 \cdot 15$ & - & p. c. \\
\hline & $5 \cdot 68$, & $6 \cdot 08$ & $5 \cdot 78$ & - & , \\
\hline $\mathrm{Cl} \ldots$ & $33 \cdot 65$, & 30.57 & $32 \cdot 71$ & $32 \cdot 39$ & , \\
\hline
\end{tabular}
$\mathrm{CO}_{2} ; 0.2188$ gram substance gave 0.2885 gram $\mathrm{AgCl}$.

III. 0.3200 gram substance gave 0.4178 gram AgCl.

Even under a pressure of only $35 \mathrm{~mm}$., it is not possible to distil this oil without traces of hydrogen chloride being given off, and this accounts for the chlorine determinations in the above analyses not agreeing so well as might be desired.

This chlorinated compound is a colourless oil, of porwerful odour, which on standing soon becomes yellowish. When pure, it distils with only slight decomposition at ordinary temperatures.

There are two possible formulæ for this substance-

I.

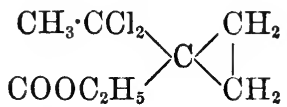

II.<smiles>COCCC(CCCl)C(C)OCO</smiles>

of which the second is certainly the most plausible.

The most probable explanation of the action of pentachloride of phosphorus on ethylic acetyltrimethylenecarboxylate is that a substance having the constitution represented by Formula $I$ is first formed according to the equation- 


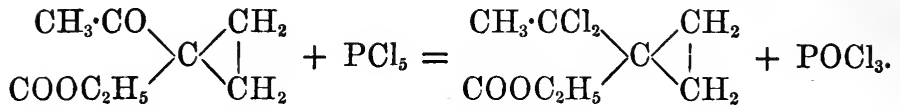

This substance is then further acted on by hydrogen chloride, which is always present; the trimethylene-ring is split, and a change takes place which may be represented by the following equation :-

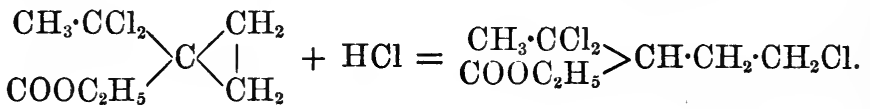

This intermediary compound then loses $1 \mathrm{~mol}$. $\mathrm{HCl}$ probably during the reaction, but certainly on distillation, when a substance results which would have the constitution represented by Formula II ; thus :-

\section{$\underset{\mathrm{COOC}}{\mathrm{CH}_{3} \cdot \mathrm{H}_{5}}>\mathrm{CH} \cdot \mathrm{CH}_{2} \cdot \mathrm{CH}_{2} \mathrm{Cl}=\underset{\mathrm{COOC}_{2} \mathrm{H}_{5}}{\mathrm{CH}_{3}}>\mathrm{CC} \cdot \mathrm{CH}_{2} \cdot \mathrm{CH}_{2} \mathrm{Cl}+\mathrm{HCl}$.}

The hydrogen chloride thus produced could then react with a further quantity of the original chlorinated compound, and thus a continuous reaction would be set ap.

The examination of the behaviour of this substance, for which we would suggest the name ethylic chlorethylchlorocrotonate, towards reducing agents has tended to confirm the above speculations. When a boiling alcoholic solution of ethylic chlorethylchlorocrotonate is treated with an excess of zinc.dust and concentrated hydrochloric acid, reduction and partial hydrolysis sets in. If the product is diluted with water, an oil is deposited, which may be separated from the aqueous layer by extraction with ether. This oil, on examination, was found to consist of an acid and a neutral substance. In order to separate these, the ethereal solution was shaken up with a slight excess of sodic carbonate, the alkaline layer separated, acidified with dilute sulphuric acid, and then allowed to stand in a cool place. After a short time a small quantity of a colourless substance crystallised out, which was filtered from the mother-liquor, washed with a little water, and dried over sulphuric acid in a vacuum. The amount of this substance, which was found to contain chlorine, was only 0.3 gram.

A determination of the chlorine gave the following result:0.1936 gram substance gave 0.1801 gram $\mathrm{AgCl}$.

$$
\begin{aligned}
& \text { Theory. } \\
& \mathrm{CH}_{3} \cdot \mathrm{CCl}: \mathrm{C}\left(\mathrm{C}_{2} \mathrm{H}_{5}\right) \cdot \mathrm{COOH} . \quad \text { Found. } \\
& \text { Cl...... 23.90 per cent. } 23.00 \text { per cent. }
\end{aligned}
$$

These numbers agree approximately with the formula of chlor- 
ethylcrotonic acid, a substance which has been obtained by Demarçay by treating ethylic ethylacetoacetate with pentachloride of phosphorus. Demarçay found the melting point of his substance to be $74-75^{\circ}$. The crystals obtained by us melted at almost the same temperature, so that it is extremely probable that the two products are identical. The formation of chlorethylcrotonic acid from ethylic chlorethylchlorocrotonate can be easily explained by the following equation :-

$$
\underset{\mathrm{COOC}_{2} \mathrm{H}_{5}}{\mathrm{CH}_{3} \cdot \mathrm{CCl}}>\mathrm{C} \cdot \mathrm{CH}_{2} \cdot \mathrm{CH}_{2} \mathrm{Cl}+\mathrm{H}_{2}=\underset{\mathrm{CQOH}}{\mathrm{CH}_{3} \cdot \mathrm{CCl}}>\mathrm{C} \cdot \mathrm{CH}_{2} \cdot \mathrm{CH}_{3}+\mathrm{C}_{2} \mathrm{H}_{5} \mathrm{Cl} \text {. }
$$

It is unfortunate that the amount of substance at our disposal was not sufficient to admit of a complete analysis, and thus prove the formula conclusively. We, however, intend to repeat these experiments at some fature date with larger quantities of material. The neutral oil formed by the reduction of ethylic chlorethylchlorocrotonate, which was separated from the chlorethylcrotonic acid as described above, has also not yet been further examined.

Experiments were next tried on the action of sodium amalgam on ethylic chlorethylchlorocrotonate. For this purpose, the pure ethereal salt was dissolved in 80 per cent. alcohol and treated with a large excess of 3 per cent. sodium amalgam, the reaction at the last being accelerated by gently warming on a water-bath. On diluting the mass with water, only a very small quantity of oil was precipitated, showing that, as was expected, hydrolysis of the reduced product had also taken place. The neutral oil was removed by shaking with a little ether, the alkaline liquid acidified with sulphuric acid, and several times extracted with pure ether. The ethereal solution, after drying and evaporating, deposited a considerable quantity of a putrid smelling oil, which, on fractioning, all distilled between $185^{\circ}$ and $205^{\circ}$.

On refractioning this, an oil was obtained boiling between $195^{\circ}$ and $200^{\circ}$, which gave the following numbers on analysis :-

0.1812 gram substance gave 0.1721 gram $\mathrm{H}_{2} \mathrm{O}$ and 0.4106 gram $\mathrm{CO}_{2}$.
Theory.
$\left(\mathrm{C}_{2} \mathrm{H}_{5}\right)_{2} \mathrm{CHI} \cdot \mathrm{COOH}$.
C..... 62.07 per cent.
Found.
H.... 10.34 ,
61.75 per cent.
O $\ldots . .27 \cdot 59$
$10 \cdot 55$
$27 \cdot 70$,

This substance appears therefore to be dicthylacetic acid. This acid has already been prepared in several ways, and its boiling point has been determined as $190^{\circ}\left(755 \mathrm{~mm} .\right.$, Fittig), and $195-197^{\circ}$ (Schnapp), temperatures which correspond sufficiently closely with that obtained for our product. 
The formation of diethylacetic acid from ethylic chlorethylchlorocrotonate takes place according to the equation-

$$
\underset{\mathrm{COOC}_{2} \mathrm{H}_{5}}{\mathrm{CH}_{3} \cdot \mathrm{CCl}}>\mathrm{C} \cdot \mathrm{CH}_{2} \cdot \mathrm{CH}_{2} \mathrm{Cl}+2 \mathrm{H}_{2}+\mathrm{H}_{2} \mathrm{O}=\underset{\mathrm{C}_{2} \mathrm{H}_{5} \cdot \mathrm{OH}+2 \mathrm{HCl}}{\underset{\mathrm{COOH}}{\mathrm{CH}_{3} \cdot \mathrm{CH}_{2}}>\mathrm{CH} \cdot \mathrm{CH}_{2} \cdot \mathrm{CH}_{3}+}
$$

When treated with hydriodic acid, ethylic chlorethylchlorocrotonate undergoes a similar reduction. 3 grams of the pure ethereal salt, 10 c.c. of fuming hydriodic acid, and 1 gram of amorphous phosphorus were heated in a sealed tube for four hours at $200^{\circ}$. The product from three such tubes was neutralised with sodic "carbonate, freed from ethyl iodide by distillation in steam, and the residue extracted with ether, to remove any unchanged oil. The alkaline solution was then acidified, and several times extracted with ether. The ethereal solution, after drying and evaporating, deposited a colourless oil, which on fractioning distilled between $180^{\circ}$ and $200^{\circ}$, and on repeating the operation, by far the greater part came over between $190^{\circ}$ and $195^{\circ}$, and possessed all the properties of diethylacetic acid.

\section{Action of Ethylene Bromide on Ethylic Acetonedicarboxylate.}

Ethylic Acetyltrimethylenedicarboxylate,

$\mathrm{COOC}_{2} \mathrm{H}_{5} \cdot \mathrm{CH}_{2} \cdot \mathrm{CO} \cdot \mathrm{C} \cdot \mathrm{COOC}_{2} \mathrm{H}_{5}$

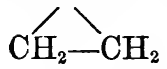

In studying this decomposition, a mixture of 40 grams of ethylic acetonedicarboxylate and 38 grams of ethylene bromide was slowly added to a well-cooled solution of 9 grams of sodium in 100 grams of absolute alcohol, and the whole allowed to boil gently on a waterbath for 10 hours. During the reaction, a considerable quantity of a white precipitate settled out, which on examination was found to contain not only bromide of sodium, but also a considerable quantity of carbonate. In order to isolate the principal product of the reaction, the excess of alcohol was distilled off, the residue mixed with water, and the resulting oil extracted several times with ether. The ethereal solution after well washing, drying over calcic chloride, and distilling, deposited about 30 grams of a yellowish oil, which was then submitted to distillation, at first under the ordinary pressure, till the thermometer had risen to $180^{\circ}$, when the distillation was continued under reduced pressure $(150 \mathrm{~mm}$.).

Below $100^{\circ}$, a considerable quantity of a colourless oil distilled over, which on examination was found to consist for the most part of ethylic acetoacetate, produced by the partial hydrolysis of some of the ethylic acetonedicarboxylate, together with a little ethylene bromide. 
The oil distilled under reduced pressure $(150 \mathrm{~mm}$.) boiled for the most part between $130^{\circ}$ and $240^{\circ}$, and this on refractioning split up into two principal fractions, $130-200^{\circ}$ and $200-240^{\circ}$. The second of these, which contains the primary product of the action of ethylene bromide on ethylic acetonedicarboxylate, was several times very carefully fractioned under the same pressure $(150 \mathrm{~mm}$.), when the pure substance was at last obtained as a very thick, almost colourless oil, boiling at $223-224^{\circ}(150 \mathrm{~mm})$. Analyses showed this to be ethylic acetyltrimethylenedicarboxylate.

I. $0 \cdot 1686$ gram substance gave $0 \cdot 1120$ gram $\mathrm{H}_{2} \mathrm{O}$ and 0.3590 gram $\mathrm{CO}_{2}$.

II. $0 \cdot 1962$ gram substance gave $0 \cdot 1286$ gram $\mathrm{H}_{2} \mathrm{O}$ and $0 \cdot 4180$ gram $\mathrm{CO}_{2}$.

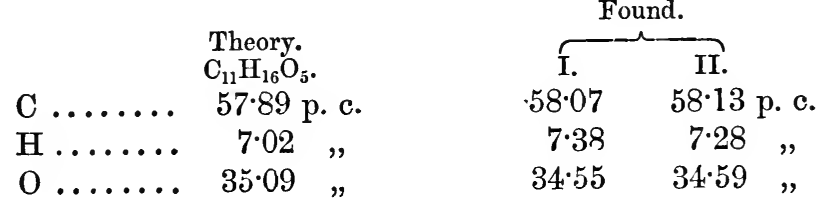

Ethylic acetyltrimethylenedicarboxylate when freshly distilled is an almost colourless syrup, of a peculiar odour, somewhat resembling that of pyruvic acid. It is a very unstable substance, a fact which probably accounts for the smallness of the yield obtained in the above reaction (about 10 per cent. of the theoretical). It combines with phenylhydrazine, and in alcoholic solution gives a violet coloration with ferric chloride.

The fraction $130-200^{\circ}$ (150 mm.), which is always formed in considerable quantity in the above reaction, was next carefally fractioned under the ordinary pressure. After two or three distillations, by far the greater part passed over between $185^{\circ}$ and $200^{\circ}$, and on examination proved to be ethylic acetyltrimethylenecarboxylate. It was not directly analysed, but converted by hydrolysis into the corresponding acid, and this into its silver salt (as described on p. 848). The silver salt, after twice recrystallising from water, was obtained in the characteristic form already described (loc. cit., p. 833). An analysis gave the following numbers, showing it to be argentic acetyltrimethylenecarboxylate.

0.24.33 gram substance gave 0.1112 gram Ag.

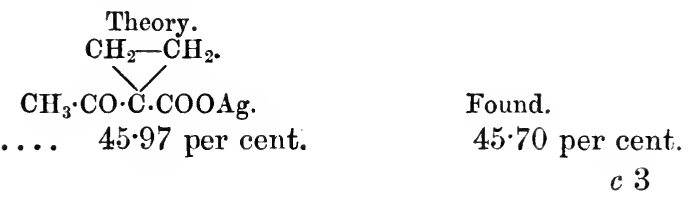


Ethylic acetyltrimethylenecarboxylate is produced from ethylic acetyltrimethylenedicarboxylate, by partial hydrolysis and subsequent splitting off of carbonic anhydride, thus :-

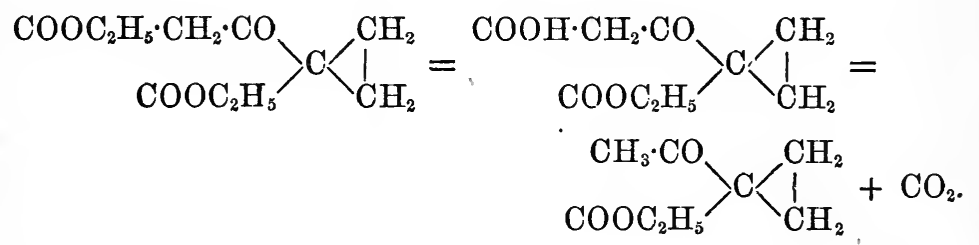

Acetyltrimethylenedicarboxylic Acid, $\mathrm{COOH} \cdot \mathrm{CH}_{2} \cdot \mathrm{CO} \cdot \mathrm{C} \cdot \mathrm{COOH}$.<smiles>[CH]1CCC1</smiles>

In preparing acetyltrimethylenedicarboxylic acid from its ethereal salt, considerable difficulty was experienced owing to the instability of the acid, and it was only after several experiments had been made, that small quantities of this substance were obtained in a fairly pure condition.

The following method was at length found to give the best results:-Pure ethylic acetyltrimethylenedicarboxylate was mixed with a strong solution of twice the calculated quantity of pure potash in methyl alcohol, and the resulting yellow solution allowed to stand at the ordinary temperature for about 12 days. The product was then diluted with water, acidified with dilute sulphuric acid, and several times extracted with ether. The yellowish-coloured ethereal solution after well washing with water, drying over calcic chloride and distilling, deposited a dark-yellowish oil, which almost entirely solidified on standing for some days over sulphuric acid in a vacuum.

This crude substance was next roughly separated from motherliquor by spreading it out on a porous plate, and allowing it to remain for several days; it was then broken up and washed with small quantities of chloroform, until all the impure mother-liquor had been dissolved out, leaving a white crystalline solid. This is nearly pure acetyltrimethylenedicarboxylic acid.

\section{Analysis.}

I. 0.1796 gram substance gave 0.0791 gram $\mathrm{H}_{2} \mathrm{O}$ and 0.3188 gram $\mathrm{CO}_{2}$.

II 0.1540 gram substance gave 0.0670 gram $\mathrm{H}_{2} \mathrm{O}$ and 0.2723 gram $\mathrm{CO}_{2}$. 


\begin{tabular}{|c|c|c|c|}
\hline & & & \\
\hline & $\begin{array}{l}\text { Theory. } \\
\mathrm{C}_{7} \mathrm{H}_{8} \mathrm{O}_{5} \text {. }\end{array}$ & I. & II. \\
\hline$c \ldots \ldots$ & 48.83 p. c. & $48 \cdot 41$ & $48 \cdot 22$ p. c. \\
\hline$H \ldots . . .$. & $4.65 \quad$ & $4 \cdot 89$ & $4.83 \quad$ \\
\hline $0 \ldots \ldots$ & 46.52, & $46 \cdot 70$ & $46 \cdot 95$ \\
\hline
\end{tabular}

Acetyltrimethylenedicarboxylic acid melts at about $175^{\circ}$ with decomposition into carbonic anhydride and an oil of ethereal odour, probably acetyltrimethylene. It is easily soluble in most of the usual solvents, with the exception of water and chloroform, in which it dissolves sparingly at the ordinary temperature.

The silver salt of this acid was prepared by dissolving the acid in a slight excess of ammonia, allowing the solution to stand over sulphoric acid in a vacuum until neutral, and then precipitating with nitrate of silver. It is a white amorphous mass, sparingly soluble in water. For analysis, it was collected on a filter, well washed with water, and dried over sulphuric acid in a vacuum. A combustion of this salt gave the following results :-

0.2157 gram substance gave 0.0354 gram $\mathrm{H}_{2} \mathrm{O} ; 0.1767$ gram $\mathrm{CO}_{2}$; Ag was lost.

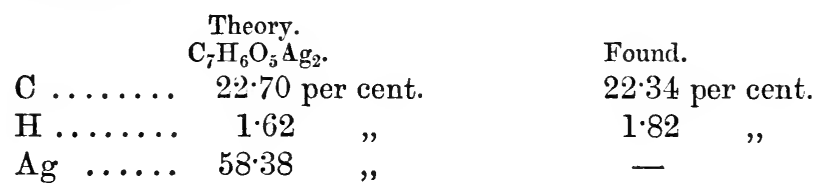

When heated in a combustion tube, the silver salt decomposes all at once as soon as the temperature reaches a certain point, with evolution of a considerable quantity of gas, and deposition of a voluminous mass of fine threads of silver, which cling to the top of the tube, and thus prevented the estimation of the silver in the above analysis. Owing to lack of material, we were unfortunately not able to repeat this determination.

In order to show more clearly the relation of acetyltrimethylenedicarboxylic acid to the monocarboxylic acid, the action of water on the former was also studied, and it was found that this acid, on boiling with water, was split up into acetopropyl alcohol and 2 mols. of carbonic anhydride, in the same way that acetyltrimethylenemonocarboxylic acid, under similar treatment, fields acetopropyl alcohol and $\mathrm{L}$ mol. of carbonic anhydride.

The experiment was carried out as follows:-2 grams of the pure acid were dissolved in a small quantity of hot water, and the solution thus obtained boiled with a reflux condenser until the evolution of carbonic anhydride had ceased. Excess of potassic carbonate 
was then added, and the resulting milky liquid extracted about 20 times with small quantities of ether. The ethereal solution was then dried over potassic carbonate, filtered and evaporated, when a considerable quantity of a thick colourless oil was obtained, which showed all the properties of acetopropyl alcohol. After allowing it to stand for a short time over sulphuric acid in a vacuum, it was analysed with the following result :-

0.1128 gram substance gave 0.1027 gram $\mathrm{H}_{2} \mathrm{O}$ and 0.2415 gram $\mathrm{CO}_{2}$.

\begin{tabular}{|c|c|c|}
\hline $\mathrm{CH}_{3} \cdot \mathrm{CO}$ & $\begin{array}{l}\text { Theory. } \\
\mathrm{CH}_{2} \cdot \mathrm{CH}_{2} \cdot \mathrm{CH}_{2} \cdot \mathrm{OH} \text {. }\end{array}$ & Found. \\
\hline $\mathrm{C} \ldots \ldots$ & 58.82 per cent. & 58.39 per cent. \\
\hline$\cdots \cdot$ & $9 \cdot 81$ & $10 \cdot 16$ \\
\hline 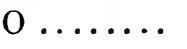 & $31: 37$ & $31 \cdot 45$ \\
\hline
\end{tabular}

This result clearly shows that the substances described above, as produced by the action of ethylene bromide on ethylic acetonedicarboxylate, are really derivatives of acetyltrimethylenecarboxylic acid.

This research was carried out partly at Prof. v. Baeyer's laboratory in Munich, and partly at Prof. Dixon's private laboratory at Owens College, Manchester. 
[Reprinted from the Journal of the Chemical Society, January, 1887. Vol. LI.]

THE SYNTHETICAL FORMATION OF CLOSED CARBONCHAINS. PART II. ON SOME DERIVATIVES OF TETRAMETHYLENE.

By W. H. Perkin, Jun., Ph.D.

Is Part I of this paper, which I had the honour of laying before the Society last year (Trans., 1885, 801), I gave a short description of some derivatives of trimethylene,<smiles>CCC</smiles>

and I now desire to communicate to the Society the results of my experiments on the synthetical formation of compounds containing the tetramethylene-ring,<smiles>C1CCC1</smiles>

These derivatives may be easily obtained by reactions exactly similar to those employed for the formation of trimethylene-derivatives.

Thus, ethylic malonate when treated with sodic ethylate and trimethylene bromide is converted into the ethereal salt of tetramethylenedicarboxylic acid $[1,1]^{*}$ -

$2 \mathrm{CHNa}\left(\mathrm{COOC}_{2} \mathrm{H}_{5}\right)_{2}+\mathrm{CH}_{2} \mathrm{Br} \cdot \mathrm{CH}_{2} \cdot \mathrm{CH}_{2} \mathrm{Br}=$ $\mathrm{CH}_{2}<\mathrm{CH}_{2}^{\mathrm{CH}_{2}}>\mathrm{C}\left(\mathrm{COOC}_{2} \mathrm{H}_{5}\right)_{2}+\mathrm{CH}_{2}\left(\mathrm{COOC}_{2} \mathrm{H}_{5}\right)_{2}+2 \mathrm{NaBr}$.

If, however, ethylic acetoacetate be substituted for ethylic malonate

* With regard to the nomenclature of these ring-bodies, see Part $I$ of this paper (Trans., 1886, 806). 
in the above reaction, a different series of compounds is formed, a description of which must be reserved for a future paper.

This paper has, for convenience sake, been divided into the following chapters:-

I. Preparation and properties of tetramethylenedicarboxylic acid $(1,1)$, tetramethylenemonocarboxylic acid, and their ethereal salts.

II. Tetramethylenetetracarboxylic acid $(1,1,2,2)$, and tetramethylenedicarboxylic acid $(1,2)$.

III. Diacetyltetramethylenedicarboxylic acid $(1,1,2,2)$.

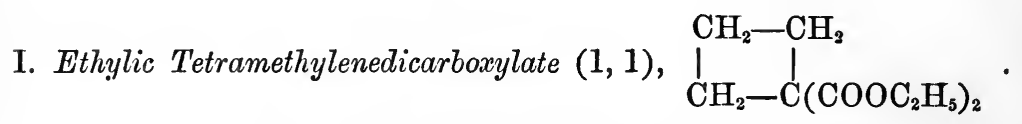

In order to prepare this ethereal salt, the following method, which 1 give in full, has usually been employed.

14.5 grams of sodium are dissolved in abont 160 grams of absolute alcohol, and when thoroughly cold a mixture of 100 grams of ethylic malonate and 64 grams of trimethylcne bromide is slowly poured in, the whole being well shaken and cooled during the operation. During the mixing, the sodium compound of ethylic malonate generally separates out as a white gelatinous mass, but this soon disappears, especially on warming, a clear liquid being formed. On allowing this to stand, sodic bromide is precipitated, and the temperature gradually rises, the reaction in most cases being so violent as to cause the alcohol to boil; as soon as it has cooled down, the decomposition is generally at an end. In order to test this, a small quantity is taken out, diluted with a little water, and tested with litmus-paper, when it should have a perfectly neutral reaction.

If, however, it should still be alkaline, it is gently warmed on a water-bath till the reaction is complete.

In order to isolate the product, the whole is dissolved in about 2-3 times its bulk of water, and extracted two or three times with ether.* On distilling off the ether, a colourless oil remains, which is next roughly purified by distillation with steam; by this means all the ethylic tetramethylenedicarboxylate and regenerated ethylic malonate are driven over, a thick oil consisting principally of ethylic pentanetetracarboxylate remaining in the retort.

The distillate is then extracted twice or thrice with ether, the ethereal solution washed, once with water and once with dilute carbonate of soda solution, and dried over calcic chloride. On distilling off the ether, about 110 grams of a colourless oil are left, which is further puritied by fractional distillation. Below $175^{\circ}$, a

* Saturating with common salt, before extracting with ether, has been found very advantageous. 
small quantity of unchanged trimethylene bromide passes over, then the temperature rises rapidly to $190^{\circ}$, between which and $240^{\circ}$ about 60 grams of oil, consisting of a mixture of ethylic malonate and tetramethylenedicarboxylate distils over.

In order to separate these ethereal salts; it is necessary to fraction repeatedly and carefully with a reflux apparatus. In this way 26-28 grams of nearly pure ethylic tetramethylenecarboxylate may be obtained, boiling between $218^{\circ}$ and $223^{\circ}(720 \mathrm{~mm}$.). When operating with larger quantities, a yield of about $30-34$ grams has been obtained from every 100 grams ethylic malonate. Varying the proportions of the different ingredients in this reaction does not appear to affect the yield very much.

The following three experiments were tried in order to determine this :-

Used.

I. II. III.

Yield, p.c.

Ethylic malonate..... 2 mol. 1 mol. 1 mol. $\quad$ I.... 30

Trimethylene bromide . 1 " $2, .1$, II... 33

Sodium.......... $2 " 1$ " $2 " 2, \quad$ III.... 28

Pure ethylic tetramethylenedicarboxylate boils at $220-221^{\circ}$ (720 mm.).

The following numbers were obtained on analysis :-

I. 0.2200 gram substance gave 0.4799 gram $\mathrm{CO}_{2}$ and 0.1605 gram $\mathrm{H}_{2} \mathrm{O}$.

II. 0.1618 gram substance gave 0.3561 gram $\mathrm{CO}_{2}$ and 0.1170 gram $\mathrm{H}_{2} \mathrm{O}$.

\section{Found.}

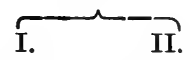

C..... $59.50 \quad 60.03$ per cent.

H $\ldots . .8 \cdot 10$

O..... $32 \cdot 40$
Theory.

$\mathrm{C}_{3} \mathrm{H}_{6}: \mathrm{C}\left(\mathrm{COOC}_{2} \mathrm{H}_{5}\right)_{2}$.

60.00 per cent.

8.00

$32 \cdot 00 \quad "$

Ethylic tetramethylenedicarboxylate is a colourless mobile liquid having a slight odour of camphor. It is not acted on by bromine at ordinary temperatures, but on boiling, the colour of the bromine disappears, torrents of hydrogen bromide being given off.

The density and magnetic rotation determinations of this compound, which were carried out by my father, gave the following results :-

$$
\begin{aligned}
& d \frac{9^{\circ}}{9^{\circ}}=1.05328 . \\
& d \frac{15^{\circ}}{15^{\circ}}=1 \cdot 04817 . \\
& d_{25^{\circ}}^{25^{\circ}}=1.04051 .
\end{aligned}
$$


Magnetic Rotations.

\begin{tabular}{c|c|c}
$t$. & Sp. rotation. & Mol. rotation. \\
\hline $20 \cdot 3$ & $0 \cdot 9320$ & $9 \cdot 921$ \\
$20 \cdot 1$ & $0 \cdot 9337$ & $9 \cdot 939$ \\
$19 \cdot 9$ & $0 \cdot 9374$ & $9 \cdot 977$ \\
$19 \cdot 6$ & $0 \cdot 9348$ & $9 \cdot 946$ \\
$17 \cdot 6$ & $0 \cdot 9372$ & $9 \cdot 955$ \\
$17 \cdot 6$ & $0 \cdot 9336$ & $9 \cdot 918$ \\
$17 \cdot 6$ & $0 \cdot 9341$ & $9 \cdot 922$ \\
\hline Average $18 \cdot 9$ & $0 \cdot 9346$ & $9 \cdot 940$
\end{tabular}

Dr. Gladstone was so kind as to determine the refractive equivalent, and gave me the following account of his results:-

\begin{tabular}{c|c|c|c|c}
\hline$t$. & Sp. gr. & $\mu_{\mathrm{A} .}$ & $\mu_{\mathrm{D} .}$ & $\mu_{\mathrm{H} .}$ \\
\hline 26 & $1 \cdot 0383$ & $1 \cdot 4353$ & $1 \cdot 4330$ & $1 \cdot 4477$ \\
\hline
\end{tabular}

\begin{tabular}{c|c|c|c}
\hline \multicolumn{2}{c|}{ Observed. } & \multicolumn{2}{c}{ Normal calculated. } \\
\hline Ref. eq. A. & Disp. eq. H-A. & Ref. eq. A. & Disp. eq. H-A. \\
\hline $82 \cdot 46$ & $3 \cdot 76$ & $83 \cdot 2$ & $3 \cdot 78$ \\
\hline
\end{tabular}

\section{Tetramethylenedicarboxylic Acid $(1,1), \stackrel{\mathrm{CH}_{2}-\mathrm{C}(\mathrm{COOH})_{2}}{\mathrm{CH}_{2}-\mathrm{CH}_{2}}$}

The following method was employed to hydrolyse ethylic tetramethylenedicarboxylate so as to obtain the free acid :-

80 grams of the nearly pure ethereal salt was dissolved in an equal bulk of alcohol and a moderately strong solution of alcoholic potash (containing 50-60 grams $\mathrm{KOH}$ ) then run in slowly. Hydrolysis sets in immediately, the mass often becoming so hot as to cause the alcohol to boil. As soon as the violence of the reaction has subsided, the whole is heated on a water-bath for five hours, and evaporated almost to dryness. The residue is next dissolved in water, and again evaporated in order to entirely remove the alcohol; the solution of the residual potassic salt in a little water, is then acidified with dilute sulphuric acid, and repeatedly (at least 10 times) extracted with pure 
ether. The ethereal solution, after being dried over calcic chloride, is evaporated, when the new acid is left as a beautiful colourless crystalline cake. These crystals, which sometimes contain a trace of oily matter, are roughly purified by spreading them out on a porous plate, and are afterwards dissolved in a little warm ether. If this ethereal solution is allowed to evaporate spontaneously, beautifully transparent crystals of tetramethylenedicarboxylic acid soon separate, and are easily obtained pure by one recrystallisation.

The following results were obtained on analysis :-

I. 0.2307 gram substance gave 0.4225 gram $\mathrm{CO}_{2}$ and 0.1226 gram $\mathrm{H}_{2} \mathrm{O}$.

II. $0 \cdot 1238$ gram substance gave 0.2276 gram $\mathrm{CO}_{2}$ and 0.0659 gram $\mathrm{H}_{2} \mathrm{O}$.

\begin{tabular}{|c|c|c|c|}
\hline & \multicolumn{2}{|c|}{ Found. } & \\
\hline & I. & II. & $\mathrm{C}_{3} \mathrm{H}_{6}: \mathrm{C}(\mathrm{COOH})_{2}$. \\
\hline$C \ldots$. & $49 \cdot 95$ & 50.13 per cent. & 50.00 per cent. \\
\hline$H \ldots \ldots$ & $5 \cdot 90$ & $5 \cdot 91$ & $5 \cdot 56$ \\
\hline $0 \ldots$. & $44 \cdot 15$ & $43 \cdot 96$ & $44 \cdot 44$ \\
\hline
\end{tabular}

When pretty quickly heated, tetramethylenedicarboxylic acid $(1,1)$ melts at $154-156^{\circ}$; if slowly heated, however, it decomposes at a lower temperature, carbouic anhydride being given off.

It is easily soluble in water, moderately easily in ether, chloroform, and benzene, but only sparingly in light petroleum. The aqueous solution, when evaporated over sulphuric acid in a vacuum, deposits the acid in thick prisms, but the most beautiful erystals are obtained when a considerable quantity of a strong ethereal solution is allowed to evaporate spontaneously.

Professor Haushofer was kind enough to measure some of these crystals for me, and gave the following account of them :-

Tetramethylenedicarboxylic Acid.

Crystalline System. Monoclinic.

$$
\begin{gathered}
a: b: c=1 \cdot 0324: 1: 1 \cdot 1354 \\
\beta=88^{\circ} 58^{\prime} .
\end{gathered}
$$

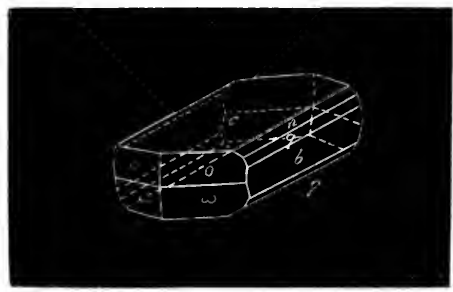


Tabular crystals of the combination-

$$
\begin{aligned}
0 \mathrm{P} & =(001)=c \\
-7 \mathrm{P} & =(771)=o \\
7 \mathrm{P} & =(77 \overline{1})=w \\
\infty \mathrm{R}_{\infty}=(010) & =b \\
\mathrm{R}_{\infty} & =(011)=q \\
\frac{1}{2} \mathrm{R} \infty & =(012)=n
\end{aligned}
$$

Cleavage perfectly conformable to the plane of symmetry $b$; on the plane $b$, the direction of extinction (with crossed Nicols) forms an angle of $38^{\circ}$ with the edge $b c$.

$$
\begin{aligned}
& \text { Measured. } \\
& c: o=(001)(771)=* 95^{\circ} 54 \\
& c: w=(00 \overline{1})(77 \overline{1})=94 \quad 32 \\
& o: o=(771)(7 \overline{7} 1)=* 8848 \\
& w: w=(77 \overline{1})(7 \overline{7} \overline{1})=8832 \\
& c: n=(001)(012)=15012 \\
& c: q=(001)(011)=13120 \\
& \overline{94^{\circ}} \overline{28^{\prime}} \\
& \text { - - } \\
& 131 \quad 23
\end{aligned}
$$

The solution of tetramethylenedicarboxylic acid in chloroform does not appear to be affected by bromine, even after standing for weeks, neither does hydrobromic acid act on the acid. Nearly the whole of the acid was recovered from a solution of it in concentrated hydrobromic acid (sp. gr. 1.83) which had been standing for six weeks, on shaking it up with ether. This is somewhat striking when it is remembered how easily trimethylenedicarboxylic acid is acted on by hydrobromic acid (Part I, page 8l4).

\section{Salts of Tetramethylenedicarboxylic Acid $(1,1)$.}

Ammonium Salt.-This salt was prepared by dissolving the acid in a slight excess of ammonia and allowing the solution to evaporate over sulphuric acid in a vacuum. It crystallises in long colourless needles, and is very easily soluble in water.

Silver Salt.-If nitrate of silver is added to a neutral solution of the ammonium salt, a soluble acid silver salt is at first produced, but on adding excess a white precipitate of the neutral salt is thrown down.

This salt, after being well washed and dried, gave the following results on analysis :-

I. 0.2595 gram substance gave 0.1904 gram $\mathrm{CO}_{2}$ aud 0.0451 gram $\mathrm{H}_{2} \mathrm{O}$.

II. 0.2265 gram substance gave 0.1359 gram silver. 
Found.

C..... 20.02 per cent.

H..... 1.94 ,

$\mathrm{Ag} \ldots 60.00 \quad$,

O..... 18.04 ,
Theory. $\mathrm{C}_{3} \mathrm{H}_{6}: \mathrm{C}(\mathrm{COOAg})_{2}$. $20 \cdot 11$ per cent. $1 \cdot 68 \quad "$ $60 \cdot 33 \quad$ " $17 \cdot 88$ "

Copper Salt.-The addition of cupric sulphate to a fairly concentrated solution of the ammonium salt produces a beautiful deepblue solution but no precipitate. If, however, this solution is warmed on a water-bath, the copper salt soon crystallises out in magnificent blue needles, which when examined under the microscopc are observed to have the form-

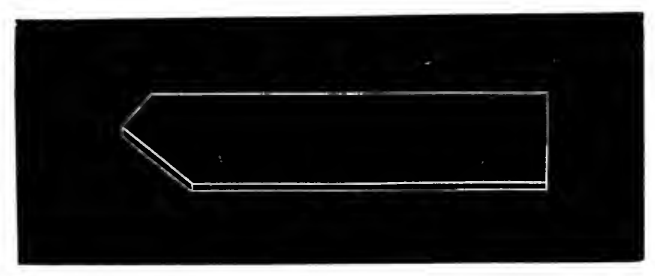

These crystals, which probably belong to the monosymmetric system, show a most beautiful play of colours when seen by polarised light.

The copper salt appears to have the formula-<smiles>CCCC1(C=O)C(=O)O[C@@H]1C=O</smiles>

\section{Analysis.}

I. (a) 0.2085 gram substance (dried over sulphuric acid) lost on heating to $150^{\circ} 0.0150$ gram.

( $\beta$ ) $0 \cdot 1935$ gram substance gave $0 \cdot 1749$ gram $\mathrm{CuO}$.

II. $0 \cdot 2640$ gram substance (dried at $150^{\circ}$ ) gave $0 \cdot 1010$ gram $\mathrm{CuO}$.

III. 0.1693 gram substance (dried over sulphuric acid) gave 0.0601 gram $\mathrm{CuO}$.

Found.

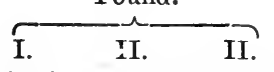

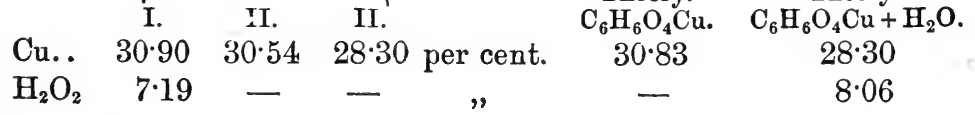

Lead Salt.-If plumbic acetate is added to a dilute solution of ammonic tetramethylenedicarboxylate, a white amorphous precipitate is 
thrown down which becomes crystalline on vigorous shaking. After filtering off, well washing with water and; drjing over sulphuric acid in a vacuum, this salt has the formula

$$
\mathrm{C}_{3} \mathrm{H}_{6}: \mathrm{C}<\mathrm{COO}>\mathrm{Cb}+\mathrm{H}_{2} \mathrm{O} \text {. }
$$

\section{Analyses.}

I. 0.2324 gram substance lost on heating to $150^{\circ}, 0.0127$ gram; thus dried, the substance gave on heating with sulphuric acid, 0.1903 gram $\mathrm{PbSO}_{4}$.

II. 0.2557 gram substance (dried at $140^{\circ}$ ) gave 0.2222 gram $\mathrm{PbSO}_{\mathbf{4}}$.

Found.

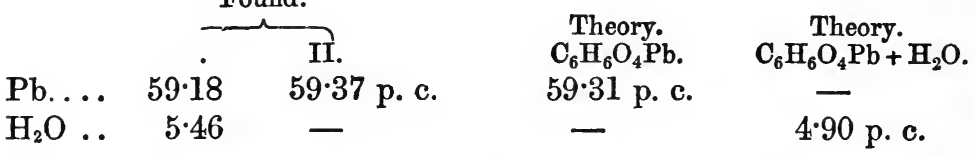

This lead salt is almost insoluble in cold water, and very sparingly soluble in hot water.

If the hot concentrated solution is allowed to cool, the salt crystallises out in four-sided prisms.

Barium Salt.-If baryta-water is added to the solution of tetramethylenedicarboxylic acid in water, no precipitate is formed at first, but on adding excess a white apparently amorphous precipitate is thrown down which is the neutral barium salt of the acid. After being well washed, and dried over sulphuric acid, it gave the following results on analysis :-

0.4002 gram substance lost on heating at $150-160^{\circ}, 0.0232$ gram, and gave on treating with sulphuric acid, 0.3117 gram $\mathrm{BaSO}_{4}$.

$$
\begin{aligned}
& \text { Theory. } \\
& \text { Ba..... } 45.82 \text { per cent. } \\
& \mathrm{H}_{2} \mathrm{O} \ldots .5 \cdot 77 \text {, }
\end{aligned}
$$

This salt, when examined under the microscope, is found not to be amorphous, but to consist of a mass of slender needles. It is sparingly soluble in water.

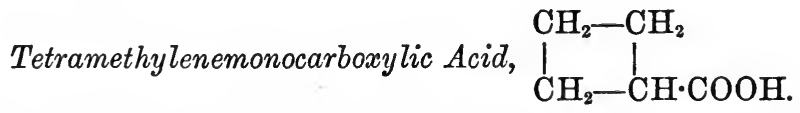

When tetramethylenedicarboxylic acid $[1,1]$ is heated to a few degrees above its melting point, it is rapidly decomposed with evolu- 
tion of carbonic anhydride and formation of tetramethylenemonocarboxylic acid, according to the equation-

$$
\mathrm{CH}_{2}<\mathrm{CH}_{2}^{\mathrm{CH}_{2}}>\mathrm{C}(\mathrm{COOH})_{2}=\mathrm{CH}_{2}<\mathrm{CH}_{2}^{\mathrm{CH}_{2}}>\mathrm{CH} \cdot \mathrm{COOH} \text {. }
$$

In order to effect this decomposition, the dicarboxylic acid is heated in a small Würtz flask at $210-220^{\circ}$. As soon as the acid has melted, carbonic anhydride begins to be evolved and a colourless oil distils over, very little residue remaining in the retort. The distillate when fractioned comes over almost entirely between $188^{\circ}$ and $193^{\circ}$ (if the dicarboxylic acid was pure), and on refractioning this once or twice the new acid is easily obtained pure as a colourless oil boiling constantly at $191^{\circ}(720 \mathrm{~mm}$.).

The analyses gave the following numbers :-

I. 0.1846 gram substance gave 0.4082 gram $\mathrm{CO}_{2}$ and $0 \cdot 1361$ gram $\mathrm{H}_{2} \mathrm{O}$.

II. $0 \cdot 2260$ gram substance gave $0 \cdot 4950$ gram $\mathrm{CO}_{2}$ and $0 \cdot 1691$ gram $\mathrm{H}_{2} \mathrm{O}$.

III. 0.1376 gram substance gave 0.3026 gram $\mathrm{CO}_{2}$ and 0.0991 gram $\mathrm{H}_{2} \mathrm{O}$.

\begin{tabular}{|c|c|c|c|c|c|c|}
\hline \multirow{2}{*}{ C.... } & \multicolumn{4}{|c|}{ Found. } & \multirow{2}{*}{\multicolumn{2}{|c|}{$\begin{array}{c}\text { Theory. } \\
\mathrm{C}_{3} \mathrm{H}_{6}: \mathrm{CH} \cdot \mathrm{COOH} . \\
60 \cdot 00 \text { per cent. }\end{array}$}} \\
\hline & $\begin{array}{c}\text { I. } \\
60 \cdot 30\end{array}$ & $\begin{array}{l}\text { II. } \\
59 \cdot 74\end{array}$ & $\begin{array}{c}\text { III. } \\
59 \cdot 97\end{array}$ & & & \\
\hline H ... & $8 \cdot 19$ & $8 \cdot 31$ & $8 \cdot 00$ & ” & $8 \cdot 00$ & , \\
\hline $0 \ldots$ & $31 \cdot 51$ & 31.95 & $32 \cdot 03$ & , & 32.00 & , \\
\hline
\end{tabular}

Tetramethylenemonocarboxylic acid is a colourless oil, of penetrating and very disagreeable odour, somewhat resembling that of butyric acid. It does not solidify when cooled down to $0^{\circ}$. It is sparingly soluble in water, but mixes in all proportions with alcohol, ether, \&c. It is easily oxidised when treated with permanganate of potash in alkaline solution, oxalic acid being formed.

Tetramethylenemonocarboxylic acid is not acted on by bromine at ordinary temperatures. The difficulty with which it is attacked by this reagent is very remarkable. On submitting a mixture of 5 grams of acid, 8 grams of bromine, and 20 grams of chloroform to direct sunlight in a sealed tube for 18 days, no apparent change had taken place, and on opening the tube only the slightest trace of hydrobromic acid could be detected. The tube was then sealed up again and heated at $100^{\circ}$ for two hours; even after this treatment, only a very small quantity of hydrobromic acid had been formed. After heating for five hours at $150^{\circ}$, however, the colour of the bromine had entirely disappeared, a colourless liquid being formed, and on opening the tube 
torrents of hydrogen bromide were evolved. The product when evaporated deposits a heavy brominated acid, the examination of which I am still engaged on.

Tetramethylenemonocarboxylic acid is isomeric with angelic acid, methylcrotonic acid, allylacetic acid, dimethylacrylic acid, and several other unsaturated acids.

Only two salts of tetramethylenemonocarboxylic acid have been examined, namely, the silver and calcium salts.

The silver salt is easily obtained by adding nitrate of silver to a neutral solution of the ammonium salt as a heavy white precipitate sparingly soluble in water.

\section{Anaylsis.}

0.2579 gram substance gave 0.2740 gram $\mathrm{CO}_{2}, 0.0804$ gram $\mathrm{H}_{2} \mathrm{O}$, and 0.1348 gram Ag.

Found.

C $\ldots \ldots .28 .98$ per cent.

H ..... $3 \cdot 46$,

$\mathrm{Ag} \ldots \ldots .52 \cdot 23 \quad$,

$0 \ldots \ldots 15 \cdot 33$,
Theory.

$\mathrm{C}_{5} \mathrm{H}_{7} \mathrm{AgO}_{2}$.

28.98 per cent.

$3 \cdot 38$ "

$52 \cdot 17 \quad$

$15 \cdot 47$,

The calcium salt of the acid is prepared by boiling the acid with calcic carbonate and water, filtering, and concentrating the filtrate on a water-bath till it becomes of a syrupy consistency. On standing, this deposits the salt in long silky needles, which are very soluble in water.

After recrystallising and allowing to dry by exposure to air for four or five days, this substance was found to have the formula-

$$
\mathrm{C}_{10} \mathrm{H}_{14} \mathrm{O}_{4} \mathrm{Ca}+5 \mathrm{H}_{2} \mathrm{O} \text {. }
$$

\section{Analyses.}

I. 0.3288 gram dried at $150^{\circ}$ for half an hour lost 0.0889 gram.

II. $0.4473, \quad " 120 \quad$ ", 0.0922, III. $0.3195 "$ treated with sulphuric acid gave 0.1277 gram $\mathrm{CaSO}_{4}$.

\begin{tabular}{|c|c|c|c|c|}
\hline & \multicolumn{3}{|c|}{ Found. } & \multirow{2}{*}{$\begin{array}{l}\text { Theory. } \\
\mathrm{C}_{10} \mathrm{H}_{11} \mathrm{O}_{4} \mathrm{Ca}+5 \mathrm{H}_{2} \mathrm{O}\end{array}$} \\
\hline & I. & II. & III. & \\
\hline & - & - & $11 \cdot 75$ p.c. & $12 \cdot 19$ p. c. \\
\hline 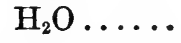 & $27 \cdot 04$ & $27 \cdot 09$ & -, & $27 \cdot 48$, \\
\hline
\end{tabular}

This salt slowly loses some of its water of crystallisation when exposed to the air for a long time. A sample which had been thus exposed for three weeks, was found to contain 24.59 per cent. $\mathrm{H}_{2} \mathrm{O}$. 
Placed over sulphuric acid in a vacuum, it loses all of its water of crystallisation in seven days.

Two analyses of this salt dried at $150^{\circ}$, gave the following results :-

I. 0.3349 gram substance gave $0 \cdot 1922$ gram $\mathrm{CaSO}_{4}$.

II. 0.3208 gram substance gave 0.1830 gram $\mathrm{CaSO}_{4}$.

Found.

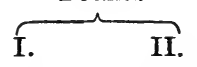

Ca..... $16.88 \quad 16.82$ per cent.
Theory.

$\left(\mathrm{C}_{3} \mathrm{H}_{6}: \mathrm{CH} \cdot \mathrm{COO}\right)_{2} \mathrm{Ca}$. 16.80 per cent.

On distilling this salt with lime, a gas is given off, which, however, does not appear to be tetramethylene. I hope in a short time to be able to obtain this gas in sufficient quantity for analysis and examination.

Tetramethylenecarboxylic acid, being one of the most simply constituted of the ring compounds yet obtained, it was thought that interesting results might be obtained from the study of its physical properties. The results of the sp. gr. and magnetic rotation determinations, which were performed by my father, are the following :-

$$
\begin{aligned}
& d \frac{15^{\circ}}{15^{\circ}}=1 \cdot 05480, \\
& d \frac{20^{\circ}}{20^{\circ}}=1 \cdot 05116, \\
& d \frac{25^{\circ}}{25^{\circ}}=1 \cdot 04761 .
\end{aligned}
$$

Magnetic Rotations.

\begin{tabular}{c|c|c}
$t$. & Sp. rotation. & Mol. rotation. \\
\hline 18 & $0 \cdot 9561$ & $5 \cdot 046$ \\
18 & $0 \cdot 9586$ & $5 \cdot 059$ \\
18 & $0 \cdot 9548$ & $5 \cdot 039$ \\
\hline Averages 18 & $0 \cdot 9565$ & $5 \cdot 048$
\end{tabular}

I submitted a very pure sample of this acid to Dr. Gladstone, who - was kind enough to examine it optically. The following are the results of his experiments :-

\begin{tabular}{c|c|c|c|c}
\hline$t$. & Sp. gr. & $\mu_{\mathrm{A}} \cdot$ & $\mu_{\mathrm{D} \cdot}$ & $\mu_{\mathrm{H} \cdot}$ \\
\hline $25 \cdot 5$ & $1 \cdot 046$ & $1 \cdot 4351$ & $1 \cdot 4403$ & $1 \cdot 4561$ \\
\hline
\end{tabular}




\begin{tabular}{c|c|c|c}
\hline \multicolumn{2}{c|}{ Observed. } & \multicolumn{2}{c}{ Normal calculated. } \\
\hline Ref. eq. A. & Disp. eq. H - A. & Ref. eq. A. & Disp. eq. H - A. \\
\hline 41.60 & $2 \cdot 00$ & $41 \cdot 6$ & $1 \cdot 89$ \\
\hline
\end{tabular}

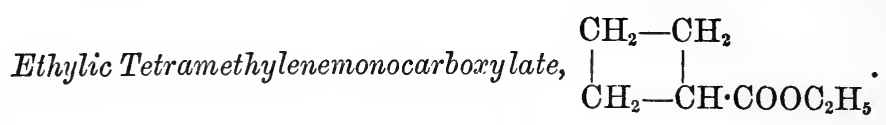

This ethereal salt was prepared as follows :-20 grams of the dry silver salt of tetramethylenemonocarboxylic acid was mixed with 25 grams of ethyl iodide and 50 grams of pure ether, and heated on a water-bath for about two hours. At the end of this time, all the silver salt was decomposed, iodide of silver being precipitated. This was removed by filtering, and once or twice extracted with ether.

On carefully distilling off the ether, a colourless oil remained behind, which on the first distillation went over between $148^{\circ}$ and $155^{\circ}$; by repeated fractioning, the ethereal salt was easily obtained pure, boiling at $151-151 \cdot 5^{\circ}(720 \mathrm{~mm}$.$) . Analyses :-$

I. $0 \cdot 1450$ gram substance gave 0.1193 gram $\mathrm{H}_{2} \mathrm{O}$ and 0.3478 gram $\mathrm{CO}_{2}$.

II. $0 \cdot 1410$ gram substance gave $0 \cdot 1210$ gram $\mathrm{H}_{2} \mathrm{O}$ and $0 \cdot 3390$ gram $\mathrm{CO}_{2}$.

\begin{tabular}{|c|c|c|c|}
\hline \multicolumn{3}{|c|}{ Found. } & \multirow{3}{*}{$\begin{array}{c}\text { Theory. } \\
\mathrm{C}_{3} \mathrm{H}_{6}: \mathrm{CH} \cdot \mathrm{COOC} \mathrm{H}_{5} \\
65 \cdot 62 \text { per cent. }\end{array}$} \\
\hline & I. & II. & \\
\hline & $65 \cdot 411$ & 65.57 per cent. & \\
\hline & $9 \cdot 14$ & $9 \cdot 53$ & $9 \cdot 38$ \\
\hline$\ldots$ & $25 \cdot 45$ & $24: 90$ & $25 \cdot 00$ \\
\hline
\end{tabular}

Ethylic tetramethylenemonocarboxylate is a limpid liquid, of agreeable odour; it does not solidify at $0^{\circ}$.

\section{Trimethylene Iodide, $\mathrm{CH}_{2} \mathrm{I} \cdot \mathrm{CH}_{2} \cdot \mathrm{CH}_{2} \mathrm{I}$.}

During the course of these experiments, it was thought that if in place of trimethylene bromide a compound were employed capable of reacting more energetically, such as trimethylene iodide, that a better yield of ethylic tetramethylenedicarboxylate would be obtained.

In order to test this, it was first necessary to find some simple method for preparing trimethylene iodide; after several experiments, it was observed that trimethylene bromide is almost quantitatively 
converted into the corresponding iodide by simply digesting it with alcoholic potassic iodide; thus :-

\section{$\mathrm{CH}_{2} \mathrm{Br} \cdot \mathrm{CH}_{2} \cdot \mathrm{CH}_{2} \mathrm{Br}+2 \mathrm{KI}=\mathrm{CH}_{2} \mathrm{I} \cdot \mathrm{CH}_{2} \cdot \mathrm{CH}_{2} \mathrm{I}+2 \mathrm{KBr}$.}

In preparing the iodide, 50 grams of pure trimethylene bromide are heated on a water-bath with 100 grams of alcohol and $90-100$ grams of finely powdered potassic iodide for about an hour. After boiling for a few minutes, potassic bromide begins to be deposited, and at the end of the reaction the mass becomes almost solid; water is now added, and the heavy oil which separates is extracted once or twice with ether. The ethereal solntion, which is generally of a brownish colour, is then well washed with water to dissolve out potassic iodide and alcohol, dried over calcic chloride, and freed from iodine by shaking with a small quantity of mercury. On distilling off the ether, nearly pure trimethylene iodide remains. In order to obtain a perfectly pure product, the oil thus obtained is next fractioned under diminished pressure $(250 \mathrm{~mm}$.) ; on the first distillation, almost the whole of it passes over between $175^{\circ}$ and $183^{\circ}$ as a heavy oil of peculiar odour, which, if discoloured by iodine, is again treated with a little mercury and refractioned. Pure trimethylene iodide is a colourless oil boiling at $179^{\circ}(250 \mathrm{~mm}$.). An analysis gave the following numbers :-

0.3048 gram substance gave 0.4820 gram AgI.

$\begin{array}{ll}\text { I } \ldots \ldots & \text { Found. } \\ 85 \cdot 46 \text { per cent. } & \text { Theory. } \mathrm{C}_{3} \mathrm{H}_{6} \mathrm{I}_{2} . \\ & 85 \cdot 81 \text { per cent. }\end{array}$

Trimethylene iodide boils at $215-220^{\circ}(720 \mathrm{~mm}$.) almost without decomposition. It does not solidify at $-10^{\circ}$. The sp. gr. of a specimen dried ever phosphoric anhydride was determined by my father with the following results :-

$$
\begin{aligned}
& d \frac{4^{\circ}}{4^{\circ}}=2.59617, \\
& d_{\frac{15^{\circ}}{15^{\circ}}}=2.57612, \\
& d_{\frac{25^{\circ}}{25^{\circ}}}=2.56144 .
\end{aligned}
$$

On trying the action of this iodide on ethylic malonate, it was soon found it presented no advantage as compared with the bromide. Not only was the yield of ethylic tetramethylenedicarboxylate no better, the boiling point of the latter and of the iodide being nearly the same, it was much more difficult to obtain a pure product. This method was therefore abandoned.

A short notice on trimethylene iodide was published in the Berichte 
$(18,221)$, and shortly afterwards (ibid., 18, 519) Louis Henry stated that he also had prepared this compound in a way similar to that described above, and remarked that Frennd (Monatsh. Chem., 2, 640) had also obtained it by heating trimethylene glycol with hydriodic acid.

Louis Henry states that trimethylene iodide boils at $224^{\circ}$ (763 mm.), and does not solidify at $-20^{\circ}$. He also makes some interesting remarks on the stability of this compound as compared with ethylene iodide.

\section{Theoretical Remarks.}

In the foregoing, the substances produced by the action of trimethylene bromide on ethylic malonate have, without comment, been taken as being derivatives of tetramethylene. This is, however, not necessarily the case. There is another interpretation of this reaction, which at first sight is quite as probable, and that is, that these compounds are really nothing more than allyl-derivatives, thus: ethylic tetramethylenedicarboxylate, tetramethylenedicarboxylic acid, and tetramethylenemonocarboxylic acid might be ethylic allylmalonate, allylmalonic acid, or allylacetic acid respectively.

Such derivatives could easily be formed in the following way:-

The first product of the action of trimethylene bromide on ethylic malonate would be undoubtedly ethylic bromopropylmalonate, thus :-

\section{$\mathrm{CHNa}\left(\mathrm{COOC}_{2} \mathrm{H}_{5}\right)_{2}+\mathrm{CH}_{2} \mathrm{Br}^{\circ} \mathrm{CH}_{2} \cdot \mathrm{CH}_{2} \mathrm{Br}=$ $\mathrm{CH}_{2} \mathrm{Br} \cdot \mathrm{CH}_{2} \cdot \mathrm{CH}_{2} \cdot \mathrm{CH}\left(\mathrm{COOC}_{2} \mathrm{H}_{5}\right)_{2}+\mathrm{NaBr}$.}

In the second phase of the reaction, two changes can take place, that is: $(\alpha)$ the bromine-atom can combine with the hydrogen-atom of the $\mathrm{CH}$-group, in which case ethylic tetramethylenedicarboxylate would be formed, or $(\beta)$ hydrogen bromide may be eliminated in the following way :-

\section{$\mathrm{CH}_{2} \mathrm{Br} \cdot \mathrm{CH}_{2} \cdot \mathrm{CH}_{2} \cdot \mathrm{CH}\left(\mathrm{COOC}_{2} \mathrm{H}_{5}\right)_{2}=\mathrm{CH}_{2}: \mathrm{CH} \cdot \mathrm{CH}_{2} \cdot \mathrm{CH}\left(\mathrm{COOC}_{2} \mathrm{H}_{5}\right)_{2}$ $+\mathrm{HBr}$.}

ethylic allylmalonate being produced.

This being the case, it will be necessary, before going further, to prove that the products of the action of trimethylene bromide on ethylic malonate are really tetramethylene-derivatives, and not allylderivatives.

Here the proof is fortunately much easier than was the case with ethylic trimethylenedicarboxylate and ethylic vinylmalonate described in Part I of this research (Trans., 1886, 817), all the corresponding allyl-derivatives having already been prepared and examined, and it is therefore only necessary to compare these side by side with the 
tetramethylene compounds, in order to show at a glance the remarkable differences existing between them.

This is shown very clearly on examining the following table of the boiling and melting points of the two series of compounds :-

(a.) Boiling Points.

\begin{tabular}{|c|c|c|c|}
\hline & (760 mm.). & & (760 mm.). \\
\hline 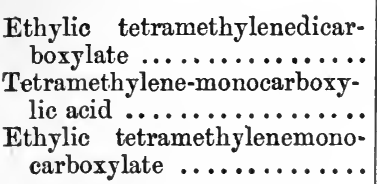 & $\begin{array}{l}224-225^{\circ} \\
194-195 \\
154-155\end{array}$ & $\begin{array}{l}\text { Ethylic allylmalonate } \\
\text { Allylacetic acid...... } \\
\text { Ethylic allylacetate .. }\end{array}$ & $\begin{array}{c}219-220^{\circ} \\
183^{\circ} \\
142-144^{\circ}\end{array}$ \\
\hline
\end{tabular}

( $\beta$.) Melting Points.

\begin{tabular}{|c|c|c|c|c|}
\hline $\begin{array}{l}\text { Tetramethylene }- \text { dicarboxylic } \\
\quad \text { acid } \ldots \ldots \ldots \ldots \ldots \ldots \ldots \ldots\end{array}$ & $154-156^{\circ}$ & Allylmalonic acid . & $\cdots$ & $103^{\circ}$ \\
\hline
\end{tabular}

These two melting points show a variation of $50^{\circ}$. In order, however, to establish the great difference between tetramethylenedicarboxylic acid and allylmalonic acid, I submitted some beautiful crystals of the latter to Professor Haushofer, who kindly measured them for me with the following results :-

\section{Monallylmalonic Acid.}

Crystalline system : triclinic.

Small brilliant crystals resembling rhombohedrons, of which the faces $c, p$, and $q$ were observed; cleavage perfect conformably to the surface $q$.

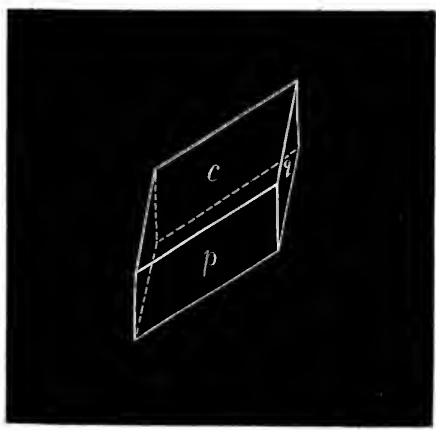




$$
\begin{gathered}
\text { Measured. } \\
p: q=43^{\circ} 58^{\prime} \text { (front) } \\
q: c=972 \\
p: c=114^{\circ} 39
\end{gathered}
$$

On comparing the crystalline form of this acid with that of tetramethylenedicarboxylic acid (given on page 5), it is at once evident that the two acids cannot possibly be identical.

Professor Franchimont (Rec. Trav. Chim., 1885, 398 and 399) has lately published a series of researches on the action of pure nitric acid on organic compounds, and, among others, on the derivatives of malonic acid. He found that malonic acid and its monosubstituted derivatives, such as ethyl-allylmalonic acid, \&c., were completely decomposed when treated with pure nitric acid, with elimination of 2 mols. $\mathrm{CO}_{2}$. The disubstituted malonic acids, such as diethylmalonic acid, \&c., were on the contrary not acted on at all when left in contact with this acid. In connection with these experiments, Professor Franchimont was kind enough to examine the behaviour of tetramethylenedicarboxylic acid when treated with pure nitric acid, and found that this compound, even after standing for days with nitric acid, showed no signs of decomposition, and after 10 days' exposure, he was able, on extracting with ether, to regain nearly the whole of the tetramethylenedicarboxylic acid nnchanged.

These experiments can only be explained on the assumption that the tetramethylenedicarboxylic acid is a disubstituted malonic acid of the formula $\underset{\mathrm{COOH}}{\mathrm{COOH}}>\mathrm{C}<\mathrm{CH}_{2}^{\mathrm{CH}_{2}}>\mathrm{CH}_{2}$, and they therefore go far to. prove that this compound is really a tetramethylene-derivative.

An equally conclusive proof of the constitution of this acid car be deduced from the study of the magnetic rotations of ethylic tetramethylenedicarboxylate and tetramethylenemonocarboxylic acid. The magnetic rotation of ethylic tetramethylenedicarboxylate is $9 \cdot 940$, that of tetramethylenecarboxylic acid is $5 \cdot 048$. The difference between the magnetic rotation of these two substances and those of the isomeric allyl* derivatives is very striking. Thus :

The magnetic rotation of the allylmalonate $=11 \cdot 281$.

$$
, \quad, \quad \text { allylacetic acid }=6.426 \text {. }
$$

Here the differences between the magnetic rotation of ethylic allymalonate and ethylic tetramethylenedicarboxylate, and between those of allylacetic acid and tetramethylenecarboxylic acid, are 1.378 resp. 1.341, a further proof that these compounds cannot possibly be identical.

The magnetic rotations of these tetramethylene-derivatives agree

* W. H. Perkin, Trans., 1884, 44, 508, 576, and 1886, 49, 211. 
much more closely with those of the corresponding saturated compounds in the fatty series, allowing of course in all cases for the difference of $\mathrm{H}_{2}=0.508$. Thus :

$$
\begin{aligned}
\text { Ethylic propymalonate }-2 \mathrm{H} & =9 \cdot 859 . \\
\text { Valeric acid }-2 \mathrm{H} & =5 \cdot 000 .
\end{aligned}
$$

It would appear from the experiments which have up to the present time been carried out by my father, that the value of $\mathrm{CH}_{2}$ in a ring is slightly different from the value of $\mathrm{CH}_{2}$ in an open chain.

The study of the magnetic rotation of these tetramethylenederivatives also shows that both tetramethylenedicarboxylic and tetramethylenemonocarboxylic acids are derivatives of the same group of elements, that is $\mathrm{CH}_{2}<\mathrm{CH}_{2} \mathrm{CH}_{2}>\mathrm{CH}_{2}$.

Thus if the value of ethylic malonate $-2 \mathrm{H}=6.902$ be abstracted from the value of ethylic tetramethylenedicarboxylate (9.940), we get 3.038 as the value of the group $-\mathrm{CH}_{2}>\mathrm{CH}_{2}$.

If a similar operation be performed with tetramethylenemonocarboxylic acid, we get the following :-

Tetramethylenemonocarboxylic acid (5.048)-Acetic acid $-2 \mathrm{H}$ $(2.017)=3.031=$ value of $-\mathrm{CH}_{2}>\mathrm{CH}_{2}$, a number closely agreeing with the preceding one.

Dr. Gladstone's measurements of the refraction and dispersion equivalents of ethylic tetramethylenedicarboxylate and tetramethylenemonocarboxylic acid also show closely that these must be saturated compounds. Dr. Gladstone has given me the following explanation of the results of his determinations (pp. 4, 11, 12).

"The refraction and dispersion equivalents of ethylic tetramethylenedicarboxylate indicate that it is a saturated body.

"Tetramethylenecarboxylic acid.-These figures closely agree with those of a saturated compound, an allyl compound gives much higher figures. (The refraction equivalent of allylacetic acid is $43 \cdot 25$, and the dispersion equivalent $2 \cdot 41$.)"

There can therefore scarcely be a doubt, but that the compounds produced by the action of trimethylene bromide on ethylic malonate are really derivatives of tetramethylene.

II. Tetramethylenetetracarboxylic acid $(1,1,2,2,) \underset{\mathrm{CH}_{2}-\mathrm{C}(\mathrm{COOH})_{2}}{\mathrm{CH}_{2}-\mathrm{C}(\mathrm{COOH})_{2}}$.

If the sodium compound of ethylic malonate be treated with ethylene bromide, the principal product of the reaction is ethylic 
trimethylenedicarboxylate $(1,1)$, as was shown in Part $I$ of this research, Trans., 1885, p. 807.*

This compound is formed according to the following equation, one molecule of ethylene bromide reacting with one molecule of ethylic malonate :-

$$
{ }_{2}^{\mathrm{COOC}_{2} \mathrm{H}_{5}}>\mathrm{CHNa}+\mathrm{C}_{2} \mathrm{H}_{4} \mathrm{Br}_{2}=\underset{\mathrm{COOC}_{2} \mathrm{H}_{5}}{\mathrm{COOOC}_{2} \mathrm{H}_{5}}>\mathrm{C}_{\mathrm{CH}_{2}}^{\mathrm{COOH}_{1}}+
$$

Ethylic trimethylenedicarboxylate.

$$
\mathrm{COOC}_{2} \mathrm{H}_{5}>\mathrm{CH}_{2}+2 \mathrm{NaBr} \text {. }
$$

This ethereal salt is, however, not the only product of the reaction, as there is always a small quantity of an oil of high boiling point formed at the same time: this remains behind in the retort during the purification of the crude product, after the ethylic trimethylenedicarboxylate has distilled over. (See Part I, Trans., 1885, p. 808.)

This oil, after being carefully fractioned under diminished pressure, proved to be the product of the action of $1 \mathrm{~mol}$. of ethylene bromide on 2 mols. of the sodium compound of ethylic malonate, thus :-

$$
2 \mathrm{CHNa}\left(\mathrm{COOC}_{2} \mathrm{H}_{5}\right)_{2}+<\underset{\mathrm{CH}_{2} \mathrm{Br}}{\mathrm{CH}_{2} \mathrm{Br}}=<_{\mathrm{CH}_{2} \cdot \mathrm{CH}\left(\mathrm{COOC}_{2} \mathrm{H}_{5}\right)_{2}}^{\mathrm{CH}_{2} \cdot \mathrm{CH}\left(\mathrm{COOC}_{2} \mathrm{H}_{5}\right.}+2 \mathrm{NaBr} \text {. }
$$

This substance, for which I propose the name ethylic butane- $\omega_{2} \omega_{2}$ tetracarboxylate, $\uparrow$ can with advantage be used for the synthesis of tetramethylene compounds.

When mixed with sodic ethylate, two of its hydrogen-atoms are directly displaced by sodium, forming a disodium compound which when treated with bromine is converted quantitatively into the ethereal salt of tetramethylenetetracarboxylic acid $(1,1,2,2)$, thus :-

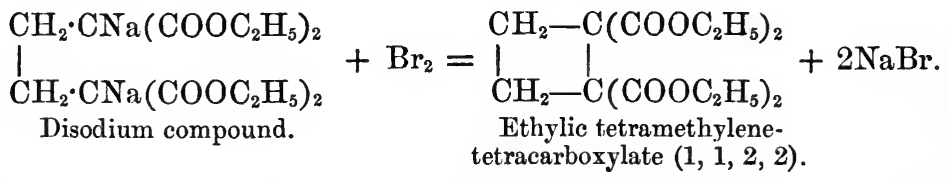

On hydrolysis, the free acid is easily obtained, and this in its turn when heated at $180^{\circ}$, splits up into tetramethylenedicarboxylic acid $(1,2)$ and 2 mols. $\mathrm{CO}_{2}$, thus :-

* See also Berichte, 17, 54, 323; 18, 1734; 19, 1049. Fittig and Roeder, Annalen, 227, 13.

+ With regard to the nomenclature, see Baeyer (Ber., 17, 961). 


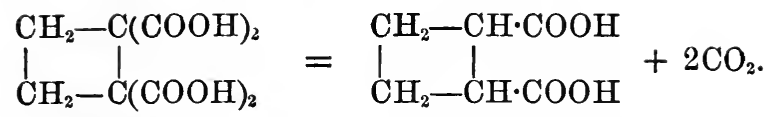

Tetramethylenetetracarboxylic acid $(1,1,2,2)$. dicarboxylic acid $(1,2)$.

As this reaction appeared to be of special interest, it was worked out very carefully, the following being the details of the processes used.

\section{Ethylic Butane- $\omega_{2} \omega_{2}$-Tetracarboxylate, \\ $\left(\mathrm{COOC}_{2} \mathrm{H}_{5}\right)_{2} \mathrm{CH} \cdot \mathrm{CH}_{2} \cdot \mathrm{CH}_{2} \cdot \mathrm{CH}\left(\mathrm{COOC}_{2} \mathrm{H}_{5}\right)_{2}$.}

In order to obtain this substance, which is the starting point in the following syntheses, the crude product of the action of ethylene bromide on ethylic malonate (prepared as described in Trans., 1885, p. 807) is submitted to distillation in steam until oily drops cease to come over. By this means, all the ethylic trimethylenedicarboxylate, together with unchanged ethylene bromide and ethylic malonate, are carried over by the steam, a small quantity of a thick yellowish oil being left in the retort, which can be easily separated from the watery liquid by extracting once or twice with ether. After drying over calcic chloride and distilling of the ether, the crude ethylic butanetetracarboxylate is obtained as a thick yellowish oil, which is next purified by fractioning under diminished pressure $\left(225 \mathrm{~mm}\right.$.) ; it then nearly all goes over between 250 and $290^{\circ}$ the first time, and on repeatedly fractioning a colourless oil is obtained boiling constantly at $275-280^{\circ}$. This gives the following numbers on analysis :-

$0 \cdot 1620$ gram substance gave 0.1118 gram $\mathrm{H}_{2} \mathrm{O}$ and 0.3280 gram $\mathrm{CO}_{2}$.

Found.

C . .... $55 \cdot 22$ per cent.

H.... $7 \cdot 66^{\circ}$,

O ... $37 \cdot 12$,
Theory. $\mathrm{C}_{16} \mathrm{H}_{26} \mathrm{O}_{8}$.

55.49 per cent.

$7 \cdot 51 \quad$,

$36 \cdot 99 \quad$,

Ethylic butane- $\omega_{2} \omega_{2}$-tetracarboxylate is a thick, colourless oil of feeble odour; it does not solidify at $0^{\circ}$. The yield obtained by the above method is very small; 10 grams from 300 grams of ethylic malonate has rarely been exceeded.

The boiling points of this compound incidentally observed are

$$
\begin{aligned}
\text { At } 100 \mathrm{~mm} . & =248-252^{\circ} \\
, 225, " & =275-280 .
\end{aligned}
$$

In order to prove that this ethereal salt really has the constitution 
assigned to it at the beginning of this chapter, it was next converted into butanetetracarboxylic acid by hydrolysis.

This acid is decomposed when heated to $200^{\circ}$ into adipic acid, and 2 mols. $\mathrm{CO}_{2}$, thus :-

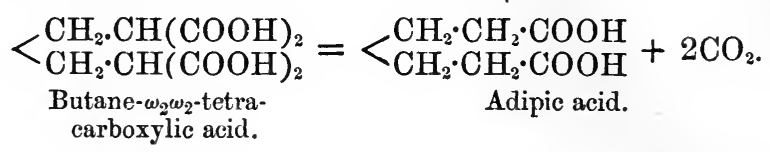

Thus affording a direct proof of the constitution of butanetetracarboxylic acid.

The hydrolysis of the ethereal salt of this acid is best carried out in the following way :-

The pure ethereal salt is mixed with a slight excess of alcoholic potash and heated for about four hours to boiling. Water is then added, the whole evaporated, nearly to dryness, on a water-bath, and the residue dissolved in water. The alkaline solution is next carefully neutralised with acetic acid, and the lead salt of the acid precipitated by adding excess of acetate of lead. After collecting the lead salt and washing it well with water, to remove the soluble salts, it is suspended in water and decomposed with sulphuretted hydrogen. In order to obtain the free acid, the lead sulphide is filtered off and the solution evaporated over sulphuric acid in a vacuum. In this way, the butanetetracarboxylic acid is obtained as a thick colourless oil which appears to crsstallise on long standing.

The analysis of the silver salt, prepared by precipitating a neutral solution of the ammonium salt with nitrate of silver, well washing and drying over sulphuric acid in a vacuum, gave the following numbers, agreeing approximately with the formula

$$
(\mathrm{COOH})_{2} \mathrm{CH} \cdot \mathrm{CH}_{2} \cdot \mathrm{CH}_{2} \cdot \mathrm{CH}(\mathrm{COOH})_{2} \text {. }
$$

$0 \cdot 1083$ gram substance gave 0.0696 gram silver.

$$
\begin{aligned}
& \text { Found. Theory. } \\
& \mathrm{Ag} \ldots . .64 .27 \text { per cent. } \quad 65.22 \text { per cent. }
\end{aligned}
$$

If butanetetracarboxylic acid is heated in a small Würtz flask at $200^{\circ}$, until the evolution of carbonic anhydride ceases, and the brownish residue is then distilled, a thick colourless oil is obtained, which on long standing almost entirely solidifies to crystals of adipic acid. In order to purify these crystals, they were roughly freed from the mother-liquor by spreading out on a porous plate, and then dissolved in a small quantity of water. This solution was allowed to evaporate over sulphuric acid in a vacuum, when beautiful colourless crystals melting at $146-148^{\circ}$ were obtained. (Adipic acid melts at $149^{\circ}$.) 
Unfortunately the quantity was too small for analysis, but there can be no doubt, from the appearance, melting point, and general properties that the substance was really adipic acid.

\section{Tetramethylenetetracarboxylic acid $(\mathrm{1}, 1,2,2), \stackrel{\mathrm{CH}_{2}-\mathrm{C}(\mathrm{COOH})_{2}}{\mathrm{CH}_{2}-\mathrm{C}(\mathrm{COOH})_{2}}$}

If the ethereal solution of $1 \mathrm{~mol}$. ethylic butanetetracarboxylate is mixed with an ethereal solution of sodic ethylate ( 2 mols.), and the whole allowed to remain for a short time, the disodium compound of the above ethereal salt is precipitated in colourless flocks; this, after collecting and washing with a mixture of alcohol and ether, is approximately pure, giving the following number on analysis :-

0.2221 gram substance gave 0.0778 gram $\mathrm{Na}_{2} \mathrm{SO}_{4}$.

\section{Found. \\ Theory.}

Na..... 11.35 per cent. $\quad 11 \cdot 79$ per cent.

This disodium compound is fairly stable and dissolves in water forming a clear solution, which however on standing or on the addition of an acid is decomposed, an oil, probably unchanged ethylic butanetetracarboxylate, separating out.

This disodium compound, when treated with bromine, is converted into ethylic tetramethylenetetracarboxylate. This synthesis of the tetramethylene-ring is carried out in the following way:- 21 grams of pure ethylic butanetetracarboxylate are dissolved in about 100 grams of pure dry ether, and then mixed with an ethereal solution of sodic ethylate containing 3 grams of sodium; ; the whole being allowed to stand about five minutes to ensure the complete precipitation of the sodium compound. 10 grams of pure bromine are now slowly added through a dropping funnel, the whole being well agitated and cooled during the operation. Each drop of bromine becomes instantly decolorised as it comes in contact with the sodium compound, but the last drop of bromine should cause a faint but permanent yellowish coloration of the solution; as soon as this takes place, the reaction is complete. After standing for about an hour, water is added, the ethereal solution separated from the aqueous layer, well washed with water and carbonate of soda solution, and dried over calcic chloride. On distilling off the ether, the crude ethylic tetramethylenetetracarboxylate is obtained as a thick colourless oil, which was not further purified,

* Prepared by dissolving the calculated quantity of sodium in as little hot alcchol as possible, and then while still hot, pouring in about five times the volume of pure dry ether. 
but used as it was for preparing the free tetramethylenetetracarboxylic acid. This was done in the following way :-

The crude ethereal salt was mixed with a solution of 16 grams of pure potash dissolved in a little alcohol, and boiled for about eight hours. Water was then added and the solution evaporated nearly to dryness on a water-bath, in order to drive off the alcohol as completely as possible. The residue was then dissolved in water, neutralised with acetic acid, and the lead salt of the acid precipitated by adding acetate of lead. The thick white precipitate thus obtained was then carefully washed with water, until quite free from soluble inorganic matter, suspended in water, and decomposed with sulphuretted hydrogen. After filtering off the precipitated sulphide of lead, the filtrate was allowed to evaporate over sulphuric acid in a vacuum, when a colourless oil was left, which, after a short time, solidified to a mass of crystals. These were first purified by spreading out on a porous plate, then washed once or twice with benzene, and dried over sulphuric acid in a vacuum.

The analysis gave the following results :-

0.0675 gram substance gave 0.0231 gram $\mathrm{H}_{2} \mathrm{O}$ and 0.1015 gram $\mathrm{CO}_{3}$.

Found.

$\begin{array}{lrc}\text { C..... } & 41 \cdot 01 \text { per cent. } \\ \text { H.... } & 3 \cdot 78 \quad, \\ \text { O } \ldots \ldots & 55 \cdot 21 \quad,\end{array}$

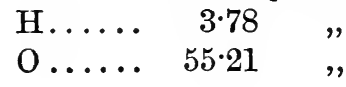

Theory.

$\mathrm{C}_{4} \mathrm{H}_{4}(\mathrm{COOH})_{4}$.

$41 \cdot 38$ per cent.

$3 \cdot 45$

$55 \cdot 17$ "

Tetramethylenetetracarboxylic acid $(1,1,2,2)$ is a colourless crystalline mass, which melts at $145-150^{\circ}$, being at the same time decomposed into tetramethylenedicarboxylic acid $(1,2)$ and carbonic anhydride. It is very easily soluble in water, alcohol, and ether, but more sparingly in benzene and light petroleum. The aqueous solution has a strongly acid reaction.

\section{Tetramethylenedicarboxylic acid $(1,2), \stackrel{\mathrm{CH}_{2}-\mathrm{CH} \cdot \mathrm{COOH}}{\mathrm{CH}_{2}-\mathrm{CH} \cdot \mathrm{COOH}}$.}

Tetramethylenetetracarboxylic acid $(1,1,2,2)$, when heated a few degrees above its melting point, is rapidly decomposed into carbonic anhydride and tetramethylenedicarboxylic acid (1, 2), as described above. Daring this process, however, a slight decomposition appears to take place, as the resulting mass is generally somewhat brownish, and it was thought possible that if a solution of the acid, instead of the dry substance, was heated up to the required temperature, this might be avoided and a cleaner product obtained. A fairly concen- 
trated solution of the tetracarboxylic acid in water was therefore heated in a sealed tube for two days at $180-200^{\circ}$. On opening the tube, a considerable pressure was noticeable due to carbonic anhydride. In order to isolate the new acid, the aqueous solution, which was nearly colourless, was evaporated on a water-bath to a syrupy consistence; this, however, showed no signs of crystallising, even after standing for days over sulphuric acid in a vacuum.

The following method of purification was then resorted to with good results:-The crude acid was dissolved in about three times its weight of absolute alcohol, and saturated with hydrogen chloride. After standing for a few hours, water was added, and the oily drops which separated were extracted with ether. The ethereal solution, after well washing with water and dilute sodic carbonate, was distilled on a water-bath, when the ethereal salt of tetramethylenedicarboxylic acid was left as a yellowish oil. This was first distilled under diminished pressure $(100 \mathrm{~mm}$.), and then fractioned under the ordinary pressure. In this way, a colourless oil was obtained boiling at $238-242^{\circ}(720 \mathrm{~mm}$.), which gave the following numbers on analysis :-

0.1711 gram substance gave 0.1290 gram $\mathrm{H}_{2} \mathrm{O}$ and 0.3754 gram $\mathrm{CO}_{2}$.

\begin{tabular}{|c|c|c|}
\hline C..... & $\begin{array}{l}\text { Found. } \\
59.83 \text { per cent. }\end{array}$ & $\begin{array}{c}\text { Theory. } \\
\mathrm{C}_{4} \mathrm{H}_{6}\left(\mathrm{COOC}_{2} \mathrm{H}_{5}\right)_{2} . \\
60 \cdot 00 \text { per cent. }\end{array}$ \\
\hline & $8.38 \quad "$ & $8 \cdot 00$ \\
\hline & $31 \cdot 79$ & $32 \cdot 00$ \\
\hline
\end{tabular}

Ethylic tetramethylenedicarboxylate $(1,2)$, is a colourless oil of feeble odour, boiling at $238-242^{\circ}$. It is isomeric with ethylic tetramethylenedicarboxylate $(1,1)$, which however boils at a much lower temperature, viz., $223^{\circ}$.

In order to obtain the free acid, the ethereal salt was boiled with an excess of alcoholic potash for three hours, the product diluted with water, evaporated on a water-bath to drive off the alcohol, and then acidified with dilute sulphuric acid. The resulting solution was several times extracted with pure ether, the ethereal solution dried over calcic chloride, and the ether distilled off, when an almost colourless crystalline mass was left, consisting of nearly pure tetramethylenedicarboxylic acid $(1,2)$. This was further purified by dissolving it in a small quantity of water, filtering, and allowing the solution to evaporate over sulphuric acid in a vacuam. In this way, colourless feathery crystals were obtained, which, after drying at $12 u^{\circ}$, gave the following numbers on analysis:- 
I. 0.1108 gram substance gave 0.0570 gram $\mathrm{H}_{2} \mathrm{O}$ and 0.2016 gram $\mathrm{CO}_{2}$.

II. 0.1477 gram substance gave 0.0797 gram $\mathrm{H}_{2} \mathrm{O}$ and 0.2689 gram $\mathrm{CO}_{2}$.

\begin{tabular}{|c|c|c|c|c|}
\hline \multicolumn{3}{|c|}{ Found. } & \multirow{2}{*}{\multicolumn{2}{|c|}{$\begin{array}{c}\text { Theory. } \\
\mathrm{C}_{4} \mathrm{H}_{6}(\mathrm{COOH})_{2} \text {. } \\
50 \cdot 00 \text { per cent. }\end{array}$}} \\
\hline C. & $\begin{array}{c}\text { I. } \\
49 \cdot 62\end{array}$ & $\begin{array}{l}\text { II. } \\
49 \cdot 65 \text { per cent. }\end{array}$ & & \\
\hline & $5 \cdot 70$ & $5.99 \quad$, & $5 \cdot 56$ & , \\
\hline & $44 \cdot 68$ & $44: 36$ & $44: 44$ & $\eta$ \\
\hline
\end{tabular}

Tetramethylenedicarboxylic acid $(1,2)$ melts at $130^{\circ}$. It is easily soluble in alcohol, ether, and water, but only sparingly in light petroleum and benzene. It is isomeric with tetramethylenedicarboxylic acid $(1,1)$ (p. 4), which melts at $154-156^{\circ}$. The difference between these two acids is most apparent from their behaviour on heating. Tetramethylenedicarboxylic acid $(1,1$ ) decomposes below $170^{\circ}$ into carbonic anhydride and tetramethylenemonocarboxylic acid, whereas tetramethylenedicarboxylic acid $(1,2)$ is not changed at this temperature, but at $300^{\circ}$, it is converted into its anhydride, thus :-

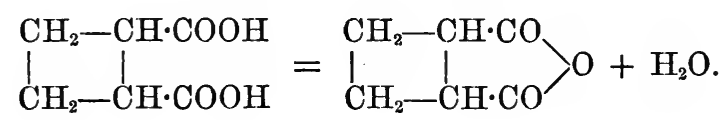

Tetramethylenedicarboxylic acid $(1,2)$ is also isomeric with ethylfumaric acid, diacrylic acid, tetrylenedicarboxylic acid, allymalonic acid, \&c. It is easily oxidised by permanganate of potash in alkaline solution, oxalic acid being formed.

The salts of tetramethylenedicarboxylic acid $(1,2)$ are well characterised.

The silver salt is easily obtained on adding nitrate of silver to a neutral solution of the ammonium salt, as a white amorphous precipitate, sparingly soluble in water. The analysis gave the following numbers :-

0.0965 gram of substance gave 0.0575 gram of $\mathrm{Ag}$.

\begin{tabular}{|c|c|}
\hline c. & $\begin{array}{l}\text { Found. } \\
59.58 \text { per cent. }\end{array}$ \\
\hline
\end{tabular}

The most characteristic salt, however, is the barium salt. If baric chloride is added to a fairly concentrated solution of the ammonium. salt, no precipitate is formed and the liquid remains clear. If heat be now applied, as soon as the boiling point is reached, the barium salt is precipitated as a colourless crystalline powder, which, when examined 
under the microscope, is found to consist of six-sided plates, having the form

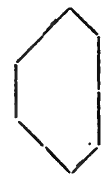

This barium salt in the crystalline form is sparingly soluble in both hot and cold water.

A solution of the ammonium salt behaves as follows when treated with the following salts:-

Plumbic acetate, white precipitate, sparingly soluble in water.

Cupric sulphate, added to a cold solution of the ammonium salt, gives no precipitate. On boiling, however, the copper salt is thrown down as a light-green amorphous mass.

$\left.\begin{array}{l}\text { Calcic chloride } \\ \text { Zincic sulphate }\end{array}\right\}$ No precipitates.

\section{Anhydride of Tetramethylenedicarboxylic Acid $(1,2)$.}

Tetramethylenedicarboxylic acid, when heated at $300^{\circ}$ for half an hour, is completely converted into its anhydride. On distilling the product, a colourless oil is obtained, which solidifies on cooling to a hard crystalline mass of the nearly pure anhydride. After powdering, washing with a little ether, and drying over sulphuric acid in a vacuum, the following results were obtained on analysis :-

0.1206 gram substance gave 0.0552 gram $\mathrm{H}_{2} \mathrm{O}$ and 0.2531 gram $\mathrm{CO}_{2}$.

\begin{tabular}{|c|c|c|}
\hline C . . & $\begin{array}{l}\text { Found. } \\
57 \cdot 23 \text { per cent. }\end{array}$ & $\begin{array}{l}\text { Theory. } \mathrm{C}_{4} \mathrm{H}_{6}: \mathrm{C}_{2} \mathrm{O}_{3} . \\
57 \cdot 14 \text { per cent. }\end{array}$ \\
\hline & 5.09 & 4.76 \\
\hline $0 \ldots$. & $37 \cdot 68$ & $38 \cdot 10$ \\
\hline
\end{tabular}

This anhydride, purified as described above, melts at $76-78^{\circ}$. It is easily soluble in alcohol, but more sparingly in ether and benzene. In contact with water, it is only very slowly decomposed at ordinary temperatures, but on boiling it is rapidly converted into "tetramethylenedicarboxylic acid.

If this anhydride is heated with resorcinol for some time at $250^{\circ}$, a reddish-coloured melt is obtained, which, when dissolved in water and treated with ammonia, gives the fluorescein reaction in the most beautiful manner.

Tetramethylenedicarboxylic acid $(1,2)$ and its anhydride are more 
especially interesting, as they are those derivatives of tetramethylene which correspond with phthalic acid and its anhydride in the aromatic series.

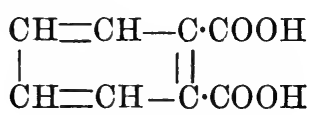

Phthalic acid.<smiles>C=CC=C1C=COOC1=O</smiles>

Phthalic anhydride.
$\mathrm{CH}_{2}-\mathrm{CH} \cdot \mathrm{COOH}$<smiles>CCC(C)C(=O)O</smiles>

Tetramethylenedicarboxylic acid $(1,2)$.<smiles>O=C1OOC2CCC12</smiles>

Anhydride.

On comparing these two series of compounds, it will be seen that there is a marked resemblance in some of their properties.

\section{Diacetyltetramethylenedicarboxylic Acid $(1,2,1,2)$,}

$$
<\mathrm{CH}_{2} \cdot \mathrm{CH}\left(\mathrm{COCH}_{3}\right) \cdot \mathrm{COOH}
$$

If ethylic sodacetacetate is treated with ethylene bromide, ethylic acetyltrimethylenecarboxylate is formed (Trans., 1885, 829). Besides this ethereal salt, however, another substance can be isolated, which corresponds very closely with ethylic butane- $\omega_{2} \omega_{2}$-tetracarboxylate, 2 mols. of ethylic sodacetoacetate reacting with $1 \mathrm{~mol}$. of ethylene bromide, thus :-

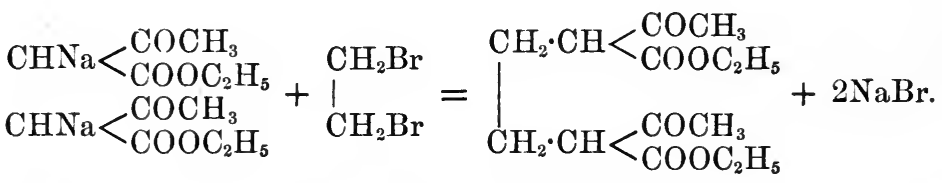

The examination of this compound, which has received the name ethylic diacetyladipate, has been carried out jointly with $M$. Obrembsky, and I hope to have the honour of laying a full account of it before the Society in a short time.

If this substance is treated with sodic ethylate, a disodium compound is formed which, on the addition of iodine, is converted into ethylic diacetyltetramethylenedicarboxylate $(1,2,1,2$,) thus :-

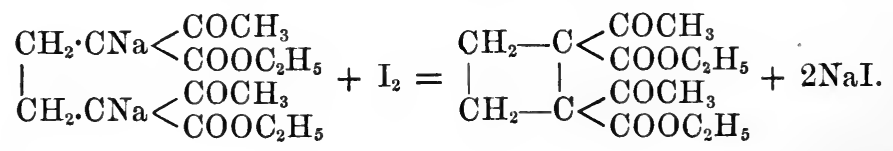

By hydrolysis, this ethereal salt can easily be converted into diacetyltetramethylenedicarboxylic acid, which is a beautifully crystal. 
line substance melting at $210^{\circ}$. This synthesis is best carried out in the following way:-

Pure ethylic diacetyladipate is dissolved in pure ether, and then the calculated amount of an ethereal solution of sodic ethylate* poured in. As soon as the sodium compound is completely formed, the calculated quantity of iodine, dissolved in ether, is added, the whole being well cooled during the operation. At first the colour of the iodine disappears instartly, but after about two-thirds has been added the reaction becomes much more sluggish, and it rarely happens that the whole of the iodine solution is decolorised.

In order to isolate the product of the reaction, water is added and sufficient sulphurous acid to decolorise the liquid completely. The ethereal solution is then separated from the aqueous layer, well washed with water, dried over calcic chloride, and the ether distilled off. The residual oil was not further purified but directly converted into the free acid by hydrolysis. For this purpose, the crude oil was boiled with an excess of a moderately concentrated solution of alcoholic potash, for about three hours, the product evaporated to dryness on a water-bath, and the residue dissolved in water. The brownish solution when acidified with dilute sulphuric acid deposited an uninviting tarry mass, which it was found almost impossible to purify. After many trials, the following method was found to give the best results. The brown alkaline solution obtained by hydrolysis with potash, as described above, was carefully neutralised with dilute sulphuric acid, boiled with freshly ignited animal charcoal, and filtered. The filtrate, which was still somewhat dark coloured, was then acidified with dilute sulphuric acid and well agitated in a stoppered bottle at a temperature of about $40-50^{\circ}$. In this way almost all the resinous matter balled together, leaving a nearly colourless solution of diacetyltetramethylenedicarboxylic acid. In carrying out this method, it is of course necessary to have sufficient water present to dissolve the acid, but too much should be avoided, as a less pure product is then obtained. In order to extract the acid from this solution, the liquid after filtering was four or five times shaken up with ether, and the ethereal solution well dried over chloride of calcium. On distilling off the ether, the acid was precipitated in the form of hard yellowish crusts, and was easily obtained pure by crystallisation from water.

Diacetyltetranethylenedicarboxylic acid $(1,2,1,2)$ crystallises from water in colourless glittering plates which contain 2 mols. $\mathrm{H}_{2} \mathrm{O}$ :-

I. 0.1509 gram substance gave 0.0853 gram $\mathrm{H}_{2} \mathrm{O}$ and 0.2520 gram $\mathrm{CO}_{2}$.

* See footnote, p. 21. 
II. 0.3015 gram substance dried at $80-90^{\circ}$ lost 0.0406 gram $\mathrm{H}_{2} \mathrm{O}$. III. 0.3664 gram substance dried at $100-110^{\circ}$ lost 0.0498 gram $\mathrm{H}_{2} \mathrm{O}$.

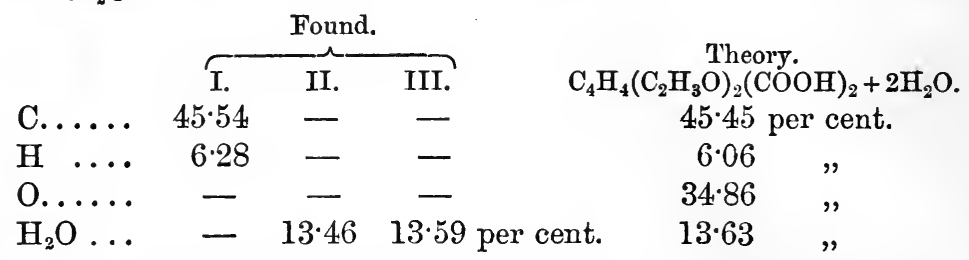

These crystals part with their water of crystallisation slowly when placed over sulphuric acid in a vacuum, but much more quickly at $80^{\circ}$; they then lose their lustre altogether and become opaque. The analysis of the dry substance crystallised once from ether gave the following results :-

0.1740 gram substance gave 0.3335 gram $\mathrm{CO}_{2}$ and 0.0874 gram $\mathrm{H}_{2} \mathrm{O}$.

\begin{tabular}{|c|c|c|}
\hline - & $\begin{array}{l}\text { Found. } \\
52 \cdot 27 \text { per cent. }\end{array}$ & $\begin{array}{l}\text { Theory. } \mathrm{C}_{10} \mathrm{H}_{12} \mathrm{O}_{6} . \\
52.63 \text { per cent. }\end{array}$ \\
\hline ... & 5.58 & $5 \cdot 26$ \\
\hline • & $42 \cdot 15$ & $42 \cdot 11$ \\
\hline
\end{tabular}

Diacetyltetramethylenedicarboxylic acid melts at $210^{\circ}$, being at the same time decomposed into carbonic anhydride and oils of high boiling poiut and disagreeable odour; as the quantity of material at my disposal was but small, these could not be further examined. The acid is easily soluble in alcohol, ethyl acetate, and hot water, but little soluble in benzene, light petroleum, chloroform, and cold water.

In conclusion, I append the following list of the substances described in the paper :-

\begin{tabular}{|c|c|c|c|}
\hline Name. & $\begin{array}{l}\text { Boiling } \\
\text { point. }\end{array}$ & $\begin{array}{l}\text { Boiling point } \\
\text { (720 mm.) } \\
\text { uncorr. }\end{array}$ & $\begin{array}{l}\text { Boiling point } \\
\text { of ethereal } \\
\text { salt ( } 720 \\
\text { mm.) uncorr. }\end{array}$ \\
\hline Tetramethylenemonocarboxylic acid ... & - & 191 & $151-151 \cdot 5^{\circ}$ \\
\hline Tetramethylenedicarboxylic acid $(1,1)$.. & $154-156^{\circ}$ & - & $220-221^{\circ}$ \\
\hline Anhydride of tetramethylenedicarboxy- & $130^{\circ}$ & - & $238-242$ \\
\hline lic acid $(1,2) \ldots \ldots \ldots \ldots \ldots$ & $76-78^{\circ}$ & - & - \\
\hline $\begin{array}{l}\text { Diacetylt etramethylenedicarboxylic acid } \\
\qquad(1,1,2,2) \ldots \ldots \ldots \ldots \ldots \ldots \ldots \ldots \ldots \ldots\end{array}$ & $210^{\circ}$ & - & - \\
\hline $\begin{array}{l}\text { Tetramethylene tetracarboxylic acid } \\
(1,1,2,2) \ldots \ldots \ldots \ldots \ldots \ldots \ldots \ldots \ldots \ldots\end{array}$ & $145-150^{\circ}$ & - & - \\
\hline Butane- $\omega_{2} \omega_{2}$-tetracarboxylic acid .... & $145-150$ & & 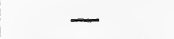 \\
\hline
\end{tabular}


[Reprinted from the Journal of the Chemical Society, March, 1887. Vol. LI.]

THE SYNTHETICAL FORMATION OF CLOSED CARBONCHAINS. PART II, continued. SOME DERIVATIVES OF TETRAMETHYLENE.

\section{By H. G. Colman, B.Sc., and W. H. Perkin, Jun., Ph.D.}

IN a former communication to this Journal on the tetramethylenederivatives (Perkin, this vol., p. 1), it was shown that substances of this nature are obtained when the sodium compound of ethyl malonate is heated with trimethylene bromide, thus :-

$2 \mathrm{CHNa}\left(\mathrm{COOC}_{2} \mathrm{H}_{5}\right)_{2}+\mathrm{CH}_{2} \mathrm{Br} \cdot \mathrm{CH}_{2} \cdot \mathrm{CH}_{2} \mathrm{Br}$

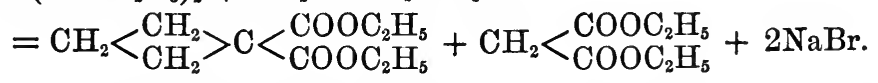

The ethyl tetramethylenedicarboxylate thus formed on hydrolysis 
yields the corresponding acid, which, when distilled, splits up into tetramethylenemonocarboxylic acid and $\mathrm{lmol} . \mathrm{CO}_{2}$ :-

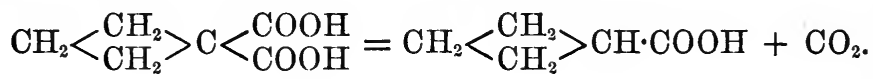

The proof that this acid really has the constitution here assigned to it, was discussed in the above-mentioned paper.

In order to accomplish, however, the chief end of this research, namely, the formation of tetramethylene itself, it was now necessary to eliminate another mol. of $\mathrm{CO}_{2}$.

The first method which suggested itself is the dry distillation of ealcium tetramethylenemonocarboxylate with lime :-

$$
\begin{aligned}
\left(\mathrm{CH}_{2}<\mathrm{CH}_{2} \mathrm{CH}_{2}>\mathrm{CH} \cdot \mathrm{COO}\right)_{2} \mathrm{Ca}+\mathrm{Ca}(\mathrm{OH})_{2}=2 \mathrm{CH}_{2}<\mathrm{CH}_{2}>\mathrm{CH}_{2} \\
+2 \mathrm{CaCO}_{3} .
\end{aligned}
$$

We have therefore carefully studied this reaction, and found, contrary to our expectations, that no tetramethylene is formed in this way. The examination of the products of this decomposition has led, however, to the isolation of some interesting derivatives of tetramethylene, a short account of which we wish to bring before the Society.

When calcium tetramethylenemonocarboxylate is distilled with lime, gas is evolved, and at the same time a light brown oil distils over. The gas proved on analysis to be a mixture of ethylene, hydrogen, marsh-gas, and carbonic oxide, and the oil was found to contain two new ketones, namely, ditetramethylene ketone and methyltetramethylene ketone.

The first of these two ketones is evidently formed by the decomposition of the calcium salt alone, according to the equation-

$$
\left(\mathrm{CH}_{2}<\mathrm{CH}_{2} \mathrm{CH}_{2}>\mathrm{CH} \cdot \mathrm{COO}\right)_{2} \mathrm{Ca}=\left(\mathrm{CH}_{2}<\mathrm{CH}_{2} \mathrm{CH}_{2}>\mathrm{CH}\right)_{2} \mathrm{CO}+\mathrm{CaCO}_{3} \text {, }
$$

a reaction exactly similar to the formation of benzophenone by the dry distillation of calcium benzoate.

The second of these compounds, that is, methyltetramethylene ketone, $\mathrm{CH}_{3} \cdot \mathrm{CO} \cdot \mathrm{CH}<\mathrm{CH}_{2}>\mathrm{CH}_{2}$, is evidently formed by a much more complicated reaction, in which one tetramethylene-gronp must in some way be reduced to methyl.

At first sight the formation of this compound seems somewhat singular, but several similar cases have been noticed in the fatty series. Thus calcium butyrate gives on distillation not only butyrone or dipropyl ketone, but also methylpropyl ketone (Friedel, Annalen, $108,125)$. Calcium isobutyrate, in like manner, gives a mixture of 
diisopropyl ketone and methylisopropyl ketone (Barbaglia and Gucci, Ber., 13, 1572).

Daring these experiments, it was thought necessary, for reasons which will be stated later on, to prepare tetramethylene aldehyde.

This substance is obtained in the usual way by the distillation of a mixture of calcium tetramethylenemonocarboxylate with calcium formate-

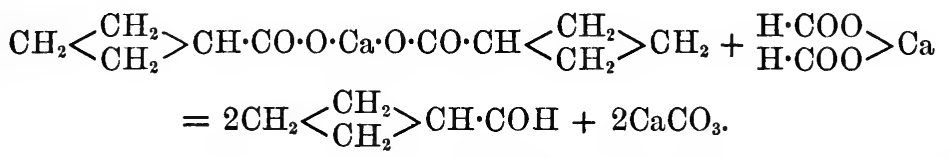

The following is a detailed description of the experiments performed during this investigation.

The distillation of the calcium tetramethylenemonocarboxylate and lime was carried out as follows :-

The calcium salt and the lime were well ground up together and filled into a broad piece of combustion tubing, which was closed at one end. This was then heated in an ordinary combustion furnace, beginning from the sealed end.

After a short time, and long before the tube had become red hot, a considerable amount of gas came off, which, after passing through a cooled flask, in order to collect the oil, was led into an apparatus described below. As a preliminary examination of a small quantity of the gas showed that it was a mixture, part of which was absorbed by bromine, a special apparatus was constructed, so as to subject the gas to the action of bromine until nothing further should be dissolved.

The accompanying figure shows the arrangement of this apparatus, and the following are the details of construction.

A and B are two Woulffe's bottles of 5 litres capacity, each having two necks. Through one neck of each passes a tube $a a^{\prime}$, to which, just above the india-rubber stopper, a side tube is fused, the latter being bent down as shown, and connected by india-rubber tubing with a glass tube reaching down below the bottom of the Woulffe's bottle. At the india-rubber joints are screw pinchcocks, $b$ and $c$. The tubes $a a^{\prime}$ are connected by bent glass tubes to the Y-tube $d$, and thus to the vessel C. The india-rubber joints at the ends of this $\mathrm{Y}$-tube are closed by pinchcocks $e$ and $f$. The vessel $\mathrm{C}$ is always kept full of water.

In the second mouth of each Woulffe's bottle is another tube, bent at right angles, connected with a bulb tube, $g$, and each of these bulb tubes is connected by the other end to the tube $h$, which is $30 \mathrm{~cm}$. long, and nearly filled with broken glass and bromine (50 grams). In each 
of the bulb tubes is placed a small quantity of water, so that they serve to indicate the speed at which the gas is passing, and also to keep back to some extent the bromine vapour carried along with the gas.

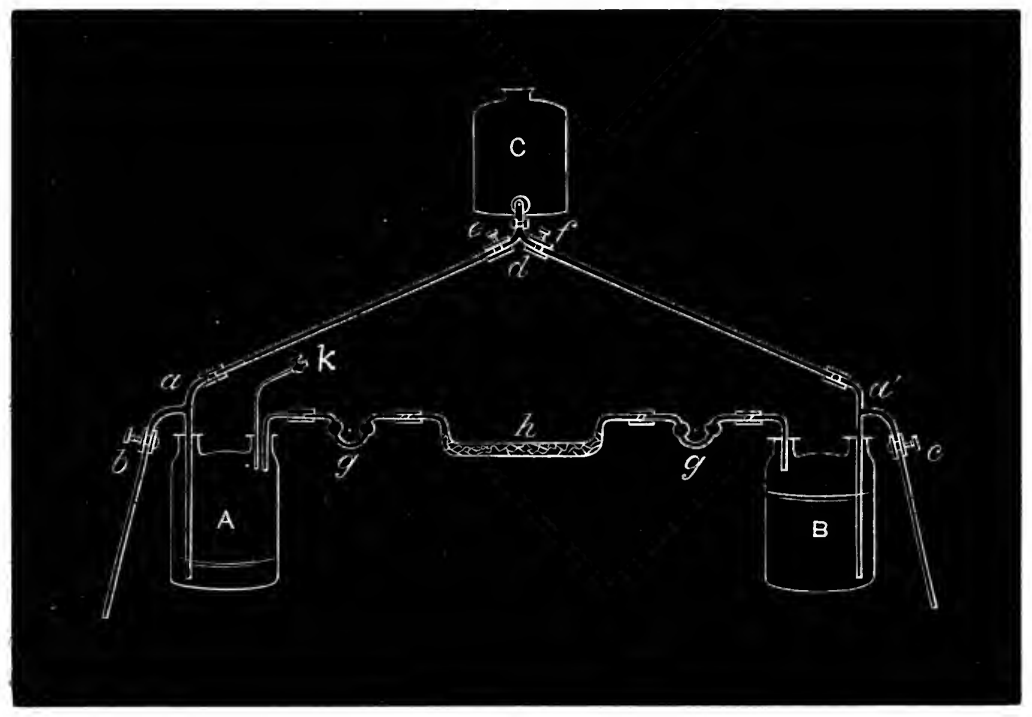

In the second mouth of the bottle $A$ is another tube, by means of which the gas to be examined is passed into the apparatus. When not in use this is closed by the india-rubber tube and pinchcock $k$.

All the india-rubber joints are carefully wired, and those which have at any time to be in contact with bromine vapour are previously soaked in paraffin.

Before using the apparatus, it is necessary to drive out the air. The vessels $\mathrm{A}$ and $\mathrm{B}$, and tubes $a a^{\prime}$, and all connections are filled with water, carbonic anhydride is then passed in through $k$, the pinchcock $b$ being opened to allow water to siphon out as fast as gas comes in. When $\mathrm{A}$ is about half full of gas, $k$ and $b$ are closed, the pinchcock $e$ is opened, and water thus passes into $A$, and forces the gas through the indicator $g$, over the bromine, into the Woulffe'e bottle, B. The pincheock $c$ is also opened, so that as gas comes into $\mathrm{B}$ water siphons out. When as much gas as possible has been driven from $\mathrm{A}$ to $\mathrm{B}, e$ and $c$ are closed, and $f$ opened, and one of the indiarubber stoppers in $\mathrm{B}$ slightly loosened, by which means the gas is forced out into the air. When all is out the stopper is forced tightly in again, and this series of operations repeated four or five times, by which time all the air has been driven out and replaced by carbonic anhydride. 
In order to pass the gas from the distillation tube into the apparatus, the tube leading from the condensing flask is connected with one end of a T-joint, a second end being connected to the Woulffe's bottle, A, by the tube $k$, and the third connected to a tube dipping. under mercury, and closed by a pinchcock. When the tube is heated the latter pinchcock is opened, and the gas evolved collected over mercury until it is found to be free from air, when $k$ is opened, and the other pinchcock closed. The gas then passes into $A$, and $b$ is opened to allow water to siphon out. When the gas ceases to come off, $k$ and $b$ are closed, and $e$ and $c$ opened, and the gas forced from A to $\mathbf{B}$. When A is empty, $e$ and $c$ are closed, and $f$ and $b$ opened. Water thus runs into $B$, and siphons out of $A$, so that the gas is forced back from $\mathrm{B}$ over the bromine again into $\mathrm{A}$; $f$ and $b$ are then closed, and $e$ and $c$ opened, and this cycle of operations repeated as often as required.

The apparatus worked in a way which left nothing to be desired, never causing the slightest trouble after it had once been put together. The operation of reversing only takes a quarter of a minute, and, as will be seen, simply consists in closing two pinchcocks and opening two others. By using screw pinchcocks the flow of the gas can be regulated very exactly. To keep the vessel $\mathrm{C}$ full of water, it was connected with the water-tap by an india-rubber tube.

On passing the gas over the bromine for the first time, the volume was reduced to about one-third. A further very slight absorption took place the second time, but although it was passed over four times more, no further absorption took place.

During the operation, a small quantity of bromine vapour is carried along with the gas, though the water in the indicators serves to retain the greater part.

To disconnect the tube $h$, containing the bromine and absorbed gas, the india-rubber tube between the indicator nearest $\mathrm{A}$ and the tube $h$ is closed by a pinchcock, the gas having previously been passed into A. The tube $h$ can then be taken away, and the unabsorbed gas is thus prevented from escaping, and no air can get into it. When any of the gas is required for analysis, it can be taken out through the tube $k$.

\section{Nature of the Absorbed Gas.}

In order.to determine as far as possible the nature of that part of the gas which was absorbed by the bromine, the contents of the tube, which still contained a considerable excess of bromine, were treated with sodium thiosulphate solution, until an almost colourless heavy oil remained. This was extracted with ether, the ethereal solution dried over calcium chloride, filtered, and the ether distilled off. Nearly. 
20 grams of a brownish oil were thus obtained, which the subsequent examination showed to be almost pure ethylene bromide.

At the first distillation, the whole went over between $125^{\circ}$ and $135^{\circ}$, and on redistillation 10 grams of oil were easily obtained, boiling constantly at $131 \cdot 5^{\circ}$.

A bromine determination made by Carius' method gave the following numbers :-

$$
\begin{aligned}
& \text { Substance, 0.4576; AgBr, 0.9061; Ag, 0.0044. } \\
& \text { Calculated for } \\
& \mathrm{BrCH}_{2} \cdot \mathrm{CH}_{2} \mathrm{Br} \text {. Found. } \\
& \text { Br ........ 85.11 84.96 }
\end{aligned}
$$

That the oil really was ethylene bromide was also shown by heating it with alcoholic potash, when a gas was evolved with the characteristic odour of acetylene, which gave a reddish-brown precipitate with ammoniacal cuprous chloride.

The formation of ethylene, unaccompanied by other unsaturated hydrocarbons, by the dry distillation of calcium tetramethylenemonocarboxylate with lime, is interesting, inasmuch as it is probably a product of decomposition of the tetramethylene first formed, thus :-

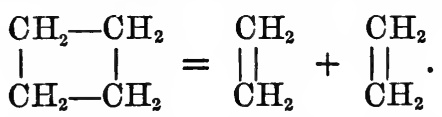

It is, however, remarkable that this decomposition should take place at so low a temperature.

\section{Examination of the Gas unabsorbed by Bromine.}

A preliminary examination was made in order to determine approximately the constituents of this gas, and for this purpose it was subjected to the action of various absorbing agents. After the absorption of carbonic anhydride by caustic potash, the gas was found to be acted on by ammoniacal cuprous chloride, showing the presence of carbonic oxide, and to be absorbed in a very marked manner by spongy palladium, indicating the presence of hydrogen.

Quantitative experiments were then made with the following results :-

I. 76.3 c.c. were reduced after absorption by potash to 74.9 c.c.

This 74.9 c.c., on treatment with ammoniacal cuprous chloride, lost further $1 \cdot 1$ c.c.

38.4 c.c. of residue when passed over spongy palladium were reduced to $7 \cdot 2$ c.c.

II. A second determination of the carbonic anhydride and carbonic oxide was made with the following results :- 
$72 \cdot 6$ c.c. were reduced after absorption by potash to $71 \cdot 6$ c.c.

This 71.6 c.c., on treatment with ammoniacal cuprous chloride, lost further 1.2 c.c.

The composition of the gas found from these analyses was as follows :-

\begin{tabular}{|c|c|c|}
\hline & \multirow{2}{*}{\multicolumn{2}{|c|}{ 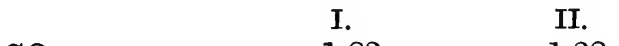 }} \\
\hline & & \\
\hline $\begin{array}{l}\mathrm{CO}_{2} \ldots \ldots \ldots \ldots \ldots \\
\mathrm{CO} \ldots \ldots \ldots \ldots \ldots\end{array}$ & $1 \cdot 44$ & $1 \cdot 65$ \\
\hline \multirow{3}{*}{ 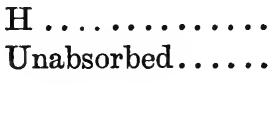 } & $78 \cdot 84$ & - \\
\hline & $18 \cdot 14$ & - \\
\hline & $100 \cdot 25$ & - \\
\hline
\end{tabular}

The unabsorbed part of the gas, as will be seen later on, was methane, together with a certain amount of air introduced from the tap-water.

From these experiments, it seems that no tetramethylene was formed.

It next appeared necessary to repeat these experiments with a sample of gas prepared at as low a temperature as possible; for this purpose, a mixture of 5 grams of calcium tetramethylenemonocarboxylate with 10 grams of slaked lime was very gently heated in a combustion furnace, and the gas evolved collected in tubes. A plug of lime in the front of the tube, which was not heated, served to absorb the oil formed.

I.

\begin{tabular}{|c|c|c|c|c|}
\hline$=$ & Volume. & Pressure. & Temp. & $\begin{array}{l}\text { Vol. at } 0^{\circ} \\
\text { and } 1 \mathrm{M} . \\
\text { pressure. }\end{array}$ \\
\hline $\begin{array}{l}\text { Original gas employed } \ldots \ldots \ldots \ldots \\
\text { After addition of air and oxygen.. } \\
\text { After explosion } \ldots \ldots \ldots \ldots \ldots \ldots \\
\text { After absorption of } \mathrm{CO}_{2} \ldots \ldots \ldots \ldots\end{array}$ & $\begin{array}{l}114 \cdot 12 \\
384 \cdot 42 \\
354 \cdot 13 \\
349 \cdot 95\end{array}$ & $\begin{array}{l}144 \cdot 27 \\
413 \cdot 11 \\
377 \cdot 80 \\
371 \cdot 30\end{array}$ & $\begin{array}{l}10 \cdot 1 \\
10 \cdot 2 \\
10 \cdot 2 \\
10 \cdot 8\end{array}$ & $\begin{array}{r}15 \cdot 32 \\
153 \cdot 15 \\
130 \cdot 52 \\
128 \cdot 60\end{array}$ \\
\hline
\end{tabular}

II.

\begin{tabular}{|c|c|c|c|c|}
\hline & Volume. & Pressure. & Temp. & $\begin{array}{l}\text { Vol. at } 0^{\circ} \\
\text { and } 1 \mathrm{M} \text {. } \\
\text { pressure. }\end{array}$ \\
\hline $\begin{array}{l}\text { Original gas employed } \ldots \ldots \ldots \ldots . . \\
\text { After addition of air and oxygen.. } \\
\text { After explosion } \ldots \ldots \ldots \ldots \ldots \ldots . \\
\text { After absorption of } \mathrm{CO}_{2} \ldots \ldots \ldots \ldots\end{array}$ & $\begin{array}{l}118 \cdot 53 \\
385 \cdot 37 \\
354 \cdot 64 \\
348 \cdot 52\end{array}$ & $\begin{array}{l}139 \cdot 42 \\
403 \cdot 94 \\
372 \cdot 06 \\
369 \cdot 56\end{array}$ & $\begin{array}{l}13 \cdot 3 \\
1.3 \cdot 8 \\
14 \cdot 8 \\
13 \cdot 1\end{array}$ & $\begin{array}{r}15 \cdot 76 \\
148 \cdot 53 \\
125 \cdot 31 \\
122 \cdot 91\end{array}$ \\
\hline
\end{tabular}


Into these tubes, a coke bullet, saturated with bromine, was then passed, in order to absorb the ethylene, and it was found that the volume was reduced to one-third. After the removal of the excess of bromine by a potash bullet, the gas was analysed by Bunsen's method with the result given in the preceding tables.

Analysis I was made from a sample taken at the beginning, and Analysis II from a sample taken at the end of the operation.

In calculating the results, it was thought that the very laborious after-explosions with hydrogen might be avoided by directly determining the amount of carbonic oxide by absorption, and then subtracting this from the volume of gas employed, making corresponding corrections in the above numbers.

The amount of $\mathrm{CO}$ in several samples was found to vary from 1.5 to 1.6 per cent.

The gas used in Analysis I was found to contain $3 \cdot 6$ per cent. of air, the amount being determined by absorption of the oxygen by pyrogallol.

Allowing for these corrections, the following is the result of the two analyses :-

\begin{tabular}{|c|c|c|}
\hline & I. & II. \\
\hline Air ............ & $3 \cdot 60$ & - \\
\hline Hydrogen $\ldots . .$. & $84 \cdot 08$ & $84 \cdot 4$ \\
\hline Methane ....... & $10 \cdot 84$ & $13 \cdot 3$ \\
\hline Carbonic oxide .. & $1 \cdot 60$ & $1 \cdot 6$ \\
\hline & $100 \cdot 12$ & $9 \cdot 3$ \\
\hline
\end{tabular}

It appears therefore that, even with these precautions, no tetramethylene is formed.

\section{Examination of the Oil.}

The oil formed by the dry distillation was now carefully examined.

In order to free it from water, it was first dissolved in a little ether, the excess of water separated by a separating funnel, and the ethereal solution dried over calcium chloride. After filtering and distilling off the ether, about 20 grams of a dark brownish oil remained; this was submitted to fractional distillation, when nearly the whole went over between $100^{\circ}$ and $250^{\circ}$ as a colourless oil, which possessed in a marked manner the pungent odour of peppermint. On refractionating, a small quantity of a limpid oil, smelling somewhat like petroleum, came over below $120^{\circ}$; the quantity was unfortunately too small for examination. On further distillation, it was noticed that the thermometer remained for a short time fairly constant at about $135^{\circ}$, and then again at about $200^{\circ}$, and for this reason two chief fractions, $120-150^{\circ}$ and $180-210^{\circ}$ were collected. 
The fraction boiling from $180^{\circ}$ to $210^{\circ}$, which weighed about 8 grams, was first examined. After twice refractionating, by far the greater part passed over between 200 and $210^{\circ}$, and on repeated distillation an oil was at last obtained which boiled constantly at $204-205^{\circ}$. On analysis, this gave the following numbers:-

I. Substance, 0.1497 gram; $\mathrm{CO}_{2}, 0.4283$ gram; $\mathrm{H}_{2} \mathrm{O}, 0.1394$ gram.

IJ. Substance, 0.1045 gram; $\mathrm{CO}_{2}, 0.2990$ gram; $\mathrm{H}_{2} \mathrm{O}, 0.0980$ gram.

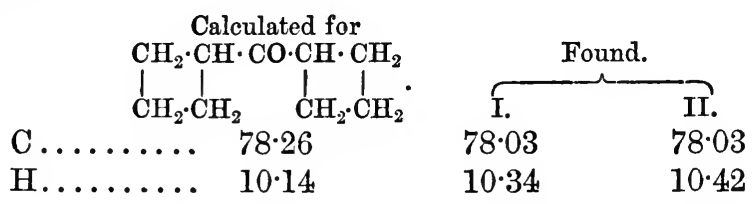

It thus appears that this compound is ditetramethylene ketone, which would be the normal product of the dry distillation of calcium tetramethylenemonocarboxylate alone. .

$$
\left(\mathrm{CH}_{2}<\mathrm{CH}_{2}^{\mathrm{CH}_{2}}>\mathrm{CH} \cdot \mathrm{COO}\right)_{2} \mathrm{Ca}=\left(\mathrm{CH}_{2}<\mathrm{CH}_{2}>\mathrm{CH}\right)_{2} \mathrm{CO}+\mathrm{CaCO}_{3} \text {. }
$$

This ketone is a colourless oil, with a pungent peppermint-like odour, boiling at $204-205^{\circ}$. It combines easily with sodium hydrogen sulphite, forming a white, beautifully crystalline compound, which is fairly soluble in water, and instantly decomposed by acids. The formation of this compound is rather remarkable, as usually only methyl ketones combine with sodium hydrogen sulphite.

With acetate of phenylhydrazine, the dilute alcoholic solution of the ketone gives a yellow oily precipitate, which is probably the hydrazine compound, but as it did not crystallise it was not further examined. It has often been noticed in such cases that when the hydrazine compound is an oil, the hydroxylamine-derivative can be used with advantage. In order to test this, a solution of 1 gram of the ketone (b. p. 202-208 ) in dilute alcohol was allowed to stand with an excess of hydroxylamine solntion for eight days. Water and a few drops of dilute hydrochloric acid were then added, and the oil which separated extracted two or three times with ether.

After washing with water, drying over calcium chloride, and distilling off the ether, a colourless syrup was obtained, which did not crystallise until it had stood for four weeks over sulphuric acid in a vacuum. It was purified by spreading it out on a porons plate, and was then obtained as a white crystalline solid, melting at $51^{\circ}$. The following results were obtained on analysis :-

Substance, 0.2066; $\mathrm{CO}_{2}, 0.5341 ; \mathrm{H}_{2} \mathrm{O}, 0.1881$. 
For nitrogen: substance, $0.3200 ; \mathrm{N}=24.5$ c.c. ; temp. $=8.5^{\circ}$; bar., $758 \mathrm{~mm}$.*

\begin{tabular}{|c|c|c|}
\hline & $\begin{array}{c}\text { Calculated for } \\
\mathrm{C}_{9} \mathrm{H}_{15} \mathrm{NO} \text {. }\end{array}$ & Found. \\
\hline C. . & . $\quad 70.59$ & $70 \cdot 50$ \\
\hline H ... & $9 \cdot 80$ & $10 \cdot 11$ \\
\hline$\ldots$. & $9 \cdot 15$ & $9 \cdot 19$ \\
\hline
\end{tabular}

Ditetramethylene ketone is isomeric with the following compounds :-

Phorone, b. p. $196^{\circ}$.

Isophorone, b. p. 208-212 .

Camphorphorone, b. p. 202-205․

Diallylacetone, b. p. $175^{\circ}$.

Bromine reacts easily with ditetramethylene ketone, but always with evolution of hydrogen bromide, a proof that it really contains two tetramethylene-rings, and is not an unsaturated compound.

Ditetramethylene ketone is interesting as being that derivative of tetramethylene which corresponds with benzophenone in the benzene series.

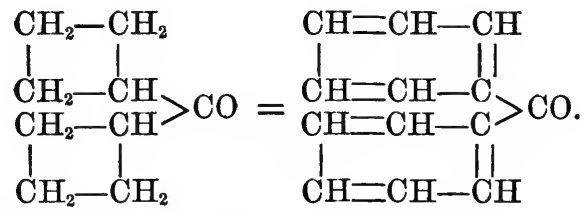

Ditetramethylene ketone.

Benzophenone.

The fraction of the original oil boiling at $120-150^{\circ}$ was next examined.

This fraction being much smaller than that of the fraction $180-210^{\circ}$, considerable difficulty was experienced in obtaining from it a substance having a constant boiling point. After repeated refractioning, however, an oil was finally isolated boiling at 136$137^{\circ}$, possessing in an even more marked degree than the ditetramethylene ketone the odour of peppermint. On analysing it the following numbers were obtained:-

I. Substance, 0.1084 gram ; $\mathrm{CO}_{2}, 0.2888$ gram; $\mathrm{H}_{2} \mathrm{O}, 0.0981$ gram.

II. Substance, 0.1374 gram; $\mathrm{CO}_{2}, 0.3661$ gram; $\mathrm{H}_{2} \mathrm{O}, 0.1262$ gram.

* The nitrogen determination was made with a portion which had remained for four days over sulphuric acid in a vacuum, but which had not then crystallised. 


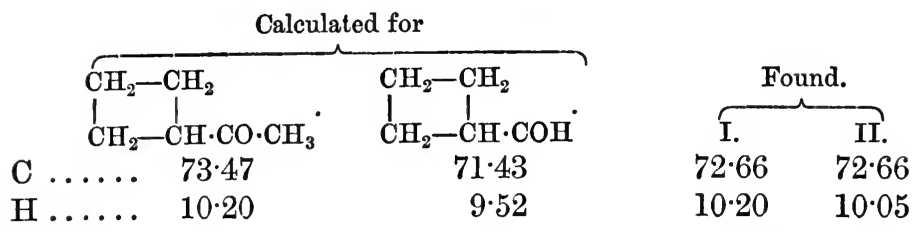

Although these numbers are not so good as could be desired, there can be no doubt that this substance is methyl tetramethylene ketone, or acetyltetramethylene.

The only substance which has an analogous constitution is tetramethylene aldehyde, and in order to prove that these were really different tetramethylene aldehyde was prepared and examined, as will be shown in the next section.

Tetramethylene ketone combines very easily with sodium hydrogen sulphite, forming a beautifully crystalline substance, easily soluble in water. When treated with phenylhydrazine, a yellow compound is produced, which is probably the hydrazine compound. An oxime is also easily produced when the alcoholic solution of the ketone is treated with hydroxylamine, but as this could not be obtained in a crystalline condition it was not further examined.

\section{Tetramethylene Aldehyde, $\mathrm{CH}_{2}<\mathrm{CH}_{2}>\mathrm{CH} \cdot \mathrm{COH}$.}

In order to prepare this compound, equal parts of calcium tetramethylenemonocarboxylate and calcium formate were finely powdered, intimately mixed, and filled into a combustion-tube drawn out at one end. The tube was then carefully heated, when a light oil distilled over, which was collected in a flask inserted in a freezing mixture.

The following equation represents the formation of the aldehyde :-

$\left(\mathrm{CH}_{2}<\mathrm{CH}_{2} \mathrm{CH}_{2}>\mathrm{CH} \cdot \mathrm{COO}\right)_{2} \mathrm{Ca}+(\mathrm{H} \cdot \mathrm{COO})_{2} \mathrm{Ca}$

$$
=2 \mathrm{CH}_{2}<\mathrm{CH}_{2} \mathrm{CH}_{2}>\mathrm{CH} \cdot \mathrm{COH}+2 \mathrm{CaCO}_{3} \text {. }
$$

During the distillation, a quantity of gas was evolved, and, particularly towards the end of the operation, a heavy brownish oil distilled over, accompanied by thick white fumes.

In fractioning the product, it was easily separated into two portions, one boiling between $100^{\circ}$ and $130^{\circ}$ and the other between $130^{\circ}$ and $230^{\circ}$. The fraction boiling between $100^{\circ}$ and $130^{\circ}$ was then well shaken with sodium hydrogen sulphite, when in a short time almost the whole solidified to a mass of crystals. These were pressed, dried on 
a porous plate, and washed with a little alcohol, when a beautiful, silky crystalline mass was left. This compound was next dissolved in a little water, and mixed with an excess of sodiam hydrogen carbonate solution, which caused decomposition and precipitation of the aldehyde. The latter was extracted with ether, and the ethereal solution rapidly dried over calcium chloride; the ether distilled off, and the residual oil fractioned.

In this way a colourless oil was obtained possessing the characteristic pungent odour of the aldehydes; on twice refractioning, it boiled at $115-117^{\circ}$. The following is the result of an analysis :-

$$
\begin{aligned}
& \text { Substance, 0.1417; } \mathrm{CO}_{2}, 0.3696 ; \mathrm{H}_{2} \mathrm{O}, 0.1256 \text {, } \\
& \text { Calculated for } \\
& \mathrm{CH}_{2}<\mathrm{CH}_{2}>\mathrm{CH} \cdot \mathrm{COH} \text {. } \\
& \text { C.......... } 71 \cdot 43 \\
& \text { H .......... } 9.53 \\
& \text { Found. } \\
& 71 \cdot 13 \\
& 9 \cdot 86
\end{aligned}
$$

The odour of tetramethylene aldehyde closely resembles that of isobutylaldehyde. It possesses all the usual properties of an aldehyde. When shaken with a solution of rosaniline hydrochloride which has been decolorised by sulphur dioxide, a purple coloration is produced. It reduces ammoniacal silver nitrate with great ease, and combines readily with phenylhydrazine and hydroxylamine. When heated with alcoholic potash, it seems to behave more like an aromatic than a fatty aldehyde. A small quantity of the substance, when heated in this way, was transformed into a neutral compound and an oily acid; the latter possessed unmistakably the odour of tetramethylenemonocarboxylic acid.

The reaction is therefore probably the following :-

$$
\begin{aligned}
& 2 \mathrm{CH}_{2}<\mathrm{CH}_{2}^{\mathrm{CH}_{2}}>\mathrm{CH} \cdot \mathrm{COH}+\mathrm{KOH}=\mathrm{CH}_{2}<\mathrm{CH}_{2}^{\mathrm{CH}_{2}}>\mathrm{CH} \cdot \mathrm{CH}_{2} \mathrm{OH}+ \\
& \mathrm{CH}_{2}<\mathrm{CH}_{2} \mathrm{CH}_{2}>\mathrm{CH} \cdot \mathrm{COOK} \text {. }
\end{aligned}
$$

Owing to the small amount of material at our disposal, it was, unfortunately, not possible to oxidise this aldehyde, and thus obtain tetramethylenemonocarboxylic acid in quantity sufficient to prove its identity; but from the above there would appear to be no grounds for doubting that this substance is really tetramethylene aldehyde. 
THE SYNTHETICAL FORMATION OF CLOSED CARBON-CHAINS. 
[Reprinted from the Journal of the Chemical Society, September, 1887. Vol. LI.]

The Synthetical Formation of Closed Carbon-chains. Part II. (continued). On the Action of Trimethylene Bromide on the Sodium Compounds of Ethylic Acetoacetate, Benzoylacetate, Paranitrobenzoylacetate and Acetonedicarboxylate.

$$
\text { By W. H. Perkin, Jun., Ph.D. }
$$

Is a paper on "Tetramethylene-derivatives," which I brought before the notice of the Society a short time since (this vol., p. 1), I showed that when the sodium-derivative of ethylic malonate is digested with trimethylene bromide, ethylic tetramethylenedicarboxylate is formed thus :-

$$
\begin{aligned}
& { }_{2} \mathrm{COOC}_{2} \mathrm{H}_{5}>\mathrm{CHNa}+\mathrm{BrCH}_{2} \cdot \mathrm{CH}_{2} \cdot \mathrm{CH}_{2} \mathrm{Br}= \\
& \underset{\mathrm{COOC}_{2} \mathrm{H}_{5}}{\mathrm{COOC}_{2} \mathrm{H}_{5}}>\mathrm{C}<\mathrm{CH}_{2}^{\mathrm{CH}_{2}}>\mathrm{CH}_{2}+\underset{\mathrm{COOC}_{2} \mathrm{H}_{5}}{\mathrm{COOC}_{2} \mathrm{H}_{5}}>\mathrm{CH}_{2}+2 \mathrm{NaBr} .
\end{aligned}
$$

Whilst engaged on these experiments, it seemed probable that if ethylic acetoacetate or benzoylacetate were substituted for ethylic malonate, various tetramethylene-derivatives, of constitntion similar to the above, might be obtained. Thus, in the case of ethylic acetoacetate, ethylic acetyltetramethylenecarboxylate should be formed :-

$$
\begin{aligned}
& { }_{2} \mathrm{COOC}_{2} \mathrm{CH}_{5} \cdot \mathrm{CO}>\mathrm{CHNa}+\mathrm{BrCH}_{2} \cdot \mathrm{CH}_{2} \cdot \mathrm{CH}_{2} \mathrm{Br}= \\
& \underset{\mathrm{COO}}{\mathrm{CH}_{2} \cdot \mathrm{CO}}>\mathrm{C}<\mathrm{CH}_{2}^{\mathrm{CH}_{2}}>\mathrm{CH}_{2}+\underset{\mathrm{COOC}_{2} \mathrm{H}_{5}}{\mathrm{CH}_{3} \cdot \mathrm{CO}}>\mathrm{CH}_{2}+2 \mathrm{NaBr} .
\end{aligned}
$$

In a short account given of these experiments (Ber., 16, 1787), it was assumed that the products thas obtained were tetramethylenederivatives. After careful study, however, it was found that these substances, from their decompositions and general reactions, must be quite differently constituted. The reaction between ethylic sodacetoacetate and trimethylene bromide evidently takes place in two distinct stages.

In the first place $1 \mathrm{~mol}$. of trimethylene bromide reacts with $1 \mathrm{~mol}$. of ethylic sodacetoacetate, forming ethylic $\omega$-bromopropylacetoacetate, thus:-

$\underset{\mathrm{COOC}_{2} \mathrm{H}_{5}}{\mathrm{CH}_{3} \cdot \mathrm{CO}}>\mathrm{CHNa}+\mathrm{BrCH}_{2} \cdot \mathrm{CH}_{2} \cdot \mathrm{CH}_{2} \mathrm{Br}=$

$\underset{\mathrm{COOC}}{\mathrm{CH}_{3} \cdot \mathrm{CO}}>\mathrm{CH} \cdot \mathrm{CH}_{2} \cdot \mathrm{CH}_{2} \cdot \mathrm{CH}_{2} \mathrm{Br}+\mathrm{NaBr}$.

Ethylic w-bromopropylacetoacetate. 
In the second phase of the reaction this intermediate product simply loses $1 \mathrm{~mol}$. of hydrogen bromide, and is converted into the compound $\mathrm{C}_{9} \mathrm{H}_{14} \mathrm{O}_{3}$,

$$
\underset{\mathrm{COOC}_{2} \mathrm{H}_{5}}{\mathrm{CH}_{3}} \cdot \mathrm{CO} \cdot \mathrm{CH}_{2} \cdot \mathrm{CH}_{2} \cdot \mathrm{CH}_{2} \mathrm{Br}=\mathrm{C}_{9} \mathrm{H}_{14} \mathrm{O}_{3}+\mathrm{HBr} \text {. }
$$

This change can take place in three different ways.

(1.) The bromine-atom can combine with the hydrogen-atom of the second $\mathrm{CH}_{2}$-group, producing a substance of the formula

$$
\underset{\mathrm{COOC}_{2} \mathrm{H}_{5}}{\mathrm{CH}_{3} \cdot \mathrm{CO}}>\mathrm{CH} \cdot \mathrm{CH}_{2} \cdot \mathrm{CH}: \mathrm{CH}_{2}
$$

(ethylic allylacetoacetate).

(2). The bromine-atom can combine with the hydrogen-atom of the $\mathrm{CH}$-group, in which case ethylic acetotetramethylenecarboxylate,<smiles>CCCC(CC)(CC)OCOC</smiles>

would be formed; or (3) ethylic $\omega$-bromopropylacetoacetate is first transformed into its labile form,

\section{$\mathrm{CH}_{3} \cdot \mathrm{C} \cdot \mathrm{OH}$ $\mathrm{COOC}_{2} \mathrm{H}_{\dot{5}} \cdot \mathrm{C}^{\prime} \cdot \mathrm{CH}_{2} \cdot \mathrm{CH}_{2} \cdot \mathrm{CH}_{2} \mathrm{Br}$,}

and then hydrogen bromide is eliminated according to the equation-

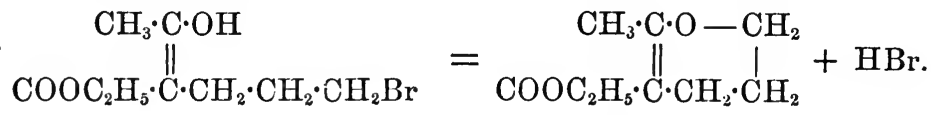

That the substance produced in this reaction is: (1) not ethylic allylacetoacetate, is easily proved by directly comparing it with the latter compound, which can easily be prepared synthetically by treating ethylic sodacetoacetate with allyl iodide,

$$
\begin{aligned}
& \underset{\mathrm{COOC}_{2} \mathrm{H}_{5}}{\mathrm{CH}_{3} \cdot \mathrm{CO}}>\mathrm{CHNa}+\mathrm{I} \cdot \mathrm{CH}_{2} \cdot \mathrm{CH}: \mathrm{CH}_{2}= \\
& \underset{\mathrm{COOH}}{\mathrm{CH}_{2} \cdot \mathrm{CO}}>\mathrm{CH} \cdot \mathrm{CH}_{2} \cdot \mathrm{CH}: \mathrm{CH}_{2}+\mathrm{NaI} \text {. }
\end{aligned}
$$

The greatest differences are found to exist between the two products, as will be shown in detail further on.

That the compound $\mathrm{C}_{9} \mathrm{H}_{14} \mathrm{O}_{3}$ is not ethylic acetotetramethylenecarboxylate is easily shown in the following way. If this substance be mixed with a concentrated solution of hydrogen bromide, it dissolves, and on standing is decomposed into alcohol, carbonic anhydride, and a cetobutyl bromide, thus :- 


\section{$\mathrm{C}_{9} \mathrm{H}_{14} \mathrm{O}_{3}+\mathrm{HBr}+\mathrm{H}_{2} \mathrm{O}=\mathrm{CH}_{3} \cdot \mathrm{CO} \cdot \mathrm{CH}_{2} \cdot \mathrm{CH}_{2} \cdot \mathrm{CH}_{2} \cdot \mathrm{CH}_{2} \mathrm{Br}$ \\ Acetobutyl bromide.}

$$
+\mathrm{CO}_{2}+\mathrm{C}_{2} \mathrm{H}_{5} \mathrm{OH} \text {. }
$$

Now, if the substance $\mathrm{C}_{9} \mathrm{H}_{14} \mathrm{O}_{3}$ be ethylic acetotetramethylenecarboxylate, this decomposition can only be explained on the assumption that the tetramethylene-ring is first split by the action of the hydrogen bromide thas :-

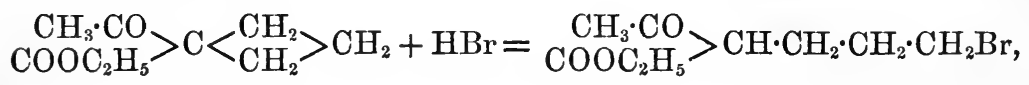

in which case ethylic $\omega$-bromopropylacetoacetate would first be formed, and then be further decomposed into alcohol, carbonic anhydride, and acetobutyl bromide.

It has, however, been shown (this vol., p. 6) that the tetramethylenering is not split even when submitted for weeks to the action of the most concentrated solution of hydrogen bromide, and it is therefore necessary to assume that the product of the action of trimethylene bromide on ethylic sodacetoacetate is not a tetramethylene-derivative, but has the constitution

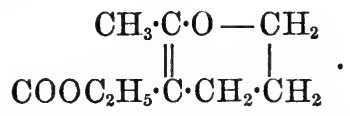

This is shown still more clearly from the following decompositions.

The ethereal salt, $\mathrm{C}_{9} \mathrm{H}_{14} \mathrm{O}_{3}$, when submitted to hydrolysis, is easily transformed into the corresponding acid, $\mathrm{C}_{7} \mathrm{H}_{10} \mathrm{O}_{3}$. If this acid be now boiled with water for a few minutes, it is quantitatively decomposed into acetobutyl alcohol and carbonic anhydride,

$$
\mathrm{C}_{7} \mathrm{H}_{10} \mathrm{O}_{3}=\mathrm{CO}_{2}+\mathrm{CH}_{3} \cdot \mathrm{CO} \cdot \mathrm{CH}_{2} \cdot \mathrm{CH}_{2} \cdot \mathrm{CH}_{2} \cdot \mathrm{CH}_{2} \cdot \mathrm{OH}+\mathrm{H}_{2} \mathrm{O} \text {, }
$$

a reaction which cannot be accounted for on the assumption that the acid is a tetrametbylene-derivative. If the acid, however, contain an oxygen carbon-chain, as represented above for its ethereal salt, this decomposition is very simply explained, as the acid is then simply a carboxylic acid of the anhydride of acetobutyl alcohol, and naturally decomposed on boiling with water into this alcohol and carbonic anhydride. The reaction would then take place according to the following equations:-

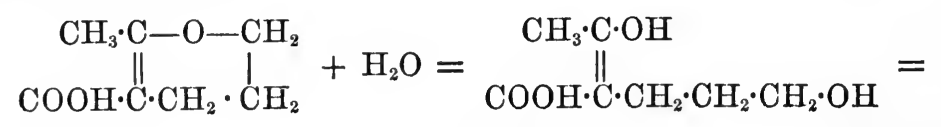

\footnotetext{
$\mathrm{CH}_{3} \cdot \mathrm{CO}$

$\mathrm{COOH} \cdot \stackrel{\mathrm{CH}}{\mathrm{C}} \cdot \mathrm{CH}_{2} \cdot \mathrm{CH}_{2} \cdot \mathrm{CH}_{2} \cdot \mathrm{OH}=$
}

$\mathrm{CH}_{3} \cdot \mathrm{CO}$

${\stackrel{\mathrm{CH}}{2} \cdot \mathrm{CH}_{2} \cdot \mathrm{CH}_{2} \cdot \mathrm{CH}_{2} \cdot \mathrm{OH}}+\mathrm{CO}_{2}$. 
With regard to the nomenclature of these and other similarly constituted substances, I would suggest that the fully saturated oxygen carbon-rings, of which these substances are derivatives, should be named according to the following scheme:-

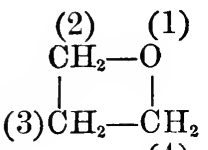

(4)

Tetrone.
(3)<smiles>C1CCCCCC1</smiles>

(5)

(2)

Pentone.
(3)<smiles>CCCCCCCCO[Ge]</smiles>

(5)

Hexone.

An acid of the formula

$$
\mathrm{CH}_{3} \cdot \mathrm{C} \cdot \mathrm{O}--\mathrm{CH}_{2}
$$

would therefore be methyldehydrohexonecarboxylic acid, $[2(2,3), 3]$.

In continuing this research, experiments were next made on the action of trimethylene bromide on the sodium-derivative of ethylic benzoylacetate, and it was found that in this case also tetramethyleuederivatives were not formed. The principal product of the reaction, an ethereal salt of the formula $\mathrm{C}_{14} \mathrm{H}_{16} \mathrm{O}_{3}$, evidently has the constitution-<smiles>CCCCCOC(=O)OCC</smiles>

and is therefore ethylic phenyldehydrohexonecarboxylate, $[2(2,3), 3\rfloor$.

Its formation is represented by an equation similar to that given above for the formation of ethylic methyldehydrohexonecarboxylate from trimethylene bromide and ethylic sodacetoacetate. This can be proved in exactly the same way as in the case of the ethylic methyldehydrohexonecarboxylate. If the ethereal salt, $\mathrm{C}_{14} \mathrm{H}_{16} \mathrm{O}_{3}$, be submitted to hydrolysis, it is easily converted into the corresponding acid, $\mathrm{C}_{12} \mathrm{H}_{12} \mathrm{O}_{3}$. This acid when treated with a concentrated solution of hydrogen bromide is decomposed into carbonic anhydride and benzoylbutyl bromide,

$$
\mathrm{C}_{12} \mathrm{H}_{12} \mathrm{O}_{3}+\mathrm{HBr}=\mathrm{CO}_{2}+\mathrm{C}_{6} \mathrm{H}_{5} \cdot \mathrm{CO} \cdot \mathrm{CH}_{2} \cdot \mathrm{CH}_{2} \cdot \mathrm{CH}_{2} \cdot \mathrm{CH}_{2} \mathrm{Br},
$$

and when boiled with waterit yields carbonic anhydride and benzoylbutyl alcohol,

$$
\mathrm{C}_{12} \mathrm{H}_{12} \mathrm{O}_{3}+\mathrm{H}_{2} \mathrm{O}=\mathrm{CO}_{2}+\mathrm{C}_{6} \mathrm{H}_{5} \cdot \mathrm{CO} \cdot \mathrm{CH}_{2} \cdot \mathrm{CH}_{2} \cdot \mathrm{CH}_{2} \cdot \mathrm{CH}_{2} \cdot \mathrm{OH},
$$

reactions which are the counterpart of those which occur in the formation of acetobutyl alcohol and its bromide from methyldehydrohexonecarboxylic acid and its ethereal salt as described above. It is 
therefore evident that the acid, $\mathrm{C}_{12} \mathrm{H}_{12} \mathrm{O}_{3}$, must be represented by the formula-<smiles>CCCC(OC(=O)O)C(C)(C)C</smiles>

and be termed phenyldehydrohexonecarboxylic acid $[2,(2,3), 3]$.

As will be shown in the experimental part of this paper, results similar to these were also obtained when ethylic paranitrobenzoylacetate was substituted in the above reaction.

Experiments on the action of trimethylene bromide on ethylic acetonedicarboxylate, which were conducted with the kind permission of Professor H. v. Pechmann, were first initiated in the hope of obtaining hexamethylene-derivatives, thus :-

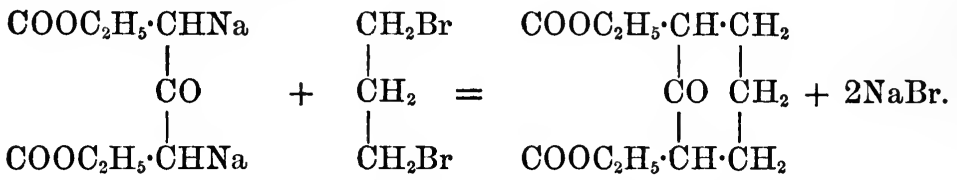

The further examination of the substances formed in this reaction has, however, shown that they are not hexamethylene-derivatives, but that in this case also hexone-derivatives result. It is most probable that this curious reaction takes place according to the following equation :-
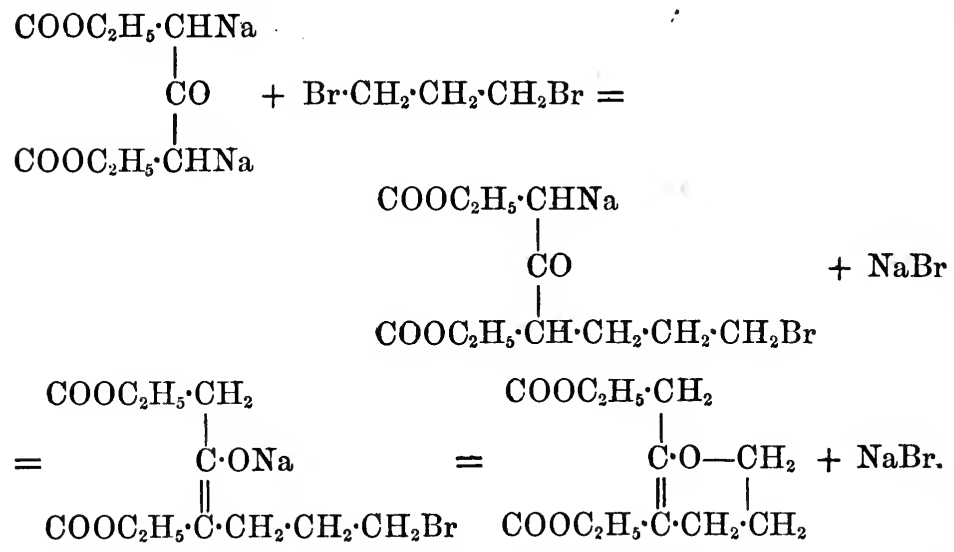

The product of this reaction is therefore ethylic methyldehydrohexonedicarboxylate, $[2,(2,3), w, 3]$. That tbis is the case is easily proved in the following way :-

Ethylic methyldehydrohexonedicarboxylate when submitted to hydrolysis, is first transformed into its acid ethylic salt, $\mathrm{C}_{10} \mathrm{H}_{14} \mathrm{O}_{5}$. If 
now this acid salt be distilled, it is decomposed into carbonic anhydride and ethylic methyldehydrohexonecarboxylate, which latter substance is identical with the compound obtained by the action of trimethylene bromide on ethylic sodacetoacetate. This interesting decomposition is clearly exhibited in the following equation :-

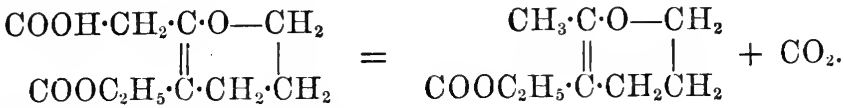

Hydric ethylic methyldehydro-

hexonedicarboxylate.

If this hydric ethylic methyldehydrohexonedicarboxylate is treated further with alkalis, it is converted into the dicarboxylic acid, $\mathrm{C}_{8} \mathrm{H}_{10} \mathrm{O}_{5}$. This dicarboxylic acid when boiled with water is easily resolved into 2 molecules of carbonic anhydride and acetobutyl alcohol, thus :-

\section{$\mathrm{COOH} \cdot \mathrm{CH}_{2} \cdot \mathrm{C} \cdot \mathrm{O}-\mathrm{CH}_{2}$ \\ $\mathrm{COOH} \cdot \mathrm{C} \cdot \mathrm{CH}_{2} \cdot \mathrm{CH}_{2}+\mathrm{H}_{2} \mathrm{O}=2 \mathrm{CO}_{2}+$}

$\mathrm{CH}_{3} \cdot \mathrm{CO} \cdot \mathrm{CH}_{2} \cdot \mathrm{CH}_{2} \cdot \mathrm{CH}_{2} \cdot \mathrm{CH}_{2} \cdot \mathrm{OH}$,

Methyl dehydrohexone-

dicarboxylic acid.

a reaction exactly similar to the formation of acetobutyl alcohol, and one molecule of carbonic anhydride from methyldehydrohexonemonocarboxylic acid described above.

In the early part of this introduction it was stated that the reaction between trimethylene bromide and the various sodium compounds employed could be also explained on the assumption that allyl-derivatives were formed, and this would also explain several of the reactions of these substances. It is therefore neressary to show that these compounds are not allyl-derivatives. This is easily done by comparing first of all their physical properties with those of the allyl compounds they might be supposed to be identical with, thus :-

Melting Points.

\begin{tabular}{|c|c|c|c|}
\hline $\begin{array}{l}\text { Methyldehydrohexonecar- } \\
\text { boxvlic acid }\end{array}$ & & Allylacetoacetic acid . & Enstable. \\
\hline 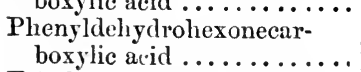 & $142-144^{\circ}$ & Allylbenzoylacetic acid .... & $\left\{\begin{array}{l}122^{\circ} \\
125^{\circ}\end{array}\right\}(?)$ \\
\hline $\begin{array}{l}\text { Ethylic methyldehydroliex- } \\
\text { onecarboxylate } \ldots . . . . .\end{array}$ & $+9^{\circ}$ & Ethylic allylacetoacetate .. & Oil. \\
\hline $\begin{array}{l}\text { Ethylic plienyldehydrohex- } \\
\text { onecarboxylate .......... }\end{array}$ & $59-60^{\circ}$ & Ethylic allylbenzoylacetate. & Oil. \\
\hline $\begin{array}{l}\text { Ethylic paranitrophenyldehy- } \\
\text { drohexonecarboxylate ... }\end{array}$ & $62-63$ & $\begin{array}{l}\text { Ethylic allylparanitroben- } \\
\text { zovlacctate }\end{array}$ & $45-46^{\circ}$ \\
\hline $\begin{array}{l}\text { Paranitrophenyldehydrohex- } \\
\text { onecarboxylic acid........ }\end{array}$ & $172^{\circ}$ & $\begin{array}{c}\text { Allyl paranitrobenzoylace- } \\
\text { tic acid .............. }\end{array}$ & Cnstable. \\
\hline
\end{tabular}




\section{Boiling Points.}

\begin{tabular}{|c|c|c|c|}
\hline $\begin{array}{l}\text { Ethylic methyldehydrohex- } \\
\text { onecarboxylate .......... }\end{array}$ & $226-227^{\circ}$ & Ethylic allylacetoacetate .. & $213-214^{\circ}$ \\
\hline Methyldehydrohexone ...... & $109^{\circ}$ & Allylacetone. & $128-130$ \\
\hline Phenyldehydrohexone ...... & $258-260^{\circ}$ & Allylacetophenone....... & $235-238$ \\
\hline
\end{tabular}

It is thus seen that these two series of substances differ most widely from each other, and cannot possibly be identical. The same differences become directly apparent on studying the chemical properties (see Ber., 16, 1797).

The careful study of the physical properties of these. substances shows, however, that there are still several curious facts in connection with them which require explanation.

Thus whilst methyldehydrohexone (b. p. $109^{\circ}$ ) boils $20^{\circ}$ lower than allylacetone (b. p. $128-130^{\circ}$ ), the ethereal salt of the carboxylic acid of methyldehydrohexone (ethylic methyldehydrohexonecarboxylate, b. p. $226-227^{\circ}$ ) boils $12^{\circ}$ higher than the ethereal salt of the carboxylic acid of allylacetone (ethylic allylacetoacetate, b. p. $213-214^{\circ}$ ). The introduction of the same group, $\mathrm{COOC}_{2} \mathrm{H}_{5}$, has in the one case raised the boiling point $117^{\circ}$, in the other case only $84^{\circ}$. Again, as methyldehydrohexone boils nearly $20^{\circ}$ lower than allylacetone, phenyldehydrohexone should boil at a lower temperature than allylacetophenone, but it boils $20^{\circ}$ higher. It is difficult to find an explanation for these discrepancies.

The study of the magnetic rotation of these substances has also given very curious results. The magnetic rotation of methyldehydrohexone is 6.074 . Now, if ethylic methyldehydrohexonecarboxylate be really the ethereal salt of the carboxylic acid of methyldehydrohexone, it should have a magnetic rotation differing from that substance by the value of the group $\mathrm{COOC}_{2} \mathrm{H}_{5}-\mathrm{H}$. The value of this group is easily calculated by subtracting from the magnetic rotation of ethylic malonate $(7 \cdot 410)$ the magnetic rotation of ethylic acetate $(4 \cdot 462)$, when $\mathrm{COOC}_{2} \mathrm{H}_{5}-\mathrm{H}=2 \cdot 948$. Or a value still more suited to the case in point can be obtained by deducting the magnetic rotation of acetone $(3 \cdot 514)$ from that of ethylic acetoacetate $(6 \cdot 501)$ when $\mathrm{COOC}_{2} \mathrm{H}_{3}-\mathrm{H}=2 \cdot 987$. In calculating the magnetic rotation of ethylic methyldehydrohexonecarboxylate we get methyldehydrohexone $(6.074)+\mathrm{COOC}_{2} \mathrm{H}_{5}-\mathrm{H}(2.987)=9.061=$ calculated magnetic rotation of ethylic methylhydrohexonecarboxylate. But the value actually found was $10 \cdot 069$, a difference of one whole number from the calculated; this is altogether unaccountable.

A further consideration which has perhaps some connection with this remarkable discrepancy is the following:-The magnetic rotation 
of acetobutyl alcohol is 6.502 , that of its anhydride, methyldehydrohexone, is 6.074 . Therefore the loss of water involved in the formation of the anhydride has reduced the magnetic rotation by 0.428 . But when acetobutyl alcohol is converted into its anhydride, it is supposed that the following changes take place:-

\section{$\mathrm{CH}_{3} \cdot \mathrm{CO} \cdot \mathrm{CH}_{2} \cdot \mathrm{CH}_{2} \cdot \mathrm{CH}_{2} \cdot \mathrm{CH}_{2} \mathrm{OH}=$}

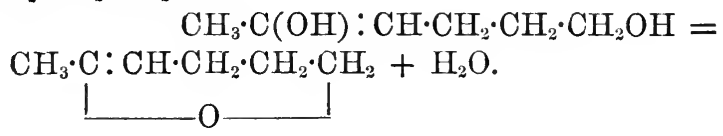

Now the diminution in magnetic rotation $(0.428)$ can explain the dehydration which occurs, but it does not account at all for the fact that methyldehydrohexone is an unsaturated compound, in which case its magnetic rotation should be expected to be one whole number more, or about $7 \cdot 074$. The only theory which can be put forward at present to meet these difficulties is that methyldehydrohexone exists in two forms, namely, ( $\alpha$ ) magnetic rotation $7 \cdot 074$, as in ethylic methyldehydrohexonecarboxylate; and ( $\beta$ ) magnetic rotation 6.074 , as in the anhydride of acetobutyl alcohol. This would account for the high rotation of ethylic methyldehydrohexonecarboxylate which would be calculated thus: $\alpha$-methyldehydrohexone $(7 \cdot 074)+\mathrm{COOC}_{2} \mathrm{H}_{5}-\mathrm{H}=$ $(2 \cdot 987)=10 \cdot 061=$ calc. magnetic rotation. This agrees with the number actually found $=10.069$. On distilling methyldehydrohexonecarboxylic acid, some remarkable change must take place, the $\alpha$-methyldehydrohexone present in this acid being converted into the $\beta$-modification.

From these considerations it is evident that although the constitution of these various substances has been explained in a satisfactory way, from a chemical point of view, there are still several curious points of physical interest which are at present unexplained. It is hoped that further experiment will throw some light on, and afford a satisfactory explanation of these physical difficulties.

\section{Ethylic Methyldehydrohexonecarboxylate,<smiles>CCC(C)=C(C)CCCCOC(=O)OC</smiles>

In the preparation of this substance the following method has been found to give the best results:-23 grams of sodium is dissolved in 250-270 grams of absolute alcohol. To this solution of sodic ethylate, when cold, a mixture of 130 grams of ethylic acetoacetate and 105 grams of trimethylene bromide is slowly added, the whole being well cooled during the operation. The clear solution formed is then heated to boiling on a water-bath in a flask connected with a 
reflux condenser. In a short time the liquid becomes cloudy, owing to the separation of sodium bromide, and after about four hours' boiling, the reaction is usually at an end. In order to be sure of this, it is only necessary to mix a few drops of the solution with a little water, and test this with litmus-paper when, if the decomposition is finished, the product will show a neutral reaction. Sufficient water is now added to dissolve all the precipitated sodic bromide, and the mass is extracted about three times with ether. The ethereal solution is then well washed with water, dried over calcic chloride, and the ether distilled off. The residual slightly yellowish-coloured oil, which should weigh 140 to 150 grams, is then submitted to careful fractional distillation. The following fractions are obtained :-

$$
\begin{aligned}
& 100-170=8 \text { grams. } \\
& 170-200=36 \quad, \\
& 200-240=80 \quad, \\
& \text { Residue }=6 \quad,
\end{aligned}
$$

The fraction 170-200 consists of regenerated ethylic acetoacetate, that boiling at $200-240^{\circ}$ contains the ethylic methyldehydrohexonecarboxylate, and gives on further fractioning $45-50$ grams boiling between $216-228^{\circ}$. This fraction is pure enough for ordinary purposes.

Pure ethylic methyldehydrohexonecarboxylate boils at $222-223^{\circ}$ (720 mm.) and $225-226^{\circ}(760 \mathrm{~mm}$.$) .$

Analysis gave the following results :-

I. $0 \cdot 1519$ gram substance gave $0 \cdot 1142$ gram $\mathrm{H}_{2} \mathrm{O}$ and 0.3528 gram

\begin{tabular}{|c|c|c|c|}
\hline \multirow[b]{3}{*}{ C....... } & \multirow{2}{*}{$\begin{array}{l}\text { Theory. } \\
\mathrm{C}_{9} \mathrm{H}_{14} \mathrm{O}_{3} \text {. }\end{array}$} & \multicolumn{2}{|c|}{ Found. } \\
\hline & & I. & II. \\
\hline & 63.53 per cent. & $63 \cdot 52$ & 63.35 per cent. \\
\hline $\mathrm{H} \ldots \ldots$ & $8 \cdot 24$ & $8 \cdot 35$ & $8 \cdot 19$ \\
\hline $0 \ldots \ldots$ & $28 \cdot 33$ & $28 \cdot 13$ & $28 \cdot 46$ \\
\hline
\end{tabular}
$\mathrm{CO}_{2}$.

II. $0 \cdot 1808$ gram substance gave $0 \cdot 1333$ gram $\mathrm{H}_{2} \mathrm{O}$ and 0.4201 gram $\mathrm{CO}_{2}$.

A vapour-density determination in aniline vapour by Hofman's method gave-

$$
\begin{array}{ll}
\text { D. . . . . . . . . . . . . . } & 6 \cdot 21 \\
\text { Theory, } \mathrm{C}_{9} \mathrm{H}_{14} \mathrm{O}_{3} \ldots \ldots \ldots & 5 \cdot 88
\end{array}
$$

Ethylic methyldehydrohexonecarboxylate is a colourless oil having

* In the samples used for analysis, the slight trace of bromine which is almost invariably present in this ethereal salt when prepared as above, was first remored by treatment with zinc-dust and acetic acid. 
a disagreeably penetrating, camphor-like smell. When cooled to $0^{\circ}$ it solidities to a white, crystalline mass which melts again at $9^{\circ}$. It is isomeric with ethylic allylacetoacetate which boils at $213-214^{\circ}$ (see p. 708). As this product of the reaction of trimethylene bromide on ethylic acetoacetate was thought to be of special interest, a very pure sample was carefully prepared and submitted to physical examination. The determinations of the specific gravity and magnetic rotation of this substance, which were performed by my father, gave the following results :-

I. Sp. gr. determinations

$$
\begin{aligned}
& \text { Preparation I. } \\
& d_{\frac{15^{\circ}}{15^{\circ}}}=1 \cdot 06457 \\
& \text { Preparation II. } \\
& d \frac{15^{\circ}}{15^{\circ}}=1.06840 \text {, } \\
& d_{\overline{2} 5^{\circ}}^{25^{\circ}}=1.05840 \\
& d \frac{20^{\circ}}{20^{\circ}}=1 \cdot 06470 \text {, } \\
& d \frac{25^{\circ}}{25^{\circ}}=1.06137 \text {. }
\end{aligned}
$$

Magnetic Rotations. I.

\begin{tabular}{c|c|c}
$t$. & Sp. rotation. & Mol. rotation. \\
\hline $15 \cdot 0$ & $1 \cdot 1512$ & $10 \cdot 212$ \\
$15 \cdot 2$ & $1 \cdot 1473$ & $10 \cdot 178$ \\
$15 \cdot 2$ & $1 \cdot 1465$ & $10 \cdot 172$ \\
$15 \cdot 6$ & $1 \cdot 1472$ & $10 \cdot 180$ \\
$15 \cdot 8$ & $1 \cdot 1456$ & $10 \cdot 167$ \\
$12 \cdot 0$ & $1 \cdot 1561$ & $10 \cdot 240$ \\
$12 \cdot 0$ & $1 \cdot 1531$ & $10 \cdot 212$ \\
$12 \cdot 0$ & $1 \cdot 1517$ & $10 \cdot 200$ \\
\hline $14 \cdot 1$ & $1 \cdot 1498$ & $10 \cdot 195$
\end{tabular}

Magnetic Rotations. II.

\begin{tabular}{c|c|c}
\hline \multicolumn{1}{c|}{$t}$. & Sp. rotation. & Mol. rotation. \\
\hline $20 \cdot 4$ & $1 \cdot 1419$ & $10 \cdot 130$ \\
$18 \cdot 8$ & $1 \cdot 1399$ & $10 \cdot 102$ \\
$18 \cdot 8$ & $1 \cdot 1424$ & $10 \cdot 125$ \\
$18 \cdot 8$ & $1 \cdot 1429$ & $10 \cdot 129$ \\
$16 \cdot 7$ & $1 \cdot 1443$ & $10 \cdot 127$ \\
$16 \cdot 7$ & $1 \cdot 1438$ & $10 \cdot 123$ \\
$16 \cdot 7$ & $1 \cdot 144$ & $10 \cdot 128$ \\
\hline $18 \cdot 1$ & $1 \cdot 1428$ & $10 \cdot 123$ \\
\hline
\end{tabular}


Dr. Gladstone has kindly determined the refraction equivalent of these preparations, together with that of the isomeric ethylic allylacetoacetate. He gave me the following account of his results :-

\section{Preparation $I$.}

\begin{tabular}{l|c|c|c|c|c|c|c}
\hline & Temp. & $\mu_{\mathrm{A} .}$ & $\mu_{\mathrm{D} .}$ & $\mu_{\mathrm{H} .}$ & $\begin{array}{c}\text { Specific } \\
\text { refrac- } \\
\text { tion. }\end{array}$ & $\begin{array}{c}\text { Specific } \\
\text { disper- } \\
\text { sion. } \\
\cdot\end{array}$ & $\begin{array}{c}\text { Refrac- } \\
\text { tion } \\
\text { equiva- } \\
\text { lent. }\end{array}$ \\
\hline $\begin{array}{c}\text { Ethylic methylde- } \\
\text { hydrohexonecar- } \\
\text { boxylate ......... }\end{array}$ & 13 & $1 \cdot 4743$ & $1 \cdot 4818$ & $1 \cdot 5078$ & 0.4446 & 0.0314 & $75 \cdot 58$ \\
$\begin{array}{c}\text { Ethylic allylaceto- } \\
\text { acetate............ }\end{array}$ & 13.5 & $1 \cdot 4356$ & $1 \cdot 4410$ & $1 \cdot 4593$ & 0.4383 & 0.0210 & $\mathbf{7 4 . 5 1}$ \\
\hline
\end{tabular}

"If we take Landolt's values for the three elements, $\mathrm{C}_{9} \mathrm{H}_{14} \mathrm{O}_{3}$ should be $72 \cdot 2$. Now bodies belonging to the allyl series are about $2 \cdot 0$ above normal, which fairly accounts for the value of ethylic allylacetoacetate. The ethylic methyldehydrohexonecarboxylate is evidently of higher refraction and much higher dispersion, suggesting the presence of a still larger proportion of carbon in a highly refracting condition."

Preparation $I I$.

\begin{tabular}{|c|c|c|c|c|c|c|c|c|}
\hline \multirow{2}{*}{$t$. } & \multirow{2}{*}{ Sp. gr. } & \multirow{2}{*}{$\mu_{\mathrm{A}}$} & \multirow{2}{*}{$\mu_{\mathrm{D}}$} & \multirow{2}{*}{$\mu_{\mathrm{H}}}$. & \multicolumn{2}{|c|}{ Observed. } & \multicolumn{2}{|c|}{ Calculated. } \\
\hline & & & & & $\begin{array}{c}\text { Ref. eq. } \\
\text { A. }\end{array}$ & $\begin{array}{c}\text { Disp. eq. } \\
\text { H-A. }\end{array}$ & $\begin{array}{c}\text { Ref. eq. } \\
\text { A. }\end{array}$ & $\begin{array}{c}\text { Disp. eq. } \\
\mathbf{H}-\mathbf{A} .\end{array}$ \\
\hline $24 \cdot 5$ & $1 \cdot 0605$ & $1 \cdot 4697$ & $1 \cdot 4772$ & $1 \cdot 5033$ & $75 \cdot 29$ & $5 \cdot 39$ & $72 \cdot 2$ & $3 \cdot 26$ \\
\hline
\end{tabular}

"This agrees substantially with the previous specimen both in refraction and dispersion. The figures are very high, and I do not know how to interpret them."

In spite of the close agreement in magnetic rotation of these two preparations, it was thought, for reasons which are explained in the introduction (p. 708), that these numbers must still be considerably too high, and that the substance prepared in this way was not pure enough for physical measurements.

A very pure sample of ethylic methyldehydrohexonecarboxylate was therefore prepared from the silver salt of the acid by means of 
ethyl iodide. In doing this 50 grams of ethylic methyldehydrohexonecarboxylate (b. p. 221-228 ) were hydrolysed by boiling for four hours on a water-bath with 40 grams of pure potash dissolved in pure methyl alcohol. The alcohol was then distilled off, first on a water-bath and then over a naked flame, and the resulting nearly colourless solution carefully acidified in the cold with very dilute sulphuric acid $(1: 6)$. In this way, the methyldehydrohexonecarboxylic acid was thrown down as a beautifully white, crystalline precipitate, which after collecting, well washing with water, and drying on a porous plate, was obtained as a colourless, crystalline powder, melting at $118-119^{\circ}$. This was dissolved in dilute ammonia, the solution carefully neutralised with nitric acid, and precipitated with nitrate of silver. The silver salt after washing and drying over sulphuric acid in a vacuum was mixed with an excess of ethyl iodide, dissolved in ten times its volume of pure dry ether, and boiled for four hours on a water-bath. The precipitated iodide of silver was filtered off from the ethereal solution, and washed two or three times with pure ether. The filtrate was then shaken with a little sodic carbonate solution, dried over potassic carbonate, and the ether distilled off, when an almost colourless oil remained, which on twice refractioning boiled constantly at $2266^{\circ}-227^{\circ}(760 \mathrm{~mm}$.), and gave the following result on analysis :-

0.1663 gram substance gave 0.1238 gram $\mathrm{H}_{2} \mathrm{O}$, and 0.3864 gram $\mathrm{CO}_{2}$.

\begin{tabular}{|c|c|c|}
\hline C $\ldots$. & $\begin{array}{l}\text { Theory. } \\
63.53 \text { per cent. }\end{array}$ & $\begin{array}{l}\text { Found. } \\
63 \cdot 37 \text { per cent. }\end{array}$ \\
\hline $\mathrm{H}$ & $8 \cdot 24$ & $8 \cdot 27$ \\
\hline . & $28 \cdot 33$ & $28 \cdot 36$ \\
\hline
\end{tabular}

The determination of the magnetic rotation of this preparation gave the following results:-

$\begin{array}{rlrl}\text { Sp. gr. } & d \frac{9^{\circ}}{9^{\circ}}=1.0744 & & d_{\overline{20^{\circ}}}^{20^{\circ}}=1.0660, \\ d \frac{15^{\circ}}{15^{\circ}}=1.0696 & d_{\overline{25^{\circ}}}^{25^{\circ}}=1.0626 .\end{array}$

Magnetic Rotation.

\begin{tabular}{c|c|c}
\multicolumn{1}{c|}{$t}$. & Sp. rotation. & Mol. rotation. \\
\cline { 1 - 2 } $24 \cdot 0$ & $1 \cdot 1314$ & $10 \cdot 050$ \\
$24 \cdot 0$ & $1 \cdot 1349$ & $10 \cdot 082$ \\
$23 \cdot 5$ & $1 \cdot 1349$ & $10 \cdot 080$ \\
$23 \cdot 5$ & $1 \cdot 1334$ & $10 \cdot 065$ \\
\hline $23 \cdot 7$ & $1 \cdot 1336$ & $10 \cdot 069$
\end{tabular}


The number 10.069 (which agrees fairly well with that obtained by the other measurements) is probably the true magnetic rotation of ethylic methyldehydrohexonecarboxylate.

Ethylic methyldehydrohexonecarboxylate does not combine with phenylhydrazine. In order to prove this, 4 grams of the pure ethereal salt were mixed with 3 grams of phenylhydrazine and gently heated on a water-bath for half-an-hour. The product, which was a comparatively limpid mass, was distilled in steam until no more oil* came over, and the distillate extracted several times with ether. The ethereal solution after drying over calcic chloride and distilling off the ether deposited 3 grams of a slightly yellowish oil, which on distilling proved to be unchanged ethylic methyldehydrohexonecarboxylate.

When the experiments on the action of trimethylene bromide on ethylic sodacetoacetate were first instituted, it was very important to show that ethylic methyldehydrohexonecarboxylate did not contain a hydrogen-atom displaceable by sodium, i.e., that it was not a monosubstituted ethylic acetoacetate such as ethylic allylacetoacetate,

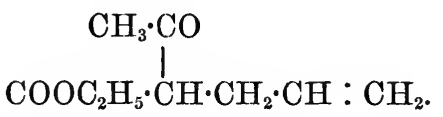

This was determined by treating 10 grams of the pure ethereal salt with $1 \cdot 4$ grams of sodium (as sodic ethylate) and $7 \cdot 5$ grams of benzyl chloride. After heating on a water-bath for some hours, the product was found to consist of a mixture of benzyl ethyl ether,

$$
\mathrm{C}_{6} \mathrm{H}_{5} \cdot \mathrm{CH}_{2} \cdot \mathrm{O} \cdot \mathrm{C}_{2} \mathrm{H}_{5} \text {, }
$$

and unchanged ethylic methyldehydrohexonecarboxylate.

A few experiments were also made on the action of phosphorous pentachloride on ethylic methyldehydrohexonecarboxylate.

35 grams of the pure ethereal salt was mixed with 70 grams of chloroform and then 45 grams of phosphorous pentachloride slowly added. After each addition of the pentachloride, vigorous reaction set in, which had to be moderated by careful cooling. Towards the end the whole was warmed gently, mixed with water to decompose the phosphorous oxychloride and unchanged pentachloride, extracted with ether, and the ethereal solution washed with dilute sodic carbonate. On distilling off the ether and chloroform, a colourless oil remained behind, which after repeated fractioning under reduced pressure $\left(200 \mathrm{~mm}\right.$.) boiled constantly at $212-215^{\circ}$.

Two chlorine determinations gave the following results, agreeing with the formula $\mathrm{C}_{9} \mathrm{H}_{13} \mathrm{O}_{2} \mathrm{Cl}$ :-

* Only traces of phenylhydrazine were carried over during the operation. 
I. 0.2883 gram substance gave 0.2103 gram $\mathrm{AgCl}$.

II. $0 \cdot 3681$

$0 \cdot 2745 \quad$,

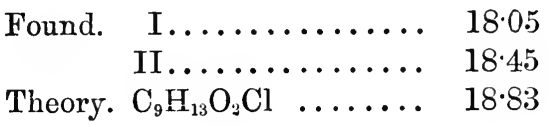

As this substance has not been further examined, no idea can be formed as to its constitution.

\section{Methyldehydrohexonecarboxylic Acid,}

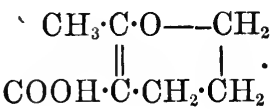

This acid is easily obtained from its ethylic salt by hydrolysis with alcoholic potash. The fraction boiling between $215^{\circ}$ and $230^{\circ}$ is mixed with a fairly concentrated solution of alcoholic potash (containing twice the theoretical quantity of $\mathrm{KOH}$ ) and boiled on a water-bath for six hours. The alcohol is then distilled off, the residue dissolved in a little water, gently evaporated on a water-bath to get rid of the last traces of alcohol, well cooled, and acidified with dilute sulphuric acid. In this way methyldehydrohexonecarboxylic acid is precipitated as a beautifully white, crystalline mass, which after collecting, well washing with cold water, and drying on a porous plate, is nearly pure.

To obtain the pure acid, this product is dissolved in a little boiling benzene or light petroleum (b. p. $70-90^{\circ}$ ), from which solution it crystallises on cooling in beautiful, colourless needles.

\section{Analyses.}

I. $0 \cdot 1964$ gram substance gave 0.1254 gram $\mathrm{H}_{2} \mathrm{O}$ and 0.4252 gram $\mathrm{CO}_{2}$.

II. $0 \cdot 1803$ gram substance gave 0.1154 gram $\mathrm{H}_{2} \mathrm{O}$ and 0.3894 gram $\mathrm{CO}_{2}$.

III. 0.2191 gram substance gave 0.1462 gram $\mathrm{H}_{2} \mathrm{O}$ and 0.4 .768 gram $\mathrm{CO}_{2}$.
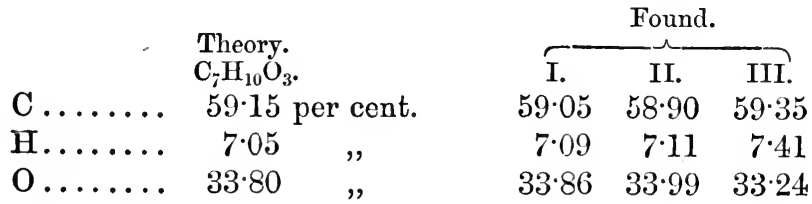

Methyldehydrohexonecarboxylic acid melts at $119^{\circ}$, but decomposes at a somewhat higher temperature into carbonic anhydride and methyldehydrohexone, as will be described later on. If, however, a small quantity of the acid is rapidly heated in a test-tube, it distils 
almost without decomposition, and solidifies on the cooler portions of the tube in beautiful, leaf-like crystals. It is easily soluble in hot benzene, light petroleum, alcohol, chloroform and ether, but more sparingly so in these solvents in the cold. When mixed with hot water, it dissolves readily, and the solution, if rapidly cooled, deposits the greater part of the acid in beautiful, colourless needles. On boiling with water, methyldehydrohexonecarboxylic acid is rapidly decomposed into carbonic anhydride and acetobutyl alcohol (see p. 718). In alkaline solutions this acid is very stable, and can be boiled for hours with strong potash without any apparent decomposition.

When heated at $200^{\circ}$ with strong aqueous ammonia, it is split up into carbonic anhydride, acetobutyl alcohol, and a volatile base, which from its odour and reactions would appear to be a derivative of pyridine.

I have not further examined this base, but from its method of preparation it is probable that it is closely related to the interesting base obtained by Lipp (Ber., 18, 3282) by treating ethylic $\omega$-bromo$\mathrm{CH}_{3} \cdot \mathrm{CO} \cdot \mathrm{CH} \cdot \mathrm{COOC}_{2} \mathrm{H}_{5}$

propylacetoacetate,

${ }_{\mathrm{CH}}^{\mathrm{C}} \cdot \mathrm{CH}_{2} \cdot \mathrm{CH}_{2} \mathrm{Br}^{\circ}$, with alcoholic ammonia.

Methyldehydrohexonecarboxylic acid dissolves easily in concentrated aqueous solution of hydrogen bromide (sp. gr. 1.83), and if the solution be quickly extracted with ether, a brominated acid is obtained, which, however, is very unstable and cannot be obtained in a pure state; but if the acid solution be allowed to stand at ordinary temperatures, carbonic anhydride is slowly evolved, and if after some hours water be adajed, a heavy oil is precipitated; this, after extracting with ether, seemed to be nearly pure acetobutyl bromide, $\mathrm{CH}_{3} \cdot \mathrm{CO} \cdot \mathrm{CH}_{2} \cdot \mathrm{CH}_{2} \cdot \mathrm{CH}_{2} \cdot \mathrm{CH}_{2} \mathrm{Br}$ (see p. 721).

When exposed to the vapour of bromine, methyldehydrohexonecarboxylic acid appears in the first case to form an unstable additive product. By the further action of the bromine, substitution takes place with evolution of hydrogen bromide. 2.036 grams of acid, after standing in an atmosphere of bromine for two days had taken up 4.767 grams of bromine $=234$ per cent. calculated for the equation

$$
\mathrm{C}_{7} \mathrm{H}_{10} \mathrm{O}_{3}+4 \mathrm{Br}_{2}=\mathrm{C}_{7} \mathrm{H}_{6} \mathrm{Br}_{4} \mathrm{O}_{3}+4 \mathrm{HBr}=232 \text { per cent. }
$$

The product of this reaction was a thick, uninviting syrup, which could not be obtained in a crystalline form, and therefore was not analysed.

Salts of Methyldehydrohexonecarboxylic Acid.

Ammonium Salt.-To prepare this salt the pure acid is dissolved 
in a slight excess of ammonia, and the solution allowed to evaporate to dryness over sulphuric acid in a vacuum. It is a white, crystalline solid, very easily soluble in water.

Silver Salt, $\mathrm{C}_{7} \mathrm{H}_{9} \mathrm{O}_{3}$ Ag. - - If silver nitrate is added to a neutral solution of ammonium methyldehydrohexonecarboxylate, a white, crystalline precipitate of the silver salt is thrown down. This, after collecting, well washing with water, and drying over sulphuric acid in a vacuum, gave the following numbers on analysis :-

I. 0.2997 gram substance gave 0.1010 gram $\mathrm{H}_{2} \mathrm{O}, 0.3705$ gram $\mathrm{CO}_{2}$, and $0.1299 \mathrm{gram} \mathrm{Ag}$.

II. 0.4668 gram substance gave 0.2027 gram Ag.

\begin{tabular}{|c|c|c|c|c|c|}
\hline & & & $\mathrm{F}$ & & \\
\hline & $\begin{array}{l}\text { Theory } \\
\mathrm{C}_{7} \mathrm{H}_{9} \mathrm{O}_{3} \mathrm{~A}\end{array}$ & & I. & II. & \\
\hline C...... & $33 \cdot 73$ & cent. & $33 \cdot 64$ & - & per cent. \\
\hline $\mathrm{H} \ldots$ & $3 \cdot 62$ & , & $3 \cdot 74$ & - & \# \\
\hline $\mathrm{Ag} \ldots$ & $43 \cdot 37$ & , & $43 \cdot 24$ & $43 \cdot 41$ & $"$ \\
\hline $0 \ldots$ & $19 \cdot 27$ & , & $19 \cdot 28$ & - & $"$ \\
\hline
\end{tabular}

This silver salt dissolves fairly easily in boiling water, and crsstallises on cooling in beautiful, leafy tufts. It is very stable, and is only very slowly decomposed on boiling with water or exposing to light.

Copper Salt.-Copper methyldehydrohexonecarboxylate is precipitated on the addition of cupric sulphate to the neutral solution of the ammonium salt as a light-green, amorphous precipitate almost insoluble in water. It was collected, well washed with water, and dried over sulphuric acid in a vacuum.

\section{Analyses.}

I. 0.3337 gram substance gave 0.0720 gram $\mathrm{CuO}$.

II. $0.4020, \quad, \quad 0.0870$,

Found.

\begin{tabular}{|c|c|c|c|}
\hline & & & \\
\hline & I. & II. & $\left(\mathrm{C}_{7} \mathrm{H}_{9} \mathrm{O}_{3}\right)_{2} \mathrm{Cu}+\mathrm{H}_{2} \mathrm{O}$. \\
\hline$\cdots$ & $17 \cdot 22$ & $17 \cdot 28$ p.c. & 17.42 p. с. \\
\hline
\end{tabular}

This copper salt, therefore, appears to contain 1 mol. $\mathrm{H}_{2} \mathrm{O}$. Unfortunately this could not be determined by a direct experiment, as the salt when heated begins to decompose at $90-100^{\circ}$.

Action of Water on Methyldehydrohexonecarboxylic Acid. Formation of Acetobutyl Alcohol, $\mathrm{CH}_{3} \cdot \mathrm{CO} \cdot \mathrm{CH}_{2} \cdot \mathrm{CH}_{2} \cdot \mathrm{CH}_{2} \cdot \mathrm{CH}_{2} \cdot \mathrm{OH}$.

When recrystallising methyl dehydrohexonecarboxylic acid from hot water, it was noticed that if the boiling was carried on for any 
length of time carbonic anhydride was evolved, and on cooling no crystals separated out. As the decomposition of the acid, which evidently takes place here, might throw some light on its constitution, it was carefully studied. 20 grams of the pure acid was boiled with 70 grams of distilled water in a vessel, provided with a reversed condenser, the end of which was connected with a flask containing barytawater. The acid slowly dissolved with evolution of a considerable quantity of gas, which on passing through the baryta-water instantly caused the precipitation of baric carbonate. After boiling for about half an hour no more gas was given off, and the reaction was complete. The solution now contains acetobutyl alcohol, which is extracted in the following way.

The colourless liquid, after filtering, is treated with anhydrous potassic carbonate until thoroughly saturated, and allowed to stand for a short time, the greater part of the acetobntyl alcohol has then separated as a thick oily layer on the surface of the liquid. This is extracted ten times with ether, the ethereal solution dried over potassic carbonate, filtered, and the ether distilled off. The residue, which consists of almost pure acetobutyl alcohol, on rapid distillation passes over between $226-227^{\circ}$, almost without decomposition. On analysis the following results were obtained :-

I. $0 \cdot 1974$ gram substance gave $0 \cdot 1834$ gram $\mathrm{H}_{2} \mathrm{O}$ and 0.4497 gram $\mathrm{CO}_{2}$.

II. $0 \cdot 1711$ gram substance gave $0 \cdot 1480$ gram $\mathrm{H}_{2} \mathrm{O}$ and $0 \cdot 4042$ gram $\mathrm{CO}_{2}$.

Theory.

$\mathrm{CH}_{3} \cdot \mathrm{CO} \cdot \mathrm{CH}_{2} \cdot \mathrm{CH}_{2} \cdot \mathrm{CH}_{2} \cdot \mathrm{CH}_{2} \cdot \mathrm{OH}$.

C .... 62.07 per cent.

H ... 10.34 ,

O ... $27 \cdot 59 \quad$,

Found.

$\begin{array}{ccc}\overbrace{\text { I. }} & \text { II. } & \\ 62 \cdot 12 & 62 \cdot 21 & \text { per } \\ 10 \cdot 33 & 10 \cdot 09 \quad, \\ 27 \cdot 55 & 27 \cdot 70 \quad ",\end{array}$

Acetobutyl alcohol is a thick, colourless oil, which distils at 226$227^{\circ}$ (760 mm.), with the formation of traces of an anhydride, probably methyldehydrohexone. It mixes with water in all proportions, forming a solution of burning but slightly sweet taste. It does not reduce Fehling's solution, or an ammoniacal solution of silver oxide in the cold. On warming with these reagents, a very slow reduction takes place in the case of the Fehling's solution, but the silver solution is, on the contrary, very quickly reduced, a bright mirror being formed on the sides of the vessel containing it.

Phenylhydrazine hydrochloride, dissolved in a solution of sodic acetate, produces at first a turbidity only on the addition to an aqueous solution of acetobutyl alcohol; in a short time, however, the 
phenylhydrazine compound separates out in large, yellow, oily drops. Several attempts were made with the object of obtaining this interesting substance in a crystalline condition suitable for analysis, but without success.

When mixed with concentrated nitric acid, acetobutyl alcohol is not attacked in the cold; on warming gently, a dark pink coloration is first formed, and on further heating oxidation rapidly sets in, torrents of red fumes being evolved.

An aqueous solution of acetobutyl alcohol, if treated with iodine and potash, gives quantities of iodoform.

Acetobutyl alcohol has been subjected to physical examination by my father, with the following results :-

Sp. gre.

$$
\begin{aligned}
d \frac{4^{\circ}}{4^{\circ}} & =0.99771, \\
d \frac{5^{\circ}}{15^{\circ}} & =0.98947, \\
d_{\frac{25^{\circ}}{25^{\circ}}} & =0.98270 .
\end{aligned}
$$

Magnetic Rotations.

\begin{tabular}{c|c|c}
$t$. & Sp. rotation. & Mol. rotation. \\
\hline $19 \cdot 5$ & $0 \cdot 99101$ & $6 \cdot 492$ \\
$19 \cdot 5$ & $0 \cdot 9972$ & $6 \cdot 515$ \\
$17 \cdot 5$ & $0 \cdot 9969$ & $6 \cdot 504$ \\
$17 \cdot 5$ & $0 \cdot 9960$ & $6 \cdot 498$ \\
\hline $18 \cdot 5$ & $0 \cdot 9953$ & $6 \cdot 502$ \\
\hline
\end{tabular}

From its method of preparation, and the numbers obtained as its magnetic rotation, there can scarcely be a doubt that the acetobutyl alcohol described above really has the constitution assigned to it, but as it was most important in explaining the constitution of these methyldehydrohexone-derivatives to be perfectly certain on this point, it was thought that some additional evidence of its constitution was necessary.

If acetobutyl alcohol were oxidised with chromic acid, the first product which should result would be acetobutyric acid, thus :-

$$
\begin{aligned}
& \mathrm{CH}_{3} \cdot \mathrm{CO} \cdot \mathrm{CH}_{2} \cdot \mathrm{CH}_{2} \cdot \mathrm{CH}_{2} \cdot \mathrm{CH}_{2} \cdot \mathrm{OH}+\mathrm{O}_{2}= \\
& \qquad \mathrm{CH}_{3} \cdot \mathrm{CO} \cdot \mathrm{CH}_{2} \cdot \mathrm{CH}_{2} \cdot \mathrm{CH}_{2} \cdot \mathrm{COOH}+\mathrm{H}_{2} \mathrm{O},
\end{aligned}
$$

and on further oxidation with an excess of the oxidising agent, acetic 
and succinic acids should be formed, according to Popoff's rule (Anualen, 161, 285) -

\section{$\mathrm{CH}_{3} \cdot \mathrm{CO} \cdot \mathrm{CH}_{2} \cdot \mathrm{CH}_{2} \cdot \mathrm{CH}_{2} \cdot \mathrm{CH}_{2} \cdot \mathrm{OH}+5 \mathrm{O}=\mathrm{CH}_{3} \cdot \mathrm{COOH}+$ $\mathrm{COOH} \cdot \mathrm{CH}_{2} \cdot \mathrm{CH}_{2} \cdot \mathrm{COOH}+\mathrm{H}_{2} \mathrm{O}$.}

Acetobutyric acid has been prepared by Wolff (Annalen, 216, 129), who describes it as a thick, oily liquid, boiling at $274-275^{\circ}$.

On oxidising acetobutyl alcohol with the calculated quantity of chromic acid and sulphuric acid, I obtained an oily acid which possessed all the properties of acetobutyric acid. As, however, without working with large quantities of acetobutyl alcohol it would have been difficult to prove definitely the identity of the two acids, it was thought more satisfactory to examine the end products of the oxidation of this alcohol. To this purpose 4 grams of pure acetobutyl alcohol was dissolved in 20 grams of water, and then slowly mixed with a slight excess of a solution of potassic dichromate in dilute sulphuric acid. As soon as the oxidation, which set in at once, was complete, the product was submitted to distillation in steam until the condensed water showed neutral reaction. The distillate was then digested with a slight excess of baric carbonate, filtered, and the filtrate evaporated to dryness, when a considerable quantity of a white residue remained. On the addition of silver nitrate to a clear solution of this salt in water, a beautiful, crystalline salt was thrown, down, which possessed all the properties of acetate of silver. It was filtered off, washed with water, dried over sulphuric acid in a vacuum, and analysed, with the following result:-

0.4657 gram substance gave 0.3010 gram Ag.

$$
\begin{aligned}
& \text { Theory. } \\
& \mathrm{CH}_{3} \cdot \mathrm{COOAg} \text {. Found. } \\
& \text { Ag....... 64.66 64.64 per cent. }
\end{aligned}
$$

Acetic acid was therefore one of the products of the oxidation of acetobutyl alcohol.

The residue remaining in the retort, after distilling off the acetic acid, was made slightly alkaline by the addition of caustic potash, well boiled until all the chromium had been precipitated, the precipitate filtered off and washed. The filtrate was then rendered slightly acid with dilute sulphuric acid, evaporated to dryness on a waterbath, and the finely powdered residue extracted several times with hot alcohol. The alcoholic solution was evaporated, the dry residue again extracted with alcohol and evaporated, when a white, crystalline substance was obtained, showing all the properties of succinic acid. After once recrystallising from water, colourless crystals were obtained, melting at $180-182^{\circ}\left(\right.$ succinic acid $\left.180^{\circ}\right)$. The solution in water gave, on 
the addition of ferric chloride, the characteristic, brown-red precipitate. In order to prove that this acid really was succinic acid, it was heated in a small retort for some time to its boiling point, and then distilled. After reheating and redistilling, a colourless mass of succinic anhydride was obtained, melting at $118-119^{\circ}$. This was further characterised by conversion into succinglfluoresceïn by heating with resorcinol.

The end products of the oxidation of acetobutyl alcohol are therefore acetic acid and succinic acid, a proof that this substance has the formula $\mathrm{CH}_{3} \cdot \mathrm{CO} \cdot \mathrm{CH}_{2} \cdot \mathrm{CH}_{2} \cdot \mathrm{CH}_{2} \cdot \mathrm{CH}_{2} \cdot \mathrm{OH}$.

Under the name acetobutyl alcohol, a substance has already been described by Lipp (Ber., 18, 3280), who prepared it by boiling ethylic $\omega$-bromopropylacetoacetate with dilute hydrochloric acid-

\section{$\mathrm{CH}_{3} \cdot \mathrm{CO} \cdot \mathrm{CH} \cdot \mathrm{CH}_{2} \cdot \mathrm{CH}_{2} \cdot \mathrm{CH}_{2} \mathrm{Br}$ $\mathrm{COOC}_{2} \mathrm{H}_{5}+2 \mathrm{H}_{2} \mathrm{O}=$$$
\mathrm{CH}_{3} \cdot \mathrm{CO} \cdot \mathrm{CH}_{2} \cdot \mathrm{CH}_{2} \cdot \mathrm{CH}_{2} \cdot \mathrm{CH}_{2} \cdot \mathrm{OH}+\mathrm{HBr}+\mathrm{C}_{2} \mathrm{H}_{5} \cdot \mathrm{OH}+\mathrm{CO}_{2} \text {. }
$$

Lipp describes it as a fairly mobile oil, smelling of camphor and boiling at $154-155^{\circ}(718 \mathrm{~mm}$.), whereas the acetobutyl alcohol obtained by me was a thick, almost odourless oil, boiling at $226-227^{\circ}$, or as much as $70^{\circ}$ higher than Lipp's substance.

As acetobutyl alcohol undoubtedly should be formed by the reaction used by Lipp, it is probable that he has made some slip in describing this substance. This is the more likely from the fact that the reduction product of this alcohol, hexylene glycol,

\section{$\mathrm{CH}_{3} \cdot \mathrm{CH}(\mathrm{OH}) \cdot \mathrm{CH}_{2} \cdot \mathrm{CH}_{2} \cdot \mathrm{CH}_{2} \cdot \mathrm{CH}_{2} \cdot \mathrm{OH}$,}

as prepared by Lipp, has approximately the same boiling point as that obtained by me from my acetobutyl alcohol.

In order to render more complete these experiments on the decomposition of methyldehydrohexonecarboxylic acid, by boiling with water, some quantitative determinations of the amount of carbonic anhydride produced during the reaction have been made. A weighed quantity of pure acid was boiled with water in a Würtz flask connected with a reflux condenser, and the carbonic anhydride evolved, after passing through a drying-bottle containing sulphuric acid, collected in a Geissler's potash-bulb. At the end of the operation the carbonic anhydride still left in the apparatus was swept out by means of a current of air, free from carbonic anhydride, and the potash-bulbs, after standing for some time, weighed. Two experiments were made with the following results :- 
I. $3 \cdot 1042$ grams substance gave $0 \cdot 9441$ gram $\mathrm{CO}_{2}$.

II. $2 \cdot 7940$

"

$"$

0.8500
9

Found.

$$
\mathrm{CO}_{2} \ldots \ldots . \quad \begin{array}{ccc}
\text { Theory. } & \text { I. } & \text { II. } \\
30.98 & 30 \cdot 44 & 30 \cdot 43 \text { per cent. }
\end{array}
$$

The amount of acetobutyl alcohol formed, as determined by saturating with carbonate of potash and repeatedly extracting with ether, was found in the two experiments to be about $4 \cdot 2$ grams, whereas the calculated amount is 4.81 grams.

It is therefore seen that the decomposition of the acid into acetobutyl alcohol and carbonic anhydride on boiling with water is a quantitative one.

\section{$\delta$-Hexyleneglycol, $\mathrm{CH}_{3} \cdot \mathrm{CH}(\mathrm{OH}) \cdot \mathrm{CH}_{2} \cdot \mathrm{CH}_{2} \cdot \mathrm{CH}_{2} \cdot \mathrm{CH}_{2} \cdot \mathrm{OH}$.}

$\delta$-Hexyleneglycol was first prepared by Lipp (Ber., 18, 3282), who obtained it by reducing his acetobutyl alcohol with sodium amalgam. As this substance, and more especially its dibromide, were of great value for the further development of these researches on the synthetical formation of closed carbon-chains, I have repeated Lipp's experiments with my acetobutyl alcohol. 10 grams of the pure alcohol were dissolved in a little water and treated with 50 grams of 3 per cent. sodium amalgam, as described by Lipp. As soon as the reduction was finished, the product was saturated with carbonic anhydride, and extracted several times with ether. After drying over potassic carbonate and distilling off the ether, a thick oil remained, which on twice refractioning boiled fairly constantly at $228-233^{\circ}$, and gave the following results on analysis :-

0.1527 gram substance gave 0.1649 gram $\mathrm{H}_{2} \mathrm{O}$ and 0.3411 gram

\begin{tabular}{|c|c|c|}
\hline & $\begin{array}{l}\text { Theory. } \\
\mathrm{C}_{6} \mathrm{H}_{14} \mathrm{O}_{2} \text {. } \\
61 \cdot 01 \text { per cent. }\end{array}$ & $\begin{array}{l}\text { Found. } \\
60.92 \text { per cent. }\end{array}$ \\
\hline & 11.86 & $11 \cdot 98$ \\
\hline & $27 \cdot 13$ & $27 \cdot 10$ \\
\hline
\end{tabular}
$\mathrm{CO}_{2}$.

This substance agrees in all its properties with the $\delta$-hexyleneglycol described by Lipp.

\section{$\delta$-Hexylene Dibromide, $\mathrm{CH}_{3} \cdot \mathrm{CHBr} \cdot \mathrm{CH}_{2} \cdot \mathrm{CH}_{2} \cdot \mathrm{CH}_{2} \cdot \mathrm{CH}_{2} \mathrm{Br}$.}

This substance is easily prepared in the following way:-Pure o-hexyleneglycol is dissolved in an excess of fuming hydrobromic acid, and the mixture heated in a sealed tube at $120^{\circ}$ for two 
hours. The contents of the tube are then cooled to $0^{\circ}$, resaturated with hydrogen bromide, and again heated in a sealed tube as before at $120^{\circ}$ for two hours. The product, which consists of two layers, is extracted with ether, dried over calcic chloride, and rapidly fractioned under $100 \mathrm{~mm}$. pressure. It is then easily obtained pure, boiling at $153-154^{\circ}$ (100 mm.).

\section{Anailysis.}

0.2390 gram substance gave 0.3654 gram AgBr.

$$
\begin{aligned}
& \text { Theory. } \\
& \mathrm{C}_{6} \mathrm{H}_{12} \mathrm{Br}_{2} \text {. Found. } \\
& \text { Br..... 65.58 per cent. 65.34 per cent. }
\end{aligned}
$$

$\delta$-Hexylene bromide is a heavy, colourless liquid, which, on standing - for any length of time, undergoes slight decomposition, at the same time turning slightly dark-coloured. A detailed description of this interesting substance is reserved for a future paper.

\section{Methyldehydrohexone, $\stackrel{\mathrm{CH}_{3} \cdot \mathrm{C} \cdot \mathrm{O}-\mathrm{CH}_{\mathrm{CH}} \cdot \mathrm{CH}_{2}}{\mathrm{CH}_{2}}$}

If pure methyldehydrohexonecarboxylic acid is gently heated in a small retort to a temperature of $140-150^{\circ}$ decomposition sets in,* carbonic anhydride is evolved, and a colourless oil distils over which consists of nearly pure methyldehydrohexone. This reaction takes place according to the following equation:-

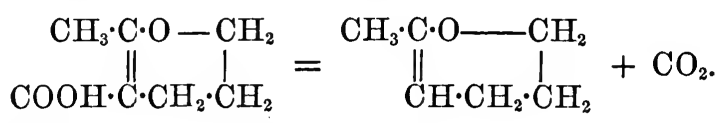

As a considerable quantity of methyldehydrohexone was required for physical measurements and other experiments, as much as 30 grams of the pure acid were operated on. This amount on slow distillation gave $19^{\cdot} 8$ grams of oil (theory $20 \cdot 7$ grams), which on carefully fractioning nearly all passed over between $109-110^{\circ}$ as a colourless, limpid liquid, which on analysis gave the following results :-

I. 0.2246 gram substance gave 0.2091 gram $\mathrm{H}_{2} \mathrm{O}$ and 0.6015 gram $\mathrm{CO}_{2}$.

II. 0.1557 gram substance gave 0.1466 gram $\mathrm{H}_{2} \mathrm{O}$ and 0.4186 gram $\mathrm{CO}_{2}$.

III. 0.1731 gram substance gave $0 \cdot 1594$ gram $\mathrm{H}_{2} \mathrm{O}$ and $0 \cdot 4640$ gram $\mathrm{CO}_{2}$.

* This acid begins to give off carbonic anhydride at $128-130^{\circ}$, the decomposition bcing very rapid at $145-150^{\circ}$. 


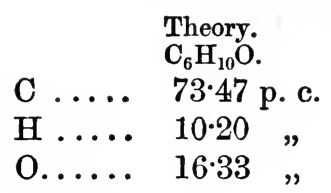

Theory.

C ..... $73 \cdot 47$ p. c.

O..... 16.33,
Found.

$\begin{array}{ccc}\text { I. } & \text { II. } & \text { III. } \\ 73 \cdot 04 & 73 \cdot 32 & 73 \cdot 10 \text { p. c. } \\ 10.34 & 10.46 & 10.23 \quad \text { " } \\ 16.62 & 16.22 & 16.67 \quad \text { ", }\end{array}$

Methyldehydrohexone is a colourless, mobile, ethereal-smelling liquid which boils constantly at $109-109.5^{\circ}$. In preparing it, it is very important to start from pure acid. If crude methyldehydrohexonecarboxylic acid is used, peculiar condensation-products of high boiling point are formed in large quantity; they have not been further examined. Their formation appears to bedue to the presence of a trace of sulphuric acid, which is difficult to completely wash out of the crude acid.

Methyldehydrohexone being the anhydride of acetobutyl alcohol, a careful study of its behaviour towards water seemed interesting. 5 c.c. of the pure substance when shaken with an equal volume of water did not appear to dissolve at all, but on standing for 24 hours the volume of the upper layer (that is of the methyldehydrohexone) had diminished to $3 \cdot 6$ c.c. and in another 24 hours to $2 \cdot 4$ c.c. After standing for about four days, the upper layer had entirely disappeared, a clear solution being formed. This on saturation with potassic carbonate deposited a quantity of a colourless oil, which on extracting with ether, drying over potassic carbonate, and fractioning, boiled almost constantly at $226-228^{\circ}$, and was evidently pure acetobutyl alcohol. The analysis gave the following results :-

0.1317 gram substance gave 0.1233 gram $\mathrm{H}_{2} \mathrm{O}$ and 0.3004 gram $\mathrm{CO}_{2}$.

\begin{tabular}{|c|c|c|}
\hline $\mathrm{CH}_{3} \cdot \mathrm{CO} \cdot \mathrm{C}$ & $\begin{array}{l}\text { Theory. } \\
\mathrm{I}_{2} \cdot \mathrm{CH}_{2} \cdot \mathrm{CH}_{2} \cdot \mathrm{CH}_{2} \cdot \mathrm{OH} \text {. }\end{array}$ & Fou \\
\hline C....... & 62.07 per cent. & $62 \cdot 20$ per cent. \\
\hline & $10 \cdot 34 \quad "$ & $10 \cdot 40$ \\
\hline $0 \ldots \ldots$ & $27 \cdot 59$ & $27 \cdot 40$ \\
\hline
\end{tabular}

The formation of acetobutyl alcohol from methyldehydrohexone by the action of water takes place according to the following equation:-

$$
\underset{\mathrm{CH}}{\|} \cdot \mathrm{CH}_{2} \cdot \mathrm{CH}_{2}^{\mathrm{CH}_{3}} \cdot \mathrm{C} \cdot \mathrm{O}-\mathrm{H}_{2} \mathrm{O}=\mathrm{CH}_{3} \cdot \mathrm{CO}
$$

This transformation takes place much more rapidly when methyldehydrohexone is warmed with water, and is then usually finished in a few hours. 
The magnetic rotation of methyldehydrohexone has been determined by my father with the following results :-

Specimen I, b. p. $109-109 \cdot 5^{\circ}$.

Sp. gr.

$$
\begin{aligned}
d \frac{4^{\circ}}{4^{\circ}} & =0.92272, \\
d \frac{15^{\circ}}{15^{\circ}} & =0.91278, \\
d \frac{25^{\circ}}{25^{\circ}} & =0.90502 .
\end{aligned}
$$

\begin{tabular}{c|c|c}
$t$. & Sp. rotation. & Mol. rotation. \\
\hline $17 \cdot 5$ & $1 \cdot 0123$ & $6 \cdot 051$ \\
$17 \cdot 5$ & $1 \cdot 0156$ & $6 \cdot 071$ \\
$18 \cdot 0$ & $1 \cdot 0126$ & $6 \cdot 056$ \\
$18 \cdot 0$ & $1 \cdot 0121$ & $6 \cdot 052$ \\
\hline $17 \cdot 7$ & $1 \cdot 0131$ & $6 \cdot 057$
\end{tabular}

This specimen was then carefully refractioned and found to boil at the same temperature. It was again remeasured with the following results :-

$$
d \frac{15^{\circ}}{15^{\circ}}=0.91225 \text {. }
$$

\begin{tabular}{c|c|c}
\hline$t$. & Sp. rotation. & Mol. rotation. \\
\hline $\begin{array}{c}22 \cdot 5 \\
22 \cdot 5\end{array}$ & $1 \cdot 0115$ & $\begin{array}{c}6 \cdot 072 \\
1 \cdot 0130\end{array}$ \\
\hline $22 \cdot 5$ & $1 \cdot 0122$ & $6 \cdot 074$ \\
\hline
\end{tabular}

Action of Hydrogen Bromide on Ethylic Methyldehydrohexonecarboxylate.

Formation of Acetobutyl Bromide, $\mathrm{CH}_{3} \cdot \mathrm{CO} \cdot \mathrm{CH}_{2} \cdot \mathrm{CH}_{2} \cdot \mathrm{CH}_{2} \cdot \mathrm{CH}_{2} \mathrm{Br}$.

Ethylic methyldehydrohexonecarboxylate dissolves readily in a strong solution of hydrogen bromide, producing a colourless liquid which, however, on standing soon turns brown, with evolution of carbonic anhydride. In studying the product of this reaction, the pure ethereal salt was mixed with two or three times its volume of hydrobromic acid (sp. gr. 1.83), and then allowed to stand until the evolution of carbonic anhydride had ceased, which was the case after 
about two days. On the addition of water, the dark, brownishcoloured product deposited a heavy oil which was taken up in ether and well washed first with water and then with a dilute solution of sodic carbonate. After drying over calcic chloride and distilling off the ether, the residue was purified by rapid distillation. In this way a colourless oil was obtained which boiled constantly at $214-215^{\circ}$, and gave the following numbers on analysis:-

I. 0.1453 gram substance gave 0.1509 gram AgBr.

II. $0 \cdot 2498$

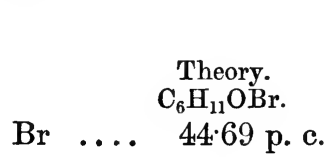

$0 \cdot 2538$

\section{Found.}

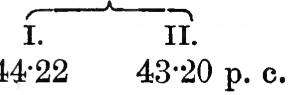

Acetobutyl bromide is a colourless oil having a penetrating and disagreeable camphor-like odour. On standing in the air it gives off a small quantity of hydrogen bromide and gradually becomes darkly coloured. When boiled with water or dilute hydrochloric acid it seems to be slowly converted into acetobutyl alcohol.

It is probably identical with the substance obtained by Lipp (Ber., 18, 3282) by distilling his acetobutyl alcohol with hydrogen bromide solution.

Action of Trimethylene Bromide on Ethylic Benzoylacetate.

$$
\text { Ethylic Phenyldehydrohexonecarboxylate, } \mathrm{COOC}_{2} \mathrm{H}_{5} \cdot \mathrm{C}_{6} \cdot \mathrm{CH}_{5} \cdot \mathrm{CH}_{\mathrm{CH}_{2}}^{-\mathrm{CH}_{2}} \cdot{ }^{\mathrm{CH}_{2}}
$$

This substance, a short description of which appeared some time ago (Ber., 16, 1790), is prepared in the following way:-A solution of 2.5 grams of sodium in 30 grams of absolute alcohol, after well cooling, is mixed with 20 grams of pure ethylic benzoylacetate and then heated to gentle boiling on a water-bath with 21 grams of trimethylene bromide. The reaction sets in almost immediately, and is usually finished in two or three hours, as is shown by the liquid becoming perfectly neutral to test-paper. At this stage of the decomposition a substance containing bromine, probably ethylic $\omega$-bromopropylbenzoylacetate,

$$
\mathrm{C}_{6} \mathrm{H}_{5} \cdot \mathrm{CO}
$$

$\mathrm{COOC}_{2} \mathrm{H}_{5} \cdot \mathrm{CH} \cdot \mathrm{CH}_{2} \cdot \mathrm{CH}_{2} \cdot \mathrm{CH}_{2} \mathrm{Br}$,

present, which by the further action of sodic ethylate is transformed into ethylic phenyldehydrohexonecarboxylate. To effect this transformation, the product is well cooled, mixed with a second quantity of sodic ethylate (containing, as before, 2.5 grams of sodium), and 
heated again on a water-bath until the product, on testing, shows neutral reaction. This is usually the case in about five or six hours. The brownish-coloured mass̀, after cooling, is filtered from the precipitated sodium bromide, the precipitate well washed with absolute alcohol, and the filtrate distilled on a water-bath until nearly all the alcohol has been removed. The residue is then mixed with water, and the oil which separates extracted two or three times with ether. The ethereal solution after washing, drying over calcic chloride, and evaporating, leaves a dark-coloured oil behind which, after standing for some days over sulphuric acid in a vacuum, deposits a quantity of long crystals. These are separated from the mother-liquor by filtering with the aid of a vacuum pump and washing with small quantities of ether. The resulting almost colourless mass is dissolved in as small a quantity of warm ether as possible, and the solution allowed to evaporate slowly at the ordinary temperature, when the new substance separates in long, colourless prisms which, after drying over sulphuric acid in a vacuum, gave the following numbers on analysis :-

I. $0 \cdot 1866$ gram substance gave 0.4929 gram $\mathrm{CO}_{2}$ and $0 \cdot 1197$ gram $\mathrm{H}_{2} \mathrm{O}$.

II. $0 \cdot 1973$ gram substance gave 0.5214 gram $\mathrm{CO}_{2}$ and $0 \cdot 1240$ gram $\mathrm{H}_{2} \mathrm{O}$.

\begin{tabular}{|c|c|c|c|}
\hline \multirow{2}{*}{\multicolumn{2}{|c|}{$\begin{array}{l}\text { Theory. } \\
\mathrm{C}_{14} \mathrm{H}_{16} \mathrm{O}_{3} \text {. }\end{array}$}} & \multicolumn{2}{|c|}{ Found. } \\
\hline & & I. & II. \\
\hline & $72 \cdot 41$ p. c. & $72 \cdot 04$ & 72.07 p. c. \\
\hline & 6.89 & $7 \cdot 13$ & 6.98 \\
\hline $0 \ldots \ldots$ & $20 \cdot 69$, & $20 \cdot 83$ & $20 \cdot 95$ \\
\hline
\end{tabular}

Ethylic phenyldehydrohexonecarboxylate melts at $59-60^{\circ}$ and distils at a high temperature almost without decomposition. It is readily soluble in most of the usual solvents, and can be obtained by the slow evaporation of its solutions in ether, bisulphide of carbon, and light petroleum in magnificent, colourless, prisms. Professor Haushofer was kind enough to measure some crystals which were grown in a saturated ethereal solution of the substance. He gave me the following description of the results of his experiments :- 
Ethylic Phenyldehydrohexonecarboxylate.

Crystalline system. Monoclinic.

$$
\begin{gathered}
a: b: c=1.0017: 1: 0.5914 . \\
\beta=68^{\circ} 34^{\prime} .
\end{gathered}
$$

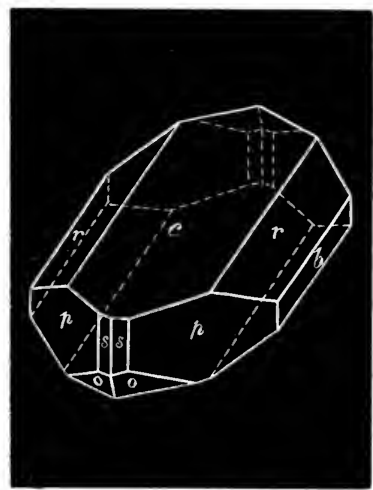

Moderately large bat incompletely developed crystals of the combination $0 \mathrm{P}(c), \infty \mathrm{P}(p), \mathbf{R} \infty(r), \cos \infty(b), \mathbb{P}_{\frac{1}{2}}(o), \infty \mathbb{R}_{\frac{1}{2}}(s)$. The faces $s$ and $o$ are generally very small; $c$ and $r$ generally run into one another, the angle between them becoming rounded.

$$
\begin{aligned}
& \text { Measured. } \\
& c: p=* 105^{\circ} 30^{\prime} \\
& p: p=* 94 \quad 0 \\
& c: r=* 151 \quad 10 \\
& r: r=12230 \\
& r: r=5746 \\
& s: s=1300 \\
& o: o=1321 \\
& r: p=12413
\end{aligned}
$$

\begin{tabular}{|c|c|}
\hline 一 & - \\
\hline - & - front \\
\hline - & - \\
\hline $122^{\circ}$ & $20^{\prime}$ top \\
\hline 57 & 40 side \\
\hline 130 & 0 \\
\hline 132 & 4 \\
\hline 124 & 16 \\
\hline
\end{tabular}

Phenyldehydiohexonecarboxylic

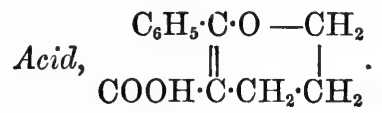

Calculated.

To obtain this acid, 5 grams of ethylic phenyldehydrohexonecarboxylate is boiled with a fairly concentrated solution of alcoholic potash (containing 2 grams of $\mathrm{KOH}$ ) for about four hours on a water-bath. After distilling off the alcohol, a little water is added, the almost colourless solution gently evaporated until the last trace of alcohol has been driven off, and then filtered. On acidifying the filtrate with dilute sulphuric acid, the new acid is precipitated as a white, crystal- 
line mass. This is collected, well washed with water, dried on a porous plate, and recrystallised from ether. It is thus obtained in beautiful, colourless prisms which, after drying over sulphuric acid in a vacuum, gave the following numbers :-

$0 \cdot 1914$ gram substance gave 0.4930 gram $\mathrm{CO}_{2}$ and $0 \cdot 1041$ gram $\mathrm{H}_{2} \mathrm{O}$.

\begin{tabular}{|c|c|c|}
\hline & $\begin{array}{l}\text { Theory. } \\
\mathrm{C}_{12} \mathrm{H}_{12} \mathrm{O}_{2} \text {. } \\
70.59 \text { per cent. }\end{array}$ & $\begin{array}{l}\text { Found. } \\
70.25 \text { per cent. }\end{array}$ \\
\hline & $5 \cdot 88$ & $6 \cdot 04$ \\
\hline & 23.53 & $23 \cdot 71$ \\
\hline
\end{tabular}

Phenyldehydrohexonecarboxylic acid melts at about $142-144^{\circ}$ with evolution of carbonic anhydride. It is easily soluble in alcohol, chloroform, benzene, carbon bisulphide, ether, and light petroleum, almost insoluble in cold water. When in a very finely-divided state it dissolves, however, to a considerable extent in boiling water, and crystallises out again on cooling in fine, colourless needles. The most beautiful crystals are obtained when a concentrated ethereal solution of the acid is allowed to evaporate at ordinary temperatures.

Professor Haushofer, who was kind enongh to examine some of these, gave the following description of them :-

Phenyldehydrohexonecarboxylic Acid.

Crystalline System. Monoclinic.

$$
\begin{gathered}
a: b: c=2 \cdot 6379: 1: 3 \cdot 3984 . \\
\beta=74^{\circ} 44^{\prime} .
\end{gathered}
$$

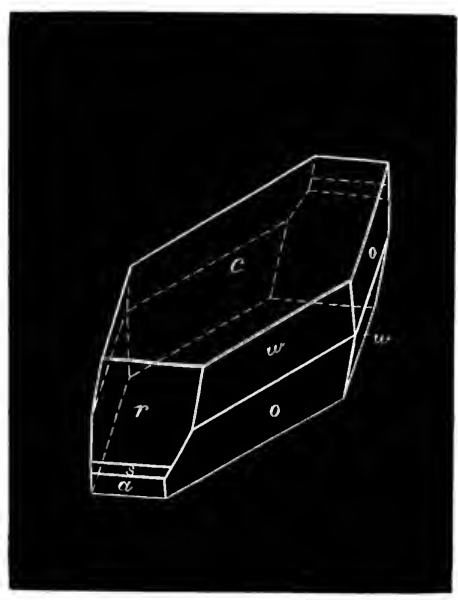


Beautifully lustrous, well-developed crystals of the combination $0 \mathrm{P}(c),-\mathrm{P}(w), \mathrm{P}(o),-\mathrm{P}_{\infty}(r)-2 \mathrm{P}_{\infty}(s) \cos (a)$. In many crystals the face $s$ is missing, whilst on others it completely disappears into $a$; neither of these faces admit of accurate measurement as they are, as a rule, flawed and rounded; $c$ also shows many flaws.

\begin{tabular}{|c|c|c|c|c|}
\hline \multicolumn{3}{|c|}{ Measured. } & \multicolumn{2}{|c|}{ Calculated. } \\
\hline$c: w=$ & * $110^{\circ}$ & $54^{\prime}$ & - & - \\
\hline$c: o=$ & $* 100$ & 40 & - & - \\
\hline$o: o=$ & $* 46$ & 28 & - & - \\
\hline$w: w=$ & 58 & 10 & $58^{\circ}$ & $16^{\prime}$ \\
\hline$c: r=$ & 137 & 1 & 137 & 0 \\
\hline$w: r=$ & 119 & 5 & 119 & $c^{2}$ \\
\hline$r: a=$ & 148 & 15 & 148 & $\varepsilon$ \\
\hline$c: a=$ & $105 \mathrm{a}$ & oprox. & 105 & 1 \\
\hline$o: w=$ & 138 & 40 & 138 & 50 \\
\hline$w: o=$ & 148 & 27 & 148 & 26 \\
\hline$o: a=$ & 106 & 50 & 106 & 4 \\
\hline$c: s=$ & $124 \mathrm{a}$ & pprox. & 124 & \\
\hline
\end{tabular}

The plane of the optical axis is the plane of symmetry, $\infty \mathrm{R} \infty$. The polarisation figure of one axis is visible on the face $c$ (in the obtuse angle $\beta$ ).

\section{Salts of Phenyldehydrohexonecarboxylic Acid.}

Phenyldehydrohexonecarboxylic acid dissolves easily in alkalis, alkaline carbonates, and ammonia. Its solution in a slight excess of ammonia was allowed to stand for some hours over sulphuric acid in a vacuum until quite neutral, and then the following salts prepared by precipitation with various reagents.

The silver salt is thrown down on the addition of nitrate of silver as a white, crystalline precipitate which, after collecting, well washing with water, and drying over sulphuric acid in a vacuum, was analysed with the following result:-

0.2735 gram substance gave 0.0952 gram silver.

$$
\begin{aligned}
& \text { Theory. } \\
& \mathrm{C}_{12} \mathrm{H}_{11} \mathrm{O}_{2} \mathrm{~A} \text {. Found. } \\
& \text { Ag..... } \quad 34 \cdot 73 \text { per cent. } \quad 34.81 \text { per cent. }
\end{aligned}
$$

This silver salt dissolves, though not easily, in boiling water, and separates out again on cooling in beautiful, colourless needles.

The copper salt is obtained as a light-green precipitate on adding a solution of cupric sulphate to the ammonium salt.

The lead salt is a white precipitate insoluble in water; calcic and 
baric chlorides give no precipitate when added to a solution of the ammonium salt.

$$
\text { Phenyldehydrohexone, } \quad \|_{\mathrm{CH} \cdot \mathrm{CH}_{2} \cdot \mathrm{CH}_{2}}^{\mathrm{C}_{6} \mathrm{H}_{5} \cdot \mathrm{C} \cdot \mathrm{O}-\mathrm{CH}_{2}}
$$

When phenyldehydrohexonecarboxylic acid is heated a few degrees above its melting point, rapid decomposition into carbonic anhydride and phenyldehydrohexone takes place thas:-

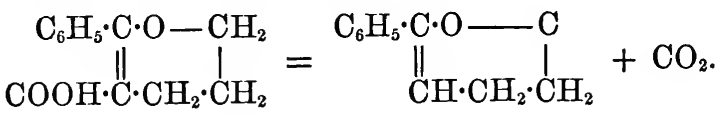

In order to examine the products of this reaction, the pure acid was heated in a small Wurtz flask in a metal bath at about $200^{\circ}$ until the evolution of carbonic anhydride had ceased, and the resulting oil fractioned. On the first distillation, the whole passed over between $235-255^{\circ}$, and on refractioning once or twice the pure substance was obtained as a colourless oil boiling constantly at $249-251^{\circ}(720 \mathrm{~mm}$.).

\section{Analyses.}

I. 0.1712 gram substance gave 0.1166 gram $\mathrm{H}_{2} \mathrm{O}$ and 0.5164 gram $\mathrm{CO}_{2}$.

II. $0 \cdot 1645$ gram substance gave $0 \cdot 1138$ gram $\mathrm{H}_{2} \mathrm{O}$ and 0.4952 gram

\begin{tabular}{|c|c|c|c|}
\hline \multirow{2}{*}{\multicolumn{2}{|c|}{$\begin{array}{l}\text { Theory. } \\
\mathrm{C}_{1} \mathrm{H}_{1} \mathrm{O} \text {. }\end{array}$}} & \multicolumn{2}{|c|}{ Found. } \\
\hline & & I. & II. \\
\hline C & 82.50 p. c. & $82 \cdot 27$ & $82 \cdot 10$ p. c. \\
\hline $\mathrm{H}$ & $7 \cdot 50$, & $7 \cdot 56$ & $7 \cdot 69$, \\
\hline $0 \ldots .$. & $10 \cdot 00$ & $10 \cdot 17$ & $10 \cdot 21$ \\
\hline
\end{tabular}
$\mathrm{CO}_{2}$.

Phenyldehydrohexone is a thick, colourless, aromatic-smelling oil, which boils almost without decomposition at $249-251^{\circ}$. After long standing it polymerises and becomes quite gelatinous. It does not appear to combine with phenylhydrazine. It is insoluble in cold water, but on long boiling a part seems to dissolve, probably being converted into benzoylbutyl alcohol. When dissolved in concentrated aqueous hydrogen bromide, phenyldehydrohexone is converted quantitatively into benzoylbutyl bromide, thus :-

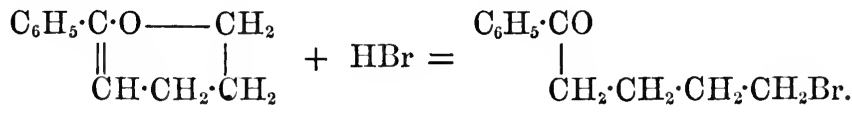


Action of Hydrogen Bromide on Phenyldehydrohexonecarboxylic Acid and Phenyldehydrohexone.

Formation of Benzoylbutyl Bromide, $\mathrm{C}_{6} \mathrm{H}_{5} \cdot \mathrm{CO} \cdot \mathrm{CH}_{2} \cdot \mathrm{CH}_{2} \cdot \mathrm{CH}_{2} \cdot \mathrm{CH}_{2} \mathrm{Br}$.

Phenyldehydrohexonecarboxylic acid dissolves easily in concentrated aqueous hydrogen bromide (sp. gr. 1.83) with evolution of heat. If the solution be allowed to stand, much carbonic anhydride is evolved, and in about one hour the whole solidifies to a mass of crystals of benzoylbutyl bromide. These are filtered off on a vacuumpump, washed with a small quantity of water, and dried on a porous tile over sulphnric acid in a vacuum. They are then further purified by recrystallisation from petroleum ether (b. p. $45-70^{\circ}$ ).

\section{Analyses.}

I. 0.1683 gram substance gave 0.0328 gram $\mathrm{H}_{2} \mathrm{O}$, and 0.3360 gram $\mathrm{CO}_{2}$.

II. 0·2928 gram substance gave 0.2288 gram $\mathrm{AgBr}$.

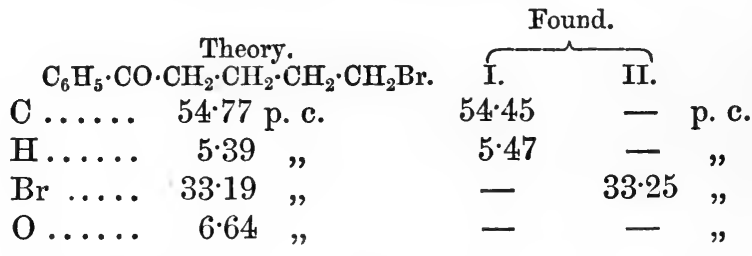

Benzoylbutyl bromide melts at $61^{\circ}$. It is easily soluble in alcohol, benzene, chloroform, carbon bisulphide, ether, and light petroleam, almost insoluble in water. It crystallises from dilute alcohol in glistening plates, which have very much the appearance of benzoic acid. From light petroleum it crystallises in six-sided plates, which have the form

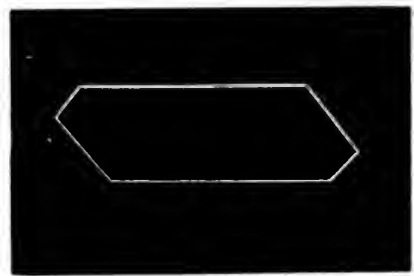

When warmed with alcoholic potash benzoylbutyl bromide is transformed into phenyldehydrohexone.

$$
\mathrm{C}_{6} \mathrm{H}_{5} \cdot \mathrm{CO} \cdot \mathrm{CH}_{2} \cdot \mathrm{CH}_{2} \cdot \mathrm{CH}_{2} \cdot \mathrm{CH}_{2} \mathrm{Br}=\mathrm{C}_{\mathrm{CH} \cdot \mathrm{CH}_{2} \cdot \mathrm{CH}_{2}}^{\mathrm{C}_{5} \cdot \mathrm{C} \cdot \mathrm{O}-\mathrm{CH}_{2}}+\mathrm{HBr} \text {. }
$$


Benzoylbutyl bromide is most easily prepared from phenyldehydrohexone. If this substance is dissolved in twice its volume of highly concentrated aqueous hydrogen bromide and then cooled, the whole solidifies to a mass of crystals of benzoylbutyl bromide, which when treated as described above are easily obtained pure.

\section{Action of Water on Phenyldehydrohexonecarboxylic Acid.}

When boiled with water for any length of time, phenyldehydrohexonecarboxylic acid is split up into benzoylbutyl alcohol and water, thus :-

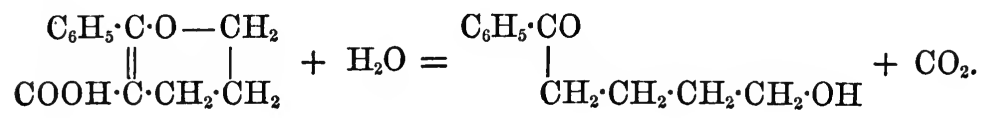

It is necessary to have the acid in as finely divided a state as possible, in order that it may dissolve in the water, and this is easily brought about by dissolving it in dilute ammonia and then carefully reprecipitating with dilute sulphuric acid. The fine powder thus obtained is collected, washed with water, and well agitated with boiling water until it completely dissolves. On boiling this solution in a reflux apparatus decomposition sets in, and carbonic anhydride is rapidly evolved, the reaction being generally finished in about six or eight hours. The resulting clear solution on the addition of anhydrous potassic carbonate, yields the benzoylbutyl alcohol in oily drops; it is extracted four or five times with ether, and dried over potassic carbonate. On evaporating this ethereal solution, a colourless oil is obtained which in a short time solidifies to a crystalline cake. This, after drying rapidly on a porous plate over sulphuric acid in a vacuum, was analysed with the following result:-

0.1134 gram substance gave 0.3010 gram $\mathrm{CO}_{2}$ and 0.0798 gram $\mathrm{H}_{2} \mathrm{O}$.

\begin{tabular}{|c|c|c|}
\hline $\begin{array}{l}\mathrm{C}_{6} \mathrm{H}_{5} \cdot \mathrm{CO} \cdot \mathrm{C} \\
\mathrm{C} \ldots \ldots\end{array}$ & $\begin{array}{l}\text { Theory. } \\
\mathrm{H}_{2} \cdot \mathrm{CH}_{2} \cdot \mathrm{CH}_{2} \cdot \mathrm{CH}_{2} \cdot \mathrm{OH} \text {. } \\
74 \cdot 27 \text { per cent. }\end{array}$ & $\begin{array}{l}\text { Found. } \\
74.51 \text { per cent. }\end{array}$ \\
\hline H..... & $7 \cdot 86$ & $7 \cdot 80$ \\
\hline $0 \ldots \ldots$ & $17 \cdot 97$ & $17 \cdot 69$ \\
\hline
\end{tabular}

If this alcohol is left for a few hours over sulphuric acid in a vacuum, the crystals gradually disappear and give place to a colourless oil. The small quantity of crystals at my disposal had thus in four days completely liquefied, and therefore I was not able to take a melting point or examine the properties. I hope, however, to be able to fill up these details at a later date. The change which takes place 
here is simply elimination of water and formation of the anhydride, phenyldehydrohexone, thas :-

$$
\mathrm{C}_{6} \mathrm{H}_{5} \cdot \mathrm{CO} \cdot \mathrm{CH}_{2} \cdot \mathrm{CH}_{2} \cdot \mathrm{CH}_{2} \cdot \mathrm{CH}_{2} \cdot \mathrm{OH}=\mathrm{C}_{6} \mathrm{H}_{5} \cdot \mathrm{C} \cdot \mathrm{O}-\mathrm{CH}_{2} \cdot \mathrm{CH}_{2}^{\mathrm{CH}_{2}}+\mathrm{H}_{2} \mathrm{O} .
$$

An analysis of the substance obtained after the benzoylbutyl alcohol had stood over sulphuric acid for seven days, gave the following numbers :-

0.1574 gram substance gave 0.1071 gram $\mathrm{H}_{2} \mathrm{O}$ and 0.4701 gram $\mathrm{CO}_{2}$.

\begin{tabular}{|c|c|c|}
\hline C ...... & $\begin{array}{l}\text { Theory. } \\
\mathrm{C}_{11} \mathrm{H}_{12} \mathrm{O} \text {. } \\
82.50 \text { per cent. }\end{array}$ & $\begin{array}{l}\text { Found. } \\
82.07 \text { per cent. }\end{array}$ \\
\hline H..... & $7 \cdot 50$ & $7 \cdot 56 \quad$ \\
\hline$\ldots$ & $10 \cdot 00$ & $10 \cdot 37$ \\
\hline
\end{tabular}

This substance is identical with the phenyldehydrohexone obtained by the dry distillation of phenjldehjdrohexonecarboxylic acid, as described previously (p. 731). When dissolved in concentrated aqueous hydrogen bromide it combines with it to form benzoylbutyl bromide, which from its melting point and other properties was found to be identical with that obtained previously (p. 732).

It is curious that acetobutyl alcohol and benzoylbutyl alcohol should differ so remarkably in stability, the former even on distillation being only to a very slight extent converted into its anhydride, whereas the latter suffers this change completely at the ordinary temperature.

On the Action of Trimethylene Bromide on the Sodium-derivative of Ethylic Paranitrobenzoylacetate.

If the sodium compound of ethylic paranitrobenzoylacetate is treated with trimethylene bromide, the paranitro-derivative of ethylic phenyldehydrohexonecarboxylate is formed, the reaction taking place in a way precisely similar to that which has already been described in the last section. The only difference being that ethylic paranitrobenzoylacetate is substituted for ethylic benzoylacetate.

As ethylic paranitrobenzoylacetate, and more especially its sodiumderivative (Trans., 1886, 440), are such beautifully crystalline substances, it seemed that in this case it would be more easy to follow the mechanism of the reaction with trimethylene bromide; this was found to be the case.

Before describing these experiments I should like to thank Dr. 
Gustar Bellenot for the valuable help he rendered me in carrying them out.

Ethylic Paranitrophenyldehydrohexonecarboxylate,

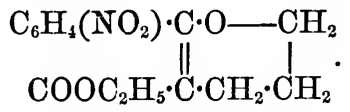

In order to obtain this compound 10 grams of the pure sodium compound of ethylic paranitrobenzoylacetate (ibid., 448), 8 grams of trimethylene bromide, and 30 grams of absolute alcohol were heated at $100^{\circ}$ in a sealed tube for three or four hours, after which time a quantity of sodium bromide had separated out and the mixture had become neutral. 0.9 gram of sodium dissolved in the least possible quantity of absolute alcohol was then added, the tube resealed, and once more heated at $100^{\circ}$ for four hours. The tube was then opened, and the alcohol distilled off. On adding water to the remainder, the product of the reaction separates as a thick, brownish oil, which was extracted several times with ether. After drying over calcic chloride and distiliing off the ether, a dark-brown liquid remains behind, the greater part of which solidifies on standing over sulphuric acid in a vacuum. The crystals were spread on a porous plate in order to free them roughly from mother-liquor, and afterwards dissolved in a little warm ether. An equal volume of light petroleum was then added and the mixture allowed to evaporate slowly at ordinary temperatures. Magnificent, lustrous, yellow crystals were thus obtained which on analysis gave the following results :-

I. 0.2664 gram substance gave 0.1300 gram $\mathrm{H}_{2} \mathrm{O}$ and 0.5889 gram $\mathrm{CO}_{2}$.

Ia. 0.1703 gram substance gave $8 \cdot 2$ c.c. N. $t=15^{\circ} ;$ bar. $=720 \mathrm{~mm}$.

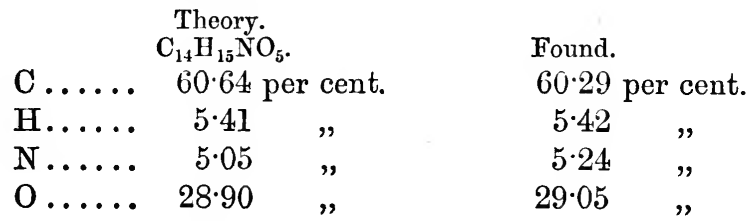

Ethylic paranitrophenyldehydrohexonecarboxylate melts at $62-63^{\circ}$. It is easily soluble in ether, benzene, and alcohol, sparingly so in light petroleum, insolable in water.

Professor Haushofer has kindly examined this beautiful substance crystallographically, and given me the following account of his experiments :- 
Ethylic Paranitrophenyldehydrohexonecarboxylate.

Crystalline system. Monoclinic.

$$
\begin{gathered}
a: b: c=2 \cdot 3530: 1: 1 \cdot 8530 . \\
\beta=80^{\circ} 42^{\prime} .
\end{gathered}
$$

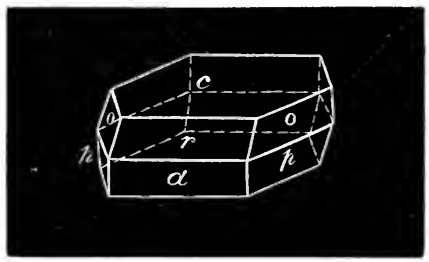

Small tabular crystals of the combination,

$$
\begin{aligned}
& 0 \mathrm{P}=(001)=c \\
& \infty \mathrm{P}=(110)=p \\
& \infty \mathrm{P}_{\infty}=(100)=a \\
& -\mathrm{P}_{\infty}=(101)=r \\
& -\mathrm{P}=(111)=o
\end{aligned}
$$

Generally the basal plane predominates, elongated in the direction of the axis $b$. The face $c$ is usually flawed or slightly curved. The faces $o$ and $p$ are incompletely developed. The plane of the optical axis is the plane of symmetry; one axis is approximately normal to the face $c$.

\begin{tabular}{lrllll} 
& \multicolumn{3}{c}{ Measured. } & \multicolumn{2}{c}{ Calculated. } \\
$p: c=(110)(001)$ & $* 93^{\circ}$ & $40^{\prime}$ & & - & - \\
$a: p=(100)(110)$ & $* 113$ & 18 & & - & - \\
$p: p=(110)(1 \overline{1} 0)$ & - & - & & $46^{\circ}$ & $36^{\prime}$ \\
$c: o=(001)(111)$ & $* 119$ & 34 & & - & - \\
$c: a=(001)(100)$ & 99 & 17 & 99 & 18 \\
$c: r=(001)(101)$ & 145 & 21 & 145 & 25 \\
$r: o=(101)(111)$ & 126 & 36 & 126 & 50
\end{tabular}

Paranitrophenyldehydrohexonecarboxylic Acid,

$\mathrm{C}_{6} \mathrm{H}_{4}\left(\mathrm{NO}_{2}\right) \cdot \mathrm{C} \cdot \mathrm{O}-\mathrm{CH}_{2}$ $\mathrm{COOH} \cdot \stackrel{\|}{\mathrm{C}} \cdot \mathrm{CH}_{2} \cdot \stackrel{\mathrm{CH}}{\mathrm{C}} \cdot$

To prepare this acid, the pure ethylic salt was mixed with a tolerably concentrated solution of alcoholic potash and allowed to stand for several hours, by which means it is easily and completely hydrolysed. Sufficient water to effect solution is added to this potassic salt, the whole, if necessary, filtered, and the filtrate acidified with dilute sulphuric acid, when the new acid is precipitated in the form of 
yellow flakes, which are best extracted with ether. After drying over calcic chloride and filtering, the ether is evaporated, the crude acid thus being left behind as a yellow crust, which after several times recrystallising from benzene is obtained in the form of thick, colourless needles. These on analysis gave numbers which agree well with the formula $\mathrm{C}_{12} \mathrm{H}_{11} \mathrm{NO}_{5}$.

0.2265 gram substance gave 0.0869 gram $\mathrm{H}_{2} \mathrm{O}$ and 0.4788 gram $\mathrm{CO}_{2}$.

0.2467 gram substance gave $13 \cdot 5$ c.c. $\mathrm{N} . \quad t=12^{\circ}$; bar. $=718 \mathrm{~mm}$.

\begin{tabular}{|c|c|c|c|c|}
\hline $\mathrm{C}$ & \multicolumn{2}{|c|}{ Theory. } & \multicolumn{2}{|l|}{ Found. } \\
\hline $\mathrm{H} \ldots \ldots$ & $4 \cdot 41$ & , & $4 \cdot 26$ & , \\
\hline$\ldots$ & $5 \cdot 62$ & " & $5 \cdot 61$ & " \\
\hline $0 \ldots \ldots$ & $32 \cdot 14$ & $"$ & $32 \cdot 49$ & " \\
\hline
\end{tabular}

Paranitrophenyldehydrohexonecarboxylic acid when crystallised from benzene melts at $172^{\circ}$. It is easily soluble in benzene, ether, alcohol, and light petroleum, but crystallises best from benzene. The crystalline acid is almost insoluble in water. If, however, it is carefully precipitated from its solution in dilute ammonia by the addition of dilute acids, a seemingly amorphous precipitate is obtained which dissolves to a considerable extent in boiling water, and crystallises on cooling in long, colourless, glistening needles, which fuse at $183^{\circ}$. It will be seen that the melting point given here is $10^{\circ}$ higher than that given for the same substance crystallised from benzene.

Phenyldehydrohexonecarboxylic acid has, as I have already shown, the same peculiarity, the thick prisms of this substance obtained from ether fusing at $142-144^{\circ}$, whereas the needles obtained from water melt at $149-150^{\circ}$.

Paranitrophenyldehydrohexonecarboxylic acid when heated above $200^{\circ}$ is split up into carbonic anhydride and a neutral substance, which, however, has not been further examined.

The salts of paranitrophenyldehydrohexonecarboxylic acid are very stable. A solution of the ammonium salt is easily obtained by dissolving the free acid in a slight excess of ammonia, and then allowing the liquid to stand over sulphuric acid in a vacuum until it reacts quite neutral.

If nitrate of silver be added to this solution, a slightly yellow, amorphous silver salt is thrown down. This after collecting, well washing with water, and drying first in a vacuum and then at $100^{\circ}$, gave the following analytical results :-

0.1990 gram substance gave 0.0601 gram Ag. 


$$
\begin{aligned}
& \text { Theory. } \\
& \mathrm{C}_{12} \mathrm{H}_{10} \mathrm{NO}_{5} \mathrm{Ag} \text {. Found. } \\
& \text { Ag...... 30.34 per cent. } 30.20 \text { per cent. }
\end{aligned}
$$

This silver salt dissolves in hot water, and crystallises out on cooling in light yellow needles.

On adding a solution of acetate of copper to the ammonium salt, the copper salt is precipitated as a light-green, flocculent precipitate.

The lead salt is a white, amorphous precipitate almost insoluble in water.

The main object in conducting these experiments with ethylic paranitrobenzoylacetate was to prove that when the sodium-derivatives of this and analogously constituted substances are treated with dibromides such as ethylene and trimethylene bromides, the reaction really takes place between two molecules of the sodium compound and one of the bromide. Thas in the case of trimethylene bromide the reaction is :

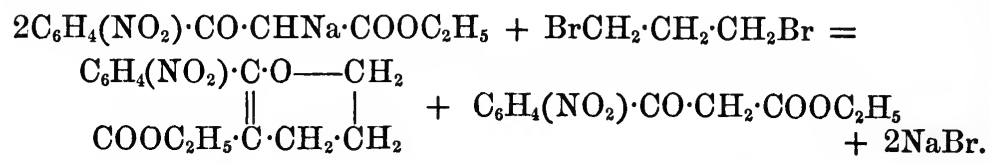

The experiment was carried out in the following way :-

An intimate mixture of 5 grams of the pure sodium-derivative of ethylic paranitrobenzoylacetate, 4 grams of trimethylene bromide, and about 10 grams of alcohol were heated in a sealed tube at $100^{\circ}$ until the reaction was complete (about six to eight hours). The product after gently warming on a water-bath to free it from alcohol, was treated with water and several times extracted with ether. The ethereal solution was carefully dried over calcic chloride and distilled, when a brownish-coloured, thick oil remained behind, a trace of which dissolved in alcohol and treated with ferric chloride gave a deep brownishred coloration, showing the presence of considerable quantities of ethylic paranitrobenzoylacetate. To the solution of this oil in pure alcohol, 0.5 gram of sodium dissolved in alcohol was added. In this way, a dark-brown solution was formed, from which, on the addition of an equal volume of pure ether, a yellowish-brown sodium compound was slowly precipitated. After standing for 24 hours this was collected, washed with a mixture of ether and alcohol, and analysed with the following result:-

$$
\begin{array}{cc}
\text { Theory. } & \\
\mathrm{C}_{6} \mathrm{H}_{4}\left(\mathrm{NO}_{2}\right) \cdot \mathrm{CO} \cdot \mathrm{CHNa} \cdot \mathrm{COOC}_{2} \mathrm{H}_{5} \text {. } & \text { Found. } \\
\mathrm{Na} \ldots . . & 9 \cdot 88 \text { per cent. }
\end{array}
$$

This substance was therefore the sodium-derivative of ethylic paranitrobenzoylacetate. The amount obtained in this way weighed 
upwards of 2 grams, whereas the yield calculated for the above equation should be 2.5 grams. The filtrate from this sodium compound was now mixed with more ether, the ethereal solution well washed with water, dried over calcic chloride, and distilled, when a brownish oil was obtained, which, after long standing over sulphuric acid in a vacuum, almost entirely solidified. The crystals, after spreading on a porous plate and recrystallising from ether and light petroleum, were easily purified, and then showed all the properties of ethylic paranitrophenyldehydrohexonecarboxylate. From this result it is seen that the formation of this substance takes place according to the equation given above.

\section{Action of Trimethylene Bromide on the Disodium-derivative of Ethylic Acetonedicarboxylate.}

Ethylic Methyldehydrohexonedicarboxylate,

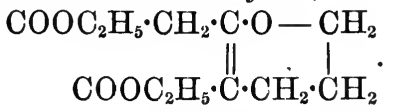

In studying this reaction, a mixture of 200 grams of ethylic acetonedicarboxylate and 205 grams of trimethylene bromide was slowly added to a cold solution of 46 grams of sodium dissolved in 500 grams of absolute alcohol, care being taken to cool the mass well during the mixing, as otherwise a violent reaction, difficult to control, is apt to set in. On warming the resulting clear solution on a water-bath, sodium bromide soon begins to separate out, and after boiling for 10 hours in a reflux apparatus, the reaction may be considered as being at an end. Water is now added, and the oily product of the reaction separated from the aqueous solution by extraction with ether. The ethereal solution is next evaporated, and the almost colourless oil thus obtained, submitted to distillation in a rapid current of steam until no more oil passes over with the water. In this way the product was easily separated into two nearly equal parts, each of which weighed about 90 grams. The ethylic methyldehydrohexonedicarboxylate which remains behind in the retort is extracted with ether, the ethereal solution washed with a little dilute sodic carbonate, and dried over calcic chloride. After distilling off the ether, an almost colourless oil is obtained, which is purified by fractioning under reduced pressure $(150 \mathrm{~mm}$.). At the first distillation nearly the whole passed over between $225-250^{\circ}$, leaving a small residue in the retort, and on repeatedly fractioning the distillate, an oil was at last obtained, which boiled constantly at $238-240^{\circ}$ (150 mm.). The analysis of this fraction gave the following results :- 
0.2172 gram substance gave 0.1497 gram $\mathrm{H}_{2} \mathrm{O}$ and 0.4730 gram $\mathrm{CO}_{2}$.

\begin{tabular}{|c|c|c|}
\hline & $\begin{array}{l}\text { Theory. } \\
\mathbf{C}_{12} \mathrm{H}_{18} \mathrm{O}_{5 .} \\
59.50 \text { per cent. }\end{array}$ & $\begin{array}{l}\text { Found. } \\
59 \cdot 40 \text { per cent. }\end{array}$ \\
\hline & $7 \cdot 44 \quad$ & $7 \cdot 65$ \\
\hline & 33.06 & $32 \cdot 95$ \\
\hline
\end{tabular}

Ethylic methyldehydrohexonedicarboxylate is a thick, colourless oil, having a disagreeable smell somewhat resembling that of ethylic methyldehydrohexonemonocarboxylate. This oil, when dissolved in alcohol gives, on the addition of a drop of ferric chloride, a beautiful violet coloration; this is, however, probably due to traces of ethylic acetonedicarboxylate, which appear always to be present in samples of ethylic methyldehydrohexonedicarboxylate prepared by the above reaction.

When mixed with alcoholic potash, a yellow solution is formed, and on heating hydrolysis rapidly sets in.

It now seemed interesting to examine the oil which distilled over with the steam during the purification of the crude product of the action of trimethylene bromide on ethylic acetonedicarboxylate, as described above. For this purpose, the aqueous distillate was extracted with ether, the ethereal solution dried over calcic chloride, filtered, the ether distilled off, and the resulting colourless oil fractioned. It was thus easily separated into two principal fractions, $170-200^{\circ}$ and $200-235^{\circ}$, of which the latter was the larger. This fraction, $200-235^{\circ}$, yielded by repeated fractioning a beautiful, colourless oil, which boiled constantly at $223-224^{\circ}(720 \mathrm{~mm}$.), and on analysis proved to be ethylic methyldehydrohexonecarboxylate.

0.2081 gram substance gave 0.1572 gram $\mathrm{H}_{2} \mathrm{O}$ and 0.4800 gram $\mathrm{CO}_{2}$.

\begin{tabular}{|c|c|c|}
\hline & $\begin{array}{l}\text { Theory. } \\
\mathrm{C}_{9} \mathrm{H}_{14} \mathrm{O}_{3} \text {. } \\
63 \cdot 53 \text { per cent. }\end{array}$ & $\begin{array}{l}\text { Found. } \\
62.91 \text { per cent. }\end{array}$ \\
\hline & $8 \cdot 23$ & $8 \cdot 39$ \\
\hline & $28 \cdot 23$ & $28 \cdot 70$ \\
\hline
\end{tabular}

In order to characterise this substance it was subjected to hydrolysis, and the free acid obtained recrystallised from benzene. It then melted at $119^{\circ}$, and showed all the properties of methyldehydrohexonecarboxylic acid. An analysis of the silver salt gave the following numbers :-

0.2805 gram substance gave 0.1209 gram of silver $=43 \cdot 10$ per cent.; theory $\mathrm{C}_{7} \mathrm{H}_{9} \mathrm{O}_{3} \mathrm{Ag}=43 \cdot 38$ per cent. 
Ethylic methyldehydrohexonecarboxylate is probably produced. from the dicarboxylate by partial hydrolysis and elimination of carbonic anhydride, as described below.

The fraction $170-200^{\circ}$ of the original oil, on repeated distillation, was found to boil for the most part between $175-185^{\circ}$, and to consist of nearly pure ethylic acetoacetate. It was not thought necessary to analyse this, its reactions being so well known that identification is a matter of ease. It is evidently formed from ethylic acetonedicarboxylate by partial hydrolysis and elimination of carbonic anhydride, thus:-

\section{$\mathrm{COOC}_{2} \mathrm{H}_{5} \cdot \mathrm{CH}_{2} \cdot \mathrm{CO} \cdot \mathrm{CH}_{2} \cdot \mathrm{COOC}_{2} \mathrm{H}_{5}=\mathrm{COOH} \cdot \mathrm{CH}_{2} \cdot \mathrm{CO} \cdot \mathrm{CH}_{2} \cdot \mathrm{COOC}_{2} \mathrm{H}_{5}$ $=\mathrm{CH}_{3} \cdot \mathrm{CO} \cdot \mathrm{CH}_{2} \cdot \mathrm{COOC}_{2} \mathrm{H}_{5}+\mathrm{CO}_{2}$.}

a reaction which always takes place when ethylic acetonedicarboxylate is treated with alkalis.

Hydrolysis of Ethylic Methyldehydrohexonedicarboxylate.

(1.) Hydric Ethylic Methyldehydrohexonedicarboxylate, $\mathrm{COOH} \cdot \mathrm{CH}_{2} \cdot \mathrm{C} \cdot \mathrm{O}-\mathrm{CH}_{2}$ $\mathrm{COOC}_{2} \mathrm{H}_{5} \cdot \stackrel{\|}{\mathrm{C}} \cdot \mathrm{CH}_{2} \cdot \stackrel{\mathrm{C} \mathrm{CH}_{2}}{ } \cdot$

The hydrolysis of ethylic methyldehydrohexonedicarboxylate takes place in two stages. In the first place an acid ethereal salt of the formula given above is formed, which then by the further action of alkalis is finally converted into the dicarboxylic acid :

(1.)

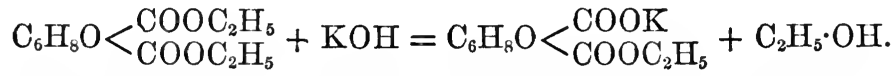

$$
\begin{aligned}
& \mathrm{C}_{6} \mathrm{H}_{8} \mathrm{O}<{ }_{\mathrm{COOC}_{2} \mathrm{H}_{5}}^{\mathrm{COOK}}+\mathrm{KOH}=\mathrm{C}_{6} \mathrm{H}_{8} \mathrm{O}<<_{\mathrm{COOK}}^{\mathrm{COOK}}+\mathrm{C}_{2} \mathrm{H}_{5} \cdot \mathrm{OH} \text {. }
\end{aligned}
$$

In preparing the hydric ethylic salt, pure ethylic methyldehydrohexonedicarboxylate is mixed with a large excess of a fairly concentrated solution of alcoholic potash, and allowed to stand at the ordinary temperature, until a sample taken out dissolves completely in $\mathrm{H}_{2} \mathrm{O}$ (10 to 14 hours). In order to isolate the new compound, dilute sulphuric acid ( 1 acid to $5 \mathrm{H}_{2} \mathrm{O}$ ) is added in excess, care being taken to keep the liquid cool during the operation. The product is then extracted five or six times with ether, the ethereal solution washed until free from alcohol, dried over calcic chloride, and the ether distilled off at as low a temperature as possible. A thick oil is thus obtained, which, after standing for some days over sulphuric acid in a vacuum, almost completely solidifies to a mass of crystals. These 
are roughly separated from oily impurities by spreading out on a porous plate, then washed with a little pure ether, and finally completely dissolved in this solvent. If this solution is allowed to evaporate slowly at ordinary temperatures, the acid ethylic salt separates out in magnificent, colourless, transparent crystals, which after drying over sulphuric acid in a vacuum, gave the following numbers on analysis :-

I. 0.2060 gram substance gave 0.1240 gram $\mathrm{H}_{2} \mathrm{O}$ and 0.4208 gram $\mathrm{CO}_{2}$.

II. $0 \cdot 1870$ gram substance gave $0 \cdot 1155$ gram $\mathrm{H}_{2} \mathrm{O}$ and 0.3805 gram $\mathrm{CO}_{2}$.

\begin{tabular}{|c|c|c|c|c|}
\hline \multirow{2}{*}{\multicolumn{3}{|c|}{$\begin{array}{c}\text { Theory. } \\
\mathrm{C}_{6} \mathrm{H}_{8} \mathrm{O}<<_{\mathrm{COOC}}^{\text {COOH }} \mathrm{H}_{5}\end{array}$}} & \multicolumn{2}{|c|}{ Found. } \\
\hline & & & I. & II. \\
\hline C ..... & 56.07 & cent. & $55 \cdot 71$ & $55 \cdot 49$ \\
\hline H ..... & $6 \cdot 55$ & , & $6 \cdot 69$ & $6 \cdot 86$ \\
\hline $0 \ldots \ldots$ & $37 \cdot 38$ & ", & $37 \cdot 60$ & $37 \cdot 65$ \\
\hline
\end{tabular}

It melts at $114^{\circ}$, is easily soluble in alcohol and ether, and sparingly so in hot water. If the hot aqueous solution be allowed to cool slowly the substance crystallises out in beantiful, colourless, four-sided needles. The alcoholic solution gives no coloration with ferric chloride, and it is for this reason that it appeared to me likely that the beautiful coloration produced in solutions of the diethylic salt by ferric chloride was caused by the presence of traces of ethylic acetonedicarboxylate. The best solvent to crystallise the acid ethylic salt from is pure ether. In this way most magnificent crystals can be obtained. Professor Haushofer was kind enough to measure these, and gave me the following account of his experiments.

Hydric Ethylic Methyldehydrohexonedicarboxylate.

Crystalline system. Triclinic.

$$
\begin{gathered}
a: b: c=0.7741: 1: 0.3371 . \\
\alpha=89^{\circ} \quad 40^{\prime} \\
\beta=98^{\circ} \quad 18^{\prime} \\
\gamma=89^{\circ} \quad 50^{\prime}
\end{gathered}
$$




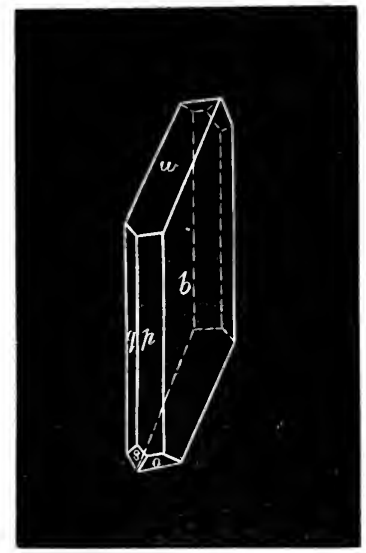

Colourless, lustrous crystals of the combination-

$$
\begin{aligned}
\infty \breve{\mathrm{P}} \infty & =(010)=b \\
\mathrm{P}^{\prime} & =(11 \overline{1})=w \\
\infty \mathrm{P}_{1}^{\prime} & =(110)=p \\
\mathrm{P}_{1} & =(1 \overline{1} \overline{1})=o \\
\infty_{1}^{\prime} & =(1 \overline{1} 0)=q \\
\breve{P}_{1} & =(3 \overline{1} \overline{1})=s
\end{aligned}
$$

Development tabular to the face $b$. The face $q$ was only observed on a single crystal, it was very small, but sharply developed. The faces $b$ are frequently rounded, and sometimes deviate some degrees from the parallel. Cleavage moderately distinct, parallel to $w$.

Measured.

$$
\begin{aligned}
& w: b=(111)(010)=* 105^{\circ} 27^{\prime} \\
& w: p=(111)(110)=* 123 \quad 19 \\
& p: o=(110)(11 \overline{1})=* 113 \quad 56 \\
& p: q=(110)(1 \overline{1} 0)=* 104 \quad 48 \\
& p: b=(110)(010)=* 127 \quad 19 \\
& o: b=(11 \overline{1})(010)=10833 \\
& q: o=(1 \overline{1} 0)(11 \overline{1})=90 \quad 20 \\
& q: w=(1 \overrightarrow{1} 0)(111)=102 \quad 26 \\
& s: p=(3 \overline{1} \overline{1})(110)=117 \quad 40 \\
& s: o=(3 \overline{1} \overline{1})(11 \overline{1})=134 \quad 42 \\
& : b=(3 \overline{1} \overline{1})(010)=77 \quad 58 \\
& s: q=(3 \overline{1} \overline{1})(1 \overline{1} 0)=135 \quad 49
\end{aligned}
$$

Calculated.

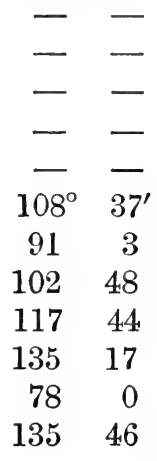

The results of the measurements of the angles do not absolutely exclude the possibility of the crystals belonging to the monoclinic system, whilst the smallness and brittleness of the crystals prevented 
the optical properties being sufficiently determined to decide the crystalline system with certainty; the direction of the only cleavage plane, however, leaves scarcely any doubt as to the crystals belonging to the triclinic system.

This acid ethylic sait dissolves easily in alkalis, and gives well characterised salts, of which the silver salt has been carefully prepared and examined. This salt is thrown down on adding nitrate of silver to a neutral solution of the ammonium salt as a white, curdy precipitate sparingly soluble in water. It was collected, well washed with water, and dried over sulphuric acid in a vacuum.

\section{Analysis.}

0.2330 gram substance gave 0.0870 gram $\mathrm{H}_{2} \mathrm{O}, 0.3168$ gram $\mathrm{CO}_{2}$, and 0.0786 gram Ag.

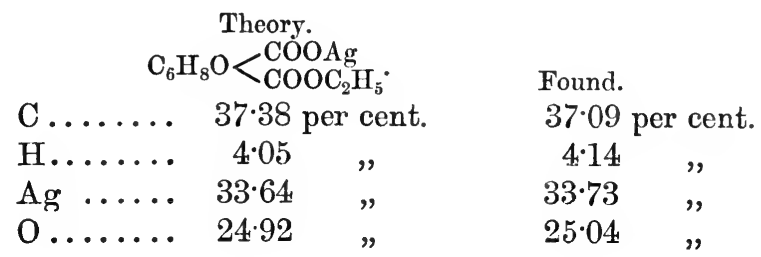

(2.) Methyldehydrohexonedicarboxylic Acid,

$\mathrm{COOH} \cdot \mathrm{CH}_{2} \cdot \mathrm{C} \cdot \mathrm{O}-\mathrm{CH}_{2}$ $\mathrm{COOH} \cdot \stackrel{\mathrm{C} \cdot \mathrm{CH}_{2} \cdot \mathrm{CH}_{2}}{\cdot}$

This acid may be obtained by the hydrolysis of the acid ethylic salt, but it was usually prepared directly from ethylic methyldehydrohexonedicarboxylate. The pure ethylic salt is mixed with an excess of alcoholic potash and heated to boiling for three or four hours on a water-bath. The slightly brownish-coloured solution is then diluted with twice its volume of water, and evaporated in an open dish on a water-bath until all the alcohol has been expelled. After filtering and acidifying with dilute sulphuric acid, the solution is allowed to stand 24 hours in a cool place, at the end of which time it is usually found that a crystalline crust, consisting of the nearly pure dicarboxylic acid, has separated on the bottom of the vessel containing the liquid. This is collected, washed with a little water, and dried on a porous plate (A). The mother-liquors are then extracted four or five times with ether, the ethereal solution washed, dried over calcic chloride, and the ether distilled off. A further quantity of impure dicarboxylic acid is thus obtained, usually as a thick oil, which, however, after standing for some days over sulphuric acid in a vacuum, deposits a quantity of crystals. These are roughly separated from impurities by spreading out on a porous plate, then 
washed with a little ether, and mixed with the crop (A) first obtained. The whole is now dissolved in a little hot water, and the solution filtered. On standing, the dicarboxylic acid soon begins to crystallise out in colourless plates, which after collecting, washing with water, and drying over sulphuric acid in a vacuum, gave the following numbers on analysis :-

I. 0.1489 gram substance gave 0.0756 gram $\mathrm{H}_{2} \mathrm{O}$ and 0.2819 gram $\mathrm{CO}_{2}$.

II. 0.1757 gram substance gave 0.0889 gram $\mathrm{H}_{2} \mathrm{O}$ and 0.3306 gram $\mathrm{CO}_{2}$.

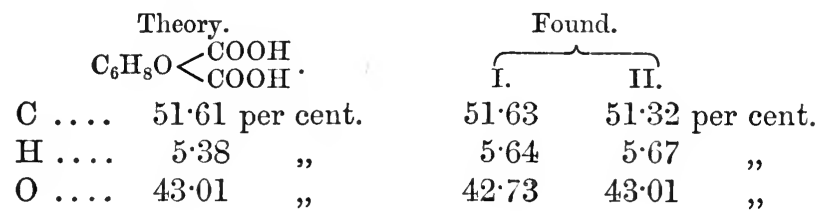

Methyldehydrohexonedicarboxylic acid melts at about $185-190^{\circ}$ with decomposition, evolution of carbonic anhydride, and formation of a curiously reddish-coloured liquid. This red coloration appears to be always produced when the pure acid is heated above its melting point, and is very characteristic. When heated in a test-tube the acid gives off carbonic anhydride, and an oil is formed which has in a marked degree the peculiar odour of methyldehydrohexone.

Methyldehydrohexonedicarboxylic acid is easily soluble in hot water and hot alcohol, but sparingly soluble in ethyl acetate, ether, and benzene. It crystallises from water in beautiful, colourless plates, which under the influence of polarised light show a fine play of colours. The forms of the crystals are generally the following :-

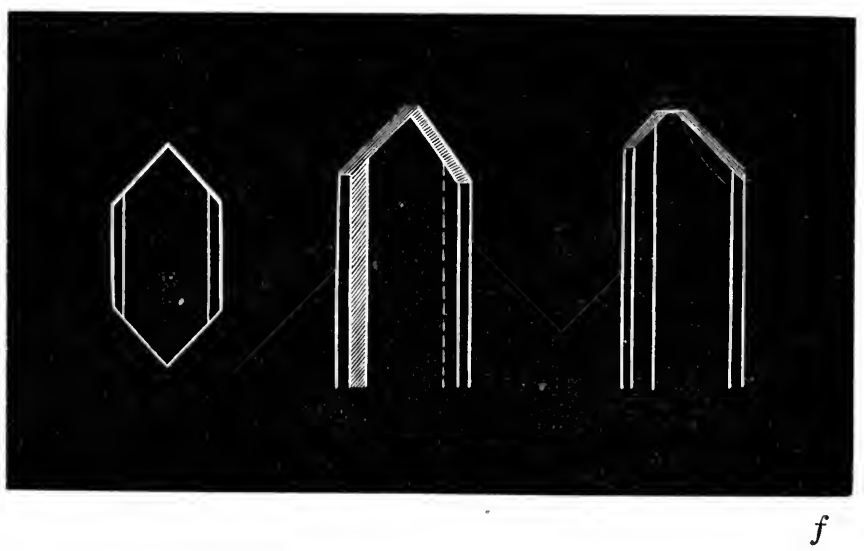


Formation of Ethylic Methyldehydrohexonecarboxylate from Hydric Ethylic Methyldehydrohexonedicarboxylate.

Hydric ethylic methyldehydrohexonedicarboxylate when heated is easily decomposed with evolution of carbonic anhydride and formation of ethylic methyldebydrohexonecarboxylate, thus :-

$$
\mathrm{C}_{6} \mathrm{H}_{8} \mathrm{O}<\underset{\mathrm{COOC}_{2} \mathrm{H}_{5}}{\mathrm{COOH}}=\mathrm{C}_{6} \mathrm{H}_{8} \mathrm{O}<\frac{\mathrm{COOC}}{\mathrm{CH}_{5}}+\mathrm{CO}_{2} \text {. }
$$

In carrying out this decomposition, the pure acid ethylic salt is transferred to a Wurtz flask and gradually heated in a metal-bath to $200^{\circ}$ until the evolution of carbonic anhydride ceases. The resulting oil when fractioned, distils constantly between $223^{\circ}$ and $225^{\circ}$, and is pure ethylic methyldehydrohexonecarboxylate.

\section{Analysis.}

0.1519 gram of substance gave 0.1135 gram $\mathrm{H}_{2} \mathrm{O}$ and 0.3526 gram $\mathrm{CO}_{2}$.

\begin{tabular}{|c|c|c|}
\hline & $\begin{array}{l}\text { Theory. } \\
\mathrm{C}_{9} \mathrm{H}_{14} \mathrm{O}_{3} \text {. } \\
63.53 \text { per cent. }\end{array}$ & $\begin{array}{l}\text { Found. } \\
63 \cdot 26 \text { per cent. }\end{array}$ \\
\hline$H$ & $8 \cdot 24$ & $8 \cdot 22$ \\
\hline & $28 \cdot 23$ & $28 \cdot 52$ \\
\hline
\end{tabular}

As it was important to be sure of the identity of this ethereal salt, it was converted into the corresponding acid by hydrolysis with alcoholic potash (as described under the heading "Methyldehydrohexonecarboxylic Acid," p. 715). The acid obtained after recrystallisation from benzene melted at $119-120^{\circ}$, and gave the following numbers on analysis:-

$0 \cdot 1764$ gram substance gave 0.1132 gram $\mathrm{H}_{2} \mathrm{O}$ and 0.3810 gram $\mathrm{CO}_{2}$.

\begin{tabular}{|c|c|c|}
\hline . & $\begin{array}{l}\text { Theory. } \\
\mathrm{C}_{7} \mathrm{H}_{10} \mathrm{O}_{3} \text {. } \\
59 \cdot 15 \text { per cent. }\end{array}$ & $\begin{array}{l}\text { Found. } \\
58: 90 \text { per cent. }\end{array}$ \\
\hline & $7 \cdot 04$ & $7 \cdot 12$ \\
\hline & $33 \cdot 80$ & $33 \cdot 98$ \\
\hline
\end{tabular}

The formation of ethylic methyldehydrohexonecarboxylate from hydric ethylic methyldehydrohexonedicarboxylate not only shows the close relation existing between these two substances, but also proves conclusively that the acid ethylic salt mentioned above must have the formula

$$
\mathrm{COOH} \cdot \mathrm{CH}_{2} \cdot \mathrm{C} \cdot \mathrm{O}-\mathrm{CHOC}_{2} \mathrm{H}_{5} \mathrm{C} \cdot \mathrm{CH}_{2} \cdot \mathrm{CH}_{2} \text {, and not }
$$

$\mathrm{COOC}_{2} \mathrm{H}_{5} \cdot \mathrm{CH}_{2} \cdot \mathrm{C} \cdot \mathrm{O}-\mathrm{CH}_{2}$ 
In the latter case on elimination of carbonic anhydride an ethereal salt of the formula $\mathrm{COOC}_{2} \mathrm{H}_{5} \cdot \mathrm{CH}_{2} \cdot \mathrm{C} \cdot \mathrm{O}-\mathrm{CH}_{2}$

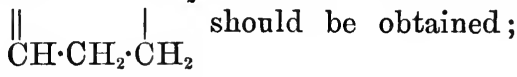
this would be isomeric, and not identical, with ethylic methyldehydrohexonecarboxylate.

\section{Action of Water on Methyldehydrohexonedicarboxylic Acid.}

In the introduction it was mentioned that methyldehydrohexonedi. carboxylic acid when boiled with water was decomposed into acetobutyl alcohol and two molecules of carbonic anhydride, thus :--

$$
\mathrm{C}_{6} \mathrm{H}_{8} \mathrm{O}<\mathrm{COOH}+\mathrm{H}_{2} \mathrm{O}=\mathrm{CH}_{3} \cdot \mathrm{CO} \cdot \mathrm{CH}_{2} \cdot \mathrm{CH}_{2} \cdot \mathrm{CH}_{2} \cdot \mathrm{CH}_{2} \cdot \mathrm{OH}+2 \mathrm{CO}_{2} \text {. }
$$

About 5 grams of the pure acid was dissolved in a small quantity of hot water and the solution then heated to boiling in a flask connected with a reversed condenser until the evolution of carbonic anhydride had ceased. The resulting liquid was saturated with anhydrous potassic carbonate, the oil thus precipitated extracted about ten times with pure ether, the ethereal solution dried over potassic carbonate, filtered, and the ether distilled off. In this way a colourless syrup was obtained, which, after standing for some time over sulphuric acid in a vacuum to free it from traces of ether, gave the following approximate numbers on analysis :-

0.1314 gram substance gave 0.1206 gram $\mathrm{H}_{2} \mathrm{O}$ and 0.3041 gram $\mathrm{CO}_{2}$.

\begin{tabular}{|c|c|c|c|c|}
\hline \multicolumn{3}{|c|}{$\begin{array}{l}\text { Theory. } \\
\mathrm{CH}_{3} \cdot \mathrm{CO} \cdot \mathrm{CH}_{2} \cdot \mathrm{CH}_{2} \cdot \mathrm{CH}_{2} \cdot \mathrm{CH}_{2} \cdot \mathrm{OH} \text {. }\end{array}$} & \multirow{2}{*}{\multicolumn{2}{|c|}{$\begin{array}{l}\text { Found. } \\
63 \cdot 11 \text { per cent. }\end{array}$}} \\
\hline C ..... & $62 \cdot 07$ & cent. & & \\
\hline & $10 \cdot 34$ & $"$ & $10 \cdot 19$ & $"$ \\
\hline O. & $27 \cdot 59$ & $"$ & $26 \cdot 70$ & 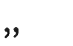 \\
\hline
\end{tabular}

Owing to the small amount of material at my disposal it, was not possible to purify this ketone alcohol by fractional distillation, but a qualitative comparison of the substance obtained as above with a specimen prepared from methyldehydrohexonemonocarboxylic acid (p. 715) showed conclusively that the two were identical. In order to be sure that the reaction really took place according to the equation just given, a determination was made of the amount of carbonic anhydride evolved when a definite amount of the acid was decomposed by boiling with water, with the following results :-

$2 \cdot 4811$ gram substance gave $1 \cdot 1432$ gram $\mathrm{CO}_{2}$. Theory for $2 \mathrm{CO}_{2}=$ 47.32 per cent. Found $46 \cdot 07$ per cent. 
The amount of acetobutyl alcohol formed was not determined.

The principal part of this research was carried out in Professor A. v. Baeyer's laboratory in Munich. Through the kindness of Professors Roscoe and Dixon, I was subsequently enabled to fill in some of the details at the Owens College, Manchester. 
THE SYNTHETICAL FORMATION OF CLOSED CARBONCHAINS. PART III. SOME DERIVATIVES OF PENTAMETHYLENE.

$$
\text { By W. H. Perkin, Jun., Ph.D. }
$$

IN Part II of this research (this vol., p. 1), I showed that when the sodium compound of ethylic malonate is treated with trimethylene bromide, ethylic tetramethylenedicarboxylate $(1,1)$ is formed thus :-

$2 \mathrm{CHNa}\left(\mathrm{COOC}_{2} \mathrm{H}_{5}\right)_{2}+\mathrm{BrCH}_{2} \cdot \mathrm{CH}_{2} \cdot \mathrm{CH}_{2} \mathrm{Br}=$

$$
\mathrm{CH}_{2}<\mathrm{CH}_{2} \mathrm{CH}_{2}>\mathrm{C}\left(\mathrm{COOC}_{2} \mathrm{H}_{5}\right)_{2}+\mathrm{CH}_{2}\left(\mathrm{COOC}_{2} \mathrm{H}_{5}\right)_{2}+2 \mathrm{NaBr} \text {. }
$$

I also mentioned that this substance was not the only product of the reaction, a high boiling oil being also formed which always remained behind in the retort on distilling off the ethylic tetramethylenedicarboxylate.

The further examination of this oil has shown that it is ethylic pentane- $\omega_{2} \omega_{2}$-tetracarboxylate, the product of the direct action of $\mathrm{l}$ mol. of trimethylene bromide on 2 mols. of the sodium compound of ethylic malonate; thus :-

$2 \mathrm{CHNa}\left(\mathrm{COOC}_{2} \mathrm{H}_{5}\right)_{2}+\mathrm{BrCH}_{2} \cdot \mathrm{CH}_{2} \cdot \mathrm{CH}_{2} \mathrm{Br}=$ $\left(\mathrm{COOC}_{2} \mathrm{H}_{5}\right)_{2} \mathrm{CH} \cdot\left(\mathrm{CH}_{2}\right)_{3} \cdot \mathrm{CH}\left(\mathrm{COOC}_{2} \mathrm{H}_{5}\right)_{2}+2 \mathrm{NaBr}$. Ethylic pentane- $\omega_{2} \omega_{2}$-tetracarboxylate.

This substance on hydrolysis is easily converted into the free pentanetetracarboxylic acid, and on heating the latter at $200^{\circ}$, it is resolved into normal pimelic acid and 2 mols. $\mathrm{CO}_{2}$; thus :-

$(\mathrm{COOH})_{2} \mathrm{CH} \cdot\left(\mathrm{CH}_{2}\right)_{3} \cdot \mathrm{CH}(\mathrm{COOH})_{2}=\mathrm{COOH} \cdot\left(\mathrm{CH}_{2}\right)_{5} \cdot \mathrm{COOH}+2 \mathrm{CO}_{2}$.

Pentane- $\omega_{2} \omega_{2}$-tetracarboxylic acid.

Pimelic acid.

The formation of normal pimelic acid by this simple decomposition shows at once that ethylic pentane-- $\omega_{2} \omega_{2}$-tetracarboxylate must have the constitution assigned to it above.

If this substance be now mixed with a solution of sodic ethylate in ether, a disodium compound is formed, which, when treated with bromine, is directly converted into ethylic pentamethylenetetracarboxylate $(1,1,2,2)$. This simple change takes place according to the following equation:-

$\left(\mathrm{COOC}_{2} \mathrm{H}_{5}\right)_{2} \mathrm{CNa} \cdot\left(\mathrm{CH}_{2}\right)_{3} \cdot \mathrm{CNa}\left(\mathrm{COOC}_{2} \mathrm{H}_{5}\right)_{2}+\mathrm{B}_{1_{2}}=$ Ethylic pentane- $\omega_{2} \omega_{2}$-tetracarboxylate.

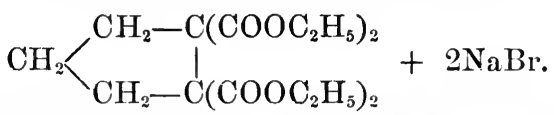

Ethylic pentamethylenetetracarbox̣late $(1,1,2,2)$. 
On hydrolysis, this substance yields the free pentamethylene tetracarboxylic acid $(1,1,2,2)$, which in its turn is easily converted (when heated at $200^{\circ}$ ) into pentamethylenedicarboxylic acid $(1,2)$ and 2 mols. $\mathrm{CO}_{2}$; thus :-

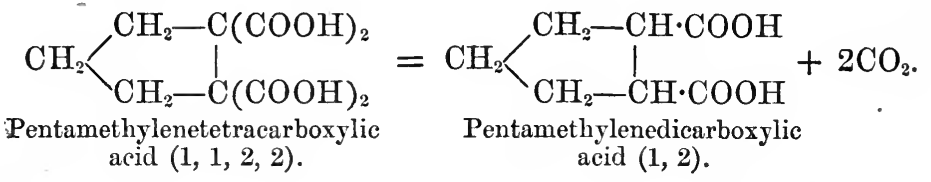

This acid is the orthodicarboxylic acid of pentamethylene, corresponding with phthalic acid of the benzene series, and is therefore easily split up on heating into its anhydride and water, thus :-

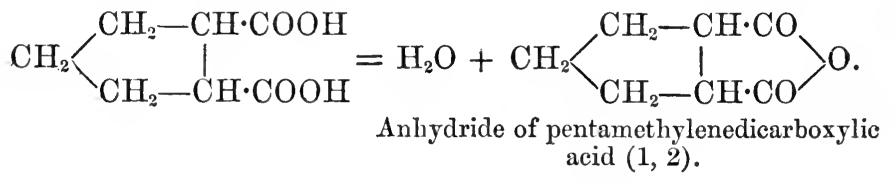

\section{Ethylic pentane- $\omega_{2} \omega_{2}$-tetracarboxylate, $\left(\mathrm{COOC}_{2} \mathrm{H}_{5}\right)_{2} \mathrm{CH} \cdot\left(\mathrm{CH}_{2}\right)_{3} \cdot \mathrm{CH}\left(\mathrm{COOC}_{2} \mathrm{H}\right)_{2}$.}

In order to prepare this substance, the following method has been found to give the best results :-

22 grams of sodium are dissolved in 250 grams of absolute alcohol, and when cool a mixture of 150 grams of ethylic malonate and 96 grams of trimethylene bromide are slowly added, care being taken that the temperature does not rise above $30^{\circ}$. On allowing this mixture to stand, a reaction soon sets in and is generally accompanied by evolution of heat sufficient to cause the alcohol to boil; as soon as this has subsided and the whole cooled down again, the decomposition is generally finished. On adding water and a few drops of dilute sulphuric acid, an oil is precipitated which is separated from the aqueous solution by extraction with ether. The ether is then distilled off and the residue subjected to a rapid distillation in a current of steam, as long as oily drops come over with the condensed water.

In this way, any unchanged trimethylene bromide and ethylic malonate, as well as the ethylic tetramethylenedicarboxylate formed in the reaction, are almost completely separated from the ethylic pentanetetracarboxylate, which at the end of the operation remains behind in the retort as a thick oily mass. This is next extracted with ether, the ethereal solution washed with a little dilute sodic carbonate, dried over calcic chloride, and the ether distilled off.

The residue, which is a slightly yellowish oil, usually weighing 
about 70 grams, is then further purified by fractioning under reduced pressure $(100 \mathrm{~mm}$.$) . By the first distillation nearly the whole distils$ over between $220^{\circ}$ and $300^{\circ}$, and after repeated fractioning an oil is obtained boiling constantly at $259-262^{\circ}(100 \mathrm{~mm}$.). The analysis gave the following results:-

I. 0.1706 gram substance gave 0.3529 gram $\mathrm{CO}_{2}$ and $0 \cdot 1200$ gram $\mathrm{H}_{2} \mathrm{O}$.

II. $0 \cdot 1525$ gram substance gave 0.3166 gram $\mathrm{CO}_{2}$ and $0 \cdot 1068$ gram $\mathrm{H}_{2} \mathrm{O}$.

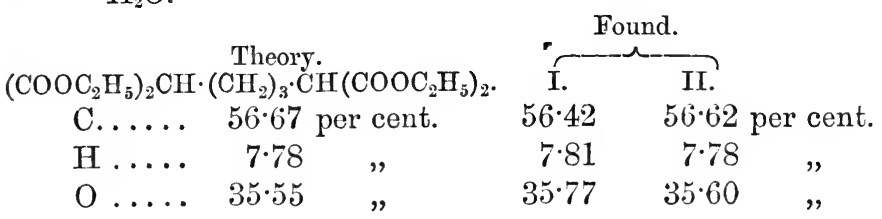

Ethylic pentane- $\omega_{2} \omega_{2}$-tetracarboxylate is a thick, colourless oil, which does not solidify at $0^{\circ}$. When quite pure, it can be distilled under ordinary pressure, with only very slight decomposition.

\section{Pentane- $\omega_{2} \omega_{2}$-tetracarboxylic Acid and Pimelic Acid, $\mathrm{COOH} \cdot\left(\mathrm{CH}_{2}\right)_{5} \cdot \mathrm{COOH}$.}

The next step was to endeavour to obtain from this ethereal salt the free pentanetetracarboxylic acid, and from this, pimelic acid by the splitting off of carbonic anbydride. For this purpose, 10 grams of the pure ethereal salt was mixed with an alcoholic solution of potash (containing 10 grams of $\mathrm{KOH}$ ), and after the violence of the reaction had subsided, the whole was heated with a reflux condenser for six hours to boiling, at the end of which time hydrolysis was complete. In order to isolate the product, water was now added and the clear solution evaporated almost to dryness on a water-bath. The residue was then mixed with an excess of dilute sulphuric acid, and extracted at least 20 times with pure ether.

This ethereal solution, after drying over calcic chloride and evaporating, deposited a slightly brownish-coloured oily acid, which is probably pentanetetracarboxylic acid, as it is easily decomposed on heating into 2 mols. $\mathrm{CO}_{2}$ and pimelic acid, as shown in the introduction. This change is best effected by heating the crude tetracarboxylic acid in an oil or metal bath at $200^{\circ}$ until the evolution of carbonic anhydriàe ceases, and then dissolving the residue in water. This solution is then filtered from a small quantity of insoluble resinous matter, and extracted five or six times with pure ether. The ethereal solntion after drying and evaporating, deposits the crude pimelic acid as a nearly colourless oil, which solidifies almost com- 
pletely on standing for some days over sulphuric acid in a vacuum. This crude substance was first roughly purified by spreading out on a porous plate and then recrystallised from water. The following results were obtained on analysis :-

0.1092 gram substance gave 0.2100 gram $\mathrm{CO}_{2}$ and 0.0730 gram $\mathrm{H}_{2} \mathrm{O}$.

\begin{tabular}{|c|c|c|c|c|}
\hline \multicolumn{3}{|c|}{$\begin{array}{l}\text { Theory. } \\
\mathrm{COOH} \cdot\left(\mathrm{CH}_{2}\right)_{5} \cdot \mathrm{COOH} \text {. }\end{array}$} & \multirow{2}{*}{\multicolumn{2}{|c|}{$\begin{array}{l}\text { Found. } \\
52 \cdot 44 \text { per cent. }\end{array}$}} \\
\hline C....... & 52.50 & cent. & & \\
\hline H....... & $7 \cdot 50$ & , & $7 \cdot 52$ & , \\
\hline $0 \ldots \ldots$ & $40 \cdot 00$ & , & $40 \cdot 04$ & " \\
\hline
\end{tabular}

This acid melts at $100-102^{\circ}$, and is undoubtedly identical with the pimelic acid obtained by Baeyer (Ber., 10, 1358) by the reduction of furonic acid with hydriodic acid and phosphorus. Schorlemmer and Dale (Annalen, 119,147) also prepared the same acid by the oxidation of suberone with concentrated nitric acid.

\section{Disodic Derivative of Ethylic Pentanetetracarboxylate,} $\left(\mathrm{COOC}_{2} \mathrm{H}_{5}\right)_{2} \mathrm{CNa} \cdot\left(\mathrm{CH}_{2}\right)_{3} \cdot \mathrm{CNa}\left(\mathrm{COOC}_{2} \mathrm{H}_{5}\right)_{2}$.

If ethylic pentanetetracarboxylate,

$$
\left(\mathrm{COOC}_{2} \mathrm{H}_{5}\right)_{2} \mathrm{CH} \cdot\left(\mathrm{CH}_{2}\right)_{3} \mathrm{CH}\left(\mathrm{COOC}_{2} \mathrm{H}_{5}\right)_{2} \text {, }
$$

is treated with excess of sodic ethylate, the two hydrogen-atoms of the $\mathrm{CH}$-groups are displaced by sodium, forming the disodium compound, $\left(\mathrm{COOC}_{2} \mathrm{H}_{5}\right) \mathrm{CNa} \cdot\left(\mathrm{CH}_{2}\right)_{3} \mathrm{CNa}\left(\mathrm{COOC}_{2} \mathrm{H}_{5}\right)_{2}$. In order to prepare this substance for analysis, a weighed quantity of ethylic pentanetetracarboxylate was dissolved in a little pure ether and mixed with an ethereal solution of sodic ethylate (2 mols.). The clear solution soon begins to deposit the disodic compound, and in a short time the whole becomes filled with a thick white precipitate. This is quickly collected, washed once or twice with a mixture of 1 pt. alcohol and 2 pts. ether and dried over sulpharic acid in a vacuum. Analysis :-

0.3754 gram substance gave $0 \cdot 1364$ gram $\mathrm{Na}_{2} \mathrm{SO}_{4}$.

$$
\begin{aligned}
& \text { Found. } \\
& \mathrm{Na} \text {.... 11.76 per cent. } \\
& \text { Theory. } \\
& \mathrm{C}_{1 ;} \mathrm{H}_{26} \mathrm{O}_{8} \mathrm{Na}_{2} \text {. } \\
& \text { 11.38 per cent. }
\end{aligned}
$$

This disodic compound is a white apparently amorphous powder, which is very easily soluble in water. On the addition of an acid, it is at once decomposed, an oil being precipitated which is probably regenerated ethylic pentanetetracarboxylate. 


\section{Pentamethylenedicarboxylic Acid $(1,2), \mathrm{CH}_{2}<\mathrm{CH}_{2}-\mathrm{CH} \cdot \mathrm{COOH}$.}

The disodium compound of ethylic pentane- $\omega_{2} \omega_{2}$-tetracarboxylate when treated with bromine is converted quantitatively into ethylic pentamethylenetetracarboxylate, as shown in the int:oduction. In carrying out this synthesis, the following method has been found to give the best results :-

18 grams of ethylic pentanetetracarboxylate are dissolved in about 50 grams of pure dry ether, and then an ethereal solution (this vol., p. 21) of sodic ethylate, containing $2 \cdot 3$ grams of sodium is slowly poured in, both ingredients being carefully cooled before mixing. After standing for about a minute to allow the sodium compound to separate out, 8 grams of dry bromine are added slowly through a dropping funnel, the mixture being well agitated and cooled during the operation. The colour of the bromine disappears instantly on coming in contact with the sodium compound, but the last drop should impart to the solution a pale yellow tint. As soon as all the bromine has been added, the product is allowed to stand for about an hour, then mixed with an equal volume of water, and the ethereal layer separated from the aqueous solution by means of a separating funnel.

After once washing with water and distilling off the cther, an almost colourless oil is obtained, which is crude ethylic pentamethylenetetracarboxylate. This was not analysed, but converted directly into the free acid by hydrolysis, and this then into the pentamethylenedicarboxylic acid $(1,2)$, which, as it is a beautifully crystalline substance, could easily be obtained pure for analysis. For this purpose, the crude ethylic pentamethylenetetracarboxylate was mixed with a solution of 15 grams of pure potash dissolved in a little alcohol, and heated for six hours to boiling on a water-bath. The mass was then dissolved in water, evaporated to expel the alcohol, the residue treated with an excess of dilute sulphuric acid, and extracted at least 20 times with pure ether.*

The ethereal solution is now very carefully dried over calcic chloride, filtered, and the ether distilled off, when the crude pentamethylenetetracarboxylic acid remains behind as a thick brownish syrup. To convert this into the dicarboxylic acid, it is now heated at $200-220^{\circ}$ until the evolution of carbonic anhydride ceases, which

* Or the acid may be isolated by means of the lead salt, as described under "Tetramethylenetetracarboxylic Acid" (this vol., p. 22). This method is, however, not to be recommended if large quantities are operated on, as the lcad salt is very difficult to filter and wash well. 
is usually the case in about half an hour. The crude residue can then be purified by dissolving it in water and decolorising with animal charcoal, as described in the Berichte $(18,3250)$; the following method, however, has since been found to give better results.

The impure substance is mixed with about three times its volume of absolute alcohol, thoroughly saturated with hydrogen chloride, and allowed to stand for three or four hours. Water is then added, and the whole extracted two or three times with ether. The ethereal solution is well washed with sodic carbonate solution, dried over carbonate of potash, and the ether distilled off. The residne is crude ethylic pentamethylenedicarboxylate. This is first roughly purified by distillation under reduced pressure (200 mm.), and then by fractionation under ordinary pressure.

After two fractionings, by far the greater part distils over between $245^{\circ}$ and $255^{\circ}$. A sample of ethylic pentamethylenedicarboxylate boiling at $24 y-252^{\circ}$ gave the following results on analysis :-

0.1370 gram substance gave 0.3090 gram $\mathrm{CO}_{2}$ and 0.1043 gram $\mathrm{H}_{2} \mathrm{O}$.

\begin{tabular}{|c|c|c|c|}
\hline \multicolumn{3}{|c|}{$\begin{array}{c}\text { Calculated for } \\
\mathrm{C}_{5} \mathrm{H}_{8}\left(\mathrm{COOC}_{2} \mathrm{H}_{5}\right)_{2} \text {. }\end{array}$} & $\begin{array}{l}\text { Found. } \\
61.51 \text { per cent. }\end{array}$ \\
\hline & $8 \cdot 41$ & , & $8 \cdot 46$ \\
\hline . & $29 \cdot 90$ & , & $30 \cdot 03$ \\
\hline
\end{tabular}

Ethylic pentamethylenedicarboxylate $(1,2)$ is a colourless oil of disagreeable odour.

In preparing free pentamethylenedicarboxylic acid it is not necessary to use the pure ethylic salt; the fraction boiling at $245-255^{\circ}$ was found to answer equally well. The hydrolysis was carried out as usual, by boiling the ethereal salt with a slight excess of alcoholic potash for three hours on a water-bath, and then evaporating to dryness. The residue was dissolved in a small quantity of water, acidified with dilute sulphuric acid, and allowed to stand in a cool place for 24 hours.

At the end of this time, the acid had almost entirely crystallised out, forming a hard cake at the bottom of the beaker; this was collected, washed with water, and recrystallised twice from water. It was thus obtained in curious nodular masses, which, after drying at $100^{\circ}$, gave the following numbers on analysis:-

I. $0 \cdot 1750$ gram substance gave $0 \cdot 1004$ gram $\mathrm{H}_{2} \mathrm{O}$ and $0 \cdot 3410$ gram $\mathrm{CO}_{2}$.

II. $0 \cdot 1508$ gram substance gave 0.0874 gram $\mathrm{H}_{2} \mathrm{O}$ and 0.2941 gram $\mathrm{CO}_{2}$.

III. 0.2318 gram substance gave 0.1330 gram $\mathrm{H}_{2} \mathrm{O}$ and 0.4484 gram $\mathrm{CO}_{2}$. 
Found.

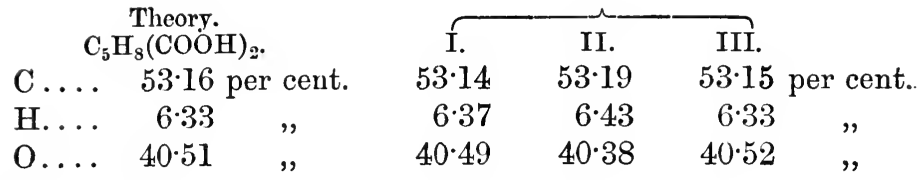

Pentamethylenedicarboxylic acid $(1,2)$ melts at $160^{\circ}$. It is easily soluble in hot water, alcohol, and ethyl acetate, sparingly in cold: water, ether, benzene, chloroform, and light petroleum.

The silver salt was prepared by precipitating the neatral solution of the ammonium salt with silver nitrate. It is a heavy, white, apparently amorphous precipitate, almost insoluble in water. For analysis, it was dried over sulphuric acid in a vacuum.

I. 0.2551 gram substance gave 0.0501 gram $\mathrm{H}_{2} \mathrm{O}, 0.2096$ gram: $\mathrm{CO}_{2}$, and $0 \cdot 1473$ gram $\mathrm{Ag}$.

II. 0·1812 gram substance gave 0.1050 gram Ag.

\begin{tabular}{|c|c|c|c|c|}
\hline \multirow{2}{*}{\multicolumn{3}{|c|}{$\begin{array}{c}\text { Theory. } \\
\left.\mathrm{C}_{5} \mathrm{H}_{3} \text { (COOAg }\right)_{2} \text {. }\end{array}$}} & \multicolumn{2}{|c|}{ Found. } \\
\hline & & & I. & II. \\
\hline$C \ldots \ldots$ & $22 \cdot 58$ & cent. & $22 \cdot 41$ & - \\
\hline$H \ldots \ldots$ & $2 \cdot 15$ & , & $2 \cdot 17$ & - \\
\hline $\mathrm{Ag} \ldots .$. & $58 \cdot 07$ & ", & $57 \cdot 74$ & $57 \cdot 94$ per cent. \\
\hline
\end{tabular}

On adding cupric sulphate to a neutral solution of the ammonium salt of pentamethylenedicarboxylic acid, the copper salt is precipitated as a beautiful green amorphous mass, which is sparingly soluble in water.

Plumbic acetate gives with a cold solution of the ammonium salt a white amorphous precipitate. If, however, the solutions are mixed boiling, the lead salt is precipitated as a white crystalline mass. This, on examining it with a microscope, is found to consist of transparent plates, grouped together like rosettes.

The most characteristic salt of pentamethylenedicarboxylic acid is the calcium salt. If calcic chloride solution is added to the cold solution of the ammonium salt, no precipitate is formed; on heating slowly to boiling, however, a colourless crystalline precipitate gradually separates out, which does not redissolve on cooling. This precipitate, when examined under the microscope, is found to consist of a mass of perfectly formed octahedrons, interspersed with examples of the combination $0 . \infty 00 s$. In these latter the surfaces $\infty 0 \infty s$ are very small, but very clearly defined, and easily seen when examined with a fairly high power. This precipitate is remarkable as. consisting only of single crystals, each of which is perfectly formed. 
Several experiments have been made with the object of obtaining a compound of the formula-<smiles>O=C(O)C(=O)C(=O)O</smiles>

by removing 4 hydrogen-atoms from pentamethylenedicarboxylic acid, but as yet without result. Oxidising agents split up this acid completely, oxalic acid being formed.

The action of bromine on pentamethylenedicarboxylic acid was therefore studied, as it was thought that in this way better results might be obtained, more especially as similar experiments to these have already been made by Hofmann, who thus succeeded in converting piperidine into pyridine. In the first experiment, I gram of pure pentamethylenedicarboxylic acid was heated in a sealed tube with 2 grams of bromine for two hours at $100^{\circ}$. On taking out the tube, no change was perceptible, and the same was the case after heating for two hours at $150^{\circ}$, but on heating at $180^{\circ}$ for four hours the acid was completely decomposed, abundance of hydrogen bromide being formed and a mass of charcoal being left behind in the tube. In experiment II, the same quantities were used, the only difference being that the acid was first dissolved in a small quantity of water. In this case, also no action took place until a comparatively high temperature had been reached, when total decomposition into hydrogen bromide and charcoal took place. It is hoped that by changing the conditions in the above experiment, better results may be obtained.

Anhydride of Pentamethylenedicarboxylic Acid, 1, 2,

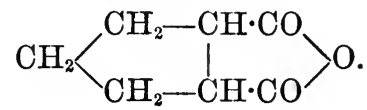

Pentamethylenedicarboxylic acid is an extremely stable compound which, if rapidly heated, can be distilled in small quantities almost without decomposition. If, however, it be kept at a temperature of $300^{\circ}$, it gradually loses water and is converted into its anhydride. In order to prepare this substance for analysis, 2 grams of the pure acid were heated in a very small retort at $300^{\circ}$ for half an hour, and the product then distilled as rapidly as possible over the naked flame. In this way an almost colourless oil condensed in the neck of the retort, which, on cooling, completely solidified to a hard crystalline mass. This was carefully ground in an agate mortar, washed with small quantities of ether, and dried first on a porous plate and then over sulphuric acid in a vacuum. The following numbers were obtained on analysis :- 
I. 0.1567 gram substance gave 0.0809 gram $\mathrm{H}_{2} \mathrm{O}$ and 0.3438 gram $\mathrm{CO}_{2}$.

II. $0 \cdot 1700$ gram substance gave 0.0871 gram $\mathrm{H}_{2} \mathrm{O}$ and 0.3716 gram $\mathrm{CO}_{2}$.

\section{Found.}

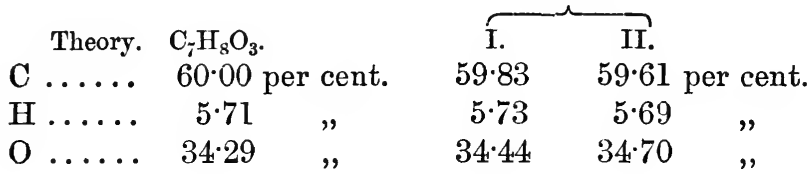

The anhydride of pentamethylenedicarboxylic acid thus prepared melts at $64-67^{\circ}$. It is fairly easily soluble in ether, benzene, chloroform, and alcohol, but only sparingly in light petroleum and bisulphide of carbon. It is almost insoluble in carbonate of soda solution at ordinary temperatures, but is more rapidly converted into the acid on heating.

If this anhydride is warmed with resorcinol and a few drops of sulphuric acid, and the melt treated with ammonia, a most beautifully fluorescing solution is produced.

Pentamethylenedicarboxylic acid and its anhydride form the third member in the series of orthodicarboxylic acids of closed carbonchains which have been obtained synthetically, thus :-

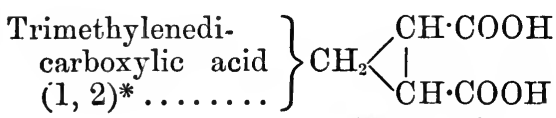

(M. p. $137^{\circ}$ ).

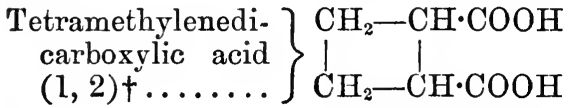

(M. p. $130^{\circ}$ ).

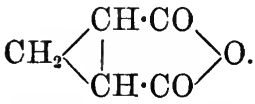

Anhyd. (m. p. $57^{\circ}$ ).

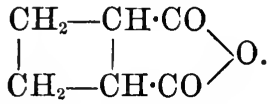

Anhyd. (m. p. 76-78 ).

Pentamethylenedicarboxylic acid $(1,2)$

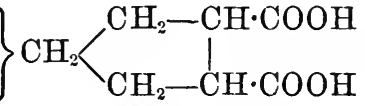

(M. p. $160^{\circ}$ ).

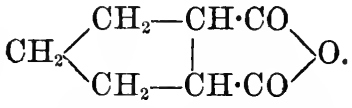

Anhyd. (m. p. 64-67 $)$.

* Conrad Guthzeit, Ber., 17, 1185.

+ This vol., pp. 22-25. 

Reprinted from the Journal of the Chemical Society, March, 1888. Vol. LIII.]

CONTRIBUTIONS FROM THE RESEARCH LABORATORY OF THE OWENS COLLEGE.

The Synthetical Formation of Closed Carbon-chains. Part III (continued). Some Derivatives of Pentamethylene.

By H. G. Colman, B.Sc., and W. H. Perkin, Junr., Ph.D.

Is a paper on Pentamethylene-derivatives communicated to the Society by one of us some time since, it was shown that a synthesis of a 5-carbon chain could be easily brought about by the following simple reactions:-If the sodium compound of ethylic malonate be 
treated with trimethylene bromide, ethylic tetramethylenedicarboxylate and ethylic pentane- $\omega_{2}-\omega_{2}$-tetracarboxylate are formed, the latter being produced according to the following reaction:-

$2 \mathrm{CHNa}\left(\mathrm{COOC}_{2} \mathrm{H}_{5}\right)_{2}+\mathrm{BrCH}_{2} \cdot \mathrm{CH}_{2} \cdot \mathrm{CH}_{2} \mathrm{Br}=$ $\left(\mathrm{COOC}_{2} \mathrm{H}_{5}\right)_{2} \mathrm{CH} \cdot\left[\mathrm{CH}_{2}\right]_{3} \cdot \mathrm{CH}\left(\mathrm{COOC}_{2} \mathrm{H}_{5}\right)_{2}+2 \mathrm{NaBr}$.

Ethylic pentane- $\omega_{2}-\omega_{2}$-tetracarboxylate.

If ethylic pentanetetracarboxylate is mixed with sodic ethylate, it yields a disodium-derivative, and this when treated with bromine or iodine yields ethylic pentamethylenetetracarboxylate, thas :-

$\left(\mathrm{COOC}_{2} \mathrm{H}_{5}\right)_{2} \mathrm{CNa} \cdot\left[\mathrm{CH}_{2}\right]_{3} \cdot \mathrm{CNa}\left(\mathrm{COOC}_{2} \mathrm{H}_{5}\right)_{2}+\mathrm{Br}_{2}=$<smiles>CCC[C](C(=O)OCC)C(=O)OCC</smiles>

Ethylic pentamethylenetetracarboxylate.

This method for the preparation of the 5-carbon chain differs widely from that employed in the case of the tetramethylene-derivatives (Trans., 51, 1), but it was resorted to because it was at the time found impossible to prepare a bromide of the formula $\mathrm{BrCH}_{2} \cdot \mathrm{CH}_{2} \cdot \mathrm{CH}_{2} \cdot \mathrm{CH}_{2} \mathrm{Br}$ which when treated with the sodium compound of ethylic malonate should have yielded pentamethylene-derivatives directly, thus :-

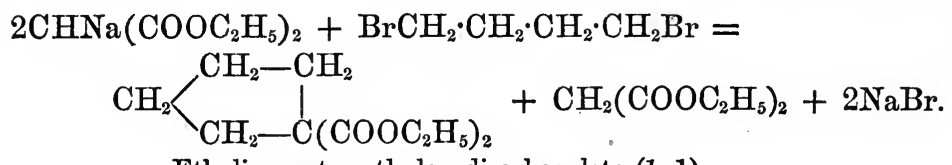

Ethylic pentamethylenedicarboxylate $(1,1)$.

This difficulty has, however, since to some extent been overcome.

In a paper brought before the Society last November (Trans., 51, 820), Freer and Perkin showed that acetyltrimethylenecarboxylic acid when boiled with water was split up into acetopropyl alcohol and carbonic anhydride, thus :-

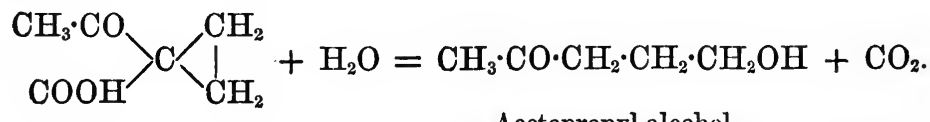

Acetopropyl alcohol.

This ketone alcohol when subjected to the action of sodium amalgam is easily converted into $\gamma$-pentylene glycol (loc. cit., p. 836) thus :-

$\mathrm{CH}_{3} \cdot \mathrm{CO} \cdot \mathrm{CH}_{2} \cdot \mathrm{CH}_{2} \cdot \mathrm{CH}_{2} \mathrm{OH}+\mathrm{H}_{2}=\mathrm{CH}_{3} \cdot \mathrm{CH}(\mathrm{OH}) \cdot \mathrm{CH}_{2} \cdot \mathrm{CH}_{2} \cdot \mathrm{CH}_{2} \mathrm{OH}$, $\gamma$-Pentylene glycol. 
and thus glycol, on heating with concentrated aqueous hydrogen bromide, yields methyltetramethylene dibromide according to the following equation :-

$\mathrm{CH}_{3} \cdot \mathrm{CH}(\mathrm{OH}) \cdot \mathrm{CH}_{2} \cdot \mathrm{CH}_{2} \cdot \mathrm{CH}_{2} \mathrm{OH}+2 \mathrm{HBr}=$

$\mathrm{CH}_{3} \cdot \mathrm{CHBr} \cdot \mathrm{CH}_{2} \cdot \mathrm{CH}_{2} \cdot \mathrm{CH}_{2} \mathrm{Br}+2 \mathrm{H}_{2} \mathrm{O}$.

Methyltetramethylene dibromide.

On treating this dibromide with the sodium compound of ethylic malonate, it was found that decomposition took place very easily, with formation of ethylic methylpentamethylenedicarboxylate $(2,1,1)$, thus :-

$2 \mathrm{CHNa}\left(\mathrm{COOC}_{2} \mathrm{H}_{5}\right)_{2}+\mathrm{CH}_{3} \cdot \mathrm{CHBr} \cdot \mathrm{CH}_{2} \cdot \mathrm{CH}_{2} \cdot \mathrm{CH}_{2} \mathrm{Br}=$<smiles>CCOC(=O)CCCC(=O)OCC</smiles>

Ethylic methylpentamethylenedicarboxylate $(2,1,1)$.

This ethereal salt, on hydrolysis, yields the free dicarboxylic acid which, when heated a few degrees above its melting point, is decomposed into carbonic anhydride and methylpentamethylenemonocarboxylic acid :-

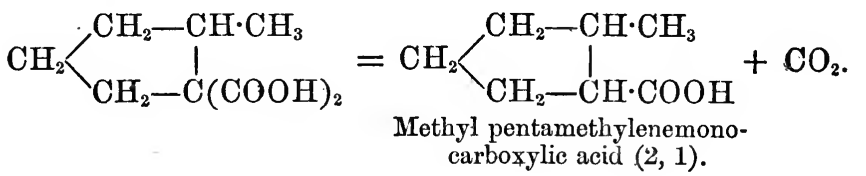

While carrying on these experiments, it was thought, that the study of the action of methyltetramethylene dibromide on the sodium compound of ethylic acetoacetate would be likely to give interesting results, and it is therefore included in this paper.

In papers on the action of trimethylene bromide on the sodium compounds of ethylic malonate and ethylic acetoacetate (Trans., 51, 1 and 702 ), it was shown that the two compounds, which are usually so analogous in their reactions, in this case gave rise to compounds entirely differently constituted. Thus when ethylic malonate is used, ethylic tetramethylenedicarboxylate, $\mathrm{CH}_{2}<\mathrm{CH}_{2}>\mathrm{C}\left(\mathrm{COOC}_{2} \mathrm{H}_{5}\right)_{2}$, is formed; whereas in the case of ethylic acetoacetate a curiuusly constituted compound-<smiles>CCOC(=O)C1CCC(C)=C1CC</smiles> 
(ethylic methyldehydrohexonecarboxylate) is produced. From these experiments it seemed probable that on treating ethylic acetoacetate with methyltetramethylene dibromide, a similarly constituted ethylic dimethylheptonecarboxylate would be formed, according to the equation-

$2 \mathrm{CH}_{3} \cdot \mathrm{CO} \cdot \mathrm{CHNa} \cdot \mathrm{COOC}_{2} \mathrm{H}_{5}+\mathrm{CH}_{3} \cdot \mathrm{CHBr} \cdot \mathrm{CH}_{2} \cdot \mathrm{CH}_{2} \cdot \mathrm{CH}_{2} \mathrm{Br}=$

$\mathrm{COOC}_{2} \mathrm{H}_{5} \cdot \mathrm{CH} \cdot \mathrm{CH}\left(\mathrm{CH}_{3}\right) \cdot \mathrm{CH}_{2} \cdot \mathrm{CH}_{3} \cdot \mathrm{CO} \cdot \mathrm{CH}_{2} \cdot \mathrm{COOC}_{2} \mathrm{H}_{5}+2 \mathrm{NaBr}$.

Ethylic dimethylheptonecarboxylate

$[2,4,3]$.

On studying this decomposition, howerer, it was soon found that this was not the case, the reaction with ethylic acetoacetate taking place in a way exactly similar to that described above with methyltetramethylene dibromide and ethylic malonate, ethylic acetylmethylpentamethylenecarboxylate being produced, thus:-

$2 \mathrm{CH}_{3} \cdot \mathrm{CO} \cdot \mathrm{CHNa} \cdot \mathrm{COOC}_{2} \mathrm{H}_{5}+\mathrm{CH}_{3} \cdot \mathrm{CHBr} \cdot \mathrm{CH}_{2} \cdot \mathrm{CH}_{2} \cdot \mathrm{CH}_{2} \mathrm{Br}=$

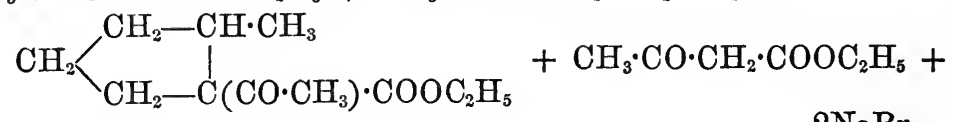

Ethylic acetylmethylpentamethylene-

$2 \mathrm{NaBr}$. carboxylate $[1,2,1]$.

That this is really the constitution of this substance is easily proved from the study of the products obtained from it by hydrolysis with alcoholic potash. When boiled with a strong solution of alcoholic potash for some hours, this ethereal salt is completely split up with formation of methylpentamethylene methyl ketone, methylpentamethylenecarboxylic acid, and other products.

This hydrolysis takes place-as in all cases when substituted ethylic acetoacetates are treated with alcoholic potash-in two distinct phases:-

I.

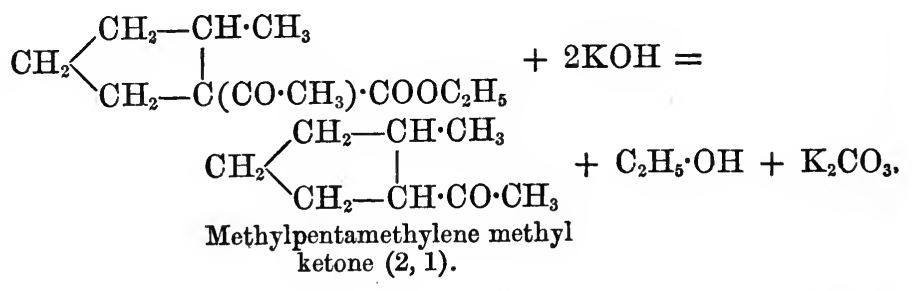




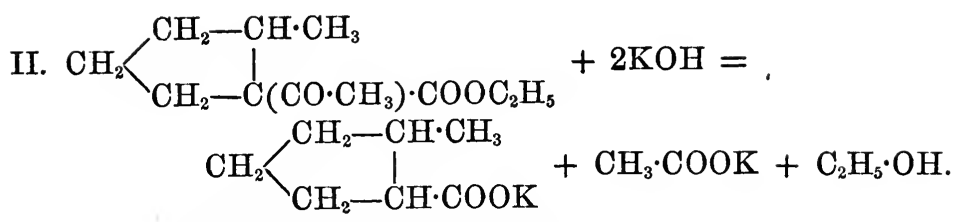

\section{Potassic methylpentamethylene- monocarboxylate $(2,1)$.}

The methylpentamethylenemonocarboxylic acid obtained thus was found to be identical with that prepared by the decomposition of methylpentamethylenedicarboxylic acid. Had the reaction between ethylic acetoacetate and methyltetramethylene dibromide taken place as indicated above, with formation of ethylic dimethylheptonecarboxylate, this ethereal salt on hydrolysis should have simply yielded the free acid, without any decomposition-products. (See Methyldehydrohexoncarboxylic acid, Trans., 51, 715.)

\section{Constitution of Acetopropyl Alcohol.}

In their description of acetopropyl alcohol (Trans., 51, 830), Freer and Perkin show that from a study of the method of preparation as well as from the numbers obtained as the magnetic rotation of this compound, that it evidently has the constitution-

\section{$\mathrm{CH}_{3} \cdot \mathrm{CO} \cdot \mathrm{CH}_{2} \cdot \mathrm{CH}_{2} \cdot \mathrm{CH}_{2} \mathrm{OH}$.}

Before starting these experiments, however, with methyltetramethylene dibromide, it was thought that it would be more satisfactory to obtain some further proof of the constitution of this important substance, as of course otherwise no definite conclusions could be formed as to the nature of the rings synthesised in the subsequent reactions. Fortunately this can easily be done in the following way :-If acetopropyl alcohol has really the constitution given above, it must evidently, on oxidation, yield levulinic acid, thus :-

$\mathrm{CH}_{3} \cdot \mathrm{CO} \cdot \mathrm{CH}_{2} \cdot \mathrm{CH}_{2} \cdot \mathrm{CH}_{2} \mathrm{OH}+\mathrm{O}_{2}=\mathrm{CH}_{3} \cdot \mathrm{CO} \cdot \mathrm{CH}_{2} \cdot \mathrm{CH}_{2} \cdot \mathrm{COOH}+\mathrm{H}_{2} \mathrm{O}$.

On making the experiment, this was found to be the case.

In carrying out this oxidation, 30 grams of acetopropyl alcohol were dissolved in 100 grams of water, and then the calculated quantity of chromic acid and sufficient dilute sulphuric acid very slowly added, the mixture being cooled with ice during the operation. The reaction was very violent, the chromic acid on coming into contact with the acetopropyl alcohol being instantly reduced.

When the oxidation was finished, the product, which smelt strongly of acetic acid, was extracted at least 10 times with pure ether, the ethereal solution dried over calcic chloride, the ether distilled off, and 
the residual oil fractioned under reduced pressure $(150 \mathrm{~mm}$.). At first a considerable quantity of acetic acid distilled over (produced by the further oxidation of levalinic acid), then the thermometer rose rapidly to $180^{\circ}$, between which temperature and $215^{\circ}$ nearly the whole passed over as a thick, slightly yellowish oil. This on refractioning boiled for the most part between $197^{\circ}$ and $203^{\circ}$ (150 mm.), and gave the following numbers on analysis, which agree with those required for levulinic acid :-

0.2501 gram substance gave 0.1552 gram $\mathrm{H}_{2} \mathrm{O}$ and 0.4790 gram $\mathrm{CO}_{2}$

\begin{tabular}{|c|c|c|c|c|}
\hline \multicolumn{3}{|c|}{$\begin{array}{l}\text { Theory. } \\
\mathrm{CH}_{3} \cdot \mathrm{CO} \cdot \mathrm{CH}_{2} \cdot \mathrm{CH}_{2} \cdot \mathrm{COOH} \text {. }\end{array}$} & \multirow{2}{*}{\multicolumn{2}{|c|}{$\begin{array}{l}\text { Found. } \\
52 \cdot 25 \text { per cent }\end{array}$}} \\
\hline$\ldots \ldots$ & $51 \cdot 72$ & & & \\
\hline . & $6: 29$ & $"$ & $6 \cdot 89$ & " \\
\hline & $41 \cdot 38$ & ", & $40 \cdot 86$ & \\
\hline
\end{tabular}

In order to be certain of the constitntion of this acid, the silver salt was prepared and analysed.

For this purpose, the fraction $197-203^{\circ}$ was dissolved in a little water, carefully neutralised with ammonia, and excess of nitrate of silver added. The white amorphous silver salt thus precipitated was collected, washed, and dissolved in a little hot water. This solution on cooling deposited the silver salt as a colourless mass, which consisted of small colourless nodules. Unfortunately, the characteristic crystals of this salt could not be obtained, owing to the smallness of the quantity at our disposal.

An analysis which was made with a portion dried over sulphuric acid in a vacuum left no doubt, however, as to the constitution of the salt.

0.2274 gram substance gave 0.2247 gram $\mathrm{CO}_{2}, 0.0711$ gram $\mathrm{H}_{2} \mathrm{O}$, and $0 \cdot 1106$ gram $\mathrm{Ag}$.

\begin{tabular}{|c|c|c|c|c|}
\hline $\mathrm{CH}_{3} \cdot \mathrm{CC}$ & $\begin{array}{l}\text { Theory } \\
\mathrm{CH}_{2} \cdot \mathrm{CH}\end{array}$ & DOAg. & \multirow{2}{*}{\multicolumn{2}{|c|}{$\begin{array}{l}\text { Found. } \\
26.94 \text { per cent. }\end{array}$}} \\
\hline C ........ & $26 \cdot 90$ & cent. & & \\
\hline $\mathrm{H}$ & $3 \cdot 14$ & $"$ & $3 \cdot 47$ & , \\
\hline Ag .. & $48 \cdot 43$ & ", & $48 \cdot 63$ & $"$ \\
\hline
\end{tabular}

Acetopropyl alcohol, prepared by boiling acetyltrimethylenecarboxylic acid with water, has therefore the constitution-

$\mathrm{CH}_{3} \cdot \mathrm{CO} \cdot \mathrm{CH}_{2} \cdot \mathrm{CH}_{2} \cdot \mathrm{CH}_{2} \mathrm{OH}$.

Methyltetramethylene Dibromide, $\mathrm{CH}_{3} \cdot \mathrm{CHBr} \cdot \mathrm{CH}_{2} \cdot \mathrm{CH}_{2} \cdot \mathrm{CH}_{2} \mathrm{Br}$.

This dibromide is best prepared in the following way:- 50 grams of acetyltrimethylenecarboxylic acid (prepared-according to the 
method given by Freer and Perkin, Trans., 51, 829) are mixed with about 150 c.c. of water, and boiled for 14 to 20 hours in a flask connected with a reflux condenser. In doing this, it has been found convenient to connect the end of the condenser with a glass tube, dipping into a cylinder 200-300 mm. long filled with mercury, as by this means not only the temperature of the boiling water is raised and the decomposition thus hastened, but also by observing the rapidity of the evolution of the carbonic anhydride it is easy to see how the reaction is progressing and when it is finished. The solution of acetopropyl alcohol thus obtained is poured out into a that porcelain dish and mixed with about 800 grams of 3 per cent. sodium amalgam. The amalgam must be added very slowly, and the reduction should take from two to three days.

In isolating the product of this reaction, some difficulty was experienced, as $\gamma$-pentylene glycol is so readily soluble in water, that even after extracting its solution 20 to 30 times with ether only about half of the amount present is obtained. In order to avoid this loss, the following method was devised :-

The solution containing the glycol and the caustic soda formed during the reduction is separated from the mercury by means of a separating funnel, thoroughly saturated with carbonic anhydride, mixed with about 10 times its volume of absolute alcohol (or purified methylated spirit), and allowed to stand in a cool place for about 24 hours. By this means, nearly all the carbonate of soda is precipitated on the bottom and sides of the vessel containing the solution as a hard crust, whilst the whole of the $\gamma$-pentylene glycol remains in the supernatant liquid; this is poured off and filtered. In order to avoid loss, the cake of carbonate of soda is broken up, well washed on a vacuum pump with absolute alcohol, and the combined alcoholic solutions very carefully fractioned with a long colonna. This precaution is very necessary, as $\gamma$-pentylene glycol is very easily carried over with the vapours of alcohol or water, and serious loss occurs if the alcohol is distilled off in the ordinary way.

The distillation is carried on until the thermometer stands at $95^{\circ}$, the residue then consisting of an aqueous solution of $\%$-pentylene glycol, in which a small quantity of carbonate of soda is dissolved. From this solution methyltetramethylene dibromide is prepared, by connecting the flask containing it with a reflux condenser, and then saturating with hydrogen bromide, the stream of gas being led in rapidly enough to raise the temperature of the liquid to the boiling point. The flask is then placed in a paraffin bath, vigorously boiled for about half an hour, a stream of hydrogen bromide being passed through all the time. When the product is cool, it is mixed with a little water and extracted four or five times with ether. The ethereal 
solution is washed with water, dried over calcic chloride, and the ether distilled off, when the crude methyltetramethylene dibromide remains behind as a dark-coloured heary liquid. On fractioning under reduced pressure $(150 \mathrm{~mm}$.), nearly the whole of this crude product passes over between $143^{\circ}$ and $150^{\circ}$, and on repeatedly fractioning a beautifully colourless liquid is obtained boiling constantly at 145$147^{\circ}$ (150 mm.).

A little hydrogen bromide is always given off during distillation, and to this the slight deficiency in bromine found on analysis is due.

Analysis.

0.2010 gram substance gave 0.3245 gram $\mathrm{AgBr}$.

$\begin{array}{ll}\text { Theory. } & \text { Found. } \\ \mathbf{C}_{5} \mathrm{H}_{10} \mathrm{Br}_{2} . & 68.65 \text { per cent. }\end{array}$

Methyltetramethylene dibromide is a heavy colourless oil possessing a powerful and unpleasant odour, resembling to some extent that of trimethylene bromide. On exposure to air and light, it gradually becomes dark coloured.

\section{Ethylic Methylpentamethylenedicarboxylate, $\mathrm{CH}_{2}\left\langle\mathrm{CH}_{2}-\underset{\mathrm{C}}{\left.\mathrm{CH}_{2}-\mathrm{COOC}_{2} \mathrm{H}_{5}\right)_{2}} \stackrel{\mathrm{CH} \cdot \mathrm{CH}_{3}}{\mathrm{CH}_{2}}\right.$.}

To prepare this ethereal salt, 13 grams of sodium was dissolved in 130 grams of absolute alcohol, and to this solution a mixture of 91 grams of ethylic malonate and 65 grams methyltetramethylene dibromide was added, care being taken to keep the whole well cooled during the mixing. When this mixture was warmed on a waterbath, a brisk reaction set in, sodium bromide separated, and it is probable that as soon as this ceased the decomposition was complete. The product was, however, as a precaution, gently boiled on a waterbath for two hours, and then tested with litmus-paper in the usual way, when it was found to have a perfectly neutral reaction.

After distilling off the excess of alcohol and adding water, an oil was precipitated, which was separated from the aqueous solution by extraction with ether, the ethereal solution well washed with water, dried over calcic chloride, and the ether distilled off. In this way an almost colourless oil was obtained, which was purified by fractional distillation. As soon as the last traces of ether had distilled over, the temperature rose rapidly to $190^{\circ}$, and the whole distilled over below $250^{\circ}$, leaving only a trace of residue.

This distillate, on several times refractioning, was easily separated into two parts, one boiling at $190-195^{\circ}$, and consisting of regenerated 
ethyl malonate, and a higher fraction boiling constantly at $243-244^{\circ}$, which was pure ethylic methylpentamethylenedicarboxylate.

\section{Analysis.}

0.1911 gram substance gave 0.1500 gram $\mathrm{H}_{2} \mathrm{O}$ and 0.4437 gram $\mathrm{CO}_{2}$.

\begin{tabular}{|c|c|c|}
\hline & $\begin{array}{l}\text { Theory. } \\
\mathrm{C}_{12} \mathrm{H}_{20} \mathrm{O}_{4} \text {. } \\
63 \cdot 15 \text { per cent. }\end{array}$ & $\begin{array}{l}\text { Found. } \\
63 \cdot 32 \text { per cent. }\end{array}$ \\
\hline & $8 \cdot 77^{1}$ & 8.73 \\
\hline & $28 \cdot 07$ & $27 \cdot 95$ \\
\hline
\end{tabular}

Ethylic methylpentamethylenedicarboxylate is a thick, colourless oil of peculiar odour, somewhat resembling that of ethylic tetramethylenedicarboxylate.

The yield of this ethereal salt obtained in the above reaction is almost quantitative.

Methylpentamethylenedicarboxylic Acid, $\mathrm{CH}_{2}\left\langle{ }_{\mathrm{CH}_{2}-\mathrm{C}(\mathrm{COOH})_{2}}^{\mathrm{CH}_{2}-} \stackrel{-}{\mathrm{CH} \cdot \mathrm{CH}_{3}}\right.$

This acid is easily prepared from its ethylic salt by hydrolysis with alcoholic potash.

For this purpose, the fraction of ethylic methylpentamethylenedicarboxylate boiling at $240-250^{\circ}$ is mixed with one and a half times the calculated quantity of potash, dissolved in pure alcohol, and the whole boiled in a reflux apparatus for $6-8$ hours. If at the end of this time a sample taken out and diluted with water is found to dissolve completely without the separation of oily particles, the hydrolysis is complete, otherwise the boiling must be continued until this result is obtained. The alcohol is now distilled off, the residue dissolved in water, and evaporated gently on a water bath until the last traces of alcohol have been driven off.

The resulting almost colourless liquid is well cooled, acidified with dilute sulphuric acid, and extracted several times with ether. After carefully drying over calcic chloride and distilling off the ether, the crude acid is obtained as a colourless crystalline cake. This is first roughly separated from traces of mother-liquor, by spreading out on a porous plate, and then purified by recrystallisation from ether. It is thus obtained in the form of magnificent colourless prisms, which, after drying over sulphuric acid in a vacuum, gave the following numbers on analysis :-

$0 \cdot 1815$ gram substance gave 0.3720 gram $\mathrm{CO}_{2}$ and 0.1157 gram $\mathrm{H}_{2} \mathrm{O}$. 


\begin{tabular}{|c|c|c|}
\hline $\mathrm{C}_{6} \mathrm{E}$ & $\begin{array}{l}\text { Theory. } \\
\mathrm{H}_{10}(\mathrm{COOH})_{2} \text {. }\end{array}$ & Found. \\
\hline$I$ & 6.97 & 7.08 \\
\hline & $37 \cdot 21$ & $37 \cdot 04$ \\
\hline
\end{tabular}

Methylpentamethylenedicarboxylic acid melts at $173-175^{\circ}$, decomposing at the same time into carbonic anhydride and methylpentamethylenemonocarboxylic acid.

It is readily soluble in alcohol, ether, and hot water, much more sparingly so in cold water. It crystallises from its solution in hot water, in curious, opaque, leaf-like crystals, but the best crystals are obtained from ether. If the concentrated ethereal solution is allowed to evaporate spontaneously, magnificent transparent prisms form on the sides and bottom of the vessel containing it. These are being crystallographically examined, and we hope shortly to be able to communicate to the Society the crystalline form and measurements of these beautiful crystals.

The silver salt of methylpentamethylenedicarboxylic acid was prepared in the usual way by adding an excess of nitrate of silver to the neutral solution of the ammonium salt. The white precipitate thus obtained was well washed with water, dried over sulphuric acid in a vacuum, and analysed.

I. 0.2888 gram of substance gave 0.2594 gram $\mathrm{CO}_{2}$ and 0.0676 gram $\mathrm{H}_{2} \mathrm{O}$.

II. 0.2817 gram of substance gave 0.1574 gram Ag.

\begin{tabular}{|c|c|c|c|c|}
\hline \multicolumn{3}{|c|}{$\begin{array}{l}\text { Theory. } \\
\mathrm{C}_{6} \mathrm{H}_{10}(\mathrm{COOAg})_{2} \\
\quad 24 \cdot 87 \mathrm{per}\end{array}$} & \multicolumn{2}{|c|}{$\begin{array}{l}\text { Found. } \\
25 \cdot 01 \text { per cent. }\end{array}$} \\
\hline . . . . & $2 \cdot 59$ & , & $2 \cdot 66$ & ״, \\
\hline$\ldots$. & $55 \cdot 96$ & $"$ & $55 \cdot 88$ & , \\
\hline
\end{tabular}

Methylpentamethylenemonocarboxylic Acid, $\mathrm{CH}_{2}\left\langle\mathrm{CH}_{2}-\underset{\mathrm{CH}}{\mathrm{CH}_{2}-\mathrm{COOH} \cdot \mathrm{CH}_{3}}\right.$.

When methylpentamethylenedicarboxylic acid is heated a few degrees above its melting point, it is rapidly decomposed into carbonic anhydride and methylpentamethylenemonocarboxylic acid. In carrying out this decomposition, the pure dicarboxylic acid was placed in a small Würtz flask, and gently heated in a metal bath, till the evolution of carbonic anhydride ceased. The residual oily monocarboxylic acid, on fractioning, distilled over completely between $218^{\circ}$ and $220^{\circ}$, and on refractioning, the substance was easily obtained pure as a colourless liquid, boiling constantly at $219.5-220.5^{\circ}$. 
Analysis.

0.1973 gram substance gave 0.4760 gram $\mathrm{CO}_{2}$ and 0.1682 gram $\mathrm{H}_{2} \mathrm{O}$.

\begin{tabular}{|c|c|c|c|c|}
\hline \multicolumn{3}{|c|}{$\begin{array}{c}\text { Theory. } \\
\mathrm{C}_{6} \mathrm{H}_{11} \text { (COOH). }\end{array}$} & \multirow{2}{*}{\multicolumn{2}{|c|}{$\begin{array}{l}\text { Found. } \\
65 \cdot 73 \text { per cent. }\end{array}$}} \\
\hline$\cdots$ & 050 & cent. & & \\
\hline . & $\begin{array}{r}9 \cdot 38 \\
25.00\end{array}$ & $"$ & $\begin{array}{r}9446 \\
24 \cdot 81\end{array}$ & \\
\hline
\end{tabular}

Methylpentamethylenemonocarboxylic acid is a thick, colourless oil of penetrating and most.unpleasant odour, somewhat resembling that of valerianic acid. It is not acted on by bromine in the cold, but when heated with bromine at $110^{\circ}$ decomposition takes place with evolution of large quantities of hydrogen bromide, showing that the acid is a saturated compound. In order to prove this still more conclusively, a very pure sample was prepared, boiling constantly at $219-219.5^{\circ}$, and submitted to physical examination by W. H. Perkin, sen. The determinations of its specific gravity and magnetic rotation gare the following numbers :-

$$
\begin{aligned}
& d \frac{15^{\circ}}{15^{\circ}}=1.02054, \\
& d \frac{20^{\circ}}{20^{\circ}}=1.01739, \\
& d \frac{25^{\circ}}{25^{\circ}}=1.01438 .
\end{aligned}
$$

Magnetic Rotation.

\begin{tabular}{c|c|c}
\hline$t$. & Sp. rotation. & Mol. rotation. \\
\hline $24 \cdot 5^{\circ}$ & $0 \cdot 9864$ & $6 \cdot 898$ \\
$24 \cdot 0$ & $0 \cdot 9853$ & $6 \cdot 905$ \\
$24 \cdot 0$ & $0 \cdot 9897$ & $6 \cdot 935$ \\
$24 \cdot 0$ & $0 \cdot 9877$ & $6 \cdot 920$ \\
\hline Average $24 \cdot 1$ & $0 \cdot 9873$ & $6 \cdot 914$ \\
\hline
\end{tabular}

In discussing the formation of tetramethylene-derivatives from ethylic malonate and trimethylene bromide (Trans., 51, 14-17), it was pointed out that by this reaction either tetramethylene or allyl derivatives might be formed.

Thus the monocarboxylic acid obtained by the hydrolysis of the product of this reaction and subsequent elimination of carbonic anhydride might be either- 

(a.) Tetramethylenecarboxylic acid:<smiles>O=C(O)C1CCCC1</smiles>

or

(b.) Allylacetic acid : $\mathrm{CH}_{2}: \mathrm{CH} \cdot \mathrm{CH}_{2} \cdot \mathrm{CH}_{2} \cdot \mathrm{COOH}$.

That the substance actually obtained was not allylacetic acid was easily proved by direct comparison with this well-known acid. In doing this, perhaps the most striking proof of the remarkable difference existing between these two acids was the study of the numbers representing the magnetic rotation of each. Thus :-

$$
\begin{aligned}
& \text { Magnetic rotation of allylacetic acid.... } 6.426 \\
& " \\
& " \\
& \left.\begin{array}{r}
\text { tetramethylenecarb- } \\
\text { oxylic acid...... }
\end{array}\right\} 5 \cdot 048 \\
& \text { Difference... } \overline{1 \cdot 378}
\end{aligned}
$$

Now, unless some decisive proof is brought to the contrary, exactly the same argument might be used with regard to the constitution of the products obtained by the action of methyltetramethylene dibromide on ethylic malonate.

The substance described as ethylic methylpentamethylenedicarboxylate might be an unsaturated compound, having one of the following formulæ:-

(1.) $\left(\mathrm{COOC}_{2} \mathrm{H}_{5}\right)_{2} \mathrm{CH} \cdot \mathrm{CH}\left(\mathrm{CH}_{3}\right) \cdot \mathrm{CH}_{2} \cdot \mathrm{CH}: \mathrm{CH}_{2}$.

(2.) $\left(\mathrm{COOC}_{2} \mathrm{H}_{5}\right)_{2} \mathrm{CH} \cdot \mathrm{CH}_{2} \cdot \mathrm{CH}_{2} \cdot \mathrm{CH}_{2} \cdot \mathrm{CH}: \mathrm{CH}_{2}$.

in which case methylpentamethylenemonocarboxylic acid would be either-
(A.) $\mathrm{COOH} \cdot \mathrm{CH}_{2} \cdot \mathrm{CH}\left(\mathrm{CH}_{3}\right) \cdot \mathrm{CH}_{2} \cdot \mathrm{CH}: \mathrm{CH}_{2}$.
(B.) $\mathrm{COOH} \cdot \mathrm{CH}_{2} \cdot \mathrm{CH}_{2} \cdot \mathrm{CH}_{2} \cdot \mathrm{CH}_{2} \cdot \mathrm{CH}: \mathrm{CH}_{2} \cdot$

Now an acid represented by Formula $\mathrm{B}$ is nothing more than allylacetic acid, $\mathrm{COOH} \cdot \mathrm{CH}_{2} \cdot \mathrm{CH}_{2} \cdot \mathrm{CH}: \mathrm{CH}_{2}$, plus $2 \mathrm{CH}_{2}$. Its magnetic rotation is therefore easily calculated, thus :-

$$
\begin{aligned}
& \text { Magnetic rotation of allylacetic acid ...... } 6.426 \\
& +2 \mathrm{CH}_{2}=2 \times 1.023 \ldots \ldots \ldots \ldots \ldots \ldots .2 .046 \\
& \text { Calc. rotation for acid B ..... } \quad 8.472
\end{aligned}
$$

An acid of the constitution represented by Formula A would have a still higher magnetic rotation (see W. H. Perkin, sen., Trans., 45, 551 ), probably about 8.590 .

The difference between these two numbers, 8.472 and 8.590 , and that actually found (6.914) is very remarkable, and shows conclusively that the acid described as methylpentamethylenecarboxylic acid cannot 
possibly be an unsaturated substance, but is really a pentamethylenederivative.

Methylpentamethylenemonocarboxylic acid is a feeble acid. It dissolves but slowly in dilute ammonia, and if this solution is allowed to stand for some days over sulphuric acid in a vacuum, the ammonium salt is completely decomposed into ammonia and the free acid which separates out in large oily drops. In preparing the sitver salt, the acid was dissolved in ammonia, neutralised with dilute nitric acid, and a small quantity of the silver salt precipitated. This was filtered off and discarded. The filtrate was then mixed with an excess of nitrate of silver, the white amorphous precipitate thas obtained collected, well washed with water, and dried on a piece of porous plate over sulphuric acid in a vacuum.

\section{Analyses.}

I. 0.2847 gram substance gave 0.1305 gram silver.

II. $0.2816 \quad " \quad, \quad 0.1295$,
Found.
Theory. $\mathrm{C}_{6} \mathrm{H}_{11} \mathrm{COOAg}$. Ag.... 45.96 per cent.

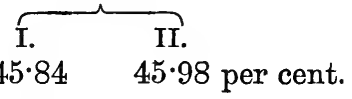

The barium salt was prepared by boiling the pure acid with an excess of baryta-water, passing carbonic anhydride, filtering, and evaporating the solution. It was thus obtained as a colourless syrup, which, even after standing for days over sulphuric acid, showed no signis of crystallising.

Action of Methyltetramethylene Dibromide on Ethylic Acetoacetate.

Ethylic Acetylmethylpentamethylenemonocarboxylate,

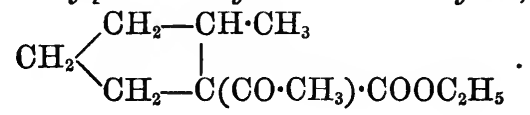

In studying this reaction, 50 grams of ethylic acetoacetate ( 2 mols.) and 46 grams of methyltetramethylene dibromide ( 1 mol.) were added to 10 grams of sodium (2 mols.) dissolved in 100 grams of absolute alcohol, and the whole heated in a flask connected with a reflux apparatus for 12 hours on a water-bath. The decomposition with ethylic acetoacetate takes place with much greater difficulty, and far less completely than when ethylic malonate is employed, so that even after long boiling the mixture still retained a decided alkaline reaction. At the end of 12 hours, the alcohol was distilled off, water 
added and the oil extracted with ether as usual. The ethereal solution was washed with water, dried over calcic chloride, and the ether distilled off, when an almost colourless oil was obtained which on repeated fractioning was easily separated into two fractions, the lower of which, boiling below $200^{\circ}$, consisted of regenerated ethylic acetoacetate, whereas the higher one, boiling at $235-240^{\circ}$, was ethylic acetylmethylpentamethylenecarboxylate.

The substance prepared in this way is quite pure enough for further experiments, but owing to the presence of small quantities of bromine, it does not give good numbers on analysis. It was, therefore, for this purpose dissolved in a little alcohol, boiled with a small quantity of the sodium compound of ethylic acetoacetate for some hours, then reprecipitated and refractioned; as thus obtained, it is a colourless oil boiling constantly at $237-238^{\circ}$. Even after this treatment, it still contained traces of bromine, which made the analytical numbers somewhat unsatisfactory :-

0.1404 gram substance gave 0.3394 gram $\mathrm{CO}_{2}$ and 0.1110 gram $\mathrm{H}_{2} \mathrm{O}$.

\begin{tabular}{|c|c|c|}
\hline & $\begin{array}{l}\text { Theory. } \\
\mathrm{C}_{11} \mathrm{H}_{18} \mathrm{O}_{3} \text {. } \\
66.67 \text { per cent. }\end{array}$ & $\begin{array}{l}\text { Found. } \\
65.93 \text { per cent. }\end{array}$ \\
\hline & $9 \cdot 09$ & $8 \cdot 79$ \\
\hline & $24 \cdot 24$ & $25 \cdot 28$ \\
\hline
\end{tabular}

Ethyic acetylmethylpentamethylenecarboxylate is a thick colourless oil boiling at $237-238^{\circ}$, and possessing a strong, but not unpleasant odour. The yield of this substance obtained by the above reaction is very good indeed.

Hydrolysis of Ethylic Acetylmethylpentamethylenecarboxylate. Formation of-

Methylpentamethylenecarboxylic Acid,<smiles>CCCC(C)C(=O)O</smiles>

and of

Methylpertamethylene Methyl Ketone, $\mathrm{CH}_{2}\left\langle\begin{array}{l}\mathrm{CH}_{2}-\mathrm{CH} \cdot \mathrm{CO} \cdot \mathrm{CH}_{3} \\ \mathrm{CH}_{2} \cdot \mathrm{CH}_{3}\end{array}\right.$

In carrying out this hydrolysis, the fraction of ethylic acetylmethyl: pentamethylenecarboxylate boiling at $235-240^{\circ}$ was mixed with a large excess of a nearly concentrated solution of potash in pure alcohol, and boiled for two hours on a water-bath in a flask connected with a reversed condenser. On dissolving the product in water, acetylmethylpentamethylene methyl ketone was precipitated in oily drops, 
and was easily separated from the solution of the potash salts by repeated extraction with ether.

The aqueous solution, which was first examined, was evaporated on a water-bath, until quite free from alcohol, acidified with dilute sulphuric acid, and the oily acid thus precipitated several times extracted with ether. The ethereal solution was well washed with water, dried over calcic chloride, and the ether distilled off, the resulting brownish-coloured oily acid being then purified by fractional distillation. It was thus easily obtained pure as a colourless oil boiling constantly at $220-221^{\circ}$, and possessing all the properties of the methylpentamethylenecarboxylic acid previously obtained from ethylic malonate and methyltetramethylene dibromide (see page 193). Analysis gave the following numbers :-

0.1856 gram substance gave 0.4442 gram $\mathrm{CO}_{2}$ and 0.1540 gram $\mathrm{H}_{2} \mathrm{O}$.

\begin{tabular}{|c|c|c|}
\hline & $\begin{array}{l}\text { Theory. } \\
\left.\mathrm{I}_{11} \text { (COOH }\right) \text {. }\end{array}$ & Found. \\
\hline & 65.62 per cent. & 65.30 per cent. \\
\hline & $9 \cdot 38$ & $9 \cdot 21$ \\
\hline & $25 \cdot 00$ & $25 \cdot 49$ \\
\hline
\end{tabular}

Although this acid was evidently identical with that obtained from ethylic malonate, it was thought necessary to prove this fact more conclusively.

The sp. gr. and magnetic rotation of this specimen was therefore examined by W. H. Perkin (sen.) with the following results :-

$$
\begin{array}{ll}
d \frac{4^{\circ}}{4^{\circ}} 1 \cdot 0256, & d \frac{15^{\circ}}{15^{\circ}} 1 \cdot 0172, \\
d \frac{10^{\circ}}{10^{\circ}} 1 \cdot 0208, & d \frac{20^{\circ}}{20^{\circ}} 1 \cdot 0139 . \\
& d \frac{25^{\circ}}{25^{\circ}} 1 \cdot 0109 .
\end{array}
$$

Magnetic Rotations.

\begin{tabular}{c|c|c}
$t$. & Sp. rotation. & Mol. rotation. \\
\hline $18^{\circ}$ & $0 \cdot 9887$ & $6 \cdot 927$ \\
18 & $0 \cdot 9943$ & $6 \cdot 966$ \\
18 & $0 \cdot 9905$ & $6 \cdot 939$ \\
18 & $0 \cdot 9908$ & $6 \cdot 941$ \\
\hline Average 18 & $0 \cdot 9911$ & $6 \cdot 943$
\end{tabular}

Although this specimen was small, and could therefore not be submitted to such repeated refractioning as was the case with the previous 
sample, still on comparing the numbers obtained in the density determination and magnetic rotation of the two products, it will be seen that they agree sufficiently well to leave no doubt as to their identity.

The ethereal solution containing the methylpentamethylene methyl ketone, which had been separated from the potash salts as described above, was next examined. After well washing with water, drying over calcic chloride, and distilling off the ether, a brownish oil of aromatic odour was left behind; on distillation, it passed over almost entirely between $165^{\circ}$ and $175^{\circ}$, and on repeated fractioning was easily obtained pure, as a colourless oil boiling constantly at $170-171^{\circ}$.

\section{Analysis.}

0.1547 gram substance gave 0.4326 gram $\mathrm{CO}_{2}$ and 0.1550 gram $\mathrm{H}_{2} \mathrm{O}$.

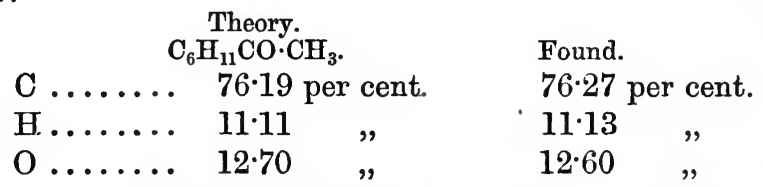

Methylpentamethylene methyl ketone is a colourless oil possessing in a very marked degree the penetrating odour of peppermint. It combines readily with phenylhydrazine and hydroxylamine, and dissolves, although with some dificulty, in bisulphite of soda.

The sp. gr. and magnetic rotalions of this interesting compound were also examined by W. H. Perkin (sen.), who gave us the following as the result of his experiments :-

$$
\begin{array}{ll}
d \frac{4^{\circ}}{4^{\circ}} 0.9222, & d \frac{15^{\circ}}{15^{\circ}} 0.9136 \\
d \frac{10^{\circ}}{10^{\circ}} 0.9174, & d \frac{20^{\circ}}{20^{\circ}} 0.9100 \\
& d \frac{25^{\circ}}{25^{\circ}} 0.9070 .
\end{array}
$$

Magnetic Rotations.

\begin{tabular}{c|c|c}
\hline$t$. & Sp. rotation. & Mol. rotation. \\
\hline $18^{\circ}$ & $1 \cdot 0450$ & $8 \cdot 026$ \\
18 & $1 \cdot 0452$ & $8 \cdot 025$ \\
18 & $1 \cdot 0432$ & $8 \cdot 012$ \\
18 & $1 \cdot 0431$ & $8 \cdot 012$ \\
\hline Average 18, & $1 \cdot 0441$ & $8 \cdot 019$ \\
\hline
\end{tabular}




\section{Action of Sodium on Methyltetramethylene Dibromidle.}

In his researches on the action of sodium on trimethylene bromide, Fremnd (Monats.; 1882, 622) obtained a gas which differed in its properties in a very marked way from ordinary propylene, and which

he therefore considered to be trimethylene,

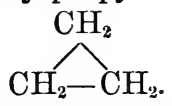

Following up this reaction, we have submitted methyltetramethylene dibromide to the action of sodium, and obtained a hydrocarbon boiling at $39-42^{\circ}$, which from its mode of formation is most probably methyltetramethylene-

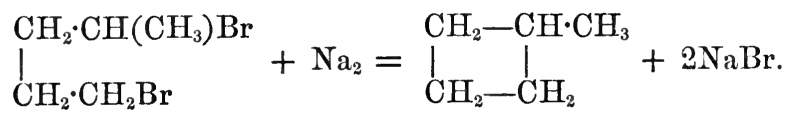

At the same time, owing to the small quantity of material at our disposal, we have not been able to prove that this really is the constitution of the substance, and we therefore use the name methyltetramethylene only provisionally, until we have been able more fully to study its properties.

If methyltetramethylene dibromide is warmed with finely divided sodium, a most violent reaction takes place, but in order to isolate the product, this must be very much moderated. After several experiments, the following method was found to give good results :-

10 grams of clean sodium were heated under toluene in a loosely stoppered flask until completely melted, the stopper then lightly inserted, and the whole vigorously shaken until the sodium had assumed the appearance of fine metallic sand.

The contents of the flask were then transferred to a Würtz flask (holding about 120-150 c.c.), fitted with a dropping funnel and supplied with a condenser, through which ice-water was run during the whole of the subsequent operation.

The toluene (20 grams) in which the sodium was suspended, was then heated nearly to boiling, the flame taken away, and a mixture of 30 grams of methyltetramethylene dibromide and 20 grams of toluene dropped in slowly through the dropping funnel. The reaction is sufficiently violent to keep the liquid boiling briskly. During the decomposition, a small quantity of liquid of low-boiling point condenses, and is collected in a small flask cooled in a freezing mixture. As soon as all the bromide has been added, the product is gently distilled, until about $\frac{1}{3}$ of the toluene has passed over. In order to separate the methyltetramethylene from this excess of toluene, the crude distillate was subjected to repeated fractioning 
from a Würtz flask provided with a very long neck, and in this way an oil was at last obtained which boiled constantly at $39-42^{\circ}$.

The combustion, which was carried out in a very long combustion tube, gave the following numbers :-

0.1864 gram substance gave 0.2454 gram $\mathrm{H}_{2} \mathrm{O}$ and 0.5830 gram $\mathrm{CO}_{2}$.

\begin{tabular}{|c|c|c|}
\hline & eory. $\mathrm{C}_{5} \mathrm{H}_{10}$. & Found. \\
\hline ..... & $85 \cdot 71$ per ce & $85 \cdot 30$ per cent \\
\hline & $14: 29$ & $14 \cdot 62$ \\
\hline
\end{tabular}

Methyltetramethylene is a very volatile substance boiling at 39$42^{\circ}$, and has an odour very similar to that of the hydrocarbons of the methane series. When mixed with very concentrated aqueous hydrogen iodide, the volume of the hydrocarbon decreased slightly during the first day, but afterwards did not appear to be acted on any further. This fact seems to show that this hydrocarbon, although it may contain a small quantity of an unsaturated compound, is itself saturated. It is isomeric with normal anylene,

\section{$\mathrm{CH}_{3} \cdot \mathrm{CH}_{2} \cdot \mathrm{CH}_{2} \cdot \mathrm{CH}: \mathrm{CH}_{2}$}

(boiling point $39-40^{\circ}$ ), but not identical, as the latter is easily and completely absorbed by concentrated aqueous hydrogen iodide with formation of the iodide of methyl propyl carbinyl.

A possible explanation of the formation of the hydrocarbon above described, by the action of sodium on methyltetramethylene dibromide was, that in the presence of small quantities of water nascent. hydrogen might be formed, which would react on the dibromide, forming pentane, $\mathrm{CH}_{3} \cdot \mathrm{CH}_{2} \cdot \mathrm{CH}_{2} \cdot \mathrm{CH}_{2} \cdot \mathrm{CH}_{3}$.

In order to avoid a reduction such as this, great care was taken to have all the materials as well as the apparatus perfectly dry, and the result of the analysis of the product obtained shows conclusively that it cannot be pentane, which requires $\mathrm{C}=83.33$ per cent., $\mathrm{H}=16 \cdot 66$ per cent. 
[Reprinted from the Journal of the Chemical Society, March, 1888. Vol. LIII.]

THE SYNTHETICAL FORMATION OF CLOSED CARBONCHAINS. Part IV. SOME DERIVATIVES OF HEXAMETHYLENE.

By Paul C. Freer, Ph.D., and W. H. Perkin (Jun.), Ph.D.

In a previous paper by one of us (Trans., 51, 704), it was shown that methyldehydrohexonecarboxylic acid when boiled with water yiclds acetobutyl alcohol, thus :- 
<smiles>CC1(C)OCCC(O)C1=O</smiles>

\section{$\mathrm{CH}_{3} \cdot \mathrm{CO} \cdot \mathrm{CH}_{2} \cdot \mathrm{CH}_{2} \cdot \mathrm{CH}_{2} \cdot \mathrm{CH}_{2} \mathrm{OH}+\mathrm{CO}_{2}$.}

This acetobutyl alcohol, when treated with sodium amalgam, is easily converted into $\delta$-hexylene glycol, thus :-

$\mathrm{CH}_{3} \cdot \mathrm{CO} \cdot \mathrm{CH}_{2} \cdot \mathrm{CH}_{2} \cdot \mathrm{CH}_{2} \cdot \mathrm{CH}_{2} \mathrm{OH}+\mathrm{H}_{2}=$

$\mathrm{CH}_{3} \cdot \mathrm{CH}(\mathrm{OH}) \cdot \mathrm{CH}_{2} \cdot \mathrm{CH}_{2} \cdot \mathrm{CH}_{2} \cdot \mathrm{CH}_{2} \mathrm{OH}$.

If this glycol is digested with concentrated aqueous hydrogen bromide, methylpentamethylene dibromide is obtained, according to the following equation:-

$$
\begin{aligned}
& \mathrm{CH}_{3} \cdot \mathrm{CH}(\mathrm{OH}) \cdot\left[\mathrm{CH}_{2}\right]_{3} \cdot \mathrm{CH}_{2} \mathrm{OH}+2 \mathrm{HBr}= \\
& \mathrm{CH}_{3} \cdot \mathrm{CHBr} \cdot\left[\mathrm{CH}_{2}\right]_{3} \cdot \mathrm{CH}_{2} \mathrm{Br}+2 \mathrm{H}_{2} \mathrm{O} \text {. }
\end{aligned}
$$

By the action of the sodium compound of ethylic malonate on this dibromide, hexamethylene-derivatives can be obtained, the reaction being exactly similar to that described in the previous paper for the formation of pentamethylene-derivatives from methyltetramethylene dibromide, thus :-

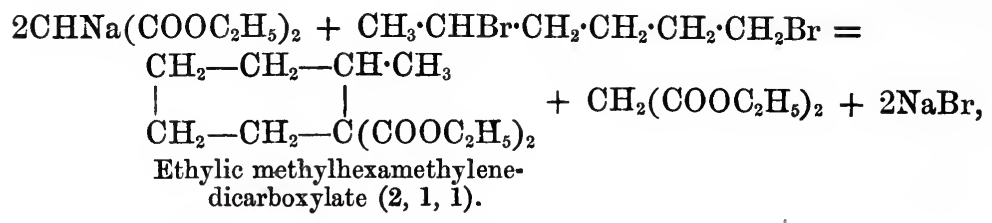

ethylic methylhexamethylenedicarboxylate being formed.

This ethereal salt on hydrolysis yields the free methylhexamethylenedicarboxylic acid which, when distilled, is decomposed into methylhexamethylenemonocarboxylic acid and carbonic anhydride, thus :-

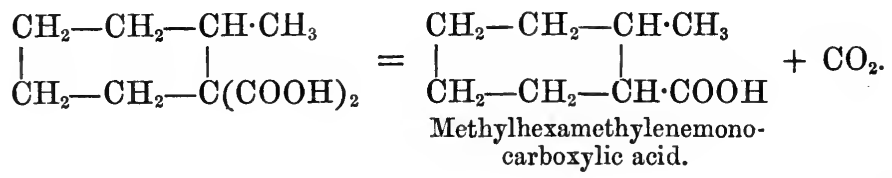

In carrying out this investigation, it was again thought that interesting results might be obtained from the study of the action of methylpentamethylene dibromide on the sodium compound of ethylic acetoacetate, in order to determine whether hexamethylene-derivatives would result, or whether oxygen-rings would be formed, as in the case of trimethylene dibromide. 
If one molecular proportion of the sodium compound of ethylic acetoacetate be digested with one molecular proportion of methylpentamethylene dibromide, a substance, $\mathrm{C}_{12} \mathrm{H}_{21} \mathrm{BrO}_{3}$, will be produced which may have either of the two following constitutional formulæ:-

\section{(1.) $\underset{\mathrm{COOC}_{2} \mathrm{H}_{5}}{\mathrm{CH}_{3} \cdot \mathrm{CO}}>\mathrm{CH} \cdot \mathrm{CH}\left(\mathrm{CH}_{3}\right) \cdot \mathrm{CH}_{2} \cdot \mathrm{CH}_{2} \cdot \mathrm{CH}_{2} \cdot \mathrm{CH}_{2} \mathrm{Br}$. \\ (2) $\mathrm{CH}_{3} \cdot \mathrm{CO}$ $\mathrm{COOC}_{2} \mathrm{H}_{5}>\mathrm{CH} \cdot \mathrm{CH}_{2} \cdot \mathrm{CH}_{2} \cdot \mathrm{CH}_{2} \cdot \mathrm{CH}_{2} \cdot \mathrm{CHBr}^{\cdot} \mathrm{CH}_{3} \cdot$}

No attempts were made to distinguish between these two formulæ, as this question has no bearing on the constitution of the substances subsequently obtained from this intermediate compound.

If this bromo-compound is treated with zinc-dust and acetic acid, the bromine-atom is easily displaced by hydrogen. In order to determine its constitution, therefore, it would only be necessary to isolate this reduction product and ascertain whether it is isomeric or identical with ethylic hexylacetoacetate; in the former case formula (1), in the latter formula (2) would be correct.

This compound, $\mathrm{C}_{12} \mathrm{H}_{21} \mathrm{BrO}_{3}$, when treated with sodic ethylate or the sodium-compound of ethylic acetoacetate, is converted into ethylic acetylmethylhexamethylene carboxylate, thus :-

$$
\begin{aligned}
\underset{\mathrm{COOC}_{2} \mathrm{H}_{5}}{\mathrm{CH}_{3} \cdot \mathrm{CO}} \mathrm{CH} \cdot \mathrm{CH}\left(\mathrm{CH}_{3}\right) \cdot \mathrm{CH}_{2} \cdot \mathrm{CH}_{2} \cdot \mathrm{CH}_{2} \cdot \mathrm{CH}_{2} \mathrm{Br}+\mathrm{C}_{2} \mathrm{H}_{5} \cdot \mathrm{ONa} \\
=\prod_{\substack{\mathrm{CH}_{2}-\mathrm{CH}_{2}-\mathrm{C}\left(\mathrm{CO} \cdot \mathrm{CH}_{3}\right) \cdot \mathrm{COOC}_{2} \mathrm{H}_{5} \\
\text { Ethylic acetylmethylhexamethylene- } \\
\text { carboxylate }(1,2,1) .}}^{\mathrm{CH}_{2}-\mathrm{CH}_{2}-\mathrm{CH} \cdot \mathrm{CH}_{3}}+\mathrm{NaBr}+\mathrm{C}_{2} \mathrm{H}_{5} \cdot \mathrm{OH} .
\end{aligned}
$$

In preparing this compound, however, it is much easier to start with 2 mols. of ethylic acetoacetate and $1 \mathrm{~mol}$. of methylpentamethylene dibromide, in which case the reaction would be represented thus :-

$$
\begin{aligned}
& { }_{2} \underset{\mathrm{COOC}_{2} \mathrm{H}_{5}}{\mathrm{CH}_{3}} \mathrm{CO} \mathrm{CHNa}+\mathrm{CH}_{3} \cdot \mathrm{CH}(\mathrm{Br}) \cdot \mathrm{CH}_{2} \cdot \mathrm{CH}_{2} \cdot \mathrm{CH}_{2} \cdot \mathrm{CH}_{2} \mathrm{Br} \\
& =\left.\right|_{\mathrm{CH}_{2}-\mathrm{CH}_{2}-\mathrm{C}\left(\mathrm{CO} \cdot \mathrm{CH}_{3}\right) \cdot \mathrm{COOC}_{2} \mathrm{H}_{5}} ^{\mathrm{CH}_{2}-\mathrm{CH}_{2}-\mathrm{CH} \cdot \mathrm{CH}_{3}} \\
& +\mathrm{CH}_{3} \cdot \mathrm{CO} \cdot \mathrm{CH}_{2} \cdot \mathrm{COOC}_{2} \mathrm{H}_{5} \\
& +2 \mathrm{NaBr} \text {. }
\end{aligned}
$$

That the product of this reaction really is a hexamethylene-derivative is shown by a study of the products resulting from its hydrolysis. When boiled with alcoholic potash, ethylic acetylmethylhexamethylenecarboxylate is easily decomposed with formation of methylhexa- 
methylene methyl ketone and methylhexamethylenecarboxylic acid, thus :-

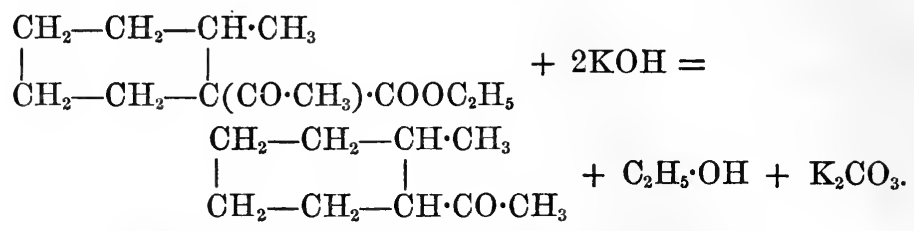
Methylhexamethylene methyl ketone $(2,1)$.

(2.)

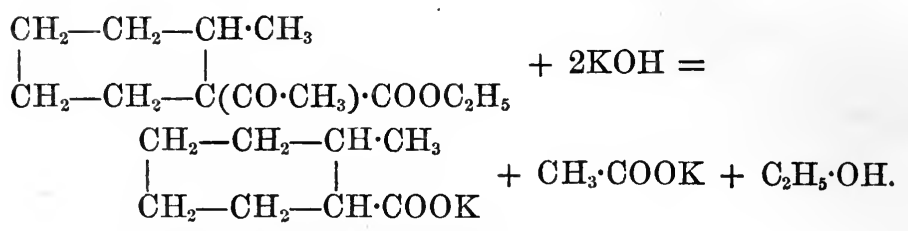

Potassic methylhexamethylenecarboxylate $(2,1)$.

The methylhexamethylenecarboxylic acid obtained in this way showed all the reactions and possessed the same properties as that obtained from ethylic malonate and methylpentamethylene dibromide.

Unfortunately, owing to the small quantity of material at our disposal no physical measurements could be made with the sample obtained from ethylic acetoacetate, which would have afforded some more definite proof as to the identity of the two acids. But from a study of the results obtained by Colman and Perkin (see previous paper) in their experiments on the action of methyltetramethylene dibromide on ethylic malonate and acetoacetate, there can scarcely be a doubt that in the present case also, hexamethylene-derivatives are formed in both the reactions described above.

\section{Methylpentamethylene Dibromide, $\mathrm{CH}_{3} \cdot \mathrm{CHBr} \cdot \mathrm{CH}_{2} \cdot \mathrm{CH}_{2} \cdot \mathrm{CH}_{2} \cdot \mathrm{CH}_{2} \mathrm{Br}$.}

The formation of methylpentamethylene dibromide' ( $\delta$-hexylene dibromide) from $\delta$-hexylene glycol has been briefly noticed in a previous paper (Trans., 51, 722).

In preparing considerable quantities of this dibromide, the following method was found to give the best results :-

$\delta$-Hexylene glycol, prepared by the reduction of acetobutyl alcohol as previously described (Trans., 51, 722), is dissolved in five times its volume of cencentrated aqueous hydrogen bromide (sp. gr. 1.85) and gently heated to boiling in a flask connected with a reflux apparatus, a slow stream of hydrogen bromide being passed into the liquid during the operation. After heating for about an hour, the whole is 
allowed to cool, diluted with water, and the precipitated dark-browncoloured bromide extracted several times with ether. The ethereal solution is then dried over calcic chloride, the ether distilled off, and the crude bromide purified by fractional distillation under reduced pressure $(100 \mathrm{~mm}$.), when it is easily obtained pure, boiling constantly at $153-154^{\circ}$ (100 mm.).

In preparing this dibromide for the following experiments, a method exactly similar to that described under "Methyltetramethylene Dibromide" (in the paper by Colman and Perkin), was first used, but afterwards it was found that as $\delta$-hexylene glycol could be so much more completely extracted from its aqueous solution by ether than \%-pentylene glycol can, the tedious method there described was not necessary.

\section{Ethylic Methylhexamethylenedicarboxylate<smiles>CCCCCC(C)C(=O)OC</smiles>

To prepare this ethereal salt, $4 \cdot 4$ grams of sodium were dissolved in 50 grams of absolute alcohol, and when quite cold mixed with 30 grams of ethylic malonate and 21 grams of methylpentamethylene dibromide, and heated on a water-bath to boiling for about five hours; at the end of this time the decomposition was complete and the mass showed neutral reaction. Water was then added, and the whole several times extracted with ether. The ethereal solution was washed till free from alcohol, dried over calcic chloride, and the ether distilled off, when 33 grams of an almost colourless oil was obtained, which was first fractioned under reduced pressure $(250 \mathrm{~mm}$.).

In this way, the product was divided into two distinct parts, an oil boiling from $150-250^{\circ}$ and a thick residue boiling at a much higher temperature, the study of which will be the snbject of a future paper. The fraction $150-250^{\circ}(250 \mathrm{~mm}$.) was then further fractioned at ordinary pressures and thus easily separated into unchanged ethylic malonate and a new substance boiling at $260-265^{\circ}$; this on analysis gave the following results :-

0.1831 gram substance gave 0.4304 gram $\mathrm{CO}_{2}$ and 0.1476 gram $\mathrm{H}_{2} \mathrm{O}$.

\begin{tabular}{|c|c|c|}
\hline $\mathrm{C}_{7} \mathrm{H}$ & $\begin{array}{l}\text { Theory. } \\
{ }_{12}\left(\mathrm{COOC}_{2} \mathrm{H}_{5}\right)_{2} \text {. }\end{array}$ & Found. \\
\hline$\cdots$ & 64.46 per cent. & 64.11 per cent. \\
\hline IT & $9 \cdot 09$ & $8 \cdot 96$ \\
\hline & $26 \cdot 44$ & $26 \cdot 93$ \\
\hline
\end{tabular}

Ethylic methylhexamethylenedicarboxylate is a thick colourless oil 
of disagreeable odour, boiling at $260-265^{\circ}$. The yield is not very good, owing to the formation of a considerable quantity of a high boiling substance. In a later experiment, where 45 grams of ethylic malonate, 34 grams of methylpentamethylene dibromide, and 6.4 grams of sodium were used, 56 grams of crude product was obtained on extraction with ether. This, on fractioning under $160 \mathrm{~mm}$. pressure, gave 39 grams of oil boiling below $250^{\circ}$; on refractioning under ordinary pressures, 20 grams of crude ethylic methylhexamethylene dicarboxylate were obtained, boiling at $245-275^{\circ}$. In both the experiments, it was found that the oil obtained boiling at $260-265^{\circ}$, and which was supposed to be pure, still contained traces of bromine which seriously affected the analytical results. The product analysed was first freed from bromine by long boiling with a little ethylic malonate and sodic ethylate, and subsequent treatment with zinc-dust and acetic acid.

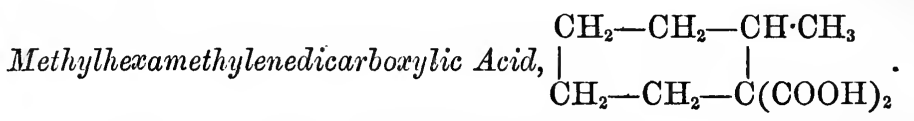

In order to obtain the free dicarboxylic acid, the fraction of the ethereal salt boiling at $258-267^{\circ}$ was mixed with an excess of alcoholic potash, containing twice the calculated quantity of pure potash dissolved in pure alcohol, and boiled on a water-bath for six hours. The excess of alcohol was then distilled off, the residue dissolved in water and evaporated till quite free from alcohol. The slightly brownish-coloured liquid was nearly neutralised with dilute sulphuric acid, filtered, the filtrate mixed with an excess of dilute sulphuric acid and several times extracted with ether. The ethereal solution, after drying and evaporating, deposited the crude acid as a thick yellowish oil, which, on standing for some days over sulphuric acid in a vacuum, almost entirely solidified. This crude product was first roughly separated from oily impurities by spreading out on a porous plate, and the resulting almost colourless crystalline mass once or twice recrystallised from water. It was thus obtained as a sandy crystalline powder, which on analysis gave the following numbers :-

I. 0.1995 gram substance gave 0.4246 gram $\mathrm{CO}_{2}$ and 0.1377 gram $\mathrm{H}_{2} \mathrm{O}$.

II. $0 \cdot 1772$ gram substance gave 0.3770 gram $\mathrm{CO}_{2}$ and 0.1217 gram $\mathrm{H}_{2} \mathrm{O}$.

\begin{tabular}{|c|c|c|c|c|}
\hline & & & & \\
\hline & $\begin{array}{l}\mathrm{I}_{12} \text { (COOH. } \\
\text { (n) }\end{array}$ & & I. & II. \\
\hline$C \ldots \ldots$ & $58 \cdot 06$ & cent. & $58 \cdot 04$ & 58.02 \\
\hline H.... & $7 \cdot 53$ & ", & $7 \cdot 67$ & $7 \cdot 63$ \\
\hline $0 \ldots \ldots$ & $34 \cdot 41$ & $"$ & $34: 29$ & $34: 35$ \\
\hline
\end{tabular}


Methylhexamethylenedicarboxylic acid melts at about $147^{\circ}$, being at the same time partially decomposed into methylhexamethylenemonocarboxylic acid and carbonic anhydride. Methylhexamethylenedicarboxylic acid is readily soluble in ether, alcohol, and hot water, but more sparingly in cold water.

This acid has, especially when not quite pure, a great tendency to form supersaturated solutions - a fact which renders its purification a matter of considerable difficulty. From its solution in water, it is slowly deposited in crystalline crusts, which consist of a mass of small hard crystals. Methylhexamethylenedicarboxylic acid shows little tendency to form large crystals, and in this respect differs in a very marked manner from the corresponding pentamethylene-derivative.

The silver salt of this acid was prepared in the usual way, by precipitating a neutral solution of the ammonium salt with nitrate of silver, collecting the white amorphous precipitate thus obtained, washing it well with water, and drying over sulphuric acid in a vacuum.

The following result was obtained on analysis :-

0.3102 gram substance gave 0.1662 gram Ag.

Theory.

$\mathrm{C}_{7} \mathrm{H}_{12}(\mathrm{COOAg})_{2}$.

Found.

54.00 per cent.

$53 \cdot 54$ per cent.

Methylhexamethylenemonocarboxylic Acid,<smiles>CC1CCCC(C(=O)O)C1C</smiles>

In preparing this acid, the pure dicarboxylic acid is melted in a small Würtz flask, and then gently heated in a metal-bath until the decomposition into carbonic anhydride and methylhexamethylenemonocarboxylic acid is complete. The residual oil on fractioning all distils over between $230^{\circ}$ and $240^{\circ}$, and on repeated fractioning the new acid is obtained pure, as a colourless oil boiling constantly at $235-236^{\circ}$.

$$
\text { Analyses :- }
$$

I. 0.1987 gram substance gave 0.4894 gram $\mathrm{CO}_{2}$ and $0 \cdot 1752$ gram $\mathrm{H}_{2} \mathrm{O}$.

II. 0.2156 gram substance gave 0.5320 gram $\mathrm{CO}_{2}$ and 0.1892 gram $\mathrm{H}_{2} \mathrm{O}$.

III. 0.1814 gram substance gave $0 \cdot 4483$ gram $\mathrm{CO}_{2}$ and $0 \cdot 1599$ gram $\mathrm{H}_{2} \mathrm{O}$. 


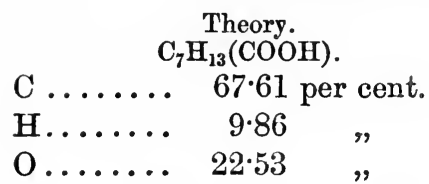

Found.

\begin{tabular}{rrr}
\multicolumn{1}{c}{ I. } & \multicolumn{1}{c}{ II. } & \multicolumn{1}{c}{ III. } \\
$67 \cdot 17$ & $67 \cdot 25$ & $67 \cdot 34$ \\
$9 \cdot 79$ & $9 \cdot 75$ & $9 \cdot 79$ \\
$23 \cdot 04$ & $23 \cdot 00$ & $22 \cdot 87$
\end{tabular}

Methylhexamethylenemonocarboxylic acid is a thick colourless oil of most penetrating and disagreeable odour, which is even more pronounced than that of the pentamethylene-derivative. It boils at $235-236^{\circ}$. .

Its sp. gr. and magnetic rotation have been determined by W. H. Perkin (sen.), who gave us the following account of his experiments :-

$$
\begin{array}{ll}
d \frac{4^{\circ}}{4^{\circ}} 1 \cdot 0079, & d \frac{15^{\circ}}{15^{\circ}} 0 \cdot 99982, \\
d \frac{10^{\circ}}{10^{\circ}} 1 \cdot 0033, & d \frac{20^{\circ}}{20^{\circ}} 0 \cdot 9966, \\
& d \frac{25^{\circ}}{25^{\circ}} 0 \cdot 9940 .
\end{array}
$$

\section{Magnetic Rotations.}

\begin{tabular}{c|c|c}
\hline \multicolumn{1}{c|}{$t}$. & Sp. rotation. & Mol. rotation. \\
\hline $18 \cdot 0^{\circ}$ & $1 \cdot 0073$ & $7 \cdot 965$ \\
$18 \cdot 0$ & $1 \cdot 0106$ & $7 \cdot 991$ \\
$18 \cdot 0$ & $1 \cdot 0103$ & $7 \cdot 989$ \\
$18 \cdot 5$ & $1 \cdot 0100$ & $7 \cdot 990$ \\
$18 \cdot 5$ & $1 \cdot 0052$ & $7 \cdot 952$ \\
$17 \cdot 0$ & $1 \cdot 0079$ & $7 \cdot 962$ \\
\hline Average $18 \cdot 0$ & $1 \cdot 0085$ & $7 \cdot 975$ \\
\hline
\end{tabular}

With regard to the constitution of this acid, the same might be said as was said of the constitution of methylpentamethylenecarboxylic acid (pp. 195-196).

From its mode of formation, this acid might be an unsaturated compound, in which case its constitation would be represented by one of the two following formulæ:-

(1.) $\mathrm{COOH} \cdot \mathrm{CH}_{2} \cdot \mathrm{CH}\left(\mathrm{CH}_{3}\right) \cdot \mathrm{CH}_{2} \cdot \mathrm{CH}_{2} \cdot \mathrm{CH}: \mathrm{CH}_{2}$.

(2.) $\mathrm{COOH} \cdot \mathrm{CH}_{2} \cdot \mathrm{CH}_{2} \cdot \mathrm{CH}_{2} \cdot \mathrm{CH}_{2} \cdot \mathrm{CH}_{2} \cdot \mathrm{CH}: \mathrm{CH}_{2}$.

The acid represented by formula (2) is allylacetic acid, plus $3 \mathrm{CH}_{2}$ or $\mathrm{COOH} \cdot \mathrm{CH}_{2} \cdot \mathrm{CH}_{2} \cdot \mathrm{CH}: \mathrm{CH}_{2}+3 \mathrm{CH}_{2}$.

Its magnetic rotation would therefore be calculated thas :- 


$$
\begin{array}{r}
\text { Magnetic rotation of allylacetic acid ...... } \\
+3 \mathrm{CH}_{2}=3 \times 1.023 \ldots \ldots \ldots \ldots \ldots \ldots
\end{array}
$$

An acid of the constitution represented in formula (2) would therefore have a calculated rotation of $9 \cdot 495$, whereas an acid with the constitution represented in formula (1) would have a rotation still larger, probably $9 \cdot 615$.

The difference between these two numbers and that actually found (i.e., 7.975) is so great as to entirely preclude the possibility of the acid described above as methylhexamethylenecarboxylic acid being an unsaturated compound.

That methylhexamethylenecarboxylic acid and methylpentamethylenecarboxylic acid are analogously constituted, can also be easily deduced from the numbers found as the magnetic rotation of each, thus :-

Magnetic rotation of methylhexamethylenecarboxylic acid $\quad 7 \cdot 975$ Magnetic rotation of methylpentamethylenecarboxylic acid $\quad 6.914$

$$
\text { Difference for } \mathrm{CH}_{2} \ldots \ldots \ldots, \quad 1 \cdot 061
$$

Now the actual value of $\mathrm{CH}_{2}$ as a difference between two analogously constitated substances is 1.023 , a number very closely agreeing with that found above.

Methylhexamethylenecarboxylic acid is a more feeble acid even than the corresponding pentamethylene-derivative. If the solution of its ammonium salt is evaporated over sulphuric acid in a vacuum, it is almost entirely decomposed into the free acid and ammonia.

In order, therefore, to prepare the silver salt, the free acid was dissolved in an excess of ammonia, neutralised with dilute nitric acid, and a small quantity of the silver salt first precipitated by the addition of a few drops of nitrate of silver. This was filtered off, and the bulk of the salt precipitated by adding an excess of silver nitrate to the filtrate. The bulky, white precipitate thus obtained, after well washing, and drying over sulphuric acid in a vacuum, gave the

\begin{tabular}{|c|c|c|c|c|}
\hline \multicolumn{3}{|c|}{$\begin{array}{c}\text { Theory. } \\
\mathrm{C}_{7} \mathrm{H}_{13}(\mathrm{COOAg}) \text {. }\end{array}$} & \multirow{2}{*}{\multicolumn{2}{|c|}{$\begin{array}{l}\text { Found. } \\
38.86 \text { per cent }\end{array}$}} \\
\hline$\ldots$ & 38.55 & cent. & & \\
\hline$\ldots$ & $5 \cdot 22$ &, & $5 \cdot 26$ & , \\
\hline $\mathrm{Ag} . .$. & $43 \cdot 37$ & $"$ & $43 \cdot 37$ & \\
\hline$O \ldots \ldots$ & $12 \cdot 85$ & , & $12 \cdot 51$ & , \\
\hline
\end{tabular}
following results on analysis :-

0.2070 gram substance gave 0.0980 gram $\mathrm{H}_{2} \mathrm{O}, 0.2950$ gram $\mathrm{CO}_{2 \text { r }}$ and 0.0898 gram Ag. 


\section{Ethylic Bromomethylpentamethyleneacetoacetate, \\ $\mathrm{CH}_{3} \cdot \mathrm{CO}$ $\mathrm{COOC}_{2} \mathrm{H}$}

This substance, which is the primary product of the action of methylpentamethylene dibromide on ethylic acetoacetate, is prepared in the following way:-

$2 \cdot 3$ grams of sodium are dissolved in 30 grams of absolute alcohol, and when quite cool mixed with 13 grams of ethylic acetoacetate and 24.5 grams of methylpentamethylene dibromide, and the whole heated on a water-bath for about two hours, at the end of which time the decomposition is usually completed. After distilling off the excess of alcohol, water is added, and the heavy oil thus precipitated is extracted two or three times with pure ether. The ethereal solution is well washed, carefully dried over calcic chloride, and the ether distilled off; in this way a colourless oil is obtained, which consists of nearly pure ethylic bromomethylpentamethyleneacetoacetate.

For the following analysis, a sample was taken which had been allowed to stand over sulphuric acid in a vacuum for some days, so as to free it completely from alcohol and ether:-

0.1944 gram substance gave 0.1242 gram AgBr.

$$
\begin{aligned}
& \text { Theory. } \\
& \mathrm{C}_{12} \mathrm{H}_{21} \mathrm{BrO}_{3} \text {. } \\
& \text { Br...... 27.31 per cent. } \quad 27 \cdot 15 \text { per cent. }
\end{aligned}
$$

Ethylic bromomethylpentamethyleneacetoacetate, when freshly prepared, is a thick, colourless oil of disagreeable odour, which on keeping, especially when exposed to the air, soon assumes a brownish colour. When boiled with sodic ethylate, it is completely converted into a new compound free from bromine; this boils at $255-260^{\circ}$, and appears to be identical with ethylic acetylmethylhexamethylenecarboxylate.

If the bromo-compound is heated in a paraffin-bath at $250-270^{\circ}$, in a flask connected with a condenser, hydrogen bromide comes off regularly for some hours. In order to examine the product of this reaction, the heating was continued until the evolution of hydrogen bromide ceased, and the residue was examined. During the experiment, the oil, which was colourless to start with, had become dark brown, bat on fractioning nearly the whole of it passed over between $255^{\circ}$ and $260^{\circ}$, as a colourless oil, which now contained traces only of bromine. It is a matter of interest to ascertain whether this substance is identical or isomeric with ethylic acetylmethylhexamethylenecarboxylate, and experiments instituted with the object of deciding this question are in progress. 
Ethylic Acetylmethylhexamethylenecarboxylate,<smiles>CCCCC(C)C(COC(=O)OCC)C(=O)OCC</smiles>

In studying the action of methylpentamethylene.dibromide on the sodium-compound of ethylic acetoacetate, the following quantities were used:-

Ethylic acetoacetate .......... 26. 0 grams.

Sodium .................. 4.6 ,

Methylpentamethylene dibromide .... 24:4 ",

The sodium was dissolved in 50 grams of absolute alcohol, and to the resulting solution of sodic ethylate, when cold, the ethylic acetoacetate and the methylpentamethylene dibromide were added. The whole was then heated on a water-bath, in a flask connected with a reflux apparatus for 20 hours. At the end of this time, the excess of alcohol was distilled off, the residue treated with water, and two or three times extracted with ether. The ethereal solution, after drying over calcic chloride and evaporating, deposited an almost colourless oil, which, when submitted to repeated fractional distillation, was easily separated into two portions, an oil boiling at $180-200^{\circ}$, and consisting of regenerated ethylic acetoacetate, and a second fraction of the boiling point $255-260^{\circ}$, which consisted of ethylic acetylmethylhexamethylenecarboxylate.

The product, even after repeated refractionation, was found to contain small quantities of bromine, amounting sometimes to as much as 2-3 per cent. In order to get satisfactory analytical numbers, it was found necessary to submit this fraction, $255-260^{\circ}$, to prolonged boiling with small quantities of the sodium compound of ethylic acetoacetate, and then, to remove the last traces, to reduction with a little zinc and acetic acid. The sample used in the following analysis boiled at $255-257^{\circ}$.

$0 \cdot 1688$ gram substance gave 0.4171 gram $\mathrm{CO}_{2}$ and $0 \cdot 1428 \mathrm{H}_{2} \mathrm{O}$.

\begin{tabular}{|c|c|c|}
\hline . & $\begin{array}{l}\text { Theory. } \\
\mathrm{C}_{12} \mathrm{H}_{20} \mathrm{O}_{3} \text {. } \\
67.92 \text { per cent. }\end{array}$ & $\begin{array}{l}\text { Found. } \\
67 \cdot 38 \text { per cent. }\end{array}$ \\
\hline . & $9 \cdot 43$ & $9 \cdot 40$ \\
\hline .. & $22 \cdot 64$ & $23 \cdot 22$ \\
\hline
\end{tabular}

Ethylic acetyl methylhexamethylenecarboxylate is a thick colourless oil of a peculiar but not disagreeable odour, strongly resembling that of the corresponding pentamethylene-derivative. The yield 
obtained is very good, but it was found impossible to get it quite free from bromine without reducing with zinc and acetic acid, except by boiling with large quantities of sodic ethylate and ethylic acetoacetate, in which case great loss is sustained owing to hydrolysis setting in.

The crude product of the reaction, however, rarely contains more than 1 or 2 per cent. of bromine and is quite pure enough for subequent experiments.

Hydrolysis of Ethylic Acetylmethylhexamethylenecarboxylate.

Methylhexamethylenecarboxylic Acid,<smiles>CCC(C)C</smiles><smiles>[CH]CC(C)C(=O)O</smiles>

Methylhexamethylene Methyl Ketone,<smiles>CCC(C)C</smiles>
$\mathrm{CH}_{2}-\mathrm{CH}_{2}-\mathrm{CH} \cdot \mathrm{CO} \cdot \mathrm{CH}_{3}$.

Just as ethylic acetylmethylpentamethylenecarboxylate when subjected to hydrolysis is split up and yields methylpentamethylenecarboxylic acid and methylpentamethylene methyl ketone (see previous paper, p. 198), so the corresponding hexamethylene-derivative when treated with alcoholic potash is converted into methylhexamethylenecarboxylic acid and methylhexamethylene methyl ketone.

In studying this decomposition, the fraction of ethylic acetylmethylhexamethylenecarboxylate boiling at $255-260^{\circ}$ was mixed with a considerable excess of very concentrated alcoholic potash, and heated on a water-bath for about four hours in a flask connected with a reflux apparatus. In a short time, the mass became quite thick owing to the separation of carbonate, so that it was found advisable from time to time to disconnect the condenser, cork up the flask, and shake well. As soon as the decomposition was complete, the product was allowed to cool, mixed with about three times its volume of water, and several times extracted with ether.

The aqueous solution, which was first examined, was evaporated on a water-bath till quite free from ether and alcohol, acidified with dilute sulphuric acid, and repeatedly extracted with pure ether. The ethereal solution after drying over calcic chloride and evaporating deposited a small quantity of a brownish-coloured oil which on distillation boiled almost entirely between $230^{\circ}$ and $240^{\circ}$. On refractioning, the greater part was obtained as a thick oil boiling at $234-237^{\circ}$. This on analysis gave the following numbers, which agree with those required for methylhexamethylenecarboxylic acid:- 
0.1763 gram substance gave 0.4340 gram $\mathrm{CO}_{2}$ and 0.1576 gram $\mathrm{H}_{2} \mathrm{O}$.

\begin{tabular}{|c|c|c|}
\hline & $\begin{array}{l}\text { Theory. } \\
\mathrm{C}_{8} \mathrm{H}_{14} \mathrm{O}_{2} \text {. } \\
67 \cdot 60 \text { per cent. }\end{array}$ & $\begin{array}{l}\text { Found. } \\
67 \cdot 14 \text { per cent. }\end{array}$ \\
\hline & $9 \cdot 86$ & $9 \cdot 93$ \\
\hline & $22 \cdot 53$ & $22 \cdot 93$ \\
\hline
\end{tabular}

The acid obtained by this reaction possesses all the properties of the methylhexamethylenecarboxylic acid obtained from ethyl malonate and methylpentamethylene dibromide. It has the same disagreeable and penetrating odour and the same boiling point $\left(234-237^{\circ}\right)$. It is unfortunate that the amount obtained was not sufficient to allow of its magnetic rotation being determined, in order thus to be able to make sure of the identity of the two samples.

The ethereal extract which had been separated from the aqueous solution of the potash salts, as described above, was next examined. After washing with water, drying over calcic chloride and evaporating, a considerable quantity of a jellowish oil was obtained, which on fractioning boiled almost constantly at $197-200^{\circ}$, and gave the following numbers on analysis :-

I. $0 \cdot 1576$ gram substance gave $0 \cdot 1630$ gram $\mathrm{H}_{2} \mathrm{O}$ and 0.4432 gram $\mathrm{CO}_{2}$.

II. $0 \cdot 1497$ gram substance gave $0 \cdot 1580$ gram $\mathrm{H}_{2} \mathrm{O}$ and $0 \cdot 4206$ gram $\mathrm{CO}_{2}$.

\begin{tabular}{|c|c|c|c|}
\hline & \multicolumn{2}{|c|}{ Found. } \\
\hline & $\begin{array}{l}\text { Theory. } \\
\mathrm{C}_{9} \mathrm{H}_{16} \mathrm{O} \text {. }\end{array}$ & I. & II. \\
\hline C.. & $77 \cdot 14$ per cent. & $76 \cdot 71$ & $76 \cdot 56$ \\
\hline & $11 \cdot 43$ & $11 \cdot 49$ & $11 \cdot 72$ \\
\hline$\ldots \ldots$ & $11 \cdot 43$ & $11 \cdot 80$ & $11 \cdot 72$ \\
\hline
\end{tabular}

Methylhexamethylene methyl ketone is a colourless oil, possessing in a concentrated state a most penetrating odour, somewhat resembling that of peppermint, but when present only in small quantities its odour reminds one of that of new-mown hay.

It combines with phenylhydrazine and apparently also with sodic bisulphite, but neither of the compounds produced have as yet been examined.

Action of Sodium on Methylpentamethylene Dibromide.

In studying this decomposition, the experiment was carried out in a way exactly similar to that described in the last paper (p. 201) in the case of methyltetramethylene dibromide.

After submitting the product to the most careful fractional distilla- 
tion, an oil was at last obtained boiling constantly at $70-71^{\circ}$; this gare the following results on analysis :-

0.2045 gram substance gave 0.2688 gram $\mathrm{H}_{2} \mathrm{O}$ and 0.6389 gram $\mathrm{CO}_{2}$.

\begin{tabular}{|c|c|c|}
\hline$\ldots \ldots$ & $\begin{array}{l}\text { Theory. } \\
\mathrm{C}_{6} \mathrm{H}_{12} \text {. } \\
85 \cdot 71 \text { per cent. }\end{array}$ & $\begin{array}{l}\text { Found. } \\
85.21 \text { per cent. }\end{array}$ \\
\hline$H \ldots . .$. & $14: 29$ & $14 \cdot 61$ \\
\hline
\end{tabular}

These numbers therefore agree with those required for methylpentamethylene, which should have been formed had the reaction taken place according to the following equation :-<smiles>CC(Br)CCCCCCC(CCBr)[Mg]Br</smiles>

At present, however, no definite proof can be given that the hydrocarbon has this constitution. It is isomeric with the hexylenes, $\mathrm{C}_{6} \mathrm{H}_{12}$, several of which are known, and which boil from $67^{\circ}$ to $73^{\circ}$, but it differs from them in this respect, that even after standing for some weeks with concentrated aqueous hydrogen iodide it is not acted on. By a further study of this interesting substance it is hoped that the question as to its constitution will be definitely settled. 


[Reprinted from the Journal of the Chemical Society, March, 1888. Vol. LIII.].

THE SYNTHETICAL FORMATION OF CLOSED CARBONCHAINS. PART V. EXPERIMENTS ON THE SYNTHESIS OF HEPTAMETHYLENE-DERIVATIVES.

By Paul C. Freer, Ph.D., and W. H. Perkin, jun., Ph.D.

WHEN ethylic malonate is treated with methyltetramethylene dibromide, the only product of the reaction is ethylic methylpentamethylenedicarboxylate, but when methylpentamethylene dibromide is used, besides ethylic methylhexamethylenedicarboxylate, a considerable quantity of a second substance is formed, which is the product of the action of $1 \mathrm{~mol}$. of methylpentamethylene dibromide on 2 mols. of ethylic malonate, thus :-

$2\left(\mathrm{COOC}_{2} \mathrm{H}_{5}\right)_{2} \mathrm{CHNa}+\mathrm{CH}_{3} \cdot \mathrm{CHBr} \cdot\left[\mathrm{CH}_{2}\right]_{3} \cdot \mathrm{CH}_{2} \mathrm{Br}=$ $\left(\mathrm{COOC}_{2} \mathrm{H}_{5}\right)_{2} \mathrm{CH} \cdot \mathrm{CH}\left(\mathrm{CH}_{3}\right) \cdot\left[\mathrm{CH}_{2}\right]_{4} \cdot \mathrm{CH}\left(\mathrm{COOC}_{2} \mathrm{H}_{5}\right)_{2}+2 \mathrm{NaBr}$.

This ethereal salt, for which we propose the name ethylic isoheptane- $w_{2} w_{2}$-tetracarboxylate, is converted by hydrolysis into the corre- 
sponding acid, and this on heating to $200^{\circ}$ is split up into carbonic anhydride and a new acid of the succinic series (methylazelaic acid)-

\section{$(\mathrm{COOH})_{2} \mathrm{CH} \cdot \mathrm{CH}\left(\mathrm{CH}_{3}\right) \cdot\left[\mathrm{CH}_{2}\right]_{4} \cdot \mathrm{CH}(\mathrm{COOH})_{2}=$}

Isoleptanetetracarboxylic acid.

\section{$\mathrm{COOH} \cdot \mathrm{CH}_{2} \cdot \mathrm{CH}\left(\mathrm{CH}_{3}\right) \cdot\left[\mathrm{CH}_{2}\right]_{5} \cdot \mathrm{COOH}+2 \mathrm{CO}_{2}$.} Methylazelaic acid.

Ethylic isoheptanetetracarboxylate was for these researches of special importance, because, as will be seen from a glance at its formula, it contains two hydrogen-atoms which, from their position with regard to the carb-ethoxyl-groups, are capable of being displaced by sodium, forming a disodium compound of the following formula :-

$$
\left(\mathrm{COOC}_{2} \mathrm{H}_{5}\right)_{2} \mathrm{CNa} \cdot \mathrm{CH}\left(\mathrm{CH}_{3}\right) \cdot\left[\mathrm{CH}_{2}\right]_{4} \mathrm{CNa}\left(\mathrm{COOC}_{2} \mathrm{H}_{5}\right)_{2} \text {. }
$$

This disodium compound when treated with iodine or bromine should be converted into ethylic methylheptamethylenetetracarboxylate, ${ }^{*}$ thus :-

$\left(\mathrm{COOC}_{2} \mathrm{H}_{5}\right)_{2} \mathrm{CNa} \cdot \mathrm{CH}\left(\mathrm{CH}_{3}\right) \cdot\left[\mathrm{CH}_{2}\right]_{4} \cdot \mathrm{CNa}\left(\mathrm{COOC}_{2} \mathrm{H}_{5}\right)_{2}+\mathrm{Br}_{2}=$<smiles>CCCCCC(CCCC)(C(=O)OCC)C(=O)OCC</smiles>

Methylheptamethylenetetracarboxylate $(1,1,2,2)$.

This ethylic salt on hydrolysis would then yield the corresponding tetracarboxylic acid, which on heating to $200^{\circ}$ would be converted into methylheptamethylenedicarboxylic acid $(1,2)$ and carbonic anhydride, thus :-

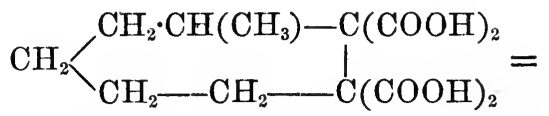

Methylheptamethylenetetracarboxylic acid $(3,1,1,2,2)$.

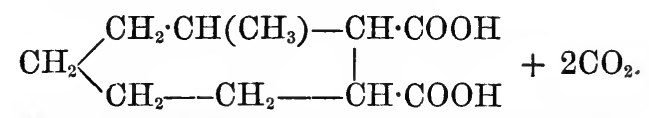

Methylheptamethylenedicarboxylic acid $(3,1,2)$.

To prove that such a change had taken place, it would be only necessary to show (1) that the resulting methylheptamethylenedicarboxylic acid differed in its properties from the methylazelaic acid produced by the hydrolysis of ethylic isoheptanetetracarboxylate

* Compare the formation of ethylic tetramethylenetet racarboxylate from ethylic butane- $\omega_{2} \omega_{2}$-tetracarboxylate (Trans., 51, 18), also of ethylic pentamethylenetetracarboxylate from ethylic pentane- $\omega_{2} \omega_{2}$-tetracarboxylate (Trans., 51, 240). 
before its treatment with sodinm and bromine; and (2) that the resulting acid was capable of forming an anhydride, thus proving that the two carboxyl-groups which at first were separated by seven carbon-atoms, were now in the ortho-position.

Two careful experiments were tried with the object of carrying out this synthesis (bromine being used), but, although, from the decolorisation of every drop of bromine as it came in contact with the sodium compound, it seemed as though the decomposition had taken place in the way desired, on examining the product it was found that no heptamethylene-ring had been formed.

The resulting ethereal salt was a mixture containing a considerable quantity of regenerated ethylic isoheptanetetracarboxylate and some very high-boiling complex products. It is impossible at the present stage of these experiments to explain what really takes place in a reaction of this kind; the only clear point is, that as far as we could tell, no trace of a heptamethylene-derivative is produced. This research does not of course prove that a closed carbon-chain of seven atoms cannot be formed; but it would nevertheless appear to throw doubt on the existence of a chain of these dimensions. Experiments are being carried on, the results of which it is hoped will clearly settle this important point.

\section{Ethylic Isoheptanetetracarboxylate, $\left(\mathrm{COOC}_{2} \mathrm{H}_{5}\right)_{2} \mathrm{CH} \cdot \mathrm{CH}\left(\mathrm{CH}_{3}\right) \cdot\left[\mathrm{CH}_{2}\right]_{4} \cdot \mathrm{CH}\left(\mathrm{COOC}_{2} \mathrm{H}_{5}\right)_{2}$.}

When the product of the action of methylpentamethylene dibromide on ethylic malonate is distilled, as described in the previous paper (p. 206), ethylic methylhexamethylenedicarboxylate passes over, whilst a thick, yellowish oil remains behind, consisting of crude ethylic isoheptanetetracarboxylate. If this crude residue be submitted to fractional distillation under a pressure of $60 \mathrm{~mm}$., nearly the whole of it will pass over without the slightest decomposition between $265^{\circ}$ and $285^{\circ}$, and on once or twice refractioning the oil is easily obtained pure boiling at $273-276^{\circ}$.

\section{Analysis.}

0.1808 gram substance gave 0.3970 gram $\mathrm{CO}_{2}$ and 0.1408 gram $\mathrm{H}_{2} \mathrm{O}$.

\begin{tabular}{|c|c|c|}
\hline$s$ & $\begin{array}{l}\text { Theory. } \\
\mathrm{C}_{20} \mathrm{H}_{34} \mathrm{O}_{8} \text {. }\end{array}$ & Found. \\
\hline 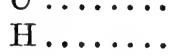 & $\begin{array}{l}89.40 \text { per cent. } \\
8.45\end{array}$ & $\begin{array}{l}59.83 \text { per cent. } \\
8.65\end{array}$ \\
\hline & $31 \cdot 84$ & $31 \cdot 52$ \\
\hline
\end{tabular}

* Compare for similar experiments, Kipping (this rol., pp. 22 and 23). 
Ethylic isoheptanetetracarboxylate is a thick, colourless syrup boiling at $273-276^{\circ}(60 \mathrm{~mm}$.).

When dissolved in ether and treated with 2 mols. of sodic ethylate, a yellowish-white sodium compound is precipitated. Sereral attempts were made to analyse this, but it was found to be impracticable, owing to its being so soluble in ether and so bygroscopic.

\section{Methylazelaic Acid, $\mathrm{COOH} \cdot \mathrm{CH}_{2} \cdot \mathrm{CH}\left(\mathrm{CH}_{3}\right) \cdot\left[\mathrm{CH}_{2}\right]_{5} \cdot \mathrm{COOH}$.}

When treated with alcoholic potash, ethylic isoheptanetetracarboxylate is readily hydrolysed. In carrying out this hydrolysis, 20 grams of the pure ethereal salt was mixed with a solution of 20 grams of pure potash dissolved in pure methyl alcohol, and boiled in a flask connected with a reflux apparatus for about six hours. The excess of alcohol was then distilled off, the residue dissolved in water, the resulting colourless solution neutralised with dilute sulphuric acid and evaporated on a water-bath till quite free from alcohol and ether. Excess of sulphuric acid was then added, and the isoheptanetetracarboxylic acid extracted at least 20 times with pure ether. The ethereal solution after drying over calcic chloride and evaporating, deposited the free acid as a thick, colourless syrup, which was not analysed, but at once converted into methylazelaic acid.

In order to do this, the flask containing the isoheptanetetracarboxylic acid was heated in a metal bath slowly to $200^{\circ}$, and kept at that temperature until the evolution of carbonic anhydride had entirely ceased. The resulting brownish-coloured oil was dissolved in a little water, boiled with a small quantity of animal charcoal, and the almost colourless solution evaporated on a water-bath. As, however, the residue, even after standing for some days over sulphuric acid in a vacuum, showed no signs of crystallisation, it was further purified by conversion into the ethereal salt. For this purpose, the crude substance was dissolved in pure ethyl alcohol, saturated with hydrogen chloride, and allowed to stand for some hours. Water was then added, the ethereal salt extracted with ether, washed with dilute carbonate of soda solution, the ether distilled off, and the residue fractioned under reduced pressure $(100 \mathrm{~mm}$.). In this way it was easily obtained pure as a thick oil boiling at $212-215^{\circ}$ (100 nim.).

Analysis.

$0 \cdot 1655$ gram substance gave $0 \cdot 1500$ gram $\mathrm{H}_{2} \mathrm{O}$ and $0 \cdot 3936$ gram $\mathrm{CO}_{2}$.

\begin{tabular}{|c|c|c|}
\hline $\mathrm{C} \ldots \ldots \ldots$ & $\begin{array}{l}\text { Theory. } \\
\mathrm{C}_{14} \mathrm{H}_{26} \mathrm{O}_{4} \text {. } \\
65 \cdot 11 \text { per cent. }\end{array}$ & $\begin{array}{l}\text { Found. } \\
64.89 \text { per cent. }\end{array}$ \\
\hline Н...... & $10.08 \quad$ & 10.07 \\
\hline $0 \ldots \ldots$ & $24 \cdot 81$ & $25 \cdot 04$ \\
\hline
\end{tabular}


The ethylic salt of methylazelaic acid is a colourless liquid of peculiar odour, and boils at $212-215^{\circ}$ under a pressure of $100 \mathrm{~mm}$. It is isomeric with ethylic sebate, which boils at $307-308^{\circ}$ under ordinary pressures.

In order to obtain methylazelaic acid, the ethereal salt was gently boiled with a slight excess of a solution of potash in pure methyl alcohol for two hours, the excess of alcohol distilled off, the product dissolved in water and the solution evaporated nearly to dryness. The residue was then dissolved in a little water, acidified with dilute sulphuric acid, and extracted two or three times with pure ether. The ethereal solution after being carefully dried over calcic chloride was evaporated, and in this way the new acid was obtained as a colourless syrup; this, after standing for some days over sulphuric acid in a vacuum, solidified to a hard cake of crystals. These were freed from a small quantity of oily mother-liquor by spreading out on a porous plate, and the acid was thus obtained quite colourless. After drying over sulphuric acid in a vacuum, it gave the following numbers on analysis :-

$0 \cdot 1334$ gram substance gave 0.1070 gram $\mathrm{H}_{2} \mathrm{O}$ and 0.2908 gram $\mathrm{CO}_{2}$.

\begin{tabular}{|c|c|c|}
\hline & $\begin{array}{l}\text { Theory. } \\
\mathrm{C}_{10} \mathrm{H}_{18} \mathrm{O}_{4} \text {. } \\
59 \cdot 41 \text { per cent. }\end{array}$ & $\begin{array}{l}\text { Found. } \\
59 \cdot 44 \text { per cent }\end{array}$ \\
\hline & 8.91 & 8.91 \\
\hline & $31 \cdot 68$ & $31 \cdot 65$ \\
\hline
\end{tabular}

Methylazelaic acid, thus prepared, melts at $43-44^{\circ}$, and when heated in small quantities in a test-tube appears to distil without decomposition. It is fairly easily soluble in boiling water, and, on cooling, the liquid becomes milky owing to the separation of the acid in oily drops. It is readily soluble in ether, alcohol, benzene, and chloroform, but cannot be recrystallised from any of these solvents, as on evaporating its solutions it is invariably obtained as a syrup which only crystallises again after standing for some time over sulphuric acid in a vacuum.

Methylazelaic acid is isomeric with sebacic acid (m.p. 197 $)$ and heptylmalonic acid (m. p. $97-98^{\circ}$ ).

\section{Salts of Methylazelaic Acid.}

Silver Salt.-To prepare this salt, the pure acid was dissolved in a little dilute ammonia, the solution allowed to stand over sulphuric acid in a vacuum till free from excess of ammonia, and the silver salt precipitated by the addition of nitrate of silver. It is thus obtained as a white amorphous mass, which after collecting on a filter, washing 
well with water, and drying over sulphuric acid in a vacuum, gave the following numbers on analysis :-

0.2380 gram substance gave 0.0833 gram $\mathrm{H}_{2} \mathrm{O}, 0.2507$ gram $\mathrm{CO}_{2}$, and $0 \cdot 1233$ gram Ag.

\begin{tabular}{|c|c|c|c|c|}
\hline \multicolumn{3}{|c|}{$\begin{array}{c}\text { Theory. } \\
\mathrm{C}_{10} \mathrm{H}_{16} \mathrm{O}_{4} \dot{\mathrm{Ag}}_{2} \text {. }\end{array}$} & \multirow{2}{*}{\multicolumn{2}{|c|}{$\begin{array}{l}\text { Found. } \\
28.80 \text { per cent. }\end{array}$}} \\
\hline .......... & $28 \cdot 84$ & r cent. & & \\
\hline H....... & $3 \cdot 84$ & , & 3.89 & , \\
\hline $\mathrm{Ag}$ & $51 \cdot 92$ & ", & $51 \cdot 80$ & $"$ \\
\hline & $15 \cdot 38$ & ", & $15 \cdot 51$ & , \\
\hline
\end{tabular}

If a neutral solution of the ammonium salt be treated with various reagents, it behaves in the following way :-

Calcic Chloride.... Added to a cold strong solution of the ammonium salt gives no precipitate; on warming however the calcium salt separates as a white curdy precipitate which when examined under the microscope presents very much the appearance of potato-starch.

Cupric Sulphate .. Gives a bluish-green precipitate almost insoluble in water.

Lead Acetate .... A white amorphous precipitate.

Zinc Sulphate .... Gives no precipitate even in strong solutions. On warming, however, the zinc salt separates as a curdy-white mass.

Baric Chloride... Gives no precipitate. The free acid dissolves readily in baryta-water, forming an easily soluble salt, not precipitated by boiling.

Magnesic Sulphate Gives no precipitate.

Cobalt Nitrate... Gives no precipitate in the cold; on gently warming the solution, a pink precipitate separates, which on boiling becomes reddishviolet.

Nickel Sulphate.. When added to a hot strong solution produces a pale-green (almost white) precipitate.

Action of Bromine on the Disodium Compound of Ethylic Isoheptanetetracarboxylate.

This experiment, which was instituted in the hope of obtaining a heptamethylene-derivative, as mentioned in the introduction, was carried out in the following way, and with the following quanti- 


\begin{tabular}{|c|c|c|}
\hline & Expt. $\mathrm{T}$. & Expt. II. \\
\hline $\begin{array}{c}\text { Ethylic isoheptanetetra- } \\
\text { carboxylate......... }\end{array}$ & 17.00 grams. & $22 \cdot 78$ grams. \\
\hline Sodium ............. & 1.93 & $2 \cdot 60$ \\
\hline Bromine. ............ & $6 \cdot 72$ & $9 \cdot 00$ \\
\hline
\end{tabular}

In both experiments, the sodium was dissolved in as little absolute alcohol as possible, the resulting sodic ethylate diluted with a large quantity of pure dry ether (about 100 c.c. in Experiment I and 200 c.c. in Experiment II), and mixed with the ethylic isoheptanetetracarboxylate. This caused the formation of a small amount of a yellowish flocculent precipitate, but the greater part of the disodium compound remained in solution. This mixture was cooled with ice, and the bromine added to it drop by drop, the whole being well agitated during the operation.

The resulting colourless product was then well washed with water, the ethereal solution evaporated, and the residual oil (which in Experiment II weighed nearly 22 grams) hydrolysed by boiling with a solution of potash in methyl alcohol. As soon as the hydrolysis was complete, the alcohol was distilled off, water added, the solution acidified with dilute sulphuric acid, and several times extracted with ether. After drying over calcic chloride and evaporating off the ether, a thick, almost colourless syrup remained, which was now heated to $200^{\circ}$ to cause the tetracarboxylic acid to split up into dicarboxylic acid and carbonic anhydride. The residue, which was slightly brownish, was then converted into its ethylic salt by treatment with ethyl alcohol and hydrogen chloride. In this way, a brownish oil was obtained which on fractioning under reduced pressure $\left(100 \mathrm{~mm}\right.$.) distilled for the most part between $200^{\circ}$ and $260^{\circ}$, leaving a small quantity of a dark brown residue. On repeated fractioning, rather more than one-half of this oil distilled between $210^{\circ}$ and $220^{\circ}$.

This oil was not analysed, but at once hydrolysed and the free acid examined. The whole quantity was boiled with a solution of potash in methyl alcohol for four hours, and the acid isolated in the usual way by acidifying with dilute sulphuric acid and extracting with pure ether. In this way, a considerable quantity of a nearly colourless syrup was obtained, which on standing for some days over sulphuric acid in a vacuum, solidified almost completely. The cake of crystals was purified from a little oily mother-liquor by spreading out on a porous plate, and after a few days became perfectly hard and colonrless. This substance on examination proved to be methylazeiaic acid. It melted at $43-44^{\circ}$, showed all the properties of this acid, and gave the following results on analysis :- 
$0 \cdot 1351$ gram substance gave 0.1075 gram $\mathrm{H}_{2} \mathrm{O}$ and 0.2935 gram $\mathrm{CO}_{2}$.

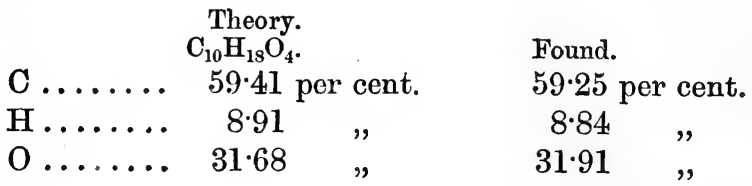

If methylheptamethylenecarboxylic acid had been present in the product of these reactions it would, on etherification with alcohol and hydrogen chloride, as described above, have yielded an ethereal salt, the boiling point of which would not have differed very much from that of ethylic methylazelate. By the hydrolysis of the fraction of this ethereal salt boiling at $210-220^{\circ}$, methylazelaic acid was obtained, but as far as we could see no trace of any other acid was present.

In order, however, to be more sure of this result, the higher and lower fractions of this ethereal salt $\left(200-260^{\circ}\right)$ were also submitted to hydrolysis, and in this way a syrupy acid was obtained, which on standing deposited a small quantity of methylazelaic acid. The mother-liquors from the crystals were now heated in a metal-bath for some time at $250-280^{\circ}$ in the hope that if any methylheptamethylenedicarboxylic acid were present it would in this way be converted into its anhydride. On treating the product with dilute ammonia, however, it readily dissolved, showing that no such change had taken place.

From these results, therefore, it is evident that the action of bromine on the disodium compound of ethylic isoheptanetetracarboxylate no heptamethylene-derivative is formed. 
SYNTHETICAL FORMATION OF CLOSED CARBON-CHAINS IN THE AROMATIC SERIES. PART I. ON SOME DERIVATIVES OF HYDRINDONAPHTHENE AND TETRAHYDRONAPHTHALENE.

$$
\text { By W. H. Perkin, Jun., Ph.D. }
$$

Is previous papers on the "Synthetical Formation of Closed Carbonchains," I have dealt exclusively with the formation of such chains in the fatty series.

In continuing these researches, it appeared to me that very interesting results might be obtained if substances belonging to the aromatic series were also experimented with. In this way it might be possible to obtain derivatives of the following hydrocarbons:-
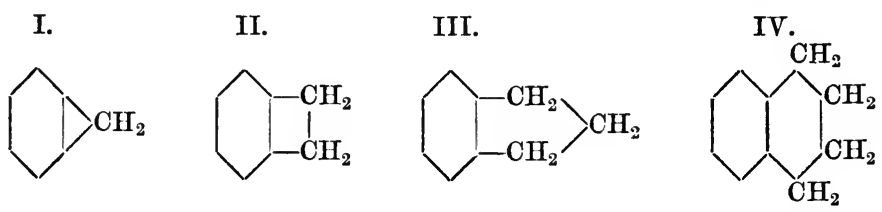

hydrocarbons which would contain, beside the benzene-ring, chains of $3,4,5$, and 6 carbon-atoms, two atoms of which would invariably be common to both rings.

Although attempts to obtain derivatives of the hydrocarbons I and II have as yet been unsuccessful, substances containing a benzene-ring and a 5-ring (III) and a benzene-ring and a 6-ring (IV) may be synthesised by the following reactions.

Ortho-xylene when treated with 2 mols. of bromine at $125^{\circ}$ is converted into ortho-xylylene dibromide, thus :-

$$
\mathrm{C}_{6} \mathrm{H}_{4}\left(\mathrm{CH}_{3}\right)_{2}+2 \mathrm{Br}_{2}=\mathrm{C}_{6} \mathrm{H}_{4}\left(\mathrm{CH}_{2} \mathrm{Br}\right)_{2}+2 \mathrm{HBr} \text {. }
$$


If now this ortho-xylylene dibromide is digested with ethylic malonate and 2 mols. of sodium ethylate; the following reaction takes place :-

$$
\begin{aligned}
& \mathrm{C}_{6} \mathrm{H}_{4}\left(\mathrm{CH}_{2} \mathrm{Br}\right)_{2}+\mathrm{Na}_{2} \mathrm{C}\left(\mathrm{COOC}_{2} \mathrm{H}_{5}\right)_{2}= \\
& \mathrm{C}_{6} \mathrm{H}_{4}<\mathrm{CH}_{2}^{\mathrm{CH}_{2}}>\mathrm{C}<\mathrm{COOC}_{2} \mathrm{COOC}_{5} \mathrm{H}_{5}+2 \mathrm{NaBr} \text {. }
\end{aligned}
$$

The product of this reaction is the ethylic salt of a dicarboxylic acid of a hydrocarbon of the formula-

$$
\mathrm{C}_{6} \mathrm{H}_{4}<\underset{\mathrm{CH}_{2}}{\mathrm{CH}_{2}}>\mathrm{CH}_{2} .
$$

The formula represents the dihydride of an unknown hydrocarbon, which stands to indole in the same relation as quinoline does to naphthalene :-
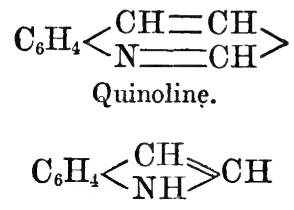

Indole.

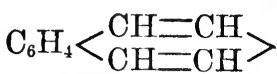

Naphthalene.<smiles>C[C]1CCCCC1</smiles>

Indonaphthene.

and which may therefore be called indonaphthene, in which case the dihydro-derivative would be hydrindonaphthene, and the product of the action of ortho-xylylene dibromide on ethylic malonate ethylic hydrindonaphthenedicarboxylate. This ethereal salt, on hydrolysis, is easily converted into the dicarboxylic acid, which on distillation is resolved into hydrindonaphthenemonocarboxylic acid and carbonic anhydride, thus :-

$$
\begin{aligned}
& \mathrm{C}_{6} \mathrm{H}_{4}<\mathrm{CH}_{2}^{\mathrm{CH}_{2}}>\mathrm{C}<_{\mathrm{COOH}}^{\mathrm{COOH}}=\mathrm{C}_{6} \mathrm{H}_{4}<_{\mathrm{CH}_{2}}^{\mathrm{CH}_{2}}>\mathrm{CH} \cdot \mathrm{COOH}+\mathrm{CO}_{2} \text {. } \\
& \text { Hydrindonaphthenedi- }
\end{aligned}
$$

The attempts to obtain the hydrocarbon direct from its monocarboxylic acid have as yet been without result, but it is hoped that in some indirect way this transformation may still be accomplished.

In continuation of these experiments, I next endeavoured to obtain synthetically derivatives of a hydrocarbon containing two rings, each of 6 carbon-atoms, such as that shown in IV (p. 1), and this, after several failures, was at length accomplished in the following way. Ethylic acetylenetetracarboxylate, when treated with 2 mols. of sodium 
ethylate, is converted into a disodium-derivative of the formula $\mathrm{CNa}_{\mathrm{CNa}\left(\mathrm{COOCO}_{2} \mathrm{H}_{5}\right)_{2}}$, and by digestion with ortho-xylylene dibromide this is easily transformed into the ethylic salt of tetrahydronaphthalenetetracarboxylic acid, thus :-

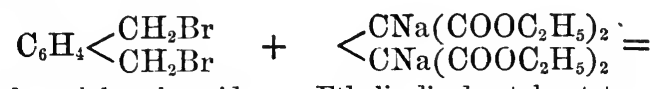

Ortho-xylylene bromide. Ethylic disodacetylenetetracarboxylate.

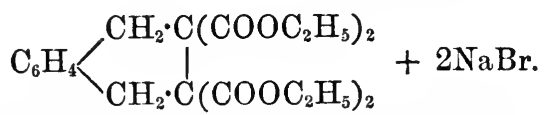

Ethylic tetrahydronaphthalenetetracarboxylate.

If this ethereal salt is treated with alcoholic potash, hydrolysis sets in, but at the same time 2 mols. of $\mathrm{CO}_{2}$ are removed, and the potassium salt of tetrahydronaphthalenedicarboxylate is formed, thus :-

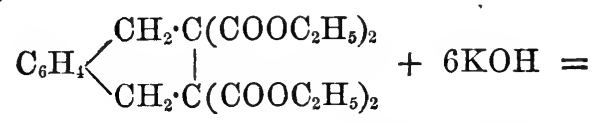

$$
\mathrm{C}_{6} \mathrm{H}_{4}\left\langle{ }_{\mathrm{CH}_{2} \cdot \mathrm{CH} \cdot \mathrm{COOK}}^{\mathrm{CH}_{2} \cdot \mathrm{CH} \cdot \mathrm{COOK}}+4 \mathrm{C}_{2} \mathrm{H}_{6} \mathrm{O}+2 \mathrm{~K}_{2} \mathrm{CO}_{3}\right. \text {. }
$$

Potassic tetrahydronaphthalenedicarboxylate.

On acidifying the aqueous solution of this salt with dilute sulphuric acid, tetrahydronaphthalenedicarboxylic acid is obtained. This acid, being an orthodicarboxylic acid similar to phthalic acid, is on heating easily converted into its anhydride and water, thus :-

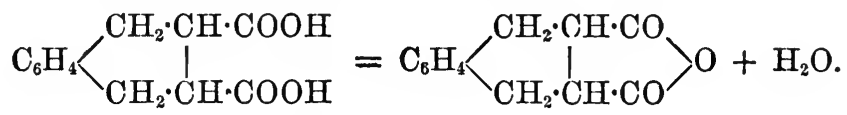

But the most interesting decomposition of this acid is that which it undergoes when its silver salt is distilled. Under these circumstances, it is completely decomposed into the anhydride of the acid and naphthalene, thus :-

$$
\begin{aligned}
& 2 \mathrm{O}_{6} \mathrm{H}_{4} \succ_{\mathrm{CH}_{2} \cdot \mathrm{CH} \cdot \mathrm{COOAg}}^{\mathrm{CH}_{2} \cdot \mathrm{CH} \cdot \mathrm{COOAg}}=\mathrm{C}_{6} \mathrm{H}_{4} \succ_{\mathrm{CH}=\mathrm{CH}}^{\mathrm{CH}=\mathrm{CH}}+ \\
& \text { Naphthalene. } \\
& \mathrm{C}_{6} \mathrm{H}_{4}\left\langle\begin{array}{l}
\mathrm{CH}_{2} \cdot \mathrm{CH} \cdot \mathrm{CO} \\
\mathrm{CH}_{2} \cdot \mathrm{CH} \cdot \mathrm{CO}
\end{array}\right\rangle \mathrm{O}+4 \mathrm{Ag}+2 \mathrm{CO}_{2}+\mathrm{H}_{2} \mathrm{O} \text {. }
\end{aligned}
$$


Now ethylic acetylenetetracarboxylate is formed from 2 mols. of ethylic malonate by the abstraction of two atoms of hydrogen. It, therefore, follows that one. should obtain the same result as the above, if ortho-xylylene dibromide were combined first with 2 mols. of ethyl malonate, and the two atoms of hydrogen were then removed. For this purpese two molecular proportions of the monosodiumderivative of ethylic malonate were treated with one molecular proportion of ortho-xylylene dibromide in the expectation of realising the following reaction :-

$$
\begin{array}{r}
\mathrm{C}_{6} \mathrm{H}_{4}\left(\mathrm{CH}_{2} \mathrm{Br}\right)_{2}+2 \mathrm{NaHC}\left(\mathrm{COOC}_{2} \mathrm{H}_{5}\right)_{2}= \\
\mathrm{C}_{6} \mathrm{H}_{4}\left[\mathrm{CH}_{2} \cdot \mathrm{CH}\left(\mathrm{COOC}_{2} \mathrm{H}_{5}\right)_{2}\right]_{2}+2 \mathrm{NaBr},
\end{array}
$$

but on examining the product it was found that ethylic hydrindonaphthenedicarboxylate had been formed, a result which can only be explained by supposing that an interchange of sodium-atoms takes place between the 2 mols. of ethylic malonate, much in the same way as in the formation of ethylic methyldehydrohexonecarboxylate from 2 mols. of the monosodium-derivatives of ethylic acetoacetate, and $1 \mathrm{~mol}$. of trimethylene bromide (Trans., 1887, 703), thus :-

$$
\begin{aligned}
\mathrm{C}_{6} \mathrm{H}_{4}< & \underset{\mathrm{CH}_{2} \mathrm{Br}}{\mathrm{CH}_{2} \mathrm{Br}}+2 \mathrm{NaHC}\left(\mathrm{COOC}_{2} \mathrm{H}_{5}\right)_{2}= \\
& \mathrm{C}_{6} \mathrm{H}_{4}<\mathrm{CH}_{2}>\mathrm{CH}\left(\mathrm{COOC}_{2} \mathrm{H}_{5}\right)_{2}+\mathrm{CH}_{2}\left(\mathrm{COOC}_{2} \mathrm{H}_{5}\right)_{2}+2 \mathrm{NaBr} .
\end{aligned}
$$

This difficulty can be got over, however, by substituting in the above reaction the sodium-derivative of ethylic monochlormalonate for that of ethylic malonate.

In this case, the following reaction takes place:-

$$
\begin{aligned}
& \mathrm{C}_{6} \mathrm{H}_{4}\left(\mathrm{CH}_{2} \mathrm{Br}\right)_{2}+2 \mathrm{NaClC}\left(\mathrm{COOC}_{2} \mathrm{H}_{5}\right)_{2}= \\
& \mathrm{C}_{6} \mathrm{H}_{4}\left[\mathrm{CH}_{2} \cdot \mathrm{ClC}\left(\mathrm{COOC}_{2} \mathrm{H}_{5}\right)_{2}\right]_{2}+2 \mathrm{NaBr} \text {, } \\
& \text { Ethylic ortho-xylylenedichlordimalonate. }
\end{aligned}
$$

ethylic ortho-xylylenedichlordimalonate being formed.

If this ethereal salt is reduced with zinc and acetic acid, the two chlorine-atoms become displaced by hydrogen and ethylic orthoxylylenedimalonate is formed, thus :-

$$
\begin{aligned}
\mathrm{C}_{6} \mathrm{H}_{4}\left[\mathrm{CH}_{2} \cdot \mathrm{ClC}\left(\mathrm{COOC}_{2} \mathrm{H}_{5}\right)_{2}\right]_{2}+ & +4 \mathrm{H}= \\
& \mathrm{C}_{6} \mathrm{H}_{4}\left[\mathrm{CH}_{2} \cdot \mathrm{CH}\left(\mathrm{COOC}_{2} \mathrm{H}_{5}\right)_{2}\right]_{2}+2 \mathrm{HCl} . \\
& \text { Ethylic ortho-xylylenedimalonate. }
\end{aligned}
$$

If this substance be now treated with two molecular proportions of sodic ethylate, the two hydrogen-atoms of the $\mathrm{CH}$ groups will be displaced by sodiam, forming a disodium-derivative, which when mixed 
with an ethereal solution of iodine is converted quantitatively into ethylic tetrahydronaphthalenetetracarboxylate, thus :-

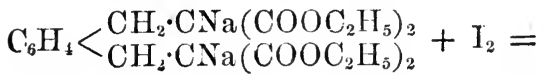

$$
\begin{aligned}
& \mathrm{C}_{6} \mathrm{H}_{4} \overbrace{\mathrm{CH}_{2} \cdot \mathrm{C}\left(\mathrm{COOC}_{2} \mathrm{H}_{5}\right)_{2}}^{\mathrm{CH}_{2} \cdot \mathrm{C}\left(\mathrm{COOC}_{2} \mathrm{H}_{5}\right)_{2}}+2 \mathrm{NaI} \text {. }
\end{aligned}
$$

\section{Orthoxylylene Dibromide, $\mathrm{C}_{6} \mathrm{H}_{4}<\mathrm{CH}_{2} \mathrm{CH}_{2}$ (2) ${ }^{\mathrm{CH}_{2}} \mathrm{Br}$ (1)}

Ortho-xylylene dibromide is best prepared in the following way :50 grams of pure ortho-xylene are placed in a large tubulated retort connected with a long reflux condenser, and after being heated carefully to $125-130^{\circ}$ by means of an oil-bath, the calculated quantity of bromine (160 grams) is allowed to drop in very slowly through a dropping funnel. Torrents of hydrogen bromide are evolved, but the liquid should remain almost colourless till the end of the operation, when it usually assumes a slightly brownish tint. It is necessary to add the bromine very slowly, otherwise the reaction is apt to become violent, in which case, if the condensing apparatus is not very good, small quantities of ortho-xylene get carried away by the hydrogen bromide rapours.

It is also important to keep the temperature of the oil-bath between $125^{\circ}$ and $130^{\circ}$ (the temperature $150-155^{\circ}$, first given in the Berichte, 17, 123, is too high), and then the resulting product is almost colourless. As soon as the reaction is over, the resulting crude dibromide is poured out into a small beaker, covered up with a watch-glass, and allowed to stand for 24 hours, when the whole solidifies to a cake of crystals. These are purified from oily motherliquor by spreading out on a porous plate, and are thus obtained almost colourless and sufficiently pure for ordinary purposes. If the reaction has been successful, the yield of this product should be about $85-90$ grams.

In order to obtain pure ortho-xylylene dibromide these crystals are washed with chloroform and then recrystallised from this solvent. An analysis gave the following numbers:-

0.2122 gram substance gave 0.3017 gram $\mathrm{AgBr}$.

$$
\begin{aligned}
& \text { Theory. } \\
& \mathrm{C}_{6} \mathrm{H}_{4}\left(\mathrm{CH}_{2} \mathrm{Br}\right)_{2} \text {. Found. } \\
& \text { Br....... } 60.60 \text { per cent. } \quad 60.50 \text { per cent. }
\end{aligned}
$$

Ortho-xylylene dibromide melts at $93^{\circ}$, and dissolves in sbout five parts of ether. It crystallises from chloroform or ether in magnifi- 
cent, large, glittering crystals, which Professor Haushofer was kind enough to examine crystallographically. He gave me the following account of his experiments :-

\section{Crystalline System: Rhombic. \\ $a: b: c=0.8581: 1: 0.5014$.}

Well developed crystals of the combination P.œP $=(111)(110)$, with tolerably smooth and even surfaces but cloudy. The prismatic faces $(p)$ very subordinate and often reduced to narrow streaks.

$$
\begin{aligned}
& \text { Measured. Calculated. } \\
& p: p=(110)(1 \overline{1} 0)=* 98 \quad 44^{\prime} \quad-\quad-\left(\begin{array}{l}
\text { brachydiagonal } \\
\text { prismatic edge }) .
\end{array}\right. \\
& p: p=(110)(\overline{1} 10)=81 \quad 16 \quad 81^{\circ} \quad 16^{\prime} \text { (macrodiagonal). }
\end{aligned}
$$

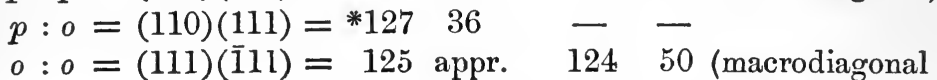

$$
\begin{aligned}
& \text { polar edge). } \\
& o: o=(111)(1 \overline{1} 1)=132 \quad 27 \quad 133 \quad 10 \text { (brachydiagonal) } .
\end{aligned}
$$

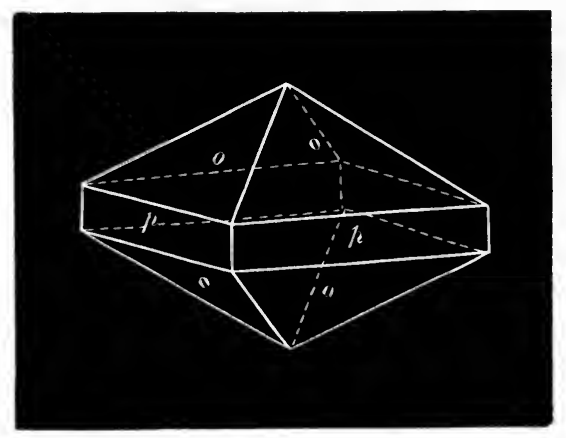

Before proceeding further, it was necessary to prove that the bromine-atoms in this product were situated one in each methyl group. This was done in the following way: 5 grams of the pure substance was mixed with an excess of sudium carbonate solution, and boiled with a reflux condenser till the oily drops had entirely disappeared, which was the case in about three hours. The solution was then filtered from a small quantity of an insoluble substance, and extracted twice with pure ether. The ethereal solution after drying, filtering, and evaporating, deposited a colourless syrup, which, on standing, solidified almost completely to a mass of crystals, melting at about $66-72^{\circ}$. To purify these, the crude mass was spread out on a porous plate, recrystallised once from ether, and analysed :-

0.1102 gram substance gave 0.0744 gram $\mathrm{H}_{2} \mathrm{O}$ and 0.2792 gram $\mathrm{CO}_{2}$. 


\begin{tabular}{|c|c|c|c|c|}
\hline $\mathrm{C}_{6} \mathrm{I}$ & $\begin{array}{l}\text { Theory } \\
\mathrm{C}_{4}\left(\mathrm{CH}_{2} \cdot \mathrm{C}\right.\end{array}$ & & Found. & \\
\hline$C \ldots \ldots$ & $69 \cdot 56$ & cent. & $69 \cdot 11$ & $r$ cent. \\
\hline$H \ldots \ldots$. & $7 \cdot 25$ & " & $7 \cdot 50$ & , \\
\hline $0 \ldots \ldots$ & $23 \cdot 19$ & ", & $23 \cdot 39$ & " \\
\hline
\end{tabular}

This substance now melted at about $62-64^{\circ}$, and proved to be identical with the phthalyl alcohol first obtained by Hessert (Ber., 12, 646). When mixed with concentrated sulphuric acid, the dark-red solution described by him was first formed; but this colour in a short time disappeared owing to the substance being completely decomposed. The constitution of this alcohol was definitely proved by the fact that when oxidised either with permanganate or with chromic mixture, it yielded phthalic acid.

In preparing and experimenting with ortho-xylylene dibromide, it is necessary to work either in a very good draught closet or, better still, in the open air, as the vapours of this substance have a most irritating and painful effect on the skin and eyes.

\section{Hydrindonaphthenedicarboxylic Acid, $\mathrm{C}_{6} \mathrm{H}_{4}<\mathrm{CH}_{2}>\mathrm{C}(\mathrm{COOH})_{2}$.}

In preparing this acid, the following method has been found to give the best results :-

0.9 gram of sodium is dissolved in 10 grams of absolute alcohol, and the solution, while still warm, is mixed with 40 c.c. of pure dry ether. To this solution, 3 grams of ethyl malonate dissolved in 10 c.c. of ether and 5 grams of ortho-xylene dibromide dissolved in 30 or 40 c.c. of ether are rapidly added, and the whole allowed to remain for about three hours at the ordinary temperature. Shortly after mixing, a thick voluminous precipitate of the sodium-derivative of ethyl malonate separates out; but this soon gives place to a heavy, sandy precipitate, which consists not only of sodium bromide, but also contains an organic sodium compound-possibly an intermediate substance of the formula $\mathrm{CH}_{2} \mathrm{Br}^{\circ} \mathrm{C}_{6} \mathrm{H}_{4} \cdot \mathrm{CH}_{2} \cdot \mathrm{CNa}\left(\mathrm{COOC}_{2} \mathrm{H}_{5}\right)_{2}$.

After standing for three hours, the ethereal solation is separated* from the precipitate by filtration, and evaporated. The residue from this, as well as the precipitate, are dissolved in a little pure alcohol and digested with an excess of alcoholic potash, using a reflux condenser until, on the addition of water, no more oil is precipitated; this usually takes place in about 10 minutes. After distilling off the alcohol, the residue is dissolved in a considerable quantity of water, gently evaporated on a water-bath to get rid of the last traces of

* If the ether be distilled off without separating the solution from the precipitate, vigorous bumping usually sets in. 
alcohol, filtered if necessary, and acidified with dilute sulphuric acid; this often causes the precipitation of a small quantity of a resinous substance, which must be filtered off before proceeding further. The clear solution is now extracted several times with pure ether, the ethereal solution dried over a little calcic chloride, and evaporated. In this way, hydrindonaphthenedicarboxylic acid is obtained as an almost colourless crystalline mass which, after removal of traces of oil by spreading it on a porous plate and recrystallising from water, is easily obtained in a pure state. The analysis gave the following numbers :-

0.1460 gram substance gave 0.0662 gram $\mathrm{H}_{2} \mathrm{O}$ and 0.3411 gram $\mathrm{CO}_{2}$.

\begin{tabular}{|c|c|c|}
\hline$y$. & $\begin{array}{l}\text { Theory. } \\
\mathrm{C}_{11} \mathrm{H}_{10} \mathrm{O}_{4} \text {. } \\
64.07 \text { per cent. }\end{array}$ & $\begin{array}{l}\text { Found. } \\
63 \cdot 72 \text { per cent. }\end{array}$ \\
\hline & 4.86 & $5.03 \quad$ \\
\hline . & $31 \cdot 07$ & $31 \cdot 25$ \\
\hline
\end{tabular}

Hydrindonaphthenedicarboxylic acid crystallises from water in rhombic plates which melt at $199^{\circ}$, and, at a slightly higher temperature, decompose rapidly into carbonic anhydride and hydrindonaphthenemonocarboxylic acid. The dicarboxylic acid is fairly easily soluble in hot water, alcohol, and ether, but much less so in cold water.

The silver salt was prepared by adding nitrate of silver to a warm neutral solution of the ammonium salt; it is obtained as a white, amorphous precipitate, which becomes crystalline on standing. After collecting, washing with a little water, and drying, first on a porous plate and then at $100^{\circ}$, it was analysed with the following results :-

I. 0.1670 gram substance gave 0.0305 gram $\mathrm{H}_{2} \mathrm{O}, 0.1920$ gram $\mathrm{CO}_{2}$, and 0.0868 gram silver.

II. $0 \cdot 1900$ gram substance gave 0.0975 gram silver.

\begin{tabular}{|c|c|c|c|c|}
\hline & & & & \\
\hline & $\begin{array}{l}\text { Theory. } \\
{ }_{1} \mathrm{H}_{8} \mathrm{O}_{4} \mathrm{~A}\end{array}$ & & I. & II. \\
\hline C.. & $31 \cdot 43$ & cent. & $31 \cdot 35$ & - \\
\hline H....... & 1.90 & , & $2 \cdot 03$ & 一 \\
\hline $\mathrm{Ag} \quad \ldots .$. & $51 \cdot 42$ & $"$ & 51.97 & $51 \cdot 32$ \\
\hline $0 \ldots \ldots$ & $15 \cdot 24$ & $"$ & $14 \cdot 65$ & - \\
\hline
\end{tabular}

Hydrindonaphthenemonocarboxylic Acid, $\mathrm{C}_{6} \mathrm{H}_{4}<_{\mathrm{CH}_{2}}^{\mathrm{CH}_{2}}>\mathrm{CH} \cdot \mathrm{COOH}$.

In order to prepare this acid, the dicarboxylic acid (1 gram) is rapidly distilled from a very small retort over a naked Bunsen flame. 
The acid first melts and decomposes with evolution of carbonic anhydride; on raising the temperature, an almost colourless oil distils over, which crystallises at once on the cool sides of the neck of the retort. No residue is left if the distillation is conducted rapidly. Hydrindonaphthenemonocarboxylic acid obtained in this way is almost pure; for the analysis - which was made with lead chromate owing to the difficulty in burning the substance-it was once recrystallised from water:-

I. 0.1642 gram substance gave 0.0945 gram $\mathrm{H}_{2} \mathrm{O}$ and 0.4451 gram $\mathrm{CO}_{2}$.

II. Details of this analysis are missing.

\begin{tabular}{|c|c|c|c|}
\hline \multirow{2}{*}{\multicolumn{2}{|c|}{$\begin{array}{l}\text { Theory. } \\
\mathrm{C}_{10} \mathrm{H}_{10} \mathrm{O}_{2} \text {. }\end{array}$}} & \multicolumn{2}{|c|}{ Found. } \\
\hline & & I. & II. \\
\hline C ... & 74.07 per cent. & $73 \cdot 91$ & $73 \cdot 67$ \\
\hline H... & $6 \cdot 17$ & $6 \cdot 39$ & $6 \cdot 70$ \\
\hline $0 \ldots . .$. & $19 \cdot 75$ & $19 \cdot 70$ & $19 \cdot 63$ \\
\hline
\end{tabular}

Some interesting experiments with hydrindonaphthenemonocarboxylic acid have been made by E. Scherks (Ber., 18, 378).

Hydrindonaphthenemonocarboxylic acid is formed directly by the action of ortho-xylylene dibromide on the sodium-derivative of ethylic acetoacetate and subsequent hydrolysis with alcoholic potash :-

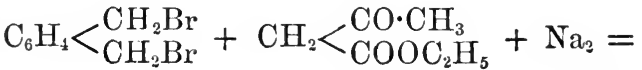

$$
\begin{aligned}
& 2 \mathrm{NaBr}+\mathrm{C}_{6} \mathrm{H}_{4}<\mathrm{CH}_{2}^{\mathrm{CH}_{2}}>\mathrm{C}<\mathrm{COOC}_{2} \mathrm{H}_{5} \cdot \mathrm{CH}_{3}
\end{aligned}
$$

$$
\begin{array}{r}
\mathrm{C}_{6} \mathrm{H}_{4}<\mathrm{CH}_{2}^{\mathrm{CH}_{2}}>\mathrm{C}<{ }_{\mathrm{COOC}}^{\mathrm{CO} \cdot \mathrm{CH}_{3}}+2 \mathrm{KOH}=\mathrm{CH}_{3} \cdot \mathrm{COOK}+ \\
\mathrm{C}_{2} \mathrm{H}_{5} \cdot \mathrm{OH}+\mathrm{C}_{6} \mathrm{H}_{4}<_{\mathrm{CH}_{2}}^{\mathrm{CH}_{2}}>\mathrm{CH} \cdot \mathrm{COOK} .
\end{array}
$$

The yield, however, in this case is considerably less than that obtained when ethyl malonate is used.

When hydrindonaphthenemonocarboxylic acid is oxidised with alkaline permanganate, it is converted into the orthocarboxylic acid of phenylglyoxylic acid (Scherks, loc. cit.)-

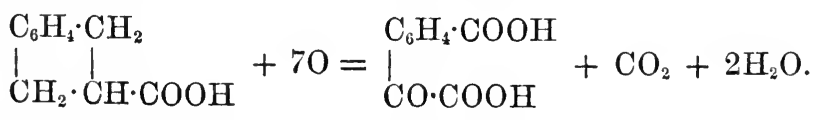

This acid, on reduction, yields phthalidecarboxylic acid :-

$$
\underbrace{\mathrm{C}_{6} \mathrm{H}_{4} \cdot \mathrm{COOH}}_{\mathrm{CO} \cdot \mathrm{COOH}}+\mathrm{H}_{2}=\underset{\mathrm{CH}(\mathrm{OH}) \cdot \mathrm{COOH}}{\mathrm{C}_{6} \mathrm{H}_{4} \cdot \mathrm{COOH}}=\underset{\mathrm{COOH} \cdot \mathrm{CH}-\mathrm{O}}{\mathrm{C}_{6} \mathrm{H}_{4} \cdot \mathrm{CO}}+\mathrm{H}_{2} \mathrm{O} \text {, }
$$


an acid which, when heated to temperatures above $180^{\circ}$, is converted into phthalide and carbonic anhydride, thus :-

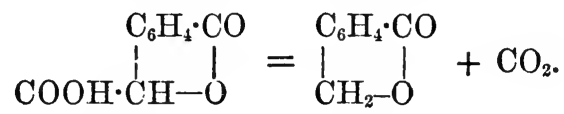

As is seen at a glance, this interesting decomposition of hydrindonaphthenemonocarboxylic acid on oxidation goes far to prove the formula ascribed to the acid in this paper.

Hydrindonaphthenemonocarboxylic acid melts at $130^{\circ}$, and, when strongly heated, distils without decomposition.

It is sparingly soluble in cold water, but dissolves in about 120 parts of boiling water. If this hot solution is allowed to cool slowly, it becomes first quite milky, and then deposits the acid in slender, colourless needles. The silver salt was prepared from the ammonium salt in the usual way. It is a bulky white precipitate, almost insoluble in cold, but apparently slightly soluble in hot water. For the analysis, it was well washed with water, and dried over sulphuric acid in a vacuum.

0.1186 gram substance gave 0.0476 gram silver.

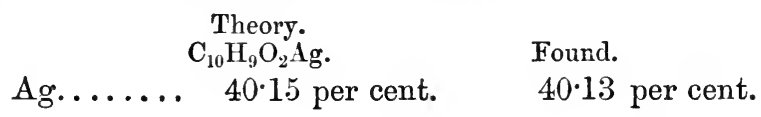

Action of Benzyl Chloride on the Sodium-derivative of Ethylic Acetylenetetracarboxylate.

\section{$\mathrm{C}_{6} \mathrm{H}_{5} \cdot \mathrm{CH}_{2} \cdot \mathrm{CH} \cdot \mathrm{COOH}$ \\ Benzylsuccinic Acid,

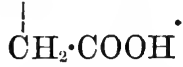

Before using ethylic acetylenetetracarboxylate for the synthesis of tetrahydronaphthalene-derivatives it was necessary to prove that the former could form a disodium-derivative when treated with sodic ethylate. To ascertain this, a quantity of the ethylic salt was dissolved in alcohol and mixed with the calculated quantity of sodic ethylate ( 2 mols.), and the resulting clear solution agitated with three times its volume of pure dry ether. This caused the precipitation of the disodium-derivative as a heavy white mass, which was collected and well washed by means of a vacuum pump, first with a mixture of alcohol and ether, and lastly with pure ether. The product was dried over sulphuric acid in a vacuum, and analysed with the following result :-

0.2323 gram substance gave 0.0889 gram $\mathrm{Na}_{2} \mathrm{SO}_{4}$. 


$$
\begin{aligned}
& \text { Theory. } \\
& \mathrm{C}_{14} \mathrm{H}_{20} \mathrm{Na}_{2} \mathrm{O}_{4} \text {. Found. } \\
& \text { Na...... 12.71 per cent. } 12.38 \text { per cent. }
\end{aligned}
$$

The action of benzyl chloride on this sodium compound was next tried. 2 grams of the latter were mixed with a little alcohol and heated in a sealed tube with excess of benzyl chloride for a short time at $150^{\circ}$. The product was diluted with water, the oil thus precipitated extracted with ether, and the ethereal solution washed, dried over calcic chloride, and evaporated; the colourless oil which remained was then hydrolysed by boiling it with excess of alcoholic potash. The product was dissolved in water, evaporated on a water-bath until all the alcohol had been driven off, acidified with dilute sulphuric acid, and extracted about 20 times with pure ether. On distilling off the ether, a slightly brownish syrup remained behind which, when heated, gave off carbonic anhydride, and was converted into a brownish oily mass; this on standing crystallised for the most part. The crystals were purified by washing with chloroform, in which they are, sparingly soluble, and then analysed with the following results :-

$$
\begin{aligned}
& \text { Theory. } \\
& \mathrm{C}_{11} \mathrm{H}_{12} \mathrm{O}_{4} \text {. Found. } \\
& \text { C ...... } 63.46 \text { per cent. } \quad 63 \cdot 14 \text { per cent. } \\
& \text { H...... } 5.77 \text { " } \\
& \text { O ..... "30.77 " }
\end{aligned}
$$

This substance was therefore benzylsuccinic acid.

I did not examine this acid fưrther, as the only reason for preparing it was to ascertain whether the sodium-atoms of the disodiumderivative of ethylic acetylenetetracarboxylate conld be displaced by organic radicles.

This reaction has, since the first publication of these results (Ber., 17, 449), been carefully studied by Bischoff and Rach (Ber., 17, 2783).

\section{Tetrahydronaphthalenedicarboxylic Acid $(\beta \beta)$,

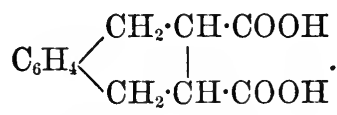

In using ortho-xylylene dibromide for the following synthetical experiments, the sparing solubility of the substance in alcohol was at first a source of trouble; it was, however, soon found that this could be got over by first dissolving the bromide in ether, adding the alcohol, and then distilling off the ether, when a supersaturated solution remains which does not crystallise for some time. In preparing tetrahydronaphthalenedicarboxylic acid, the following method was found to give good results :- 
A mixture of 1 molecular proportion of ortho-xylylene dibromide dissolved in 5 parts of alcohol, 1 molecular proportion of ethylic acetylenetetracarboxylate in 5 parts of alcohol, and 2 atomic proportions of sodium in 15 parts of alcohol were heated together in a sealed tube for six hours at $130^{\circ}$; it was found that sodium bromide separated, and on opening the tube a slight pressure was observable. The contents were emptied into a flask, mixed with a strong solution of alcoholic potash (containing 8 mols. KHO), and allowed to stand at the ordinary temperature for 12 hours; at the end of that time, the mass was gently warmed on a waterbath until a sample taken out dissolved in water without the precipitation of oily drops. The whole was then mixed with water, evaporated on a water-bath almost to dryness, again dissolved in a small quantity of water, acidified with dilute sulphuric acid, and the solution filtered from a few particles of resinous matter which had separated. The clear liquid was now extracted 10 times with pure ether (free from alcohol), the ethereal solution dried over calcic chloride, filtered and evaporated, when an almost colourless syrup was obtained consisting probably for the most part of tetrahydronaphthalenetetracarboxylic acid, $\mathrm{C}_{6} \mathrm{H}_{4} \succ_{\mathrm{CH}_{2} \cdot \mathrm{C}(\mathrm{COOH})_{2}}^{\mathrm{CH}_{2} \cdot \mathrm{C}(\mathrm{COOH})_{2}}{ }^{\mathrm{C}_{2}}$

This was not further examined, but directly transferred to a small flask and heated in an oil-bath gradually to $185^{\circ}$. During this operation, a considerable quantity of carbonic anhydride and aqueous vapour was given off, and a thick oily residue was left which, on cooling, solidified to a mass of crystals. These were pounded up, agitated with a little dry ether, filtered, and well washed with ether; they were thus obtained as a white crystalline powder, which on examination proved to be the anhydride of tetrahydronaphthalenedicarboxylic acid.

The formation of this anhydride from tetrahydronaphthalenetetracarboxylic acid is represented in the following equation:-

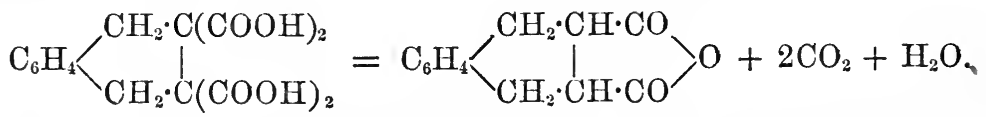

This product after recrystallising from ether gave the following numbers on analysis :-

0.2000 gram substance gave 0.5214 gram $\mathrm{CO}_{2}$ and $0.0882 \mathrm{H}_{2} \mathrm{O}$.

\begin{tabular}{|c|c|c|}
\hline & $\begin{array}{l}\text { Theory. } \\
\mathrm{C}_{12} \mathrm{H}_{10} \mathrm{O}_{3} \text {. } \\
71 \cdot 28 \text { per cent. }\end{array}$ & $\begin{array}{l}\text { Found. } \\
71 \cdot 10 \text { per cent. }\end{array}$ \\
\hline & $4.95 \quad$ & $4: 90$ \\
\hline & $23 \cdot 76$ & $24 \cdot 00$ \\
\hline
\end{tabular}


The anhydride is sparingly soluble in ether, but more readily so in alcohol and chloroform. On slowly evaporating its solution in warm ether, it crystallises in large four-sided prisms which melt at $184^{\circ}$. The yield obtained was 70 per cent. of the theoretical.

If the vapour of this anhydride is passed through a red-hot tube, a considerable quantity of naphthalene is formed, a decomposition which is referred to in the introduction. The naphthalene thus obtained was not analysed, but was sharply characterised by its melting point $\left(79^{\circ}\right)$, its odour, and by converting it into the characteristic picric acid compound ; as there could be no doubt of its identity with ordinary naphthalene, it was not thought necessary to prepare enough for analysis, which would have entailed the loss of a large quantity of valuable material.

The anhydride of tetrahydronaphthalenedicarboxylic acid is insoluble in cold water, but is slowly altered by boiling water with formation of the acid. In order to obtain the acid, the anhydride was warmed with a little sodic hydrate solution, in which it dissolves fairly easily, the solution cooled and acidified with dilute sulphuric acid. On standing, the dicarboxylic acid separates as a white crystalline powder, which when examined under the microscope is found to consist of small rhombic plates. Analysis gave the following results :-

0.1740 gram substance gave 0.4154 gram $\mathrm{CO}_{2}$ and 0.0877 gram $\mathrm{H}_{2} \mathrm{O}$.

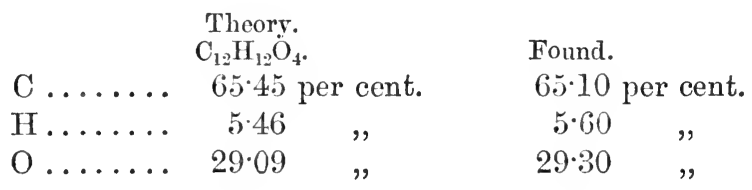

Tetrahydronaphthalenedicarboxylic acid is sparingly soluble in cold, but more easily so in hot water. It dissolves freely in chloroform, alcohol, and acetone. The pure acid melts at $199^{\circ}$, being at the same time converted into its anhydride; this is clearly shown by the fact that if the substance which has been used for determining the melting point of the acid, be allowed to cool, and then remelted, it will now melt at $184^{\circ}$, which is the melting point of the anhydride.

The silver salt of this acid is prepared by adding silver nitrate to a neutral solution of the ammonium salt, and is thus obtained as a white amorphous precipitate, which on standing becomes crystalline. It is rapidly discoloured by the action of light, and should therefore be prepared in the dark. Analysis gave the following result:-

0.1665 gram substance gave 0.2042 gram $\mathrm{CO}_{2}, 0.0338$ gram $\mathrm{H}_{2} \mathrm{O}$, and 0.0825 gram $\mathrm{Ag}$. 


\begin{tabular}{|c|c|c|c|c|}
\hline \multicolumn{3}{|c|}{$\begin{array}{c}\text { Theory. } \\
\mathrm{C}_{12} \mathrm{H}_{10} \mathrm{O}_{4} \dot{\mathrm{A}} g_{2} \text {. }\end{array}$} & \multirow{2}{*}{\multicolumn{2}{|c|}{$\begin{array}{l}\text { Found. } \\
33 \cdot 44 \text { per cent }\end{array}$}} \\
\hline C $\ldots \ldots \ldots$ & $33 \cdot 18$ & cent. & & \\
\hline H....... & $2 \cdot 30$ & " & $2 \cdot 26$ & $"$ \\
\hline $\mathrm{Ag}$ & $49 \cdot 77$ & " & 49.55 & , \\
\hline O. & 14.75 & " & 14.75 & " \\
\hline
\end{tabular}

If this silver salt is gently heated in a test-tube, it is easily decomposed, and on further heating a mixture of naphthalene and the anhydride of tetrahydronaphthalenedicarboxylic acid distils and condenses on the cooler part of the tube; there is no charring.

\section{Ethylic Ortho-xylylenedichlorodimalonate, $\dot{\mathrm{C}}_{6} \mathrm{H}_{4}\left[\mathrm{CH}_{2} \cdot \mathrm{CCl} \text { : }\left(\mathrm{COOC}_{2} \mathrm{H}_{5}\right)_{2}\right]_{2}$.}

In order to prepare this substance, pure ethylic monochloromalonate ( 2 mols.) is dissolved in the calculated quantity of sodic ethylate (2 mols.), the mixture diluted with its own volume of pure dry ether, and then slowly added to an ethereal solution of ortho-xylylene dibromide ( 1 mol.). The whole is now gently warmed on a waterbath for about three hours, at the end of which time the reaction is complete.

In order to isolate the product, water is added, the ethereal solution separated, well washed with water, dried over calcic chloride, and the ether distilled off, when a thick, almost colourless, oil remains consisting of nearly pure ethylic ortho-xylylenedichlorodimalonate. After the substance had been allowed to stand for some days over sulphuric acid in a vacuum, a chlorine determination was made with the following result:-

$$
\begin{aligned}
& \text { Theory. } \\
& \mathrm{C}_{22} \mathrm{H}_{28} \mathrm{Cl}_{2} \mathrm{O}_{8} \text {. Found. } \\
& \text { Cl ...... } 14.49 \text { per cent. } \quad 13.9 \text { per cent. }
\end{aligned}
$$

Ethylic ortho-xylylenedichlorodimalonate is a thick, almost colourless syrup, which on long standing solidifies to large, colourless crystals. It cannot be purified by distillation even in a vacuum, but when prepared by the above method it is pure enough for all further experiments.

\section{Orthophenylenediacrylic Acid, $\mathrm{C}_{6} \mathrm{H}_{4}(\mathrm{CH}: \mathrm{CH} \cdot \mathrm{COOH})_{2}$.}

The potassium salt of this acid is formed when ethylic orthoxylylenedichlorodimalonate is boiled with alcoholic potash, thus :$\mathrm{C}_{6} \mathrm{H}_{4}\left[\mathrm{CH}_{2} \cdot \mathrm{CCl}\left(\mathrm{COOC}_{2} \mathrm{H}_{5}\right)_{2}\right]_{2}+8 \mathrm{KOH}=\mathrm{C}_{6} \mathrm{H}_{4}(\mathrm{CH}: \mathrm{CH} \cdot \mathrm{COOK})_{2}+$ $4 \mathrm{C}_{2} \mathrm{H}_{5} \cdot \mathrm{OH}+2 \mathrm{KCl}+2 \mathrm{~K}_{2} \mathrm{CO}_{3}+2 \mathrm{H}_{2} \mathrm{O}$. 
This decomposition is carried out in the following way :-

Ethylic ortho-xylylenedichlorodimalonate is dissolved in a little alcohol, the solution mixed with excess of concentrated alcoholic potash (10 mols. of $\mathrm{KOH})$, and heated to boiling on a water-bath for four hours in a flask connected with a reflux condenser. During the operation, a quantity of potassic chloride and carbonate separates out as a heavy sandy precipitate, which sometimes causes bumping to such an extent that it is necessary to remove it by filtration before the hydrolysis can be proceeded with. As soon as this is completed, the alcohol is distilled off as far as possible, the residue dissolved in water, evaporated on a water-bath till all the alcohol has been driven off, and the solution filtered and acidified with dilute sulpharic acid. This canses the new acid to be precipitated in the form of light yellow flocks; when collected, well washed with water and dried, it is nearly pure. For analysis, the substance was washed with acetone, redissolved in dilute carbonate of soda, the solution boiled for some time with animal charcoal, filtered, and the acid reprecipitated with dilute sulphuric acid. In this way it was obtained as an almost colourless flocculent mass, which after very careful washing and drying gave the following numbers on analysis :-

I. 0.1365 gram substance gave 0.3306 gram $\mathrm{CO}_{2}$ and 0.0605 gram $\mathrm{H}_{2} \mathrm{O}$.

II. 0.1612 gram substance gave 0.3900 gram $\mathrm{CO}_{2}$ and 0.0723 gram $\mathrm{H}_{2} \mathrm{O}$.

\begin{tabular}{|c|c|c|c|}
\hline & Theory. & \multicolumn{2}{|c|}{ Found. } \\
\hline & $\mathrm{C}_{12} \mathrm{H}_{10} \mathrm{O}_{4}$. & I. & II. \\
\hline$C \ldots \ldots$ & 66.05 p. с. & 66.05 & 65.98 р. с. \\
\hline $\mathrm{H}$. . & $4 \cdot 59 \quad$ & $4 \cdot 96$ & 4.98 \\
\hline 0 & $29 \cdot 36$, & $28 \cdot 89$ & $29 \cdot 04$ \\
\hline
\end{tabular}

Orthophenylenediacrylic acid is very sparingly soluble in toluene, alcohol, benzene, chloroform, and ether, slightly more soluble in acetone and water. It can with difficulty be recrystallised from acetone, but as it is so very pure when prepared as above, this is not necessary.

Orthophenylenediacrylic acid melts above $300^{\circ}$, and when heated in small quantities in a test-tube it sublimes, but with considerable decomposition. In order to prepare the silver salt, the pure acid was dissolved in dilnte ammonia, carefully neutralised with dilute nitric acid, and precipitated with nitrate of silver. In this way an almost colourless insoluble silver salt was obtaincd, which after well washing and drying over sulphuric acid in a vacuum gave the following result on analysis :- 
0.2982 gram substance gave 0.1477 gram Ag.

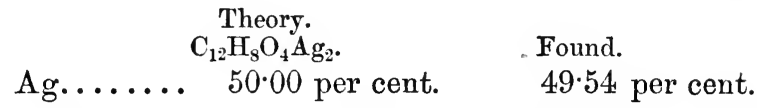

If the solution of orthophenylenediacrylic acid in dilute sodic hydrate, be treated with benzene and potassic permanganate (according to the method described by Einhorn for the preparation of orthonitrobenzoic aldehyde, Ber., 17, 119), an oil is obtained which has all the properties of an aldehyde, and which, as it gives phthalic acid on oxidation, is possibly phthalic aldehyde.

\section{Ethylic Ortho-xylylenedimalonate, $\mathrm{C}_{6} \mathrm{H}_{4}\left[\mathrm{CH}_{2} \cdot \mathrm{CH}:\left(\mathrm{COOC}_{2} \mathrm{H}_{5}\right)_{2}\right]_{2}$.}

This compound is formed, as was mentioned in the introduction, by the action of reducing agents on ethylic ortho-xylylenedichlorodimalonate. In preparing it, about $10-15$ grams of the latter are dissolved in $80-100$ c.c. of glacial acetic acid, and then zinc-dust is slowly added in small quantities at a time, the whole being vigorously shaken after each addition. It is important to add only small quantities of zinc-dust at a time, otherwise it is apt to cake together at the bottom of the flask, and thus be of little use. During the reaction, a considerable rise of temperature takes place, and after a time the liquid becomes quite thick from separation of zincic acetate. When it has reached this stage, a little water is added, and the whole warmed on a water-bath for about half an hour. The supernatant liquid is now poured off from the undissolved zinc into about three times its volume of water, and the oil thus precipitated extracted three or four times with ether. The residual zinc is also extracted four or five times with ether, the two ethereal solutions mixed together, and freed from acetic acid by washing first with water and then with dilute carbonate of soda solution. Lastly, the ethereal extract is dried over potassic carbonate, and the ether distilled off, when a colourless oil remains, consisting of nearly pure ethylic orthoxylylenedimalonate.

In preparing this substance, it often happens that the product is found still to contain chlorine; if this is the case, it is necessary to repeat the reduction until all the chlorine has been removed.

Ethylic ortho-xylylenedimalonate cannot be purified by distillation even in a vacuum, and in order to prepare it for analysis, advantage had to be taken of the sodium-derivative which it forms. A weighed quantity of the oil ( 1 mol.) when dissolved in ether and mixed with an ethereal solution of sodic ethylate ( 2 mols.) is converted into a disodium-derivative, which separates in white flocks, whereas any 
impurities present remain in solution, and can be removed by collect. ing the precipitate and washing it with ether. If the sodiumderivative thus obtained be mixed with dilute sulphoric acid, it is instantly decomposed, and pure ethylic ortho-xylylenedimalonate is set free. This after extraction with ether and washing with sodic carbonate is obtained as a thick, colourless oil. In order to be certain of the purity of the sample analysed, this operation was repeated twice, and with the product thus obtained, the following analysis was made :-

0.1365 gram substance gave 0.3140 gram $\mathrm{CO}_{2}$ and 0.0884 gram $\mathrm{H}_{2} \mathrm{O}$.

\begin{tabular}{|c|c|c|}
\hline$C \ldots \ldots$ & $\begin{array}{l}\text { Theory. } \\
\mathrm{C}_{22} \mathrm{H}_{30} \mathrm{O}_{8} \text {. } \\
62 \cdot 56 \text { per cent. }\end{array}$ & $\begin{array}{l}\text { Found. } \\
62 \cdot 73 \text { per cent. }\end{array}$ \\
\hline H. & $7 \cdot 11$ & $7 \cdot 19$ \\
\hline & $30 \cdot 33$ & $30 \cdot 18$ \\
\hline
\end{tabular}

Ethylic ortho-xylylenedimalonate thus prepared is a thick colourless syrup, insoluble in water, but easily soluble in alcohol, ether, and most of the usual solvents.

Its most important property is that when mixed with sodic ethylate it forms a disodium-derivative, and before proceeding with this research it appeared necessary to prove the constitution of this compound by analysis.

To prepare as pure a sample as possible, pure ethylic orthoxylylenedimalonate was dissolved in pure dry ether and mixed with the calculated quantity of an ethereal solution of sodic ethylate; after the precipitation was complete, the sodium-derivative was collected on a filter-pump as rapidly as possible, washed several times with ether, and then spread out on a porous plate, and transferred to a vacuum desiccator. The following is the result of analysis :-

$$
\begin{aligned}
& \text { Theory. } \\
& \mathrm{C}_{22} \mathrm{H}_{28} \mathrm{O}_{8} \mathrm{Na}_{2} \text {. Found. } \\
& \mathrm{Na} . . . . .996 \text { per cent. } \quad 9 \cdot 12 \text { per cent. }
\end{aligned}
$$

This compound is a colourless, hygroscopic mass, and for this reason the numbers obtained were rather lower than those required by theory. When mixed with acids, it is instantly decomposed with regeneration of ethylic ortho-xylylenedimalonate.

In experimenting further with the latter substance, it was not purified by conversion into the disodium-derivative and reprecipitation as described above, as this is always attended with great loss of substance, probably owing to partial hydrolysis. It was found that the crude product of the reduction of ethylic ortho-xylylenedichloro- 
malonate was sufficiently pure to render this process of purification unnecessary.

\section{Orthophenylenedipropionic Acid, $\mathrm{C}_{6} \mathrm{H}_{4}\left(\mathrm{CH}_{2} \cdot \mathrm{CH}_{2} \cdot \mathrm{COOH}\right)_{2}$.}

This acid is obtained by the hydrolysis of ethylic ortho-xylylenedimalonate with alcoholic potash, thas:-

$\mathrm{C}_{6} \mathrm{H}_{4}\left[\mathrm{CH}_{2} \cdot \mathrm{CH}\left(\mathrm{COOC}_{2} \mathrm{H}_{5}\right)_{2}\right]_{2}+6 \mathrm{KOH}=$

$$
\mathrm{C}_{6} \mathrm{H}_{4}\left(\mathrm{CH}_{2} \cdot \mathrm{CH}_{2} \cdot \mathrm{COOK}\right)_{2}+2 \mathrm{~K}_{2} \mathrm{CO}_{3}+4 \mathrm{C}_{2} \mathrm{H}_{5} \cdot \mathrm{OH} \text {. }
$$

In preparing it, ethylic ortho-xylylenedimalonate was boiled with a considerable excess of concentrated alcoholic potash for some hours on a sand-bath until hydrolysis was complete. Water was then added, the solution evaporated on a water-bath until all the alcohol had been driven off, filtered and acidified with dilute sulphuric acid; this precipitates the new acid as a yellowish-white, flocculent mass which, after collecting, drying, and washing with hot chloroform, is easily obtained pure by recrystallising once or twice from water. On analysis, the following results were obtained :-

I. 0.1357 gram substance gave 0.3237 gram $\mathrm{CO}_{2}$ and 0.0794 gram $\mathrm{H}_{2} \mathrm{O}$.

II. $0 \cdot 1811$ gram substance gave $0 \cdot 4358$ gram $\mathrm{CO}_{2}$ and $0 \cdot 1068$ gram $\mathrm{H}_{2} \mathrm{O}$.

\begin{tabular}{|c|c|c|c|}
\hline \multirow[b]{3}{*}{$C \ldots \ldots$} & \multirow[b]{2}{*}{ Theory. } & \multicolumn{2}{|c|}{ Found. } \\
\hline & & I. & II. \\
\hline & 64.86 p. с. & $65 \cdot 05$ & 65.00 p. c. \\
\hline$\ldots \ldots$ & $6 \cdot 31$, & $6 \cdot 50$ & $6.55 \quad$, \\
\hline $0 \ldots . .$. & $28 \cdot 82$, & $28 \cdot 45$ & $28 \cdot 45$ \\
\hline
\end{tabular}

Orthophenylenedipropionic acid crystallises from water in small, colourless, microscopic needles, which melt not very sharply at about $160-162^{\circ}$. The silver salt which was prepared from the ammonium salt in the usual way, is a white, amorphous, insoluble precipitate. It gave the following numbers on analysis :-

0.2121 gram substance gave 0.1045 gram Ag.

$$
\begin{aligned}
& \text { Theory. } \\
& \mathrm{C}_{12} \mathrm{H}_{12} \mathrm{O}_{4} \mathrm{Ag}_{2} \text {. } \quad \text { Found. } \\
& \text { Ag....... } \quad 49 \cdot 54 \text { per cent. } \quad 49 \cdot 27 \text { per cent. }
\end{aligned}
$$

Orthophenylenedipropionic acid appears also to be formed by the action of reducing agents on orthophenylenediacrylic acid. 


\section{Tetrabromo-orthophenylenedipropionic Acid, $\mathrm{C}_{6} \mathrm{H}_{4}(\mathrm{CHBr} \cdot \mathrm{CHBr} \cdot \mathrm{COOH})_{2}$.}

This acid is produced by the direct combination of orthophenylenediacrylic acid with 2 mols. of bromine, thus :-

$$
\mathrm{C}_{6} \mathrm{H}_{4}(\mathrm{CH}: \mathrm{CH} \cdot \mathrm{COOH})_{2}+2 \mathrm{Br}_{2}=\mathrm{C}_{6} \mathrm{H}_{4}\left(\mathrm{CHBr} \cdot{ }^{\circ} \mathrm{CHBr} \cdot \mathrm{COOH}\right)_{2} \text {. }
$$

In preparing it, pure orthophenylenediacrylic acid is allowed to stand under a glass shade containing a small beaker of bromine, for about 24 hours, when it is found to have absorbed exactly 2 molecular proportions of bromine. If care be taken to have the acid in as finely divided a condition as possible, the product obtained is almost pure. After allowing a sample thus prepared to stand for some hours over soda-lime to free it from any excess of bromine, it gave the following numbers on analysis :-

0.2943 gram substance gave 0.2930 gram $\mathrm{CO}_{2}$ and 0.0598 gram $\mathrm{H}_{2} \mathrm{O}$.

0.2443 gram substance gave 0.3400 gram AgBr.

\begin{tabular}{|c|c|c|c|c|}
\hline \multicolumn{3}{|c|}{$\begin{array}{c}\text { Theory. } \\
\mathrm{C}_{12} \mathrm{H}_{10} \mathrm{Br}_{4} \mathrm{O}_{4} .\end{array}$} & \multicolumn{2}{|l|}{ Found. } \\
\hline C....... & $26 \cdot 76$ & cent. & $27 \cdot 15$ & cent. \\
\hline$H \ldots \ldots$ & $1 \cdot 86$ & " & $2 \cdot 25$ & , \\
\hline Br....... & $59 \cdot 48$ & ", & $59 \cdot 22$ & $"$ \\
\hline$O \ldots \ldots$ & $11 \cdot 89$ & " & $11 \cdot 38$ & ", \\
\hline
\end{tabular}

A direct experiment was made to prove that the acid was really formed quantitatively from orthophenylenediacrylic acid according to the equation given above. 0.731 gram of orthophenylenediacrylic acid, after being subjected to the action of bromine and the excess of the latter removed by exposure to soda-lime, weighed 1.808 gram; 1.077 gram of bromine or $147 \cdot 3$ per cent. had therefore been absorbed, whereas theoretically an absorption of bromine equivalent to 146.3 per cent. should have taken place.

Tetrabromo-orthophenylenedipropionic acid is sparingly soluble in benzene, light petroleum, bisulphide of carbon, and chloroform. When boiled with water or alcohol, it is decomposed with elimination of hydrogen bromide. Heated in a test-tube, bromine and hydrogen bromide are evolved, and a carbonaceous residue is left.

Several experiments were next tried with the object of obtaining orthophenylenedipropiolic acid from this tetrabromide, according to the following equation:-

$\mathrm{C}_{6} \mathrm{H}_{4}(\mathrm{CHBr} \cdot \mathrm{CHBr} \cdot \mathrm{COOH})_{2}+6 \mathrm{KOH}=$

$$
\mathrm{C}_{6} \mathrm{H}_{4} \cdot(\mathrm{C} \vdots \mathrm{C} \cdot \mathrm{COOK})_{2}+4 \mathrm{KBr}+6 \mathrm{OH}_{2} \text {. }
$$


For this purpose, the action of caustic potash nnder various conditions was tried; and although on several occasions small quantities of an acid free from bromine were obtained, the amount was too small for analysis. Probably by converting the tetrabromo-acid into its ethylic salt, and then hydrolysing with the calculated quantity of alcoholic potash, better results will be obtained; experiments in this direction are in progress.

\section{Formation of Tetrahydronaphthalenedicarboxylic Acid from Ethylic Oitho-xylylenedimalonate.}

This interesting synthesis, which has already been referred to in the introduction, was carried out in the following way :-

Ethylic ortho-xylylenedimalonate was dissolved in about 10 vols. of ether and slowly added to an ethereal solution of sodic ethylate containing the latter in the proper proportion to form the disodium-derivative. An ethereal solution of the calculated quantity of iodine was then slowly run in, the whole being well agitated and cooled during the operation. At first the colour of the iodine disappeared at once, but more slowly towards the end of the reaction; after all the iodine had been added, the solution had a decidedly yellow tinge. Water was now added, the ethereal solution separated, washed with water, dried over calcic chloride, and the ether distilled off; an almost colourless oil remained which was at once hydrolysed by boiling with alcoholic potash. After evaporating the alcohol, dissolving in water, acidifying with dilute sulphuric acid, and extracting with ether, a quantity of an almost colourless syrup was obtained which, when heated to $185^{\circ}$, gave off water and carbonic anhydride, and was converted into the anhydride of tetrahydronaphthalenedicarboxylic acid; this was easily identified with that obtained by the action of ortho-xylylene dibromide on the disodium-derivative of ethylic acetylenetetracarboxylate (as described page 12 ), not only from its melting point, $184^{\circ}$, but also from the fact that when boiled with caustic soda it is converted into tetrahydronaphthalenedicarboxylic acid, melting at $199^{\circ}$, and otherwise identical in every respect with that obtained from ethylic acetylenetetracarboxylate.

I cannot finish this paper without expressing my sincere thanks to Professor Adolf v. Baeyer, in whose laboratory these experiments were carried out, not only for the many suggestions he made, but also for the valuable help he rendered in overcoming the many difficulties of the experimental part. 
[Reprinted from the Journal of the Chemical Society, January, 1888. Vol. LIII.]

\section{SYNTHETICAI FORMATION OF CLOSED CARBON-CHAINS IN THE AROMATIC SERIES. Part II.}

\section{By F. Stanley Kipping, Ph.D., D.Sc.}

Avovg the vast number of aromatic hydrocarbons, there are many which have been proved to contain two or more benzene nuclei condensed together, that is to say, they are compounds in which certain carbon-atoms are common to both rings, as for example, naphthalene, anthracene, and phenanthrene.

Other substances are known in which one or more atoms of nitrogen displace one or more atoms of carbon in the closed chain, without producing any alteration in the fundamental constitution of the compound; for instance, quinoline, acridine, and pseudoanthroline.

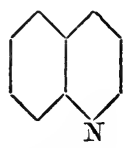

Quinoline.

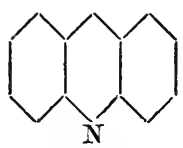

Acridine.

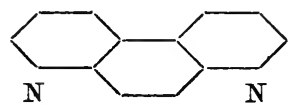

Pseudoanthroline.

Now, in examining the formulæ which, according to the present theoretical views, have been proved, both by synthetical and analytical methods, to represent the constitution of the compounds mentioned, it is at once evident that the carbon-atoms which are common to any two rings are situated in the ortho- or 1.2-position; up to the present time no compound has been obtained in which two carbon-atoms, occupying the meta- or para-position in one closed chain, enter into the formation of the adjoining one; although in the present state of our knowledge of the formation of closed chains there is no $\grave{a}$ priori reason why such compounds should not exist, and why, for instance, isomeric naphthalenes having the formulæ-
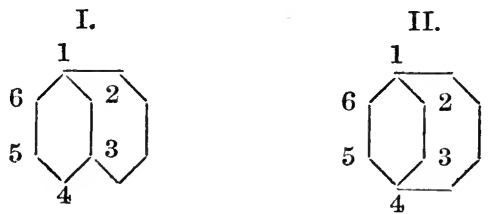

could not be formed. In formula I, two carbon-atoms in the metaposition would be common to both rings, and in formula II this would be the case with two carbon-atoms in the para-position. It seemed an interesting subject for research to attempt to synthesise 
either such hydrocarbons themselves, or some derivatives of them, in order to obtain experimental evidence as to the possibility of the existence of compounds so constituted.

In Part I of this paper (this vol., p. 1), W. H. Perkin, jun., has described the synthesis of naphthalene from ortho-xylene by a series of reactions, which forms an additional proof that condensation of the benzene-rings takes place in the ortho-position; if isomeric naphthalenes, or derivatives of such compounds, can be produced, it seemed probable that they would be obtained in an exactly similar manner when meta- or para-xylene was used in place of the ortho-compound. Acting on the kind suggestion of Perkin, I undertook to work out this research, and proceeded in the following manner: meta-xylene was treated with bromine, and the meta-xylylene bromide thus obtained was acted upon, in ethereal solution, with ethylic chloromalonate and sodic ethylate when ethylic meta-xylylenedichlorodimalonate was formed, the reaction being expressed by the following equation:-

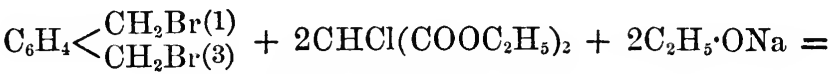

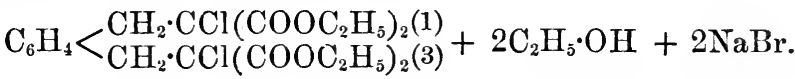

By treating this product with reducing agents, the chlorine is displaced by hydrogen, and ethylic meta-xylylenedimalonate formed, thus :-

$$
\begin{array}{r}
\mathrm{C}_{6} \mathrm{H}_{4}\left[\mathrm{CH}_{2} \cdot \mathrm{CCl}\left(\mathrm{COOC}_{2} \mathrm{H}_{5}\right)_{2}\right]_{2}+2 \mathrm{H}_{2}=\mathrm{C}_{6} \mathrm{H}_{4}\left[\mathrm{CH}_{2} \cdot \mathrm{CH}\left(\mathrm{COOC}_{2} \mathrm{H}_{5}\right)_{2}\right]_{2} \\
+2 \mathrm{HCl} .
\end{array}
$$

On adding sodic ethylate to an ethereal solution of this ethylic salt, a disodium-derivative is produced, thus:-

$\mathrm{C}_{6} \mathrm{H}_{4}\left[\mathrm{CH}_{2} \cdot \mathrm{CH}\left(\mathrm{COOC}_{2} \mathrm{H}_{5}\right)_{2}\right]_{2}+2 \mathrm{C}_{2} \mathrm{H}_{5} \cdot \mathrm{ONa}=$ $\mathrm{C}_{6} \mathrm{H}_{4}\left[\mathrm{CH}_{2} \cdot \mathrm{CNa}\left(\mathrm{COOC}_{2} \mathrm{H}_{5}\right)_{2}\right]_{2}+2 \mathrm{C}_{2} \mathrm{H}_{5} \cdot \mathrm{OH}$.

By appropriate treatment with iodine or bromine, it was thought that the sodium would be eliminated, and from this compound a tetrahydrometanaphthalene-derivative obtained according to the following equation:-

$$
\widehat{C}_{\mathrm{CH}_{2} \cdot \mathrm{CNa}\left(\mathrm{COOC}_{2} \mathrm{H}_{5}\right)_{2}}^{\mathrm{CH}_{2} \cdot \mathrm{CNa}\left(\mathrm{COOC}_{2} \mathrm{H}_{5}\right)_{2}}+\mathrm{I}_{2}=\varnothing \int_{\mathrm{CH}_{2} \cdot \mathrm{C}\left(\mathrm{COOC}_{2} \mathrm{H}_{5}\right)_{2}}^{\mathrm{CH}_{2} \cdot \mathrm{C}\left(\mathrm{COOC}_{2} \mathrm{H}_{5}\right)_{2}}+2 \mathrm{NaI},
$$

from which, by hydrolysis and removal of two carboxyl-groups, tetrahydrometanaphthalenedicarboxylic acid would be obtained- 


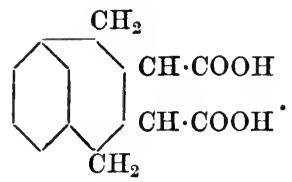

A dicarboxylic acid was in fact obtained, which was at.first thought to be the desired compound; on careful examination, however, it was found that it contained no meta-ring, but was simply metaphenylenedipropionic acid-

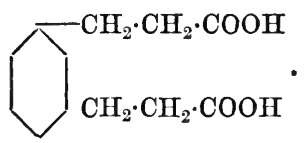

It wili be seen that this acid differs in composition from the hypothetical metanaphthalene-derivative only by two atoms of hydrogen, and that, therefore, analysis alone would be insufficient to distinguish between them with certainty; it can be proved beyond doubt, however, that no closed ring has been formed in the above reactions, for when ethylic meta-xylylenedimalonate is directly hydrolysed without previous treatment with sodic ethylate and iodine (or bromine), the dicarboxylic acid which is ultimately obtained is identical with that which was supposed to be a tetrahydrometanaphthalene-derivative.

It is difficult to explain what takes place when the above-mentioned sodium-derivative is treated with iodine (or bromine), but it would seem that the halogen destroys a portion of the substance, with formation of hydriodic (or hydrobromic) acid, which then reacts on the remainder, reproducing the ethereal salt and the respectire haloïd sodium compound.

In spite of the failure of these experiments to produce a metaclosed ring, the conclusion was not justified that a para-ring could not be formed, since, according to the present theories beld regarding the constitution of the numerous compounds containing a pyridine.ring, and of the paraquinones, a union of two atoms in the paraposition either directly or through the interposition of other atoms is assumed.

Para-xylene was therefore prepared, and submitted to a series of reactions exactly in the same way as in the case of the meta-hydrocarbon; the sodium compound of ethylic para-xylylenedimalonate was obtained, and treated with iodine (or bromine) in the hope of forming a tetrahydroparanaphthalene-derivative.

But here again no closed ring could be produced; when iodine is used the reaction which takes place appears to be similar to that suggested as the most probable in the case of the meta-compound, and the dicarboxylic acid which is the ultimate product is not a 
tetrahydroparanaphthalene-derivative, but simply paraphenylenedipropionic acid-

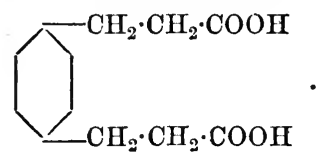

This was proved in a manner similar to that already described. When bromine is used, the reaction is quite different; substitution takes place, and ethylic para-xylylenedibromodimalonate is formed, most probably as expressed by the equation-

$$
\begin{aligned}
& 2 \mathrm{C}_{6} \mathrm{H}_{4}\left[\mathrm{CH}_{2} \cdot \mathrm{CNa}\left(\mathrm{COOC}_{2} \mathrm{H}_{5}\right)_{2}\right]_{2}+2 \mathrm{Br}_{2}= \\
& \mathrm{C}_{6} \mathrm{H}_{4}\left[\mathrm{CH}_{2} \cdot \mathrm{CBr}\left(\mathrm{COOC}_{2} \mathrm{H}_{5}\right)_{2}\right]_{2}+2 \mathrm{NaBr}+ \\
& \mathrm{C}_{6} \mathrm{H}_{4}\left[\mathrm{CH}_{2} \cdot \mathrm{CNa}\left(\mathrm{COOC}_{2} \mathrm{H}_{5}\right)_{2}\right]_{2} .
\end{aligned}
$$

From these experiments, then, the conclusion may be drawn that compounds containing rings condensed together in the meta- or paraposition are incapable of existence, otherwise they would have been formed by the methods which have been described; now this may be so for one of two reasons-either on account of the relative positions of the two rings, or because of the number of carbon-atoms which would form the reduced ring; in tetrahydrometanaphthalene, as will be seen from the figures already given, there would be seven, in tetrahydroparanaphthalene there would be eight carbon-atoms in the larger closed chain.

To help to a decision on this point, experiments were made with the object of obtaining a derivative of a compound having the formula-

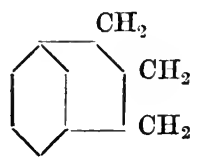

which, like naphthalene itself, would contain six atoms in each ring; a compound of this character should be obtained by treating metaxylylene bromide with 2 mols. of sodic ethylate and 1 mol. of ethylic malonate, according to the equation-

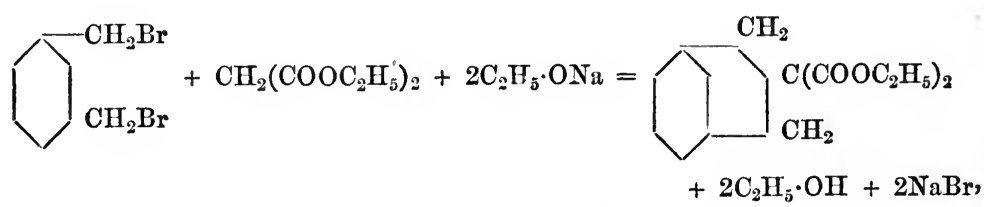

in a manner exactly similar to that in which hydrindonaphthene- 
derivatives were prepared by Perkin (see Part I, this vol., p. 7). But, although the experiment was repeated several times under varied conditions, no such compound could be obtained; the reaction was evidently of a very complicated nature, and yielded a resinous mass from which no definite product could be isolated. It seems, therefore, that the non-formation of a meta- or para-ring in any of these cases is due, not entirely to the number of atoms which would go to form the ring, but also, and perhaps wholly, to its relative position.

It is a general law in the chemistry of the aromatic compounds that no substance can be obtained in which a ring of any sort is joined to the benzene nucleus in other than the ortho-position, e.g., the coumarins, carbostyrile-derivatives, quinoxaline, \&c.; the simplest bibasic acid-phthalic-is readily converted into the anhydride, whilst the isomeric meta- and para-anhydrides have not yet been prepared.

The two isomeric meta- and para-phenylenedipropionic acids obtained in this research by reactions already described, were of considerable theoretical interest, owing to the fact that if anhydrides could be obtained from them they would contain a closed ring in the meta- and para-position respectively, as shown by the formulæ
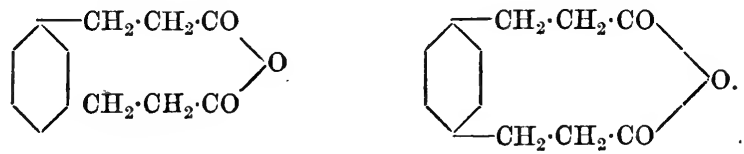

Attempts were therefore made in this direction, but without success, and it was thought that perhaps this was owing to the large number of atoms which would have to take part in the formation of the closed ring: in the fatty series, as is well known, many dicarboxylic acids can be converted into anhydrides, but whether this is possible or not depends on the relative positions of the carboxyl-groups, that is, on how many carbon-atoms go to form the anhydride-ring. To throw some light on this question, meta- and para-xylylene cyanides were prepared, and from them, by hydrolysis, the corresponding phenylenediacetic acids; it seemed possible that these compounds, containing as they do one atom of carbon less in each of the side chains, would be more capable of forming anhydrides; these would have the formulæ
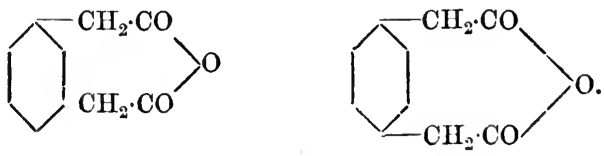
No anhydride, however, could be obtained from either of these acids, and the conclusion drawn from all the experiments briefly described above confirms the general law that no exterior ring in the meta- or para-position can be formed.

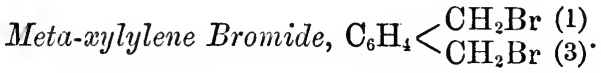

Meta-xylylene bromide was first obtained by Colson (Compt. rend., $99,40)$, by brominating boiling meta-xylene; in preparing large quantities of this substance, I have found that the following method gives the best results :- 50 grams of pure meta-xylene, placed in a retort connected with a long reflux condenser, are heated in an oilbath to $125-130^{\circ}, 160$ grams of bromine are then added by means of a dropping funnel inserted into the tubulus of the retort; at first the bromine may be added tolerably quickly, but later on, when the metaxylene is partially converted and red fumes escape up the condenser, the halogen must be added slowly, otherwise loss is incurred. The hydrogen bromide, which is evolved in large quantities during the reaction, is absorbed by conducting it into a concentrated solution of caustic soda.

When all the bromine has been added, the contents of the retort, which ought to be only slightly coloured, are poured out and left 24 hours in a cool place to crystallise; the mother-liquor is drained off, and the crystals, after lying for some time on a porous plate, are purified by recrystallisation from light petroleum; meta-xylylene bromide is thus obtained in the pure state as a colourless solid melting at $77^{\circ}$, the melting point given by Colson (loc. cit.). From 50 grams of meta-xylene the quantity of pure bromide obtained is not very large, the mother-liquor can, however, be worked up for a less pure product. In consequence of the painful effect of the vapour on the eyes, it is best when using meta-xylylene to work as much as possible in the open air.

\section{Ethylic Meta-xylylenedichlorodimalonate, $\mathrm{C}_{6} \mathrm{H}_{4}\left[\mathrm{CH}_{2} \cdot \mathrm{CCl}\left(\mathrm{COOC}_{2} \mathrm{H}_{5}\right)_{2}\right]_{2}$.}

This compound is obtained by the action of ethylic chloromalonate and sodic ethylate on meta-xylylene bromide; 4.4 grams of sodium, dissolved in as small a quantity of alcohol as possible, are mixed with about 10 volumes of pure ether, and a solution of 37.8 grams of ethylic chloromalonate in 500 c.c. pure ether slowly added; after well cooling the mixture, 25.5 grams of finely-powdered meta-xylylene bromide are thrown in, and the whole well shaken. The white precipitate which is at first formed is the sodium-derivative of ethylic 
chloromalonate, but as the meta-xylylene bromide dissolves, a lively reaction commences and the ether begins to boil, the sodiumderivative being decomposed with formation of ethylic meta-xylylene dichlorodimalonate and sodium bromide. The mixture is then heated on a water-bath for a couple of hours, great care being taken to shake occasionally, otherwise there is considerable danger of the ether boiling over from the bumping which always occurs; water is subsequently added, the ethereal solution washed and separated, dried over calcium chloride, and the ether distilled off.

Ethylic meta-xylylene dichlorodimalonate remains as a thick, yellowish oil, which even after long standing over sulphuric acid in a vacuum shows no signs of crystallising; on analysis the following results were obtained :-

I. $0 \cdot 1615$ gram substance gave $0 \cdot 1015$ gram $\mathrm{AgCl}$.

II. $0.2805 \quad, \quad, \quad 0 \cdot 1660 \quad, \quad$,

\begin{tabular}{|c|c|c|}
\hline \multirow{2}{*}{$\begin{array}{l}\text { Calculated for } \\
\mathrm{C}_{22} \mathrm{H}_{28} \mathrm{O}_{8} \mathrm{Cl}_{2} \text {. }\end{array}$} & \multicolumn{2}{|c|}{ Found. } \\
\hline & I. & II. \\
\hline .. 14.4 p.c. & $15 \cdot 5$ & $14 \cdot 6 \mathrm{p} . \mathrm{c}$. \\
\hline
\end{tabular}

The second analysis was made from a different sample, the ethereal salt used in (I) having been found to contain a trace of unchanged meta-xylylene bromide.

When the calculated quantities of ethylic chloromalonate and meta-xylylene bromide are carefully weighed, the resulting ethylic meta-xylylene dichlorodimalonate is almost pure and the yield quantitative.

\section{Etliylic Meta-xylylenedimalonate, $\mathrm{C}_{6} \mathrm{H}_{4}\left[\mathrm{CH}_{2} \cdot \mathrm{CH}\left(\mathrm{COOC}_{2} \mathrm{H}_{5}\right)_{2}\right]_{2}$.}

When the chlorine-compound described above is dissolved in about 15 volumes of glacial acetic acid and a small quantity of zinc-dust added, reduction at once commences, the mixture becoming quite warm.

The process is best carried out by adding the zinc-dust in very small quantities at a time for about an hour, the whole being constantly shaken in a long-necked flask; at the end of this time, the solution becomes very thick, owing to the formation of zinc acetate, and reduction proceeds only very slowly; water is therefore added, the mixture warmed on the water-bath, and the ethereal salt extracted with ether, not only from the solution but also from the undissolved zincdust, which retains a considerable quantity of the substance; the ethereal solution after washing, first with water, and then with sodium carbonate solution to get rid of acetic acid, is dried over anhydrous 
potassium carbonate and the ether distilled off. The oil which remains still contains a considerable proportion of the unchanged chlorinated derivative, the reduction is therefore repeated in exactly the same manner; it was found, however, that even after three such treatments a trace of chlorine is still present; the best results are obtained when only small quantities at a time are operated upon.

In order to prepare this substance pure for analysis, use is made of the sodium-derivative; when the calculated quantity of sodium, dissolved in absolute alcohol and mixed with about 10 volumes of ether, is added to a weak ethereal solution of ethylic meta-xylylene dimalonate, a white precipitate of the disodium-derivative is formed. This is washed with ether on the filter pump as quickly as possible, and then thrown into water or dilute sulphuric acid, when it is at once decomposed, yielding ethereal salt and sodium hydrate (or sulphate). The oil which separates is extracted with ether, and after drying the ethereal solution with calcium chloride, it is treated again, exactly as before, with the calculated quantity of sodic ethylate; the precipitate is washed as above, decomposed with dilute sulphuric acid, the solution extracted with ether, and the product isolated in the usual manner.

Ethylic meta-xylylenedimalonate is thus obtained in the pure state as a thick, colourless oil, which, however, could not be obtained in the crystalline form; it is readily soluble in ether, alcohol, acetic acid, \&c., bat insoluble in water.

The following results were obtained on analysis :-

$0 \cdot 1550$ gram substance gave 0.3522 gram $\mathrm{CO}_{2}$ and 0.1016 gram $\mathrm{H}_{2} \mathrm{O}$.

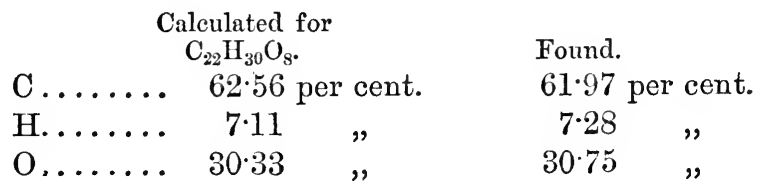

The sodium-derivative of this ethereal salt is a white solid, which, however, on account of its extremely hygroscopic nature, soon decomposes on exposure to the air. To prepare a sample for analysis, a few grams of ethylic meta-xylylenedimalonate are dissolved in a large volume of dry ether, and the calculated quantity of sodic ethylate, mixed with about 10 volumes of ether, added to the solution. The flask is tightly corked, and the mixture after being well shaken is allowed to stand, in order that the precipitate may settle; when it has completely subsided, it is washed twice by decantation with pure ether (the air being excluded as much as possible), and after draining 
off the ether as far as possible, quickly transferred to a tared stoppered weighing-bottle.

After standing in a vacuum over sulphuric acid, a sodium determination was made, from which the following result was obtained :-

0.4055 gram substance gave 0.1215 gram sodic sulphate.

$$
\begin{aligned}
& \text { Calculated for } \\
& \mathrm{C}_{22} \mathrm{H}_{29} \mathrm{Na}_{2} \mathrm{O}_{8} \text {. Found. } \\
& \mathrm{Na} \ldots . . . \quad 9 \cdot 9 \text { per cent. } \quad 9 \cdot 7 \text { per cent. }
\end{aligned}
$$

Experiments were now made to obtain a tetrahydrometanaphthalene-derivative from the disodium malonate.

The following is a description of the methods employed:-The calculated quantity of sodium, dissolved in alcohol and mixed with about 10 volumes of ether, is added to a weak ethereal solution of ethylic meta-xylylenedimalonate, and after allowing the sodium-derivative to separate out, which takes but a very few moments, a very slight excess of the calculated quantity, of iodine, also dissolved in ether, is added and the mixture vigorously shaken. The dark-brown colour of the iodine rapidly disappears, and in a few minutes the reaction is completed, the slightly brown colour of the solution being due to the small excess of the halogen used; after adding water to dissolve the sodic iodide, and removing the trace of iodine by means of dilute sulphurous acid, the colourless ethereal solution is washed with water, dried, and the ether distilled off, when the ethereal salt of a tetracarboxylic acid remains behind as a thick, brown oil. This salt is hydrolysed by boiling it for two hours on the water-bath with a solution of potash in methyl alcohol; the alcohol is then evaporated and the potassium-compound dissolved in water; on acidifying this solution with dilute sulphuric acid, a small quantity of impurity is precipitated, this is filtered off and the filtrate extracted repeatedly with ether. The acid obtained on distilling off the ether is a thick, brown oil, which, as it could not be obtained crystalline was, without purify. ing, converted into the dicarboxylic acid; this is effected by heating it in a flask in a metal-bath at $180-200^{\circ}$, a considerable amount of decomposition taking place, with a copious evolution of carbonic anhydride. When no more gas is given off, the process is at an end, and, on cooling, the contents of the flask become solid. To purify the product, it is now converted into the ethylic salt; for this purpose it is dissolved in dilute ammonia, the excess of the latter removed by standing in a vacuum over sulphuric acid, and the solution filtered to get rid of a small quantity of oil which is present; silver nitrate is then added to the filtrate, and the silver salt thus formed is washed with water on the filter-pump. After careful drying, it is suspended 
in ether, treated with an excess of ethyl iodide, and boiled on the water-bath with reflux condenser for about two hours, whereby the silver salt is completely decomposed. The silver iodide is filtered off and well washed with warm ether, the washings added to the filtrate, and the ethereal salt obtained by distilling off the ether ; it is, however, still impure, and is therefore submitted to fractional distillation under diminished pressure. A trace of ethyl iodide first comes over, the thermometer then rapidly rises, and the whole distils between $200^{\circ}$ and $250^{\circ}$ (60 mm. pressure) ; the high boiling portion is again fractioned, when almost the whole distils over without decomposition constantly at $247-250^{\circ}$ as a colourless, mobile oil. An analysis was now made, which, however, agreed equally well for either of the two formulæ--.

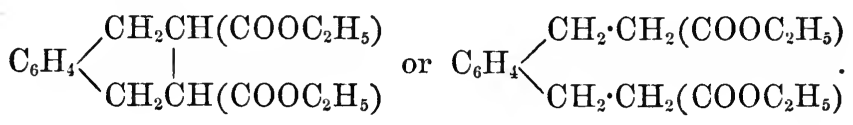

As the acid itself would probably crystallise, and could therefore be more easily obtained in a high degree of purity, further attempts to purify the ethereal salt were considered unnecessary, and it was converted into the acid by boiling with a solution of pure potash in methyl alcohol; when hydrolysis was complete, the solution was evaporated to dryness, the potassium salt dissolved in water, dilute sulphuric acid added in excess, and the organic acid extracted with ether. After isolating the product in the usmal way, and recrystallising it from boiling water, it was obtained in beautiful glistening plates; an analysis was made which again did not decide whether the ring formation had taken place or not, and other means had therefore to be employed to settle this point beyond doubt. Metaphenylenedipropionic acid was prepared according to the methods described later on, and as this substance, which must hare the formula

\section{$\mathrm{C}_{6} \mathrm{H}_{4}\left(\mathrm{CH}_{2} \cdot \mathrm{CH}_{2} \cdot \mathrm{COOH}\right)_{2}$,}

is identical with the acid obtained above, it is quite clear that no meta-ring had been formed by the action of iodine on the sodiumderivative of ethylic meta-xylylenedimalonate.

Another attempt to obtain a tetrahydrometanaphthalene-derivative was therefore made; in this case, bromine was used instead of iodine, and since there was a considerable loss from decomposition on heating the tetracarboxylic acid in the metal-bath, another method for eliminating the two carboxyl-groups was adopted. The following is an account of the manner in which the experiment was carried out : to a solution of 1.4 grams of sodium dissolved in 15 grams of absolute alcohol, and mixed with 50 c.c. of pure ether, 12 grams of ethylic meta-xylylenc- 
dimalonate in ethereal solution were added, and the mixture allowed to stand in a freezing mixture until the sodium compound had completely subsided; $4 \cdot 6$ grams of bromine were then gradually dropped in, the whole being kept well cooled. Each drop of bromine was immediately absorbed, the few last only causing the solution to assume a slightly yellow colour.

The product of this reaction was separated as already described in the experiment with iodine, and the ethereal salt hydrolysed by boiling it for two hours with alcoholic potash; 9 grams of the tetracarboxylic acid were thus obtained in the form of a brown oil which could not be got to crystallise; it was therefore mixed with about three times its weight of water, and heated in a sealed tube, first at $110^{\circ}$, then, after opening the capillary to get rid of the carbonic anhydride, at $150^{\circ}$, and finally again at $180^{\circ}$ until the evolution of gas entirely ceased.

The contents of the tube, when cool, consisted of two layers; the undermost after standing for some time solidified almost completely, whilst the upper one was filled with colourless crystals. After purification, these crystals were found to be identical with the acid obtained before-viz., metaphenylenedipropionic acid. The solid mass which formed the lower layer was dissolved in boiling water, and, after filtering, allowed to crystallise; again the same acid was proved to have been formed, and the quantity obtained was from $1 \frac{1}{2}-2$ grams. About 3 grams of a black resinous mass from which no crystalline product could be isolated remained on the filter; the mother-liquor from the acid yielded nothing but a further quantity of impure metaphenylenedipropionic acid, which took the form of a slightly yellow oil.

The result of these two experiments showed, therefore, that by acting on the sodium compound of ethyl meta-xylylenedimalonate with bromine or iodine no meta-ring is produced, and the same compound is ultimately obtained after hydrolysis and elimination of two carboxyl-groups, whether this reaction is carried out or not; the possibility that such a ring can exist is thereby rendered improbable.

\section{MLeta-xylylenedimalonic Acid, $\mathrm{C}_{6} \mathrm{H}_{4}\left[\mathrm{CH}_{2} \cdot \mathrm{CH}(\mathrm{COOH})_{2}\right]_{2}$.}

When the crude ethylic salt of this acid (see p. 27) is boiled with excess of potash in methylic alcohol until no oil is precipitated on addition of water, hydrolysis is complete; this is the case after about two hours.

The alcohol is distilled off, and the potassium salt which is obtained on evaporation to dryness is dissolved in water; the addition of sulphuric acid in excess produces a slight turbidity, the solution is 
therefore filtered, and the product extracted from the filtrate by shaking out about 10 times with pure ether. When the ether is distilled off, meta-xylylenedimalonic acid remains behind as a thick, slightly brown oil, which is readily soluble in water, alcohol, and ether. Unfortunately it could not be obtained in the crystalline form, so no analysis of it was made; that its constitution is that represented by the above formula is, however, proved by its method of formation and by its decompositions, also by a comparison with the isomeric para-acid described later on, to which it is strictly analogous.

\section{Metaphenylenedipropionic Acid, $\mathrm{C}_{6} \mathrm{H}_{4}\left(\mathrm{CH}_{2} \cdot \mathrm{CH}_{2} \cdot \mathrm{COOH}\right)_{2}$.}

On heating the tetracarboxylic acid just described, it is readily converted into metaphenylenedipropionic acid with elimination of 2 mols. of carbonic anhydride.

This conversion may be effected in two different ways:-First, meta-xylylenedimalonic acid is heated in a small flask in a metalbath; as soon as the temperature rises above $100^{\circ}$ carbonic anhydride begins to be evolved, the evolution becoming more rapid as the temperature rises; after keeping the bath at about $180^{\circ}$ for some time, no more carbonic anhydride is given off, and the change is complete. On cooling, the dark-brown oil solidifies to an almost solid cake, which is dissolved in hot water, boiled with animal charcoal, and the sclution filtered; the new acid crystallises from the filtrate in beautiful colourless plates, and is obtained pure by three recrystallisations from boiling water.

The second method for the preparation of this substance takes up far more time, but it is preferable as the yield is far better: metaxylylenedimalonic acid is dissolved in about three times its volume of water, and heated in a sealed tube for one hour at $100-120^{\circ}$; the tube is then allowed to cool, and the capillary opened to permit of the escape of the carbonic anhydride; after re-sealing, it is heated again at $150^{\circ}$ for an hour, the gas formed allowed to escape, and the contents of the tube raised to a temperature of about $180^{\circ}$ for some considerable time. The tetrabasic acid is now completely decomposed, and the liquid which was at first homogeneous is found, after cooling, to have separated into two layers; the bottom one solidifies completely whilst the top one is filled with leaf-like crystals. To purify the product, the whole is collected, and the residue, after spreading on a porous plate to remove traces of oil, is dissolved in boiling water, and the solution filtered. On cooling, pure metaphenylenedipropionic acid separates in magnificent, lustrous, colourless plates. It was dried 
over sulphuric acid, then at $100^{\circ}$, and analysed with the following result:-

0.1690 gram substance gave 0.4010 gram $\mathrm{CO}_{2}$ and 0.0985 gram $\mathrm{H}_{2} \mathrm{O}$.

\begin{tabular}{|c|c|c|c|}
\hline \multicolumn{3}{|c|}{ Calculated for } & \multirow{2}{*}{$\begin{array}{l}\text { Found. } \\
64.71 \text { per cent. }\end{array}$} \\
\hline & $64: 86$ & r cent. & \\
\hline$\ldots$ & $6 \cdot 31$ & , & $6 \cdot 47$ \\
\hline$\ldots$. & $28 \cdot 83$ & , & $28 \cdot 82$ \\
\hline
\end{tabular}

Metaphenylenedipropionic acid when pure melts at $146-147^{\circ}$; it is moderately soluble in hot, but almost insoluble in cold water, and dissolves easily in ether and alcohol. On precipitating a neutral solution of the ammonium salt with silver nitrate, the silver salt falls down as a white amorphous precipitate; after washing well and drying over sulphur:c acid in a vacuum, it was analysed with the following result :-

0.3318 gram substance gave 0.1638 gram Ag.

$$
\begin{aligned}
& \text { Calculated for } \\
& \mathrm{C}_{12} \mathrm{H}_{12} \mathrm{Ag}_{2} \mathrm{O}_{4} \text {. Found. } \\
& \text { Ag....... 4. 49.54 per cent. } 49.37 \text { per cent. }
\end{aligned}
$$

This salt is very stable, and does not darken when exposed to diffused light.

In an aqueous neutral solution of the ammonium salt, lead acetate gives a white, amorphous precipitate, copper sulphate a light, bluishgreen precipitate, and zinc sulphate a white, crystalline precipitate. Barium chloride gives no reaction.

An experiment was made to try and obtain the anbydride of this acid by heating a small quantity for half an hour in a metal-bath at a temperature of $250^{\circ}$. The acid darkened in colour, traces of water were evolved, and a slight amount of decomposition took place; on heating more strongly over the naked flame, a brown oil distilled and almost immediately solidified. After washing the product with ether on a porous plate, an almost colourless substance was obtained which showed the same melting point as the original acid; no anhydride was therefore formed.

\section{Methylic Metaphenylenedipropionate.}

This ethereal salt is obtained in the usual manner by treating argentic metaphenylenedipropionate with methyl iodide.

The pure dry silver salt is mixed with an excess of methyl iodide dissolved in 10 volumes of ether, and the mixture heated on the 
water-bath with reflux condenser for about five hours; at the end of this time, the silver salt will be entirely decomposed. To isolate the product, the silver iodide is filtered off, washed with ether, and the washings added to the filtrate; on distilling off the ether, a thick colourless oil remains behind, which in a short time solidifies to a hard cake of crystals; it is purified by spreading on a porous plate and recrystallising from dilute methyl alcohol. Methylic metaphenylenedipropionate is thus obtained pure in the form of colourless plates, and on analysis the following results were obtained :-

0.1570 gram substance gave 0.3865 gram $\mathrm{CO}_{2}$ and 0.1065 gram $\mathrm{H}_{2} \mathrm{O}$.

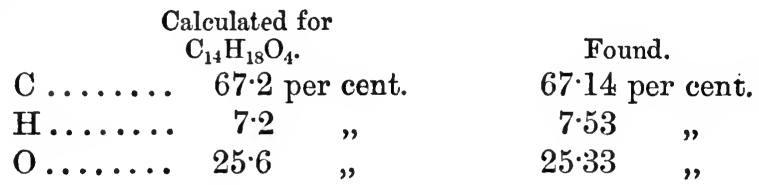

This substance is very easily soluble in ether, alcohol, and benzene, but only sparingly in cold methyl alcohol; it melts at $51^{\circ}$, and when heated in small quantities at a time distils at a high temperature almost without decomposition.

\section{Ethylic Metaphenylenedipropionate.}

This is obtained in a manner exactly similar to that used in the preparation of the methyl salt just described; it is a colourless oil, which boils at $247-250^{\circ}$ (60 $\mathrm{mm}$. pressure) without decomposition. The analysis gave the following results :-

0.1243 gram substance gave 0.3171 gram $\mathrm{CO}_{2}$ and 0.0866 gram $\mathrm{H}_{2} \mathrm{O}$.

\begin{tabular}{|c|c|c|c|}
\hline$\ldots \ldots \ldots$ & $\begin{array}{l}\text { alculated for } \\
\mathrm{C}_{16} \mathrm{H}_{22} \mathrm{O}_{4} \text {. } \\
\quad 69 \cdot 07 \text { per cent. }\end{array}$ & $\begin{array}{l}\text { Found. } \\
69 \cdot 55\end{array}$ & per cent. \\
\hline . & $7 \cdot 91$ & $7 \cdot 74$ & $"$ \\
\hline & 23.02 & $22 \cdot 71$ & $"$ \\
\hline
\end{tabular}

\section{Para-xylylene Bromide, $\mathrm{C}_{6} \mathrm{H}_{4}\left(\mathrm{CH}_{2} \mathrm{Br}\right)_{2}$.}

This is prepared by brominating para-xylylene in the same way as described under the meta-compound. When the reaction is finished, the product is allowed to stand for a short time; it then solidifies, and is purified by simply spreading on a porous plate. The yield obtained ranges from $85-90^{\circ}$ of the theoretical. The action of the vapour of this substance on the eyes is extremely painful, it is advisable, therefore, to work as much as possible in the open air. 


\section{Ethylic Para-xylylenedichlorodimalonate, $\mathrm{C}_{5} \mathrm{H}_{4}\left[\mathrm{CH}_{2} \cdot \mathrm{CCl}\left(\mathrm{COOC}_{2} \mathrm{H}_{5}\right)_{2}\right]_{2}$.}

36.8 grams of ethylic chloromalonate are dissolved in about 500 c.c. of ether, the calculated quantity of sodium dissolved in absolute alcohol and mixed with 10 volumes of ether added, and then 25 grams para-xylylene bromide thrown in and the mixture vigorously shaken. The sodium-derivative of ethyl chloromalonate first forms, but on continued shaking the para-xylylene bromide dissolves and the reaction commences; no appreciable warming of the mixture, however, is noticed.

After heating on the water-bath with reflux condenser for about four hours, care being taken on account of the great tendency of the mixture to bump, water is added to dissolve the sodium bromide, and the ethereal solution is then separated and dried. When the ether is distilled off, ethyl para-xylylenedichlorodimalonate remains as a thick, brownish oil, the amount obtained being in accordance with theory. On standing for some hours, this oil is converted into a crystalline solid mass; the adhering mother-liquor is removed by spreading on a porous plate, and the product purified by recrystallisation from alcohol. In this way the ethereal salt is obtained in large, colourless, six-sided plates; it melts at $86-87^{\circ}$, is readily soluble in alcohol, ether, light petroleum, acetic acid, \&c., but is insoluble in water. A chlorine determination gave the following result :-

0.3715 gram substance gave 0.2200 gram $\mathrm{AgCl}$.

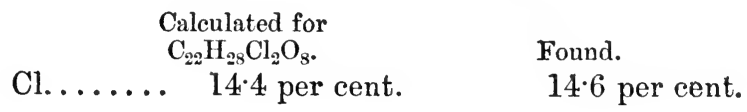

Ethylic Para-xylylenedimalonate, $\mathrm{C}_{6} \mathrm{H}_{4}\left[\mathrm{CH}_{2} \cdot \mathrm{CH}\left(\mathrm{COOC}_{2} \mathrm{H}_{5}\right)_{2}\right]_{2}$.

To prepare this substance, the chlorine-compound just described is reduced with zinc-dust and acetic acid in exactly the same way as the corresponding meta-ethereal salt. About 15 grams are dissolved in 15 c.c. of glacial acetic acid, and a small quantity of zinc-dust added; as the reduction commences, the mixture becomes warm. After constant shaking for about an hour with frequent addition of small quantities of zinc-dust, water is added and the mixture warmed on the water-bath in order to dissolve the zinc acetate; the whole is then shaken out with ether several times, the ethereal solution washed with water and sodium carbonate to get rid of the acetic acid, dried over anhydrous potassium carbonate, and the ether distilled off. Ethyl para-xylylenedimalonate remains behind: as in the case of the 
meta-derivative, however, a very considerable quantity of the chlorine compound still remains unreduced; it is necessary, therefore, to repeat the treatment with zinc-dust and acetic acid, and it was found best to work with only small quantities of the substance, and submit it to at least three reductions. It is thus obtained as a slightly yellow oil, which if allowed to remain over sulphuric acid in a vacuum, in a few days deposits a quantity of colourless crystals ; these are freed from oil by spreading them on a porons plate and washing with a trace of alcohol, when the substance is obtained quite pure ; after drying in a vacuum, an analysis was made with the following results :-

0.2285 gram substance gave 0.5215 gram $\mathrm{CO}_{2}$ and 0.1495 gram $\mathrm{H}_{2} \mathrm{O}$.

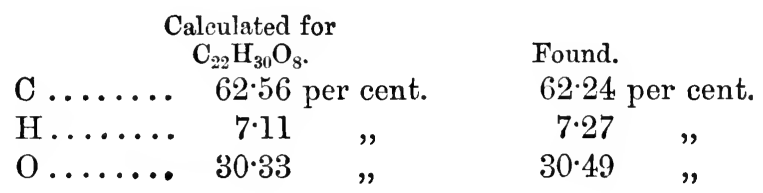

Ethylic para-xylylenedimalonate is a fine, white, crystalline substance, which melts at $51^{\circ}$; it is very readily soluble in ether, far less so in alcohol, and insoluble in water. This ethereal salt, like the corresponding meta-derivative, forms a compound with sodium, which is obtained as a white precipitate when the calculated quantity of sodic ethylate and ethyl para-xylylenedimalonate are mixed together in ethereal solution. The precipitate is washed with pure dry ether, by decantation, the same precautions being taken to exclude the air as were described in the preparation of the meta-compound, thrown on to a filter and transferred as quickly as possible to a tared stoppered weighing bottle; after standing in vacunm over sulphuric acid, a sodium estimation was made, of which the following was the result:-

0.5010 gram substance gave $0 \cdot 1520$ gram $\mathrm{Na}_{2} \mathrm{SO}_{4}$.

$$
\begin{aligned}
& \text { Calculated for } \\
& \mathrm{C}_{22} \mathrm{H}_{28} \mathrm{Na}_{2} \mathrm{O}_{8} \text {. } \\
& \text { Na...... } 9.9 \text { per cent. } \quad 9 \cdot 8 \text { per cent. }
\end{aligned}
$$

This sodium-derivative is white and extremely hygroscopic, in consequence of which it decomposes quickly on exposure to the air, and immediately on the addition of water or acids, the ethereal salt being regenerated.

It was thought that if this compound were treated with iodine or bromine a tetrahydropara-naphthalene-derivative would be formed, as shown by the following equation:- 


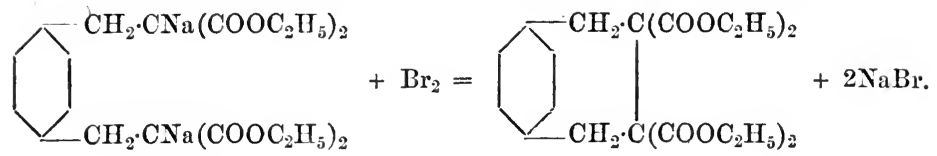

The experiment was carried ont thus : 12 grams of ethyl paraxylylenedimalonate, dissolved in about 50 c.c. of ether, were mixed with an ethereal solution of sodic ethylate, containing 1.4 grams of 'sodium, and then 5 grams of bromine slowly added. The colour of the bromine disappeared instantly on coming into contact with the sodium.compound, the last few drops, howerer, causing a yellow coloration. Water was then added to dissolve the sodium bromide, and after separating and drying the ethereal solution, the ether was distilled off ; 16 grams of a heary oil remained, which on examination was found to contain bromine. On standing for 24 hours in a cool place, crystals were deposited; these were partially separated from the mother-liquor by speading on a porous plate, and while still on the plate purified by washing with a trace of methyl alcohol, in which the oil is readily soluble, whilst the crystals are hardly dissolved at all. After crystallising from hot methyl alcohol, 4.5 grams of a fine, colourless substance were obtained, and on analysis numbers were found agreeing with the formula-

$$
\mathrm{C}_{6} \mathrm{H}_{4}\left[\mathrm{CH}_{2} \mathrm{CBr}\left(\mathrm{COOC}_{2} \mathrm{H}_{5}\right)_{2}\right]_{2} \text {. }
$$

I. 0.2370 gram substance gave 0.3990 gram $\mathrm{CO}_{2}$ and 0.1090 gram $\mathrm{H}_{2} \mathrm{O}$.

II. $0 \cdot 2900$ gram substance gave $0 \cdot 1820$ gram bromide of silver.

$$
\text { Found. }
$$

\begin{tabular}{cr}
\multicolumn{4}{c}{ Calculated. } \\
C ..... & 45.6 p. c. \\
H..... & $4.8 \%$ \\
Br .... & $27.5 \quad "$
\end{tabular}

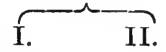

This substance is, therefore, ethylic para-xylylenedibromodimalonate; it is only sparingly soluble in cold, but readily in hat methyl alcohol, from which it crystallises on cooling in nagnificent, glittering plates, which melt at $107-108^{\circ}$. In order to prove that it really had the constitution assigned to it above, it was treated with zinc-dust and acetic acid, when it was reduced to ethyl para-xylylenedimalonate, and far more readily than the corresponding chlorinecompound. On extracting with ether, a quantity of a crystalline product was obtained, which melted at $51^{\circ}$, and possessed all the properties of ethylic para-xylylenedimalonate, there can therefore be no doubt that this substance is para-xylylenedibromodimalonate. 
Since bromine acted on the sodium-compound of ethylic paraxylylenedimalonate in this manner, an experiment was made in exactly the same way, using the calculated quantity of iodine, however, instead of bromine; the product of the reaction was a thick brown oil, which owing to the impurities present could not be got to crystallise; on heating a few drops in a test-tube, violet vapours were evolved, and traces of iodine sublimed. The analysis of the impure substance showed that it 'contained only from 2 to $2 \frac{1}{2}$ per cent. of iodine, which was present in the combined state, so that ethylic paraxylylenediiododimalonate was either not formed at all or only in very small quantities, as this compound would contain 38 per cent. of iodine. The oil was, therefore, hydrolysed and converted into the dicarboxylic acid by heating it in a sealed tube at $180^{\circ}$ until there was no further evolution of carbonic anhydride; the product was isolated, recrystallised, and when pure its melting point was found to be $223-224^{\circ}$ (the melting point of para-phenylenedipropionic acid). In this case, also, it is clear that no ring formation had taken place.

\section{Para-xylylenedimalonie Acid, $\mathrm{C}_{6} \mathrm{H}_{4}\left[\mathrm{CH}_{2} \cdot \mathrm{CH}(\mathrm{COOH})_{2}\right]_{2}$.}

When the ethereal salt of this acid, which has been already described, is boiled for about four hours on a water-bath, with excess of potash in methyl alcohol, hydrolysis is complete. The solution is then evaporated to dryness to drive off the alcohol, the residual potassium salt is dissolved in water, and the solution after acidifying with sulphuric acid is extracted repeatedly with ether. When the ethereal solution has been dried over calcium chloride, and the ether distilled off, para-xylylenedimalonic acid is obtained as a thick syrup, which on standing solidifies to a hard cake. This is spread on a porous plate in order to get rid of traces of oil, and is then warmed with water and a small quantity of animal charcoal; the filtered solution when allowed to evaporate over sulphuric acid in a vacuum, slowly deposits a finely divided crystalline powder; this is collected and dried, first on a porous plate, and then in a vacuum over sulphuric acid. The analysis gave the following results:-

0.1982 gram substance gave 0.3905 gram $\mathrm{CO}_{2}$ and 0.0885 gram $\mathrm{H}_{2} \mathrm{O}$.

\begin{tabular}{|c|c|c|}
\hline & $\begin{array}{l}\text { alculated for } \\
\mathrm{C}_{14} \mathrm{H}_{14} \mathrm{O}_{8} \text {. } \\
54.62 \text { per cent. }\end{array}$ & $\begin{array}{l}\text { Found. } \\
53.7 \text { per cent. }\end{array}$ \\
\hline & 4.5 & 4.9 \\
\hline .. & $41 \cdot 3$ & $41 \cdot 4$ \\
\hline
\end{tabular}

On heating, para-xylylenedimalonic acid melts at about $195^{\circ}$ with 
slight decomposition and evolution of carbonic anhydride; the whole mass then suddenly becomes solid, and on continuing to heat melts again at $223^{\circ}$ (the melting point of para-phenylenedipropionic acid, see below).

The silver salt was prepared by precipitating a neutral solution of the ammonium salt with silver nitrate; it is a whitè amorphous substance, and on analysis gave the following result :-

0.5843 gram substance gave 0.3393 gram silver.

$$
\begin{aligned}
& \text { Calculated for } \\
& \mathrm{C}_{14} \mathrm{H}_{10} \mathrm{Ag}_{4} \mathrm{O}_{8} \text {. Found. } \\
& \mathrm{Ag} \text {...... } 58.47 \text { per cent. } \quad 58.07 \text { per cent. }
\end{aligned}
$$

The neutral solution of the ammonium salt gives with lead acetate, barium chloride, and calcium chloride, white precipitates of the respective salts.

\section{Paraphenylenedipropionic Acid, $\mathrm{C}_{6} \mathrm{H}_{4}\left(\mathrm{CH}_{2} \cdot \mathrm{CH}_{2} \cdot \mathrm{COOH}\right)_{2}$.}

Para-xylylenedimalonic acid when heated is easily decomposed yielding paraphenylenedipropionic acid and 2 mols. of carbonic anhydride. This decomposition is best effected by heating a solution of the acid in about 3 parts of water at $180^{\circ}$ in a sealed tube until the evolution of gas ceases, exactly as is described in more detail under the corresponding meta-compound. The reaction in this case goes beautifully, and after cooling, the tube is found to be filled with hard white nodular masses of almost pure paraphenylenedipropionic acid. To purify the product, it is collected and spread on a porous plate to remove traces of oil, and is then dissolved in boiling methyl alcohol; this solution on standing for 24 hours deposits the pure acid in curious nodular aggregates, which after washing with a little alcohol and drying at $100^{\circ}$, were analysed and the following results obtained :-

\begin{tabular}{|c|c|c|}
\hline & $\begin{array}{l}\text { Calculated for } \\
\mathrm{C}_{12} \mathrm{H}_{14} \mathrm{O}_{4} \text {. } \\
64.86 \text { per cent. }\end{array}$ & $\begin{array}{l}\text { Found. } \\
64.73 \text { per cent. }\end{array}$ \\
\hline & $6 \cdot 31$ & $6 \cdot 34$ \\
\hline & $28 \cdot 83$ & $28 \cdot 93$ \\
\hline
\end{tabular}

0.1770 gram substance gave 0.4200 gram $\mathrm{CO}_{2}$ and 0.1010 gram $\mathrm{H}_{2} \mathrm{O}$.

Paraphenylenedipropionic acid melts at $223-224^{\circ}$; it is only sparingly soluble in cold methyl or ethyl alcohol, and almost insoluble in water.

The silver salt is a white, apparently amorphous precipitate which does not blacken on exposure to diffused light; it was obtained in the 
usual way by precipitating the neutral solution of the ammonium salt, and, after drying at $100^{\circ}$, analysed.

0.4300 gram substance gave on ignition 0.2125 gram silver.

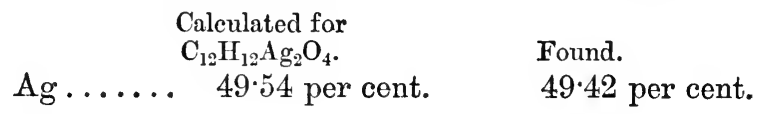

The following reactions are shown by a neutral solution of the ammonium salt:-Copper sulphate gives a faint greenish-white amorphous precipitate, sparingly soluble in cold, more readily in hot water; lead acetate throws down the lead salt as a white compound insoluble in water; zinc sulphate and mercuric chloride also give white precipitates, whilst barium chloride and calcium chloride give no precipitate.

The following experiment was then made in the hope of obtaining an anhydride of this acid:- 0.3 gram of the pure substance was heated in a test-tube in a metal-bath for half an hour at $300-320^{\circ}$, the acid darkened slightly but no appreciable quantity of water was given off; on raising the temperature considerably and as quickly as possible, the whole distilled over in the form of a thick oil, which, however, at once solidified on the colder parts of the tube, while a very small quantity of carbonaceous matter remained behind. The solid distillate was scraped out of the tube and washed on a porous plate with a small quantity of ether, when an almost colourless substance was obtained which melted at $221-224^{\circ}$; it is therefore clear that no anhydride was formed, but that, on heating, paraphenylenedipropionic acid (m. p. 223-224 $)$ distils unchanged.

\section{Methylic Paraphenylenedipropionate.}

By boiling the silver salt of the acid, suspended in ether, with excess of methyl iodide, for about four hours on a water-bath, methylic paraphenylenedipropionate is formed. The solution is filtered, the residual iodide of silver washed well with warm ether, the washings added to the filtrate, and the whole distilled, when the ethereal salt remains behind as a white, crystalline mass. It was purified by spreading on a porous plate and then recrystallising from hot methyl alcohol. The analysis gave the following results :-

$0 \cdot 16225$ gram substance gave 0.3995 gram $\mathrm{CO}_{2}$ and 0.1050 gram $\mathrm{H}_{2} \mathrm{O}$.

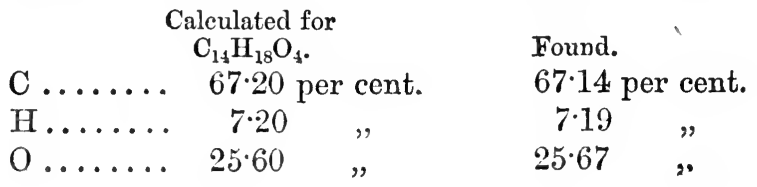


Methylic paraphenylenedipropionate is only sparingly soluble in cold methyl alcohol, and separates out from the hot solution, on cooling, in beautiful glittering plates which melt at $115^{\circ}$.

\section{Paraphenylenediacrylic Acid, $\mathrm{C}_{6} \mathrm{H}_{4}(\mathrm{CH}: \mathrm{CH} \cdot \mathrm{COOH})_{2}$.}

Ethylic ortho-xylylenedichlorodimalonate is readily decomposed on heating it with alcoholic potash into hydrochloric acid, carbonic anhydride, and orthophenylenediacrylic acid.

It was thought interesting to try a similar reaction with an analogous para-derivative. 2 grams of ethylic para-xylylenedibromodimalonate were mixed with a solution of 3 grams of caustic potash in a little alcolool, and the mixture boiled on a water-bath for four hours. After addition of water, the solution was evaporated to dryness, in order to expel the alcohol, and the residual potassium salts dissolved in water; on acidifying with dilute sulphuric acid, a yellowish flocculent substance, probably consisting of impure paraphenylenediacrylic acid, was precipitated; this was not filtered off but the whole extracted with ether, the ethereal solution dried and the ether distilled off. The semi-solid mass which remained was mixed with about 10 c.c. of water and heated in a sealed tube for eight hours at $180^{\circ}$, in order to decompose any tetrabasic acid which might be present. The slightly yellow flocculent substance thus obtained was purified by dissolving it in ammonia, boiling, with animal charcoal, and reprecipitating; lastly the substance was well washed with water, dried at $100^{\circ}$ and analysed, when the following results were obtained:-

0.2010 gram substance gave 0.4840 gram $\mathrm{CO}_{2}$ and 0.0892 gram $\mathrm{H}_{2} \mathrm{O}$.

\begin{tabular}{|c|c|c|c|c|}
\hline \multicolumn{3}{|c|}{$\begin{array}{l}\text { Calculated for } \\
\mathrm{C}_{21} \mathrm{H}_{10} \mathrm{O}_{4} \text {. }\end{array}$} & \multicolumn{2}{|c|}{$\begin{array}{l}\text { Found. } \\
65 \cdot 67 \text { per cent }\end{array}$} \\
\hline H....... & $4: 59$ & , & $4 \cdot 96$ & ", \\
\hline $0 \ldots \ldots$ & $29 \cdot 36$ & , & $29 \cdot 37$ & " \\
\hline
\end{tabular}

Paraphenylenediacrylic acid has been already obtained by Loew (Annalen, 231, 361-384) by the action of sodic acetate and acetic anhydride on terephthalic aldehyde; the product obtained from ethylic para-xylylenedibromodimalonate is identical with Loew's compound, its properties agreeing with those already given by him.

\section{Meta-xylylene Cyanide, $\mathrm{C}_{6} \mathrm{H}_{4}\left(\mathrm{CH}_{2} \cdot \mathrm{CN}\right)_{2}$.}

The method used in the preparation of this substance is as follows :-13 grams of meta-xylylene bromide are dissolved in alcohol 
and a slight excess of the calculated quantity of potassium cyanide, in aqueous solution, added. No reaction takes place at first, but, on warming, potassium bromide begins to crystallise out on the sides of the flask, the reaction being as follows:- $\mathrm{C}_{6} \mathrm{H}_{4}\left(\mathrm{CH}_{2} \mathrm{Br}\right)_{2}+2 \mathrm{KCN}$ $=\mathrm{C}_{6} \mathrm{H}_{4}\left(\mathrm{CH}_{2} \cdot \mathrm{CN}\right)_{2}+2 \mathrm{KBr}$.

By boiling on a water-bath with reflux condenser for a considerable time, the change is completed, the alcohol is then distilled off, and the residue treated with water, whereby the crude metaxylylene cyanide is precipitated as a brown oil ; after separating it from the aquenus solution by means of a separating funnel, it is dried over calcinm chloride and fractioned in a vacuum.

Thus purified, it is obtained as a colourless oil, which on cooling almost immediately solidifies to a white crystalline mass; the analyses gave the following results :-

I. 0.2410 gram substance gave 0.6775 gram $\mathrm{CO}_{2}$ and $0 \cdot 1120$ gram $\mathrm{H}_{2} \mathrm{O}$.

II. $0 \cdot 2195$ gram substance gave $0 \cdot 6155$ gram $\mathrm{CO}_{2}$ and $0 \cdot 1095$ gram $\mathrm{H}_{2} \mathrm{O}$.

III. $0 \cdot 22425$ gram substance gave 34.75 c.c. Nitrogen. Bar. $718 \mathrm{~mm}$. T. $17^{\circ}$.

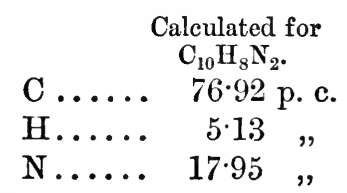

Found.

Meta-xylylene cyanide is a colourless, crystalline substance melt ing at $28-29^{\circ}$; it boils under $300 \mathrm{~mm}$. pressure at $305-310^{\circ}$, a certain amount of decomposition taking place. Ether, alcohol, and chloroform dissolve it readily; it is insoluble, however, in water and light petroleum.

\section{Metaphenylenediacelic acid, $\mathrm{C}_{6} \mathrm{H}_{4}\left(\mathrm{CH}_{2} \cdot \mathrm{COOH}\right)_{2}$.}

To obtain this acid, the orude oil formed by the action of potassium cyanide on meta-xylylene bromide is, without further purification, hydrolysed by boiling it on a water-bath with an excess of alcoholic potash solution, whereby it is converted into the potassium salt of metaphenylenediacetic acid.

Hydrolysis does not take place at all readily, and it is necessary to boil for about six hours before the cessation of the evolution of ammonia shows that the reaction is at an end. When this is the case, the alcohol is distilled off and the residue dissolved in water; on acidifying with dilute sulphuric acid, a small quantity of a brown 
resinous substance is precipitated and is filtered off; the clear solution is then extracted about 20 times with ether, and after drying the ethereal solution over calcium chloride and distilling off the ether, the acid is left in slightly yellowish crystalline crusts. The product is spread on a porous plate, and while still on the plate, the traces of oil are washed away with a few drops of ether; after two crystallisations from water, it is obtained pure in the form of colourless needles.

It was dried at $100^{\circ}$, and analysed with the following results :-

0.1697 gram substance gave 0.3830 gram $\mathrm{CO}_{2}$ and 0.0800 gram $\mathrm{H}_{2} \mathrm{O}$.

\begin{tabular}{|c|c|c|}
\hline & $\begin{array}{l}\text { aleulated for } \\
\mathrm{C}_{10} \mathrm{H}_{10} \mathrm{O}_{4} \text {. }\end{array}$ & Faund. \\
\hline & $5 \cdot 15$ & $5 \cdot 24$ \\
\hline & 32.99 & $33 \cdot 22$ \\
\hline
\end{tabular}

Metaphenylenediacetic acid melts at $170^{\circ}$; it is easily soluble in water, alcohol, and ether, but almost insoluble in light petroleum or chloroform: it crystallises from water in beautiful clusters of concentric needles. The yield obtained by hydrolysing the cyanide with alcoholic potash is almost theoretical.

On adding silver nitrate to a neutral aqueous solution of the ammonium salt, the silver salt is obtained as a white amorphous precipitate; after washing well with water, and drying first on a porous plate, and then at $100^{\circ}$, analysis gave the following result:-

0.1305 gram substance gave 0.0685 gram Ag.

$$
\begin{aligned}
& \text { Calculated for } \\
& \mathrm{C}_{10} \mathrm{H}_{8} \mathrm{Ag}_{2} \mathrm{O}_{4} \text {. Found. } \\
& \text { Ag..... } 52.8 \text { per cent. } \quad 52.5 \text { per cent. }
\end{aligned}
$$

In an aqueous solution of the ammonium salt, lead acetate gives a white amorphous precipitate, but with sulphate of zinc a crystalline substance is produced; barium and calcium chlorides do not give any reaction.

No anhydride of the acid was obtained although an experiment was made as follows : -0.2 gram of the pure acid was heated in a metalbath for 40 minutes at $300-320^{\circ}$, and then distilled by quickly raising the temperature: a small amount of carbonaceous residue was left, but almost the whole of the acid passed over as a slightly yellowish oily distillate, which condensed on the cooler parts of the tube and immediately solidified; it was purified by washing with a small quantity of ether, and a melting point taken, which was found to be $170-172^{\urcorner}$; metaphenylenediacetic acid melts 
at $170^{\circ}$, and it is therefore clear that no anhydride had been formed.

\section{Para-xylylene Cyanide, $\mathrm{C}_{6} \mathrm{H}_{4}\left(\mathrm{CH}_{2} \cdot \mathrm{CN}\right)_{2}$.}

15 grams of para-xylylene bromide are dissolved in alcohol, and 8 grams of potassium cyanide dissolved in as little water as possible are added to the solution; no reaction takes place at first, but on heating the mixture double decomposition commences, a large quantity of a bright-yellow flocculent substance separating; this is, however, not para-xylylene cyanide, but a resinous compound formed by some more complicated secondary reaction: after boiling on the water-bath with reflux condenser until the penetrating odour of the bromide is no longer perceptible, the alcohol is distilled off and water added to the residue, when the cyanide is precipitated as a dirty: white solid. On shaking with ether, it is dissolved while the insoluble resinous impurities are left; after drying, the ether is evaporated and the para-xylylene cyanide is obtained crystallised on the sides of the flask.

It may be purified by redissolving it in ether, boiling with a little animal charcoal, filtering, and allowing the filtrate to evaporate slowly: by this means well-defined crystals are obtained which after recrystallisation are quite pure. On analysis, the following results were obtained :-

I. $0 \cdot 1690$ gram substance gave $0 \cdot 4755$ gram $\mathrm{CO}_{2}$ and 0.0820 gram $\mathrm{H}_{2} \mathrm{O}$.

II. $0 \cdot 12425$ gram substance gave $19 \cdot 7$ c.c. Nitrogen. Bar. $718 \mathrm{~mm}$. $\mathrm{T}=7^{\circ}$.

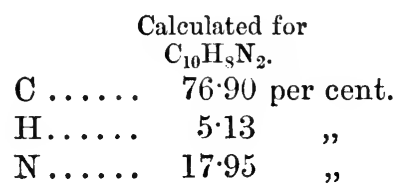

Found.

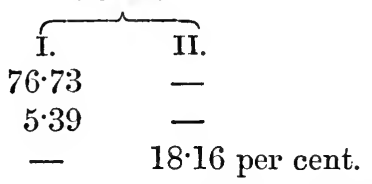

Para-xylylene cyanide is a colourless substance which crystallises in long three-sided prisms and melts at $96^{\circ}$; in whatever manner the conditions are varied, it seems impossible to avoid the formation of a large amount of the yellow resinous substance alluded to above; the yield of cyanide is, therefore, a poor one, being only about 50 per cent. of the theoretical.

\section{Paraphenylenediacetic Acid, $\mathrm{C}_{6} \mathrm{H}_{4}\left(\mathrm{CH}_{2} \cdot \mathrm{COOH}\right)_{2}$.}

When the preceding compound is hydrolysed, paraphenylenediacetic acid is obtained; for its preparation, 2 grams of the cyanide 
were added to an excess of the calculated quantity of potash dissolved in methyl alcohol, and boiled on the water-bath with reflux condenser, until no further evolution of ammonia could be observed.

After evaporating the alcohol, the residue was taken up with water and dilute sulphuric acid until the solution showed a strongly acid reaction; paraphenylenediacetic acid was thus partially precipitated together with a small quantity of brown impurity; the whole was shaken out well with ether, when the acid dissolved, leaving most of the impurities. The ethereal solution was dried and the product obtained as a crystalline crust when the ether was distilled off; the yield was almost theoretical. To purify the acid, it was dissolved in alcohol, boiled with a little animal charcoal, the filtered solution evaporated to dryness on the water-bath, and the residue twice recrystallised from water containing a trace of alcohol. Para-phenylenediacetic acid is thus obtained in fine, colourless needles, which are very readily soluble in alcohol, far less so in water or ether, and melt at $240-241^{\circ}$. The analysis gave the following results :-

0.1730 gram substance gave 0.3920 gram $\mathrm{CO}_{2}$ and 0.08850 gram $\mathrm{H}_{2} \mathrm{O}$.

\begin{tabular}{|c|c|c|c|}
\hline \multicolumn{3}{|c|}{ Calculated for } & \multirow{2}{*}{$\begin{array}{l}\text { Found. } \\
61 \cdot 80 \text { per cent }\end{array}$} \\
\hline . & $61 \cdot 86$ & cent. & \\
\hline & $5 \cdot 15$ & , & $5 \cdot 46$ \\
\hline & $32 \cdot 99$ & $"$ & $32 \cdot 74$ \\
\hline
\end{tabular}

The silver salt is precipitated on adding nitrate of silver to a neutral solution of the ammonium salt as a white amorphous mass; it was collected, well washed with water, and dried, first on a porous plate, then at $100^{\circ}$, and analysed with the following result :-

0.2805 gram substance gave 0.1473 gram silver.

$$
\begin{aligned}
& \text { Calculated for } \\
& \mathrm{C}_{10} \mathrm{H}_{8} \mathrm{Ag}_{2} \mathrm{O}_{4} \text {. Found. } \\
& \text { Ag...... } \quad 52 \cdot 8 \text { per cent. } \quad 52 \cdot 6 \text { per cent. }
\end{aligned}
$$

Paraphenylenediacetic acid is not converted into its anhydride even on heating at $300-320^{\circ}$ for half an hour, since, by quickly raising the temperature, a product distils over which after washing with a little ether showed the same melting point as the original acid.

\section{Note un the Preparation of Isophthalic Acid.}

$$
\text { Meta-xylylenediethyl Ether, } \mathrm{C}_{6} \mathrm{H}_{4}\left(\mathrm{CH}_{2} \mathrm{OC}_{2} \mathrm{H}_{5}\right)_{2} \text {. }
$$

The preparation of isophthalic acid by the oxidation of meta-xylene with potassium dichromate and dilate sulphuric acid (Fittig, Velguth, 
Annalen, 148, 11), is known to be very tedious owing to the extreme stability of this hydrocarbon, the greater portion of which remains unchanged 'even after continued boiling for days together. The following method was therefore worked out and found to give excellent results: meta-xylene is treated with the requisite quantity (2 mols.) of bromine at $125^{\circ}$, and the dibromide formed, without purification, is boiled with alcoholic potash, and thus converted into meta-xylylene diethyl ether. When this is treated with potassium dichromate and sulphuric acid, a quantitative yield of isophthalic acid is obtained; the ether was, however, first prepared in the pure state and investigated a little niore closely; for this purpose pure meta-xylylene bromide was boiled for six hours with a solution of alcoholic potash which contained twice the calculated quantity of alkali, water was then added, and the oil which settled to the bottom separated from the aqueous solution, dissolved in ether, and allowed to stand over calcium chloride; after distilling off the ether, the product remained as a dark-brown oil which was found still to contain bromine, eitter. owing to the presence of a trace of undecomposed meta-xylylene bromide, or of an intermediate compound having the formula $\mathrm{C}_{6} \mathrm{H}_{4} \mathrm{Br} \cdot \mathrm{CH}_{2} \cdot \mathrm{OC}_{2} \mathrm{H}_{5}$. It was therefore treated with zinc-dust and acetic acid, and the ether, thus freed completely from bromine, was isolated in the usual manner, and submitted to fractional distillation, when almost the whole distilled over between $235^{\circ}$ and $255^{\circ}$; this process was twice repeated and meta-xylylene diethyl ether obtained in the pure state in the form of a colourless, mobile, pleasant-smelling oil; it was analysed with the following results :-

0.1392 gram substance gave 0.3805 gram $\mathrm{CO}_{2}$ and 0.1153 gram $\mathrm{H}_{2} \mathrm{O}$.

\begin{tabular}{|c|c|c|c|c|}
\hline \multicolumn{3}{|c|}{$\begin{array}{l}\text { Calculated for } \\
\mathrm{C}_{12} \mathrm{H}_{18} \mathrm{O}_{2} \text {. }\end{array}$} & \multicolumn{2}{|c|}{$\begin{array}{l}\text { Found. } \\
74.53 \text { per cent }\end{array}$} \\
\hline & $9 \cdot 28$ & , & $9 \cdot 19$ & , \\
\hline$\ldots$ & $16 \cdot 49$ & , & $16 \cdot 28$ & ", \\
\hline
\end{tabular}

This compound boils at $246-247^{\circ}$ (712 mm. pressure, uncorr.), and does not solidify even when cooled to $0^{\circ}$; it is isomeric with the ortho-derivative prepared by Leser (Ber., 17, 1825), which boils at $247-249^{\circ}$ (720 mm. pressure).

Of course, when this ether is required for the preparation of isophthalic acid, it is quite unnecessary to purify it, the crude product of the action of alcoholic potash on the xylylene bromide is mixed directly with an excess of potassium dichromate in a large flask and sulphuric acid added; the reaction is very energetic and great heat is evolved, so that in working with large quantities it is advisable to add the acid very slowly or the mixture will be apt to froth over. 
When the oxidation is completed, which is the case in a very short time, the isophthalic acid seen as a white sandy powder on the bottom of the flask is collected and washed with water; it is, however, very difficult to get rid of the last traces of the green mother-liquor, and to obtain the product quite pure, it is dissolved in sodium carbonate, and the filtered solution acidified with sulphuric acid. After washing the precipitated isophthalic acid and drying it at $100^{\circ}$, an analysis was made with the following result :-

0.1477 gram substance gave 0.3128 gram $\mathrm{CO}_{2}$ and 0.0500 gram $\mathrm{H}_{2} \mathrm{O}$.

\begin{tabular}{|c|c|c|}
\hline & lculated for & Found. \\
\hline$C \ldots \ldots$ & $57 \cdot 77$ per cent. & $57 \cdot 75$ per cent. \\
\hline & $3 \cdot 61 \quad$ & $3 \cdot 76 \quad$, \\
\hline $\mathrm{O}$ & $38 \cdot 62$ & $38 \cdot 49$ \\
\hline
\end{tabular}

In this way, an almost theoretical yield of isophthalic acid can be obtained from meta-xylene with comparative ease and in a short time, whereas by the old method, several days were required to prepare a few grams.

This work was carried out in the laboratory of Professor A. v. Baeyer in Munich, and, before closing, I desire to express my gratitude for the kindness which was invariably shown to me, and for the interest which he took in this research. 

[Reprinted from the Journal of the Chemical Society, June, 1884. Vol. XLV.]

\title{
ON BENZOYLACETIC ACID AND SOME OF ITS DERIVATIVES. PART I.
}

\author{
By W. H. Perkin (Jun.), Ph.D., Privatdocent at the \\ University of Munich.
}

Among the many methods which have been discovered and employed in the last few years, for the synthesis of organic compounds, those which are based on the use of acetoacetic ether are among the most important, this body being useful in two ways for building up organic bodies : in the first place on account of the acid character of the hydrogen-atoms contained in the methylene-group; and in the second place, being a ketone, it is capable of entering into almost all the reactions which characterise the latter class of bodies. The replacement of the hydrogen-atoms in acctoacetic ether, by alcohol and acid radicals, has been especially studied by Wislicenus and his pupils; 
whereas the reactions which involve the ketone-group have, with few exceptions, been more or less neglected.

The reason of this is that not only are the experimental difficulties great, but there is also considerable uncertainty in interpreting the results, owing to the mobility of the different groups in acetoacetic ether. More especially does doubt exist as to the part played by the methyl-group in such reactions and condensations. For this reason it appeared to me that it would be very interesting to carefully examine the benzoylacetic ether, recently discovered by Baeyer (Ber., 15, 2705), with especial reference to reactions in which the ketonegroup takes part. Benzoylacetic ether, as was to be expected, gives in the aromatic series bodies exactly corresponding to those obtained from acetoacetic ether in the fatty series, and for this reason, as well as on account of the stability of the benzoyl-group, and the greater tendency of bodies in the aromatic series to crystallise, it seemed to be especially adapted to the study of the more complicated condensations.

Before entering into the details of this research, I should like to express my sincere thanks to Professor Baeyer, in whose laboratory it was carried out, for his kind help and advice which he always placed at my disposal during its progress, as well as for the interest which be took in all my other studies. I must also thank Herr C. Bernhart for the help which he gave me in the experimental part.

Part I of this research comprises the preparation and properties of benzoylacetic ether, benzoylacetic acid, and the compounds in which the hydrogen-atoms in the methylene-group are replaced by alcohol radicals.

\section{Preparation of Phenylpropiolic Acid.}

Cinnamic acid, as is well known, combines directly with two atoms of bromine, being thereby converted into phenyldibromopropionic acid, thus :

$$
\mathrm{C}_{6} \mathrm{H}_{5} \cdot \mathrm{CH}: \mathrm{CH} \cdot \mathrm{COOH}+\mathrm{Br}_{2}=\mathrm{C}_{6} \mathrm{H}_{5} \cdot \mathrm{CHBr} . \mathrm{CHBr} . \mathrm{COOH} \text {. }
$$

This acid, on treatment with alcoholic potash, first gives up $1 \mathrm{~mol}$ of hydrobromic acid, forming the two isomeric $\alpha$ - and $\beta$-monobromocinnamic acids according to the equation-

$$
2 \mathrm{C}_{6} \mathrm{H}_{5} \mathrm{CHBr} . \mathrm{CHBr} . \mathrm{COOH}=\underset{a-\mathrm{Monobromocinnamic} \mathrm{acid.}}{\mathrm{C}_{6} \mathrm{H}_{5} \mathrm{CBr}: \mathrm{CH} . \mathrm{COOH}}
$$


By converting these acids into ammonia salts and recrystallising once or twice from water, it is easy to separate the one from the other, the ammonia-salt of a-monobromocinnamic acid being much less soluble than that of the $\beta$-acid. Of these two bodies only the $\alpha$-acid gives a good yield of phenylpropiolic acid on treatment with alcoholic potash, the $\beta$-acid being partly transformed into monobromostyrolene and other secondary products (Barisch, J.pr. Chem. [2], 20, 180 ; Glaser, Annalen, 154, 140). It is therefore necessary before heating with potash, to transform the $\beta$-acid into the $\alpha$-acid, which is easily done by heating it some degrees above its melting point, or more simply still by etherification, in which case the ether of the $a$-acid is formed. The formation of phenylpropiolic acid from $\alpha$-monobromocinnamic acid is represented by the following equation :-

\section{$\mathrm{C}_{6} \mathrm{H}_{5} \cdot \mathrm{CBr}: \mathrm{CH} \cdot \mathrm{COOH}=\mathrm{C}_{6} \mathrm{H}_{5} \cdot \mathrm{C}: \mathrm{C} \cdot \mathrm{COOH}+\mathrm{HBr}$.}

After many experiments, however, it was found that the following modification of the above process gives the best results, at the same time rendering the somewhat troublesome transformation of the $\beta$ into the $\alpha$-monobromocinnamic acid unnecessary.

500 grams of cinnamic acid are suspended in about $1-1 \frac{1}{2}$ litres of absolute alcohol, and hydrochloric acid is passed through till the whole is dissolved and the liquid is thoroughly saturated with the gas. The product, after standing for two or three hours, in order to make sure that the reaction is complete, is poured into ice and water, and the oil which sinks to the bottom is separated by means of a tapfunnel, the watery liquid being extracted with a little ether. This oil, which consists of almost pure cinnamic ether, is next dissolved in a little ether, washed with a dilute solution of sodium carbonate, to remove traces of hydrochloric acid and unchanged cinnamic acid; and after thoroughly drying over chloride of calcium, treated with 440 grams of bromine, in small quantities at a time, care being taken to keep the liquid cool. The bromine disappears instantly without evolution of hydrobromic acid. After standing for a short time, the whole is poured into a large open dish and the ether is allowed to evaporate, when phenyldibromopropionic ether separates out in large crystals, forming a solid cake at the bottom of the dish. The crystals are then separated from the mother-liquor, pressed between filter-paper, exposed to the air for a short time to remove traces of bromine, and lastly, if necessary, crystallised from ligroin or alcohol.

The mother-liquor on evaporation deposits crystals of the ether, which are somewhat discoloured by impurities, but on recrystallisation may easily be obtained pure, and melting at $69^{\circ}$.

A weighed quantity of this body is now added to the calculated 
quantity of alcoholic potash (1 mol. of ether requires 3 mols. of potash). The reaction is so energetic that the heat evolved is generally sufficient to cause the alcohol to boil. As soon as all the ether has been added, the whole is heated to boiling for six to eight hours in a flask connected with an inverted condenser. After distilling off the alcohol, the residue, which consists of a mixture of potassic phenylpropiolate and potassic bromide, is dissolved in water and filtered, and the clear liquid is acidulated with an excess of dilute sulphuric acid, which precipitates the phenylpropiolic acid as an oil, which, however, on standing, soon solidifies, especially if well agitated from time to time. The crude acid is next filtered off, well washed with water, dissolved in carbonate of soda solution, and gently heated with animal charcoal for about half an hour on a water-bath, agitating well from time to time. After filtering off the animal charcoal, an almost colourless solution of soda-salt is obtained, which, on acidulating with sulphuric acid, deposits the phenylpropiolic acid nearly

Phenylpropiolic

Acid.

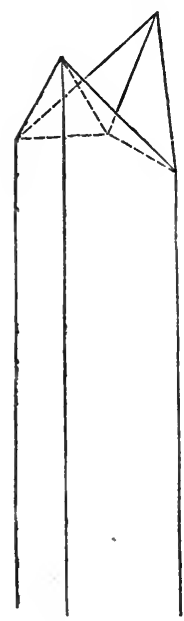
pure. If required perfectly pure, it must be crystallised from water, and is thus easily obtained in well-characterised crystals, which Professor Haushofer was kind enough to measure, and describe as follows :-

"Prismatic crystals of the rhombic system with monoclinic habit. Acute dihedral angle of the prism $66^{\circ} 50^{\prime}$. Of pyramidal faces, there appear constantly only the pair resting on an obtuse edge of the prism. Very characteristic is the twin-combination of the crystals according to the macropinacoid, whereby the hemi-pyramids of the two individuals are symmetrically joined, and form reentering angles. The entire development of the crystals is so decidedly monoclinic, that my reference of them to the rhombic system is, based solely on the directions of extinction on the prismatic faces, which directions are exactly parallel and perpendicular to the prismatic edges."

Phenylpropiolic acid is produced from the dibromophenylpropionic ether according to the following equation :-

\section{$\mathrm{C}_{6} \mathrm{H}_{5} \cdot \mathrm{CHBr} . \mathrm{CHBr} . \mathrm{COOC}_{2} \mathrm{H}_{5}+3 \mathrm{KOH}=\mathrm{C}_{6} \mathrm{H}_{5} \cdot \mathrm{C}$ : C.COOK $+2 \mathrm{KBr}+2 \mathrm{OH}_{2}$.}

By this method there is apparently no bromstyrolene formed, an if care be taken to avoid any large excess of potash in saponifying the dibromophenylpropionic ether, the yield is very good, about $80-85$ 
per cent. of the theoretical amount. If, however, excess of potash be used, the phenylpropiolic acid is easily decomposed into phenylacetylene and acetophenone.

\section{Preparation of Benzoylacetic Ether.}

The phenylpropiolic acid must now be converted into the ethylic ether, which is best done in the following way. Pure phenylpropiolic acid is dissolved in absolute alcohol, and hydrochloric acid gas is passed through the liquid till the whole is thoroughly saturated, care being taken to cool the liquid well during the operation, after which the product is left at rest for two or three hours in order to complete the reaction. The whole is then poured into ice-cold water, and the phenylpropiolic ether, which is precipitated as an oil, is two or three times extracted with ether.

The etheric solution, after washing with dilute carbonate of soda, is well dried over chloride of calcium and the ether is distilled off, when pure phenylpropiolic ether remains behind as a thick, and almost colourless oil, having a fruity smell and burning taste; it does not solidify at $0^{\circ}$. If rabbed on the skin it produces a most painful burning sensation. When quickly distilled it boils between $260^{\circ}$ and $270^{\circ}$ without undergoing much decomposition. When this ether is dissolved in common sulphuric acid it takes up the elements of water, forming ethylic benzoylacetate, thas :-

$$
\mathrm{C}_{6} \mathrm{H}_{5} \cdot \mathrm{C}: \mathrm{C} . \mathrm{COOC}_{2} \mathrm{H}_{5}+\mathrm{H}_{2} \mathrm{O}=\mathrm{C}_{6} \mathrm{H}_{5} \cdot \mathrm{CO} \cdot \mathrm{CH}_{2} \cdot \mathrm{COOC}_{2} \mathrm{H}_{5} \text {. }
$$

For the formation of benzoylacetic ether in this way, commercial sulphuric acid is somewhat too concentrated, and a better yield is obtained if a few drops of water are added to every kilogram before use.

The method of preparation is the following:-From 2 to 3 kilos. of sulphuric acid are cooled to $0^{\circ}$ in a freezing mixture, and then 100 grams of phenylpropiolic ether are slowly added, drop' by drop, so that the temperature never rises above $+3^{\circ}$, the whole being well shaken after each addition. The sulphuric acid becomes coloured slightly brownish during the reaction, and there appears always to be a little sulphurous anhydride formed, especially if a rise of temperature takes place. After standing for two or three hours at as low a temperature as possible, the product is slowly poured on powdered ice, everything being carefully cooled, and the operation being best performed by allowing the sulphuric acid to trickle into a funnel filled with ice, which is kept well stirred all the time. The watery liquid, which is filled with drops of oil, is repeatedly extracted with ether. 
The etheric solution is then quickly washed with a dilute solution of sodium carbonate, and finally dried over potassium carbonate, which, as a rule, extracts a quantity of colouring matter. After filtering from potassium carbonate and distilling off the ether, crude benzoylacetic ether remains behind as a thick yellowish oil.

In order to purify this liquid, it is next shaken with a cold dilute solution of caustic soda and a little animal charcoal, and filtered, the whole operation being performed as quickly as possible, in order to avoid decomposition of the ether. The clear alkaline solution is next mixed with ice, acidulated with dilute sulphuric acid, and well shaken with ether; and lastly this etheric solution is carefully dried over potassium carbonate, and the ether distilled off, when pure benzoylacetic ether remains behind as a colourless oil, which can easily be freed from traces of ether by leaving it for some time over sulphuric acid in a vacuum.

On analysis the following numbers were obtained:-

I. 0.2072 gram substance gave $0.1198 \mathrm{OH}_{2}$ and $0.5208 \mathrm{CO}_{2}$.

Found.
$\mathrm{C}=68 \cdot 62$ per cent.
$\mathrm{H}=6.42 \quad$,
$\mathrm{O}=24.96 \quad$,

Theory,
$\mathrm{C}_{6} \mathrm{H}_{5} \cdot$ CO.CH
$68 \cdot 75$ per cent.
6.25 cl
$25 \cdot 00 \quad "$

Benzoylacetic ether is a colourless oil having an agreeable smell, somewhat recalling that of acetoacetic ether. It does not solidify at $0^{\circ}$. If quickly fractioned most of it distils over between $265^{\circ}$ and $270^{\circ}$ without much decomposition. Under a pressure of $200 \mathrm{~mm}$. it passes over between $230^{\circ}$ and $235^{\circ}$. It should not, however, be distilled, if this operation can be avoided, as it entails considerable loss, and the crude ether is generally pure enough for most purposes.

Benzoylacetic ether is sparingly soluble in water, but mixes with ether and alcohol in all proportions. The solution in alcohol gives the same violet coloration with chloride of iron as acetoacetic ether. By boiling with water, or better with dilute sulphuric acid, it is split up into acetophenone, alcohol, and carbonic anhydride, according to the equation :-

$$
\mathrm{C}_{6} \mathrm{H}_{5} \cdot \mathrm{CO} \cdot \mathrm{CH}_{2} \cdot \mathrm{COOC}_{2} \mathrm{H}_{5}+\mathrm{H}_{2} \mathrm{O}=\mathrm{C}_{6} \mathrm{H}_{5} \mathrm{CO} \cdot \mathrm{CH}_{3}+\mathrm{CO}_{2}+\mathrm{C}_{2} \mathrm{H}_{5} \mathrm{OH} \text {. }
$$

This decomposition is exactly analogous to the splitting up of acetoacetic ether into acetone, alcohol, and carbonic anhydride. In benzoylacetic ether, just as in acetoacetic ether, the hydrogen-atoms in the $\mathrm{CH}_{2}$-group are replaceable by sodium. It appears, however, that after having replaced 1 atom of hydrogen with sodium, it is necessary 
first to exchange this for some radical such as ethyl, before it is possible to insert another.

The sodium-compound is best prepared by mixing benzoylacetic ether (4 grams) with a strong solution of sodium ethylate in alcohol, containing the calculated quantity of sodium $\left(0^{\circ} 6 \mathrm{gram}\right)$. The reaction takes place according to the equation :-

\section{$\mathrm{C}_{6} \mathrm{H}_{5} \cdot \mathrm{CO} \cdot \mathrm{CH}_{2} \cdot \mathrm{COOC}_{2} \mathrm{H}_{5}+\mathrm{NaOC}_{2} \mathrm{H}_{5}=\mathrm{C}_{6} \mathrm{H}_{5} \cdot \mathrm{CO} \cdot \mathrm{CHNa} \cdot \mathrm{COOC}_{2} \mathrm{H}_{5}$ $+\mathrm{C}_{2} \mathrm{H}_{5} \mathrm{OH}$.}

After standing for some time in the dark, the sodium-compound crystallises out in long silky needles which, however, on being exposed to the air, quickly turn brown, especially when moist.

The mother-liquor is then drawn off by means of a vacuum pump, and the crystals are pressed between filter-paper, when the sodiumcompound is obtained pure, having somewhat the appearance of cotton wool. On standing in contact with the air, however, it slowly decomposes. . If heated in the dry state it decomposes easily, yielding acetophenone and other bodies. It is readily soluble in water and alcohol, almost insoluble in ether.

The following derivatives of benzoylacetic ether were prepared by precipitation from a solution of the sodium-compound in water.

The barium compound, $\left(\mathrm{C}_{11} \mathrm{H}_{11} \mathrm{O}_{3}\right)_{2} \mathrm{Ba}$, is precipitated on adding barium acetate as a white amorphous mass, sparingly soluble in water, but more so in alcohol.

The silver compound, $\mathrm{C}_{11} \mathrm{H}_{11} \mathrm{AgO}_{3}$, is formed by adding a solution of silver nitrate. It is a white amorphous precipitate. On warming, silver is immediately precipitated; it is also easily decomposed by caustic soda.

On adding copper sulphate to the solution of the sodium salt, the copper compound, $\left(\mathrm{C}_{11} \mathrm{H}_{11} \mathrm{O}_{3}\right)_{2} \mathrm{Cu}$, is precipitated as a pale green mass, insoluble in water. In dilute soda solution it dissolves easily, forming a beautiful blue solution, from which $\mathrm{CuO}$ is precipitated on boiling.

The lead compound is precipitated, on adding lead acetate, as a white precipitate, which is also soluble in caustic soda.

Ferric chloride gives a dirty violet coloration with dilute solutions of the sodium salt, the liquid becoming turbid on standing.

\section{Benzoylacetic Acid.}

Some time since Ceresole (Ber., 15, 1871), by saponifying acetoacetic ether with dilute potash at ordinary temperatures, obtained the corresponding acetoacetic acid. It therefore appeared interesting to 
see if in this case, by saponification in the cold, benzoylacetic acid could be isolated, this appearing the more probable, as benzoylacetic ether is more stable than acetoacetic ether. Pure benzoylacetic ether was therefore dissolved in dilute aqueous potash ( $2 \frac{1}{2}-3$ per cent.), care being taken to avoid any large excess, and then left at rest at the ordinary temperature for about 24 hours. The product was then filtered, and after carefully cooling with ice, acidulated with dilute sulphuric acid, when the acid was precipitated in white flakes. After extracting with ether, the etheric solution was allowed to evaporate at the ordinary temperature, the acid remaining behind as a slightly brownish, hard, crystalline mass, which was next dissolved in dilute ammonia, shaken with a little animal charcoal, and filtered.

On acidulating with dilate sulphuric acid, and extracting with ether, the acid remained as an almost colourless crystalline mass, consisting of nearly pure benzoylacetic acid.

In order to obtain the body perfectly pure, it is dissolved in a little warm benzene (at $70^{\circ}$ ), and a trace of light petroleum added. On cooling, the acid crystallises out in small crystals, which, when seen under the microscope, appear in the form of thin transparent needles, which polarise light. The analysis gave the following numbers:-

I. 0.1519 gram substance gave 0.0675 gram $\mathrm{OH}_{2}$ and 0.3647 gram $\mathrm{CO}_{2}$.

II. 0.1492 gram substance gave 0.0695 gram $\mathrm{OH}_{2}$ and 0.3614 gram $\mathrm{CO}_{2}$.

\begin{tabular}{|c|c|c|}
\hline \multicolumn{2}{|c|}{ Found. } & Theory, \\
\hline & II. & $\mathrm{C}_{6} \mathrm{H}_{5} \cdot \mathrm{CO} . \mathrm{CH}_{2} \cdot \mathrm{COOH}$ \\
\hline$=65 \cdot 48$ & 66.06 per cent. & 65.85 per cent. \\
\hline$=4.93$ & $5 \cdot 17$ & $4 \cdot 88$ \\
\hline$=29.59$ & $28 \cdot 77$ & $29 \cdot 27$ \\
\hline
\end{tabular}

Benzoylacetic acid, several times crystallised from benzene, melts at $103-104^{\circ}$, if quickly heated, at the same time undergoing decomposition, with evolution of carbonic anhydride. (The melting point, $85-90^{\circ}$, first given in the Berichte $[16,2129]$, is too low, owing to the fact that at that time I had not sufficient substance at my disposal to purify it by repeated crystallisation.)

The acid appears to sublime slowly at ordinary temperatures, so that if preserved in a test-tube the sides, after some months, become covered with thin colourless transparent plates, consisting, apparently, of pure benzoylacetic acid. This acid is difficultly soluble in light petroleum, and crystallises from the hot solution on cooling in small flat needles. It is easily soluble in alcohol, ether, and hot benzene. On adding light petroleum to the solution in benzene the acid is almost completely precipitated in the form of slender needles. 
A small crystal of the acid dissolved in alcohol gives, on addition of a drop of ferric chloride, a beautiful reddish-violet coloration.

Benzoylacetic acid is sparingly soluble in cold water, the solution giving only a slight coloration on adding chloride of iron; it is much more easily soluble in hot water, a drop of chloride of iron producing an intense violet coloration.

Benzoylacetic acid may be formed directly, by dissolving finely powdered phenylpropiolic acid in concentrated sulphuric acid, according to the equation-

$$
\mathrm{C}_{6} \mathrm{H}_{5} \cdot \mathrm{C}: \mathrm{C} \cdot \mathrm{COOH}+\mathrm{H}_{2} \mathrm{O}=\mathrm{C}_{6} \mathrm{H}_{5} \cdot \mathrm{CO} \cdot \mathrm{CH}_{2} \cdot \mathrm{COOH} \text {. }
$$

When the acid liquid is poured on ice, a yellow amorphous mass is obtained consisting of benzoylacetic acid, mixed with a little unchanged phenylpropiolic acid.

The former acid, on heating either alone or with dilute sulphuric acid, is quantitatively decomposed into acetophenone and carbonic anhydride, thus :-

$$
\mathrm{C}_{6} \mathrm{H}_{5} \cdot \mathrm{CO} \cdot \mathrm{CH}_{2} \cdot \mathrm{COOH}=\mathrm{C}_{6} \mathrm{H}_{5} \cdot \mathrm{CO} \cdot \mathrm{CH}_{3}+\mathrm{CO}_{2} \text {. }
$$

The formation of acetophenone, by the action of concentrated potash on phenylpropiolic acid, is probably due to the latter first taking np the elements of water, being thereby converted into benzoylacetic acid, which is then decomposed by the further action of the alkali into potassium carbonate and acetophenone.

Benzoylacetic acid dissolves easily on shaking with dilute soda, sodium carbonate, or ammonia. In order to prepare the silver salt, the acid was dissolved in dilute ammonia, and the solution, after filtering, left over sulphuric acid in a vacuum till the excess of ammonia had volatilised, or, better still, carefully neutralised with pure nitric acid. On adding a solution of silver nitrate, the silver salt was precipitated in the form of a white amorphons mass, somewhat soluble in water. It was filtered off, well washed, and dried over sulphuric acid in a vacuum. The analysis gave the following numbers :-

$0 \cdot 1440$ gram substance gave $0 \cdot 0567$. gram silver.

$$
\begin{aligned}
& \text { Found. } \\
& \mathrm{Ag}=39 \cdot 37 \text { per cent }
\end{aligned}
$$

The silver salt is instantly decomposed on heating, with separation of silver.

The following salts were prepared from the ammoniacal salt, which can be obtained as a waxy crystalline mass, by allowing the aqueous 
solution to evaporate to dryness in a vacuum over sulphuric acid. The crystals are generally mixed with traces of acetophenone, produced by the spontaneous decomposition of the salt. The ammonium salt is easily soluble in water. On adding ferric chloride to a solution of the ammonium salt, a thick blackish-violet precipitate is formed, which is slowly decomposed on boiling, carbonic anhydride being evolved. F'errous sulphate does not give any precipitate. The copper salt is produced by adding a solution of copper sulphate to a fairly concentrated solution of the ammonium salt, as a light greenishyellow precipitate, which is not formed in dilute solutions. It is fairly stable, but is slowly decomposed on boiling.

The lead salt is precipitated, on adding acetate of lead, as a heary white amorphous precipitate. The calcium and barium salts are easily soluble in water.

The salts of benzoylacetic acid are difficult to obtain pure and to analyse, on account of their tendency to decomposition into acetophenone and carbonic anhydride. From the above facts it is evident that benzoylacetic acid, although not a stable body, is much more so than acetoacetic acid, which has never been obtained pure enough for direct analysis.

\section{Ethylbenzoylacetic Acid.}

It was now necessary to see whether it was really possible, as in the case of acetoacetic ether, to replace the hydrogen atoms in the methylene-group, by alcohol-radicals, such as methyl, ethyl, \&c., and thus to obtain higher homologues of benzoylacetic acid. The first experiment tried was the action of ethyliodide on the sodium-compound of benzoylacetic ether, in the hope of getting ethylbenzoylacetic ether, according to the following equation:-

$$
\begin{gathered}
\mathrm{C}_{6} \mathrm{H}_{5} \cdot \text { CO.CHNa.COOC } \mathrm{H}_{5}+\mathrm{C}_{2} \mathrm{H}_{5} \mathrm{I}=\mathrm{C}_{6} \mathrm{H}_{5}+\mathrm{CO} \cdot \mathrm{CH}\left(\mathrm{C}_{2} \mathrm{H}_{5}\right) \cdot \mathrm{COOO}_{2} \mathrm{H}_{5} \\
+\mathrm{NaI} .
\end{gathered}
$$

The method of procedure was the following :-

0.6 gram of sodium was dissolved in about 15 c.c. of absolute alcohol, and then mixed with 5 grams of pure benzoylacetic ether, taking care to avoid any rise of temperature. 10 grams of ethyliodide were then added, and the whole heated on a water-bath till a sample, on dilution with water, had no longer an alkaline reaction. The alcohol and excess of ethyliodide were then distilled off, the residue mixed with water, and the oily product extracted with ether. The etheric solution, after well washing and drying over carbonate of potash, left, on distilling off the ether, a thick yellowish oil, consisting probably of nearly pure ethylbenzoylacetic ether, which was, 
however, not further examined, as it appeared to be decomposed on distillation. In order to obtain the free acid, the oil was mixed with dilute alcoholic potash and left for several days at the ordinary temperature until, on diluting a sample with water, only a small quantity of oil was precipitated. The product was mixed with water and several times extracted with ether, to remove any unchanged ethylic ethylbenzoylacetate. The solution of the potash salt of the new acid was then acidulated with dilute sulphuric acid, a solid acid being thereby precipitated, which was extracted with ether. After distilling off the ether, the acid remained behind as a semi-solid mass, which was first spread on a porous plate to remove traces of oil, and then purified by dissolving it in a small quantity of dilute alcohol, from which solvent it crystallises in small needles.

After drying at $60^{\circ}$ the following numbers were obtained on analysis :-

$0 \cdot 1914$ gram substance gave $0 \cdot 1082 \mathrm{H}_{2} \mathrm{O}$ and $0 \cdot 4814 \mathrm{CO}$.

Found.
$\mathrm{C}=68 \cdot 60$ per cent.
$\mathrm{H}=6 \cdot 28 \quad$,
$\mathrm{O}=25 \cdot 12 \quad$,

$\mathrm{C}_{6} \mathrm{H}_{5} \mathrm{CO}$ Theory,
$\mathrm{C}_{2} \mathrm{H}_{5}>\mathrm{CH} . \mathrm{COOH}$.
$68 \cdot 75$ per cent.
$6.25 \quad "$
$25.00 \quad "$

It was therefore ethylbenzoylacetic acid. The acid melts at 111$115^{\circ}$ with slight decoinposition, but could not be obtained free from traces of benzoic acid, which, while rendering it difficult to get an accurate melting point, do not much influence the analytical results. It is easily soluble in alcohol, ether, and benzene, and crystallises on evaporating the etheric solution in silky needles; it is easily soluble also in ammonia, soda, and sodium carbonate solution. When warmed with dilute sulphuric acid, it is decomposed, carbonic acid being given off, and an oil remaining behind, probably consisting for the most part of ethylacetophenone.

\section{Decomposition-products of Ethylbenzoylacetic Ether.}

It next appeared interesting to determine whether the substituted benzoylacetic ethers would, on heating with alcoholic potash, undergo the same decompositions as the corresponding substituted acetoacetic ethers, which, as is well known from the researches of Wislicenus, are split up in two ways. He found, namely, that on employing a highly concentrated potash solution, the product consisted chiefly of organic acids, whereas with dilute solntions a large amount of ketone and 
only a small quantity of organic acids were obtained, according to the following equations, taking ethylacetoacetic ether as example :-

\section{I. $\mathrm{CH}_{3} \cdot \mathrm{CO} \cdot \mathrm{CH}\left(\mathrm{C}_{2} \mathrm{H}_{5}\right) \cdot \mathrm{CO}_{2} \mathrm{C}_{2} \mathrm{H}_{5}+2 \mathrm{KOH}=\mathrm{CH}_{3} \cdot \mathrm{CO}_{2} \mathrm{~K}+$ $\mathrm{C}_{2} \mathrm{H}_{5} \cdot \mathrm{CH}_{2} \mathrm{CO}_{2} \mathrm{~K}+\mathrm{C}_{2} \mathrm{H}_{5} \mathrm{OH}$. Butyric acid.}

\section{II. $\mathrm{CH}_{3} \cdot \mathrm{CO} \cdot \mathrm{CH}\left(\mathrm{C}_{2} \mathrm{H}_{5}\right) \cdot \mathrm{CO}_{2} \mathrm{C}_{2} \mathrm{H}_{5}+2 \mathrm{KOH}=\mathrm{CH}_{3} \cdot \mathrm{CO} \cdot \mathrm{CH}_{2} \cdot \mathrm{C}_{2} \mathrm{H}_{5}+$ Methylpropyl ketone.}

$$
\mathrm{C}_{2} \mathrm{H}_{5} \mathrm{OH}+\mathrm{K}_{2} \mathrm{CO}_{3} \text {. }
$$

As will be seen from the following remarks, this rule applies also to the substituted benzoylacetic ethers.

In order to examine the decomposition-products of ethylbenzoylacetic ether, the crude ether was heated on a water-bath for three hours with moderately concentrated alcoholic potash. The mixture quickly became deep brown, and acquired a smell strongly resembling that of acetophenone. At the end of the reaction water was added, and the precipitated oil was separated from the potash-salt, by extraction with ether.

The etheric solution, after well washing with water, and drying with carbonate of potash, gave, on distilling off the ether, a dark brownish oil, which was then carefully fractioned. Almost the whole quantity distilled over by the first distillation, between $215-230^{\circ}$, and gave, on oft-repeated fractioning, an oil, boiling constantly by $220-222^{\circ}$.

The analysis gave the following results :-

$0 \cdot 1588$ gram substance gave $0 \cdot 1140$ gram $\mathrm{H}_{2} \mathrm{O}$ and $0 \cdot 4687 \mathrm{CO}_{2}$.

$$
\begin{aligned}
& \text { Found. } \\
& \mathrm{C}=80 \cdot 49 \text { per cent. } \\
& \mathrm{H}=7.97 \quad \text {, } \\
& \mathrm{O}=11.54 \quad,
\end{aligned}
$$

Propylphenylketone had therefore been formed from the ethylbenzoylacetic ether by splitting off $\mathrm{CO}_{2}$ and alcohol, according to the following equation :-

$$
\begin{array}{r}
\mathrm{C}_{6} \mathrm{H}_{5} \cdot \mathrm{CO} \cdot \mathrm{CH}\left(\mathrm{C}_{2} \mathrm{H}_{5}\right) \mathrm{COOC}_{2} \mathrm{H}_{5}+2 \mathrm{KOH}=\mathrm{C}_{6} \mathrm{H}_{5} \cdot \mathrm{CO} \cdot \mathrm{CH}_{2} \cdot \mathrm{C}_{2} \mathrm{H}_{5}+ \\
\mathrm{C}_{2} \mathrm{H}_{5} \mathrm{OH}+\mathrm{K}_{2} \mathrm{CO}_{3} .
\end{array}
$$

This ketone has already been described by Schmidt and Fieberg (Ber., 6, 498), and by Burcker (Bull. Soc. Chim., 37, 4). The former obtained it by the distillation of a mixture of benzoate and butyrate of lime, the latter by the action of aluminium chloride on butyric chloride and benzene. 
It does not combine with sodium bisulphite. On oxidation with chromic acid and sulphuric acid benzoic and propionic acids are formed.

In order to examine the acids produced by the action of potash on ethylbenzoylacetic ether, the potassium salt which had been separated from the propylphenylketone, by extraction with ether, was next acidulated with dilute sulphuric acid, and distilled with steam. An almost colourless but strongly acid liquid distilled over, which possessed strongly the characteristic smell of butyric acid. The residue on cooling was extracted with ether.

On distilling off the ether a solid acid remained behind, which crystallised from water in silky plates, possessing all the properties of benzoic acid. They melted at $120^{\circ}$, aud sublimed very easily on heating, and gave the characteristic iron reaction: in order to be certain the acid was turned into the silver salt and analysed with the following result :-

0.4026 gram substance gave 0.1917 gram $\mathrm{Ag}=47 \cdot 59$ per cent. Theory for $\mathrm{C}_{6} \mathrm{H}_{5} \mathrm{COOAg}=47 \cdot 16 \quad$ "

By the action of potash on ethylbenzoylacetic ether, a second decomposition had therefore taken place, according to the following equation :-

$\mathrm{C}_{6} \mathrm{H}_{5} \mathrm{CO} \cdot \mathrm{CH}\left(\mathrm{C}_{2} \mathrm{H}_{5}\right) \cdot \mathrm{COOC}_{2} \mathrm{H}_{5}+2 \mathrm{KOH}=\mathrm{C}_{6} \mathrm{H}_{5} \mathrm{COOK}+$ $\mathrm{CH}_{2}\left(\mathrm{C}_{2} \mathrm{H}_{5}\right) \cdot \mathrm{CO}_{2} \mathrm{~K}+\mathrm{C}_{2} \mathrm{H}_{5} \mathrm{OH}$.

As in the case of substituted acetoacetic ethers, it was found on using very concentrated potash that mostly organic acids were formed, and that in order to get a good yield of phenylpropylketone, it is advisable to use the alkali, moderately dilute, and to digest for a longer time.

\section{Diethylbenzoylacetic Acid.}

From the foregoing it is clear that one of the hydrogen-atoms in the methylene-group in benzoylacetic ether may be replaced by alcohol radicals, the result being the production of homologues of this ether, exactly corresponding in the aromatic series to the substituted acetoacetic ethers in the fatty series. It remained, however, to be tested, whether the second hydrogen-atom could, in like manner, be replaced, e.g., by ethyl, producing diethylbenzoylacetic ether. This, as a matter of fact, is the case. The experiment was carried out in the following way:-

A mixture of 1.2 gram of sodium (dissolved in about 20 c.c. of absolute alcohol), 10 grams benzoylacetic ether, and 10 grams ethyliodide, were heated in a sealed tube for about three hours to $100^{\circ}$. 
At the end of this time, the product having become neutral, the alcohol was distilled off, then 1.2 gram of sodium (dissolved as before in 20 c.c. of absolute alcohol) added to the residue, and the whole was heated with 10 grams of ethyliodide three or four hours to $100^{\circ}$.

The reaction takes place in two phases. In the first place ethylbenzoylacetic ether is formed, which is then further acted on by the sodium ethylate and ethyliodide, forming diethylbenzoylacetic ether, according to the following equation :-

$$
\begin{aligned}
& \mathrm{C}_{6} \mathrm{H}_{5} \cdot \mathrm{CO} \cdot \mathrm{C}\left(\mathrm{C}_{2} \mathrm{H}_{5}\right) \mathrm{Na} \cdot \mathrm{COOC}_{2} \mathrm{H}_{3}+\mathrm{C}_{2} \mathrm{H}_{5} \mathrm{I}= \\
& \mathrm{C}_{6} \mathrm{H}_{5} \cdot \mathrm{CO} \cdot \mathrm{C}\left(\mathrm{C}_{2} \mathrm{H}_{5}\right)_{2} \cdot \mathrm{COOC}_{2} \mathrm{H}_{5}+\mathrm{NaI} .
\end{aligned}
$$

At the end of the reaction the alcohol is distilled off, the residue diluted with water, and extracted several times with ether. After distilling off the ether the diethylbenzoylacetic ether remains behind as a thick dark brown oil, which, as it could not be purified by distillation, was directly saponified and the acid examined.

For this purpose the ether was dissolved in alcohol, and after adding very dilute alcoholic potash, allowed to stand for some weeks at ordinary temperatures. The product was then diluted with water, and any unsaponified diethylbenzoylacetic ether removed by extracting several times with ether. On acidulating the solution of the potash salt with dilute sulphuric acid, an oily acid was precipitated, which, on standing for some time and continually stirring, became quite solid. It was once or twice extracted with ether, and the ethereal solution dried over calcium chloride. On distilling off the ether, diethylbenzoylacetic acid remained behind as a brownish crystalline mass, which was spread on a porous plate to remove traces of oil. The acid, which was now only slightly yellowish coloured, was purified by dissolving in dilute ammonia and agitating with animal charcoal. On filtering and acidulating with dilute sulphuric acid, the acid was obtained in the form of an almost colourless crystalline solid. Although the saponification was conducted at ordinary temperatures, and with very dilute potash, the diethylbenzoylacetic acid always contained traces of benzoic acid, which spoilt the analytical numbers and melting point.

0.2121 gram substance gave 0.1325 gram $\mathrm{H}_{2} \mathrm{O}$ and 0.5417 gram $\mathrm{CO}_{2}$.

Found.

$\mathrm{C}=69 \cdot 66$ per cent.

$\mathrm{H}=6.94 \quad$,

$\mathrm{O}=23 \cdot 40$
Theory $\mathrm{C}_{6} \mathrm{H}_{5}$.CO.C. $\left(\mathrm{C}_{2} \mathrm{H}_{5}\right)_{2} \cdot \mathrm{COOH}$.

70.90 per cent.

$7 \cdot 27$

$21 \cdot 81$

The acid melts at $128-130^{\circ}$. Heated either alone or with dilute 
sulphuric acid, it gives off carbonic anhydride, and an oil is left behind, probably diethylacetophenone.

Diethylbenzoylacetic acid is easily soluble in ammonia, soda, or sodic carbonate. On account of the difficulty of getting the acid pure, the salts were not further examined, it appearing probable that better results would be obtained on studying the decomposition-products of diethylbenzoylacetic ether.

\section{Decomposition-products of Diethylbenzoylacetic Ether.}

In order to examine these products, crude diethylbenzoylacetic ether was heated on a water-bath with dilute alcoholic potash for about five hours, by which means the ether was completely decomposed. After distilling off the alcohol and diluting with water, an oil was precipitated, which was separated from the solution of the potash salts by extracting with ether. The aqueous solutions were then gently warmed on a water-bath to get rid of the ether, acidulated with dilute sulphuric acid, and distilled in steam. A strongly acid slightly turbid distillate came over, which smelt strongly of the higher members of the acetic series, and probably contained diethylacetic acid. The residue on cooling deposited an acid, in the form of feathery crystals, which were collected on a filter and washed with water. The acid crystallised from water in silky plates, which possessed all the properties of benzoic acid. They melted at $120^{\circ}$, and gave the characteristic reaction with ferric chloride. In order to be certain, the acid was turned into the ammonia salt and precipitated with silver nitrate. An analysis of the silver salt gave the following numbers :-

0.2994 gram substance gave 0·1420 gram silver.
Found.
$\mathrm{Ag}=47 \cdot 43$ per cent.
Theory $\mathrm{C}_{6} \mathrm{H}_{5} \mathrm{COOAg}$. $47 \cdot 16$ per cent.

Diethylbenzoylacetic ether is therefore decomposed on heating with dilute alcoholic potash, partly into benzoic acid and diethylacetic acid, according to the equation :-

\section{$\mathrm{C}_{6} \mathrm{H}_{5} \cdot$ CO.C $\left(\mathrm{C}_{2} \mathrm{H}_{5}\right)_{2} \cdot \mathrm{COOC}_{2} \mathrm{H}_{5}+2 \mathrm{KOH}=\mathrm{C}_{6} \mathrm{H}_{5} \cdot \mathrm{COOK}+$ $\mathrm{CH}\left(\mathrm{C}_{2} \mathrm{H}_{5}\right)_{2} \cdot \mathrm{CO}_{2} \mathrm{~K}+\mathrm{C}_{2} \mathrm{H}_{5} \mathrm{OH}$.}

The neutral oils, produced by the action of potash on diethylbenzoylacetic ether which had been separated from the potash salts by extraction with ether, were next examined. After drying over calcium chloride and distilling off the ether, a dark brownish oil remained behind, which was fractioned. The principal portion which 
distilled over between $220^{\circ}$ and $235^{\circ}$ gave, after several times carefully fractioning, an oil boiling constantly at $229-231^{\circ}$ (at $710 \mathrm{~mm}$.), which gave the following numbers on analysis, showing that diethylacetophenone had been formed:-

0.1711 gram substance gave $0.1371 \mathrm{H}_{2} \mathrm{O}$ and 0.5099 gram $\mathrm{CO}_{2}$.

\begin{tabular}{|c|c|c|}
\hline $\mathrm{C}=$ & $\begin{array}{l}\text { Found. } \\
81 \cdot 28 \text { per cent. }\end{array}$ & $\begin{array}{c}\text { Theory } \mathrm{C}_{6} \mathrm{H}_{5} \cdot \mathrm{CO} \cdot \mathrm{CH}\left(\mathrm{C}_{2} \mathrm{H}_{5}\right)_{2} \text {. } \\
81 \cdot 81 \text { per cent. }\end{array}$ \\
\hline $\mathrm{H}=$ & $8 \cdot 90$ & $9 \cdot 09$ \\
\hline $\mathrm{O}=$ & $9 \cdot 82$ & $9 \cdot 09$ \\
\hline
\end{tabular}

Diethylacetophenone is a thick colourless oil, which does not soli-. dify at $0^{\circ}$, the smell of which closely resembles that of acetophenone. Bromine acts easily on the solution in glacial acetic acid, on warming, quantities of hydrobromic acid being given off. The product is a colourless oil, which was not further examined, as it could not be purified by distillation, owing to decomposition, and on standing for some weeks over sulphuric acid in vacuo, it did not crystallise. The formation of diethylacetophenone by the action of potash on diethylbenzoylacetic ether may be expressed by the following equation:-

$$
\begin{array}{r}
\mathrm{C}_{6} \mathrm{H}_{5} \cdot \mathrm{CO} \cdot \mathrm{CH}\left(\mathrm{C}_{2} \mathrm{H}_{5}\right)_{2} \cdot \mathrm{COOC}_{2} \mathrm{H}_{5}+2 \mathrm{KOH}= \\
\mathrm{C}_{6} \mathrm{H}_{5} \cdot \mathrm{CO} \cdot \mathrm{CH}\left(\mathrm{C}_{2} \mathrm{H}_{5}\right)_{2}+\mathrm{K}_{2} \mathrm{CO}_{3}+\mathrm{C}_{2} \mathrm{H}_{5} \mathrm{OH} .
\end{array}
$$

\section{Allylbenzoylacetic Acid.}

This acid and its decomposition-products were more especially examined, on account of their isomerism with benzoyltetramethylenecarbonic acid and benzoyltetramethylene obtained by the action of trimethylene bromide on benzoylacetic ether (an account of which I hope in a short time to be able to have the honour of laying before the Society), it being for several reasons interesting to determine any differences which might exist between these two bodies. Allylbenzoylacetic ether was prepared by the action of allyliodide on the sodium compound of benzoylacetic ether in the following way :-

10 grams of benzoylacetic ether were mixed with $1 \cdot 2$ gram of sodium (dissolved in about 15 c.c. of absolute alcohol), and the whole, after adding an excess of allyliodide heated on a water-bath for about four hours, or better still in a sealed tube to $100^{\circ}$ for two hours, after which the mass had acquired a neutral reaction. The alcohol was then distilled off, the residue diluted with water, and several times extracted with ether. After drying over chloride of calcium and distilling off the ether, allylbenzoylacetic ether remained behind as a thick brownish oil, which, as it could not be distilled without decomposition, was not directly analysed. 
In order to obtain allylbenzoylacetic acid, the crude ether was dissolved in alcohol, a little dilute alcoholic potash added, and the whole allowed to stand at ordinary temperatures for three weeks. The product was then diluted with water, and any unsaponified ether removed by extraction with ether.

The solution of the potash salts was then acidulated with dilute sulphuric acid, and once or twice extracted with ether. After distilling off the ether, the acid remained behind as a brownish crystalline mass, which was first roughly purified by spreading out on a porous plate.

In this manner the acid was obtained as a yellowish crystalline mass, which was very easily soluble in most of the ordinary solvents, and for this reason could not be satisfactorily recrystallised from any of them. It was therefore dissolved in dilute ammonia, and after shaking with animal charcoal and filtering, reprecipitated and analysed with the following result:-

0.1570 gram substance gave $0.0787 \mathrm{H}_{2} \mathrm{O}$ and $0.4005 \mathrm{CO}_{2}$.

\begin{tabular}{|c|c|c|}
\hline ; = & $\begin{array}{l}\text { Found. } \\
69.57 \text { per cent. }\end{array}$ & $\begin{array}{c}\text { Calculated, } \\
\mathrm{C}_{6} \mathrm{H}_{5} \cdot \mathrm{CO} \cdot \mathrm{CH}\left(\mathrm{CH}_{2} \cdot \mathrm{CH}: \mathrm{CH}_{2}\right) \cdot \mathrm{CO}_{2} \mathrm{H} . \\
70.58 \text { per cent. }\end{array}$ \\
\hline $\mathrm{I}=$ & 5.57 & $5 \cdot 78$ \\
\hline & $24 \cdot 86$ & $23 \cdot 53$ \\
\hline
\end{tabular}

The acid always seems to contain traces of benzoic acid, which spoil the analytical numbers and melting point. It melted at 122$125^{\circ}$, and on further heating either alone or with dilute sulphuric acid, gave off carbonic anhydride and an oil, probably allylacetophenone. I hope later on, if possible, to find some other more suitable means of saponifying diethyl- and allyl-benzoylacetic ethers, and of thus obtaining the acids in a purer state.

\section{Decomposition-products of Allylbenzoylacetic Ether.}

The best proof of the formation of allylbenzoylacetic ether in the foregoing experiments was the study of its decomposition-products. In order to examine these, 10 grams of crude allylbenzoylacetic ether were heated for two or three hours with dilute alcoholic potash on a water-bath, by which means it was completely decomposed. After distilling off the alcohol, the residue was diluted with water, and in order to remove the neutral oils from the solution of the potassium salts, it was several times extracted with ether. The ethereal solution, after drying over calcium chloride and distilling off the ether, deposited 5 grams of a dark-brown oil, which was then fractioned.

The principal quantity distilled over between $230^{\circ}$ and $240^{\circ}$, and 
gave on oft-repeated fractionation, an oil boiling constantly at 235 $238^{\circ}$ (at $710 \mathrm{~mm}$. pressure), which on analysis gave numbers agreeing with those required for allylacetophenone.

$0 \cdot 1248$ gram substance gave 0.0852 gram $\mathrm{H}_{2} \mathrm{O}$ and $0.3757 \mathrm{CO}_{2}$.

Found.

$\mathbf{C}=82 \cdot 10$ per cent.

$\mathrm{H}=7.58 \quad$,

$\mathrm{O}=10.32 \quad$,

$$
\begin{gathered}
\text { Theory, } \\
\mathrm{C}_{8} \mathrm{H}_{5} \cdot \mathrm{CO} \cdot \mathrm{CH}_{2} \cdot \mathrm{CH}_{2} \cdot \mathrm{CH}: \mathrm{CH}_{2} \text {. } \\
82 \cdot 50 \text { per cent. } \\
7 \cdot 50 \quad " \\
10 \cdot 00 \quad "
\end{gathered}
$$

Allylacetophenone is formed by the action of alcoholic potash on allylbenzoylacetic ether according to the following equation:$\mathrm{C}_{6} \mathrm{H}_{5} \cdot \mathrm{CO} \cdot \mathrm{CH}\left(\mathrm{CH}_{2} \cdot \mathrm{CH}: \mathrm{CH}_{2}\right) \cdot \mathrm{CO}_{2} \mathrm{C}_{2} \mathrm{H}_{5}+2 \mathrm{KOH}=$

$$
\mathrm{C}_{6} \mathrm{H}_{3} \cdot \mathrm{CO} \cdot \mathrm{CH}_{2} \cdot \mathrm{CH}_{2} \cdot \mathrm{CH}: \mathrm{CH}_{2}+\mathrm{K}_{2} \mathrm{CO}_{3}+\mathrm{C}_{2} \mathrm{H}_{5} \mathrm{OH} \text {. }
$$

Allylacetophenone is a colourless oil, boiling without decomposition at $235-238^{\circ}$ (710 $\mathrm{mm}$.).

It has a curious smell, something like that of camphor, and a burning taste. It is insoluble in water, but dissolves in most of the other solvents. It does not appear to combine with acid sodium sulphite.

The potash salts, which were separated from the allylacetophenone by extraction with ether, were evaporated nearly to dryness on a water-bath, acidulated with dilute sulphuric acid, and distilled in steam. The distillate, which had a strong acid reaction, was filled with drops of an unpleasantly smelling oil, which dissolved easily in a solution of sodium carbonate, and consisted probably of allylacetic acid.

The residue in the retort, on cooling, deposited a quantity of colourless crystals, which were filtered off and recrystallised from water. They then melted at 118-120 reddish-brown precipitate, and showed all the rroperties of benzoic acid. It is therefore probable that part of the allylbenzoylacetic ether had been saponified by the action of the alcoholic potash into allylacetic acid, benzoic acid, and alcohol, according to the following equation :-

$\mathrm{C}_{6} \mathrm{H}_{5} \cdot \mathrm{CO} \cdot \mathrm{CH}\left(\mathrm{CH}_{2} \cdot \mathrm{CH}: \mathrm{CH}_{2}\right) \cdot \mathrm{CO}_{2} \mathrm{C}_{2} \mathrm{H}_{5}+2 \mathrm{KOH}=$ $\mathrm{C}_{6} \mathrm{H}_{5} \mathrm{CO}_{2} \mathrm{~K}+\mathrm{CH}_{2}: \mathrm{CH} \cdot \mathrm{CH}_{2} \cdot \mathrm{CH}_{2} \cdot \mathrm{CO}_{2} \mathrm{~K}+\mathrm{C}_{2} \mathrm{H}_{5} \mathrm{OH}$.

Action of Bromine on Allylacetophenone.

Allylacetophenone being an unsaturated body, it appeared interesting to try the action of bromine on it, in the hope of obtaining a 
crystalline addition-product. The experiment was carried out in the following way :-

Pure allylacetophenone was dissolved in glacial acetic acid, and after weighing the mixture, bromine slowly run in.

The colour of the bromine disappeared instantly at first; after a time, however, much more slowly, without the evolution of hydro. bromic acid, the mixture becoming warm, rendering it necessary to cool the mass with water.

As soon as the colour of the bromine was permanent, the whole was re-weighed, when it was found that 1 mol. of allylacetophenone had taken up two atoms of bromine, according to the equation-

\section{$\mathrm{C}_{6} \mathrm{H}_{5} \cdot \mathrm{CO} \cdot \mathrm{CH}_{2} \cdot \mathrm{CH}_{2} \cdot \mathrm{CH}: \mathrm{CH}_{2}+\mathrm{Br}_{2}=\mathrm{C}_{6} \mathrm{H}_{5} \cdot \mathrm{CO} \cdot \mathrm{CH}_{2} \cdot \mathrm{CH}_{2} \cdot \mathrm{CHBr} \cdot \mathrm{CH}_{2} \mathrm{Br}$.}

In order to isolate the product, the acid solution was poured into water, allylacetophenone dibromide being precipitated as a heary oil, which was extracted with ether.

The ethereal solution was then washed once or twice with dilate sodic carbonate to remove any acetic acid, and finally dried over potassic carbonate. After distilling off the ether, a thick colourless oil remained behind, which, after standing over sulphuric acid in vacuo for 14 days, did not crystallise.

An analysis of this crude product gave the following numbers:-

0.3180 gram substance gave 0.3812 gram $\mathrm{AgBr}=51.01$ per cent. $\mathrm{Br}$.

Theory for $\mathrm{C}_{6} \mathrm{H}_{5} \cdot \mathrm{CO} \cdot \mathrm{CH}_{2} \cdot \mathrm{CH}_{2} \cdot \mathrm{CHBr} . \mathrm{CH}_{2} \mathrm{Br}=50 \cdot 00$ per cent.

If the solution of allylacetophenone dibromide in glacial acetic acid be further treated with bromine, at the same time warming the mixture on a water-bath, the colour of the mixture rapidly disappears, large quantities of hydrobromic acid being given off. In this experiment 4 grams of allylacetophenone dibromide, dissolved in glacial acetic acid, were treated with 1 gram of bromine, the whole being warmed on a water-bath till the reddish colour had disappeared.

On pouring the liquid into water, an oil was precipitated which was extracted with ether.

After washing the ethereal solution with sodium carbonate and distilling off the ether, a thick oil remained behind, which, on standing for some days over sulphuric acid in a vacuum, deposited a quantity of crystals. These were roughly separated from the oily products by spreading them out on a porous plate, and finally once or twice recrystallised from dilute alcohol.

The analysis gave the following result:-

0.4196 gram of substance gave 0.587 gram $\mathrm{AgBr}=59.53$ per cent. $\mathrm{Br}$. Theory for $\mathrm{C}_{11} \mathrm{H}_{11} \mathrm{OBr}_{3}=60.15$ 
The substance was therefore bromallylacetophenone dibromide. This body crystallises from dilute alcohol in four-sided prisms which melt at $121-122^{\circ}$.

It is easily soluble in alcohol, benzene, chloroform, ether, and carbon disulphide. In caustic soda, it dissolves with a yellow colour. 
BENZOYLACETIC ACID AND SOME OF ITS DERIVATIVES. Part II.

By W. H. Perkin, Jun., Ph.D., Privatdocent at the University of Munich.

IN Part I of this research (Trans., 1884, 170), I described the preparation and properties of ethylic benzoylacetate and of benzoylacetic acid, as well as of some compounds in which the hydrogen-atoms of the methylene group of the former are displaced by alcoholic radicles, such as ethyl-, diethyl-, and allyl-benzoylacetic ether, together with the products of their hydrolysis. Before describing the more complicated reactions in which ethylic benzoylacetate takes part, I should like to give the results of the experiments I have made with some of these compounds since the publication of Part I (Trans., 1884, 170).

It has been found that although the substituted etheric salts of the benzoylacetic acids cannot be distilled without decomposition under the ordinary pressure, they can easily be obtained pure by distillation under diminished pressure. Using an apparatus similar to that described by Thorne, I have succeeded in purifying the monethyland allyl-derivatives described in the previous paper. If the crude product of the action of ethyl iodide on ethylic benzoylacetate is distilled under a pressure of $225 \mathrm{~mm}$., a small quantity of acetophenone passes over first, and then the temperature rises rapidly to $220^{\circ}$, between which and $240^{\circ}$ nearly the whole comes over as an almost colourless oil, leaving but a small residue. On repeatedly refractioning this oil under the same pressure, it distils mostly between $230-235^{\circ}$. The portion used for analysis boiled at $231-232^{\circ}$ at $225 \mathrm{~mm}$.

0.1550 gram substance gave 0.1020 gram $\mathrm{H}_{2} \mathrm{O}$ and 0.4016 gram $\mathrm{CO}_{2}$. 
Found.

C ... 70.66 per cent.

$\mathrm{H}, \ldots .7 \cdot 31 \quad$,

O ...222.03 ,
Theory.

$\mathrm{C}_{6} \mathrm{H}_{5} \cdot \mathrm{CO} \cdot \mathrm{CH}\left(\mathrm{C}_{2} \mathrm{H}_{5}\right) \cdot \mathrm{COOC}_{2} \mathrm{H}_{5}$.

70.91 per cent.

$7 \cdot 27$

$21 \cdot 82$

Ethylic ethylbenzuylacetate is a colourless, strongly refracting oil possessing an aromatic odour and burning taste. When pure, it appears to distil at the ordinary pressure almost without decomposition. The boiling points of the pure substance were incidentally noticed as being-

$$
\begin{aligned}
& 210-211^{\circ} \text { at } 90 \mathrm{~mm} . \\
& 223-224 \quad, 150 ", \\
& 231-232 \quad, 225 "
\end{aligned}
$$

Ethylic ethylbenzoylacetate is insoluble in potassic hydroxide solution, but appears to give a sodium compound on mixing it with sodic ethoxide. On continued boiling, no condensation-product is formed corresponding with the dehydrobenzoylacetic acid, which is so easily obtained by heating ethylic benzoylacetate for a short time by itself. This reaction I hope to describe in Part III of this paper. Ethylic acetoacetate and ethylacetoacetate behave in a precisely similar way. On passing ethylic acetoacetate through a tube at a dull red heat, it is almost completely decomposed, yielding as much as 20 per cent. of its condensation-product, dehydracetic acid, whereas ethylic ethylacetoacetate passes through almost unaltered, no trace of the analogous acid being formed.

Ethylic allylbenzoylacetate may also be purified in exactly the same way as ethylic ethylbenzoylacetate. On submitting the crude product of the action of allyl iodide on ethylic benzoylsodacetate to distillation nnder a pressure of $225 \mathrm{~mm}$., nearly the whole passes over between $225-245^{\circ}$ as a peculiar greenish-coloured oil, with a slight reddish fluorescence. This coloration can only be removed by repeated fractioning, and even then it often returns on standing.

Pure ethylic allylbenzoylacetate boils at-

$$
\begin{aligned}
& 220^{\circ} \text { under } 100 \mathrm{~mm} \text {. pressure. } \\
& \begin{array}{lllll}
226-227^{\circ} & , & 130 & , & \quad, \\
240-241 & , & 225 & , & \quad,
\end{array}
\end{aligned}
$$

As it is difficult to burn, it is necessary that the combustion should be done with chromate of lead. The following numbers were obtained :-

0.1491 gram substance gave 0.0902 gram $\mathrm{H}_{2} \mathrm{O}$ and 0.3924 gram $\mathrm{CO}_{2}$. 
Found.

C ... $71 \cdot 77$ per cent.

H .... 6.7.2

O .... $21 \cdot 51$

Theory.

$\mathrm{C}_{6} \mathrm{H}_{5} \cdot \mathrm{CO} \cdot \mathrm{CH}\left(\mathrm{CH}_{2} \cdot \mathrm{CH}: \mathrm{CH}_{2}\right) \cdot \mathrm{COOC}_{2} \mathrm{H}_{5}$.

$72 \cdot 41$ per cent.

$20 \cdot 69$
,

,

Ethylic allylbenzoylacetate possesses the peculiar disagreeable odour Laracteristic of most allyl compounds. It does not solidify at $0^{\circ}$. The solution in acetic acid takes up bromine readily, especially if warmed, and without evolution of hydrobromic acid.

Ethylic benzoylacetate itself cannot be purified by distillation under diminished pressure, the greater part being decomposed, owing to the comparative slowness with which distillation takes place. When distilled as rapidly as possible (at $200 \mathrm{~mm}$.) a portion passes over between $220-225^{\circ}$, consisting of nearly pure ethylic benzoylacetate, but a large residue is left containing considerable quantities of dehydrobenzoylacetic acid.

Having obtained ethylic monethyl- and allyl-benzoylacetates in a pure state, it was next thought necessary to repeat the hydrolysis with potash, in order, if possible, to obtain the acids in a pure state, but even when very dilute alcoholic potash was used, the acids were always mixed with benzoic acid, in some cases to a very considerable extent.

It was then thought that hydrolysis with sulphuric acid might give better results, and several experiments were therefore tried, asing ethylic benzoylacetate itself in the first case.

On mixing ethylic benzoylacetate with 20 times its volume of concentrated sulphuric acid, and allowing it to remain for some time, the mixture becomes thick and dark-coloured, sruall quantities of carbonic anhydride and sulphurous anhydride being given off.

After standing for 14 days, the product was poured into ice water; this caused the precipitation of a thick dark brownish oil, which partly solidified on prolonged agitation. This uninviting body was separated from the aqueous solution by filtration, washed well with water, and then spread out on a porous plate; in this way the dark brown oil was almost completely remored from the crystals in two or three days, leaving them as a hard yellowish crystalline crust. After recrystallising it several times from warm benzene, the substance was easily obtained pure, melting between $103-104^{\circ}$, and presenting all the appearance of benzoylacetic acid.

On adding a drop of ferric chloride to the alcoholic solution, it. gave the beautiful violet coloration mentioned in Part I, and the acid itself on dry distillation split up quantitatively into carbonic anhydride and acetophenone. There can be no doubt, therefore, that the substance was pure benzoylacetic acid. Experiments were next tried 
to ascertain if ethylic ethylbenzoylacetate could also be hydrolysed in the same way. A small quantity of this ethereal salt was mixed with nearly three times its volume of concentrated sulphuric acid, and allowed to remain for three weeks. The solution became brownishcoloured, but not nearly so dark as in the case of ethylic benzoylacetate itself; on adding water, an almost colourless oil was precipitated, which was removed by shaking with ether, and on evaporating the ether, a Jellowish oil remained behind; this when exposed over sulphuric acid in a vacuum, deposited crystals, apparently ethylbenzoylacetic acid. The quantity, however, was unfortunately too small for analysis, so that it will be necessary to repeat the experiment with more material before I can be certain that this method is of general application for the hydrolysis of ethylic benzoylacetate derivatives.

Part II of this research comprises the preparation and properties of ethylic nitrosobenzoylacetate, dibenzoylacetic acid, di- and tribenzoylmethane, the reduction of ethylic benzoylacetate, the action of phosphorous pentachloride on ethylic benzoylaoetate, and the condensation of the latter with benzaldehyde.

\section{Ethylic Benzoylnitrosoacetate.}

By the action of nitrous acid on ethylic acetoacetate, Victor Meyer obtained ethylic nitrosoacetoacetate, according to the equation-

$\mathrm{CH}_{3} \cdot \mathrm{CO} \cdot \mathrm{CH}_{2} \cdot \mathrm{COOC}_{2} \mathrm{H}_{5}+\mathrm{NOOH}=\mathrm{CH}_{3} \cdot \mathrm{CO} \cdot \mathrm{C}(\mathrm{N} \cdot \mathrm{OH}) \cdot \mathrm{COOC}_{2} \mathrm{H}_{5}$ $+\mathrm{H}_{2} \mathrm{O}$.

After a series of adinirable researches, he proved that the formula of this compound was not $\mathrm{CH}_{3} \cdot \mathrm{CO} \cdot \mathrm{CH}(\mathrm{NO}) \cdot \mathrm{COOC}_{2} \mathrm{H}_{5}$, and therefore did not contain the nitroso-group $\mathrm{NO}$, as was at first supposed, but that it was in reality an isonitroso-body, having the constitution $\mathrm{CH}_{3} \cdot \mathrm{CO} \cdot \mathrm{C}(\mathrm{N} . \mathrm{OH}) \cdot \mathrm{COOC} \mathrm{H}_{5}$, and containing the isonitrosoradicle: $\mathrm{N} . \mathrm{OH}$.

With the exception of ethylic malonate, ethylic acetoacetate is the only compound which is capable of being converted into an isonitrosoderivative; for the substituted acetoacetates, such as ethylic ethylacetoacetate, $\mathrm{CH}_{3}$. $\mathrm{CO} . \mathrm{CH}\left(\mathrm{C}_{2} \mathrm{H}_{5}\right) \cdot \mathrm{COOC}_{2} \mathrm{H}_{5}$, possessing only one hydrogen-atom which is capable of being substituted by any group, cannot combine with the dyad radicle $\mathrm{NOH}$.

The nitroso-derivative of the whole molecule is therefore never formed by the action of nitrous acid on such bodies, but the ethereal salt being first hydrolysed, carbonic anhydride is given off, and the nitroso-ketone remains behind :-

$$
\mathrm{CH}_{3} \cdot \mathrm{CO} \cdot \mathrm{CH}\left(\mathrm{C}_{2} \mathrm{H}_{3}\right) \cdot \mathrm{COOC}_{2} \mathrm{H}_{5}+\mathrm{NOOH}=\mathrm{CH}_{3} \cdot \mathrm{CO} \cdot \mathrm{C}\left(\mathrm{C}_{2} \mathrm{H}_{5}\right): \mathrm{NOH}
$$

Ethylic ethylacetoacetate. Nitroscethylacetone.

$$
+\mathrm{C}_{2} \mathrm{H}_{5} \mathrm{OH}+\mathrm{CO}_{2} \text {. }
$$


It therefore appeared interesting to try the action of nitrous acid on ethylic benzoylacetate and some of its derivatives, in order to determine whether they would follow the same law or not.

After several trials, the following was found to be the best method of preparing ethylic nitrosobenzoylacetate:-

Ethylic benzoylacetate is first dissolved in dilute sodic hydroxide solution, filtered, mixed with an excess of sodic nitrite, and cooled down below $0^{\circ}$ in a freezing mixture. Dilute sulphuric acid is then slowly added from a burette until the mixture is decidedly acid, care being taken that the liquid does not get warm. After a short time, more sodic hydroxide solution is added; this dissolves all that was precipitated by the sulphuric acid, producing an intense yellow solution, a sign that a nitroso-body has been formed. Last of all, the ethylic nitrosobenzoylacetate is precipitated by adding an excess of sulphuric acid. It separates as an oil, which, however, on agitating soon solidifies to long colourless needles. These are collected, well washed with water, and separated from any oily matter by spreading them out on a porous plate. In order to purify it, it is dissolved in a little hot alcohol, and water added until it becomes milky. The whole is then warmed up again and allowed to stand, when pure ethylic nitrosobenzoylacetate separates out in beautiful long colourless needles; on analysis', these gave the following numbers :-

I. 0.1711 gram substance gave 0.0813 gram $\mathrm{H}_{2} \mathrm{O}$ and 0.3729 $\mathrm{CO}_{2}$.

II. $0 \cdot 1566$ gram substance gave 0.0730 gram $\mathrm{H}_{2} \mathrm{O}$ and 0.3423 gram $\mathrm{CO}_{2}$.

I. 0.2617 gram substance gave 16 c.c. $\mathrm{N}$, bar. $=718 \mathrm{~mm} ., t .=21^{\circ}$. II. $0.2154 \quad, \quad, \quad 12.5$ c.c. $\mathrm{N}$, bar. $=716 \mathrm{~mm}$., $t .=$ $18^{\circ}$.

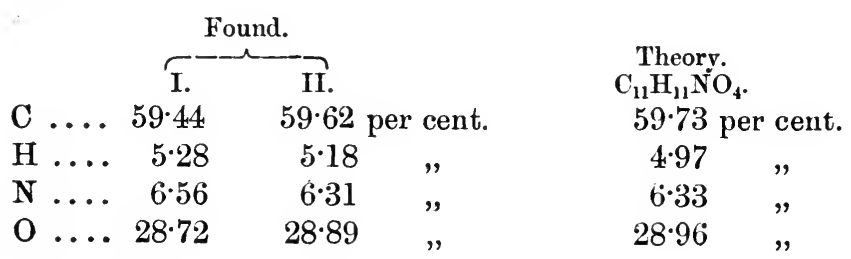

From its analogy to ethylic nitrosoacetoacetate, this compound must have the formula $\mathrm{C}_{6} \mathrm{H}_{5} \cdot \mathrm{CO} \cdot \mathrm{C}(\mathrm{N} \cdot \mathrm{OH}) \cdot \mathrm{COOC}_{2} \mathrm{H}_{5}$.

Ethylic nitrosobenzoylacetate melts at $120-121^{\circ}$. It is easily soluble in alcohol, ether, benzene, and chloroform, sparingly in water. It dissolves easily in alkalis, forming a yellow solution like all nitrosobodies, and on acidifying is reprecipitated unchanged. It is decomposed by dry distillation with separation of charcoal and formation 
of hydrocyanic acid. A heavy oil passes over, which soon solidifies, and then melts at $110-114^{\circ}$; it is perhaps impure benzoic acid.

On treating ethylic nitrosoacetoacetate with alkalis, it is decomposed in the same way as ethylic acetoacetate itself, Jielding nitrosoacetone instead of acetone. It was thought likely, therefore, that ethylic nitrosobenzoylacetate under the same conditions would give nitrosoacetophenone.

In order to test this, 3 grams of ethylic nitrosobenzoylacetate were dissolved in dilute potash, and allowed to remain for some days at the ordinary temperature, when the yellow solution of the nitrosoderivative became quite colourless. The product was then cooled in a freezing mixture, acidified with sulphuric acid, and extracted with 'ether. On evaporating the ethereal solution, it deposited a colourless crystalline substance, which contained but traces of nitrogen, and therefore could not be nitrosoacetophenone. It is easily soluble in warm water, and on cooling crystallises in small thick prisms, which were collected, dried over sulphuric acid in a vacuum, and analysed, with the following results :-

I. 0.1797 gram substance gave 0.0684 gram $\mathrm{H}_{2} \mathrm{O}$ and 0.3962 gram $\mathrm{CO}_{2}$.

II. $0 \cdot 1247$ gram substance gave $0 \cdot 0477$ gram $\mathrm{H}_{2} \mathrm{O}$ and $0 \cdot 2747$ gram $\mathrm{CO}_{2}$.

\begin{tabular}{|c|c|c|c|}
\hline \multicolumn{3}{|c|}{ Found. } & \multirow{2}{*}{$\begin{array}{l}\text { Theory. } \\
\mathrm{C}_{9} \mathrm{H}_{8} \mathrm{O}_{4} \cdot \\
60 \cdot 00 \text { per cent }\end{array}$} \\
\hline & $\begin{array}{c}\text { I. } \\
60 \cdot 13\end{array}$ & II. & \\
\hline $\mathrm{H}$ & $4 \cdot 22$ & $4 \cdot 25$ & $4 \cdot 44$ \\
\hline $\mathrm{O}$ & $35 \cdot 65$ & $35 \cdot 67$ & 35.55 \\
\hline
\end{tabular}

The compound therefore appears to have the formula $\mathrm{C}_{9} \mathrm{H}_{8} \mathrm{O}_{4}$. It crystallises from water in small prisms melting at $125^{\circ}$. It dissolves easily in alkalis, forming a colourless solution. On precipitating the neutral solution of the ammonium salt with silver nitrate, the silver salt was obtained as a voluminous white precipitate, which after being dried over sulphuric acid in a vacuum gave the following numbers on analysis :-

$0 \cdot 3050$ gram substance gave 0.1147 gram $\mathrm{Ag}=37 \cdot 61$ per cent. Theory, $\mathrm{C}_{y} \mathrm{H}_{7} \mathrm{O}_{4} \mathrm{Ag}=37 \cdot 63 \quad$ "

I hope at some future time to be able to examine more thoroughly this curious reaction, and if possible to determine the constitution of the body $\mathrm{C}_{9} \mathrm{H}_{8} \mathrm{O}_{1}$. 


\section{Dibenzoylacetic Acid.}

In the preceding chapters, it has been shown that ethylic benzoylacetate is capable of forming derivatives in which the bydrogen atoms of the methylene group are displaced by alcoholic radicles such as ethyl, allyl, \&c., in exactly the same way as ethylic acetoacetate. It was thought likely that very interesting results might be obtained if the acid chlorides such as benzoyl chloride were substituted for the iodides of the alcoholic radicles in the above reaction. If the sodium compound of ethylic benzoylacetate be heated with benzoyl chloride, the ethyl salt of dibenzoylacetic acid is formed according to the equation-

$\mathrm{C}_{6} \mathrm{H}_{5}$.CO.CHNa.COOC $\mathrm{H}_{5}+\mathrm{C}_{6} \mathrm{H}_{5} \mathrm{COCJ}=$

$\left(\mathrm{C}_{6} \mathrm{H}_{5} \cdot \mathrm{CO}\right)_{2} \cdot \mathrm{CH} \cdot \mathrm{COOC}_{2} \mathrm{H}_{5}+\mathrm{NaCl}$.

This ethereal salt on hydrolysis gives dibenzoylacetic acid, $\left(\mathrm{C}_{6} \mathrm{H}_{5} . \mathrm{CO}\right)_{2} \mathrm{CH} . \mathrm{COOH}$.

When boiled with dilute acids, dibenzoylacetic acid is easily split up into acetophenone, benzoic acid, and carbonic anhydride, thus$\left(\mathrm{C}_{6} \mathrm{H}_{5} \cdot \mathrm{CO}\right)_{2} \mathrm{CH} . \mathrm{COOH}+\mathrm{H}_{2} \mathrm{O}=\mathrm{C}_{6} \mathrm{H}_{5} \cdot \mathrm{CO} \cdot \mathrm{CH}_{3}+\mathrm{C}_{6} \mathrm{H}_{5} \cdot \mathrm{COOH}+\mathrm{CO}_{2}$.

If boiled with water, a second reaction takes place at the same time, by which dibenzoylmethane is formed according to the equation-

$$
\begin{aligned}
& \left(\mathrm{C}_{6} \mathrm{H}_{5} \cdot \mathrm{CO}\right)_{2} \mathrm{CH} . \mathrm{COOH}=\left(\mathrm{C}_{6} \mathrm{H}_{5} \cdot \mathrm{CO}\right)_{2} \mathrm{CH}_{2}+\mathrm{CO}_{2} \text {. } \\
& \text { Dibenzoylmethane. }
\end{aligned}
$$

In the preparation of ethylic dibenzoylacetate, it is not necessary to use the pure dry sodium compound of ethylic benzoylacetate and act on it with benzoyl chloride in ethereal solution, as was done in the first experiments, but we may proceed in the way usually employed in 'such syntlieses, that is, by mixing ethylic benzoylacetate with sodic ethylate, and allowing benzoyl chloride to act directly on the product in the following way: $-0.6 \mathrm{gram}$ of sodinm is dissolved in absolute alcohol, well cooled, and then mixed with 4 grams of ethylic benzoylacetate. 3 grams of benzoyl chloride are now added drop by drop, the whole being well cooled in a mixture of ice and salt. The reaction is very energetic, each drop of benzoyl chloride instantly producing a precipitate of chloride of sodium, and so much heat is dereloped that the alcohol will boil if a refrigerating mixture is not used. After all the benzoyl chloride has been added, the whole is warmed on a water-bath for about a quarter of an hour to insure the reaction being complete, and the greater part of the alcohol is distilled off; water is then added, and the product allowed to re- 
main for some time, in order to insure the decomposition of any slight excess of benzoyl chloride. The watery liquid is next extracted with ether once or twice, the ethereal solution well shaken with carbonate of soda to remove benzoic acid, dried over potassium carbonate, and the ether distilled off. A thick dark brown oil remains behind. This was not further purified, although it is possible that by fractioning in a vacuum the ethylic dibenzoylacetate might be obtained in a pure state, as in the case of the ethyl- and allyl-benzoylacetates.

In order to obtain the free acid, the crude ethereal salt was hydrolysed by warming with an excess of alcoholic potash for about ten minutes on a water-bath. The mixture became dark coloured, and on adding water almost all of it dissolved, forming a dark brownish solution; this when acidified deposited the crude dibenzoylacetic acid as a thick oil, which became nearly solid on agitating for a short time. The product was collected, well washed with water, and dissolved in as little boiling alcohol ( 80 per cent.) as possible. On cooling, the acid crystallised out in colourless nodules, and was easily obtained pure and free from benzoic acid by repeated crystallisation. On analysis the following numbers were obtained :-

0.2257 gram substance gave 0.0925 gram $\mathrm{H}_{2} \mathrm{O}$ and 0.5926 gram $\mathrm{CO}_{2}$.

\begin{tabular}{|c|c|c|}
\hline C .... & $\begin{array}{l}\text { Found. } \\
71 \cdot 61 \text { per cent. }\end{array}$ & $\begin{array}{l}\text { Calculated. } \\
\mathrm{C}_{16} \mathrm{H}_{12} \mathrm{O}_{4} \cdot \\
7 \mathrm{l} \cdot 64 \text { per cent. }\end{array}$ \\
\hline ... & 4.55 & $4 \cdot 48$ \\
\hline & $23 \cdot 84$ & $23 \cdot 88$ \\
\hline
\end{tabular}

This substance was therefore dibenzoylacetic acid, $\left(\mathrm{C}_{6} \mathrm{H}_{5} \cdot \mathrm{CO}\right)_{2} \mathrm{CH} . \mathrm{COOH}$.

The acid melts at $109^{\circ}$. When rapidly heated in small quantities, some of it distils undecomposed, but the greater part is split up into dibenzoylmethane and carbonic anhydride according to the equation-

$$
\begin{aligned}
& \left(\mathrm{C}_{6} \mathrm{H}_{5} \cdot \mathrm{CO}\right)_{2} \cdot \mathrm{CH} \cdot \mathrm{COOH}=\mathrm{CH}_{2}\left(\mathrm{CO}_{6} \mathrm{C}_{6} \mathrm{H}_{5}\right)_{2}+\mathrm{CO}_{2} \text {. } \\
& \text { Dibenzoylacetic acid. Dibenzoylmethane. }
\end{aligned}
$$

Dibenzoylacetic acid is sparingly soluble in water and cold alcohol, but easily so in hot alcohol, benzene, and ether. The solutiou in alcohol gives a dirty red coloration on the addition of ferric chloride. The acid dissolves in concentrated sulphuric acid, producing a yellow solution, which, however, becomes colourless on warming. It dissolves slowly in solntions of ammonia, sodic hydroxide, and sodic carbonate, and is reprecipitated on the addition of an acid. In order to obtain a neutral solution of the ammonium salt, the pure acid was 
dissolved in a slight excess of dilute ammonia, and then allowed to stand over sulphuric acid in a vacuum until the excess of ammonia had evaporated. The salt itself is very easily soluble in water; on boiling the solution, carbonic anhydride is given off, and the smell of acetophenone becomes noticeable. Several of the salts of dibenzoylacetic acid were prepared by precipitating the solution of the ammonim salt with various reagents. The silver salt is obtained on adding a solution of nitrate of silver as a white curdy precipitate, sparingly soluble in water. For analysis, the substance was dried at $80^{\circ}$.

$0 \cdot 1154$ gram substance gave 0.0327 gram silver.

Found.

$\mathrm{Ag}$.... 28.34 per cent.
Theory. $\left(\mathrm{C}_{6} \mathrm{H}_{5} \mathrm{CO}\right)_{2} \mathrm{CH} . \mathrm{COOAg}$. $28 \cdot 72$ per cent.

A solution of cupric sulphate precipitates the copper salt as a light yellowish-green mass, which appears to be somewhat soluble in hot water and alcohol. Prolonged boiling, however, decomposes the salt, cupric oxide being precipitated. This copper salt dissolves easily in ammonia, yielding a beautiful blue solution. The barium salt is soluble in water, and is prepared by shaking the finely powdered acid with baryta-water and a little alcohol for some time. After precipitating the excess of baryta by passing carbonic anhydride and filtering, a solution of the salt is obtained, which, however, is partly decomposed by evaporation. The zinc salt is also easily soluble in water. With a solution of the ammonium salt, acetate of lead gives a white curdy precipitate of the lead salt. With a solution of sulphate of nickel, the nickel salt is obtained as a light green precipitate soluble in excess of sulphate of nickel. Ferric chloride gives a brownish-red coloration with dilute solutions of the ammonium salt; with more concentrated solutions, however, the ferric salt is precipitated as a dirty reddish precipitate somewhat soluble in hot water. Ferrous sulphate gives the same reaction. Chloride of tin precipitates the tin salt as a heavy white mass sparingly soluble in water.

If dibenzoylacetic acid is mixed with alcoholic potash, a yellowishgreen solution is formed which is decomposed by boiling, benzoic acid and a neutral body being formed. On distilling dibenzoylacetic acid with soda-lime, it is split up into benzene and an oily substance, which appears to be acetophenone.

In order to obtain pure ethylic dibenzoylacetate, the pure acid was dissolved in absolute alcohol, and hydrogen chloride passed through the solution until it was thoroughly saturated, the whole being kept well cooled during the operation. On adding water, an oil was precipitated, which, on distillation, came over almost entirely between 
$205-220^{\circ}$ as a colourless oil smelling strongly of ethyl benzoate. The pure ether can easily be prepared, however, by decomposing the silver salt of the acid with ethylic iodide in ethereal solution. After filtering from the precipitated iodide of silver and evaporating on a water-bath, ethylic dibenzoylacetate remains behind as a thick colourless oil of a faint fruity odour; it does not solidify when cooled down to $-10^{\circ}$.

Several experiments were next made in the hope of obtaining tribenzoylacetic acid by treating ethylic dibenzoylacetate with sodic ethylate and benzoyl chloride as expressed in the equation-

$$
\begin{aligned}
\left(\mathrm{C}_{6} \mathrm{H}_{5} \cdot \mathrm{CO}\right)_{2} \mathrm{CNa} \mathrm{COOC}_{2} \mathrm{H}_{5}+ & \mathrm{C}_{6} \mathrm{H}_{5} \cdot \mathrm{COCl}=\left(\mathrm{C}_{6} \mathrm{H}_{5} \cdot \mathrm{CO}\right)_{3} \mathrm{C} \cdot \mathrm{COOC}_{2} \mathrm{H}_{5} \\
& +\mathrm{NaCl} .
\end{aligned}
$$

In this way an almost colourless oil is obtained which, however, is unfortunately completely split up on hydrolysis, nothing but benzoic acid being formed.

As it appeared probable that ethylic tribenzoylacetate was really formed in the first place, an experiment was very carefully made with pure ethylic dibenzoylacetate, and the product freed from excess of benzoyl chloride and benzoic acid by shaking with dilute sodic carbonate was dried over carbonate of potash and analysed.

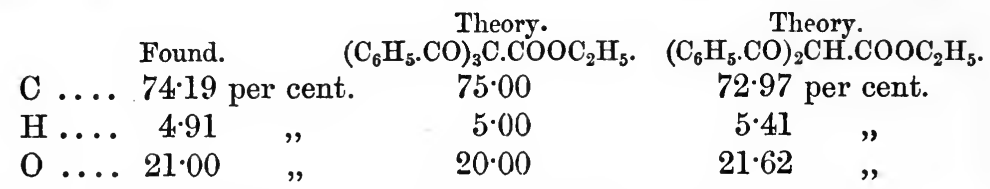

T'his ethereal salt is a very thick yellowish oil, which unfortunately could not be made to crystallise. On boiling it with dilute sulphuric acid, acetophenone and a solid acid, probably benzoic acid, are formed. It dissolves in alcoholic potash and is reprecipitated on adding a few drops of water, whereas ethylic dibenzoylacetate is only precipitated from its solution in alcoholic potash on the addition of a much larger quantity of water.

\section{Decomposition-products of Dibenzoylacetic Acid.}

\section{Dibenzoylmethane.}

As dibenzoylacetic acid contains two carbonyl-groups, it seemed probable that interesting results might be obtained by carefully examining its decomposition-products. The acid was therefore treated with several reagents ; first of all with dilute sulphuric acid in the hope of simply splitting off carbonic anhydride, and thus obtaining dibenzoylmethane according to the equation-

$$
\left(\mathrm{C}_{6} \mathrm{H}_{5} \cdot \mathrm{CO}\right)_{2} \mathrm{CH} \cdot \mathrm{COOH}=\mathrm{CO}_{2}+\mathrm{CH}_{2}\left(\mathrm{CO} \cdot \mathrm{C}_{6} \mathrm{H}_{5}\right)_{2} \text {. }
$$


The experiment was carried out in the following way:-A mixture of pure dibenzoylacetic acid with an excess of dilute sulphuric acid $(1: 2)$ was heated for about five hours in a flask connected with a reflux condenser; at the end of this a test-tube containing barytawater was fixed, to serve as a rough indicator both of the quantity of carbonic anhydride and the rate at which it was given off.

The acid soon began to decompose and swam about in the liquid at first as a semi-solid mass, but later on in large oily drops, a considerable quantity of carbonic anhydride being evolved. At the end of the reaction, the contents of the flask, which smelt strongly of acetophenone, were subjected to distillation by steam; in this way a considerable quantity of a colourless oil passed over and was extracted by shaking with ether. On distilling off the ether and subsequent fractioning, nearly the whole of the residual oil passed over between $195-205^{\circ}$, and without doubt consisted of nearly pure acetophenone. In order to be certain, it was shaken with a solution of orthonitrobenzaldehyde in dilute sodic hydroxide, when a considerable quantity of indigo was precipitated after the mixture had been standing for some time.

On cooling, the residue in the flask deposited a quantity of glittering crystals which were collected and dried. They melted at $120^{\circ}$ and showed all the properties of benzoic acid, with which they were no doubt identical.

Dibenzoylacetic acid is therefore decomposed by boiling with dilute sulphuric acid into benzoic acid, carbonic anhydride, and acetophenone according to the equation--

$\left(\mathrm{C}_{6} \mathrm{H}_{5} \cdot \mathrm{CO}\right)_{2} \mathrm{CH} \cdot \mathrm{COOH}+\mathrm{H}_{2} \mathrm{O}=\mathrm{C}_{6} \mathrm{H}_{5} \cdot \mathrm{COOH}+\mathrm{C}_{6} \mathrm{H}_{5} \cdot \mathrm{CO} \cdot \mathrm{CH}_{3}+\mathrm{CO}_{2}$.

In repeating this reaction more carefully, it was noticed that if the boiling with sulphuric acid were stopped before all the dibenzoylacetic acid had been decomposed, a small quantity of a solid body, insoluble in sodic carbonate, could be isolated; when this is dissolved in alcohol and ferric chloride added, it gives a beautiful reddish-violet coloration. As, however, only very small quantities of this compound could be obtained by this method, several experiments were tried in order to improve the yield, and it was found that simple boiling with distilled water gave the desired result.

Pure dibenzoylacetic acid was boiled with water in a flask connected with a reflux condenser as long as carbonic anhydride continued to come off in any quantity (about $4-5$ hours).

The acid melted slowly and sank to the bottom of the flask as a heavy, slightly brownish oil which on cooling partially solidified, the liquid becoming filled with needles of benzoic acid. A dilute solution of sodic carbonate was now added, and the whole well 
shaken and allowed to stand for some time until all the benzoic acid had dissolved. After filtering and washing with water a small quantity of a semi-solid mass remained, smelling strongly of acetophenone; the latter can be roughly separated from the crystals by spreading them out on a porous plate or by pressing between blottingpaper. The crude product thus obtained was then dissolved in a little hot methyl alcohol from which it crystallised on cooling in beautiful flat plates; these are easily obtained pure by recrystallisation.

The analysis gave numbers agreeing with the formula --

$$
\mathrm{CH}_{2}\left(\mathrm{CO} . \mathrm{C}_{6} \mathrm{H}_{5}\right)_{2} \text {. }
$$

0.1967 gram substance gave 0.0952 gram $\mathrm{H}_{2} \mathrm{O}$ and 0.5772 gram $\mathrm{CO}_{2}$.

Found.

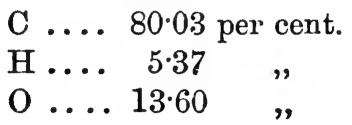

Calculated.

$\mathrm{CH}_{2}\left(\mathrm{CO}_{6} \mathrm{C}_{5}\right)_{2}$.

80.36 per cent.

$5 \cdot 36$

$14 \cdot 28$.

This compound was therefore dibenzoylmethane or benzoylacetophenone. It melts at $81^{\circ}$ and distils above $200^{\circ}$ apparently without the least decomposition. It is easily soluble in ether, alcohol, and chloroform, the alcoholic solution, on the addition of a drop of ferric chloride, giving an intense reddish-violet coloration which resembles very closely that produced by benzoylacetic acid itself. If the solution in hot methyl alcohol is cooled slowly, it crystallises most beautifully; it can, however, best be obtained in large crystals by allowing the ethereal solution to evaporate slowly. Professor Haushofer was kind enough to measure these crystals, and gave me the following description of them :-

Crystalline system rhombic:

$$
a: b: c=?: 1: 1 \cdot 38 \text {. }
$$

Small crystals with rhomboïdal base and extended in the direction of the makrodiagonal on which the surface $0 \mathrm{P}(c), \quad c \overline{\mathrm{P}} \infty(a), \stackrel{\mathrm{P}}{\infty}(r$ and an undetermined makrodome, $d$, were observed. The only surfaces fit for measurement were $a, r$, and in some crystals $c$. The

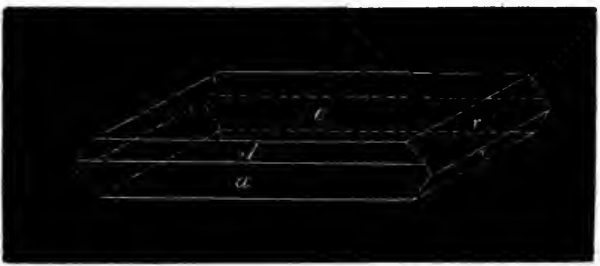


surface $d$ is smooth, curved, and appears only on one-half of the crystals (with respect to the basal section). The surface $c$ is also generally curved.

$\begin{array}{ccc} & \text { Measured. } & \text { Calculated. } \\ r: r & 108^{\circ} 18^{\prime} & - \\ c: r & 125^{\circ} 54^{\prime} & 125^{\circ} 5 \mathrm{I}^{\prime} \\ a: r & 90^{\circ} & 90^{\circ}\end{array}$

The compound is remarkable by the strong dispersion in its axes. The medial line is the vertical axis. The makropinacoïd is the plane of the optical axis for the red, and the brachypinacoild for the blue. In the polariscope with white light, the surface $c$ shows the interference figure of rhombic titanic acid (brookite).

Dibenzoylmethane is easily soluble in alkalis, particalarly in alcobolic potash, and is reprecipitated unchanged on acidifying. It combines easily with phenylhydrazine, forming a solid substance which, however, was not further examined.

\section{Tribenzoylmethane.}

Dibenzoylmethane, $\mathrm{CH}_{2}\left(\mathrm{CO} . \mathrm{C}_{6} \mathrm{H}_{5}\right)_{2}$, contains a methylene-group associated with two carbonyl-groups, and for this reason is soluble in alkalis, forming metallic salts, the methylene-group in this body behaving in exactly the same way as the corresponding one in ethylic benzoylacetate, $\mathrm{C}_{6} \mathrm{H}_{5} \cdot \mathrm{CO} \cdot \mathrm{CH}_{2} \cdot \mathrm{COOC}_{2} \mathrm{H}_{5}$. It appeared probable, therefore, that by further treatment with sodic ethylate and benzoyl chloride a tribenzoylmethane might be formed according to the equation-

$$
\mathrm{CHNa}\left(\mathrm{CO} . \mathrm{C}_{6} \mathrm{H}_{5}\right)_{2}+\mathrm{C}_{6} \mathrm{H}_{5} \cdot \mathrm{COCl}=\mathrm{CH}\left(\mathrm{CO}_{6} \mathrm{C}_{6} \mathrm{H}_{5}\right)_{3}+\mathrm{NaCl} \text {. }
$$

This is in reality the case, as will be seen from the following experiment: 2 grams of pure dibenzoylmethane were mixed with a solution of 0.2 gram of sodium in absolute alcohol, and then as soon as all had completely dissolved 1.4 gram of henzoyl chloride was added drop by drop. Sodic chloride was immediately precipitated, the mixture becoming warm. After all the benzoyl chloride had been added, the product was heated on a water-bath for about ten minutes to ensure the reaction being complete; a quantity of water was then added, when a solid body separated; this was collected, well washed with water, and spread out on a porous plate to remove a small quantity of oil adhering to the crystals.

The compound was easily purified by recrystallisation from alcohol, and gave the following numbers on analysis :-

$0 \cdot 1 \varepsilon 49$ gram substance gave 0.0864 gram $\mathrm{H}_{2} \mathrm{O}$ and 0.5443 gram $\mathrm{CO}_{2}$. 
Found.

C ... 80.28 per cent.

H .... 5.19 ,

O ... 14.53,
Theory.

$\mathrm{CH}\left(\mathrm{CO} . \mathrm{C}_{6} \mathrm{H}_{5}\right)_{3}$.

$80 \cdot 49$

$4 \cdot 87$

$14 \cdot 63$

This substance, therefore, is tribenzoylmethane. On heating, it melts at $224-225^{\circ}$, and sublimes at a higher temperature almost without decomposition; in this respect it seems to be an extremely stable compound. Tribenzoylmethane is almost insoluble in eold alcohol,' more easily soluble in hot, and crystallises from this solvent on cooling in small, almost microscopic, colourless needles. The hot alcoholic solution gives an orange coloration on adding a drop of ferric chloride. It is fairly easily soluble in benzene and carbon bisulphide, and crystallises in small needles on evaporating the solution. In chloroform and ether, on the contrary, it is sparingly soluble, and in light petroleum almost insoluble, It dissolves readily in alcoholic potash, and on adding water forms a colourless solution from which acids precipitate the original compound unchanged. On warming with concentrated sulphuric acid, it is coloured yellow. Nitric acid appears to entirely decompose it. Experiments were next made in the hope of obtaining tetrabenzoylmethane by the further action of sodic ethylate and benzoyl chloride on tribenzoylmethane, thas:-

$$
\mathrm{CNa}\left(\mathrm{CO} . \mathrm{C}_{6} \mathrm{H}_{5}\right)_{3}+\mathrm{C}_{6} \mathrm{H}_{5} \cdot \mathrm{COCl}=\mathrm{C}\left(\mathrm{CO} . \mathrm{C}_{6} \mathrm{H}_{5}\right)_{4}+\mathrm{NaCl} \text {. }
$$

By this means, a small quantity of a colourless body was obtained which, after being extracted several times with boiling alcohol, melted between $260-270^{\circ}$, and gave the following results on analysis:-

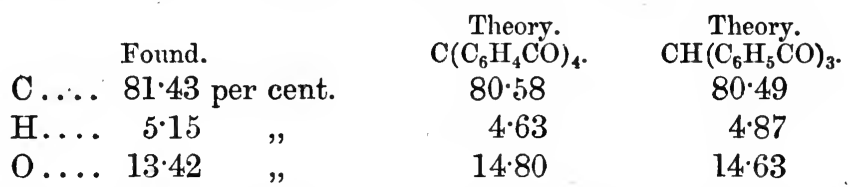

As will be seen from the above, the analysis of such a substance does not show whether it is a tetra- or tri-benzoylmethane. The only fact, which seems to prove that tetrabenzoylmethane had been formed is that the product is far more sparingly soluble in alcoholic potash than tribenzoylmethane. I hope at some future time to be able to continue. the study of these interesting compounds, and if possible to improve the methods of preparing them.

\section{Reduction of Ethylic Benzoylacetate.}

Benzoylacetic acid being a ketonic acid, it was thought that it would be interesting to study the action of nascent hydrogen on it, in order, , if possible, to obtain $\beta$-phenyllactic acid according to the equation- 


\section{$\mathrm{C}_{6} \mathrm{H}_{5} \cdot \mathrm{CO} \cdot \mathrm{CH}_{2} \cdot \mathrm{COOH}+\mathrm{H}_{2}=\mathrm{C}_{6} \mathrm{H}_{5} \cdot \mathrm{CH}(\mathrm{OH}) \cdot \mathrm{CH}_{2} \cdot \mathrm{COOH}$.}

In this reaction, it is better to use ethylic benzoylacetate than the free acid; the following method gives good results.

Ethylic benzoylacetate is dissolved in dilute sodic hydroxide solution, care being taken to avoid any large excess of alkali, and then $\mathbf{5}$ per cent. sodium amalgam is added in small portions at a time. The amalgam liquefies at first without evolution of hydrogen, the liquid becoming so warm that it is necessary to cool it continually. After about ten hours, hydrogen begins to be evolved, and the reaction is finished. The product is then filtered from a small amount of a resinous body (which will be described later on), the filtrate acidulated with dilute sulphuric acid, and extracted several times with ether. On distilling off the ether, an almost colourless oily acid remains, which does not solidify even after long standing in a vacuum over sulphuric acid. In order to purify it, the whole is converted into the barium salt by agitation with baryta-water, the excess of baryta removed by carbonic anhydride, and the precipitated barium carbonate filtered off. The colourless solution of the barium salt of the acid so obtained, is acidified with hydrochloric acid, shaken several times with ether, and the ethereal solution dried over chloride of calcium. On distilling off the ether, a very thick colourless oil is left; and this, on standing for some time, solidifies almost completely, especially after scratching the sides of the vessel containing it with a sharp glass rod. It may be purified by crystallising it once or twice from benzene, when it is obtained in beantiful colourless nodules consisting of fine needles. It melts at $93-94^{\circ}$, and gave the following numbers on analysis :-

0.2005 gram substance gave $0 \cdot 1100$ gram $\mathrm{H}_{2} \mathrm{O}$ and 0.4760 gram $\mathrm{CO}_{2}$.

\begin{tabular}{|c|c|c|}
\hline & $\begin{array}{l}\text { Found. } \\
64.75 \text { per cent. }\end{array}$ & $\begin{array}{c}\text { Theory. } \\
\mathrm{C}_{6} \mathrm{H}_{5} \cdot \mathrm{CH}(\mathrm{OH}) \cdot \mathrm{CH}_{2} \cdot \mathrm{COOH} \text {. } \\
65 \cdot 06 \text { per cent. }\end{array}$ \\
\hline & $6 \cdot 09$ & 6.03 \\
\hline & $21 \cdot 16$ & 28.91 \\
\hline
\end{tabular}

The substance was therefore $\beta$-phenyllactic acid, and was further identified by an analysis of the silver salt, which is easily obtained by precipitating a neutral solution of the ammonium salt with nitrate of silver, as a white curdy precipitate, somewhat soluble in water.

0.3360 gram substance gave 0.1330 gram $\mathrm{Ag}=39.58$ per cent. Theory: $\mathrm{C}_{6} \mathrm{H}_{5} \cdot \mathrm{CH}(\mathrm{OH}) \cdot \mathrm{CH}_{2} \cdot \mathrm{COOAg}=39 \cdot 56 \quad$ "

On heating this silver salt, it decomposes into silver carbonic anhydride and cinnamene, almost without charring. The acid itself, when 
carefully heated, splits up almost quantitatively into water and cinnamic acid, according to the equation -

$\mathrm{C}_{6} \mathrm{H}_{5} \cdot \mathrm{CH}(\mathrm{OH}) \cdot \mathrm{CH}_{2} \cdot \mathrm{COOH}=\mathrm{C}_{6} \mathrm{H}_{5} \cdot \mathrm{CH}: \mathrm{CH} \cdot \mathrm{COOH}+\mathrm{H}_{2} \mathrm{O}$.

This acid has all the properties of the 3 -phenyllactic acid prepared by Glaser (Annalen, 147, 86) by the action of sodium amalgam on phenylchlorolactic acid, $\mathrm{C}_{6} \mathrm{H}_{5} . \mathrm{CH}(\mathrm{OH}) . \mathrm{CHCl} . \mathrm{COOH}$, its formation from ethylic benzoylacetate being a proof that the hydroxyl group is really in the $\beta$-position. The same acid is also formed by boiling phenylbromopropionic acid, $\mathrm{C}_{6} \mathrm{H}_{5} \cdot \mathrm{CHBr} \mathrm{CH}_{2} \cdot \mathrm{COOH}$, with water (Fittig and Binder, Aunalen, 195, 138).

By the action of nascent hydrogen on ethylic benzoylacetate, there is always a certain amount of a resinous substance formed which separates out of the alkaline solution during the reduction, and can easily be freed from the sodic salt of the $\beta$-phenyllactic acid by filtration and washing with water. As in some cases a considerable amount of this compound was formed, it was thought that it might contain something definite, and was therefore carefully examined. The filter-papers were extracted with ether, and the ethereal solution filtered and evaporated; by this means a very thick dark-brown oil was left behind, which, after standing for several days, partly crystallised. The crystals were easily obtained colourless and free from oil, by repeated washings with small quantities of ether in which they are difficultly soluble. Last of all they were dissolved in a little hot alcohol, from which they crystallise on cooling in thick colourless prisms melting at $102^{\circ}$.

The analysis gave the following result :-

I. $0 \cdot 1665$ gram substance gave 0.0768 gram $\mathrm{H}_{2} \mathrm{O}$ and 0.4581 gram $\mathrm{CO}_{2}$.

II. 0.2082 gram substance gave 0.0906 gram $\mathrm{H}_{2} \mathrm{O}$ and 0.5720 gram $\mathrm{CO}_{2}$.

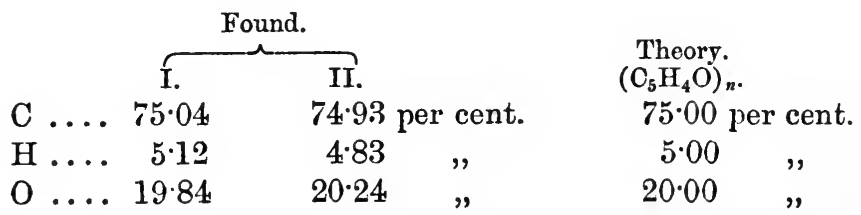

This substance therefore has the formula $\left(\mathrm{C}_{5} \mathrm{H}_{4} \mathrm{O}\right)_{n}$, most likely $\mathrm{C}_{10} \mathrm{H}_{8} \mathrm{O}_{2}$.

When warmed with alcoholic putash, it yields an intense magentared solution; and on filtering and acidifying a colourless crystalline acid is precipitated. The alcoholic solution does not give any colour reaction with ferric chloride. 
A small quantity of this body warmed with concentrated sulphuric acid dissolves, forming a pinkish solution with a slight greenish fluorescence. If, after standing for a short time, this solution be poured into water, a whitish amorphous substance separates; this forms an intense blue-green solution on the addition of an excess of sodic hydroxide. If the original substance, however, be more strongly heated with sulphuric acid, the pinkish colour first produced becomes browner, and at last of a deep olive-green. On pouring this olive-green solution into water, a bright yellowish liquid is produced, which, on the addition of sodic hydroxide, becomes of a beautiful purple. This reaction is extremely delicate, and is produced by mere traces of substance. This compound is isomeric with a substance which was obtained by the action of sodium on acetate of phenyl, a reaction which W. R. Hodgkinson and I studied some time ago (Trans., 1880, 487). Curiously enough the latter also gives the same characteristic reaction when warmed with potash; the red colour is destroyed, however, on diluting with water, which is not the case with the body described above.

\section{Action of Phosphorous Pentachloride on Ethylic Benzoylacetate.}

Geuther (Zeitschr.f. Chem., 1871, 237), who was the first to study the action of pentachloride of phosphorus on ethylic acetoacetate, found that when it was treated with an excess of the former it was resolved into the chlorides of two isomeric chlorcrotonic acids, namely, $\beta$-chlor- $\alpha$-crotonic acid, $\mathrm{CH}_{3} . \mathrm{CCl}: \mathrm{CH} . \mathrm{COOH}$, and the $\beta$-chloro$\beta$-crotonic acid, $\mathrm{CH}_{2}: \mathrm{CCl} . \mathrm{CH}_{2}$. $\mathrm{COOH}$.

On decomposing these chlorides with water, and distilling with steam, the $\beta$-chloro- $\beta$-crotonic acid distils over first, and then the $\beta$-chlor- $\alpha$-crotonic acid. As the methyl group of the ethylic acetoacetate must have taken part in the formation of $\beta$-chloro- $\beta$-crotonic acid, supposing the formula $\mathrm{CH}_{2}: \mathrm{CCl} . \mathrm{CH}_{2} \cdot \mathrm{COOH}$ to be correct, it appeared interesting to repeat these experiments with ethylic benzoylacetate, in which the presence of the phenyl group would shut out the possibility of the formation of any acid analogous in constitution to $\beta$-chloro- $\beta$-crotonic acid.

In order to test this, 10 grams of ethylic benzoylacetate was dis. solved in 25 grams of oxychloride of phosphorus, and 25 grams of phosphorous pentachloride slowly added. As the reaction appeared to take place very slowly at ordinary temperatures, the mixture becoming only slightly warm, the whole was heated for about half an hour on a water-bath, when the pentachloride slowly disappeared, hydrogen chloride being given off. As soon as the reaction was over, the oxychloride of phosphorus was distilled off on a water-bath in a 
vacuum, the latter portions carrying over a small quantity of a crystalline substance which sublimed into the condenser in yellow needles; these, however, were not further examined. The residue, consisting of a dark-brown oil, was poured into ice-water and allowed to remain for some time, in order that acid chlorides and any excess of pentachloride of phosphorus might be entirely decomposed. An excess of sodic carbonate was then added, and the whole gently warmed with animal charcoal on a water-bath for abont fifteen minutes, and filtered. On acidifying the filtrate, which was nearly colourless, it deposited a crystalline acid; this was collected, well washed, and recrystallised twice from alcohol. It was thus obtained perfectly pure in beautiful glittering plates, which, on analysis, gave the following result:-

$0 \cdot 1798$ gram substance gave 0.0665 gram $\mathrm{H}_{2} \mathrm{O}$ and 0.3901 gram $\mathrm{CO}_{2}$. $0 \cdot 2424$ , , $0 \cdot 3080 \mathrm{AgCl}$.

Found.

C ... $59 \cdot 17$ per cent.

H ... $4 \cdot 10$,

Cl ... 19.47 ,

O $\ldots .17 \cdot 26 \quad$ "
Theory. $\mathrm{C}_{9} \mathrm{H}_{7} \mathrm{O}_{2} \mathrm{Cl}$.

$59 \cdot 18$ per cent.

$\begin{aligned} 3.84 & \text { " } \\ 19 \cdot 45 & \quad \\ 17.53 & \text { " }\end{aligned}$

The compound was therefore chlorocinnamic acid, produced according to the equations-

\section{I. $\mathrm{C}_{6} \mathrm{H}_{5} \cdot \mathrm{CO} \cdot \mathrm{CH}_{2} \cdot \mathrm{COOC}_{2} \mathrm{H}_{5}+2 \mathrm{PCl}_{5}=\mathrm{C}_{6} \mathrm{H}_{5} \cdot \mathrm{CCl}_{2} \cdot \mathrm{CH}_{2} \cdot \mathrm{COCl}$ $+\mathrm{C}_{2} \mathrm{H}_{5} \mathrm{Cl}+2 \mathrm{POCl}_{3}$.}

\section{II. $\mathrm{C}_{6} \mathrm{H}_{5} \cdot \mathrm{CCl}_{2} \cdot \mathrm{CH}_{2} \cdot \mathrm{COCl}+\mathrm{H}_{2} \mathrm{O}=\mathrm{C}_{6} \mathrm{H}_{5} \cdot \mathrm{CCl}$ : $\mathrm{CH} \cdot \mathrm{COOH}+2 \mathrm{HCl}$. $\beta$-Chlorocinnamic acid.}

By this reaction, therefore, $\beta$-chlorocinnamic acid should be obtained. There are two monochlorocinnamic acids known, the $\alpha$-monochlorocinnamic acid, $\mathrm{C}_{6} \mathrm{H}_{5} . \mathrm{CH}: \mathrm{CCl} . \mathrm{COOH}$, and the $\beta$-monochlorocinnamic acid, $\mathrm{C}_{6} \mathrm{H}_{5} . \mathrm{CCl}: \mathrm{CH} . \mathrm{COOH}$. These were obtained by Jutz (Ber., 15, 788), in a way exactly similar to that employed by Glaser (Annalen, 143, 330), in the preparation of the monobromocinnamic acids, namely, by heating phenyldichloropropionic acid with potash :-

2. $\mathrm{C}_{6} \mathrm{H}_{5} \cdot \mathrm{CHCl} . \mathrm{CHCl} \cdot \mathrm{COOH}+4 \mathrm{KOH}=\mathrm{C}_{6} \mathrm{H}_{5} \cdot \mathrm{CH}: \mathrm{CCl} \cdot \mathrm{COOK}$

Phenyldichloropropionic acid.

Potassic $\alpha$-chlorocinnamate.

$$
+\mathrm{C}_{6} \mathrm{H}_{5} \cdot \mathrm{CCl}: \mathrm{CH} \cdot \mathrm{COOK}+2 \mathrm{KCl}+4 \mathrm{H}_{2} \mathrm{O} \text {. }
$$

Potassic $\beta$-chlorocinnamate.

He separated these acids by means of the potash salts, the salt of 
the $\alpha$-acid being sparingly soluble in water, whereas the salt of the $\beta$-acid is easily soluble. The $\alpha$-chlorocinnamic acid, crystallised from water, melts at $137-138^{\circ}$, crystallised from light petroleum at $142^{\circ}$. $\beta$-Chlorocinnamic acid melts at $114^{\circ}$. $\alpha$-Chlorocinnamic acid is also formed by heating phenylchlorolactic acid with water at $120-200^{\circ}$ :-

\section{$\mathrm{C}_{6} \mathrm{H}_{5} \cdot \mathrm{CH}(\mathrm{OH}) \cdot \mathrm{CHCl} \cdot \mathrm{COOH}=\mathrm{C}_{6} \mathrm{H}_{5} \cdot \mathrm{CH}: \mathrm{CCl} \cdot \mathrm{COOH}+\mathrm{H}_{2} \mathrm{O}$.}

Phenylchlorolactic acid. $\quad a$-Chlorocinnamic acid.

This is a proof that the chlorine is in the $\alpha$-position, there being no doubt as to the constitution of phenylchlorolactic acid. A further proof of the constitution of $\alpha$-chlorocinnamic acid is its formation by the action of acetic anhydride on a mixture of benzaldehyde with sodic monochloracetate, according to the equation-

$\mathrm{C}_{6} \mathrm{H}_{6} \cdot \mathrm{COH}+\mathrm{CH}_{2} \mathrm{Cl} \cdot \mathrm{COONa}=\mathrm{C}_{6} \mathrm{H}_{3} \cdot \mathrm{CH}$ : CCl.COONa $+\mathrm{H}_{2} \mathrm{O}$.

(Plöchl, Ber., 15, 1945).

The chlorocinnamic acid obtained from ethylic benzoylacetate by the action of pentachloride of phosphorus in the way just described, melts at $142^{\circ}$, and possesses, strangely enough, all the properties of a-chlorocinnamic acid, with which it is no doubt identical, although, according to the equation given above, $\beta$-chlorocinnamic acid should be produced.

The formation of $\alpha$-chlorocinnamic acid from ethylic benzoylacetate in this way is difficult to explain; one is forced to come to the conclusion that the $\beta$-acid is first formed, and afterwards, under the influence of such powerful reagents as pentachloride and oxychloride of phosphorus, that a molecular change takes place, whereby the $\beta$-acid is transformed into the $\alpha$-acid. In the case of the monobromocinnamic acids, the $\beta$-acid can with the greatest ease be transformed into the $\alpha$-acid by distillation, or by heating with hydriodic acid, the change being almost quantitative. Still stranger is the fact that the $\beta$-monobromocinnamic acid on etherification is converted quantitatively into the ethyl salt of $\alpha$-monobromocinnamic acid. This mobility of the bromine-atom makes it appear possible that these compounds are merely physical isomerides, and not chemical isomerides in the true sense of the word.

\section{Condensation of Ethylic Benzoylacetate with Benzaldehyde.}

The hydrogen-atoms of the methylene group in ethylic acetoacetate are very easily substituted by condensation with aldehydes, according to the equation (Claisen, Annalen, 218, 170)-

$\mathrm{CH}_{3} \cdot \mathrm{CO} \cdot \mathrm{CH}_{2} \cdot \mathrm{COOEt}+\mathrm{R} \cdot \mathrm{CHO}=\mathrm{CH}_{3} \cdot \mathrm{CO} \cdot \mathrm{C}(\mathrm{CH} \cdot \mathrm{R}) \cdot \mathrm{COOEt}+\mathrm{H}_{2} \mathrm{O}$. 
This condensation is best brought about by mixing the ethylie acetoacetate with the calculated amount of the aldehyde, and then after cooling in a freezing mixture, saturating the whole with hydrogen chloride. After standing for about two days, the reaction is complete, the resulting product, e.g., in the case of the condensation of ethylic acetoacetate with benzaldehyde, consisting of a mixture of two isomeric ethylic acetylbenzylchloracetates-

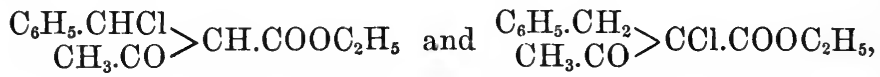

which are easily separated by crystallisation from light petroleum. Both these compounds are decomposed by distillation, yielding hydrogen chloride and ethylic acetylbenzalacetate-

$$
\begin{aligned}
& \mathrm{C}_{6} \mathrm{H}_{5} . \mathrm{CH} \\
& \mathrm{C}_{6} \mathrm{H}_{3} \cdot \mathrm{CO} . \mathrm{COOC}_{2} \mathrm{H}_{5} .
\end{aligned}
$$

Claisen explains this condensation by supposing that the aldehyde is first converted by the hydrochloric acid into an unstable chlorhydrol, R. $\mathrm{CH}(\mathrm{OH}) \mathrm{Cl}$, which then acts on the etiıylic acetoacetate in the following way :-

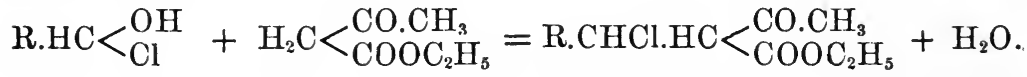

On substituting benzoylacetate for ethylic acetoacetate, it was found that condensation with aldehydes took place in precisely the same way. As it appeared interesting to prove the analogy between ethylic benzoylacetate and acetoacetate in this respect also, the condensation of the former with benzaldehyde was further studied.

10 grams of ethylic benzoylacetate were mixed with 5 grams of benzaldehyde, and the whole, after being cooled below $0^{\circ}$ in a freezing mixture, was completely saturated with hydrogen chloride. The product soon became very thick and milky, from the formation of minute glohules of water, which, after standing for 24 hours at the ordinary temperature, settled out in large drops on the side of the vessel.

The dark brown oily product was dissolved in ether, well washed, first with water and then with sodic carbonate solution, to remove the excess of hydrochloric acid, and finally dried over potassic carbonate. After filtering and distilling off the ether, a dark brownish very thick oil remained; this was distilled as rapidly as possible under diminished pressure $(200 \mathrm{~mm}$.), only a small residue being left. The distillate, which was nearly colourless, was distilled again, under the same pressure, the first portions, consisting of acetophenone and unchanged benzaldehyde, being rejected; afterwards a thick oil came over, which rapidly solidified on standing. 
The latter was dissolved in hot methyl alcohol, from which on cooling it crystallised in beautiful colourless needles, easily obtained pure by recrystallisation.

The analysis of the crystals, dried at $70-80^{\circ}$, gave the following numbers :-

I. $0 \cdot 2 \cdot 243$ gram of substance gave 0.1195 gram $\mathrm{H}_{2} \mathrm{O}$ and 0.6321 gram $\mathrm{CO}_{2}$.

II. 0.2022 gram of substance gave 0.1040 gram $\mathrm{H}_{2} \mathrm{O}$ and 0.5665 gram $\mathrm{CO}_{2}$.

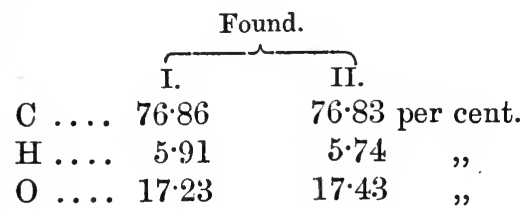

$$
\begin{aligned}
& \text { Theory. } \\
& \mathrm{C}_{6} \mathrm{H}_{5} . \text { CO.C }<_{\text {COOC }}{ }_{2} \mathrm{H}_{5} \text {. } \\
& 77 \cdot 14 \text { per cent. } \\
& 5 \cdot 72 \quad \text {, } \\
& 17 \cdot 14,
\end{aligned}
$$

The compound is therefore ethylic benzalbenzoylacetate. Ethylic benzalbenzoylacetate crystallises from methyl alcohol in long colourless glittering prisms, which melt at $98-99^{\circ}$. It is pretty easily soluble in hot methyl alcohol, sparingly so in cold. Like ethylic acetylbenzalacetate, it crystallises very readily. It separates from a mixture of benzene and light petroleum in flat plates, which polarise light splendidly. It crystallises best, however, from ether. If the ethereal solution is allowed to evaporate slowly, the sides and bottom of the vessel containing it become covered with large transparent glittering prisms, which do not lose their lustre on drying. Professor Haushofer, who kindly measured some of the crystals, gave me the following description of them :-

Crystalline system monoclinic:

$$
a: b: c=1 \cdot 2730: 1: 0 \cdot 7461 . \quad \beta=86^{\circ} 36^{\prime} .
$$

Pale yellow lustrous crystals, forming the combinations $\operatorname{cof} \cos (a)$, $\operatorname{cs} \mathrm{P}(p),-\mathrm{P} c \rho(r), \mathrm{P} c s(s)$, extended in the direction of the ortho-

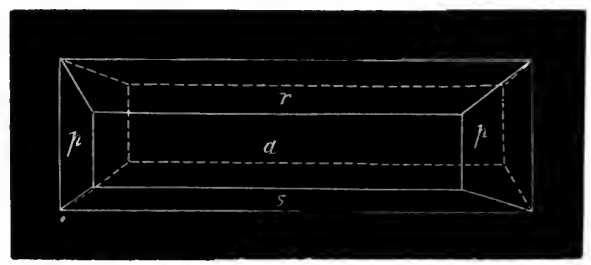

diagonal, often also tabular on the surface $a$. The surfaces of this zone are smooth, but as a rule indented, and the measurements are therefore somewhat irregular. 


\begin{tabular}{|c|c|}
\hline $\begin{array}{r}\text { Measured. } \\
. n=103^{\circ} 36^{\prime}\end{array}$ & Calculated. \\
\hline$r: p=10937$ & - \\
\hline$s: r=11920$ & - \\
\hline$r: a=12257$ & $122^{\circ} 53^{\prime}$ \\
\hline$p: a=12830$ & $128 \quad 12$ \\
\hline$s: a=11753$ & $117 \quad 47$ \\
\hline$s: p=10640$ & $106 \quad 45$ \\
\hline
\end{tabular}

Ethylic benzalbenzoylacetate dissolves in sulphuric acid, producing a yellow solution, which, however, becomes colourless again on standing or gently warming. The solution of this body in chloroform is only very slowly attacked by bromine.

Several saponification experiments were next made, in the hope of obtaining benzylideneacetophenone, according to the equation-

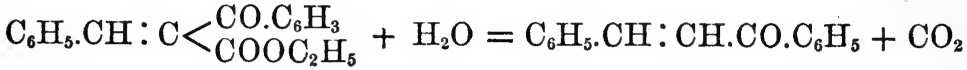

$$
\begin{aligned}
& +\mathrm{C}_{2} \mathrm{H}_{5} \mathrm{OH}
\end{aligned}
$$

but withont result. When ethylic benzalbenzoylacetate was heated with dilute hydrochloric acid in a sealed tube at $160^{\circ}$ for seven hours, it was decomposed in part, producing a yellow oil. On opening the tube, large quantities of carbonic anhydride escaped, and on fractioning the product a considerable amount was obtained boiling below $210^{\circ}$, and consisting of a mixture of benzaldehyde and acetophenone. The thermometer was then removed, and the residue distilled in a vacuum, when a thick oil came over, which solidified on cooling. After crystallisation from methyl alcohol, it melted at $98^{\circ}$, and showed all the properties of ethylic benzalbenzoylacetate. Negative results were also obtained on boiling it with potash, only small quantities of benzoic acid being produced.

Claisen (Annalen, 218, 173) obtained similar results on trying to obtain ethylideneacetone and crotonic acid by saponifying ethylic acetylethylideneacetate, $\underset{\mathrm{CH}_{3} . \mathrm{CO}}{\mathrm{CH}_{3} . \mathrm{CH}}>\mathrm{C} . \mathrm{COOC}_{2} \mathrm{H}_{5}$, with potash, and attributes this to the fact of the latter class of bodies being so easily decomposed into their components, and thus giving only aldehyde and the decomposition-products of ethylic acetoacetate, respectively ethylic benzoylacetate on hydrolysis. Another method employed by Claisen to effect the condensation of aldehydes with ethylic acetoacetate was by heating a mixture of the two bodies in a sealed tube with acetic anhydride. In order to see whether this also took place in the case of etbylic benzoylacetate, 10 grams of the latter, 5 grams of benzaldehyde, and an excess of acetic anhydride, were heated together in a sealed tube at $180-200^{\circ}$ for six hours. On opening the 
tube a large quantity of carbonic anhydride escaped. The product, which was a dark brown oil, was then directly fractioned. Below $100^{\circ}$, a small quantity of a limpid oil came over, which, after washing and refractioning, distilled almost entirely between $70^{\circ}$ and $80^{\circ}$ as a light oil, smelling strongly of ethylic acetate. After the excess of acetic anhydride and acetic acid had distilled over, the thermometer rose rapidly to $180^{\circ}$, and between that and $210^{\circ}$ a considerable quantity of a mixture of benzaldehyde and acetophenone came over. The residue was then distilled under diminished pressure, when the latter portions, which were collected separately, partially solidified on cooling. The crystals were first roughly freed from oily matter, by spreading them out on a porous plate, and then purified by crystallisation, once from methyl alcohol and once from ether.

The analysis gave the following numbers :-

0.1863 gram substance gave 0.0976 gram $\mathrm{H}_{2} \mathrm{O}$ and 0.5254 gram $\mathrm{CO}_{2}$.

Found.

C .... 76.92 per cent.

H.... 5.82 ,

O... $17 \cdot 26$,
Theory.<smiles>CCOC(=O)C(OCC)c1ccccc1</smiles>

$77 \cdot 14$ per cent.

$5 \cdot 72$

$17 \cdot 14$,

The crystals melted at $98^{\circ}$, and the body was therefore ethylic benzalbenzoylacetate. The yield by this method was not nearly so good as by the one previously described. It is possible that the temperature employed $\left(180-200^{\circ}\right)$ was too high, and that some of the product was again resolved into its components, the ethylic benzoylacetate being further decomposed into acetophenone and carbonic anhydride. 

[Reprinted from the Journal of the Chemical Society, May, 1885. Vol. XLVII.]

ON BENZOYLACETIC ACID AND SOME OF ITS DERIVATIVES. PART III.

By W. H. Perkin (Jun.), Ph.D., Privatdocent at the University of Munich.

In the first two parts of this research, I described a number of simpler derivatives of ethylic benzoylacetate, showing it to be analogous to ethylic acetoacetate in almost every respect. In the third part, I wish to bring before the Society an account of some of the more complicated reactions into which this body enters, namely, the preparation and properties of ethylic dibenzoylsuccinate, ethylic monobenzoylsuccinate, and dehydrobenzoylacetic acid. 


\section{Ethylic Dibenzoylsuccinate.}

By the action of iodine on the sodium compound of ethylic acetoacetate, Harrow (Trans., 1878, 425) obtained ethylic diacetylsuccinate, according to the equation-

$$
\underset{\mathrm{CH}_{3} \text { CO.CHNa.COOEt }}{\mathrm{CH}_{3} \text { CO.CHNa.COOEt }}+\mathrm{I}_{2}=\underset{\mathrm{CH}_{3} \cdot \mathrm{CO} . \mathrm{CH} \cdot \mathrm{COOEt}}{\mathrm{CH}_{3} \cdot \mathrm{CO} \cdot \mathrm{CH} \cdot \mathrm{COOEt}}+2 \mathrm{NaI} \text {. }
$$

On boiling this compound with dilute sulphuric acid, it is partly hydrolysed, giving off one molecule of alcohol, and forming the ethylic salt of carbopyrotritaric acid, thus :-

$$
\mathrm{C}_{12} \mathrm{H}_{18} \mathrm{O}_{6}=\mathrm{C}_{10} \mathrm{H}_{12} \mathrm{O}_{5}+\mathrm{C}_{2} \mathrm{H}_{5} . \mathrm{OH} \text {. }
$$

If the saponifying action is continued further, this ethereal salt is split up into carbopyrotritaric acid and alcohol,-

$$
\mathrm{C}_{10} \mathrm{H}_{12} \mathrm{O}_{5}+\mathrm{H}_{2} \mathrm{O}=\mathrm{C}_{8} \mathrm{H}_{8} \mathrm{O}_{5}+\mathrm{C}_{2} \mathrm{H}_{5} . \mathrm{OH} \text {. }
$$

This acid, which Harrow supposes to have the constitution-

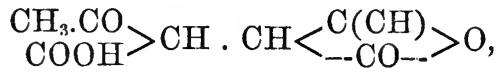

is resolved, on further heating with dilute sulphuric acid, into pyrotritaric acid and carbonic anhydride, thus :-

$$
\mathrm{C}_{8} \mathrm{H}_{8} \mathrm{O}_{5}=\mathrm{C}_{7} \mathrm{H}_{8} \mathrm{O}_{3}+\mathrm{CO}_{2} \text {. }
$$

On account of this latter reaction, Harrow assigns the following constitution to pyrotritaric acid :-

$$
\mathrm{CH}_{3} \cdot \mathrm{CO} \cdot \mathrm{CH}(\mathrm{COOH}) \cdot \mathrm{CH}: \mathrm{C}: \mathrm{CH}_{2} \text {. }
$$

Considering the complicated nature of these reactions, it appeared to be interesting to repeat them with ethylic benzoylacetate, in order to determine whether similar compounds would be produced, corresponding with carbopyrotritaric and pyrotritaric acids.

It was necessary, in the first place, to prepare ethylic dibenzoylsuccinate, which can easily be effected by a method analogous to that employed by Harrow for preparing ethylic diacetylsuccinate, namely, by the action of iodine on ethylic benzoylsodacetate. 4.7 grams of the finely powdered sodium compound were mixed with dry ether, and 2.8 grams of iodine added in small quantities at a time, the whole being well shaken and cooled after each addition. At first the colour of the iodine disappears instantly, but towards the end of the reaction much more slowly, the mixture becoming slightly brownish.

The ethereal solution is filtered from the precipitated sodic iodide, washed with water and a little sulphurous acid in order to remove any slight excess of iodine, and then dried over calcium chloride. On 
distilling off the ether, a brownish mass remains, which becomes almost entirely solid when exposed for about 24 hours over sulphuric acid in a vacuum. These crystals are spread out on a porous plate, in order to remove as much of the mother-liquor as possible, washed on a filter, with small quantities of ether, until an almost colourless mass remains behind, and purified by two crystallisations from alcohol.

0.1630 gram substance gave 0.0890 gram $\mathrm{H}_{2} \mathrm{O}$ and $0.4113 \mathrm{CO}_{2}$.

\begin{tabular}{|c|c|c|}
\hline C. . & $\begin{array}{l}\text { Found. } \\
68.82 \text { per cent. }\end{array}$ & $\begin{array}{l}\text { Theory, } \mathrm{C}_{22} \mathrm{H}_{22} \mathrm{O}_{6} \text {. } \\
\quad 69 \cdot 11 \text { per cent. }\end{array}$ \\
\hline & 6.06 & $5 \cdot 76$ \\
\hline $0 \ldots$ & $25 \cdot 12$ & $25 \cdot 13$ \\
\hline
\end{tabular}

The compound is therefore ethylic dibenzoylsuccinate, formed according to the equation-

$$
\begin{aligned}
& \mathrm{C}_{6} \mathrm{H}_{5} \text {.CO.CHNa.COOEt } \\
& \mathrm{C}_{6} \mathrm{H}_{5} \text {.CO.CHNa.COOEt }+\mathrm{I}_{2}={ }_{\mathrm{C}_{6} \mathrm{H}_{5} \text {.CO.CH.COOEt }}+2 \mathrm{NaI} \text {. }
\end{aligned}
$$

Ethylic dibenzoylsuccinate melts at $128-130^{\circ}$, and when heated in a test-tube it is decomposed, a carbonaceous residue being left. It is sparingly soluble in cold alcohol, but easily in hot, being deposited from this solution on cooling in short thick prisms; it may be obtained perfectly colourless by repeated recrystallisation. It is easily soluble in ether and benzene, crystallising in small needles on evaporation.

The behaviour of ethylic dibenzoylsuccinate with concentrated sulphuric acid is very characteristic. The finely powdered substance dissolves in the acid, forming an almost colourless solution, and on adding water is reprecipitated apparently unchanged. When the solution is heated, it becomes first reddish and then olive-green, but if the heating is continued the colour changes to a bluish-red. If this solution when cold is poured into a little water, a brownish liquid is obtained, with a strong blue fluorescence; an excess of water destroys the coloration altogether. As ethylic benzoylsuccinate contains two $\mathrm{CH}$ groups which are each joined on both sides to carbonyl groups, it appeared very probable that it might yield a disodium compound on treatment with sodium ethoxide, according to the equation-

$$
\underset{\mathrm{C}_{6} \mathrm{H}_{5} \cdot \mathrm{CO} \cdot \mathrm{C}_{5} \mathrm{H}_{5} \cdot \mathrm{CO} \cdot \mathrm{CH} \cdot \mathrm{COOEt}}{\mathrm{C}_{1}}+2 \mathrm{NaOEt}={ }_{\mathrm{C}_{6} \mathrm{H}_{5} \cdot \mathrm{CO} \cdot \mathrm{CNa}_{5} \cdot \mathrm{COOEt}}^{\mathrm{C}_{6} \mathrm{H}_{5} \mathrm{CO} \cdot \mathrm{CNa} \cdot \mathrm{COOEt}}+2 \mathrm{EtOH} .
$$

This was in reality found to be the case, the compound being best formed in the following way: 0.3 gram of sodium is dissolved in the smallest possible quantity of alcohol, and while still warm an excess of pure dry ether is added, the whole being thoroughly mixed, by 
means of a glass rod, until the liquid becomes clear, or only slightly turbid. An ethereal solution of 2.5 grams of ethylic dibenzoylsuccinate is then added, and the whole allowed to stand for some time. The sodium compound slowly separates out as a slightly. yellowish mass, and as soon as the precipitation is finished it is collected by means of a vacuum pump, and well washed, first, with a mixture of alcohol and ether, and lastly, with ether, in which the compound is almost insoluble. The disodium compound was thus obtained as an almost colourless mass, which, after standing for some hours over sulphuric acid in a vacuum, gave the following numbers on analysis:-

0.4434 gram substance gave $0.1575 \mathrm{Na}_{2} \mathrm{SO}_{4}$.

$\begin{array}{cl}\text { Found. } & \text { Theory. } \\ \mathrm{Na} \ldots \mathrm{11} \cdot 49 \text { per cent. } & 10.80 \text { per cent. }\end{array}$

When perfectly dry, this compound is fairly stable, but if it contains a little alcohol it quickly takes up moisture from the air, and is decomposed. It is fairly soluble in hot alcohol, sparingly in the cold. On adding a drop of ferric chloride to the alcoholic solution, a dirty red coloration is formed. Cupric acetate precipitates the copper salt as a light-green amorphous mass. The sodium compound is easily decomposed by dilute acids, the original substance being apparently regenerated.

If this compound is treated with an ethereal solution of iodine, sodium iodide is instantly precipitated, and on filtering and evaporating the ethereal solution a thick brownish oil is obtained, which is probably ethylic dibenzoylfumarate produced according to the equation-

$$
\begin{aligned}
& \mathrm{C}_{6} \mathrm{H}_{5} \text {.CO.CNa.COOEt } \quad \mathrm{C}_{6} \mathrm{H}_{5} \text {.CO.C.COOEt }
\end{aligned}
$$

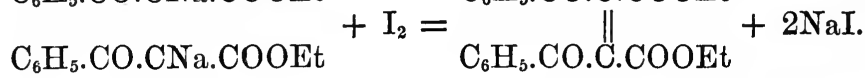

The oil, unfortunately, would not crystallise, and therefore a good analysis could not be obtained. The solution in chloroform, however, decolorised bromine instantly, without evolution of hydrobromic acid, showing that it is an unsaturated compound.

\section{Decomposition Products of Ethylic Dibenzoylsuccinate.}

On mixing ethylic dibenzoylsuccinate with alcoholic potash, it dissolves, producing an intensely yellow solution. If after allowing it to remain for some time, or gently warming, the product be acidulated with dilute sulphuric acid, a yellow amorphous acid is precipitated; unfortunately, however, this could not be obtained in a crystalline state, as it is extremely soluble in all solvents except water. In order to purify it as far as possible, it was dissolved in ammonia, and 
fractionally precipitated with dilute acid; this treatment having been repeated several times, the product was finally converted into the silver salt, and analysed with the following result:-

0.3047 gram substance gave 0.1171 gram Ag.

$$
\begin{array}{cc}
\text { Found. } & \text { Theory, } \mathrm{Ag}_{2} \mathrm{C}_{18} \mathrm{H}_{12} \mathrm{O}_{6} \text {. } \\
\mathrm{Ag} \text {... 38.4.3 per cent. } & 40.00 \text { per c̀ent. }
\end{array}
$$

Although these numbers do not agree very closely with theory, it is yet probable that the substance is in reality impure dibenzoylsuccinic acid. On adding ferric chloride to the solution of the acid in alcohol, or that of the ammonium salt, a peculiar dark-green precipitate is formed, closely resembling that obtained with sulphuric acid.

The acid dissolves in concentrated sulphuric acid, producing a yellowish solution which becomes of a beautiful crimson on warming. The acid gives acetophenone when distilled with sodalime. From the above experiments, it appeared unlikely that better results would be obtained by saponifying with other alkalis, such as baryta-water, and it was thought probable that, as in the case of ethylic diacetylsuccinate, dilute acids might be emplcyed with greater advantage. The following method of hydrolysis was finally adopted.

Pure ethylic dibenzoylsuccinate was boiled with an excess of dilute sulphuric acid $(1: 2)$ for about six hours in a flask connected with a reflux condenser, at the end of which a test-tube containing barytawater was fixed. The mass became more and more darkly coloured, but very little carbonic anhydride was given off. When cold, the product was collected, washed with water, well shaken with a warm solution of sodic carbonate, and filtered. The residual tarry mass was then washed with water, again boiled with dilute sulphuric acid for six hours, and the extraction with sodic carbonate repeated as before. The greater part dissolved, leaving a thick black residue, from which mere traces of an organic acid were obtained on repeating the treatment with sulphuric acid.

The different alkaline solutions were mixed and acidified with dilute sulphuric acid. This produced a precipitate which, after being recrystallised several times from dilute acetic acid (70 per cent.), was obtained pure in the form of small colourless needles which gave the following numbers on analysis :-

I. $0 \cdot 1862$ gram substance gave $0.0664 \mathrm{H}_{2} \mathrm{O}$ and $0.4752 \mathrm{CO}_{2}$.

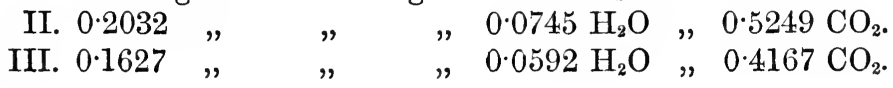


Found.

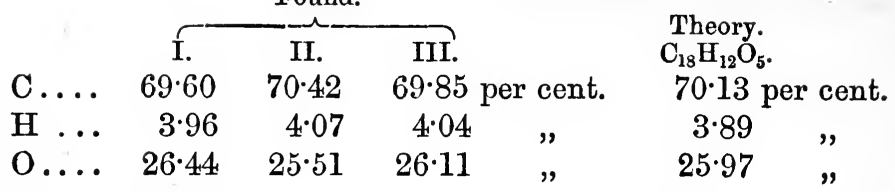

The formula of this acid is therefore $\mathrm{C}_{18} \mathrm{H}_{12} \mathrm{O}_{5}$, and it is formed from ethylic dibenzoylsuccinate by the removal of 2 mols. of alcohol, thus :-

$$
\underset{\substack{\mathrm{C}_{23} \mathrm{H}_{22} \mathrm{O}_{6}+\mathrm{H}_{2} \mathrm{O} \\ \text { Ethylic dibenzoyl- } \\ \text { succinate. }}}{\mathrm{C}_{18} \mathrm{H}_{12} \mathrm{O}_{5}}+2 \mathrm{C}_{2} \mathrm{H}_{5} \mathrm{OH} .
$$

its formation corresponding precisely with that of carbopyrotritaric acid from ethylic diacetylsuccinate :-

$$
\mathrm{C}_{12} \mathrm{H}_{18} \mathrm{O}_{6}+\mathrm{H}_{2} \mathrm{O}=\mathrm{C}_{8} \mathrm{H}_{8} \mathrm{O}_{5}+2 \mathrm{C}_{2} \mathrm{H}_{5} . \mathrm{OH} \text {. }
$$

This new acid melts at $236-238^{\circ}$. It is easily soluble in ether, alcohol, benzene, light petroleum, and glacial acetic acid, less so in a 70 per cent. solution of the latter solvent, from which it crystallises in small needles. The alcoholic solution gives an orange coloration with ferric chloride.

This acid, $\mathrm{C}_{18} \mathrm{H}_{12} \mathrm{O}_{5}$, dissolves in concentrated sulphuric acid, forming a colourless solution, which however acquires a beautiful violet colour when warmed. Heated by itself in a test-tube, it first melts and then sublimes, water being given off at the same time. The sublimate is crystalline, but apparently insoluble in anımonia, and is perhaps the anhydride of the acid. The acid $\mathrm{C}_{18} \mathrm{H}_{12} \mathrm{O}_{5}$ is easily decomposed on distilling it with soda-lime, acetophenone being formed. Its alcoholic solution does not appear to react with phenylhydrazine or hydroxylamine. It is, however, a strong acid, dissolving easily in ammonia, sodic hydroxide, or sodic carbonate solutions. The ammonium salt was prepared by dissolving the acid in ammonia and then allowing the solution to stand over sulphuric acid in a vacuum until the excess of alkali had evaporated. On adding silver nitrate to this solution, a white precipitate of the silver salt was formed; this, when dried over sulphuric acid in a vacuum, gave the following numbers on analysis :-

0.4817 gram substance gave 0.1939 gram silver.

Theory.

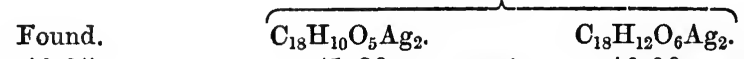

Ag. ... $\quad 40.25$ per cent. $\quad 41.29$ per cent. $\quad 40.00$ per cent.

This result not being satisfactory, a complete analysis of the silver salt was made with the following result:- 
0.2683 gram substance gave 0.400 $\mathrm{CO}_{2}, 0.0562 \mathrm{H}_{2} \mathrm{O}$ and $0.1087 \mathrm{Ag}$.

\begin{tabular}{lrl}
\multicolumn{4}{c}{ Found. } \\
C .... & $40 \cdot 66$ per cent. \\
H ... & $2 \cdot 32$ \\
Ag ... & $40 \cdot 52 \quad "$ \\
O .... & $16.50 \quad "$
\end{tabular}

\begin{tabular}{|c|c|c|}
\hline $\begin{array}{c}\mathrm{C}_{18} \mathrm{H}_{10} \mathrm{O}_{5} \mathrm{Ag}_{2 \cdot} \cdot \mathrm{Al} \cdot 44 \\
\end{array}$ & $\begin{array}{r}\mathrm{C}_{18} \mathrm{H}_{12} \mathrm{O}_{6} \mathrm{Ag}_{2} \\
40.00 \mathrm{per}\end{array}$ & \\
\hline 1.91 & $2 \cdot 22$ & ", \\
\hline $41 \cdot 29$ & $40^{\circ} 00^{\circ}$ & ", \\
\hline $15 \cdot 35$ & $17 \cdot 77$ & " \\
\hline
\end{tabular}

This silver salt is very stable, being only slowly discoloured on standing in the air by daylight. The solution of the ammonium salt gives the following reactions:-Barium chloride precipitates the barium salt as a white amorphous mass, insoluble in water, but slightly soluble in alcohol. The calcium salt is thrown down by calcium chloride as a heavy, white, amorphous powder insoluble in water. Dried at $100^{\circ}$ and analysed, it gave the following result :-

0.2625 gram substance gave 0.0949 gram $\mathrm{CaSO}_{4}$.

$$
\begin{aligned}
& \text { Found. } \quad \overbrace{18} \mathrm{H}_{10} \mathrm{O}_{5} \mathrm{Ca} \text {. } \mathrm{C}_{18} \mathrm{H}_{12} \mathrm{O}_{6} \mathrm{Ca} \text {. } \\
& \text { Ca.... 10.63 per cent. 11.56 per cent. } 10.99 \text { per cent. }
\end{aligned}
$$

The copper salt is precipitated on the addition of cupric sulphate as a light green, almost colourless mass, which is very sparingly soluble in water. It dissolves in ammonia and sodic hydroxide solution with a blue colour.

On boiling these solutions, the salt is decomposed, copper oxide being precipitated.

Ferric chloride produces a reddish-brown precipitate, very much resembling that produced in solutions of benzoic acid or its salts.

Ferrous sulphate gives no precipitate.

On adding a solution of acetate of lead, the lead salt is thrown down as a heary white mass, almost insoluble in water.

If a solution of zinc sulphate be added to the ammonia salt, the whole remains clear for some time, but after standing the zinc salt slowly separates out as a white powder which is somewhat soluble in hot water.

The nickel salt is formed on adding a solution of sulphate of nickel as a light-green precipitate, which is soluble in ammonia, producing a beautiful blue solution.

Action of Acetic Anhydride on the Acid $\mathrm{C}_{18} \mathrm{H}_{12} \mathrm{O}_{5}$.

In order to obtain some clue as to the constitution of this acid, sevoral experiments were tried with different reagents, mostly without 
result. On heating it with acetic anhydride, however, it was found that the acid easily gave up the elements of water, being converted into an indifferent body according to the equation-

$$
\mathrm{C}_{18} \mathrm{H}_{12} \mathrm{O}_{5}=\mathrm{C}_{18} \mathrm{H}_{10} \mathrm{O}_{4}+\mathrm{H}_{2} \mathrm{O} \text {. }
$$

In order to examine this new compound, the pureacid, $\mathrm{C}_{18} \mathrm{H}_{12} \mathrm{O}_{5}$, was heated with an excess of acetic anhydride for about an hour in a flask connected with a reflux condenser. On cooling, the solution which had become slightly yellowish was diluted with water and allowed to. remain until the excess of acetic anhydride had dissolved. During this operation, a beautifully crystalline substance separated, which was collected, well washed with water, recrystallised once from acetic acid, and analysed, with the following result:-

0.1961 gram substance gave 0.0670 gram $\mathrm{H}_{2} \mathrm{O}$ and 0.5329 gram $\mathrm{CO}_{2}$.

\begin{tabular}{|c|c|c|}
\hline C. & $\begin{array}{l}\text { Found. } \\
74 \cdot 11 \text { per cent. }\end{array}$ & $\begin{array}{l}\text { Calculated. } \\
\mathrm{C}_{18} \mathrm{H}_{10} \mathrm{O}_{4} \cdot \\
74: 48 \text { per cent. }\end{array}$ \\
\hline$T$ & $3 \cdot 79$ & $3 \cdot 45$ \\
\hline & $22 \cdot 10$ & 22.07 \\
\hline
\end{tabular}

It melts at $254-255^{\circ}$ with slight decomposition. If heated carefully in a test-tube, the greater part sublimes, but considerable decomposition takes place, and a black residue is left. The sublimate is crystalline, and probably identical with that obtained by treating the acid $\mathrm{C}_{18} \mathrm{H}_{12} \mathrm{O}_{5}$ in the same way. The compound $\mathrm{C}_{18} \mathrm{H}_{10} \mathrm{O}_{4}$ is sparingly soluble in hot alcohol, yielding a solution with a most beautiful violet fluorescence very much like that exhibited by anthracence. It is nearly insoluble in cold alcohol. The solution in hot alcohol gives an orange coloration with ferric chloride. The substance crystallises beautifully from its solution in hot glacial acetic acid, which on cooling deposits it in glittering plates. It is easily soluble in hot benzene, and on adding light petroleum is precipitated in a beautifully crystalline condition. It is easily soluble in chloroform, but only sparingly in ether or bisulphide of carbon. It dissolves in concentrated sulphuric acid, yielding a yellowish-green solution which changes to a violet colour on warming.

The compound $\mathrm{C}_{18} \mathrm{H}_{10} \mathrm{O}_{4}$ is neutral, being almost insoluble in ammonia and potash solution, and apparently quite insoluble in sodic carbonate solution.

\section{Action of Potash on the Compound $\mathrm{C}_{18} \mathrm{H}_{10} \mathrm{O}_{4}$.}

If this substance is warmed with fairly concentrated alcoholic potash, it dissolves without becoming discoloured or any apparent decomposi- 
tion taking place. In order to further examine this reaction, the liquid was heated for ten minutes on a water-bath, and then diluted with water, when the whole dissolved, forming a slightly yellowish solution. On adding dilute sulphuric acid, a solid acid was precipitated, which was collected, well washed with water, dissolved in alcohol, and filtered; in this way a small quantity of unchanged $\mathrm{C}_{18} \mathrm{H}_{10} \mathrm{O}_{4}$ was easily removed. On evaporation, the filtrate deposited a solid crystalline acid, which after recrystallising from dilute acetic acid was analysed with the following result :-

0.1865 gram substance gave 0.0668 gram $\mathrm{H}_{2} \mathrm{O}$ and 0.4758 gram $\mathrm{CO}_{2}$.

\begin{tabular}{|c|c|c|}
\hline & $\begin{array}{l}\text { Found. } \\
69.58 \text { per cent. }\end{array}$ & $\begin{array}{l}\text { Calculated. } \\
\mathrm{C}_{18} \mathrm{H}_{12} \mathrm{O}_{5} \text {. } \\
70.13 \text { per cent. }\end{array}$ \\
\hline $\mathrm{H}$ & $3 \cdot 98$ & $3 \cdot 89$ \\
\hline & $26 \cdot 44$ & $25 \cdot 97$ \\
\hline
\end{tabular}

This substance was, therefore, the original acid, $\mathrm{C}_{18} \mathrm{H}_{12} \mathrm{O}_{5}$, formed from the compound $\mathrm{C}_{18} \mathrm{H}_{10} \mathrm{O}_{4}$ by the addition of water according to the equation-

$$
\mathrm{C}_{18} \mathrm{H}_{12} \mathrm{O}_{4}+\mathrm{H}_{2} \mathrm{O}=\mathrm{C}_{18} \mathrm{H}_{12} \mathrm{O}_{5} \text {. }
$$

It melted at $230-235^{\circ}$, and had all the properties of the acid obtained by the action of dilnte sulphuric acid on ethylic dibenzoylsuccinate.

\section{Constitution of the Acid $\mathrm{C}_{18} \mathrm{H}_{10} \mathrm{O}_{5}$.}

Harrow by the action of dilute sulphuric acid on ethylic diacetylsuccinate (Trans., 1878, 428) obtained carbupyrotritaric acid, $\mathrm{C}_{8} \mathrm{H}_{8} \mathrm{O}_{5}$, which from his analyses of its salts would seem to be a monobasic acid. When boiled with potash, however, it is converted into the potash salt of a bibasic acid, $\mathrm{C}_{8} \mathrm{H}_{10} \mathrm{O}_{6}$, which gives salts of the formula $\mathrm{C}_{8} \mathrm{H}_{8} \mathrm{O}_{6} \mathrm{M}_{2}$.

The acid $\mathrm{C}_{15} \mathrm{H}_{12} \mathrm{O}_{5}$ is, however, either bibasic or else very easily converted into a bibasic acid, as it gives directly salts containing two atoms of the metal; unfortunately from the analyses it is not clear whether these have the formula $\mathrm{C}_{18} \mathrm{H}_{10} \mathrm{O}_{5} \mathrm{MI}_{2}$ or $\mathrm{C}_{18} \mathrm{~N}_{12} \mathrm{O}_{6} \mathrm{M}_{2}$. It is, however, possible that these acids, $\mathrm{C}_{18} \mathrm{H}_{12} \mathrm{O}_{5}$ and $\mathrm{C}_{8} \mathrm{H}_{8} \mathrm{O}_{5}$, are in reality anhydro-acids, the former acid when dissolved in ammonia or alkalis being converted into salts of an unstable acid, $\mathrm{C}_{18} \mathrm{H}_{14} \mathrm{O}_{6}$.

Since the publication of an abstract of this research (Ber., 17, 59), the carbopyrotritaric acid has been more thoroughly examined by Knorr (Ber., 17, 2863), who considers it to be a bibasic acid, forming salts of the formula $\mathrm{C}_{8} \mathrm{H}_{6} \mathrm{O}_{5} \mathrm{Ag}_{2}$. On account of the formation of ethylic carbopyrotritarate by the action of concentrated sulphuric 
acid on ethylic diacetylsuccinate in the cold, according to the equation-

$$
\mathrm{C}_{12} \mathrm{H}_{18} \mathrm{O}_{6}-\mathrm{H}_{2} \mathrm{O}=\mathrm{C}_{12} \mathrm{H}_{16} \mathrm{O}_{5} \text {, }
$$

Knorr comes to the conclusion that carbopyrotritaric acid must have the following constitution:-

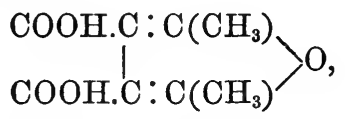

it being, in fact, dimethylfurfuranedicarboxylic acid. Since the publication of Knorr's research, I have tried the action of concentrated sulphuric acid on ethylic dibenzoylsuccinate, and found that it behaves in exactly the same way as the diacetylsuccinate, although, unfortunately, having very little material at my command, I have not been able to analyse the intermediate product corresponding with the ethylic carbopyrotritarate.

Ethylic dibenzoylsuccinate dissolves easily in concentrated sulphuric acid, the mixture becoming slightly warm and brownish coloured. If water be added to it after standing for 15 hours, an oil is precipitated which soon becomes solid. This was extracted with ether, and on allowing the solution to evaporate, a beautiful substance was obtained crystallising in long thick prisms melting at $85^{\circ}$. This is undoubtedly the diethyl salt of the acid $\mathrm{C}_{18} \mathrm{H}_{12} \mathrm{O}_{5}$, as on saponification with potash it gives the acid in a perfectly pure state, melting at $236-237^{\circ}$. In order to explain the formation of this acid, $\mathrm{C}_{18} \mathrm{H}_{12} \mathrm{O}_{5}$, it is necessary in the first place to suppose the formula of ethylic dibenzoylsuccinate transformed into an isomeric unstable modification, thus :-

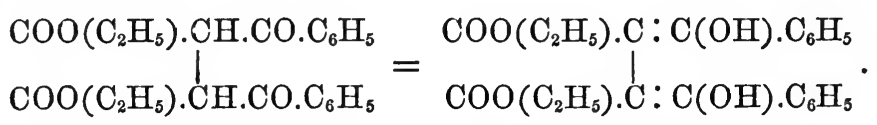

On treating this with sulphuric acid, water is eliminated, the diethyl salt of diphenylfurfuranedicarboxylic acid being formed, which therefore has the following constitution :-

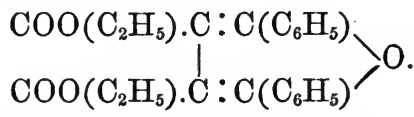

On saponification, this salt gives the acid $\mathrm{C}_{18} \mathrm{H}_{12} \mathrm{O}_{5}$, thus :-

$$
\mathrm{C}_{22} \mathrm{H}_{20} \mathrm{O}_{5}+2 \mathrm{H}_{2} \mathrm{O}=\mathrm{C}_{18} \mathrm{H}_{12} \mathrm{O}_{5}+2 \mathrm{C}_{2} \mathrm{H}_{5} . \mathrm{OH} \text {. }
$$

The acid $\mathrm{C}_{18} \mathrm{H}_{12} \mathrm{O}_{5}$ is, therefore, without doubt, in reality diphenylfurfuranedicarboxylic acid.

The compound $\mathrm{C}_{18} \mathrm{H}_{10} \mathrm{O}_{4}$, produced by the action of acetic anhydride 
on diphenylfurfuranedicarboxylic acid, is probably the anhydride of the acid, it being reconverted into the same by the action of alkalis. Its constitution would, therefore, be represented by the formula-

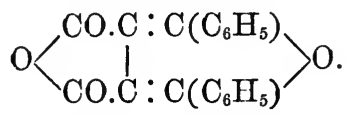

A further examination of these compounds is, however, necessary before these points can be settled.

In the paper published in the Berichte (loc. cit.), the splitting off of the water was supposed to take place between the carboxyl-groups and the hydroxyl-groups in the compound $\mathrm{COOH} . \mathrm{C}: \mathrm{C}(\mathrm{OH}) \mathrm{Ph}$

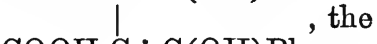
$\mathrm{COOH} . \mathrm{C}: \mathrm{C}(\mathrm{OH}) \mathrm{Ph}$ acid $\mathrm{C}_{18} \mathrm{H}_{12} \mathrm{O}_{5}$ being a mono $(\gamma)$ lactone and the compound $\mathrm{C}_{18} \mathrm{H}_{10} \mathrm{O}_{4}$ a $\operatorname{di}(\gamma)$ lactone of dibenzoylsnccinic acid, and therefore having the formulæ-

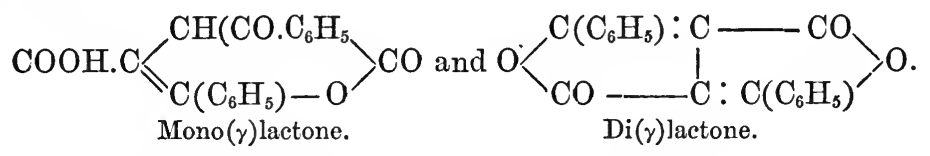

From Knorr's experiments, however, it would seem that his explanation of the formation of these bodies is the most probable, and that hey are furfurane-derivatives and not lactones.

\section{Ethylic Monobenzoylsuccinate.}

The examination of this substance was undertaken in the hope of throwing further light on the constitution of the compounds mentioned in the preceding section. If the acid $\mathrm{C}_{18} \mathrm{H}_{12} \mathrm{O}_{5}$ is the $\gamma$-lactone of dibenzoylsuccinic acid, it was thought probable that the corresponding $\%$-lactone of monobenzoylsuccinic acid might be obtained by the action of dilute sulphuric acid on ethylic monobenzoylsuccinate, thus :-

$$
\mathrm{CO}\left(\mathrm{C}_{6} \mathrm{H}_{5}\right) \cdot \mathrm{CH}<\mathrm{CH}_{2} \cdot \mathrm{COOH}
$$

Benzoylsuccinic acid.

\section{$\mathrm{C}(\mathrm{OH})\left(\mathrm{C}_{6} \mathrm{H}_{5}\right): \mathrm{C}<_{\mathrm{CH}_{2} . \mathrm{COOH}}^{\mathrm{COOH}}$}

Do. unstable form.

$$
\text { COOH.C }<{ }_{\mathrm{CH}_{2} . \mathrm{CO}}^{\mathrm{C}\left(\mathrm{C}_{6} \mathrm{H}_{5}\right)}>\mathrm{O} \text {. }
$$

$\gamma$-Lactone of benzoylsuccinic acia.

Ethylic benzoylsuccinate is easily obtained by treating the sodium compound of ethylic benzoylacetate with ethylic monochloracetate.

$\mathrm{CO}\left(\mathrm{C}_{6} \mathrm{H}_{5}\right) \cdot \mathrm{CHNa} \cdot \mathrm{COO}\left(\mathrm{C}_{6} \mathrm{H}_{5}\right)+\mathrm{CH}_{2} \mathrm{Cl} \cdot \mathrm{COO} \cdot \mathrm{C}_{2} \mathrm{H}_{5}=$ $\mathrm{CO}\left(\mathrm{C}_{6} \mathrm{H}_{3}\right) \cdot \mathrm{CH}\left(\mathrm{COO} \cdot \mathrm{C}_{2} \mathrm{H}_{5}\right) \cdot \mathrm{CH}_{2} \cdot \mathrm{COO} \cdot \mathrm{C}_{2} \mathrm{H}_{5}+\mathrm{NaCl}$. 
It was prepared as follows :- 2.5 grams of sodium were dissolved in 30 grams of absolute alcohol, and then after well cooling, 20 grams of ethylic benzoylacetate and 12 grams of ethylic monochloracetate slowly added. The reaction takes place very slowly in the cold, but rapidly on a water-bath, the mixture after heating for two hours becoming perfectly neutral. In order to isolate the product, the alcohol was distilled off, and the residue then dissolved in water; by this means a heavy oil separated out which was extracted with ether. After drying over calcium chloride and distilling off the ether, a thick brownish oil was obtained, which unfortunately could not be made to crystallise.

On trying to distil this in a vacuum, it was found that only a small quantity went over undecomposed, the principal part frothing up and charring, a quantity of gas being at the same time given off. It is, however, comparatively easy to distil small quantities of about 5 grams, if the distillation is rapidly conducted. The distillates from several such operations were united and fractioned once or twice, when an oil was obtained boiling at $260-265^{\circ}$ (160 mm.); this gave the following numbers on analysis, agreeing with the formula of ethylic benzoylsuccinate:-

0.1557 gram substance gave 0.0905 gram $\mathrm{OH}_{2}$ and $0.3695 \mathrm{CO}_{2}$.

\begin{tabular}{|c|c|c|}
\hline C. & $\begin{array}{l}\text { Found. } \\
64.72 \text { per cent. }\end{array}$ & $\begin{array}{l}\text { Theory. } \\
64.75 \text { per cent. }\end{array}$ \\
\hline & $6.46 \quad$, & $6 \cdot 47$ \\
\hline $0 \ldots$. & $28 \cdot 82$ & $28 \cdot 78$ \\
\hline
\end{tabular}

As thus obtained, it is a thick, almost colourless, oil, of an agreeable aromatic smell, somewhat resembling that of ethylic benzoate: It boils at $260-265^{\circ}$ (at $160 \mathrm{~mm}$.). The pure compound is very stable, and may be distilled at the ordinary pressure, apparently without much decomposition. It is insoluble in water, but mixes with alcohol, ether, and the usual solvents. As on examination it was found that the crude product consisted of almost pure ethylic benzoylsuccinate, and as the distillation entailed great loss of valuable material, the crude compound was used in the following reactions and decompositions, after the excess of ethylic benzoylacetate had been removed by shaking the ethereal solution several times with dilute potash solution.

On the addition of a drop of ferric chloride to the solution of ethylic benzoylsuccinate in alcohol a beautiful claret colour is produced. If heated for some hours on an oil-bath at about $250^{\circ}$, ethylic benzoylsuccinate is decomposed, lower boiling aromatic oils being formed, which, however, could not be further examined, on account of the smallness of the quantity obtained. It dissolves in concentrated 
sulphuric acid, producing a yellow solution, which becomes intensely red when heated.

It is decomposed by heating with alcoholic potash, a violet solution being produced, which on acidifying deposits a solid acid melting at $118-120^{\circ}$, probably benzoic or benzoylpropionic acid.

Like ethylic dibenzoylsuccinate, ethylic monobenzoylsuccinate gives a sodium compound when treated with sodium ethoxide.

This compound is easily prepared in the following way:- $0.3 \mathrm{gram}$ of sodium is dissolved in a small quantity of absolute alcohol, and then pure dry ether added until the whole has nearly dissolved. A solution of 3.5 grams of ethylic benzoylsuccinate in ether is then poured in, and the whole allowed to remain for some time. The mixture becomes violet coloured, and a gelatinous precipitate slowly separates, which is collected, and washed, first with a mixture of ether and alcohol, and lastly with pure ether. Dried over sulphuric acid in a vacuum, it gave the following numbers:-

0.2601 gram substance gave 0.0680 gram $\mathrm{Na}_{2} \mathrm{SO}_{4}$.

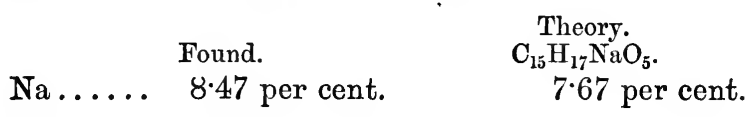

It is a hard, almost colourless, apparently amorphous mass, which is moderately soluble in alcohol. If cupric acetate is added to this solution, the copper derivative is precipitated as a pale green amorphous mass, almost insoluble in water, but soluble in sodic hydroxide, producing a blue solution. Nitrate of silver and acetate of lead give white precipitates with the alcoholic solution of the sodium derivative. The sodium compound is very stable, much more so than the disodium derivative of ethylic dibenzoylsuccinate. The powdered substance is easily decomposed by treatment with acids, a yellowish oil being precipitated, probably regenerated ethylic monobenzoylsuccinate.

\section{Decomposition Products of Ethylic Benzoylsuccinate.}

Hydrolysis with Baryta-water.-Before examining the products of the hydrolysis with dilute sulphuric acid, it appeared to be interesting to study the action of alkalis, and for this purpose, experiments were made with potash, but as this did not appear to give promising results, baryta-water was afterwards substituted for it, the method employed being the following :-

Ethylic benzoylsuccinate was heated in a flask with an excess of a concentrated solution of barium hydroxide, by which means hydrolysis rapidly set in, the reaction being complete in about an hour. During the operation the oil disappeared, without the liquid becoming 
much discoloured, and a heavy sandy precipitate of a brownish colour slowly separated. This was collected, washed with water, and decomposed with hydrochloric acid, when traces of carbonic anhydride were given off. After extracting several times with ether, a dark brown oil was obtained, which began to deposit crystals after some time. These were separated from the oily mother-liquor by extracting with chloroform, and thus obtained in a perfectly colourless state, melting at $180^{\circ}$, and having all the properties of succinic acid. In order to confirm this, the silver salt was prepared and analysed with the following result :-

0.2024 gram substance gave 0.1306 gram silver.

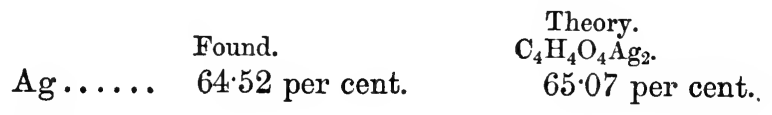

On acidifying with hydrochloric acid the filtrate from the precipitated baric succinate, it gave a quantity of a beautifully crystalline acid, which was collected, well washed, and recrystallised once or twice from water. It was thus obtained in silky needles melting at $118-120^{\circ}$, and was therefore either benzoic or benzoylpropionic acid. A silver salt was made and analysed with the following result:-

0.1976 gram substance gave 0.0936 gram silver.

$$
\begin{aligned}
& \text { Found. } \quad \mathrm{C}_{6} \mathrm{H}_{5} \cdot \mathrm{COOA} \text { Ah. } \quad \mathrm{C}_{6} \mathrm{H}_{5} \cdot \mathrm{CO}^{\text {Theory. }} \mathrm{CH}_{2} \cdot \mathrm{CH}_{2} \cdot \mathrm{COOAg} \text {. } \\
& \mathrm{Ag} \text {.... } \quad 47.37 \text { per cent. } \quad 47 \cdot 16 \text { per cent. } \quad 37.82 \text { per cent. }
\end{aligned}
$$

The acid was evidently benzoic acid.

Ethylic benzoylsuccinate is therefore decomposed by boiling with concentrated baryta water into benzoic and succinic acids, thus :$\mathrm{C}_{6} \mathrm{H}_{5} \cdot \mathrm{CO} \cdot \mathrm{CH}\left(\mathrm{COO} \cdot \mathrm{C}_{2} \mathrm{H}_{5}\right) \cdot \mathrm{CH}_{2} \cdot \mathrm{COO} \cdot \mathrm{C}_{2} \mathrm{H}_{5}+3 \mathrm{H}_{2} \mathrm{O}=\mathrm{C}_{6} \mathrm{H}_{5} \cdot \mathrm{COOH}+$
$\mathrm{C}_{2} \mathrm{H}_{4}(\mathrm{COOH})_{2}+2 \mathrm{C}_{2} \mathrm{H}_{5} \cdot \mathrm{OH}$.

In this respect it differs somewhat from ethylic acetylsuccinate, which on hydrolysis with dilute baryta-water, is spiit up into acetylpropionic acid and carbonic anhydride, only a small quantity of acetic acid and succinic acid being formed (Conrad, Annalen, 118, 218).

Ethylic benzoylsuccinate, if treated with dilute baryta-water, might also be split up in this way into benzoylpropionic acid and carbonic anhydride.

Hydrolysis with Dilute Sulphuric Acid.-Ethylic benzoylsuccinate was heated with a large excess of dilute sulphuric acid (1:2) in a flask connected with a reflux condenser, at the end of which a test-tube containing baryta-water was attached. The oil became 
more and more darkly coloured, and large quantities of carbonic anhydride were given off, until at the end of about 15 hours most of the ethereal salt had dissolved, a small quantity of a tarr'y substance being left. On cooling, the liquid became filled with glittering crystals, which were collected, well washed with water, roughly separated from oily mother-liquor by spreading out on a porous plate, and then recrystallised once or twice from water, animal charcoal being used to decolorise the liquid. In this way, the body was obtained in glittering plates melting at $117^{\circ}$, and closely resembling benzoic acid. The analysis, however, showed the body to be benzoylpropionic acid.

0.2230 gram substance gave 0.1132 gram $\mathrm{OH}_{2}$ and 0.5487 gram $\mathrm{CO}_{2}$.

\begin{tabular}{|c|c|c|}
\hline & $\begin{array}{l}\text { Found. } \\
67 \cdot 12 \text { per cent. }\end{array}$ & $\begin{array}{l}\text { Theory. } \\
\mathrm{C}_{10} \mathrm{H}_{10} \mathrm{O}_{3} \text {. } \\
67 \cdot 41 \text { per cent. }\end{array}$ \\
\hline & $5 \cdot 64 \quad$, & $5 \cdot 62 \quad$ \\
\hline & $27 \cdot 24$ & $26 \cdot 97$ \\
\hline
\end{tabular}

Ethylic benzoylsuccinate splits up, therefore, on boiling with dilute sulphuric acid into benzoylpropionic acid and carbonic anhydride according to the equation-

$$
\begin{gathered}
\mathrm{CO}\left(\mathrm{C}_{6} \mathrm{H}_{5}\right) \cdot \mathrm{CH}\left(\mathrm{COO} \cdot \mathrm{C}_{2} \mathrm{H}_{5}\right) \cdot \mathrm{CH}_{2} \cdot \mathrm{COO} \cdot \mathrm{C}_{2} \mathrm{H}_{5}+2 \mathrm{H}_{2} \mathrm{O}= \\
\mathrm{CO} \cdot \mathrm{C}_{2} \mathrm{H}_{5} \cdot \mathrm{CH}_{2} \cdot \mathrm{CH}_{2} \cdot \mathrm{COOH}+\mathrm{CO}_{2}+2 \mathrm{C}_{2} \mathrm{H}_{5} \cdot \mathrm{OH} .
\end{gathered}
$$

Benzoylpropionic acid has already been obtained (1) by the action of aluminic chloride on a mixture of succinic anhydride and benzene (Burcker, Bull. Soc. Chim., 35, 17), and by the reduction of benzoylacrylic acid (Pechmann, Ber., 14, 889). The acid obtained from ethylic benzoylsuccinate agrees with that produced by the above two reactions in every respect.

By modifying the conditions in the hydrolysis of ethylic benzoylsuccinate with dilute.sulphuric acid, the same results were always obtained, no lactone ever being formed. The reason for this is that the $\mathrm{C}_{2} \mathrm{H}_{5}$ group of the COO. $\mathrm{C}_{2} \mathrm{H}_{5}$, which is joined to the $\mathrm{C}_{6} \mathrm{H}_{5} . \mathrm{CO} . \mathrm{CH}$, in benzoylsuccinic ether, is always split off first, forming the unstable acid $\mathrm{C}_{6} \mathrm{H}_{5}$. CO.CH(COOH). $\mathrm{CH}_{2}$. COO. $\mathrm{C}_{2} \mathrm{H}_{5}$, which then naturally loses carbonic anhydride, and is converted into ethylic benzoylpropionate, this being then further hydrolysed by the action of the sulphuric acid. Analogous results were obtained by Conrad (Ber., 11, 2177) by the action of dilute sulphuric acid on ethylic acetylsuccinate, acetylpropionic acid, ethylic acetylpropionate and carbonic anhydride being the products of the reaction. 


\section{Dehydrobenzoylacetic Acid.}

Among the many reactions in which ethylic acetoacetate takes part, there are few more interesting than the condensation which this body undergoes on prolonged boiling; by this treatment, 2 molecules appear to combine, with elimination of 2 molecules of alcohol and formation of dehydracetic acid, according to the equation-

$$
2 \mathrm{CH}_{3} \text {.CO. } \mathrm{CH}_{2} \text {. COO. } \mathrm{C}_{2} \mathrm{H}_{5}=\mathrm{C}_{8} \mathrm{H}_{8} \mathrm{O}_{4}+2 \mathrm{EtOH} \text {. }
$$

This acid, the constitution of which, in spite of many experiments, still remains to be explained, was first obtained by Geuther (Zeit. $f$. Chem., 1866,8) by passing carbonic anhydride over the heated sodium derivative of ethylic acetoacetate. During the reaction, a quantity of ethylic acetoacetate distils over, and the sodium salt of dehydracetic acid remains in the retort. On dissolving the latter in water and acidifying with dilute sulphuric acid, the acid is set free, and by repeated recrystallisation from water can easily be obtained pure in colourless leafy crystals melting at $109^{\circ}$.

Geuther proved this compound to be a monobasic acid, forming salts of the formula $\mathrm{C}_{8} \mathrm{H}_{7} \mathrm{O}_{4} \mathrm{M}$. At a more recent date, Oppenheim and Precht (Ber., 9, 324, 1100) undertook the further investigation of the acid, and succeeded in preparing a number of derivatives, which they carefully examined, and from the results proposed the following as the most probable constitutional formula :-

$$
\mathrm{CH}_{3} \text {.CO.CH.C }<\underset{\mathrm{C}(\mathrm{OH})}{\mathrm{C}(\mathrm{COOH})} \geqslant \mathrm{CH} \text {. }
$$

This formula presupposes that, in the formation of this acid from 2 molecules of the acetoacetate, the methyl-group of one molecule takes part in the reaction, whereas the methyl-group of the second molecule remains intact. In order to test the accuracy of this formula, it appeared interesting to determine whether it would be possible under the same conditions to obtain a similar body from ethylic benzoylacetate, in which case the two phengl-groups would be excluded from taking part in the condensation.

The constitation of an aromatic compound of this kind would probably be much easier to determine, and if it were found that its derivatives behaved in a way exactly analogous to those of dehydracetic acid itself, it would be legitimate to suppose that the methyl-groups of the two molecules of ethylic acetoacetate which went to form dehydracetic acid did not take part in the reaction.

In the first experiments on ethylic benzoylacetate, it was noticed that on distilling this body rapidly a great part went over as a colourless oil, a considerable quantity of a black tarry mass being left 
behind, partly soluble in carbonate of soda solution on continued agitation. On filtering and acidifying the alkaline solution, an acid was deposited in the form of brownish flakes; this by repeated recrystallisation from alcohol was obtained pure in the form of long yellow needles. As it was thought possible that this might be the desired acid, numerous experiments were made in order to find some means of obtaining it in larger quantity. The following process was found to give the best results :-

Ethylic benzoylacetate was first heated to boiling in portions of $10-15$ grams in test-tubes or small retorts for $7-8$ minutes, during which operation the mass gradually became darker, whilst a quantity of lower boiling oil was given off; this was collected for further examination. On cooling, the residue generally solidified to a brownish cake of crystals. The distillate from a number of such operations was first examined. On fractioning, the principal portion passed over between $70^{\circ}$ and $110^{\circ}$, then the thermometer rose rapidly to $180^{\circ}$, between which temperature and $210^{\circ}$ a considerable quantity distilled; a small residue was left, which appeared to consist in great part of unchanged ethylic benzoylacetate. The fraction $70-110^{\circ}$, which had a strongly acid reaction, was mixed with dry carbonate of potash, and after remaining for some time, distilled on a water-bath. On further fractioning, the distillate went over almost to the last drop between 78 and $80^{\circ}$, and possessed all the properties of common alcohol. The residual potash salt was then acidulated with dilute sulphoric acid and distilled in steam; a strongly acid liquid passed over, which showed all the reactions of acetic acid. The fraction $70-110^{\circ}$ was therefore a mixture of alcohol and acetic acid.

On refractioning the fraction $180-210^{\circ}$, nearly the whole distilled over between $190-200^{\circ}$, and was from its odour easily recognised as acetophenone. This was confirmed by warming it with a solution of orthonitrobenzaldehyde in dilute sodic hydroxide, when a copious precipitate of indigo was slowly formed.

The solid residue from these experiments was then boiled for a considerable time with alcohol and animal charcoal, and filtered, the new acid crystallising out in long yellow needles on cooling. On distilling off the alcohol from the mother-liquor of these crystals, it deposited a quantity of a dark brown oil; on heating this again in the way described above, it gave a new quantity of acid. This treatment was repeated until the residual oil on further heating no longer solidified on cooling, but remained behind as a blackish tarry mass, which, however, still contained considerable quantities of the acid. In order to extract this, the whole was dissolved in a little alcohol, enough potash added to neutralise the acid, the product diluted with water, and several times extracted with ether. On acidifying the solution of the 
potash salt, the acid was deposited in a crude state, and was treated. with alcohol and animal charcoal as described above.

The different crops of crystals were then mixed together, crystallised several times from alcohol, and thus obtained pure. The analysis gave the following numbers:-

I. 0.1879 gram substance gave 0.0715 gram $\mathrm{H}_{2} \mathrm{O}$ and 0.5082 gram $\mathrm{CO}_{2}$.

II. 0.2577 gram substance gave 0.0958 gram $\mathrm{H}_{2} \mathrm{O}$ and 0.6978 gram $\mathrm{CO}_{2}$.

III. 0.1941 gram substance gave 0.0746 gram $\mathrm{H}_{2} \mathrm{O}$ and 0.5241 gram $\mathrm{CO}_{2}$.

IV. 0.1974 gram substance gave 0.0740 gram $\mathrm{H}_{2} \mathrm{O}$ and 0.5319 gram $\mathrm{CO}_{2}$.

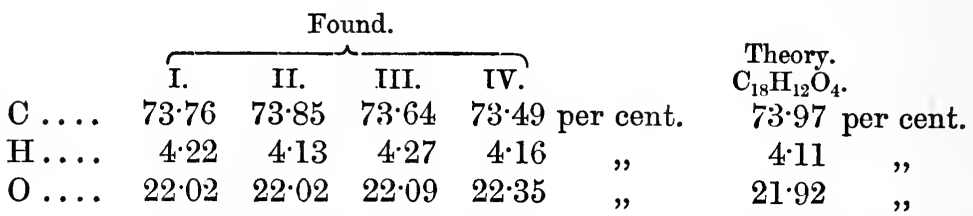

This substance, for which I propose the name dehydrobenzoylacetic acid, has therefore the formula $\mathrm{C}_{18} \mathrm{H}_{12} \mathrm{O}_{4}$, and is formed from 2 molecules of ethylic benzoylacetate in a way exactly analogous to the formation of dehydracetic acid from ethylic acetoacetate, according to the equation-

$$
2 \mathrm{C}_{6} \mathrm{H}_{5} \cdot \mathrm{CO} \cdot \mathrm{CH}_{2} \cdot \mathrm{COO} \cdot \mathrm{C}_{2} \mathrm{H}_{5}=\mathrm{C}_{18} \mathrm{H}_{12} \mathrm{O}_{4}+2 \mathrm{C}_{2} \mathrm{H}_{5} \cdot \mathrm{OH} \text {. }
$$

Ethylic benzoylacetate. Dehydrobenzoylacetic acid.

This acid crystallises from its solution in hot alcohol in long yellow needles which melt at $171-172^{\circ}$. It is moderately soluble in hot alcohol, but only sparingly in the cold. On the addition of a drop of ferric chloride to the hot alcoholic solution, it gives a deep orange-red coloration. Dehydrobenzoylacetic acid dissolves easily in benzene, chloroform, bisulphide of carbon, and ether, but sparingly so in light petroleum. On evaporating these solutions, the acid crystallises out beautifully in long yellow needles. The solution in bisulphide of carbon does not decolorise bromine in the cold, and only very slowly on boiling, hydrogen bromide being given off. This behaviour is exactly analogous to that of dehydracetic acid, which, when treated with bromine, forms a substitution-product, $\mathrm{C}_{8} \mathrm{H}_{7} \mathrm{O}_{4} \mathrm{Br}$, and not an additive product, as might be expected, considering the small percentage of hydrngen which the acid contains.

Dehydrobenzoylacetic acid dissolves in concentrated sulphuric acid with an olive-green colour; on warming, a beautiful violet solution is 
obtained, which, when examined with the spectroscope, gives exactly the same absorption-spectrum as indigo.

The acid is not acted on, even by prolonged heating with acetic anhydride, being precipitated unchanged on adding water, a proof that the dehydrobenzoylacetic acid contains no hydroxyl-groups.

The acid chars when submitted to dry distillation, a quantity of gas being given off; but if heated with soda-lime, acetophenone is formed.

Dehydrobenzoylacetic acid readily combines with phenylhydrazine, forming an intensely yellow compound, which is difficult to purify, and has not as yet been analysed. The pure acid dissolves easily in ammonia, soda, and sodic carbonate, forming yellow solutions.

The ammonium salt was obtained in the usual way, by allowing the solution of the acid in ammonia to stand over sulphuric acid in a vacuum until the excess of alkali had been removed. The following salts were prepared by precipitation:-Silver nitrate canses the precipitation of the silver salt as a yellowish-white flocculent mass, which is very stable and only slowly acted on by light. It was collected, well washed with water, dried at $100^{\circ}$, and analysed with the following result :-

0.2949 gram substance gave 0.0818 gram $\mathrm{H}_{2} \mathrm{O}, 0.5874$ gram $\mathrm{CO}_{2}$, and 0.0786 gram silver.

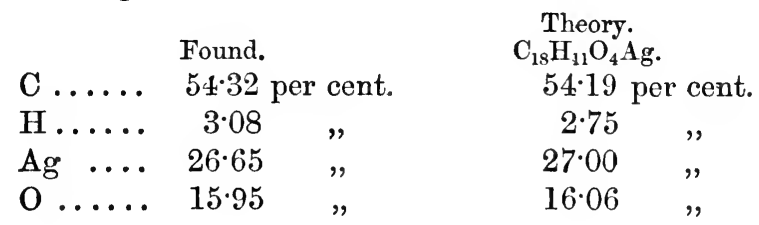

Dehydrobenzoylacetic acid is therefore a monobasic acid. The ferric salt is thrown down as a beautiful scarlet precipitate on adding ferric chloride to a solution of the ammonium salt. Ferrous sulphate, on the contrary, gives a blackish-violet precipitate, the behaviour with these two reagents being a very characteristic reaction for the acid. The copper salt is obtained, on adding cupric sulphate, as a light greenish precipitate, soluble in ammonia. The lead, calcium, barium, zinc, and tin salts are heavy white precipitates, almost insoluble in water. Nickel sulphate gives, with a solution of the ammonium salt, a light green almost colourless precipitate, soluble in ammonia. All these salts are extremely stable.

If ethylic benzoylacetate is boiled as described above for about eight minutes, the only condensation-product formed in any quantity is dehydrobenzoylacetic acid. If, however, the boiling is continued 
for a longer time, the reaction becomes more and more complicated, higher condensation-products being formed.

In order to study these, and, if possible, to determine their constitution, the ethlic benzoylacetate was heated in small retorts in quantities of from 10 to 15 grams for about half an hour, during which time alcohol and acetophenone were given off continuously and fairly regularly. The black tarry residue, which became semi-solid on cooling, was found to contain at least two condensation-products besides dehydrobenzoylacetic acid; these were separated as follows :In order to remove the dehydrobenzoylacetic acid the mass was first treated with a moderately concentrated solution of ammonia and allowed to stand, the whole being well stirred from time to time. After filtering off the aqueous solution of the ammonium salt, the residue was well washed with water, and then several times extracted with hot alcohol; this removed all the colouring matter, and left a yellowish amorphous mass, which will be described further on.

The dark brown alcoholic solution was digested for a long time with animal charcoal, filtered, and the bulk of the alcohol distilled off. On cooling, a beautifully crystalline body separated, which was purified by repeated recrystallisation.

The analysis gave numbers agreeing with the formula $\left(\mathrm{C}_{9} \mathrm{H}_{6} \mathrm{O}_{2}\right)_{n}$.

I. 0.2343 gram substance gave 0.0941 gram $\mathrm{H}_{2} \mathrm{O}$ and 0.6374 gram $\mathrm{CO}_{2}$.

II. $0 \cdot 1746$ gram substance gave $0 \cdot 0709$ gram $\mathrm{H}_{2} \mathrm{O}$ and 0.4722 gram $\mathrm{CO}_{2}$.

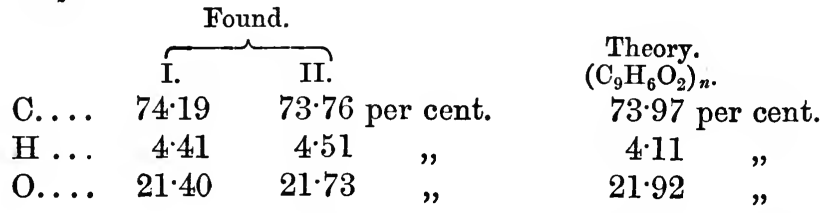

This compound probably has the formula $\mathrm{C}_{27} \mathrm{H}_{18} \mathrm{O}_{6}$; being formed from 3 mols. of ethylic benzoylacetate by the elimination of 3 mols. of alcohol, according to the equation-

$$
3 \mathrm{C}_{6} \mathrm{H}_{5} \cdot \mathrm{CO} \cdot \mathrm{CH}_{2} \text {. COO. } \mathrm{C}_{2} \mathrm{H}_{5}=\mathrm{C}_{27} \mathrm{H}_{18} \mathrm{O}_{6}+3 \mathrm{C}_{2} \mathrm{H}_{5} \text {. OH. }
$$

This substance melts at $273-275^{\circ}$. It is sparingly soluble in cold alcohol, more easily in hot, and crystallises on cooling in beautiful silky plates, which strongly resemble anthracene, and give a beautiful play of colours with polarised light. It is almost insoluble in benzene, light petroleum, and acetone. In ether and carbon bisulphide, it is somewhat soluble, and on evaporation crystallises in glittering plates. The solution in carbon bisulphide is only very slowly acted on by bromine, hydrogen bromide being given off. On adding an 
ethereal solution of sodium ethoxide to the solution of the substance in warm ether, a voluminous precipitate of a sodium compound is thrown down; this is decomposed by treatment with acids with regeneration of the original compound.

This substance, however, is not a true acid. It dissolves easily in dilute alcoholic soda or sodium ethoxide, but is entirely reprecipitated on passing carbonic anhydride, so that in this case a sodium compound, corresponding with that of ethylic benzoylacetate is formed, and not the sodium salt of an acid. If the solution in alcoholic potash is allowed to remain for some time, the compound is slowly but completely decomposed. On diluting the product with water, and acidifying with dilute sulphuric acid, an acid is precipitated which has all the properties of benzoylacetic acid. It melts at $80-85^{\circ}$, gives the characteristic violet coloration with ferric chloride, and is decomposed into carbonic anhydride and acetophenone on heating. This acid appears to be produced almost quantitatively by the action of potash on the substance $\mathrm{C}_{27} \mathrm{H}_{18} \mathrm{O}_{6}$.

If it is boiled with alcoholic potash, benzoic acid, acetophenone, and other bodies are formed. Heated in a test-tube, the compound $\mathrm{C}_{27} \mathrm{H}_{18} \mathrm{O}_{6}$ sublimes partly unchanged, but most of it is decomposed, with separation of oily bodies, probably acetophenone. When distilled with zinc-dust, a light boiling body is given off, which burns with a strongly luminous flame, and appears to be benzene. It is not decomposed by boiling with dilute sulphuric acid. It dissolves in concentrated sulphuric acid with a faint yellowish colour, which is not perceptibly changed on warming. It is easily attacked by hot concentrated nitric acid, and on adding water to the product a yellowish mass is precipitated, which crystallises beautifully from alcohol, and is probably a nitro-derivative. On warming the compound $\mathrm{C}_{27} \mathrm{H}_{18} \mathrm{O}_{6}$ with phenylhydrazine, it yields an intensely yellow body, which, however, was not further examined.

The yellow amorphous compound, mentioned above (p. 281) as remaining when the compound $\mathrm{C}_{27} \mathrm{H}_{18} \mathrm{O}_{6}$ was dissolved by treating the crude product with alcohol, was then further examined.

This substance melts above $300^{\circ}$. It is insoluble in most of the usnal solvents, and can therefore be easily separated from impurities by repeated extraction with them. It is thus obtained as a light yellow amorphous mass, which gave the following numbers on analysis :-

I. 0.1243 gram substance gave 0.04 .60 gram $\mathrm{H}_{2} \mathrm{O}$ and 0.3380 gram $\mathrm{CO}_{2}$.

II. 0.1556 gram substance gave 0.0586 gram $\mathrm{H}_{2} \mathrm{O}$ and 0.4239 gram $\mathrm{CO}_{2}$. 
Found.

\begin{tabular}{|c|c|c|c|c|c|c|}
\hline & I. & & II. & & \multirow{2}{*}{\multicolumn{2}{|c|}{$\begin{array}{l}\text { Theory. } \\
\left(\mathrm{C}_{9} \mathrm{H}_{6} \mathrm{O}_{2}\right)_{n} \text {. } \\
73397 \text { per cent }\end{array}$}} \\
\hline & $74: 16$ & cent. & $74: 29$ & r cent. & & \\
\hline & $4 \cdot 11$ & $"$ & $4 \cdot 18$ & " & $4 \cdot 11$ & " \\
\hline & $21 \cdot 73$ & $"$ & $21 \cdot 53$ & , & 21.92 & " \\
\hline
\end{tabular}

The compound appears to be homogeneous, the above analyses having been made with two distinct preparations. It is possible that it may have the formula $\mathrm{C}_{36} \mathrm{H}_{24} \mathrm{O}_{8}$, being a condensation-product of $4 \mathrm{mols}$. of ethylic benzoylacetate, $4 \mathrm{C}_{6} \mathrm{H}_{5} \cdot \mathrm{CO} \cdot \mathrm{CH}_{2} \cdot \mathrm{COO} \cdot \mathrm{C}_{2} \mathrm{H}_{5}=\mathrm{C}_{36} \mathrm{H}_{24} \mathrm{O}_{8}+$ $4 \mathrm{C}_{2} \mathrm{H}_{5} . \mathrm{OH}$. It is an acid, and dissolves easily in sodium ethoxide, being reprecipitated by dilute sulphuric acid, but not by carbonic anhydride. It is also partially soluble in ammonia. On boiling it with sodium ethoxide, a deep red solution is formed; this, when acidified, deposits a small quantity of an acid, the alcoholic solution of which gives a violet coloration with ferric chloride. The following is an extremely characteristic reaction or this substance. If the merest trace of it is mixed with concentrated sulphuric acid, it dissolves to a yellowish solution; and on warming this the colour changes slowly to a reddishbrown and then to a reddish-violet. If this solntion be further heated, a most intense bluish-violet coloration is produced, which becomes purple on strongly heating. The bluish-violet coloration is most beautiful, and strongly resembles methylene-blue. The colour disappears on diluting with water.

Owing to the difficulty of obtaining any quantity of these last two condensation-products, I have not examined them further, but confined my attention to the dehydrobenzoylacetic acid.

\section{Ethylic Dehydrobenzoylacetate.}

In order to prepare this ethereal salt, silver dehydrobenzoylacetate was digested on a water-bath for two hours with excess of ethyl iodide and a little dry ether; in this way the salt was completely decomposed. After collecting and extracting the precipitated iodide of silver several times with ether, and evaporating the ethereal solution, a semi-solid mass was obtained, which was first spread on a porous plate to free the crystals from oily matter, and then several times recrystallised from benzene. When analysed, it gave the following results :-

$0 \cdot 1843$ gram of substance gave 0.0858 gram $\mathrm{H}_{2} \mathrm{O}$ and 0.5050 gram $\mathrm{CO}_{2}$.

Found.

$\begin{array}{rrr}\text { C . . . . . } & 74.73 & \text { per cent. } \\ \text { H ..... } & 5 \cdot 17 \\ \text { O ..... } & 20.00 \quad,\end{array}$

$\begin{array}{rr}\text { H.... } & 5 \cdot 17 \\ \text { O.... } & 20 \cdot 00\end{array}$

Theory. $\mathrm{C}_{17} \mathrm{H}_{11} \mathrm{O}_{2} \cdot \mathrm{COOC}_{2} \mathrm{H}_{5}$. 75.00 per cent.

$5 \cdot 20$, 20.00, 
This compound is, therefore, ethylic dehydrobenzoylacetate. It crystallises from benzene in almost colourless glittering needles which melt at $159^{\circ}$, and show a beautiful play of colours when examined with the polariscope. If carefully heated, it appears to distil almost withont decomposition.

It is moderately soluble in alcohol, acetone, benzene, and bisulphide of carbon, more sparingly in ether and light petroleum. The solution in alcohol gives a reddish-brown coloration on the addition of a drop of ferric chloride. Warmed with alcoholic potash, it is easily saponified, dehydrobenzoylacetic acid being generated.

Its alcoholic solution when mixed with phenylhydrazine yields an intensely yellow compound which, however, has not yet been obtained pure enough for analysis ; its formation is, however, a proof that the dehydrobenzoylacetic acid contains at least one carbonyl-group.

If an ethereal solution of ethylic dehydrobenzoylacetate is treated with an ethereal solution of sodic ethylate, an intensely yellow sodium compound is precipitated; but, unfortunately, ethylic dehydrobenzoylacetate is so sparingly soluble in ether, that it was found impossible to obtain the compound in quantity sufficient for analysis.

In the course of some experiments on dehydracetic acid, the results of which I hope to have the honour of laying before the Society at some future time, it was found that the methyl salt of this acid possesses the property of forming a sodium compound of the formula $\mathrm{C}_{8} \mathrm{H}_{6} \mathrm{O}_{4} \mathrm{CH}_{3} \mathrm{Na}$, in which one hydrogen of the methyl compound is directly replaced by sodium.

It is extremely probable, therefore, that the sodium compound of ethylic dehydrobenzoylacetate is similarly constituted.

\section{Action of Potassic Hydroxide on Dehydrobenzoylacetic Acid.}

Dehydracetic acid is, as is well known, easily split up by heating with alkalis, into acetone, acetic acid, and carbonic anhydride, according to the equation-

$$
\mathrm{C}_{8} \mathrm{H}_{8} \mathrm{O}_{4}+3 \mathrm{H}_{2} \mathrm{O}=\mathrm{CH}_{3} \cdot \mathrm{CO} \cdot \mathrm{CH}_{3}+2 \mathrm{CH}_{3} \cdot \mathrm{COOH}+\mathrm{CO}_{2} \text {. }
$$

It appeared likely, therefore, that by treating dehydrobenzoylacetic acid in the same way, similar decomposition-products would be produced; thus affording evidence that this acid and dehydracetic acid were analogous compounds.

In order to test this, the action of potash on dehydrobenzoylacetic acid was carefully studied in the following way:-

The pure acid was first dissolved in dilute alcoholic potash, and after an excess of a concentrated solution of the latter had been added, the whole was heated to boiling on a water-bath for several hours. 
During the reaction the mass became gradually darker; when finished, the smell of acetophenone was easily recognisable.

In order to isolate the products, the alcohol was first slowly distilled off, water was then added, and the whole distilled in a current of steam. The distillate, which smelt strongly of acetophenone, contained small particles of oil, which were separated from the watery liquid by shaking with ether. On evaporating the ether, a colourless oil remained, which, on fractioning, went over entirely between 195$200^{\circ}$, and was undoubtedly acetophenone. This was confirmed by shaking it with a solution of orthonitrobenzaldehyde in dilute soda; in a short time an indigo-blue precipitate separated in considerable quantity.

The alkaline solution which was freed from the acetophenone in the way above described was acidified with dilute sulphuric acid, and again distilled, when an acid liquid passed over which smelt distinctly of acetic acid; the quantity, however, was so small that an analysis could not be performed. The residne in the retort was then several times extracted with ether; and on evaporating the solution, a darkbrown semi-solid acid was obtained. This was first separated from oily matter by spreading it out on a porous plate, and then recrystallised from water. In this way, it was obtained in colourless needles melting at $118-120^{\circ}$, and possessing all the properties of benzoic acid, with which it was undoubtedly identical.

Dehydrobenzoylacetic acid is therefore decomposed on heating with alcoholic potash into acetophenone, acetic acid, benzoic acid, and carbonic anhydride, according to the equation-

\section{$\mathrm{C}_{18} \mathrm{H}_{12} \mathrm{O}_{4}+3 \mathrm{H}_{2} \mathrm{O}=\mathrm{C}_{6} \mathrm{H}_{5} \cdot \mathrm{CO} \cdot \mathrm{CH}_{3}+\mathrm{CH}_{3} \cdot \mathrm{COOH}+\mathrm{C}_{6} \mathrm{H}_{5} \cdot \mathrm{COOH}$ $+\mathrm{CO}_{2}$.}

The reaction is perfectly analogous to the formation of 2 mols. of acetic acid and one of acetone by the action of potash on dehydracetic acid.

In the course of these experiments, it was noticed that when the boiling with potash was continued for a short time only, the benzoic acid obtained was always mixed with a certain quantity of another acid, the alcoholic solution of which gave a violet coloration with ferric chloride, strongly resembling that produced by benzoylacetic acid. In order, if possible, to obtain this acid in larger quantity, the action of potash on dehydrobenzoylacetic acid was tried in the cold, and it was found that it was completely decomposed if mixed with a concentrated solution of alcoholic potash, and allowed to remain for 12 hours. The mass became dark reddish-brown, and in a short time a considerable quantity of a potash salt crystallised out in glittering plates. On adding water, the whole dissolrcd, forming a reddish. 
brown solution, which smelt only very faintly of acetophenone. In order to isolate the products of the reaction, the alkaline liquid was well cooled, acidified with dilute sulphuric acid, and once or twice extracted with ether. On allowing the ethereal solution to eraporate, a quantity of a dark-brownish acid was left, which was purified from oily matter by spreading it out on a porous plate, and thus obtained as a slightly yellowish, hard, crystalline mass. This was dissolved in dilute ammonia, well shaken with pure animal charcoal, and filtered. In this way an almost colourless solution of the ammonium salt was obtained, which was acidified with dilute sulphuric acid and extracted with ether. The ethereal solution, on evaporation, deposited a faintly yellowish-coloured crystalline acid; this after drying over sulphuric acid in a vacuum, gave the following numbers on analysis :-

0.1796 gram of substance gave 0.0841 gram $\mathrm{H}_{2} \mathrm{O}$ and 0.432 gram $\mathrm{CO}_{2}$.

\begin{tabular}{|c|c|c|c|}
\hline & $\begin{array}{l}\text { Found. } \\
65.62 \text { per cent. }\end{array}$ & . & $\begin{array}{l}\text { Theory. } \\
\mathrm{C}_{9} \mathrm{H}_{8} \mathrm{O}_{3} \text {. } \\
65.85 \text { per cent. }\end{array}$ \\
\hline $\mathrm{H} .$. & $5 \cdot 23 \quad$ & & $4 \cdot 88$ \\
\hline O. & $29 \cdot 15$ & & $29 \cdot 27$ \\
\hline
\end{tabular}

This acid has, therefore, the same constitution as benzoylacetic acid, and is undoubtedly identical with it. It melted at $83-85^{\circ}$ (after recrystallising from benzene at $98-100^{\circ}$ ), and exhibited all the properties of benzoylacetic acid. The solution in alcohol gives an intense violet coloration with ferric chloride, and the free acid, when distilled, is split up into acetophenone and carbonic anhydride. In order to be certain that the acid obtained from the dehydrobenzoylacetic acid really had the constitution $\mathrm{C}_{9} \mathrm{H}_{8} \mathrm{O}_{3}$, it was converted into the silver salt, and analysed with the following result:-

$0 \cdot 1463$ gram substance gave 0.0592 gram silver.

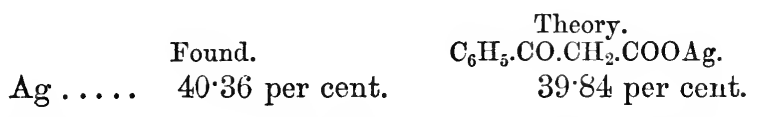

There is, therefore, no doubt that dehydrobenzoylacetic acid, when treated with alcoholic potash in the cold, is converted into benzoylacetic acid according to the equation-

$$
\mathrm{C}_{18} \mathrm{H}_{12} \mathrm{O}_{4}+2 \mathrm{H}_{2} \mathrm{O}=2 \mathrm{C}_{6} \mathrm{H}_{5} \cdot \mathrm{CO} \cdot \mathrm{CH}_{2} \cdot \mathrm{COOH} \text {. }
$$

This reaction is exactly the reverse of its formation from 2 mols. of ethylic benzoylacetate.

In order to determine whether this decomposition really did take 
place according to the above reaction, some quantitative experiments were made, and the amount of benzoylacetic acid produced weighed. 3 grams of pure dehydrobenzoylacetic acid were mixed with a concentrated solution of alcoholic potash, and allowed to stand at the ordinary temperature until a very faint smell of acetophenone was perceptible. The product was then treated as described above. After evaporating the ethereal extract of the acid formed in a weighed dish, 3.15 grams of crude product were obtained (calculated for the above equation 3.3 grams). After spreading it out on a porous plate, and thus removing a trace of oily matter, the almost colourless acid left weighed nearly 3 grams. It was then treated with ammonia and animal charcoal, and gave $2 \cdot 8$ grams of nearly pure benzoylacetic acid.

In order to be sure that this acid was benzoylacetic acid, it was heated in a retort until the whole was decomposed, and the resulting oil dissolved in ether and shaken with a dilute solution of sodic hydroxide. On acidifying the alkaline solution and extracting with ether, a very small quantity of benzoic acid was obtained. The neutral oil formed by the decomposition showed the boiling point of $195^{\circ}$, and was undoubtedly acetophenone. It is probable therefore that on boiling dehydrobenzylacetic acid with potash benzoylacetic acid is first formed, which is then further decomposed into benzoic acid, acetic acid, acetophenone, and carbonic anhydride.

\section{Reduction of Dehydrobenzoylacetic Acid.}

Dehydrobenzoylacetic acid, $\mathrm{C}_{18} \mathrm{H}_{12} \mathrm{O}_{4}$, besides the carboxyl-group, contains 2 atoms of hydrogen, one of which at least must be present in the ketone form, $\mathrm{CO}$, from the fact that the acid combines with phenylhydrazine. This phenylhydrazine compound could be obtained crystalline only with difficulty, and then in such small quantity that a complete analysis could not be made, but only a nitrogen determination, which seemed to show that the body had the formula $\mathrm{C}_{16} \mathrm{H}_{12} \mathrm{O}_{3} . \mathrm{N}_{2} \mathrm{H} . \mathrm{C}_{6} \mathrm{H}_{5}, \mathrm{I}$ mol. of the acid combining with 1 mol. of phenylhydrazine. In order to obtain some idea as to the condition of the fourth oxygen-atom in the molecule $\mathrm{C}_{18} \mathrm{H}_{12} \mathrm{O}_{4}$, it was next treated with reducing agents under different conditions, the best results being obtained with sodium amalgam in the following way.

Dehydrobenzoylacetic acid was dissolved in dilute sodic hydroxide and an excess of liquid sodium amalgam slowly added, the whole being well cooled during the operation, and the product allowed to stand for 24 hours in order to ensure complete reduction.

At first no evolution of hydrogen was perceptible, but towards the end of the reaction a quantity of gas was given off and a small 
amount of a resinous body formed, which was filtered off from the alkaline solution, but was too small for further examination. On acidifying the filtrate with dilute sulphuric acid, it deposited an almost colourless, amorphous acid, which was collected, well washed with water, and spread out on a porous plate to free it from traces of oily matter. This crude product was dissolved in a little boiling benzene and then light petroleum boiling at $70-80^{\circ}$ added antil a slight turbidity was produced. On standing, this solution deposited a quantity of a new acid in colourless plates, which were obtained pure by recrystallisation from benzene and light petroleum.

The analysis gave the following numbers :-

I. $0 \cdot 1665$ gram substance gave $0 \cdot 0761$ gram $\mathrm{H}_{2} \mathrm{O}$ and $0 \cdot 4750$ gram $\mathrm{CO}_{2}$.

II. 0.1485 gram substance gave 0.0669 gram $\mathrm{H}_{2} \mathrm{O}$ and 0.4247 gram $\mathrm{CO}_{2}$.

III. 0.1782 gram substance gave 0.0723 gram $\mathrm{H}_{2} \mathrm{O}$ and 0.5097 gram $\mathrm{CO}_{2}$.

\begin{tabular}{|c|c|c|c|c|c|c|}
\hline \multirow[b]{3}{*}{ C .... } & \multicolumn{3}{|c|}{ Found. } & - & \multirow{2}{*}{\multicolumn{2}{|c|}{$\begin{array}{l}\text { Theory. } \\
\mathrm{C}_{18} \mathrm{H}_{12} \mathrm{O}_{3} \text {. }\end{array}$}} \\
\hline & I. & II. & III. & & & \\
\hline & $77 \cdot 81$ & 77.99 & 78.09 & cent. & $78 \cdot 26$ & $r$ cent. \\
\hline & 5.07 & $5 \cdot 00$ & $4: 51$ & , & 4.35 & ", \\
\hline $\mathrm{O}$ & $17 \cdot 12$ & $17 \cdot 01$ & $17 \cdot 40$ & " & $17 \cdot 39$ & ” \\
\hline
\end{tabular}

This acid, therefore, has the formula $\mathrm{C}_{18} \mathrm{H}_{12} \mathrm{O}_{3}$, and is produced from dehydrobenzoylacetic acid by the addition of 2 atoms of hydrogen and the elimination of the elements of water according to the equation-

$$
\mathrm{C}_{18} \mathrm{H}_{12} \mathrm{O}_{4}+\mathrm{H}_{2}=\mathrm{C}_{18} \mathrm{H}_{12} \mathrm{O}_{3}+\mathrm{H}_{2} \mathrm{O} \text {. }
$$

The yield of this acid is very small, and it appears to be formed only under certain conditions, the principal product of the reaction being the acid $\mathrm{C}_{18} \mathrm{H}_{14} \mathrm{O}_{4}$, which will be described later on. In this reduction, by far the greater part of the dehydrobenzoylacetic acid is entirely decomposed, a quantity of oily and resinous product being formed, which render the purification of the acids very difficult. It is curious that a considerable quantity of benzaldehyde always appears to be formed during the reduction. The acid $\mathrm{C}_{18} \mathrm{H}_{12} \mathrm{O}_{3}$ melts at $112^{\circ}$. It is easily soluble in alcohol, benzene, ether, chloroform, and bisulphide of carbon, almost insoluble in light petroleum. It is difficult to obtain it well crystallised, a mixture of benzene and light petroleum being the best solvents to crystallise it from. The bisulphide of carbon solution does not decolorise bromine.

The acid dissolves in concentrated sulphuric acid with an intense orange coloration which, on warming, first disappears and then turns to a greenish-brown. 
Apparently it is a very stable body, and is not affected by potassium permanganate in the cold, and only slowly on warming. Heated by itself in a test-tube, it is decomposed into carbonic anhydride and high boiling oils, a considerable quantity of carbonaceous matter being left behind. No acetophenone is formed when it is distilled with sodalime.

The acid dissolves in soda and ammonia slowly in the cold, more rapidly on heating. The sodium salt is best prepared by dissolving the acid in a little alcohol, neutralising with soda, and allowing the solution to evaporate over sulphuric acid in a vacuum. Thus obtained it is an almost colourless syrup easily soluble in water; the solution, exposed for a long time over sulphuric acid in a vacuum, slowly deposits crystals. The quantity of this acid, $\mathrm{C}_{18} \mathrm{H}_{12} \mathrm{O}_{3}$, which $\mathrm{I}$ obtained was unfortunately insufficient to allow of the further examination and analysis of its salts. The products of the reduction of dehydracetic acid were first examined by Oppenheim and Precht (Ber., 9, 1101) who, by treating this body with zinc and hydrochloric acid, obtained an acid melting at $187^{\circ}$; this, however, they did not further examine, only stating that it was not a simple reductionproduct, but that it appeared to contain less oxygen than the dehydracetic acid. Schibbye (Inaugural Dissertation, Würzburg, 1882), who during some experiments on dehydracetic acid, repeated this reaction, and analysed the acid and its barium salt, gave to the former the formula $\mathrm{C}_{8} \mathrm{H}_{10} \mathrm{O}_{3}$, it being formed from dehydracetic acid according to the equation-

$$
\mathrm{C}_{8} \mathrm{H}_{8} \mathrm{O}_{4}+2 \mathrm{H}_{2}=\mathrm{C}_{8} \mathrm{H}_{10} \mathrm{O}_{3}+\mathrm{H}_{2} \mathrm{O} \text {. }
$$

If the reaction takes place in this way, it is possible that the acid $\mathrm{C}_{18} \mathrm{H}_{12} \mathrm{O}_{3}$, described above, is constituted similarly to the acid $\mathrm{C}_{8} \mathrm{H}_{10} \mathrm{O}_{3}$, in which case the constitution of the former would have to be changed to $\mathrm{C}_{18} \mathrm{H}_{14} \mathrm{O}_{3}$. I am at present engaged in some experiments to decide this point.

In order to isolate any other products of the reaction of dehydrobenzoylacetic acid, the mother-liquors from the acid $\mathrm{C}_{18} \mathrm{H}_{12} \mathrm{O}_{3}$ were next evaporated to dryness, the semi-solid residue dissolved in a little alcohol, and light petroleum boiling at $70-80^{\circ}$ added until the liquid became slightly turbid. On allowing the solution to evaporate at the ordinary temperature, a new acid crystallised out in small needles, which were collected, washed with alcohol and light petroleum, and dried at $110^{\circ}$. The analysis gave the following results:-

I. 0.1568 gram substance gave 0.0731 gram $\mathrm{H}_{2} \mathrm{O}$ and 0.4239 gram $\mathrm{CO}_{2}$.

II. $0 \cdot 1614$ gram substance gave 0.0758 gram $\mathrm{H}_{2} \mathrm{O}$ and 0.4364 gram $\mathrm{CO}_{2}$. 
III. 0.1511 gram substance gave 0.0705 gram $\mathrm{H}_{2} \mathrm{O}$ and 0.4064 gram $\mathrm{CO}_{2}$.

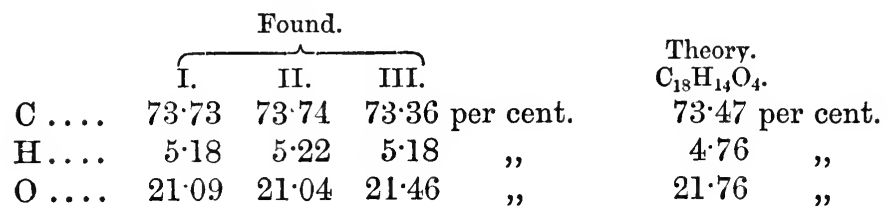

The acid, therefore, has the formula $\mathrm{C}_{18} \mathrm{H}_{14} \mathrm{O}_{4}$, and is formed from dehydrobenzoylacetic acid by the addition of 2 atoms of hydrogen, according to the equation-

$$
\mathrm{C}_{18} \mathrm{H}_{12} \mathrm{O}_{4}+\mathrm{H}_{2}=\mathrm{C}_{18} \mathrm{H}_{14} \mathrm{O}_{4} \text {. }
$$

It crystallises from a mixture of alcohol and light petroleum in small yellow needles which melt at $145-150^{\circ}$ with decomposition and evolution of carbonic auhydride. It is easily soluble in most of the usual solvents, but only sparingly in light petroleum and cold bisulphide of carbon. From its hot solution in the latter, it is deposited on cooling in nodules, seldom in well-formed crystals. The solution in bisulphide of carbon does not decolorise bromine at ordinary temperatures, but on warming hydrogen bromide is given off, and a brominated acid formed which crystallises out in small needles on cooling.

The acid $\mathrm{C}_{18} \mathrm{H}_{14} \mathrm{O}_{4}$ is moderately soluble in dilute alkalis, especially on warming, and is reprecipitated on the addition of an acid. It dissolves in concentrated sulphuric acid, forming a yellow solution, which changes to brownish-red on warming. When heated in a test-tube, it melts and gives off carbonic anhydride, a low-boiling oil distilling over with an odour like benzene; if the heating be continued the greater part chars, a small quantity of a heavy oil being formed which becomes solid on cooling, and appears to be benzoic acid. On distilling the acid with soda-lime, a small amount of an oil of low boiling point is formed, but no acetophenone.

\section{Action of Acetic Anhydride on the Acid $\mathrm{C}_{18} \mathrm{H}_{14} \mathrm{O}_{4}$.}

From the formula of this acid, it would seem to be the intermediate product in the formation of the acid $\mathrm{C}_{18} \mathrm{H}_{12} \mathrm{O}_{3}$ by the reduction of dehydrobenzoylacetic acid, thus :-

$$
\mathrm{C}_{18} \mathrm{H}_{12} \mathrm{O}_{4}+\mathrm{H}_{2}=\mathrm{C}_{18} \mathrm{H}_{14} \mathrm{O}_{4}=\mathrm{C}_{18} \mathrm{H}_{12} \mathrm{O}_{3}+\mathrm{H}_{2} \mathrm{O} \text {. }
$$

It appeared probable, therefore, that by treating it with some dehydrating agent, such as acetic anhydride, the acid $\mathrm{C}_{18} \mathrm{H}_{12} \mathrm{O}_{3}$ would be produced. In order to test this, 2 grams of the acid $\mathrm{C}_{18} \mathrm{H}_{14} \mathrm{O}_{4}$ were heated with an excess of acetic anhydride in a flask connected with 
a reflux condenser for about one hour. Water was then added, and the precipitated product after washing with water was crystallised from 80 per cent. acetic acid. The analysis gave the following result :-

I. 0.1910 gram substance gave 0.0817 gram $\mathrm{H}_{3} \mathrm{O}$ and 0.5292 gram $\mathrm{CO}_{2}$.

II. 0.2124 gram substance gave 0.0908 gram $\mathrm{H}_{2} \mathrm{O}$ and 0.5871 gram $\mathrm{CO}_{2}$.

\begin{tabular}{|c|c|c|c|}
\hline & \multicolumn{2}{|c|}{ Found. } & \\
\hline & I. & II. & $\begin{array}{l}\text { Theory. } \\
\mathrm{C}_{20} \mathrm{H}_{14} \mathrm{O}_{4} .\end{array}$ \\
\hline C. & 75.57 & $75 \cdot 39$ per cent. & 75.47 per cent. \\
\hline & 4.75 & $4.75 \quad "$ & $4.40 \quad$, \\
\hline $0 \ldots \ldots$ & $19 \cdot 68$ & $19 \cdot 86$ & $20 \cdot 13$ \\
\hline
\end{tabular}

This compound appears therefore to have the formula $\mathrm{C}_{20} \mathrm{H}_{14} \mathrm{O}_{4}$, and it would seem as if the acetic anhydride had not only acted as a dehydrating agent, but that an acetate had also been formed, according to the equation-

$$
\mathrm{C}_{18} \mathrm{H}_{14} \mathrm{O}_{4}+{ }_{2} \mathrm{CH}_{3} \cdot \mathrm{CO}>\mathrm{O}=\mathrm{C}_{20} \mathrm{H}_{14} \mathrm{O}_{4}+3 \mathrm{CH}_{3} \cdot \mathrm{COOH} .
$$

It is, however, impossible to come to any definite conclusion from this result, as the amount of material at my disposal was so small that I was unable to try this reaction again, and confirm the constitution of the compound $\mathrm{C}_{20} \mathrm{H}_{14} \mathrm{O}_{4}$ by further analyses. It crystallises from acetic acid in yellow needles which melt at $145-150^{\circ}$. Heated in a test-tube, it first melts, and then gives off carbonic anhydride and acetic anhydride. From the ease with which it gives up its acetic anhydride, it is possible that it is nothing more than a mixed anhydride of the acid $\mathrm{C}_{18} \mathrm{H}_{12} \mathrm{O}_{3}$ and acetic acid. It is easily soluble in hot alcohol, less so in cold. It dissolves easily in benzene and chloroform, but only sparingly in ether and bisulphide of carbon: in light petroleum it is almost insoluble. It is precipitated in small needles from its solution in benzene on adding light petroleum, and can be purified in this manner. It crystallises, however, best from dilute acetic acid (80 per cent.).

The alcoholic solution gives an intense scarlet coloration with ferric chloride. The compound is moderately soluble in ammonia, sodium hydroxide, and carbonate, especially on warming. It dissolves in concentrated sulphuric acid, forming an orange solution, which changes to brown on heating. This coloration does not disappear on diluting with water.

Heated with alcoholic potash, it is easily saponified, acetic acid and another acid being formed. 


\section{Action of Phosphorous Pentachloride on Dehydrobenzoylacetic Acid.}

In order, if possible, to obtain some further clue as to the constitution of dehydrobenzoylacetic acid, it was next treated with phosphorous pentachloride. By this means, not only the carboxylgroup, but also any carbonyl- or hydroxyl-groups would be acted on, and be replaced by chlorine, whereas any oxygen present in the indifferent or oxide form would remain intact, and could be easily recognised.

If dehydrobenzoylacetic acid is mixed with phosphorous pentachloride, a very energetic reaction sets in, the whole becoming very hot and most of the acid being charred, so that it was found impossible to extract any crystalline compound from the product. It was necessary therefore to moderate the violence of the reaction by diluting with oxychloride of phosphorus and cooling, the experiment being carried out in the following way :-

5 grams of dehydrobenzoylacetic acid were mixed with 30 grams of phosphorous oxychloride, and then 10 grams of phosphorous pentachloride were slowly added, the whole being kept thoroughly cooled with ice-water. The acid slowly dissolved in the mixture, forming a yellowish-brown solution, and on standing, a quantity of yellow crystals were deposited, which, however, at a later stage of the reaction, disappeared again almost entirely. After remaining for 12 hours, the product was poured into ice-water, when a yellowish semisolid mass was precipitated; this was collected, well washed with water, and dried over sulphuric acid in a vacuum. In about 48 hours, the mass, which had become hard and brittle, was powdered and extracted with small quantities of hot alcohol, in which it was sparingly soluble, and filtered. The filtrate on cooling deposited a yellowish crystalline substance, which by one or two recrystallisations from methyl alcohol, was obtained pure in colourless plates melting at $227-229^{\circ}$.

The amount of this compound obtained by the above reaction, was however, so small, that no analysis could be made. It appeared to contain only traces of chlorine. It is coloured yellow on being warmed with potash.

The residue left after extracting the above described substance with alcohol, was dissolved in boiling methyl alcohol, from which it was deposited on cooling in beautiful brownish-coloured glittering plates. On repeated recrystallisation from the same solvent it was easily obtained pure. For the analysis it was dried at $120^{\circ}$ and then gave the following results :-

I. 0.2114 gram substance gave 0.0692 gram $\mathrm{H}_{2} \mathrm{O}$ and 0.5370 gram $\mathrm{CO}_{2}$. 
II. 0.2226 gram substance gave 0.0758 gram $\mathrm{H}_{2} \mathrm{O}$ and 0.5668 gram $\mathrm{CO}_{2}$.

III. 0.1394 gram substance gave 0.0665 gram AgCl.

IV. $0 \cdot 2363$

V. $0 \cdot 2415$

,,

0.1110

" $0.1128 \quad$,

,

Found.

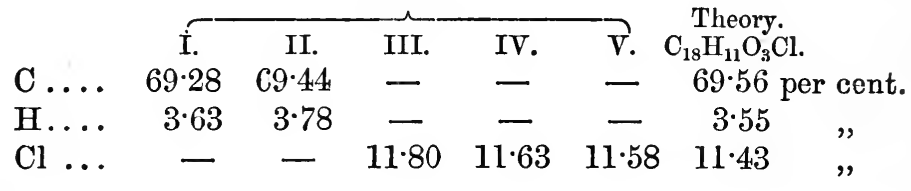

The compound, therefore, has the formula $\mathrm{C}_{18} \mathrm{H}_{11} \mathrm{O}_{3} \mathrm{Cl}$. When crystallised from methyl alcohol, it forms brownish-coloured plates melting at $150-151^{\circ}$.

It is sparingly soluble in ether, alcohol, benzene, and light petroleum, more easily in hot methyl alcohol and acetic acid. The hot alcoholic solution gives a reddish-brown coloration with ferric chloride. It dissolves with difficulty in sodium hydroxide, ammonia, and sodium carbonate, forming yellow solutions, which, when acidified, deposit a colourless amorphous acid. On boiling with alcoholic potash for a short time, however, it is easily decomposed, and on evaporating the alcohol and adding water, a dark-brownish solution of a potassium salt is obtained; this, when acidified, deposits an oily acid, which was extracted with ether. On allowing the ethereal solution to evaporate, a thick oily acid was obtained, and on standing a small quantity of a crystalline body was deposited; this, however, could not be obtained pure enough for analysis. The alcoholic solution of this acid gives an intense violet coloration with ferric chloride, very similar to that produced by benzoylacetic acid. As the formation of this latter acid appeared possible, the crude product of the above reaction was distilled, and a few drops of a brownish oil obtained, which smelt decidedly of acetophenone. The quantity was too small, however, to determine the boiling point, and I was therefore unfortunately unable to decide definitely whether benzoylacetic acid had been formed or not. It appears, however, to be extremely probable. On simply warming the compound $\mathrm{C}_{18} \mathrm{H}_{11} \mathrm{O}_{3} \mathrm{Cl}$ with alcoholic potash, it dissolves completely, and after a short time a potash salt crystallises out; this, when acidified, yields an acid, which also gives the violet coloration with ferric chloride. On prolonged boiling with alcoholic potash, however, a neutral body is formed, and only a trace of the above acid. If a solution of this chloride in ether is treated with an ethereal solution of sodium ethoxide, a beautiful yellow sodium compound is precipitated. From these experiments, it would seem 
that the componnd $\mathrm{C}_{18} \mathrm{H}_{11} \mathrm{O}_{3} \mathrm{Cl}$, is in reality a chlorinated acid of the formula $\mathrm{C}_{17} \mathrm{H}_{10} \mathrm{OCl} . \mathrm{COOH}$, and not the acid chloride $\mathrm{C}_{17} \mathrm{H}_{11} \mathrm{O}_{2} . \mathrm{COCl}$, as might be expected from the mode of formation. In the latter case we should expect that dehydrobenzoylacetic acid would be regenerated on treating it with water; or on treating it with boiling alcohol or sodium ethoxide, that the ethylic salt of the latter would be formed, reactions which as a fact do not take place. I therefore propose to name the compound $\mathrm{C}_{18} \mathrm{H}_{11} \mathrm{O}_{3} \mathrm{Cl}$, "Chlorodehydrobenzoylacetic acid."

Heated by itself in small quantities at a time, it first melts, and then at a high temperature a thick brown oil distils over, which on cooling slowly solidifies, a small quantity of a carbonaceous residue being left. The distillate after washing with a little alcohol melts at $147-149^{\circ}$, and appears to be the unchanged substance, a proof that it is very stable. Chlorodehydrobenzoylacetic acid is easily reduced by warming it with zinc-dust and acetic acid. On adding water, a pale yellowish substance is obtained, which is free from chlorine. After collecting and washing with water, the residue was dissolved in a little hot alcohol, from which it separated on cooling in amorphous flakes. On heating, this substance becomes brownish at about $130^{\circ}$, and melts at between $155-160^{\circ}$; at a higher temperature carbonic anhydride is given off, and a blackish, tarry residue is left. It dissolves in concentrated sulphuric acid, with a beautiful purple colour, but the solution soon becomes discoloured on standing. It dissolves easily in benzene, and is precipitated in yellowish flakes on adding light petroleum, but as it could not be obtained in crystalline form from these or any other solvents, it was not analysed.

By the action of phosphorous pentachloride on dehydracetic acid, Oppenheim and Precht (Ber., 9, 1100) obtained a compound of the formula $\mathrm{C}_{8} \mathrm{H}_{6} \mathrm{O}_{2} \mathrm{Cl}_{2}$, which is possibly the product of the further action of the above reagent on an unstable body $\mathrm{C}_{8} \mathrm{H}_{7} \mathrm{O}_{3} \mathrm{Cl}$, the latter being similarly constituted to chlorodehydrobenzoylacetic acid. In order to test this, the action of an excess of phosphorous pentachloride on the compound $\mathrm{C}_{18} \mathrm{H}_{11} \mathrm{O}_{3} \mathrm{Cl}$ was tried, and, as a fact, traces of a second body were obtained, which, however, owing to the small quantity of material at my dis osal, could not be further examined.

\section{Constitution of Dehydrobenzoylacetic Acid.}

Dehydrobenzoylacetic acid, $\mathrm{C}_{13} \mathrm{H}_{12} \mathrm{O}_{4}$, contains besides a carboxylgroup $\mathrm{COOH}$, and a carbonyl-group $\mathrm{CO}$, a fourth atom of oxygen, which, on account of its indifference towards acetic anhydride, reducing agents, and phosphorous pentachloride, can neither be present in the ketone form (CO) nor as hydroxyl, OH, and must therefore be 
in the ether form, i.e., joined with its two affinities to two different carbon-atoms, thus; : C.O.C :. It is clear, moreover, that this acid contains two phenyl-groups, otherwise it could not be split up into two molecules of benzoylacetic acid on treatment with alcoholic potash.

In endeavouring from these facts to establish a formula for dehydrobenzoylacetic acid, it is first important to bear in mind, that it is evidently formed by the elimination of two molecules of water from two molecules of benzoylacetic acid, thus :-

$$
\underset{\mathrm{C}_{6} \mathrm{H}_{5} \cdot \mathrm{CO} \cdot \mathrm{CH}_{2} \cdot \mathrm{COOH}}{\mathrm{C}_{2} \mathrm{H}_{5} \cdot \mathrm{CO} \cdot \mathrm{CH}_{2} \cdot \mathrm{COOH}}=\mathrm{C}_{18} \mathrm{H}_{22} \mathrm{O}_{4}+2 \mathrm{H}_{2} \mathrm{O} \text {. }
$$

Dehydrobenzoylacetic acid is, however, a monobasic acid, forming salts of the formula $\mathrm{C}_{18} \mathrm{H}_{11} \mathrm{O}_{4} \mathrm{M}$, so that it follows that the carboxylgroup of one of the molecules of benzoylacetic acid must condense in some way with the second molecule of the latter, one molecule of water being eliminated. This intermediate product then looses a second molecule of water, dehydrobenzoylacetic acid being formed. The simplest way in which this condensation of two molecules of benzoylacetic acid can take place is the following :-

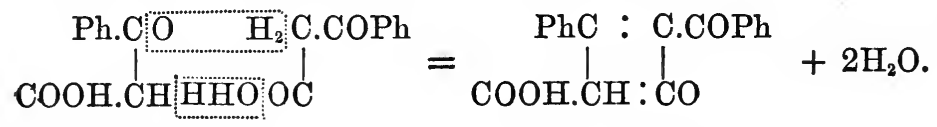

That this formula is not the right one is evident, as it not only contains two ketone-groups, but is so strongly bound together that the splitting up into two molecules of benzoylacetic acid, on simply standing in contact with potash, would be extremely difficult to account for. Besides the above, there are two other possible formulæ for dehydrobenzoylacetic acid, which can be built up on the assumption that the carboxyl-group of one molecule of benzoylacetic acid takes part in the reaction, namely:-

I.

COOH.C.CO.CH<smiles></smiles>

II.

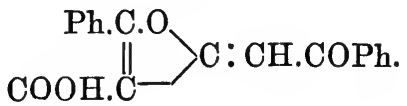

In the formation of these two formulæ, it is necessary to suppose that, as in almost all condensations of ethylic benzoylacetate and acetoacetate, the molecule first undergoes an internal change, thus :-

$$
\underset{\text { Stable form. }}{\mathrm{C}_{6} \mathrm{H}_{5} \cdot \mathrm{CO} . \mathrm{CH}_{2} \cdot \mathrm{COOH}}=\underset{\mathrm{T}_{6} \mathrm{H}_{5} \cdot \mathrm{C}(\mathrm{OH}): \mathrm{CH} . \mathrm{COOH} .}{\text { Mobile form. }}
$$


In formula I the condensation takes place between two such mobile forms, thas :-

$$
\underset{\mathrm{PhC} \cdot \mathrm{OH} \quad \mathrm{H}] \mathrm{O} . \mathrm{CPh}}{\| \mathrm{HHO} . \mathrm{OC} . \mathrm{CH}}=\underset{\mathrm{PhC}-\mathrm{O}-\mathrm{CPh}}{\|}+2 \mathrm{H}_{2} \mathrm{O} \text {. }
$$

Formula II is formed in two stages. In the first place, the carboxyl-group of one molecule of benzoylacetic acid (in the stable form) condenses with the second molecule (in the mobile form) in the following way :-

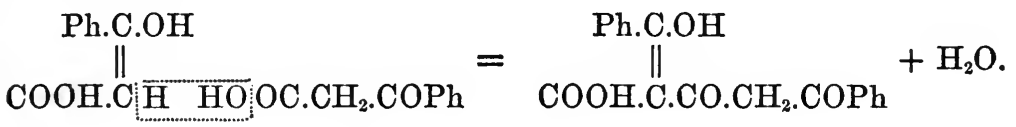

In the second part of the condensation, this intermediate body must be transformed into the mobile form, which can take place in two ways. In the first place, one of the hydrogen-atoms of the $\mathrm{CH}_{2}$ group can react with the CO-group next to the phenyl-group, when the following mobile intermediate body would be formed :-

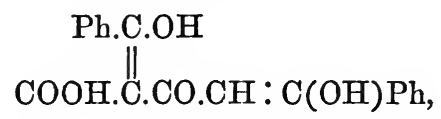

which, on removing the elements of water from the two hydroxylgroups, gives formula I.

In the second place, the molecular change can take place between the $\mathrm{CH}_{3}$-group and the other ketone-group, and thus give the following body:-

\author{
Ph.C.OH \\ COOH.C.C(OH) : CH.COPh,
}

which, on the elimination of water, gives formula II thus :-

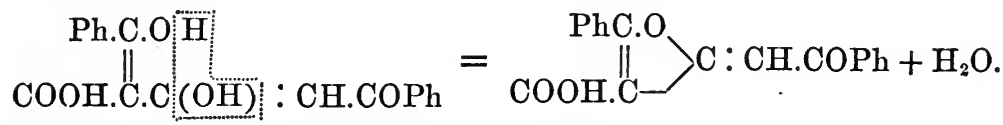

Of these two formulæ, it is evident that, apart from the complicated formation of formula II, formula I is the most likely, as it explains best of all the reactions in which the acid takes part.

I therefore suggest as the most probable constitution of the dehydrobenzoylacetic acid the following formula :- .

COOH.C.CO.CH<smiles>CCCCOc1ccccc1</smiles> 
This body is split up on treatment with alcoholic potash into two molecules of benzoylacetic acid, thus :-

$$
\begin{aligned}
& \mathrm{H} \mathrm{OH} \\
& \text { COOH.C.CO.CH COOH.CH COOH.CH }
\end{aligned}
$$

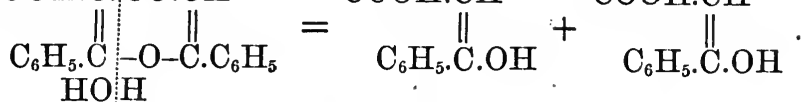

The two acids, $\mathrm{C}_{18} \mathrm{H}_{14} \mathrm{O}_{4}$ and $\mathrm{C}_{18} \mathrm{H}_{12} \mathrm{O}_{3}$, produced by the action of nascent hydrogen on dehydrobenzoylacetic acid, would therefore have the following formulæ:-

COOH.C.CH(OH).CH

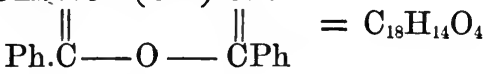

COOH.C.CH: C<smiles>C=C(CCCC)OCCO</smiles>

The formation of chlorodehydrobenzoylacetic acid, by the action of pentachloride of phosphorus on dehydrobenzoylacetic acid, undoubtedly takes place in two stages:-

I.

COOH.C.CO.CH

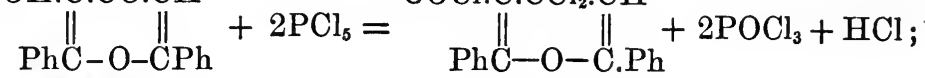

COCl.C.CCl 2 . CH

II.
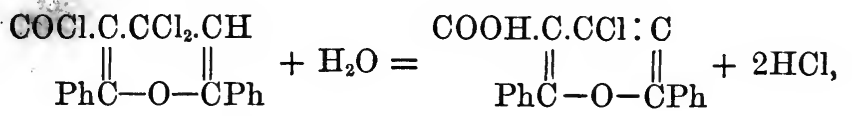

this reaction being in every way analogous to the formation of chlorocinnamic acid by the action of pentachloride of phosphorus on ethylic benzoylacetate as described in Part II (this vol., p. 257) of this research.

On comparing the properties of dehydrobenzoylacetic acid with those of the dehydracetic acid, it is evident that although some minor differences do exist, these two compounds are most probably of an analogous constitution; and as the former is extremely difficult to get in any quantity, I have commenced working on the latter, and hope at some future time to have the honour of laying the results of $\mathrm{my}$ experiments before the Society. 


\title{
BENZOYLACETIC ACID AND SOME OF ITS DERIVATIVES.
}

\author{
By W. H. Perkin (Jun.), Ph.D., and A. Calman, Ph.D.
}

IN the three previous parts of this paper, the preparation and properties of ethylic benzoylacetate and a few of its derivatives were described, and the close resemblance between these and the ethylic acetoacetate derivatives pointed out. As, however, there were still several doubtful points which required clearing up, a further examination of the derivatives of benzoylacetic acid was undertaken. The results of this investigation we now lay before the Society. Before proceeding with this research, it was thought probable that if methylic benzoylacetate were substituted for the ethylic salt as the starting point, more crystalline products might be obtained; it being a well-known fact that methylic salts crystallise more easily as a rule than ethylic.

The mode of preparing methylic benzoylacetate is exactly analogous to that of the ethylic salt (Trans., 1884, 170), the only difference being that methylic phenylpropiolate* must be substituted for ethylic phenylpropiolate.

The crude methylic benzoylacetate produced by the action of sulphuric acid on methylic phenylpropiolate is a brownish oil which does not solidify at $0^{\circ}$. To purify it, it is shaken with fairly con-

* This is easily produced by treating phenylpropiolic acid with methyl alcohol and bydrogen chloride. It is a colourless oil. 
centrated caustic potash solution and animal charcoal, and filtered, the temperature being kept as low as possible by adding ice. The filtrate, which should be perfectly clear and free from oily drops, is then mixed with ice and acidulated with dilute sulphuric acid; this causes the precipitation of an oil, which is separated from the acid liquid by agitation with pure ether. The ethereal solution, after being washed with dilute sodic carbonate, is dried over calcic chloride, filtered, and the ether distilled off; an almost colourless oil is left behind, which after standing for a week over sulphuric acid in a vacuum gave the following numbers on analysis :-

$0 \cdot 1800$ gram substance gave 0.0913 gram $\mathrm{H}_{2} \mathrm{O}$ and 0.4440 gram $\mathrm{CO}_{2}$.

Found.

C ..... $67 \cdot 27$ per cent.

H ..... $5 \cdot 62$,

O ..... 27.11 ,
Theory.

$\mathrm{C}_{6} \mathrm{H}_{5} \cdot \mathrm{CO} \cdot \mathrm{CH}_{2} \cdot \mathrm{COOCH}_{3}$.

$67 \cdot 41$

$26 \cdot 97$

Methylic benzoylacetate appears to be more stable than the ethylic salt, it is certainly not so easily decomposed by distillation.

On the addition of ferric chloride to the alcoholic solution, it gives the same beautiful violet coloration as the ethereal salt. If methylic benzoylacetate is added to an ethereal solution of sodic ethylate, and the whole allowed to remain for a short time, a thick white precipitate separates which is the sodium-derivative. In order to purify it, it is collected as rapidly as possible, well washed with dry ether, and dried over sulphuric acid in a vacuum. The following numbers were obtained on analysis :-

$0 \cdot 3470$ gram substance gave 0.1206 gram $\mathrm{Na}_{2} \mathrm{SO}_{4}$.

\begin{tabular}{|c|c|c|}
\hline & Found. & $\begin{array}{c}\text { Theory. } \\
\mathrm{C}_{6} \mathrm{H}_{5} \cdot \mathrm{CO} \cdot \mathrm{CHNa} \cdot \mathrm{COOCH}_{3} \text {. }\end{array}$ \\
\hline & 4 per cent. & 11.50 per cent. \\
\hline
\end{tabular}

Prepared as above this sodium-derivative is a white amorphous powder easily soluble in water and hot alcohol.

It is extremely stable, much more so than the sodium-derivative of ethylic benzoylacetate, and behaves much like a salt of an acid. On acidifying its aqueous solution with dilute sulphuric acid, methylic benzoylacetate is reprecipitated together with a certain anount of benzoylacetic acid.

When treated with benzyl chloride in alcoholic solution, it gave a nroduct, which after repeated fractioning boiled fairly constantly between $250^{\circ}$ and $255^{\circ}$ ( $50 \mathrm{~mm}$. pressure). It gave the following numbers on analysis :-

0.214 gram substance gave 0.59.55 grain $\mathrm{CO}_{2}$ and 0.117 gram $\mathrm{H}_{2} \mathrm{O}$. 
Found.

C ..... $75 \cdot 89$ per cent.

H .... 6.07 ,
Theory.

$\mathrm{C}_{6} \mathrm{H}_{5} \cdot \mathrm{CO} \cdot \mathrm{CH}\left(\mathrm{C}_{7} \mathrm{H}_{7}\right) \cdot \mathrm{COOCH}_{3}$. $75 \cdot 00$ per cent.

$6.25 \quad$,

$18 \cdot 75 \quad$,

This compound, therefore, was probably methylic benzylbenzoylacetate. A few other derivatives of methylic benzoylacetate were also prepared, but as they did not crystallise, and as the yield was in every case inferior to that obtained when ethylic benzoylacetate was used, the remainder of this research was carried out with the ethylic salt.

\section{Ethylic Methylbenzoylacetate.}

To prepare this compound, 11 grams of sodium were dissolved in 120 grams of absolute alcohol, and a mixture of 90 grams of ethylic benzoylacetate and 80 grams of methylic iodide were slowly added, taking care to cool well during the operation. The clear solution was then heated at $100^{\circ}$ for three hours in a soda-water bottle, when the reaction was complete. In order to isolate the product, water was added, and the liquid extracted twice or thrice with ether. The ethereal solution was then well washed with water, dried over potassic carbonate, and the ether distilled off; the dark-brownish coloured oil thus obtained was further purified by fractioning under a pressure of $300 \mathrm{~mm}$.

By the first distillation, almost the whole went over between 230$240^{\circ}$, and after repeated refractioning a colourless oil of aromatic odour was obtained, boiling constantly at $235^{\circ}(300 \mathrm{~mm})$. It gave the following results on analysis:-

$0 \cdot 1227$ gram substance gave 0.3135 gram $\mathrm{CO}_{2}$ and $0 \cdot 788$ gram $\mathrm{H}_{2} \mathrm{O}$.

\begin{tabular}{|c|c|c|}
\hline . & $\begin{array}{l}\text { Found. } \\
69 \cdot 69 \text { per cent. }\end{array}$ & $\begin{array}{l}\text { Theory. } \\
\mathrm{C}_{6} \mathrm{H}_{5} \cdot \mathrm{CO} \cdot \mathrm{CH}\left(\mathrm{CH}_{3}\right) \cdot \mathrm{COOC}_{2} \mathrm{H}_{5} \cdot \\
69 \cdot 90 \text { per cent. }\end{array}$ \\
\hline & $7 \cdot 13^{1}$ & $6 \cdot 80 \quad$, \\
\hline & $23 \cdot 18$ & $23 \cdot 40$ \\
\hline
\end{tabular}

This compound is therefore ethylic methylbenzoylacetate. It boils at-

$235^{\circ}$ under a pressure of $300 \mathrm{~mm}$.

$226-227^{\circ}$ under a pressure of $225 \mathrm{~mm}$.

Its alcoholic solution gives no coloration with ferric chloride. If sodic ethylate is added to its ethereal solution, a thick white sodiumderivative is precipitated, which is decomposed on the addition of much water. 
In order, if possible, to obtain the free methylbenzoylacetic acid, the pure ethereal salt was mixed with pure concentrated sulphuric acid, and allowed to stand at ordinary temperatures for three weeks. The product, which was slightly brownish, was then poured on ice, and the solution several times extracted with pure ether. On drying this ethereal solution over calcic chloride and evaporating, an almost colourless oil was left which did not crystallise, even after standing for several days over sulphuric acid in a vacuum, and therefore was not analysed. When treated with dilute ammonia, the greater part dissolved, leaving a small quantity of a brownish-coloured oil.

When heated in a test-tube, carbonic anhydride was given off, and the residue on fractioning went over almost entirely between $205^{\circ}$ and $215^{\circ}$ as a colourless oịl, which was without doubt ethyl phenyl ketone, $\mathrm{C}_{6} \mathrm{H}_{5} \cdot \mathrm{CO} \cdot \mathrm{CH}_{2} \cdot \mathrm{CH}_{3}$ (b. p. $210^{\circ}$ ).

The solution of the crude acid was coloured reddish-brown on the addition of a drop of ferric chloride. It is therefore almost certain that this oily acid consisted for the most part of methylbenzoylacetic acid, rendered impure by the presence of oily decomposition products which prevented it from crystallising.

Action of Phosphorous Pentachloride on Ethylic Methylbenzoylacetate.

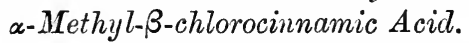

On treating ethylic benzoylacetate with pentachloride of phosphorus, it is transformed (Trans., 1885, 256) into a chlorocinnamic acid, $\mathrm{C}_{6} \mathrm{H}_{5}: \mathrm{CH}: \mathrm{CCl}: \mathrm{COOH}$, in which the chlorine-atom appears to be in the $\alpha$-position. From the method of formation, however, $\beta$-chlorocinnamic acid should be formed according to the equation-

\section{I. $\mathrm{C}_{6} \mathrm{H}_{5} \cdot \mathrm{CO} \cdot \mathrm{CH}_{2} \cdot \mathrm{COOEt}+2 \mathrm{PCl}_{5}=\mathrm{C}_{6} \mathrm{H}_{5} \cdot \mathrm{CCl}_{2} \cdot \mathrm{CH}_{2} \cdot \mathrm{COCl}$ $+\mathrm{EtCl}+2 \mathrm{POCl}_{3}$.}

\section{II. $\mathrm{C}_{6} \mathrm{H}_{5} \cdot \mathrm{CCl}_{2} \cdot \mathrm{CH}_{2} \cdot \mathrm{COCl}+\mathrm{H}_{2} \mathrm{O}=\mathrm{C}_{6} \mathrm{H}_{5} \cdot \mathrm{CCl}: \mathrm{CH} \cdot \mathrm{COOH}+2 \mathrm{HCl}$.}

It appeared therefore interesting to follow up this reaction, and in the first place to examine the action of pentachloride of phosphorus on a substituted ethylic benzoylacetate.

For this purpose, 10 grams of ethylic methylbenzoylacetate were dissolved in 50 grams of phosphorous oxychloride, and then 25 grams of phosphorous pentachloride were slowly added, the whole being well cooled with ice during the operation. After standing for about an hour, the mixture was finally heated a short time on a water-bath and then poured on ice. When all the oxychloride had been decomposed, a yellowish oil was left, which was separated from the acid liquid by extraction with ether. The ethereal solution was shaken 
with dilute sodic hydrate to remove any acids which had been formed, dried over calcic chloride and the ether distilled off.

In this way a yellowish oil was obtained which was further purified by fractioning under reduced pressure $(300 \mathrm{~mm}$.). Almost the whole of it went over in the first distillation between $225^{\circ}$ and $240^{\circ}$, and after repeated fractioning an oil was obtained boiling constantly at $230-233^{\circ}(300 \mathrm{~mm}$.). This gave the following numbers on analysis :-

I. $0 \cdot 1996$ gram substance gave 0.4700 gram $\mathrm{CO}_{2}$ and $0 \cdot 1076$ gram $\mathrm{H}_{2} \mathrm{O}$.

II. 0·1536 gram substance gave 0.0952 gram AgCl.

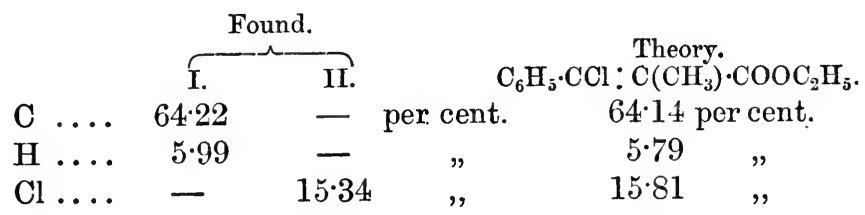

This substance therefore is ethylic $\alpha$-methyl- $\beta$-chlorocinnamate, formed according to the following equation:-
$\mathrm{C}_{6} \mathrm{H}_{5} \cdot \mathrm{CO} \cdot \mathrm{CH}\left(\mathrm{CH}_{3}\right) \cdot \mathrm{COOC}_{2} \mathrm{H}_{5}+\mathrm{PCl}_{5}=\mathrm{C}_{6} \mathrm{H}_{5} \cdot \mathrm{CCl}: \mathrm{C}\left(\mathrm{CH}_{3}\right) \cdot \mathrm{COOC}_{2} \mathrm{H}_{5}$ $+\mathrm{HCl}+\mathrm{POCl}_{3}$.

On examining the alkaline solution obtained by extracting the crude product of the action of phosphorus pentachloride on ethylic methylbenzoylacetate, it was found that when this was acidified with dilute sulphuric acid, a quantity of an organic acid was precipitated in white flakes. These when collected, dried on a porous plate, and crystallised several times from boiling light petroleum, were obtained in the form of colourless needles, which gave the following results on analysis :-

I. 0.1890 gram substance gave 0.4213 gram $\mathrm{CO}_{2}$ and 0.086 gram $\mathrm{H}_{2} \mathrm{O}$.

II. 0.2413 gram substance gáve 0•1555 gram $\mathrm{AgCl}$.

\begin{tabular}{|c|c|c|c|c|}
\hline \multirow[b]{3}{*}{ C .... } & \multicolumn{2}{|c|}{ Found. } & & \multirow{2}{*}{$\begin{array}{l}\text { Theory. } \\
\mathrm{C}_{6} \mathrm{H}_{5} \cdot \mathrm{CCl}: \mathrm{C}\left(\mathrm{CH}_{3}\right): \mathrm{COOH}\end{array}$} \\
\hline & I. & II. & & \\
\hline & $61 \cdot 07$ & - & per cent. & $60 \cdot 79$ per cent. \\
\hline $\mathrm{H} \ldots$. & $4: 58$ & - & $"$ & $5 \cdot 06$ \\
\hline $\mathrm{Cl} \ldots$ & - & 18.07 & , & $17 \cdot 95$ \\
\hline
\end{tabular}

This acid is therefore $\alpha$-methyl- $\beta$-chlorocinnamic acid. It melts at $116^{\circ}$, and can be distilled in small quantities almost without decomposition. It dissolves easily in alcohol, ether, chloroform, benzene, and acetic acid, more sparingly in cold light petroleum. When 
treated with concentrated nitric acid, crystalline nitro-derivatives are formed, whish, however, were not further examined.

The silver salt of this acid was prepared by adding silver nitrate to a neutral solution of the ammonic salt. After collecting and washing with water, it was obtained as a white mass, which when dried over sulphuric acid in a vacuum gave the following numbers on analysis :-

0.3175 gram substance gave 0.1482 gram $\mathrm{AgCl}$.

$$
\begin{aligned}
& \text { Found. } \\
& \text { Ag..... 35.20 per cent. } \\
& \mathrm{C}_{6} \mathrm{H}_{5} \cdot \mathrm{CCl}: \mathrm{C}\left(\mathrm{CH}_{3}\right) \cdot \mathrm{COOAg} \text {. } \\
& 35 \cdot 64 \text { per cent. }
\end{aligned}
$$

On heating this salt, it is decomposed, a colourless oil being given off which is probably chloromethylstyrene. In this chloromethylcinnamic acid, it is obvious that the chlorine-atom must be in the $\beta$-position, the $\alpha$-position being occupied by the methyl-group.

\section{$\alpha$-Methyl- $\beta$-phenylhydroxypropionic Acid.}

Ethylic benzoylacetate is easily reduced when treated with sodium amalgam, $\beta$-phenyllactic acid being formed (Trans., 1885, 253), thus-

$$
\begin{array}{r}
\mathrm{C}_{6} \mathrm{H}_{5} \cdot \mathrm{CO} \cdot \mathrm{CH}_{2} \cdot \mathrm{COOC}_{2} \mathrm{H}_{5}+\mathrm{H}_{2}+\mathrm{H}_{2} \mathrm{O}=\mathrm{C}_{6} \mathrm{H}_{5} \cdot \mathrm{CH}(\mathrm{OH}) \cdot \mathrm{CH}_{2} \cdot \mathrm{COOH} \\
+\mathrm{C}_{2} \mathrm{H}_{5} \cdot \mathrm{OH} .
\end{array}
$$

In order to determine whether this reaction is a general one, it was determined to try the action of reducing agents on a substituted ethylic benzoylacetate. For this purpose, pure ethylic methylbenzoylacetate was dissolved in dilute alcohol, and sodium amalgam slowly added, the whole being cooled from time to time with cold water. After 24 hours, the alkaline liquid was separated from the mercury, evaporated on a water-bath until all the alcohol had been driven off, aciảified with dilute sulphuric acid, and extracted six times with ether. On drying over calcic chloride and distilling off the ether, a thick oil was left, which did not crystallise even after remaining for some hours over sulphuric acid in a vacuum. In order to purify this substance, it was dissolved in a slight excess of barytawater, the excess of baryta precipitated by carbonic anhydride and the whole filtered. The filtrate was then acidified with dilute hydrochloric acid and again extracted with ether. On distilling off the ether, a colourless oil remained, which solidified on standing. The crystalline mass was first spread out on a porous plate to remove traces of oily matter, and then dissolved in a little boiling benzene. On cooling, the acid crystallised out in colourless needles which gave the following numbers on analysis:- 
I. $0 \cdot 1920$ gram substance gave 0.4695 gram $\mathrm{CO}_{2}$ and $0 \cdot 1175$ gram $\mathrm{H}_{2} \mathrm{O}$.

II. 0.2671 gram substance gave 0.6488 gram $\mathrm{CO}_{2}$ and 0.1666 gram $\mathrm{H}_{2} \mathrm{O}$.

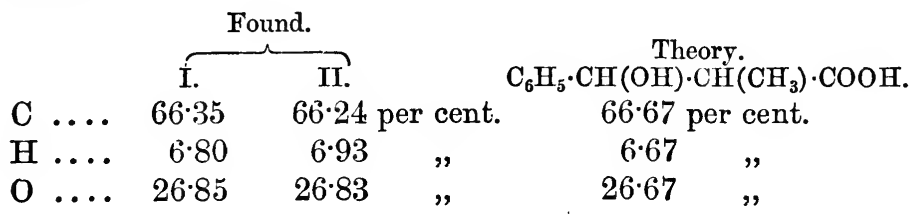

$\alpha$-Methyl- $\beta$-phenylhydroxypropionic acid when quite pure melts at $124-125^{\circ}$. It is very easily soluble in alcohol, ether, acetone, and hot water, sparingly soluble in chloroform, bisulphide of carbon, and light petroleum. It crystallises beautifully from boiling water in nodular masses, and from a solution of equal parts of light petroleum and benzene in flat needles. If the free acid is heated in a test-tube, water is given off and an oil distils over, which on cooling for the most part solidifies, and smells strongly of benzaldehyde.

When mixed with concentrated sulphuric acid, it becomes yellowish, and on warming it dissolves forming a reddish-brown solution.

The ammonium salt of $\alpha$-methyl- $\beta$-phenylhydroxypropionic acid crystallises from water in beautiful feathery needles. The silver salt is easily obtained on adding silver nitrate to a neutral solution of the ammonium salt as a heavy white crystalline precipitate, which gave the following numbers on analysis:-

$0 \cdot 2773$ gram substance gave 0.1036 gram silver.

$$
\begin{aligned}
& \text { Found. } \\
& \text { Ag..... 37.36 per cent. } \\
& \mathrm{C}_{6} \mathrm{H}_{5} \cdot \mathrm{CH}(\mathrm{OH}) \cdot \mathrm{CH}\left(\mathrm{CH}_{3}\right) \cdot \mathrm{COOAg} \text {. } \\
& 37 \cdot 63 \text { per cent. }
\end{aligned}
$$

This silver salt, when heated, was rapidly decomposed into silver, and a colourless oil, which smelt strongly of benzaldehyde.

\section{Ethylic Propylbenzoylacetate.}

This ethereal salt was prepared by heating a mixture of ethylic benzoylacetate ( 50 grams) with sodic ethylate ( $\mathrm{Na}=6$ grams) and an excess of propylic iodide (50 grams) for four hours at $100^{\circ}$ in a sodawater bottle.

The product was isolated in the same way as the ethylic methylbenzoylacetate and purified by fractioning under diminished pressure $(300 \mathrm{~mm}$.$) . In the first distillation, almost all went over between$ $240^{\circ}$ and $260^{\circ}$, and after repeated refractioning the ethylic propyl- 
benzoylacetate was obtained pure as a colourless oil, boiling constantly between $250^{\circ}$ and $252^{\circ}$ (300 mm.). On combustion it gave the following numbers:-

0.3358 gram substance gave 0.8886 gram $\mathrm{CO}_{2}$ and 0.2423 gram $\mathrm{H}_{2} \mathrm{O}$.

Found.

C ..... 72.15 per cent.

H..... 8.01 ,

O .... 20.52 ,
Theory. $\mathrm{C}_{6} \mathrm{H}_{5} \cdot \mathrm{CO} \cdot \mathrm{CH}\left(\mathrm{C}_{3} \mathrm{H}_{7}\right) \cdot \mathrm{COOC}_{2} \mathrm{H}_{5}$. $71 \cdot 79$ per cent. $7 \cdot 69$, $20 \cdot 52 \quad$

Ethylic propylbenzoylacetate is a colourless oil of aromatic odour, which does not solidify at $0^{\circ}$. It is slightly soluble in caustic potash. When treated with sodic ethylate, it gives a sodium-derivative. Its alcoholic solution gives no coloration with ferric chloride.

When quite pure, it was found to have the following boiling points :-

$$
\begin{aligned}
& 250-252^{\circ} \\
& 2300 \mathrm{~mm} .) \\
& 238-239
\end{aligned}
$$

\section{Butyl Phenyl Ketone.}

In order to obtain this ketone, ethylic propylbenzoylacetate was heated in a flask connected with a reflux condenser with dilute alcoholic potash for six hours, at the end of which time decomposition was complete. On adding water, an oil was precipitated, which was separated from the alkaline solution by extraction with ether. The ethereal solution, after being well washed with water, was dried over potassic carbonate, and the ether distilled off.

In this way a dark-brownish oil was obtained, which after repeated fractioning boiled constantly between $236^{\circ}$ and $238^{\circ}$ (720 mm.), and on analysis gave numbers, showing it to be butyl phenyl ketone:-

I. 0.1158 gram substance gave 0.3441 gram $\mathrm{CO}_{2}$ and 0.0957 gram $\mathrm{H}_{2} \mathrm{O}$.

II. $0 \cdot 1805$ gram substance gave 0.5373 gram $\mathrm{CO}_{2}$ and $0 \cdot 1528$ gram

\begin{tabular}{|c|c|c|c|c|c|}
\hline \multirow[b]{3}{*}{ C .... } & \multicolumn{2}{|c|}{ Found. } & & \multirow{2}{*}{\multicolumn{2}{|c|}{$\begin{array}{l}\text { Theory. } \\
\mathrm{C}_{6} \mathrm{H}_{5} \cdot \mathrm{CO} \cdot \mathrm{CH}_{2} \cdot \mathrm{CH}_{2} \cdot \mathrm{CH}_{2} \cdot \mathrm{CH}_{3} \cdot\end{array}$}} \\
\hline & I. & II. & & & \\
\hline & $81 \cdot 05$ & $81 \cdot 19$ & c cent. & & r cent. \\
\hline H. . & $9 \cdot 19$ & $9 \cdot 28$ & " & $8 \cdot 64$ & , \\
\hline $0 \ldots$ & $9 \cdot 75$ & 9.53 & , & $9 \cdot 88$ & ", \\
\hline
\end{tabular}
$\mathrm{H}_{2} \mathrm{O}$.

This ketone is formed from ethylic propylbenzoylacetate by hydrolysis and splitting off of carbonic anhydride according to the following equation :- 


\section{$\mathrm{C}_{6} \mathrm{H}_{5} \cdot \mathrm{CO} \cdot \mathrm{CH}\left(\mathrm{C}_{3} \mathrm{H}_{7}\right) \cdot \mathrm{COOC}_{2} \mathrm{H}_{5}+2 \mathrm{KOH}=\mathrm{C}_{6} \mathrm{H}_{5} \cdot \mathrm{CO} \cdot \mathrm{CH}_{2} \cdot \mathrm{C}_{3} \mathrm{H}_{7}+$ $\mathrm{K}_{2} \mathrm{CO}_{3}+\mathrm{C}_{2} \mathrm{H}_{5} \cdot \mathrm{OH}$.}

It is a colourless oil of agreeable aromatic odour, and boils at 236$238^{\circ}$ (720 mm.) without decomposition.

\section{Action of Phosphorus Pentachloride on Ethylic Propylbenzoylacetate.}

It was next thought interesting to try the action of phosphorus pentachloride on ethylic propylbenzoylacetate. 10 grams of the sub-

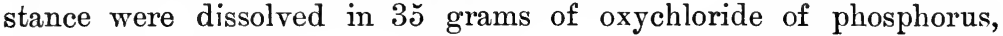
18 grams of phosphorus pentachloride were slowly added, and the whole allowed to remain until the principal reaction was over. The mass was then heated on a water-bath for half an hour, poured into ice, and from time to time stirred round until the oxychloride of phosphorus was completely decomposed. After extracting with ether, the ethereal solution was treated with dilute sodic hydrate, then dried over calcic chloride and the ether distilled off. In this way, a heavy yellow oil was obtained which after repeated fractioning in a vacuum - was obtained pure as a colourless oil boiling between $247^{\circ}$ and $249^{\circ}$ (300 mm.).

On analysis the following numbers were obtained:-

I. 0.2251 gram substance gave 0.5480 gram $\mathrm{CO}_{2}$ and 0.1438 gram $\mathrm{H}_{2} \mathrm{O}$.

II. 0.2085 gram substance gave 0.5075 gram $\mathrm{CO}_{2}$ and 0.1228 gram $\mathrm{H}_{2} \mathrm{O}$.

III. 0•1330 gram substance gave 0.0755 gram $\mathrm{AgCl}$.

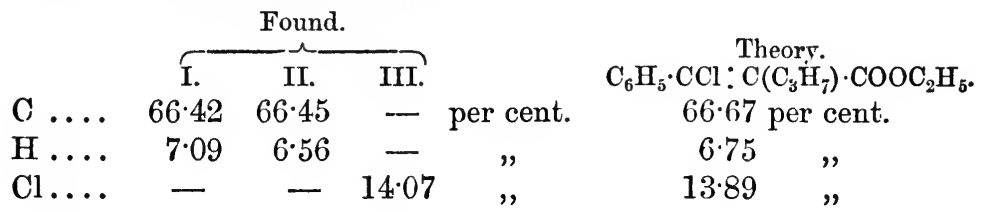

This substance was therefore ethylic $\alpha$-propyl- $\beta$-chlorocinnamate, formed according to the equation-

$$
\begin{aligned}
& \mathrm{C}_{6} \mathrm{H}_{5} \cdot \mathrm{CO} \cdot \mathrm{CH}\left(\mathrm{C}_{3} \mathrm{H}_{7}\right) \cdot \mathrm{COOC}_{2} \mathrm{H}+\mathrm{PCl}_{5}= \\
& \quad \mathrm{C}_{6} \mathrm{H}_{5} \cdot \mathrm{CCl}: \mathrm{C}\left(\mathrm{C}_{3} \mathrm{H}_{7}\right) \cdot \mathrm{COOC}_{2} \mathrm{H}_{5}+\mathrm{POCl}_{3}+\mathrm{HCl} .
\end{aligned}
$$

The alkaline solution, formed by treating the ethereal solution of the crude product of the action of phosphorus pentachloride on ethylic propylbenzoylacetate with sodic hydrate was next examined. On acidifying with dilute sulphuric acid, Jellow flakes were precipitated, which were collected and well washed with water. They were 
then dried on a porous plate and purified by several recrystallisations from light petroleum.

The following analytical results showed that the compound is $\alpha$-propyl $\beta$-chlorocinnamic acid :-

I. $0 \cdot 2030$ gram substance gave $0 \cdot 4738$ gram $\mathrm{CO}_{2}$ and $0 \cdot 1022$ gram $\mathrm{H}_{2} \mathrm{O}$.

II. 0.2246 gram substance gave 0.5289 gram $\mathrm{CO}_{2}$ and 0.1156 gram $\mathrm{H}_{2} \mathrm{O}$.

III. 0.2839 gram substance gave 0.1789 gram $\mathrm{AgCl}$.

Found.

\begin{tabular}{|c|c|c|c|c|c|}
\hline \multirow[b]{3}{*}{ C ... } & & & \multirow{3}{*}{$\begin{array}{c}\text { Theory. } \\
\mathrm{C}_{6} \mathrm{H}_{5} \cdot \mathrm{CCl}: \mathrm{C}\left(\mathrm{C}_{3} \mathrm{H}_{7}\right) \cdot \mathrm{COOH} \text {. } \\
64 \cdot 29 \text { per cent. }\end{array}$} \\
\hline & I. & II. & III. & & \\
\hline & $63 \cdot 66$ & $64: 23$ & - & per cent. & \\
\hline & $5 \cdot 62$ & $5 \cdot 72$ & - & , & $5 \cdot 80$ \\
\hline & - & - & $15 \cdot 39$ & , & $15 \cdot 63$ \\
\hline
\end{tabular}

$\alpha$-Propyl- $\beta$-chlorocinnamic acid melts at $121^{\circ}$, and sublimes in long colourless needles when carefully heated. It is easily soluble in aicohol, ether, chloroform, benzene, and acetic acid, more sparingly in cold light petroleum. By slowly cooling its solution in boiling light petroleum, it is obtained in beautiful prisms, which Professor Haushofer was kind enough to measure. He gave us the following description :-

Crystalline System. Triclinic.

$$
\begin{aligned}
& a: b: c:=0.7967: 1: 0.7403 \\
& a=122^{\circ} 33^{\prime} \\
& b=106 \quad 21 \\
& c=69 \quad 25 \\
& \infty \breve{\mathrm{P}} \infty(0 \overline{\mathbf{I}} 0)=b \\
& \infty \overline{\mathrm{P}} \infty(100)=a \\
& \text { OP }(001)=c \\
& \infty \mathrm{P}_{1}^{\prime} \quad(110)=p \\
& \text { ' } \overline{\mathrm{P}}^{\prime} \text { cs }(101)=r
\end{aligned}
$$

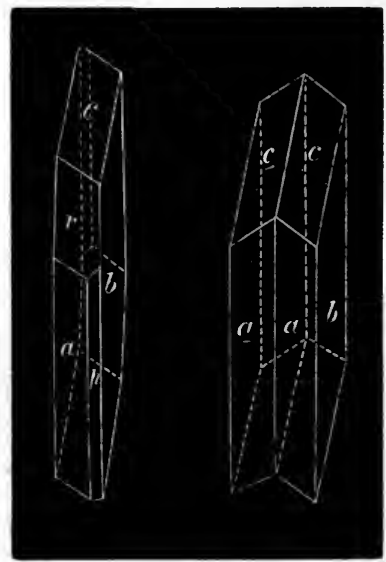

It forms tabular shaped twinned crystals on the surface $h$, with which the two surfaces $a$ form a projecting angle in front, and a re-entering angle behind of $151^{\circ} 22^{\prime}$ (measurement $151^{\circ} 22-42^{\prime}$ ). The surfaces $c$ form a projecting angle above and below a re-entering 
angle of $121^{\circ} 19^{\prime}$. The re-entering angle between the surfaces $c$ could not be measured, as the crystals were irregular at the ends.

The surfaces $b$ are generally curved, and not adapted for measurement, the edges in most of the crystals are broken up, but occasionally they are fully developed, and brilliant as the diamond. No single crystals were noticed.

The surfaces $p$ are usually wanting in the twins, often $r$ also.

\begin{tabular}{|c|c|c|c|c|}
\hline & \multicolumn{2}{|c|}{ Measured. } & \multicolumn{2}{|c|}{ Calculated. } \\
\hline$a: b=$ & $* 75^{\circ}$ & $41^{\prime}$ & - & - \\
\hline$a: c=$ & $* 96$ & 44 & - & - \\
\hline$b: c=$ & *119 & 16 & - & - \\
\hline$a: r=$ & *133 & 52 & - & - \\
\hline$b: p=$ & *140 & 39 & - & - \\
\hline$r: b=$ & 101 & 43 & $101^{\circ}$ & $56^{\prime}$ \\
\hline$c: c=$ & 121 & 19 & 121 & 19 (in the twins). \\
\hline
\end{tabular}

In convergent polarised light two systems of rings are observed on the surface $b$ of the twins, the optical axes of which belong to both the twins.

The silver salt of the acid, prepared by precipitating a neutral solution of the ammonium salt with silver nitrate, is a thick white amorphous mass, almost insoluble in water. The analysis gave the following numbers :-

0.4945 gram substance gave 0.216丂 gram $\mathrm{AgCl}$.

$$
\begin{aligned}
& \text { Found. } \\
& \text { Ag..... } 32 \cdot 95 \text { per cent. } \\
& \mathrm{C}_{6} \mathrm{H}_{5} \cdot \mathrm{CCl}: \mathrm{C}\left(\mathrm{C}_{3} \mathrm{H}_{7}\right) \cdot \mathrm{COOAg} \text {. } \\
& 32 \cdot 25 \text { per cent. }
\end{aligned}
$$

Ethylic Isopropylbenzoylacetate.

In order to obtain this substance, 50 grams of ethylic benzoylacetate were heated with 6 grams of sodium and an excess of isopropylic iodide for six hours at $100^{\circ}$. Water was then added, and the product isolated in the usual manner. On distilling the crude substance, almost all went over at $225-245^{\circ}(225 \mathrm{~mm}$.), and on repeated refractioning an oil was obtained boiling constantly at $236-237^{\circ}$ (225 mm.), which gave the following numbers on analysis:-

0.2760 gram substance gave 0.7230 gram $\mathrm{CO}_{2}$ and $0 \cdot 188$ gram $\mathrm{H}_{2} \mathrm{O}$.

Found.

C ..... 71.45 per cent.

H ..... $7 \cdot 61$,

O .... 20.94,
Theory. $\mathrm{C}_{6} \mathrm{H}_{5} \cdot \mathrm{CO} \cdot \mathrm{CH}\left[\mathrm{C}\left(\mathrm{CH}_{3}\right)_{2}\right] \cdot \mathrm{COOC}_{2} \mathrm{H}_{5}$. $71 \cdot 79$ per cent.

$7 \cdot 70 \quad$, $20 \cdot 51$ 
Ethylic isopropylbenzoylacetate is an agreeably smelling oil. Its boiling point is about $2^{\circ}$ lower than that of the normal propylic derivative previously described.

\section{Isobutyl Phenyl Ketone.}

This ketone was prepared by digesting ethylic isopropylbenzoylacetate with dilute alcoholic potash for about six hours. The brownishcoloured product was then diluted with water and several times extracted with ether. After distilling off the ether, a dark-coloured oil was left, which was easily obtained colourless by distillation. On repeatedly refractioning it, it boiled constantly at $227-228^{\circ}(720 \mathrm{~mm}$.), and gave on analysis the following numbers agreeing with the formula $\mathrm{C}_{6} \mathrm{H}_{5} \cdot \mathrm{CO} \cdot \mathrm{CH}_{2} \cdot \mathrm{CH}\left(\mathrm{CH}_{3}\right)_{2}$ :-

0.1605 gram suhstance gave 0.4775 gram $\mathrm{CO}_{2}$ and 0.1315 gram $\mathrm{H}_{2} \mathrm{O}$.

\begin{tabular}{|c|c|c|}
\hline C & $\begin{array}{l}\text { Found. } \\
81 \cdot 14 \text { per cent. }\end{array}$ & $\begin{array}{l}\text { Theory. } \mathrm{C}_{11} \mathrm{H}_{14} \mathrm{O} \text {. } \\
81.48 \text { per cent. }\end{array}$ \\
\hline H .. & $9 \cdot 10$ & $8 \cdot 64$ \\
\hline $0 \ldots \ldots$ & $9 \cdot 76$ & $9 \cdot 87$ \\
\hline
\end{tabular}

Isobutyl phenyl ketone is a colourless oil, of an agreeable aromatic odour.

This ketone has already been prepared by Popow (Annalen, 162, 153) by distilling a mixture of benzoate and valerate of calcium. Popow gives the boiling point as $225-226^{\circ}$.

Isobutyl phenyl ketone does not combine with hydrogen sodium sulphite. On oxidation with chromic acid, benzoic, isobutyric, and acetic acids are formed (Popow).

\section{Ethylic Isobutylbenzoylacetate.}

This substance was prepared in exactly the same way as the isopropyl-derivative by heating ethylic benzoylacetate ( 1 mol.) with sodic ethylate $\left(1 \mathrm{~mol}\right.$.) and an excess of isobutylic iodide at $100^{\circ}$ for six hours. On fractioning the product, almost the whole of it went over between $230^{\circ}$ and $260^{\circ}(225 \mathrm{~mm}$.) in the first distillation. Pure ethylic isobutylbenzoylacetate boils at $246-247^{\circ}(225 \mathrm{~mm}$.).

The iollowing results were obtained on analysis :-

0.2257 gram substance gave 0.5960 gram $\mathrm{CO}_{2}$ and 0.1620 gram $\mathrm{H}_{2} \mathrm{O}$.

$$
\begin{array}{lll}
\multicolumn{4}{c}{} & \text { Found. } & \mathrm{C}_{6} \mathrm{H}_{5} \cdot \mathrm{CO} \cdot \mathrm{CH}\left[\mathrm{CH}_{2} \cdot \mathrm{CH}\left(\mathrm{CH}_{3}\right)_{2}\right] \cdot \mathrm{COOC}_{2} \mathrm{H}_{5} \cdot \\
\mathrm{C} & . \ldots 2 \cdot 01 \text { per cent. } & 72 \cdot 58 \text { per cent. }
\end{array}
$$$$
\begin{array}{rrrr}
\text { H } \ldots . . . & 7.97 & , & 8.06 \\
\text { O } \ldots . . . & 20.02 & 19.35
\end{array}
$$

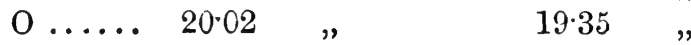


This ethereal salt has much the same properties as the isopropylderivative. The boiling point is about $10^{\circ}$ higher.

\section{Isoamyl Phenyl'Ketone.}

Ethylic isobutylbenzoylacetate is easily split up on heating it with dilute alcoholic potash into alcohol, carbonic anhydride, and isoamyl phenyl ketone, thus :-

$\mathrm{C}_{6} \mathrm{H}_{5} \cdot \mathrm{CO} \cdot \mathrm{CH}\left[\mathrm{CH}_{2} \cdot \mathrm{CH}\left(\mathrm{CH}_{3}\right)_{2}\right] \cdot \mathrm{C}^{1} \mathrm{COC}_{2} \mathrm{H}_{5}+2 \mathrm{KOH}$

$$
=\mathrm{C}_{6} \mathrm{H}_{5} \cdot \mathrm{CO} \cdot \mathrm{CH}_{2} \cdot \mathrm{CH}_{2} \cdot\left[\mathrm{CH}\left(\mathrm{CH}_{3}\right)_{2}\right]+\mathrm{K}_{2} \mathrm{CO}_{3}+\mathrm{C}_{2} \mathrm{H}_{5} \cdot \mathrm{OH} \text {. }
$$

The product of this reaction was isolated in the usual manner, and purified by fractioning. Isoamyl phenyl ketone is an aromatic smelling oil, boiling at $240-241^{\circ}(720 \mathrm{~mm}$.). Analysis :-

$0 \cdot 1870$ gram substance gave 0.561 gram $\mathrm{CO}_{2}$ and 0.156 gram $\mathrm{H}_{2} \mathrm{O}$.

\begin{tabular}{|c|c|c|}
\hline & $\begin{array}{l}\text { Found. } \\
81.82 \text { per cent. }\end{array}$ & $\begin{array}{l}\text { Theory. } \\
\mathrm{C}_{6} \mathrm{H}_{5} \cdot \mathrm{CO} \cdot \mathrm{CH}_{2} \cdot \mathrm{CH} H_{2} \cdot \mathrm{CH}\left(\mathrm{CH}_{3}\right)_{2} \cdot \\
81 \cdot 82 \text { per cent. }\end{array}$ \\
\hline & $9 \cdot 27$ & $9 \cdot 09$ \\
\hline & $8 \cdot 91$ & $9 \cdot 09$ \\
\hline
\end{tabular}

In Part III of this research (Trans., 1885, 265-266) an acid, $\mathrm{C}_{18} \mathrm{H}_{12} \mathrm{O}_{5}$, was described, which was obtained in small quantity from ethylic dibenzoylsuccinuce by prolonged boiling with dilute sulphuric acid, and which from its method of formation was thought to be similarly constituted to carbopyrotritartaric acid.

This appeared the more probable from a small experiment which was tried at the time (loc. cit., p. 271) on the action of concentrated sulphuric sulphuric acid on ethylic dibenzoylsuccinate; a compound was produced which on hydrolysis yielded the acid $\mathrm{C}_{18} \mathrm{H}_{12} \mathrm{O}_{5}$ quantitatively, and was therefore probably its ethereal salt. In order to determine the nature of this decomposition, the experiment has since been carried out on a larger scale, in the following way:-

Finely-powdered ethylic dibenzoylsuccinate was shaken with about 10 times its weight of pure concentrated sulphuric acid, in which it soon dissolved, forming a colourless solution. This was allowed to remain for 12 hours, and then poured on to ice, when a thick almost colourless oil was precipitated, and was separated from the acid liquid by extracting it with ether. The ethereal solution was then well washed with water, and dried over calcic chloride. On distilling off the ether, an almost colourless oil remained, which instantly became solid on being touched with a glass rod. The mass was then dissolved in hot alcohol, from which it crystallised on cooling in beautiful canaryyellow prisms.

The analysis gave the following numbers:- 
I. 0.1698 gram substance gave 0.4505 gram $\mathrm{CO}_{2}$ and 0.0900 gram $\mathrm{H}_{2} \mathrm{O}$.

II. 0.2250 gram substance gave 0.5974 gram $\mathrm{CO}_{2}$ and 0.1134 gram $\mathrm{H}_{2} \mathrm{O}$.

\begin{tabular}{|c|c|c|c|c|c|}
\hline \multirow[b]{3}{*}{ C .... } & & \multirow{2}{*}{\multicolumn{2}{|c|}{$\begin{array}{l}\text { Theory. } \\
\mathrm{C}_{12} \mathrm{H}_{20} \mathrm{O}_{5} \text {. }\end{array}$}} \\
\hline & I. & II. & & & \\
\hline & $72 \cdot 36$ & $72 \cdot 41$ & cent. & $72 \cdot 53$ & cent \\
\hline H. & $5 \cdot 89$ & $5 \cdot 60$ & $"$ & $5 \cdot 89$ & , \\
\hline o $\ldots$. & $21 \cdot 75$ & $21 \cdot 99$ & $"$ & 21.98 & ", \\
\hline
\end{tabular}

This substance contains 1 mol. $\mathrm{H}_{2} \mathrm{O}$ less than ethylic benzoylsuccinate, and appears to be formed according to the following equations :-<smiles>CCCCC(C(=O)OCC)C(C(=O)OCC)C(=O)OCC</smiles>

Ethylic dibenzoylsuccinate.

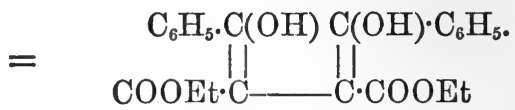

Labile form of the same.

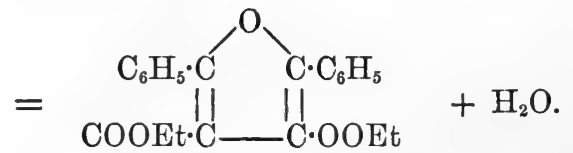

Ethylic diphenylfurfurandicarboxylate $(1,4,2,3)$.

Ethylic diphenylfurfurandicarboxylate $[1,4,2,3]$ melts at $86^{\circ}$, and is characterised particularly by the readiness with which it crystallises. It is easily soluble in chloroform, benzene, ether, alcohol, and light petroleum, and can be obtained in beautiful almost colour. less prisms, by allowing the ethereal solution to evaporate slowly. Prof. Haushofer was kind enough to measure these. He gave me the following description of these crystals :-

Crystalline System. Rhombic.

$$
a: b: c=0.9005: 1: 0.6309
$$

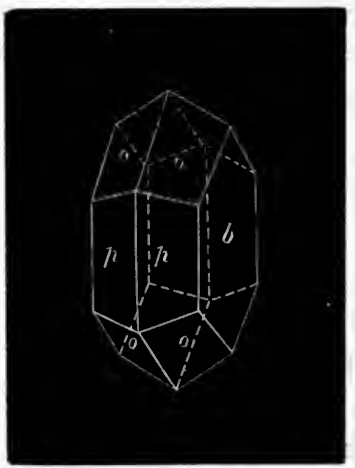


Pale sulphur-yellow transparent crystals of the combinations $\mathrm{P}(111)=o, \infty \breve{\mathrm{P}} \frac{1}{2}(210)=p, \infty \breve{\mathrm{P}} \propto(010)=b$. Short prismatic in the vertical axis, usually tabular through development of the surfaces $b$.

\begin{tabular}{|c|c|c|c|c|}
\hline $0: 0=(111)(11 \overline{1})=$ & $\begin{array}{l}\text { Calc } \\
* 86^{\circ}\end{array}$ & $\begin{array}{l}\text { lated. } \\
38^{\prime}\end{array}$ & - & - (Basal angle). \\
\hline$o: o=(111)(1 \overline{1} 1)=$ & *118 & 42 & 一 & $\begin{array}{l}\text { - (Macrodiagonal. Polar } \\
\text { angle). }\end{array}$ \\
\hline$o: o=(111)(1 \overline{1} 1)=$ & 125 & 10 & $125^{\circ}$ & $\begin{array}{c}20^{\prime} \text { (Brachydiagonal. Polar } \\
\text { angle). }\end{array}$ \\
\hline$p: p^{\prime}=(210)(2 \overline{1} 0)=$ & 131 & 32 & 131 & 35 \\
\hline$o: p=(111)(210)=$ & 130 & 37 & 130 & 47 \\
\hline$o: p^{\prime}=(111)(2 \overline{1} 0)=$ & 106 & 3 & 106 & 50 \\
\hline
\end{tabular}

The base of the plane the optical axis. In convergent polarised light the interference image of an axis (excentric in the direction of the angle $p b$ ) appears on the surface $p$.

When heated in small quantities, this ethereal salt distils almost without decomposition. Although the solution in chloroform does not decolorise bromine at ordinary temperatures, it does so easily on warming, hydrobromic acid being liberated. When treated with nitric acid, beautifully crystalline nitro-derivatives are formed. Ethylic diphenylfurfurandicarboxylate is not acted on by phosphorus pentachloride at ordinary temperatures. It dissolves in concentrated sulphuric acid, forming a colourless solution, which on heating becomes first brownish and then of a beautiful violet colour.

\section{Diphenylfurfurandicarboxylic Acid $[1,4,2,3]$.}

When ethylic dibenzoylsuccinate is boiled with dilute sulphuric acid, a small quantity of an acid is formed, which from previous analysis (Trans., 1885, 267) was found to have the formula $\mathrm{C}_{18} \mathrm{H}_{12} \mathrm{O}_{5}$.

Owing to the small yield obtained by this reaction, it was only superficially examined. The same acid can, howerer, be obtained quantitatively by the hydrolysis of ethylic diphenylfurfurandicarboxylate with alcoholic potash. It is only necessary to digest the ethereal salt 15 minutes with an excess of alcoholic potash, to completely saponify it. At the end of the reaction, water is added, and the whole evaporated until all the alcohol has been driven off. On acidifying with dilute sulphuric acid, the acid is thrown down in white flakes. After collecting and recrystallising it from acetic acid (70 per cent.), it is easily obtained pare in colourless needles, melting at $238^{\circ}$.

The following analyses confirm the formula previously assigned to this acid. 
I. $0 \cdot 1762$ gram substance gave 0.4523 gram $\mathrm{CO}_{2}$ and 0.0637 gram $\mathrm{H}_{2} \mathrm{O}$.

II. $0 \cdot 1848$ gram substance gave 0.4735 gram $\mathrm{CO}_{2}$ and 0.0726 gram $\mathrm{H}_{2} \mathrm{O}$.

\begin{tabular}{|c|c|c|c|}
\hline & \multicolumn{2}{|c|}{ Found. } & \multirow[b]{2}{*}{$\begin{array}{l}\text { Theory. } \\
\mathrm{C}_{18} \mathrm{H}_{12} \mathrm{O}_{5} \text {. }\end{array}$} \\
\hline & I. & II. & \\
\hline & $70 \cdot 01$ & 69.89 per cent. & 70.13 per cent. \\
\hline & $4 \cdot 01$ & $4 \cdot 36$ & $3 \cdot 89$ \\
\hline $\mathrm{O}$ & $25 \cdot 98$ & $25 \cdot 75$ & $25 \cdot 97$ \\
\hline
\end{tabular}

This compound is therefore diphenylfurfurandicarboxylic acid,

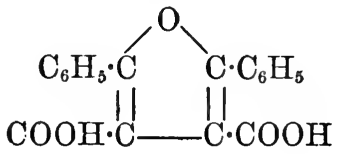

It melts at $238^{\circ}$ to a colourless liquid, which when strongly heated splits up into the compound $\mathrm{C}_{18} \mathrm{H}_{10} \mathrm{O}_{4}$ and water. This was described in Part III of this research (Trans., 1885, 268), and appears to be the anhydride of diphenylfurfurandicarboxylic acid,*

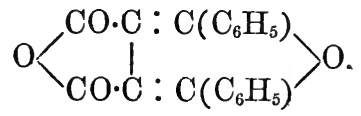

According to this interpretation, the re-formation of the acid $\mathrm{C}_{18} \mathrm{H}_{12} \mathrm{O}_{5}$ by treating this body $\mathrm{C}_{18} \mathrm{H}_{10} \mathrm{O}_{4}$ with alcoholic potash can be easily nnderstood.

The salts of diphenylfurfurandicarboxylic acid were described in Part III of this research (Trans., 1885, 268), and the analyses of the silver and calcium salts given. Owing to the small quantity of acid which could be prepared at that time, no satisfactory results were obtained by the analysis of these salts. The experiments were therefore repeated with larger quantities of material. The silver salt was prepared by fractional precipitation of the ammonium salt with silver nitrate, and analysed with the following results :-

I. 0.2235 gram substance gave 0.3380 gram $\mathrm{CO}_{2}, 0.0520$ gram $\mathrm{H}_{2} \mathrm{O}$, and 0.0925 gram Ag.

II. 0.2010 gram substance gave 0.3060 gram $\mathrm{CO}_{2}, 0.040$ gram $\mathrm{H}_{2} \mathrm{O}$, and 0.0833 gram Ag.

III. 0.2750 gram substance gave 0.1130 gram Ag.

* This is also the opinion of Knorr (Ber., 17, 2869). 
Found.

\begin{tabular}{lrrrrr} 
& \multicolumn{1}{c}{ I. } & \multicolumn{1}{c}{ II. } & III. & \\
$\mathrm{C} \ldots \ldots$ & $41 \cdot 25$ & $41 \cdot 51$ & - & p. c. \\
$\mathrm{H} \ldots \ldots$ & $2 \cdot 59$ & $2 \cdot 21$ & - & $"$ \\
$\mathrm{Ag} \ldots$ & $41 \cdot 39$ & $41 \cdot 44$ & $41 \cdot 09$ & $"$ \\
$\mathrm{O} \ldots$. & $14 \cdot 77$ & $14 \cdot 84$ & - & $"$
\end{tabular}

Old analyses.
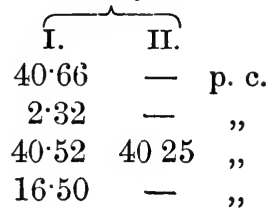

$$
\begin{array}{ccc}
\multicolumn{3}{c}{\text { Theory. } \mathrm{C}_{18} \mathrm{H}_{10} \mathrm{O}_{5} \mathrm{Ag}_{2} \text {. }} \\
\text { C....... } & 41 \cdot 44 \text { per cent. } \\
\mathrm{H} \ldots \ldots \ldots & 1 \cdot 91 \quad, \\
\mathrm{Ag} \ldots \ldots & 41 \cdot 29 \quad, \\
\mathrm{O} . \ldots \ldots & 15 \cdot 35 \quad,
\end{array}
$$

Diphenylfurfurandicarboxylic acid is therefore a simple bibasic acid forming salts of the formula $\mathrm{C}_{18} \mathrm{H}_{10} \mathrm{O}_{5} \mathrm{M}_{2}$.

From the method of formation of this acid it would at first sight appear probable that its constitution was exactly similar to that of carbopyrotritartaric acid, which Knorr has shown to be produced by the action of concentrated sulphuric acid on ethylic diacetylsuccinnate:

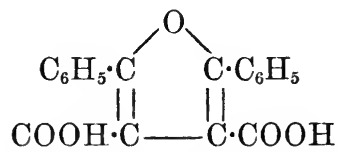

Diphenylfurfurandicarboxylic acid.

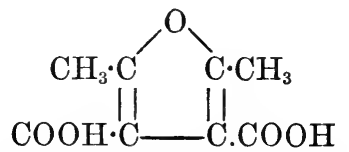

Dimethylfurfurandicarboxylic acid

(carbopy rotritartaric acid).

There is, however, one great difference between these acids; carbopyrotartaric acid is easily decomposed on heating above its melting point into pyrotritartaric acid and carbonic anhydride,

$$
\mathrm{C}_{8} \mathrm{H}_{8} \mathrm{O}_{5}-\mathrm{CO}_{2}=\mathrm{C}_{7} \mathrm{H}_{8} \mathrm{O}_{3},
$$

whereas diphenylfurfurandicarboxylic acid is simply converted into its anhydride, as described above, the monocarboxylic acid corresponding with pyrotritartaric acid not having as yet been obtained.

Lately Fittig (Ber., 18, 3410) has made it doubtful whether carbopyrotritartaric and pyrotritartaric acids really are furfurane-derivatives.

In his researches on the condensation of ethylic acetoacetate with succinic acid, he obtained an acid isomeric with carbopyrotritartaric acid, according to the equation-

$\mathrm{CH}_{3} \cdot \mathrm{CO} \cdot \mathrm{CH}_{2} \cdot \mathrm{COOC}_{2} \mathrm{H}_{5}+\mathrm{COOH} \cdot \mathrm{CH}_{2} \cdot \mathrm{CH}_{2} \cdot \mathrm{COOH}$

$$
=\mathrm{C}_{8} \mathrm{H}_{8} \mathrm{O}_{5}+\mathrm{H}_{2} \mathrm{O}+\mathrm{C}_{2} \mathrm{H}_{5} \cdot \mathrm{OH} \text {. }
$$

Methronic acid. 
which at a temperature of $200-240^{\circ}$ is converted quantitatively into pyrotritartaric acid and carbonic anhydride.

In this way, it is difficult to conceive how pyrotritartaric acid can contain two methyl groups, and Fittig therefore supposes that in the formation of ethylic carbopyrotritartrate from ethylic diacetylsuccinate condensation takes place in a way analogous to the formation of mesityl oxide from acetone.

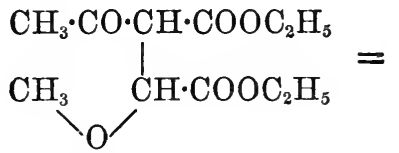

Ethylic diacetylsuccinate.

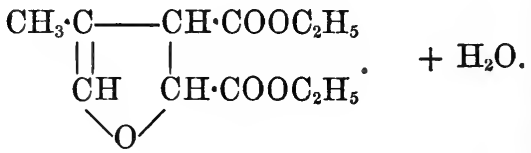

Ethylic carbopyrotritartrate.

Pyrotritartaric acid would then have the constitution-

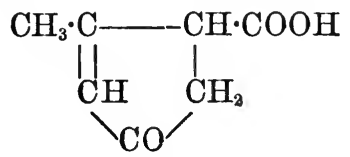

It is therefore probable that the acids obtained from ethylic diacetylsuccinate and dibenzoylsuccinate by the same reaction, i.e., by the action of dehydrating agents, are of an entirely different structure, the former being derivatives of pentamethylene, the latter of furfurane. From the experiments described above, it is scarcely possible that the acid $\mathrm{C}_{18} \mathrm{H}_{12} \mathrm{O}_{5}$ can be a lactonic acid, as Fittig believes (Ber., 18, 3411).

In conclusion, we append for convenience sake tables of the boiling points of the homologues of ethylic benzoylacetate obtained in the course of this research, and of the homologues of acetophenone prepared by the hydrolysis of these ethereal salts.

Boiling points of the Ethylic Salts of Benzoylacetic Acid and its Homologues under $225 \mathrm{~mm}$. pressure.

$\begin{array}{ccc}\text { Ethylic benzoylacetate ............. } & 220-225^{\circ} \\ , \quad \quad \text { methyl benzoylacetate....... } & 226-227 \\ " \quad \text { ethyl benzoylacetate........ } & 231-232 \\ " \quad \text { propyl benzoylacetate ........ } & 238-239 \\ " \quad \text { isopropyl benzoylacetate..... } & 236-237 \\ " \quad \text { allyl benzoylacetate. ....... } & 240-241 \\ \text { Methylic benzylbenzoylacetate....... } & 250-255(?)\end{array}$

(50 mm. pressure). 
Boiling point under $720 \mathrm{~mm}$. pressure.

Acetophenone........... 200.

Ethyl phenyl ketone ....... 210

Propyl phenyl ketone ..... 220-222

Allylacetophenone........ 235-238

Butyl phenyl ketone ....... 236-238

Isobutyl phenyl ketone...... 227-228

Diethylacetophenone....... 229--231

Isoamyl phenyl ketone ..... . 240-241

Further experiments on ethylic benzoylacetate, and more especially on the behaviour of ethylic dibenzoylsuccinate with different reagents, are in progress. 



\section{PARANITROBENZOYLACETIC ACID AND SOME OF ITS DERIVATIVES.}

By W. H. Perkin (Jun.), Ph.D., and Gostav Bellenot, Ph.D.

Is previous researches on ethylic benzoylacetate, it has been shown that it is not only capable of entering into almost all the reactions in which ethylic acetoacetate takes part, but moreover has the great advantage that its derivatives, being aromatic substances, have a much greater tendency to crystallise than those of the acetoacetate.

During the study of some of the more complicated derivatives of ethylic benzoylacetate, and more especially the benzoyl-derivatives of trimethylene and tetramethylene, it was found necessary, in order to explain the formation of these compounds, that not only the acids themselves, but also their ethereal salts should be solid, but this is not always the case when ethylic benzoylacetate is employed.

It seemed, therefore, if a nitro-derivative of ethylic benzoylacetate could be employed as the starting point instead of the ethyl salt itself, that this difficulty could be avoided, and such is, in fact, the case.

Experiments were first made with the object of nitrating ethylic benzoylacetate directly, but as it is difficult to obtain the latter free from acetophenone, benzoic acid, and other impurities, no satisfactory results could be obtained, and this node of procedure was abandoned.

It was, however, soon discovered that paranitrobenzoylacetic acid could easily be prepared by a process analogous to that for the formation of benzoylacetic acid from phenylpropiolic acid; that is, by treating paranitrophenylpropiolic acid with concentrated sulphuric acid :-

\section{$\mathrm{NO}_{2} \cdot \mathrm{C}_{6} \mathrm{H}_{4} \cdot \mathrm{C}: \mathrm{C} \cdot \mathrm{COOH}+\mathrm{H}_{2} \mathrm{O}=\mathrm{NO}_{2} \cdot \mathrm{C}_{6} \mathrm{H}_{4} \cdot \mathrm{CO} \cdot \mathrm{CH}_{2} \cdot \mathrm{COOH}$ \\ Paranitrophenylpropiolic acid. Paranitrobenzoylacetic acid.}

This fact is all the more interesting when it is remembered that ethylic orthonitrophenylpropiolate behaves so differently when treated in the same way. Baeyer (Ber., 14, 174), who studied this reaction, has shown that on treating the ortho-derivative with sulphuric acid, a simple intramolecular change takes place, ethylic isatogenate being formed :-

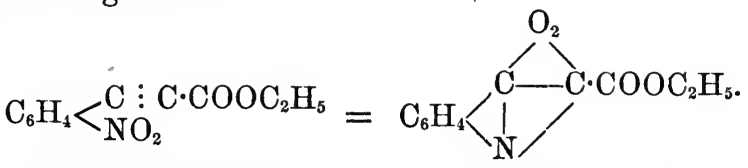

Ethylic orthonitrophenyl-

Ethylic isatogenate. propiolate. 
It seemed of interest, therefore, to prepare some of the derivatives of paranitrobenzoylacetic acid, an account of which we now desire to lay before the Society.

We have divided this research into the following sections :-

(1.) Paranitrophenylpropiolic acid.

(2.) Paranitrobenzoylacetic acid.

(3.) Methylic paranitrobenzoylacetate.

(4.) Methylic benzylparanitrobenzoylacetate.

(5.) Ethylic paranitrobenzoylacetate.

(6.) Ethylic nitrosoparanitrobenzoylacetate.

(7.) Ethylic ethylparanitrobenzoylacetate.

(8.) Ethylic allylparanitrobenzoylacetate.

(9.) Ethylic di-paranitrobenzoylsuccinate.

\section{(1.) Paranitrophenylpropiolic Acid.}

Paranitrocinnamic acid and its derivatives were first carefully examined by Drewsen (Annalen, 212, 150).

If this acid is treated with bromine, it easily takes up 1 mol. $\mathrm{Br}$ exactly in the same way as cinnamic acid does, and is converted into paranitrophenyldibromopropionic acid, $\mathrm{NO}_{2} \cdot \mathrm{C}_{6} \mathrm{H}_{4} \cdot \mathrm{CHBr} \cdot \mathrm{CHBr} \cdot \mathrm{COOH}$. If this dibromo-acid is treated with alkalis, a curious reaction takes place, no $\alpha$ - or $\beta$-monobromo-acid being formed as in the case of dibromophenylpropionic acid, but the dibromide is split up partly into paranitrophenylpropiolic acid and partly into the original paranitrocinnamic acid, bromine being given off. In preparing paranitrophenylpropiolic acid by this method, the separation of these two acids is not only a matter of considerable difficulty, but the yield of acid obtained is very small. It was found, however, that better results could be obtained by using the dibromide of the ethereal salt instead of the free acid.

The following method of preparation is based on that proposed by Drewsen :-

Ethylic paranitrophenyldibromopropionate is first prepared by mixing pure ethylic paranitrocinnamate with a slight excess of bromine. On pounding the substances together, the ethereal salt dissolves in the bromine, and in a short time the whole solidifies to a brown cake of impure ethylic paranitrophenyldibromopropionate. This is reduced to powder, spread out in the air till the excess of bromine has evaporated, and then crystallised once from alcohol. In this way it is obtained quite pure in colourless crystals, and can be used directly for the preparation of paranitrophenylpropiolic acid.

For this purpose, the crystals are dissolved in a small quantity of 
boiling alcohol, and the calculated amount of a hot alcoholic potash solution poured in until the mixture has a decidedly alkaline reaction. As soon as the reaction is finished, a slight excess of potash causes the solution to become brownish coloured, probably from the formation of azo-derivatives. After remaining for an hour or so, about twice the volume of water is added, the whole filtered, and the paranitrophenylpropiolic acid precipitated by acidifying, with dilute sulphuric acid.

The precipitate being very difficult to collect, it is better to agitate the whole repeatedly with ether. By this means also a purer product is obtained, as the paranitrocinnamic acid which is always formed during the reaction is not dissolved by the ether. The ethereal solution is well washed with water and shaken with dilute potash solution, when the paranitrophenylpropiolic acid dissolves, leaving the paranitrophenylacetylene and paranitroacetophenone-which are always present-in the ethereal solution. The acid is then reprecipitated from the aqueous potash solution and again extracted with ether.

On distilling off the ether, the paranitrophenylpropiolic acid is obtained pure in small needles, and can be used directly for the preparation of paranitrobenzoylacetic acid.

\section{(2.) Paranitrobenzoylacetic acid.}

In order to prepare ethylic paranitrobenzoylacetate, experiments were first made on the action of sulphuric acid on ethylic paranitrophenylpropiolate. 50 grams of the pure ethereal salt were dissolved in 2 kilos. of concentrated sulphuric acid which had been diluted with about 40 c.c. of water, and the whole allowed to remain for several hours at the ordinary temperature. As no reaction appeared to take place at this temperature, experiments were made with a view of ascertaining the proper conditions for the transformation, and it was ultimately found that the addition of water took place best at $35-40^{\circ}$, and required at least 12 to 15 hours before the reaction was completed. On pouring the product on to ice, a dirty whitish flocculent precipitate was formed, which was collected, well washed with water, and dried on a porous plate. The addition of a drop of ferric chloride to the alcoholic solution of this substance gave a beautiful violet coloration, a proof that the reaction had really taken place.

It was soon discovered, however, that the compound formed was not the ethereal salt of paranitrobenzoylacetic acid, but the acid itself, the ethereal salt having undergone hydrolysis by the action of the sulphuric acid. All attempts to obtain the ethereal salt directly were in vain. 
As the yield of acid obtained in this way was not good, it was necessary in order to be able to prepare larger quantities to improve the method. After numerous experiments, the following-which we give in detail-was found to give the best results.

Pure paranitrophenylpropiolic acid (50 grams) is dissolved in 2-3 kilos. of $80-85$ per cent. sulphuric acid, and the whole kept at a temperature of $35-40^{\circ}$ for 15 hours. The solution which is at first yellowish-coloured becomes darker and darker, and is at last of a deep brown. In order to determine whether the reaction is finished a sample is taken out, mixed with ice and water, and extracted with ether. The residue left on distilling off the ether is dissolved in dilute ammonia and boiled for about two minutes; this causes the separation of oily drops of paranitroacetophenone, which on cooling become solid and can be filtered off. On acidifying the filtrate, any unchanged paranitrophenglpropiolic acid is precipitated, and from the amount of this it is easy to judge how far the reaction has progressed.

When it is found that the reaction is finished, the product is poured on to ice. This causes the paranitrobenzoylacetic acid to be precipitated in whitish flakes, which must be removed from the acid liquid as rapidly as possible by extraction with ether. The ethereal solution is well washed with water, dried over calcic chloride, and the ether distilled off until crystals begin to form; these consist of nearly pure paranitrobenzoylacetic acid. The filtrate from these crystals contains, besides this acid, unchanged paranitrophenylpropiolic acid and paranitroacetophenone; these can be separated by recrystallisation from benzene. Pure paranitrobenzolyacetic acid crystallises from this solvent in almost colourless microscopic needles.

Analysis :-

I. 0.2511 gram substance gave 0.0840 gram $\mathrm{H}_{2} \mathrm{O}$ and 0.4698 gram $\mathrm{CO}_{2}$.

II. $0 \cdot 2293$ gram substance gave $13^{\circ} 2$ c.c. $\mathrm{N}$ at $16^{\circ}$ and $720 \mathrm{~mm}$. III. $0.3165 " \quad " \quad$ " 19.0 c.c. $\mathrm{N}$ at $13^{\circ}$ and $709 \mathrm{~mm}$.

\begin{tabular}{|c|c|c|c|c|c|}
\hline \multirow[b]{3}{*}{ C.... } & \multicolumn{3}{|c|}{ Found. } & . & \multirow{2}{*}{$\begin{array}{l}\text { Theory. } \\
\mathrm{NO}_{2} \cdot \mathrm{C}_{6} \mathrm{H}_{4} \cdot \mathrm{CO} \cdot \mathrm{CH} \cdot \mathrm{COOH} .\end{array}$} \\
\hline & I. & II. & III. & & \\
\hline & $51 \cdot 02$ & - & - & per cent. & \\
\hline & $3 \cdot 72$ & - & - & 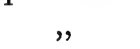 & $3 \cdot 34$ \\
\hline N.... & - & $6 \cdot 35$ & $6 \cdot 61$ & $"$ & $6 \cdot 69$ \\
\hline
\end{tabular}

This acid is formed from paranitrophenylpropiolic acid according to the equation-

$$
\mathrm{NO}_{2} \cdot \mathrm{C}_{6} \mathrm{H}_{4} \cdot \mathrm{C}: \mathrm{C} \cdot \mathrm{COOH}+\mathrm{H}_{2} \mathrm{O}=\mathrm{NO}_{2} \cdot \mathrm{C}_{6} \mathrm{H}_{4} \cdot \mathrm{CO} \cdot \mathrm{CH} \cdot \mathrm{COOH} \text {. }
$$


Paranitrobenzoylacetic acid melts at $135^{\circ}$, being at the same time decomposed into paranitroacetophenone and carbonic anhydride. It is easily soluble in alcohol, ether, bisulphide of carbon, and light petroleum, more sparingly in benzene. The alcoholic solution gives an intense reddish-brown coloration with ferric chloride.

If the finely powdered acid is warmed with water at $60^{\circ}$, it dissolves in considerable quantity and without much decomposition, crystallising out on cooling in four-sided plates. The hot aqueous solution is coloured deep reddish-brown on the addition of ferric chloride, the cold saturated solution, however, only very slightly so.

When boiled with water or dilute acids, the acid is rapidly decomposed into carbonic anhydride and paranitroacetophenone:-

$$
\mathrm{NO}_{2} \cdot \mathrm{C}_{6} \mathrm{H}_{4} \cdot \mathrm{CO} \cdot \mathrm{CH}_{2} \cdot \mathrm{COOH}=\mathrm{NO}_{2} \cdot \mathrm{C}_{6} \mathrm{H}_{4} \cdot \mathrm{CO} \cdot \mathrm{CH}_{3}+\mathrm{CO}_{2} \text {. }
$$

The preparation of pure salts of paranitrobenzoylacetic acid is a matter of great difficulty owing to their instability. The acid dissolves easily in dilute alkalis, with an intense yellow colour, the solution, however, soon becomes filled with needles of paranitroacetophenone.

The solution of the sodic salt was prepared by carefully neutralising the solution of the acid in sodic carbonate with dilute nitric acid. On the addition of silver nitrate to this, a light yellow amorphous silver salt was obtained, which was very unstable, however, and on analysis gave approximate numbers only.

The copper salt is a light green amorphous salt, which explodes if heated in the dry state.

The other salts are also very unstable.

\section{(3.) Methylic Paranitrobenzoylacetate.}

The methylic salt of paranitrobenzoylacetic acid is easily obtained by suspending the pure acid in absolute methyl alcohol, and saturating with hydrochloric acid gas, the whole being well cooled during the operation. In a short time, the methylic salt begins to crystallise out in yellow needles. After remaining for 12 hours at as low a temperature as possible, the crystals are collected and well washed with methyl alcohol. By one recrystallisation from methyl alcohol, they are easily obtained pure.

Analysis :-

$0 \cdot 2360$ gram substance gave 0.0926 gram $\mathrm{H}_{2} \mathrm{O}$ and $0 \cdot 4646$ gram $\mathrm{CO}_{2}$.

$\begin{array}{ccc} & \text { Found. } & \mathrm{NO}_{2} \cdot \mathrm{C}_{6} \mathrm{H}_{4} \cdot \mathbf{C O} \cdot \mathrm{CH}_{2} \cdot \mathrm{COOCH}_{3} \cdot \\ \mathrm{C} . \ldots . & 53 \cdot 68 & 53 \cdot 81 \\ \mathrm{H} \ldots \ldots & 4.36 & 4 \cdot 04\end{array}$


Methylic paranitrobenzoylacetate melts at $106-107^{\circ}$. It is only sparingly soluble in cold ethylic or methylic alcohol, but easily on boiling. It is easily soluble in light petroleum, benzene, and chloroform. The addition of a drop of ferric chloride to the alcoholic solution gives a brownish-violet coloration.

If the hot alcuholic solution of this ethereal salt be allowed to cool slowly, beautiful weli-formed crystals can be obtained. For the following description of these I am indebted to the kindness of Professor Haushofer.

Methylic Paranitrobenzoylacetate.

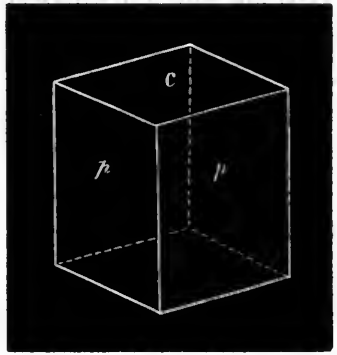

Crystalline Form. Monoclinic.

$$
\begin{aligned}
a: b: c & =0 \cdot 4993: 1 \text { (?). } \\
\beta & =56^{\circ} 5^{\prime} .
\end{aligned}
$$

The crystals form the prismatic twinned combination $0 \mathrm{P}(c), \infty \mathrm{P}(p)$ in the direction of the vertical axis.

$$
\begin{array}{r}
\text { Measured. } \\
p: p=* 135^{\circ} 0^{\prime} \\
c: p=* 121^{\circ} 3^{\prime}
\end{array}
$$

On the plane $p$, the angle made by the directions of extinction (with crossed Nicols) with the edge of the prism is about $15^{\circ}$.

Sodium compound, $\mathrm{NO}_{2} \cdot \mathrm{C}_{6} \mathrm{H}_{4} \cdot \mathrm{CO} \cdot \mathrm{CHNa} \cdot \mathrm{COOCH}_{3}$ - - If the solntion of methylic paranitrobenzoylacetate $(1 \mathrm{~mol}$.) in warm alcohol is treated with the calculated quantity ( 1 mol.) of a strong solution of sodic ethylate or methylate, a dark orange-brown solution is formed, and, on stirring, the whole solidifies to a mass of yellow erystals. These after collecting, well washing with alcohol, and drying on a porous plate in a vacuum, gave the following numbers on analysis :-

0.2690 gram substance gave 0.0768 gram $\mathrm{Na}_{2} \mathrm{SO}_{4}$.

Found.

Na..... $9 \cdot 27$ per cent.
Theory. $\mathrm{NO}_{2} \cdot \mathrm{C}_{6} \mathrm{H}_{4} \cdot \mathrm{CO} \cdot \mathrm{CHNa} \cdot \mathrm{COOCH}_{3}$. 9.38 per cent. 
The substance is, therefore, the monosodium-derivative of methylic paranitrobenzoylacetate. It is extremely stable, and behaves more like the salt of an acid than a sodium-derivative. It dissolves in water without decomposition, forming a yellow solution. It is scarcely attacked when treated with iodine.

\section{(4.) Methylic Benzylparanitrobenzoylacetate.}

It seemed to be of interest to determine whether the paranitroderivatives of benzoylacetic acid could be used for synthetical experiments with the same advantage as benzoylacetic acid itself.

For this purpose, the action of benzyl chloride on the sodium-derivative of methylic paranitrobenzoylacetate was studied. The pure sodium-derivative was heated with the calculated quantity of benzyl chloride and a little alcohol in a sealed tube for six hours at $150^{\circ}$. The product was mixed with water, and once or twice extracted with ether. After drying the ethereal solution and distilling off the ether, a thick brownish oil remained, which did not solidify even when exposed for some days over sulphuric acid in a vacuum.

On distilling this crude product in a current of steam, a small quantity of oil passed over, but the main part remained behind in the retort. This was extracted with ether, and the ethereal solution well dried; on distilling off the ether, a thick oil remained, which, however, soon solidified to a cake of crystals. These were roughly purified by spreading them out on a porous plate, and then once or twice recrystallising from dilute alcohol. Analysis :-

I. 0.1728 gram substance gave 0.4113 gram $\mathrm{CO}_{2}$ and 0.0755 gram $\mathrm{H}_{2} \mathrm{O}$.

II. $0 \cdot 3150$ gram substance gave 13 c.c. N. $t=11^{\circ}$. Bar. $=710 \mathrm{~mm}$. Found.

\begin{tabular}{|c|c|c|c|c|}
\hline \multirow[b]{3}{*}{ C..... } & & \multirow{2}{*}{$\begin{array}{l}\text { Theory. } \\
\cdot \mathrm{CO} \cdot \mathrm{CH}\left(\mathrm{C}_{7} \mathrm{H}_{7}\right) \cdot \mathrm{COOCH}_{3} \text {. }\end{array}$} \\
\hline & I. & II. & & \\
\hline & $64: 91$ & - & per cent. & $65 \cdot 17$ per cent. \\
\hline & $4 \cdot 86$ & - & $"$ & $4 \cdot 79$ \\
\hline N..... & - & $4 \cdot 57$ & , & $4 \cdot 47$ \\
\hline
\end{tabular}

The substance was therefore methylic benzylparanitrobenzoylacetate, formed according the equation-

$\mathrm{NO}_{2} \cdot \mathrm{C}_{6} \mathrm{H}_{4} \cdot \mathrm{CO} \cdot \mathrm{CHNa} \cdot \mathrm{COOCH}_{3}+\mathrm{C}_{7} \mathrm{H}_{7} \mathrm{Cl}=$

$\mathrm{NO}_{2} \cdot \mathrm{C}_{6} \mathrm{H}_{4} \cdot \mathrm{CO} \cdot \mathrm{CH}\left(\mathrm{C}_{7} \mathrm{H}_{7}\right) \cdot \mathrm{COOCH}_{3}+\mathrm{NaCl}$.

It dissolves easily in most solvents, and crystallises from dilute alcohol in colourless plates which melt at $57^{\circ}$. 


\section{(5.) Ethylic Paranitrobenzoylacetate.}

In order to prepare this ethereal salt, pure paranitrobenzoylacetic acid is suspended in an excess of absolute ethyl alcohol and the liquid thoroughly saturated with hydrogen chloride, care being taken that. the temperature does not rise above $20^{\circ}$ during the operation, otherwise decomposition is apt to set in. The product, which is a clear brownish liquid, is allowed to remain for two hours, and then poured into ice and water. This precipitates the ethereal salt in oily drops which solidify after a time; these can be collected on a filter, but it. is best to extract the whole with ether. The ethereal solution, after washing with water, drying over calcic chloride, and distilling off the ether, deposits a thick brownish oil, which gradually solidifies on standing over sulphuric acid in a vacuum; this crude product can easily be separated from paranitroacetophenone and tarry products, by dissolving it in a little boiling alcohol.

On cooling, the ethylic paranitrobenzoylacetate crystallises out in yellow needles, which may be obtained almost colourless by repeated recrystallisation. The analysis gave the following results:-

I. 0.1619 gram substance gave 0.0672 gram $\mathrm{H}_{2} \mathrm{O}$ and 0.3304 gram $\mathrm{CO}_{2}$.

II. 0.1993 gram substance gave 10.50 c.c. $\mathrm{N} . \quad t=12^{\circ}$. Bar. $=$ $709 \mathrm{~mm}$.

\begin{tabular}{|c|c|c|c|c|}
\hline \multicolumn{3}{|c|}{ Found. } & & \multirow[b]{2}{*}{$\begin{array}{l}\text { Theory. } \\
\mathrm{NO}_{2} \cdot \mathrm{C}_{6} \mathrm{H}_{4} \cdot \mathrm{CO} \cdot \mathrm{CH}_{2} \cdot \mathrm{COOC}_{2} \mathrm{H}_{5} \cdot\end{array}$} \\
\hline & I. & II. & & \\
\hline$\ldots . .$. & $55 \cdot 63$ & - & per cent. & 55.64 per cent. \\
\hline $\mathrm{H}$ & $4 \cdot 64$ & 一 & , & $4 \cdot 64$ \\
\hline $\mathrm{N}$ & - & $5 \cdot 83$ & , & $5 \cdot 90$ \\
\hline
\end{tabular}

Ethylic paranitrobenzoylacetate is easily soluble in boiling alcohol, much less so in the cold ; it crystallises, however, best from a mixture of benzene ( 1 part), and light petroleum (b. p. $60-80^{\circ}, 2$ parts). If this solution be allowed to evaporate at the ordinary temperature, beautiful transparent crystals are obtained, melting at $74-76^{\circ}$. Professor Haushofer, who was kind enough to measure these, gave me the following account of them.

Crystalline Form. Monoclinic.

$$
\begin{gathered}
a: b: c=0.3584: 1: 1 \cdot 2380 . \\
\beta=72^{\circ} 22^{\prime} .
\end{gathered}
$$

Tabular prismatic crystals developed in the direction of the clinodiagonal, with the combinations $0 \mathrm{P}(001)=c, \mathbb{P} \infty(011)=q$, $\cos (100)=a,-\mathrm{P} \infty(101)=r,-2 \mathrm{P} 2(121)=t,-4 \mathrm{P} 4(141)=s$. 
The surface $c$ well developed but usually rough; the surfaces $t$ and $s$ usually very small, scarcely visible to the naked eye.

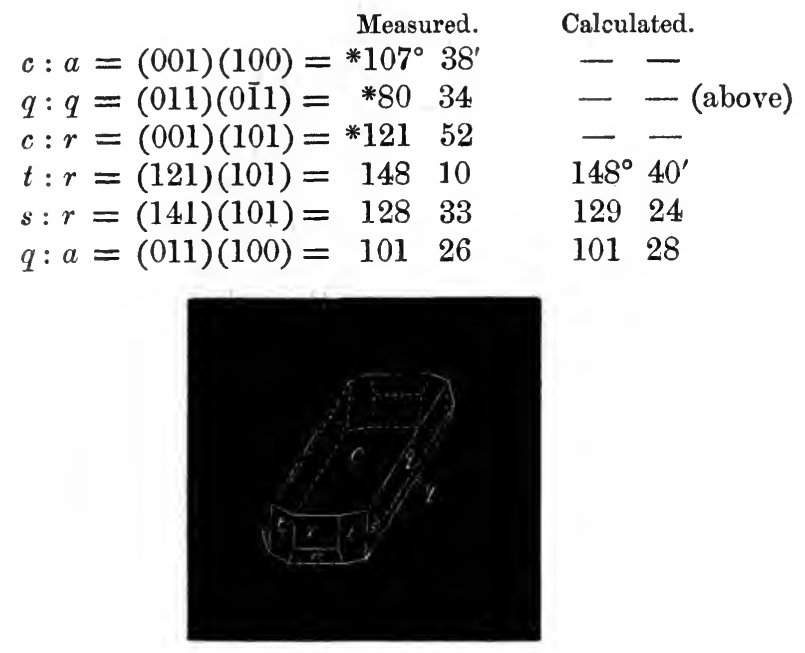

The plane of the optical axes is symmetrical. In convergent polarised light, the interference image of an axis makes its appearance on the surface $c$.

Ethylic paranitrobenzoylacetate dissolves fairly easily in sodic hydrate, especially on warming, producing a yellow solution. On the addition of acids, it is reprecipitated unchanged. Its alcoholic solution gives a beautiful brownish-violet coloration with ferric chloride.

Ethylic paranitrobenzoylacetate is easily reduced when treated with tin and hydrochloric acid in alcoholic solution, but the products formed have not been further examined.

If a strong solution of sodic ethylate $(1 \mathrm{~mol}$.) be added to the alcoholic solution of ethylic paranitrobenzoylacetate (1 mol.), an intense brownish-yellow solution is formed, which on standing solidifies to a mass of orange-coloured crystals of the sodium compound. These when collected, well washed with alcohol, and dried over sulphuric acid in a vacuum, gave the following numbers on analysis :-

0.2583 gram substance gave 0.0705 gram $\mathrm{Na}_{2} \mathrm{SO}_{4}$.

$$
\begin{array}{cc}
\text { Found. } & \begin{array}{c}
\text { Theory. } \\
\text { Na...... }
\end{array} \quad 8.86 \text { per cent. } \\
\mathrm{NO}_{2} \cdot \mathrm{C}_{6} \mathrm{H}_{4} \cdot \mathrm{CO} \cdot \mathrm{CHNa} \cdot \mathrm{COOC}_{2} \mathrm{H}_{5} \cdot
\end{array}
$$

The dry sodium compound is characterised by its intensely bitter taste. It explodes feebly when heated, paranitroacetophenone and other compounds being formed. 
It is sparingly soluble in cold water, but easily on warming, forming a deep yellow solution, which on the addition of an acid is instantly decolorised, ethylic paranitrobenzoylacetate being precipitated. If the solution of the sodinm compound is mixed with a solution of an inorganic salt, the former is completely precipitated and the solution becomes colourless. The sodium compound is fairly easily soluble in boiling alcohol, and crystallises out on cooling in small orange-yellow needles, which are much redder than the sodium compound of methylic paranitrobenzoylacetate. The addition of ferric chloride to the alcoholic solution produces a dark reddish-brown coloration; but if this reagent is added to the solution of the sodium compound in water, a light reddish precipitate is formed, which appears to be crystalline, and is probably the ferric salt of ethylic paranitrobenzoylacetate.

If nitrate of silver is added to the solution of the sodium compound in water, a light yellow, amorphous precipitate is formed which explodes when heated in the dry state. The lead compound is easily obtained as a yellow amorphous mass, on adding a solution of acetate of lead to the aqueous solution of the sodium compound. The copper compound is a yellowish-green precipitate.

The compounds of ethylic paranitrobenzoylacetate with metals are remarkable for their stability. The sodium compound behaves exactly like the salt of an acid, it dissolves in water without decomposition and can even be recrystallised from it. The sodium compound of ethylic benzoylacetate is decomposed by boiling with water, and ethylic sodacetoacetate is split ap when merely mixed with water. The sodium compound of methylic paranitrobenzoylacetate is, curiously enough, still more stable than that of the ethylic salt.

\section{(6.) Ethylic Nitrosoparanitrobenzoylacetate.}

As has been shown in previous researches (Trans., 1885, 244), ethylic benzoylacetate is easily converted into its isonitroso-derivative,

$$
\mathrm{C}_{6} \mathrm{H}_{5} \cdot \mathrm{CO} \cdot \mathrm{C}(\mathrm{NOH}) \cdot \mathrm{COOC}_{2} \mathrm{H}_{5} \text {, }
$$

when its solution in dilute sodic hydrate is treated with sodic nitrite and dilute sulphuric acid. This method, however, is not applicable to ethylic paranitrobenzoylacetate.

If the solution of the sodium compound of the ethereal salt is mixed with sodic nitrite, the former is precipitated almost entirely, and thus escapes the action of the nitrous acid when the mixtare is acidified with dilute sulphuric acid.

After several experiments, however, it was found that the desired isonitroso-derivative could easily be obtained in the following way :Pure ethylic paranitrobenzoylacetate is dissolved in dry ether and a 
stream of nitrous gas passed through the solution until it is saturated, care being taken to cool well during the operation.

After standing for a short time, a rapid current of carbonic anhydride is passed through in order to remove the excess of nitrous acid as completely as possible, and the solution is allowed to evaporate spontaneously. The semi-solid mass which remains is first roughly separated from oily impurities by spreading it out on. a porous plate, and then twice recrystallised from dilute alcohol.

Analysis :-

I. 0.1646 gram substance gave 0.0504 gram $\mathrm{H}_{2} \mathrm{O}$ and 0.2980 gram $\mathrm{CO}_{2}$.

II. $0 \cdot 1927$ gram substance gave 19 c.c. $\mathrm{N} . t=14^{\circ}$. Bar. $=716 \mathrm{~mm}$.

Found.

\begin{tabular}{|c|c|c|c|c|}
\hline \multirow{2}{*}{ C.... } & & & & \multirow{2}{*}{$\begin{array}{l}\text { Theory, } \\
\mathrm{NO}_{2} \cdot \mathrm{C}_{6} \mathrm{H}_{4} \cdot \mathrm{CO} \cdot \mathrm{C}(\mathrm{NOH}) \cdot \mathrm{COOC}_{2} \mathrm{H}_{5} \cdot \\
49 \cdot 62 \text { per cent. }\end{array}$} \\
\hline & $49 \cdot 37$ & 11. & per cent. & \\
\hline $\mathrm{H}$ & $3 \cdot 40$ & - & , & $3 \cdot 75$ \\
\hline ... & - & 10.55 & , & 10.53 \\
\hline
\end{tabular}

This compound is, therefore, ethylic nitrosoparanitrobenzoylacetate, formed according to the equation-

$\mathrm{NO}_{2} \cdot \mathrm{C}_{6} \mathrm{H}_{4} \cdot \mathrm{CO} \cdot \mathrm{CH}_{2} \cdot \mathrm{COOC}_{2} \mathrm{H}_{5}+\mathrm{NO}_{2} \mathrm{H}=$

$\mathrm{NO}_{2} \cdot \mathrm{C}_{6} \mathrm{H}_{4} \cdot \mathrm{CO} \cdot \mathrm{C}(\mathrm{NOH}) \cdot \mathrm{COOC}_{2} \mathrm{H}_{5}+\mathrm{H}_{2} \mathrm{O}$.

It is easily soluble in alcohol, ether, and acetone, less readily in benzene and light petroleum. It crystallises from dilute alcohol in colourless needles, which melt at $220^{\circ}$ with decomposition. It dissolves easily in sodic hydrate, forming a deep yellow solution, and is reprecipitated unchanged on the addition of dilute acids.

\section{(7.) Ethylic Ethylparanitrobenzoylacetate.}

In preparing homologues of ethylic paranitrobenzoylacetate it is always better to start from the pure sodium compound than from the ethereal salt itself, as the products obtained in this way are always purer.

In order to prepare ethylic ethylparanitrobenzoylacetate, the pure sodium compound was heated with an excess of ethyl iodide and a little alcohol for about three hours at $100^{\circ}$. The whole was then evaporated to dryness on a water-bath, and the residue treated with water and extracted with ether. On distilling off the ether, a thick yellowish oil was left, which solidified after standing for some time over sulphuric acid in a vacuum. The crystals were first roughly freed from impurities by spreading the mass out on a porous plate, and then recrystallised twice from dilute alcohol. 
Analysis :-

I. $0 \cdot 1100$ gram substance gave 0.0564 gram $\mathrm{H}_{2} \mathrm{O}$ and 0.2365 gram $\mathrm{CO}_{2}$.

II. $0 \cdot 1512$ gram substance gave 8 c.c. N. $t=13^{\circ}$. Bar. $=711 \mathrm{~mm}$. Found.

\begin{tabular}{|c|c|c|c|c|}
\hline & I. & II. & & $\begin{array}{l}\text { Theory. } \\
\mathrm{NO}_{2} \cdot \mathrm{C}_{6} \mathrm{H}_{4} \cdot \mathrm{CO} \cdot \mathrm{CH}\left(\mathrm{C}_{2} \mathrm{H}_{5}\right) \cdot \mathrm{COOC}_{2} \mathrm{H}_{5}\end{array}$ \\
\hline C.... & $58 \cdot 63$ & - & per cent. & 58.86 per cent. \\
\hline & $5 \cdot 69$ & - & , & $5 \cdot 66$ \\
\hline N... & - & $5 \cdot 50$ & 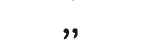 & $5 \cdot 28$ \\
\hline
\end{tabular}

Ethylic ethyl paranitrobenzoylacetate crystallises from dilute alcohol in colourless plates which melt at $39-40^{\circ}$. It is easily soluble in alcohol, ether, light petroleum, benzene, and carbon bisulphide.

All attempts to obtain the corresponding ethyl paranitrobenzoylacetic acid by hydrolysis were fruitless, paranitrobenzoic acid always being formed, even when very dilute potash was used. No better results were obtained with sulphuric acid at ordinary temperatures.

If ethylic ethylparanitrobenzoylacetate is heated with concentrated sulphuric acid at $100^{\circ}$, carbonic anhydride is given off, and, on adding water and extracting with ether, a yellowish oil is obtained; this on long standing becomes solid. It appears to be ethyl paranitroacetophenone, $\mathrm{NO}_{2} \cdot \mathrm{C}_{6} \mathrm{H}_{4} \cdot \mathrm{CO} \cdot \mathrm{CH}_{2} \cdot \mathrm{C}_{2} \mathrm{H}_{5}$.

The quantity was unfortunately too small for analysis.

\section{(8.) Ethyl Allylparanitrobenzoylacetate.}

This ethereal salt was prepared in exactly the same way as the ethylic ethylparanitrobenzoylacetate, allyl iodide being used instead of ethyl iodide. The crude product obtained was purified by repeated crystallisation from dilute alcohol.

Analysis :-

I. $0 \cdot 2290$ gram substance gave 0.1129 gram $\mathrm{H}_{2} \mathrm{O}$ and $0.5 \mathrm{l} 00$ gram $\mathrm{CO}_{2}$.

II. $0 \cdot 1695$ gram substance gave $7 \cdot 9$ c.c. $\mathrm{N}$. $t=14^{\circ}$. Bar. $=$ $717 \mathrm{~mm}$.

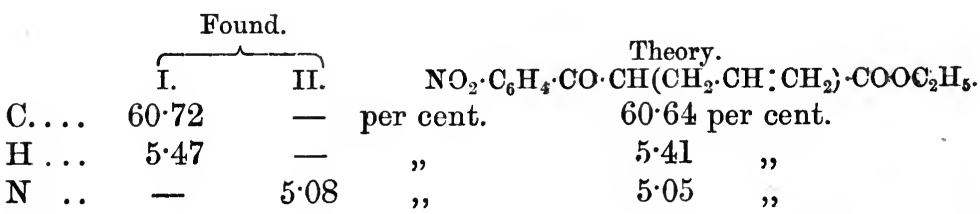

Ethylic allylparanitrobenzoylacetate melts at $45-46^{\circ}$. A number 
of experiments were made with the object of obtaining the corresponding allylparanitrobenzoylacetic acid, but without result; paranitrobenzoic acid always being formed. When heated with concentrated sulphuric acid at $100^{\circ}$, ethylic allylparanitrobenzoylacetate is decomposed into carbonic anhydride and a ketone, probably allylparanitroacetophenone, $\mathrm{NO}_{2} \cdot \mathrm{C}_{6} \mathrm{H}_{4} \cdot \mathrm{CO} \cdot \mathrm{CH}_{2} \cdot \mathrm{CH}_{2} \cdot \mathrm{CH}: \mathrm{CH}_{2}$.

\section{(9.) Ethylic Diparanitrobenzoylsuccinate,}

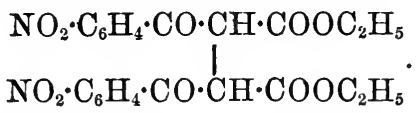

This compound is formed when the sodium-derivative of ethylic paranitrobenzoylacetate is treated with iodine:

$2\left(\mathrm{NO}_{2} \cdot \mathrm{C}_{6} \mathrm{H}_{4} \cdot \mathrm{CO} \cdot \mathrm{CHNa} \cdot \mathrm{COOC}_{2} \mathrm{H}_{5}\right)+\mathrm{I}_{2}=$

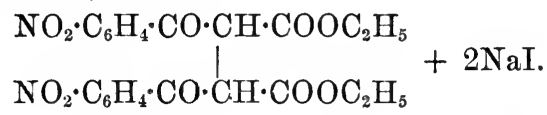

in a way exactly analogous to the formation of ethylic dibenzoylsuccinate from the sodium compound of ethylic benzoylacetate. To prepare it, the pure sodium compound of ethylic paranitrobenzoylacetate is first finely powdered and then agitated with an ethereal solution of iodine, containing the calculated quantity. If this solution be added by degrees, it will be seen that the colour of the iodine disappears rapidly at first, but at last only after long shaking, and it will be found usually that the whole amount is not decolorised. The product is then mixed with water and sulphurous acid, the ethereal solution separated, dried over calcic chloride, and evaporated. In this way, a yellow oil is obtained which, after standing over sulphuric acid in vacuum, deposits crystals. These must be separated from the oily mother-liquor by spreading out on a porous plate, and then recrystallised once or twice from dilute alcohol.

The analysis gave the following result :-

I. 0.2344 gram substance gave 0.0941 gram $\mathrm{H}_{2} \mathrm{O}$ and 0.4805 gram $\mathrm{CO}_{2}$.

II. 0.2001 gram substance gave $10 \cdot 7$ c.c. $\mathrm{N} . \quad t=17^{\circ}$. Bar. $=$ $712 \mathrm{~mm}$.

Found.

\section{I.}

C.... $55 \cdot 90$

H... $4: 48$

$\mathrm{N} \ldots-$
II.

- per cent.

$5 \cdot 82$
Theory. $\left(\mathrm{NO}_{2} \cdot \mathrm{C}_{6} \mathrm{H}_{4} \cdot \mathrm{CO}\right)_{2} \mathrm{C}_{2} \mathrm{H}_{2}\left(\mathrm{COOC}_{2} \mathrm{H}_{5}\right)_{2}$. 55.93 per cent.

4.24 5.92 
Ethylic diparanitrobenzoylsuccinate crystallises from dilute alcohol in colourless needles which melt at $180^{\circ}$. It is easily soluble in alcohol, ether, benzene, and light petroleam. If a solution of this substance in dry ether is mixed with an ethereal solution of sodic ethylate, a white amorphous compound is thrown down, which has without doubt the formula-

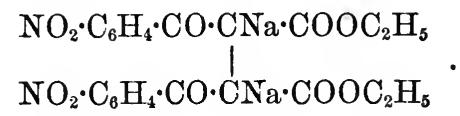

When ethylic diparanitrobenzoylsuccinate is treated with concentrated sulphuric acid, it is converted into a new compound, which is probably ethylic diparanitrophenylfurfurandicarboxylate. Owing to want of material, however, the reaction was not followed up.

From these experiments, it can be seen that ethylic paranitrobenzoylacetate may be used with advantage instead of ethylic benzoylacetate, the derivatives formed being solid and therefore easy to purify.

In a short time, we hope to be able to communicate to the Society the experiments on the formation of trimethylene- and tetramethylene-derivatives, which were made with this substance. 


\title{
DEHYDRACETIC ACTD.
}

\author{
By W. H. Perkix, Jun., Ph.D.
}

DeHYDRAGETIC acid was first prepared by Geuther in the year 1866 , who obtained it by passing a current of carbonic anhydride over the sodium compound of ethylic acetoacetate. From the analyses of the acid itself and also of its barium and sodium salts, Geuther showed that this new compound was a monobasic acid of the formula $\mathrm{C}_{8} \mathrm{H}_{8} \mathrm{O}_{4}$. He supposed it to be formed by the dehydration of acetic acid, probably thus-

$$
4 \mathrm{CH}_{3} \cdot \mathrm{COOH}=\mathrm{C}_{8} \mathrm{H}_{8} \mathrm{O}_{4}+4 \mathrm{H}_{2} \mathrm{O},
$$

bence the name dehydracetic acid.

Geuther does not appear to have examined this acid further, and it is to Oppenheim and Precht (Ber., 9,324) that the first careful study of it is due. These chemists showed that when dehydracetic acid is treated with alkalis it is decomposed into acetone, acetic acid, and carbonic anhydride, thus :-

$$
\mathrm{C}_{8} \mathrm{H}_{8} \mathrm{O}_{4}+3 \mathrm{H}_{2} \mathrm{O}=\mathrm{CO}_{2}+\mathrm{CH}_{3} \cdot \mathrm{CO} \cdot \mathrm{CH}_{3}+2 \mathrm{CH}_{3} \cdot \mathrm{COOH} \text {. }
$$

Besides these products, they obtained by the hydrolysis of dehydracetic acid with baryta-water a small quantity of a crystalline substance very much resernbling orcinol, and this led Beilstein to place dehydracetic acid in the aromatic series (Handluch der organischen ('hemie, 1527-1528).

Oppenheim and Precht also prepared the methylic and ethylic salts of dehydracetic acid, chlorodehydracetic acid, $\mathrm{C}_{8} \mathrm{H}_{7} \mathrm{ClO}_{4}$, bromodehydracetic acid, $\mathrm{C}_{8} \mathrm{H}_{7} \mathrm{BrO}_{4}$, and by reduction with zinc and hydrochloric acid a new acid of the formula $\mathrm{C}_{8} \mathrm{H}_{10} \mathrm{O}_{3}$.

By the action of pentachloride of phosphorus on dehydracetic acid, they obtained a remarkable chloride of the formula $\mathrm{C}_{8} \mathrm{H}_{6} \mathrm{O}_{2} \mathrm{Cl}_{2}$, the formation of which led them to imagine that dehydracetic acid contained two hydroxyl-groups. For this reason they proposed the formula-

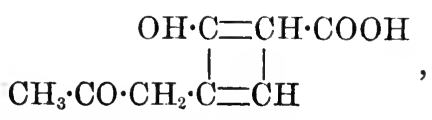

as best representing the constitution of this acid.

At the suggestion of Wislicenus, Shibbye (Dissertation, Würzburg, $1882,5)$, at a later date, repeated the experiments of Oppenheim and Precht on the action of alkalis on dehydracetic acid, and showed that 
besides acetone, acetic acid, and carbonic anhydride, considerable quantities of malonic acid were also formed.

As these results were still insufficient to definitely prove the constitution of dehydracetic acid, I thought it would be interesting to carry these experiments further, and wish now to bring before the Society the results I have obtained.

Dehydracetic acid, $\mathrm{C}_{8} \mathrm{H}_{8} \mathrm{O}_{4}$, contains besides the carboxyl-group, two oxygen-atoms, which may either be present as $\mathrm{CO}, \mathrm{OH}$, or $\mathrm{C} \cdot \mathrm{O} \cdot \mathrm{C}$.

When treated with hydroxylamine or phenylhydrazine, one oxygen. atom is easily displaced, forming the derivatives $\mathrm{C}_{7} \mathrm{H}_{8} \mathrm{O}_{3}(\mathrm{CNOH})$ and $\mathrm{C}_{8} \mathrm{H}_{8} \mathrm{O}_{3}\left(\mathrm{~N}_{2} \mathrm{H} \cdot \mathrm{C}_{6} \mathrm{H}_{5}\right)$ respectively, a proof that one of the oxygenatoms is present as $\mathrm{CO}$; but all attempts to displace a second atom by the above groups were fruitless.

Dehydracetic acid does not give an acetyl-compound when treated with acetic anhydride, even after long boiling, and it is therefore clear that the fourth oxygen-atom is not present as hydroxyl, but that it is most probably united by each of its affinities to a different carbon-atom thus: C.O.C. This view is borne out by the reduction experiments of Oppenheim and Precht, as also by the behaviour of dehydracetic acid when treated with phosphorus pentachloride. In order to obtain some further clue as to the arrangement of the atoms in this interesting acid, the hydrolysis with alkalis was next very carefully studied.

In a paper on dehydrobenzoylacetic acid (Trans., 1885, 284) it was shown that when this acid is treated with alkalis, the first change which takes place is a quantitative decomposition into 2 mols. of benzoylacetic acid, thus :-

$$
\underset{\substack{\text { Dehydrobenzoyl- } \\ \text { acetic acid. }}}{\mathrm{C}_{18} \mathrm{H}_{12} \mathrm{O}_{4}}+2 \mathrm{H}_{2} \mathrm{O}=\underset{\text { Benzoylacetic acid. }}{2 \mathrm{C}_{6} \mathrm{H}_{5} \cdot \mathrm{CO} \cdot \mathrm{CH}_{2} \cdot \mathrm{COOH} .}
$$

This result is very important for two reasons :-Firstly, it shows that the dehydrobenzoylacetic acid is really formed by the splitting off of 2 mols. of water from 2 mols. of benzoylacetic acid; and secondly, it proves that the two $\mathrm{C}_{6} \mathrm{H}_{5}$-groups are intact in the dehydrobenzoylacetic acid, and that all changes which take place in its formation from benzoylacetic acid must take place within the group $-\mathrm{CO} \cdot \mathrm{CH}_{2} \cdot \mathrm{COOH}$. In determining the constitution of dehydracetic acid, it was clearly just as important to determine whether it contained two methyl-groups, in which case the formula proposed by Oppenheim and Precht would be incorrect.

If dehydracetic acid be very carefully hydrolysed with potash, it is possible to isolate an acid, which is without doubt acetoacetic acid; although naturally owing to the instability of the latter no quantita- 
tive experiments could be made. From this result, it is legitimate to suppose that this acid, when treated with potash, in a way exactly analogous to dehydrobenzoylacetic acid, is first split up into 2 mols. of acetoacetic acid, thus :-

$$
\mathrm{C}_{8} \mathrm{H}_{8} \mathrm{O}_{4}+2 \mathrm{H}_{2} \mathrm{O}=2 \mathrm{CH}_{3} \cdot \mathrm{CO} \cdot \mathrm{CH}_{2} \cdot \mathrm{COOH} \text {. }
$$

This being the case, it is clear that dehydracetic acid must contain two methyl-groups, in the same way as dehydrobenzoylacetic acid contains two phenyl-groups.

The next question is: How is dehydracetic acid formed from 2 mols. of acetoacetic acid?

Dehydracetic acid being a monobasic acid, it naturally follows that one of the carboxyl-groups of one of the molecules of acetoacetic acid must take part in the reaction. Now, as the methyl-groups remain intact, this change can only take place by the condensation of the carboxyl-group of one molecule with the methylene-group of the other molecule, thus :-

\section{$\mathrm{CH}_{3} \cdot \mathrm{CO} \cdot \mathrm{CH}_{2} \cdot \mathrm{COOH} \quad \mathrm{CH}_{3} \cdot \mathrm{CO} \cdot \mathrm{CH} \cdot \mathrm{COOH}$ $\mathrm{CH}_{3} \cdot \mathrm{CO} \cdot \mathrm{CH}_{2} \cdot \mathrm{COOH}=\mathrm{CH}_{3} \cdot \mathrm{CO} \cdot \mathrm{CH}_{2} \cdot \mathrm{CO}+\mathrm{H}_{2} \mathrm{O}$.}

In the formation of dehydracetic acid from this intermediate compound, one more molecule of water is split off; the simplest way of effecting this being thus*:-
$\begin{aligned} & \mathrm{CH}_{3} \cdot \mathrm{CO} \cdot \mathrm{CH} \cdot \mathrm{COOH} \\ & \mathrm{CH}_{3} \cdot \mathrm{CO} \cdot \mathrm{CH}_{2} \cdot \mathrm{CO}\end{aligned}=$
$\mathrm{CH}_{3} \cdot \mathrm{C}(\mathrm{OH}): \mathrm{C} \cdot \mathrm{COOH}$ $\mathrm{CH}_{3} \cdot \mathrm{C}(\mathrm{OH}): \mathrm{CH} \cdot \stackrel{\mathrm{C} O}{ }$

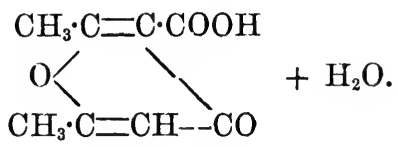

The most probable formula for dehydracetic acid would therefore be-<smiles>CC1=CC(=O)C(C(=O)O)O1</smiles>

In considering how best to obtain evidence in support of this conclusion, it struck me that there was a certain resemblance between this formula and the formula given to cumalinic acid by

v. Pechmann, namely :

$\mathrm{COOH} \cdot \mathrm{C}=\mathrm{CH}-\mathrm{O}$

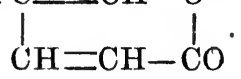

* This change is fully.discussed in a paper on "Dehydrobenzoylacetic Acid" (Trans., 1885, 295-297). 
The striking relation of this acid to chelidonic and meconic acids did not occur to me till afterwards.

If the methyl salt of cumalinic acid is treated with ammonia, it is converted, as v. Pechmann has shown in his beautiful researches (Ber., 17, 2391), into hydroxynicotinic acid, thus :-

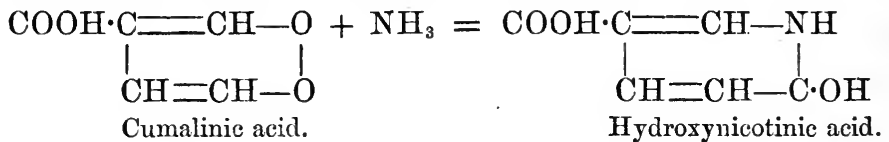

It was therefore possible that dehydracetic acid under similar conditions might also yield pyridine-derivatives. The experiment has shown that this is indeed the case.

If methylic dehydracetate is treated with ammonia or aniline, reaction immediately sets in, and pyridine-derivatives are formed, the decomposition being quantitative.

The products formed by the action of ammonia I have prepared but not analysed, as, curiously enough, Haitinger, at the same time as myself, investigated the action of ammonia on dehydracetic acid, and has reserved the further study of these products.

When methylic dehydracetate is warmed with aniline, two substances, $\mathrm{C}_{15} \mathrm{H}_{15} \mathrm{NO}_{3}$ and $\mathrm{C}_{13} \mathrm{H}_{13} \mathrm{NO}$, are formed, of which the former is undoubtedly the primary product.

The reaction probably takes place in two stages, in a way similar to that shown by v. Pechmann in the case of the cumalinic acid.

I.

$\mathrm{COOCH}_{3} \cdot \mathrm{C}-\mathrm{CO}-\mathrm{CH}$
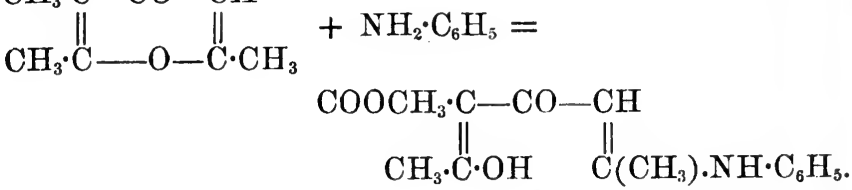

II.
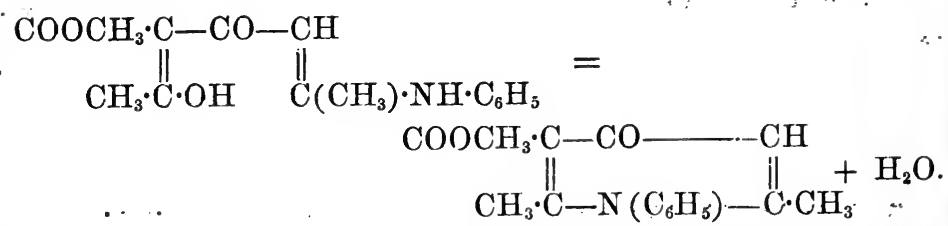

'During this synthesis, partial hydrolysis of the compound $\mathrm{C}_{13} \mathrm{H}_{15} \mathrm{NO}_{3}$ and decomposition into, carbonic anhydride and the substance $\mathrm{C}_{13} \mathrm{H}_{13} \mathrm{NO}$ takes place. The latter has therefore the constitution-:<smiles>Cc1ccccc1</smiles> 


\section{If the group}

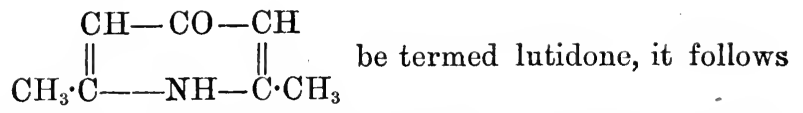
that the primary product of the action of aniline on methylic dehydracetate is methylic phenyllutidinecarboxylate, whereas the substance $\mathrm{C}_{13} \mathrm{H}_{13} \mathrm{NO}$ is phenyllutidone.

A most interesting synthetical proof of the constitution given above to dehydracetic acid has lately been carried out by Conrad and Guthreit (Ber., 19, 19, and 20,151 and 154). These chemists found that when the copper salt of ethylic acetoacetate is treated with phosgene, the following decomposition takes place :-

$$
\begin{aligned}
& \underset{\mathrm{CH}_{3} \cdot \mathrm{CO}}{\mathrm{COOC}} \mathrm{H}_{5}>\mathrm{CH} \cdot \mathrm{Cu} \cdot \mathrm{CH}<\mathrm{CO}_{\mathrm{COCH}} \mathrm{CH}_{3} \mathrm{COC}_{5}+\mathrm{COCl}_{2}= \\
& \underset{\mathrm{CH}_{3} \cdot \mathrm{CO}}{\mathrm{COOC}_{2} \mathrm{H}_{5}}>\mathrm{CH} \cdot \mathrm{CO} \cdot \mathrm{CH}<\underset{\mathrm{CO} \cdot \mathrm{CH}_{3}}{\mathrm{COOC}_{2} \mathrm{H}_{5}}+\mathrm{CuCl}_{2} \text {. }
\end{aligned}
$$

This substance does not appear to exist as such, but spontaneously loses a molecule of water, and is converted into ethylic carboxyldehydracetate thus :-

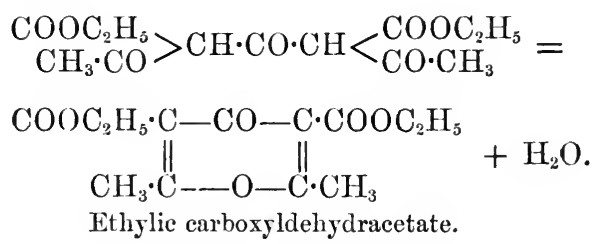

If this compound is treated with aniline, ethylic phenyllutidonedicarboxylate is found-

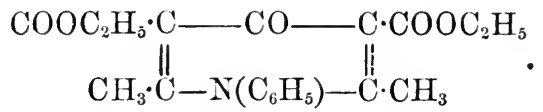

The free acid when heated to $270^{\circ}$ is converted into phengllutidone, a substance identical with that which $\mathrm{I}$ obtained by the action of aniline on methylic dehydracetate. This elegant synthesis by Conrad and Guthzeit proves without doubt that dehydracetic acid really has the formula given by me above.

The group pyrone, ${ }_{\mathrm{CH}}^{\mathrm{CH}}-\mathrm{CO}-\mathrm{O}-\mathrm{CH}$ (Haitinger and Zieben, Monxtsh., 6,283 ), besides being present in dehydracetic acid, is the basis of a number of interesting acids, among them chelidonic acid and meconic acid. 
The relation of the most important representatives of this class to one another is clearly seen when their constitutional formulæ are placed side by side, thus :-<smiles>O=c1ccocc1</smiles>

Pyrove.

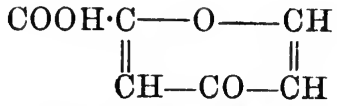

Pyronecarboxylic acid.

Comanic acid.<smiles>O=C(O)c1ccc(C(=O)O)c(=O)o1</smiles>

Pyronedicarboxylic acid.

Chelidonic acid.<smiles>O=C[C@H]1O[C@H]2OC(=O)[C@H]1O[C@H]2O</smiles>

Oxypyronecarboxylic acid. Comenic acid.<smiles>C[C@@H]1O[C@@H](C)O[C@H](C)[C@@H]1OC(=O)[O-]</smiles>

Dimethylpyronecarboxylic acid. Dehydracetic acid.
$\mathrm{COOH} \cdot \mathrm{C}-\mathrm{O}-\mathrm{C} \cdot \mathrm{COOH}$<smiles>C=CC(=O)O</smiles>

Oxypyronedicarbexylic acid. Meconic acid.<smiles>CC(=O)[C@H]1O[C@](C)(C(=O)O)[C@H](C(=O)O)O[C@H]1C</smiles>

Dimethylpyronedicarboxylic acid.

\section{Preparation of Dehydracetic Acid.}

In preparing dehydracetic acid, Oppenheim and Precht's method, which consists in passing the vapours of ethylic acetoacetate through a tube heated to dull redness, was after a few slight modifications found to give the best results.

The details of this process are the following:-A piece of combustion tabe, 50 inches long, and bent about 18 inches from one end to an angle of $120^{\circ}$, is filled up to the bend with small pieces of pumice, and heated in a combustion furnace to dull redness. 200 grams of ethylic acetoacetate are then slowly distilled through this tabe, from a distilling flask directly connected to the tube by means of a cork, and the product which comes over is collected in a well-cooled flask placed at the other end of the tube. A dark-brown oil is thus obtained, which on cooling should solidify almost entirely to a mass of crystals of dehydracetic acid. In order to isolate the acid, the crude product is rapidly distilled from a retort, until the temperature rises to $195^{\circ}$, and the residue is then poured into a dish and allowed to solidify. The dark-brown cake formed in this way is next broken up, and digested with an excess of a fairly strong solution of sodic 
carbonate and a little animal charcoal for a short time, and filtered hot. The filtrate, on cooling and acidifying with hydrochloric acid, deposits the dehydracetic acid as a slightly brownish-coloured crystalline powder, which, after being collected, well washed, and recrystallised from water is easily obtained pure.

The distillate from the crude dehydracetic acid, boiling below $195^{\circ}$, yields on fractioning a considerable quantity of ethylic acetoacetate, which, when again passed through the tube, gives a further quantity of acid. With a little practice it is easy to obtain by this method from 58-66 grams of pure dehydracetic acid from 200 grams of ethylic acetoacetate. Most of the dehydracetic acid used in this research was obtained from Meister, Lucius, and Brünning, through the kindness of Herr v. Gerichten, and I take this opportunity of thanking these gentlemen for their liberality.$$
\mathrm{C}_{8} \mathrm{H}_{7} \mathrm{BrO}_{4}=\mathrm{COOH} \cdot \mathrm{C}-\mathrm{CO}-\mathrm{CBr}
$$ \\ Monobromodehydracetic Acid,<smiles>C=C(C)OC(=C)C</smiles>

Monobromodehydracetic acid was first obtained by Oppenheim and Precht (Ber., 9, 1101) by treating dehydracetic acid with bromine. As the yield of the brominated acid obtained by this method is small, several experiments were made with the object of improving it. The following modification was found to give good results :-

5 grams of dehydracetic acid are dissolved in about 50 grams of chloroform, a slight excess of bromine, and two or three crystals of iodine added, and the whole warmed in a water-bath at about $50^{\circ}$ for a minute or two. As soon as the reaction has once set in, it continues at the ordinary temperature, and is usually finished in about 12 hours. The liquid is then decanted from any crystals which may have separated, and is allowed to evaporate at the ordinary temperatures in an open dish, when a slightly yellowish crystalline mass is left behind, consisting of a mixture of monobromodehydracetic acid and unchanged dehydracetic acid. These two aciùs may be easily separated by recrystallisation from alcohol, in which the latter is much more easily soluble than the former.

The monobromodehydracetic acid thus obtained is mixed with any crystals which may have separated from the chloroform solution during the preparation, and is purified by two crystallisations from alcohol.

The analysis gave the following numbers:-

0.3258 gram substance gave 0.2458 gram $\mathrm{AgBr}$.

Found.

Br...... $32 \cdot 11$ per cent.
Theory. $\mathrm{C}_{8} \mathrm{H}_{7} \mathrm{BrO}_{4}$.

$32 \cdot 38$ per cent. 
Monobromodehydracetic acid melts at $137^{\circ}$ (Oppenheim and Precht give $134^{\circ}$ ). It is easily soluble in hot alcohol, chloroform, benzene, and light petroleum, but only sparingly in alcohol and light petroleum in the cold.

It crystallises from the hot solution in methyl alcohol, on copling slowly, in beantiful plates and prisms, having the following forms:-

$$
\text { I. II. III. }
$$

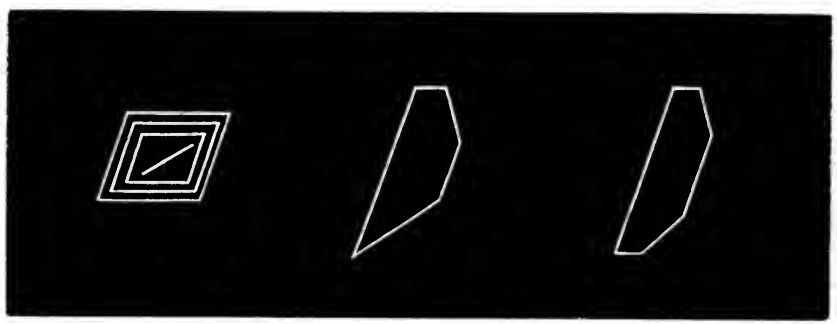

When the hot solution in methyl alcohol is rapidly cooled, curiously striated four-sided plates (I) are produced, but on slowly crystallising the forms lose the striated appearance and become well developed mostly in the forms II and III.

$$
\text { Hydroxydelydracetic Acid, } \mathrm{C}_{8} \mathrm{H}_{7} \mathrm{O}_{4} \cdot \mathrm{OH}=\stackrel{\mathrm{COOH} \cdot \mathrm{C}-\mathrm{CO}-\mathrm{C} \cdot \mathrm{OH}}{\mathrm{CH}_{3} \cdot \mathrm{C}-\mathrm{O}-\stackrel{\mathrm{C} \cdot \mathrm{CH}_{3}}{\|} .}
$$

This interesting compound is the first product of the acticn of potash on monobromodehydracetic acid, thus :-

$$
\mathrm{C}_{8} \mathrm{H}_{7} \mathrm{BrO}_{4}+\mathrm{KOH}=\mathrm{C}_{8} \mathrm{H}_{7} \mathrm{O}_{4} \cdot \mathrm{OH}+\mathrm{KBr} \text {. }
$$

To prepare it, pure monobromodehydracetic acid is dissolved in a little alcoholic potash, then mixed with an excess of concentrated alcoholic potash, and the whole allowed to stand 14 days at a temperature of $35-40^{\circ}$. The mass which is at first quite thick, soon becomes more fluid, and at the end of the operation again thick and brownish coloured. In order to isolate the product, water and dilute sulphuric acid are added, when a solid acid separates, which is collected and well washed with water. The mother-liquors, on repeated extraction with ether, give a further quantity of this acid, which is, however, not so pure. The crude product obtained in this way is a mixture of hydroxydehydracetic acid and unchanged monobromodehydracetic acid. These two acids can be easily separated by treatment with chloroform, in which the hydroxy-acid is almost insoluble The crestalline mass which remains undissolved is then 
once recrystallised from dilute alcohol, and thus obtained as a white sandy crystalline precipitate, which, on analysis, gave the following results :-

I. 0.2381 gram substance gave 0.0947 gram $\mathrm{H}_{2} \mathrm{O}$ and 0.4535 gram $\mathrm{CO}_{2}$.

II. 0.2060 gram substance gave 0.0831 gram. $\mathrm{H}_{2} \mathrm{O}$ and 0.3927 gram $\mathrm{CO}_{2}$.

\begin{tabular}{|c|c|c|c|c|}
\hline & \multicolumn{2}{|c|}{ Found. } & \\
\hline & I. & II. & $\mathrm{C}_{8} \mathrm{H}_{8} \mathrm{O}_{5}$ & \\
\hline $\mathrm{C}$. & $51 \cdot 95$ & 51.99 per cent. & $52 \cdot 17$ & r cent. \\
\hline H . . & $4 \cdot 42$ & $4 \cdot 48$ & $4 \cdot 35$ & ," \\
\hline $\mathrm{O} \ldots \ldots$ & $4.3 \cdot 63$ & $43 \cdot 53$ & $43 \cdot 47$ & , \\
\hline
\end{tabular}

Hydroxydehydracetic acid, when rapidly heated, melts at $250-255^{\circ}$ with decomposition, if slowly heated, the acid begins to decompose and char at $210^{\circ}$. It is fairly soluble in hot ethyl alcohol and methyl alcohol, but almost insoluble in .cold water, chloroform, light petroleum, benzene, and acetone. The hot solutions in alcohol or water deposit the acid on cooling in the form of a heavy sandy precipitate, which when examined under the microscope is found to consist of a mass of small but badly formed four-sided crystals.

The alcoholic or aqueous solution gives an intense violet coloration on the addition of a drop of ferric chloride. When carefully heated in small quantities, this acid sublimes with slight decomposition. Hydroxydehydracetic acid dissolves easily in alkalis. The silver salt appears curiously enough to have the constitution $\mathrm{C}_{8} \mathrm{H}_{6} \mathrm{O}_{5} \mathrm{Ag}_{2}$, which would point to the acid being bibasic. It is intended to further study this interesting acid, and experiments on the subject are already in progress.

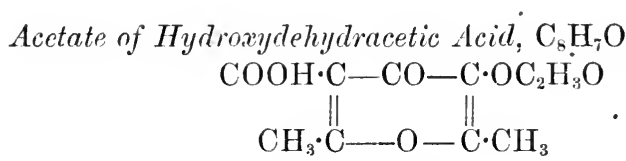

In order to prepare this substance, a small quantity of hydroxydehydracetic acid was boiled with an excess of acetic anhydride for two hours, the bulk of the acetic anhydride distilled off, and the clear solution after cooling mixed with water. As soon as the excess of acetic anhydride had been decomposed, the whole was extracted several times with ether, the ethereal solution well washed, and allowed to evaporate at ordinary temperatures. In this way, a colourless crystalline mass was obtained, which, after washing with a little water and drying on a porous plate over sulphuric acid in a vacuum, gave the following numbers on analysis:- 
0.2155 gram substance gave 0.0885 gram $\mathrm{H}_{2} \mathrm{O}$ and 0.4180 gram $\mathrm{CO}_{2}$.

Found.

$\begin{array}{ccc}\text { C ..... } & 52.90 & \text { per cent. } \\ \text { H..... } & 4.56 \quad, \\ \text { O .... } & 42 \cdot 54 \quad,\end{array}$
Theory. $\mathrm{C}_{10} \mathrm{H}_{10} \mathrm{O}_{6}$.

53.09 per cent.

$4 \cdot 42$

$42 \cdot 48$

This acetate melts at $165-167^{\circ}$. It is easily soluble in hot alcohol, less so in benzene, chloroform, ether, and bisulphide of carbon. From the hot solution in methyl alcohol, it separates on cooling as a hard sandy mass, which when seen under the microscope is found to consist of beautifully formed crystals, mostly having the following forms :-

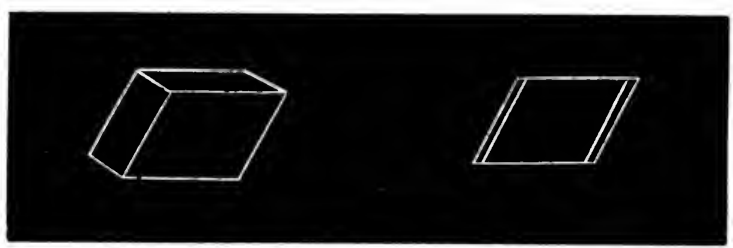

When boiled with water or alcohol for any length of time, it is slowly decomposed, hydroxydehydracetic acid being apparently regenerated. The acetate gives no coloration with ferric chloride. When heated in a test-tube, acetic anhydride is split off, and a small quantity of a white sublimate is obtained, which gives a violet coloration with ferric chloride, and consists therefore probably of regenerated hydroxydehydracetic acid.

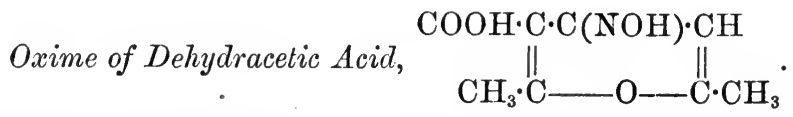

Dehydracetic acid combines readily with hydroxylamine, if its sodium salt be allowed to remain in contact with a solution of this compound. In a short time, a crystalline substance begins to separate, and after standing for 24 hours the reaction is complete. In order to isolate the product, the solution is slightly acidified with dilute sulphuric acid, and the precipitate thus thrown down is collected, washed with water, and repeatedly recrystallised from alcohol or dilute acetic acid, or light petroleum. In this way, colourless needles are obtained, melting at about $171-173^{\circ}$ with decomposition.

Analysis :-

0.1874 gram substance gave 0.0812 gram $\mathrm{H}_{2} \mathrm{O}$ and 0.3640 gram $\mathrm{CO}_{2}$. 
$0 \cdot 2261$ gram substance gave 15.5 c.c. $\mathrm{N}, t=15^{\circ}$, bar. $=720 \mathrm{~mm}$.

\begin{tabular}{|c|c|c|}
\hline & $\begin{array}{l}\text { Found. } \\
52.97 \text { per cent. }\end{array}$ & $\begin{array}{l}\text { Theory. } \\
\mathrm{C}_{7} \mathrm{H}_{8} \mathrm{O}_{3} \mathrm{CNOH} \text {. } \\
52 \cdot 46 \text { per cent. }\end{array}$ \\
\hline & $4 \cdot 81 \quad$ & 4.92 \\
\hline & $7 \cdot 60^{\circ}$ & $7 \cdot 65$ \\
\hline . . & $34 \cdot 62$ & $34 \cdot 97$ \\
\hline
\end{tabular}

This substance is formed from dehydracetic acid according to the equation-

$$
\mathrm{C}_{8} \mathrm{H}_{8} \mathrm{O}_{4}+\mathrm{NH}_{2} \cdot \mathrm{OH}=\mathrm{C}_{8} \mathrm{H}_{8} \mathrm{O}_{3}(\mathrm{NOH})+\mathrm{H}_{2} \mathrm{O} .
$$

This oxime is easily soluble in alcohol, benzene, and acetic acid, but only sparingly in cold light petroleum. The alcoholic solution gives an intense violet coloration with ferric chloride. Odernheimer(Ber., 17, 2087) has also prepared the oxime of dehydracetic acid. In addition to the properties given above, he found that the alcoholic solution was precipitated by silver nitrate, an unstable white precipitate of the silver salt being formed. He also prepared a barium salt by precipitating a neutral solution of the oxime with baric chloride.

Pheinglhydrazinedehydrucetic Acid,

$$
\mathrm{COOH} \cdot \mathrm{C}-\mathrm{C}\left(\mathrm{N}_{2} \mathrm{H} \cdot \mathrm{C}_{6} \mathrm{H}_{5}\right)-\mathrm{CH}
$$<smiles>C=C(C)OC(=C)C</smiles>

The phenylhydrazine-derivative of dehydracetic acid may be prepared in several ways. It was first obtained by treating the sodium salt of dehydaracetic acid with a solution of phenylhydrazine acetate, and then acidifying with dilute sulpharic acid, but the easiest way of preparing it is to add an excess of phenylhyảrazine to the warm alcoholic solution of dehydracetic acid, and then allowing it to stand. In a short time, a yellow crystalline solid separates out, which after collecting, well washing with alcohol, and recrystallising from alcohol, is easily obtained pure.

Analysis :-

$0 \cdot 2069$ gram substance gave $0 \cdot 1041$ gram $\mathrm{H}_{2} \mathrm{O}$ and $0 \cdot 4940$ gram $\mathrm{CO}_{2}$.

0.2634 gram substance gave 26 c.c. $\mathrm{N}, t=18^{\circ}$; bar. $=719 \mathrm{~mm}$.

Found.

C ..... $65 \cdot 12$ per cent.

H..... 5.59,

N.... 10.79 ,

O.... 18.50 ,
Theory. $\mathrm{C}_{8} \mathrm{H}_{8} \mathrm{O}_{3} \mathrm{~N}_{2} \mathrm{H} \cdot \mathrm{C}_{6} \mathrm{H}_{5}$.

$65 \cdot 12$ per cent.

$5 \cdot 42$

$10 \cdot 85$

$18 \cdot 61$ 
This substance is formed from dehydracetic acid according to the equation-

$$
\mathrm{C}_{8} \mathrm{H}_{8} \mathrm{O}_{4}+\mathrm{NH}_{2} \cdot \mathrm{NH} \cdot \mathrm{C}_{6} \mathrm{H}_{5}=\mathrm{C}_{8} \mathrm{H}_{8} \mathrm{O}_{3} \cdot \mathrm{N}_{2} \mathrm{H} \cdot \mathrm{C}_{6} \mathrm{H}_{5}+\mathrm{H}_{2} \mathrm{O} \text {. }
$$

When rapidly heated, it melts at $207^{\circ}$ with decomposition, but when slowly heated it begins to decompose at $200^{\circ}$. It is moderately easily soluble in hot benzene, alcohol, and chloroform, but only sparingly in light petroleum. From the hot solution in benzene or alcohol, it crystallises on cooling in beautiful brilliant yellow crystals, which when examined under the microscope are found to have the following forms :-

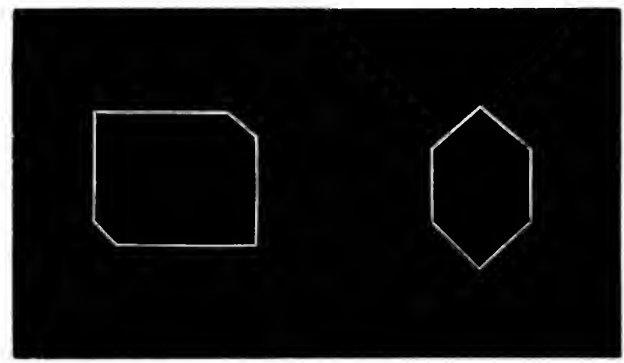

It dissolves with difficulty in ammonia and sodic carbonate solutions, and is reprecipitated by acids apparently unchanged.

Haitinger (Ber., 18, 453) suggests that these products of the action of hydroxylamine and phenylhydrazine on dehydracetic acid may have constitutions different from those given above. He thinks it very probable that these basic substances may have acted in the same way as ammonia, producing compounds of the constitution-

$\mathrm{COOH} \cdot \mathrm{C}-\mathrm{CO}-\mathrm{CH}$

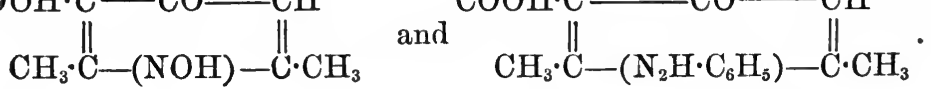

This is quite possible, although improbable, and I hope by further experiments to be able to distinguish between these formulæ.

\section{Action of Potash on Delydracetic Acid.}

In the introduction to this paper, I mentioned that the first change which dehydracetic acid underwent when treated with potash was decomposition into 2 mols. of acetoacetic acid, thus :-

$$
\mathrm{C}_{8} \mathrm{H}_{8} \mathrm{O}_{4}+2 \mathrm{H}_{2} \mathrm{O}=2 \mathrm{CH}_{3} \cdot \mathrm{CO} \cdot \mathrm{CH}_{2} \cdot \mathrm{COOH} \text {. }
$$

The experiments which were made to prove this were conducted in the following way:- 
Pure dehydracetic acid was first dissolved in a little dilute alcoholic potash, then an excess of a concentrated alcoholic potash solution added, and the whole allowed to stand for 14 days. The mass soon became brownish coloured and quite thick, but towards the end of the reaction it was again more fluid. The thick stage appears to be brought about by the separation of the potassium salt of dehydracetic acid; this at a later stage gives place to the potassium salt of acetoacetic acid, which is much more soluble.

In order to isolate the product of the reaction, the whole is dissolved in water, acidified with dilute sulpharic acid, and filtered from any unchanged dehydracetic acid which may separate out. The filtrate is then extracted several times with ether, the ethereal solution washed with water, dried over calcic chloride, and evaporated at as low a temperature as possible. A small quantity of dehydracetic acid, which generally crystallises out during this operation, can be easily removed by treating the residue with a small quantity of water and filtering, when the dehydracetic acid remains undissolved. The filtrate which contains the acetoacetic acid gives the characteristic violet coloration on adding ferric chloride. If this solution be extracted with pure ether, a small quantity of a yellowish-coloured oil is obtained, which shows all the reactions of acetoacetic acid. In order to obtain it as pure as possible, it was allowed to stand for some time over sulphuric acid in a vacuum, and then further characterised by distilling with water. In this way, carbonic anhydride was evolved and a distillate obtained, which, when treated with orthonitrobenzaldehyde and dilute soda, gave the indigo reaction rery distinctly, thus proving the presence of acetone. The acetoacetic acid had been decomposed into carbonic anhydride and acetone thus :-

\section{$\mathrm{CH}_{3} \cdot \mathrm{CO} \cdot \mathrm{CH}_{2} \cdot \mathrm{COOH}=\mathrm{CH}_{3} \cdot \mathrm{CO} \cdot \mathrm{CH}_{3}+\mathrm{CO}_{2}$.}

The quantity of acetoacetic acid obtained in this way is naturally very small, owing to its instability.

It is an interesting fact that Conrad and Guthzeit (Ber., 20, 152) also obtained a substance by the action of potash on ethylic dimethylpyronedicarboxylate, which gave a violet coloration with ferric chloride. They consider it probable that in this case acetoacetic acid was also produced by the hydrolysis.

$$
\text { Methylic Dehydracetate, } \quad \mathrm{COOCH}_{3} \cdot \mathrm{C}-\mathrm{CO}-\mathrm{CH}
$$

The methylic ether of dehydracetic acid used in the following experiments was prepared by Oppenheim and Precht's method (Ber., 9, 324), that is, by treating the silver salt of dehydr- 
acetic acid with methyl iodide, and extracting the product with ether. The yield obtained in this way is far from satisfactory, owing to the difficulty of preparing the silver salt in a pure condition, but up to the present I have not been able to improve on this method, the ordinary methods of etherification with alcohol and bydrochloric acid, \&e., being useless in the case of dehydracetic acid. It is, however, very essential, in preparing this methylic salt from the silver salt, to have all the materials scrupulously dry, otherwise no methylic salt at all is obtained.

Methylic dehydracetate melts at $91^{\circ}$, as Oppenheim and Precht liave shown. In experimenting with it, I have found it to possess most remarkable properties. It is easily soluble in water, producing a solution which has a decidedly acid reaction to litmus-paper. When treated with potash it is instantly saponified, even in the cold. If a solution of methylic dehydracetate $(1 \mathrm{~mol}$.) in pure dry ether is mixed with an ethereal solution of sodic ethylate ( 1 mol.), a pink sodium compound is precipitated, which, after being collected, well washed with ether, and dried over sulphuric acid in a vacuum, gave the following numbers on analysis :-

I. $0 \cdot 1440$ gram substance gave $0 \cdot 0476$ gram $\mathrm{Na}_{2} \mathrm{SO}_{4}$.

II. $0 \cdot 2414$

$$
\text { " }
$$

"

0.0788

Found.
I.
II.
Na..... $10 \cdot 71$
$10 \cdot 60$ per cent.
Theory.
$\mathrm{C}_{5} \mathrm{II}_{6}(\mathrm{Na}) \mathrm{O}_{4} \mathrm{CH}_{3}$.
$11 \cdot 27$ per cent.

It is possible that this sodium compound has the formula

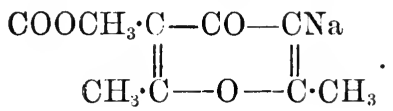

The hydrogen-atom of the $\mathrm{CH}$-group in methylic dehydracetate may, on account of its position between the ketone-group - $\mathrm{CO}-$ and the ether-group $\equiv \mathrm{C}-\mathrm{O}-\mathrm{C} \equiv$, have an acid character like the hydrogen-atoms of the $\mathrm{CH}_{2}$-group in ethylic acetoacetate. It does not appear possible, however, by acting on ethylic or methylic dehydracetate with ethyl iodide to obtain substituted dehydracetic acids (see Shibbye, Inaugural Dissertation, Wurzbiirg, 1882, page 12), for this the sodium compound is too unstable. The sodium compound dissolves easily in water, forming a pinkish-coloured solution. On quickly adding acid to this and extracting at once with ether, I once succeeded in obtaining colourless crystals melting at $85-90^{\circ}$, and which consisted probably of impure methylic dehydracetate. On repeating this experiment several times, however, I have 
since always obtained an uninviting brownish resin, which resisted all attempts to get it in a crystalline form.

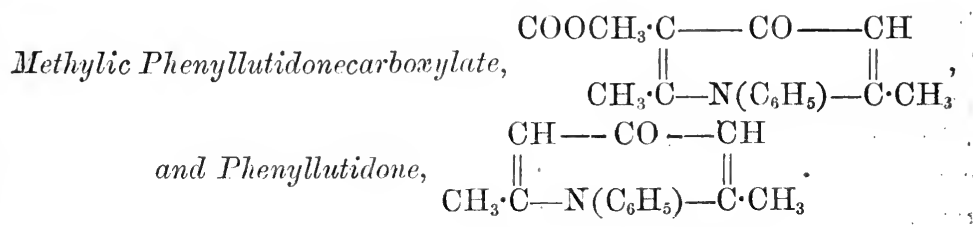

Methylic dehydracetate when treated with aniline is converted almost immediately into methylic phenyllutidonecarboxylate and phenyllutidone, as described in the introduction. In order to effect this change, pure methylic dehydracetate dissolved in a little methyl alcohol, is mixed with about twice its weight of aniline, and warmed gently for about 15 seconds to $50^{\circ}$. An equal bulk of water is then added, and the whole allowed to stand in a cool place for 12 hours. At the end of this time, it is found that a small quantity of a substance crystallising in long, colourless needles has separated. This, after collecting, washing with water, and once recrystallising, is easily obtained pure.

The analysis gave the following numbers:-

0.1472 gram substance gave 0.0775 gram $\mathrm{H}_{2} \mathrm{O}$ and 0.3750 gram $\mathrm{CO}_{2}$.

$$
\begin{aligned}
& \text { Theory. } \mathrm{C}_{15} \mathrm{H}_{15} \mathrm{NO}_{3} \text {. } \\
& \text { C..... 70.04 per cent. } \\
& \text { H .... } 5.83 \text {, }
\end{aligned}
$$

Found.

$69 \cdot 66$ per cent.

$5.69 \quad$,

Methylic phenyllutidonecarboxylate is insoluble in sodic carbonate solution. On boiling with alcoholic potash, it is easily saponified, a colourless acid being produced, which has, however, not yet been further examined.

The principal product of the action of aniline on methylic dehydracetate is, however, not methylic phenyllutidonecarboxylate, but a substance which remains dissolved in the mother-liquor after this has crystallised out. If these mother-liquor's are evaporated on a water-bath, the solution becomes yellower and yellower, and at last quite deep orange-coloured. This is due to the formation of a colouring matter, and if the eraporation is carried on to dryness, a reddish amorphous mass with a magnificent green lustre remains, which appears to be this colouring matter in an almost pure state. No attempt has been made to isolate and purify this substance, owing to the small amount of material at command.

In order, however, to avoid the formation of this colouring matter, the solution was first acidified with dilute hydrochloric acid, and then 
evaporated almost to dryness on a water-bath. The yellow colouring matter does not appear to be formed when acid solutions are emplosed, the residue left on evaporation being an almost colourless syrup, which did not crystallise after standing for some hours. The whole was therefore rendered slightly alkaline by the addition of potash solution and allowed to stand in a cool place for some hours. After about six hours, a quantity of needle-shaped crystals had separated, which, when collected, washed with a little water, and recrystallised from this solvent, were easily obtained pure.

Analysis :-

0.1392 gram substance gave 0.0833 gram $\mathrm{H}_{2} \mathrm{O}$ and 0.4023 gram $\mathrm{CO}_{2}$.

$$
\begin{aligned}
& \text { Theory. } \\
& \mathrm{C}_{13} \mathrm{H}_{13} \text { NO. Found. } \\
& \text { C . ..... } 78.39 \text { per cent. } \quad 78.82 \text { per cent. } \\
& \mathrm{H} \ldots .66 .54 \quad, \quad 6.65 \text {, }
\end{aligned}
$$

Th1s substance is phenyllutidone.

Phenyllutidone melts at $196-197^{\circ}$. It is easily soluble in warm water and alcohol, less so in benzene. From the hot solution in water or benzene, it crystallises on cooling in colourless needles. This substance is identical with the phenyllutidone obtained by Conrad and Guthzeit (Ber., 20, 161), by heating phenyllutidonedicarboxylic acid at $270^{\circ}$. The platinochloride is easily prepared by adding platinum chloride to the solution of phenyllutidone in hydrochloric acid. It is thus obtained as a yellow powder, which crystallises from water in yellow needles; these, when examined under the microscope, are found to have the following forms:-

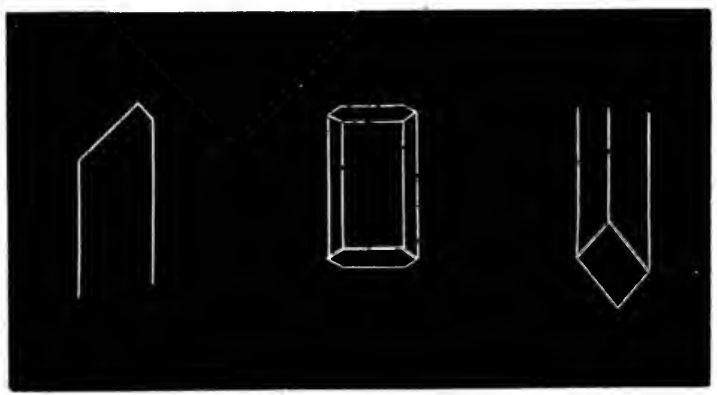

Analysis :-

0.3017 gram substance gave 0.1080 gram $\mathrm{H}_{2} \mathrm{O}, 0.4286$ gram $\mathrm{CO}_{2}$, and 0.0726 gram $\mathrm{Pt}$. 
Theory.

$\left(\mathrm{C}_{13} \mathrm{H}_{13} \mathrm{NO}\right)_{2}, \mathrm{H}_{2} \mathrm{PtCl}_{6}$.

C ....... 38.52 per cent.

$\mathrm{H} \ldots \ldots .3 \cdot 46$

Pt ..... 24:32
Found.

38.68 per cent.

3.97 ,

24.09 ,

This platinochloride has also been prepared by Conrad and Guthzeit from their phenyllutidone from phenyllutidonedicarboxylic acid. Both salts were found to be identical, not only in appearance but also in behaviour.

I am at present engaged on further experiments on dehydracetic acid, the results of which I hope to be able to bring before the Society in a short time. 



\section{ON SOME DERIVATIVES OF ANTHRAQUINONE.}

\section{By A. G. Perkin and W. H. Perkin, jun., Ph.D.}

DURIvg some experiments on the sulphonic acids of anthraquinone it was observed that the sodium salts of these acids were decomposed in a somewhat remarkable way when submitted to dry distiliation. As the compounds formed appeared to be of considerable interest, we have investigated them more closely.:

The sodium salt employed in the following experiments was the socalled "silver salt" which is produced in such large quantities in alizarin factories, and which consists of nearly pure sodium anthraquinonemonosulphonate.

The specimen used was soluble in water, forming a clear nentral solution, which gave no coloration with caustic soda, and thus showed the absence of hydroxyanthraquinone-derivatives. Dried at $120^{\circ}$ and ignited with nitrate of ammonia, 0.2005 gram gave 0.0467 gram $\mathrm{Na}_{2} \mathrm{SO}_{4}=23 \cdot 29$ per cent. ; calculated for $\mathrm{C}_{14} \mathrm{H}_{7} \mathrm{O}_{2} \cdot \mathrm{SO}_{3} \mathrm{Na}=22 \cdot 97$.

The dry distillation of this salt was carried out in an iron tube $8^{\prime \prime} \times 2^{\prime \prime}$ welded together at one end. A piece of gas piping, bent at a right angle, was screwed into the aperture of this tube, and its open end passed through a loose wooden lid into a large cylindrical copper vessel which served as a condenser. The retort was charged with about 150 grams of salt, and heated over an ordinary fire. After a few minutes, yellowish-brown vapours were evolved which easily condensed in the receiver, and these continued to come off until the end of the operation, the whole distillation usually occupying abont half an hour.

The distillate was a brownish, fluffy mass, mixed with a small quantity of a slightly acid liquid containing sulphur in suspension. On unscrewing the connecting tube it was usually found to contain a snall quantity of long yellow needles easily recognisable as anthraquinone.

The yield of distillate was small-on an average only about 20 per cent. of the weight of salt employed. From 55-60 per cent. of residue remained in the retort as a hard, brittle, carbonaceous mass; this, when extracted with water, yielded a strongly alkaline, green solution, which was found to consist almost entirely of sodium sulphide containing a little ferrous sulphide.

The distillate, which proved to be a mixture of several compounds, was digested for some time with boiling baryta-water. A portion dissolved, producing a blood-red solution which was separated from 
the insoluble residue by filtration. The filtrate, after standing for some time, was again filtered, and then treated with dilute hydrochloric acid, which caused the precipitation of a small quantity of a straw-coloured, gelatinous substance.

This was thrown on a filter, well washed with water, and dried. After repeated recrystallisation from coal-tar naphtha, it was obtained pure in the form of pale-yellow needles, which on analysis gave the following numbers :-

0.2058 gram substance gave $0.0693 \mathrm{H}_{2} \mathrm{O}$ and $0.5641 \mathrm{CO}_{2}$.

\begin{tabular}{|c|c|c|}
\hline & $\begin{array}{l}\text { Found. } \\
74.7 \check{\text { per cent. }}\end{array}$ & $\begin{array}{l}\text { Theory : } \mathrm{C}_{14} \mathrm{H}_{8} \mathrm{O}_{3} \text {. } \\
75.00 \text { per cent }\end{array}$ \\
\hline H ... & $3 \cdot 74$ & 3.57 \\
\hline $0 \ldots$ & $21 \cdot 51$ & $21 \cdot 43$ \\
\hline
\end{tabular}

It was therefore metahydroxyanthraquinone, the compound produced by fusing anthraquinonemonosulphonic acid or metabromoanthraquinone with potash (Graebe and Liebermann, Annalen, 160, $141)$, and agreed in properties with this compound in every respect. It melted at about $300^{\circ}$ (metahydroxyanthraquinone $302^{\circ}$ ), and gave an easily soluble barium salt (distinction from orthohydroxyanthraquinone).

The residue insoluble in baryta-water was repeatedly extracted with boiling glacial acetic acid, nntil the extracts became of a pale, yellowish colour. These extracts, when filtered from the insoluble residue and cooled, deposited a dirty yellowish substance, which was separated from the mother-liquors, well dried, and sublimed. The sublimate, which appeared to consist chiefly of anthraquinone, was extracted with small quantities of hot coal-tar naphtha, in order to free it from traces of a more soluble substance, and the residue repeatedly recrystallised from the same solvent. On analysis it gave the following numbers, showing it to be anthraquinone :-

0.1207 gram substance gave $0.0448 \mathrm{H}_{2} \mathrm{O}$ and $0.3554 \mathrm{CO}_{2}$.

Found.

C ...... 80.30 per cent.

H ........ $4: 12$

O $\ldots \ldots \ldots 15 \cdot 58$
Theory : $\mathrm{C}_{14} \mathrm{H}_{8} \mathrm{O}_{2}$.

$80 \cdot 77$ per cent.

$\begin{array}{rr}3 \cdot 84 & , \\ 15 \cdot 39 & \text { " }\end{array}$

This compound crystallised in yellow needles, melting at 271$273^{\circ}$ (anthraquinone melts at $273^{\circ}$ ). On heating it in a test-tube it sublimed in the beautiful way so characteristic of anthraquinone, and had, moreover, all the properties of that compound.

On evaporating the mother-liquors from the above crystals they deposited a small quantity of metahydroxyanthraquinone which had 
escaped extraction in the first instance. The residue, after having been extracted a few times with glacial acetic acid as described above, was digested with large quantities of glacial acetic acid and filtered, this operation being repeated until the whole was dissolved. On cooling the solutions thus obtained a yellowish-red substance separated ont, which under the microscope was seen to consist for the most part of minute crystals. By carefully recrystallising this substance several times from the same solvent, it was obtained pure in the form of a spongy mass of minute reddish-yellow needles, which sometimes had a silky lustre. The greatest care was necessary in order to obtain this substance well crystallised, the method found to give the best results being the following:-A saturated solution in boiling acetic acid was filtered into a flask standing on a sand-bath and containing a small quantity of boiling acid, the funnel being so arranged that the greater portion was heated by the vapour evolved. After filtration the lamp was removed, and the whole allowed to stand in a place free from draught. The separation of the substance, which commenced after several minutes, was peculiar, reminding one somewhat of a fungoid growth. It started at the bottom of the vessel in small spongy tufts which gradually grew until the whole liquid was filled with crystals. If the solution be quickly cooled, or agitated during cooling, the substance is deposited in a more or less gelatinous condition. The following numbers were obtained on analysis, each sample being from a different preparation.

I. 0.1248 gram substance gave $0.0390 \mathrm{H}_{2} \mathrm{O}$ and $0.3425 \mathrm{CO}_{2}$.

II. $0.1745 \quad " \quad, \quad 0.0526 \mathrm{H}_{2} \mathrm{O}, 0.4815 \mathrm{CO}_{2}$.

III. $0.1358 \quad " \quad " \quad$ " $\quad 0.0420 \mathrm{H}_{2} \mathrm{O}, \quad 0.3728 \mathrm{CO}_{2}$.

1V. $0.1415 \quad " \quad " \quad 0.0437 \mathrm{H}_{2} \mathrm{O}, 0.3899 \mathrm{CO}_{2}$.

V. $0.1354 \quad " \quad, \quad 0.0418 \mathrm{H}_{2} \mathrm{O} \quad, \quad 0.3740 \mathrm{CO}_{2}$.

Found.

\begin{tabular}{|c|c|c|c|c|c|c|}
\hline I. & II. & III. & IV. & V. & $\mathrm{C}_{28} \mathrm{H}_{14} \mathrm{O}_{6}$. & $\mathrm{C}_{14} \mathrm{H}_{8} \mathrm{O}_{3}$. \\
\hline $1 \cdot 84$ & 75 & 75 & 75. & 75.33 p.c. & 75.34 & $75.00 \mathrm{p}$ \\
\hline $3 \cdot 46$ & $3 \cdot 3$ & & 3 . & $3 \cdot 43$ & $3 \cdot 14$ & 3.57 \\
\hline $91 \cdot 70$ & $21 \cdot 40$ & $21 \cdot 38$ & $21 \cdot 55$ & $21 \cdot 24$ & $21 \cdot 52$ & $21 \cdot 43$ \\
\hline
\end{tabular}

This compound therefore appears to have the formula $\mathrm{C}_{28} \mathrm{H}_{14} \mathrm{O}_{6}$, rather than that of $\mathrm{C}_{14} \mathrm{H}_{8} \mathrm{O}_{3}$, which is also borne out by its properties. It is practically insoluble in all the usual solvents in the cold, and only very sparingly soluble in boiling acetic acid, from which it seems to crystallise best. For ordinary purposes it is therefore only necessary to extract the crude product from the destructive distillation of the sodium anthraquinoncmonosulphate with alcohol or acetic acid 
until the more soluble portion has been removed, and to use for further reactions the sparingly soluble residue, which consists of the compound $\mathrm{C}_{28} \mathrm{H}_{14} \mathrm{O}_{6}$ in an almost pure state. On distillation with zinc-dust, the compound $\mathrm{C}_{28} \mathrm{H}_{14} \mathrm{O}_{6}$ gave anthracene-recognised by its melting point $\left(215^{\circ}\right)$, and from the fact that it gave anthraquinone on oxidation. When heated, it melts at a temperature much above $300^{\circ}$, to a reddish-black liquid, which on further heating sublimes as a reddish powder, only a small quantity of charcoal being left. It dissolves in concentrated sulphuric acid in the cold, forming an intense magenta-coloured solution, from which on the addition of water it is precipitated unchanged in the form of a transparent yellow jelly. When heated to a high temperature with fuming sulphuric acid, it dissolves, forming an orange-coloured solution resembling anthraquinonesulphonic acid, with which, however, it is not identical. At very high temperatures a small quantity of a colourless, crystalline sublimate was obtained. This readily dissolved in a hot solution of sodium carbonate, and on addition of acid, extraction with ether, and evaporation of the ethereal extract, colourless needles were obtained, which were easily recognised as phthalic acid.

A boiling mixture of nitric and sulphuric acids dissolves it, and on addition of water a white substance is precipitated, which separates in an amorphous condition from its boiling solution in ordinary solvents. The compound $\mathrm{C}_{28} \mathrm{H}_{14} \mathrm{O}_{6}$ is, curiously enough, absolutely insoluble in alkalis. When fused with caustic soda it dissolves, forming a violet mass, which has the properties of sodium alizarate, and which, on solntion in water and treatment with acid, gives a yellow precipitate; this dyes mordanted cloth in a way perfectly analogous to alizarin.

As it was important to be sure of the formation of alizarin, a quantity of the red body was heated with a strong solution of caustic soda in an iron tube at $180^{\circ}$, and the sodium alizarate, after dilution with water and filtering, purified by conversion into the lime salt. On acidifying this salt with hydrochloric acid, a jellowish mass was precipitated, which after being collected, well washed with water, and recrystallised, was found to melt at $277^{\circ}$ (alizarine melts at $275^{\circ}$ ). The identity of these two substances was further proved.by the similarity of the spectra of their solutions.

Heated with zinc-dust and caustic soda the compound $\mathrm{C}_{28} \mathrm{H}_{14} \mathrm{O}_{6}$ dissolves, and forms a blood-red solution, which on exposure to the air is decolorised, the original compound being reprecipitated. In this respect the substance very much resembles anthraquinone. 


\section{Oxidation of the Compound $\mathrm{C}_{28} \mathrm{H}_{14} \mathrm{O}_{6}$.}

If a boiling saturated solution of this substance in glacial acetic acid be treated with chromic acid, a violent reaction sets in, and, on cooling, a whitish substance separates. As it was thought that the examination of this reaction might give some clue to the constitution of the compound $\mathrm{C}_{28} \mathrm{H}_{14} \mathrm{O}_{6}$, it was carried out on a large scale in order to obtain the white substance in sufficient quantity for analysis. For this purpose the boiling solution of the red compound was treated with chromic acid, in small quantities at a time, until it was no longer reduced. The boiling was then continued for a short time, and the solution allowed to cool. After standing for some hours, the green solution had deposited a quantity of a white crystalline substance, which was collected, well washed with water, and dried at $100^{\circ}$. After being purified by several crystallisations from coal-tar naphtha, it was obtained in the form of colourless transparent plates melting at $292-295^{\circ}$. On analysis the following numbers were obtained :-

I. $0 \cdot 1546$ gram substance gave 0.0439 gram $\mathrm{H}_{2} \mathrm{O}$ and 0.3988 gram $\mathrm{CO}_{2}$.

II. $0 \cdot 1196$ gram substance gave $0 \cdot 0327$ gram $\mathrm{H}_{2} \mathrm{O}$ and 0.3096 gram $\mathrm{CO}_{2}$.

\begin{tabular}{|c|c|c|c|}
\hline & \multicolumn{2}{|c|}{ Found. } & \multirow[b]{2}{*}{ Theory. } \\
\hline & I. & II. & \\
\hline & $70 \cdot 35$ & 70.59 per cent. & 70.59 per cent \\
\hline & $3 \cdot 15$ & $3 \cdot 03$ & $2 \cdot 52$ \\
\hline & $26 \cdot 50$ & $26 \cdot 38$ & $26 \cdot 89$ \\
\hline
\end{tabular}

The compound has therefore the formula $\mathrm{C}_{14} \mathrm{H}_{6} \mathrm{O}_{4}$, and is formed from the compound $\mathrm{C}_{28} \mathrm{H}_{14} \mathrm{O}_{6}$ according to the equation-

$$
\mathrm{C}_{28} \mathrm{H}_{14} \mathrm{O}_{6}+\mathrm{O}_{3}=2 \mathrm{C}_{14} \mathrm{H}_{6} \mathrm{O}_{4}+\mathrm{H}_{2} \mathrm{O} \text {. }
$$

It is almost insoluble in boiling benzene, slightly soluble in boiling coal-tar naphtha and acetic acid, more soluble in boiling aniline; on cooling, it separates from the latter, in the form of transparent needles. It is quite insoluble in alkalis, and can therefore contain no hydroxyl-group.

When heated it first melts to a straw-coloured liquid, and then sublimes almost without decomposition. It yields anthracene when distilled with zinc-dnst. On warming with phenylhydrazine it yields a red substance, which, however, has not been obtained in sufficient quantity for analysis. The hot alcoholic solution of the compound $\mathrm{C}_{14} \mathrm{H}_{6} \mathrm{O}_{4}$ gives, on the addition of ferric chloride, a reddish-brown coloration. When heated with strong alcoholic potash, a violet solution is first 
obtained, which, however, again becomes colourless on shaking with air. If the heating be repeated, a reddish-brown solution is obtained.

If the substance is heated with aqueous potash and a trace of zinc-dust, a violet solution is produced, which on the addition of more of the reducing agent changes to olive-green. On agitating with air, the solution becomes first violet and then colourless, the original compound being reprecipitated. With zinc-dust and ammonia a violet solution is produced, which becomes colourless in contact with the air.

From the study of these properties, it seems that the compound $\mathrm{C}_{14} \mathrm{H}_{6} \mathrm{O}_{4}$ must have the following formula:-<smiles></smiles>

it being an orthoquinone of anthraquinone.

The red substance $\mathrm{C}_{28} \mathrm{H}_{14} \mathrm{O}_{6}$ has therefore probably the constitution-

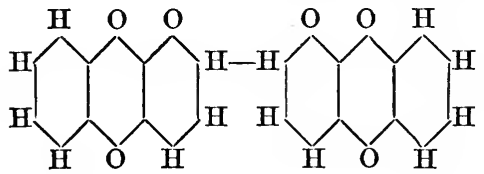

being a derivative of di-anthracene.

These formulæ, however, require further experimental proof before they can be looked upon as being definitely settled. It is also intended to investigate the behaviour of the $\alpha$ - and $\beta$-salts of anthraquinonedisulphonic acid when submitted to dry distillation. 


\section{ON SOME DERIVATIVES OF ANTHRAQUINONE.}

\section{By A. G. Perkin and W. H. Perkin, Jun., Ph.D.}

IN a previous paper on this subject, which we had the honour of communicating to the Society some time since (Trans., 1885, 47, 679), we described a series of experiments on the products of the destructive distillation of sodium anthraquinonemonosulphonate.

This salt, when heated in an iron tabe, yielted a brownish-red distillate, which on examination was found to contain metahydroxyanthraquinone, anthraquinone, and a peculiar red substance, which after purification by recrystallisation gave numbers agreeing with the formula $\mathrm{C}_{28} \mathrm{H}_{14} \mathrm{O}_{6}$; it is nearly insoluble in all the usual solvents, and, curiously enough, in alkalis also.

On oxidation with chromic acid, it yields a white crystalline substance of the formula $\mathrm{C}_{14} \mathrm{H}_{6} \mathrm{O}_{4}$, and when distilled with zinc-dust anthracene is produced, proving the substance to be an anthracenederivative. The remarkable character of this decomposition of sodium anthraquinonemonosulphonatc, has led us to continue our experiments, with a view to obtain some clue as to the constitution of the compound $\mathrm{C}_{28} \mathrm{H}_{14} \mathrm{O}_{6}$. The extreme difficulty, however, of obtaining it in any quantity, owing to the tediousness of its preparation and purification, has prevented us from making as thorough an examination of the subject as we could have wished. We hope at some future date to be able to complete these experiments, and to establish without doubt the constitution of these derivatives.

Before proceeding with the examination of the red substance $\mathrm{C}_{28} \mathrm{H}_{14} \mathrm{O}_{6}$, we thought it advisable to again analyse it, in order that there might be no doubt as to its formula. A quantity was therefore prepared and very carefully purified by repeated recrystallisations from glacial acetic acid. The pure substance was thus obtained as an orange, satiny, crystalline mass, which after careful drying at 110$120^{\circ}$ gave the following results on analysis :- 
0.1609 gram substance gave 0.0495 gram $\mathrm{H}_{2} \mathrm{O}$ and 0.4450 gram $\mathrm{CO}_{2}$.

\begin{tabular}{|c|c|c|}
\hline & $\begin{array}{l}\text { Theory. } \\
\mathrm{C}_{28} \mathrm{H}_{14} \mathrm{O}_{6} \text {. } \\
75 \cdot 34_{1} \text { per cent. }\end{array}$ & $\begin{array}{l}\text { Found. } \\
75.37 \text { per cent. }\end{array}$ \\
\hline$\ldots$ & $3 \cdot 14$ & $3 \cdot 42$ \\
\hline & $21 \cdot 52$ & $21 \cdot 21$ \\
\hline
\end{tabular}

If a hot dilute solution of this substance in glacial acetic acid is slowly cooled, groups of small, orange-coloured crystals separate, just visible to the naked eye ; these when collected, washed, and dried, present the appearance of beautiful, satiny, orange-coloured flakes. If hot saturated solutions are rapidly cooled, the substance is deposited as an orange-coloured gelatinous mass.

In the previous paper it was stated that this compound, $\mathrm{C}_{28} \mathrm{H}_{14} \mathrm{O}_{6}$, was con verted into alizarin on fusion with potash. As it was important to be quite sure of this, the experiment was repeated with somewhat larger quantities, the decomposition being conducted in the following manner :-About 2 grams of $\mathrm{C}_{28} \mathrm{H}_{14} \mathrm{O}_{6}$ were heated with a very concentrated solution of caustic potash at $180^{\circ}$ for about 12 hours in a closed iron tube. The contents of the tube, which in appearance very much resembled an ordinary alizarin melt, were extracted with boiling water, the violet-coloured solution filtered from a small quantity of insoluble matter, and a little slaked lime added. The alizarate of lime thus precipitated was collected, well washed, suspended in water, decomposed with a little dilute hydrochloric acid, and the orange precipitate obtained was purified by recrystallisation from benzene. The product consisted of red needles melting at about $285^{\circ}$, and showing all the properties of alizarin. On analysis it gave the following numbers :-

0.1107 gram substance gave 0.0398 gram $\mathrm{H}_{2} \mathrm{O}$ and 0.2821 gram $\mathrm{CO}_{2}$.

\begin{tabular}{|c|c|c|}
\hline & $\begin{array}{l}\text { Theory. } \\
\mathrm{C}_{14} \mathrm{H}_{8} \mathrm{O}_{4} \text {. } \\
70.00 \text { per cent. }\end{array}$ & $\begin{array}{l}\text { Found. } \\
69.50 \text { per cent. }\end{array}$ \\
\hline & $3 \cdot 33 \quad$, & 3.97 \\
\hline & $26 \cdot 66$ & $26 \cdot 53$ \\
\hline
\end{tabular}

The filtrate from the alizarate of lime was of a reddish colour, with a green fluorescence; on treatment with hydrochloric acid, it deposited a very small quantity of a nearly white precipitate, which when washed on a filter changed to a dirty green. On fusion with potash and treatment of the fused mass with water, a solution was obtained having all the properties of potassic alizarate.

This substance is therefore intermediate between the red substance and alizarin ; it is probably identical with a substance, $\mathrm{C}_{28} \mathrm{H}_{14} \mathrm{O}_{7}$, which 
will be described later on, under the heading "Action of Nitric Acid on $\mathrm{C}_{28} \mathrm{H}_{14} \mathrm{O}_{6}$." When fused with potash, $\mathrm{C}_{28} \mathrm{H}_{14} \mathrm{O}_{6}$ is probably first oxidised to $\mathrm{C}_{28} \mathrm{H}_{14} \mathrm{O}_{7}$, which then takes up the elements of water, and is converted into alizarin, thus :-

$$
\mathrm{C}_{28} \mathrm{H}_{14} \mathrm{O}_{7}+\mathrm{H}_{2} \mathrm{O}=2 \mathrm{C}_{14} \mathrm{H}_{8} \mathrm{O}_{4} \text {. }
$$

That such a change really takes place is all the more probable, from the fact that the substance $\mathrm{C}_{28} \mathrm{H}_{14} \mathrm{O}_{7}$, when fused with potash, also gives alizarin.

The residue from the fusion, insoluble in water, was crystallised from acetic acid, and thus obtained in orange needles, which were found to consist of unchanged $\mathrm{C}_{28} \mathrm{H}_{14} \mathrm{O}_{6}$.

$0 \cdot 1491$ gram substance gave 0.0470 gram $\mathrm{H}_{2} \mathrm{O}$ and 0.4097 gram $\mathrm{CO}_{2}$.

\begin{tabular}{|c|c|c|}
\hline & $\begin{array}{l}\text { Theory. } \\
\mathrm{C}_{28} \mathrm{H}_{14} \mathrm{O}_{6} \text {. } \\
75 \cdot 34 \text { per cent. }\end{array}$ & $\begin{array}{l}\text { Found. } \\
75.01 \text { per cent }\end{array}$ \\
\hline & $3 \cdot 14$ & $3 \cdot 50$ \\
\hline & $21 \cdot 52$ & $21 \cdot 49$ \\
\hline
\end{tabular}

Action of Sulphuric Acid on $\mathrm{C}_{28} \mathrm{H}_{14} \mathrm{O}_{6}$.

Cold Nordhausen sulphuric acid dissolves the red substance, forming a dirty green solution, which in contact with the air absorbs moisture, and deposits the unchanged substance as a transparent jelly. When the solntion in Nordhausen acid is heated to $190^{\circ}$, the colour gradually changes to a brownish-red, and if at the end of about half an hour the mixture is poured into water, it dissolves to a clear solution, which on cooling sets to an opaque jelly. All attempts to free this mixture from sulphuric acid by treatment with the carbonates of lead or barium failed, owing to the salts of the sulphonic acid being insoluble.

If the mixture of the sulphonic acid and sulphuric acid is neutralised with potash and slowly evaporated, the dark solution deposits a small quantity of a crystalline salt, which under the microscope is seen to consist of long needles; these when heated to $100^{\circ}$ fuse to a gummy mass. Fused with strong potash in an iron tube for some hours, and then decomposed with hydrochloric acid, a substance is obtained, which dyes mordanted cloth shades intermediate between those produced by anthrapurpurin and flavopurpurin.

\section{Action of Nitric Acid on $\mathrm{C}_{28} \mathrm{H}_{14} \mathrm{O}_{6}$.}

On gradually adding the red substance, $\mathrm{C}_{28} \mathrm{H}_{14} \mathrm{O}_{6}$, to cold fuming nitric acid of sp. gr. 1.5, it dissolves without evolution of red fumes, 
forming a bromine-coloured solution. In examining the product of this reaction, the mass was allowed to stand for a short time, and then diluted with water. This caused the precipitation of a yellowishwhite, amorphous substance, which was filtered off, well washed, and dried. It was then dissolved in a little hot aniline or nitrobenzene, filtered, and boiling alcohol added drop by drop to the hot solution until the mixture showed a tendency to become milky. On allowing this to cool slowly, minute crystals were deposited, which, after collecting, washing with alcohol, and drying at $110^{\circ}$, gave the following numbers on analysis :-

I. 0.1366 gram substance gave 0.0418 gram $\mathrm{H}_{2} \mathrm{O}$ and 0.3658 gram $\mathrm{CO}_{2}$.

II. 0.1650 gram substance gave 0.0505 gram $\mathrm{H}_{2} \mathrm{O}$ and 0.4420 gram $\mathrm{CO}_{2}$.

\begin{tabular}{|c|c|c|c|}
\hline \multirow[b]{3}{*}{$C \ldots \ldots$} & \multirow{2}{*}{$\begin{array}{l}\text { Theory. } \\
\mathrm{C}_{28} \mathrm{H}_{14} \mathrm{O}_{7} \text {. }\end{array}$} & \multicolumn{2}{|c|}{ Found. } \\
\hline & & I. & II. \\
\hline & 72.72 p. с. & $73 \cdot 01$ & 73.05 p. c. \\
\hline & 3.03 & $3 \cdot 40$ & $3 \cdot 40$ \\
\hline $\mathrm{O}$ & $24 \cdot 24 \quad$, & 23.59 & 23.55 \\
\hline
\end{tabular}

This substance has therefore the formula $\mathrm{C}_{28} \mathrm{H}_{14} \mathrm{O}_{7}$, being produced from the red substance $\mathrm{C}_{23} \mathrm{H}_{14} \mathrm{O}_{6}$ by the simple addition of oxygen, thus :-

$$
\mathrm{C}_{28} \mathrm{H}_{14} \mathrm{O}_{6}+\mathrm{O}=\mathrm{C}_{28} \mathrm{H}_{14} \mathrm{O}_{7} \text {. }
$$

Crystallised from a mixture of aniline or nitrobenzene and alcohol, this substance $\mathrm{C}_{28} \mathrm{H}_{14} \mathrm{O}_{7}$ appears under the microscope as groups of short, colourless needles. From hot acetone, however, in which it is only very slightly soluble, it can be obtained in larger crystals. It.is slightly soluble in coal-tar naphtha, more soluble in acetic acid. When heated, it melts at a high temperature to a yellow liquid, which on cooling solidifies to a hard, crystalline mass. At higher temperatures it chars, only a very small quantity subliming in microscopic needles. $\mathrm{C}_{28} \mathrm{H}_{14} \mathrm{O}_{7}$ dissolves readily in hot aniline or nitrobenzene, and the solutions on cooling deposit small plates of a brownish colour. These substances, which are evidently additive compounds of $\mathrm{C}_{28} \mathrm{H}_{14} \mathrm{O}_{7}$ with aniline and nitrobenzene, have not as yet been analysed. They are readily obtained pure by adding cautiously to the hot solution in aniline or nitrobenzene about twice the volume of benzene. On cooling, the compound separates out in glistening plates. On boiling with alcohol, they are quickly split up into their constituents.

Boiled with zinc-dust and potash solution, $\mathrm{C}_{28} \mathrm{H}_{14} \mathrm{O}_{7}$ slowly dissolves, forming a reddish-brown solution, which on shaking with air instantaneously decolorises with precipitation of a white, gelatinous substance. 
The following reaction is characteristic of this substance:-If a trace be boiled with a strong solution of potash in methyl alcohol in a test-tube, it will gradually dissolve, with an olive-green coloration. On continued boiling, the solution becomes darker and darker coloured as the methyl alcohol evaporates, the green gradually changing to a beautiful brownish-pink. On adding water, a violet solution is obtained, which does not decolorise on shaking with air, and therefore possibly consists of potassic alizarate.

\section{Action of Nitric and Sulphuric Acids on $\mathrm{C}_{28} \mathrm{H}_{14} \mathrm{O}_{6}$.}

If $\mathrm{C}_{28} \mathrm{H}_{14} \mathrm{O}_{6}$ is treated with a mixture of nitric and sulphuric acids, a reaction quite different from the above takes place. In studying this, a small quantity of substance was boiled with a mixture of equal parts of nitric and sulphuric acids for a short time, the product poured into water, and the precipitate well washed and dried. In order to purify it, it was dissolved in hot acetic anhydride, the solution poured into an equal bulk of hot alcohol, and allowed to cool slowly. A beautiful yellow, crystalline powder was thus obtained, which on heating with potassium and then testing with ferrous and ferric chlorides, was found to contain a considerable amount of nitrogen. We have not as yet analysed this nitro-derivative, but its properties resemble very much those of the nitroanthraquinones except that it does not sublime when heated, but is alnost entirely decomposed, leaving a black residue. If heated with sulphuric acid, a violent reaction sets in, and on diluting the product with water, a red-violet precipitate is obtained, which dissolves in potash with a blue colour. When boiled with sodium sulphide, a brick-red amido-compound is obtained, resembling amidoanthraquinone, with which, however, it does not. appear to be identical.

\section{Fusion of $\mathrm{C}_{28} \mathrm{H}_{14} \mathrm{O}_{7}$ with Potash.}

In carrying out this experiment, the substance was heated in a closed iron tube with concentrated potash solution for 12 hours at $180^{\circ}$. The product was then boiled with water, filtered, a small quantity of lime added to the violet-blue filtrate, again filtered, and the residual violet powder washed with water and decomposed with hydrochloric acid.

The orange-coloured precipitate formed was washed with water; dried, and once or twice recrystallised from benzene. Red needles were thus obtained which melted at about $285^{\circ}$, and showed all the properties of alizarin. The analysis gave the following numbers:- 
0.1471 gram substance gave 0.0488 gram $\mathrm{H}_{2} \mathrm{O}$ and 0.3749 gram $\mathrm{CO}_{2}$.

\begin{tabular}{|c|c|c|}
\hline C. . & $\begin{array}{l}\text { Theory. } \\
\mathrm{C}_{14} \mathrm{H}_{8} \mathrm{O}_{4} \text {. } \\
70.00 \text { per cent. }\end{array}$ & $\begin{array}{l}\text { Found. } \\
69.57 \text { per cent. }\end{array}$ \\
\hline & $3 \cdot 33^{1}$ & 3.62 \\
\hline & $26 \cdot 66$ & $26 \cdot 81$ \\
\hline
\end{tabular}

The filtrate from the alizarate of lime was a red solution with a green fluorescence, which on acidifying gave a white precipitate, changing to green on washing. This substance, of which only a minute quantity was obtained, appeared to be unattacked $\mathrm{C}_{28} \mathrm{H}_{14} \mathrm{O}_{7}$.

That portion of the product which was insoluble in water, after repeated recrystallisation from glacial acetic acid, appeared under the microscope as orange-red needles, giving with sulphuric acid a reddish-violet solution, and in all other respects showing the properties of the red substance $\mathrm{C}_{25} \mathrm{H}_{14} \mathrm{O}_{6}$. Dried at $120^{\circ}$, it gave the following results on analysis :-

0.0859 gram substance gave 0.0269 gram $\mathrm{H}_{2} \mathrm{O}$ and 0.2365 gram $\mathrm{CO}_{2}$.

\begin{tabular}{|c|c|c|}
\hline & $\begin{array}{l}\text { Theory. } \\
\mathrm{C}_{28} \mathrm{H}_{14} \mathrm{O}_{6} \text {. } \\
75 \cdot 34 \text { per cent. }\end{array}$ & $\begin{array}{l}\text { Found. } \\
75.09 \text { per cent. }\end{array}$ \\
\hline $1 \ldots \ldots$ & $3 \cdot 14$ & $3 \cdot 48$ \\
\hline .. & $21 \cdot 52$ & $21 \cdot 43$ \\
\hline
\end{tabular}

Two distinct reactions therefore take place when $\mathrm{C}_{28} \mathrm{H}_{14} \mathrm{O}_{7}$ is fused with potash.

In the first place the elements of water are taken up, $1 \mathrm{~mol}$. of $\mathrm{C}_{28} \mathrm{H}_{14} \mathrm{O}_{7}$ splitting up into 2 mols. of alizarin, thus :-

$$
\mathrm{C}_{28} \mathrm{H}_{14} \mathrm{O}_{7}+\mathrm{H}_{2} \mathrm{O}=2 \mathrm{C}_{14} \mathrm{H}_{8} \mathrm{O}_{4},
$$

whereas at the same time a certain amount of reduction takes place, some of the $\mathrm{C}_{28} \mathrm{H}_{14} \mathrm{O}_{7}$ being reduced to the original red substance, thus:-

$$
\begin{aligned}
& \mathrm{C}_{28} \mathrm{H}_{14} \mathrm{O}_{7}+\mathrm{H}_{2}=\mathrm{C}_{28} \mathrm{H}_{14} \mathrm{O}_{6}+\mathrm{H}_{2} \mathrm{O} . \\
& \text { Action of Chromic Acid on } \mathrm{C}_{28} \mathrm{H}_{14} \dot{\mathrm{O}}_{7} \text {. }
\end{aligned}
$$

In the first paper on these anthraquinone-derivatives (loc. cit., p. 683), we showed that when the red substance $\mathrm{C}_{28} \mathrm{H}_{14} \mathrm{O}_{6}$ is oxidised with chromic acid in acetic solution it is converted into a white substance of the formula $\mathrm{C}_{14} \mathrm{H}_{6} \mathrm{O}_{4}$, thus :-

$$
\mathrm{C}_{28} \mathrm{H}_{14} \mathrm{O}_{6}+\mathrm{O}_{3}=2 \mathrm{C}_{14} \mathrm{H}_{6} \mathrm{O}_{4}+\mathrm{H}_{2} \mathrm{O} \text {. }
$$


On considering the results just described, it appeared likely that the substance $\mathrm{C}_{28} \mathrm{H}_{14} \mathrm{O}_{7}$ was really the first product of the action of oxidising agents on $\mathrm{C}_{28} \mathrm{H}_{14} \mathrm{O}_{6}$, and that this, therefore, on further oxidation should yield the same substance, $\mathrm{C}_{14} \mathrm{H}_{6} \mathrm{O}_{4}$, as is obtained direct by the action of chromic acid on $\mathrm{C}_{28} \mathrm{H}_{14} \mathrm{O}_{6}$. In order to decide this point, a small quantity of the substance $\mathrm{C}_{28} \mathrm{H}_{14} \mathrm{O}_{7}$ was dissolved in hot glacial acetic acid, and treated with chromic acid until the violent reaction which set in at first had subsided. On allowing the green solution to cool, a white, crystalline powder was deposited. This was collected, washed, dried, and several times recrystallised from glacial acetic acid.

In this way the product was easily separated into two portions, one of which was considerably more soluble in acetic acid than the other.

The less soluble portion on analysis gave the following numbers:-

0.1583 gram substance gave 0.0385 gram $\mathrm{H}_{2} \mathrm{O}$ and 0.4100 gram $\mathrm{CO}_{2}$.

\begin{tabular}{|c|c|c|}
\hline & $\begin{array}{l}\text { Theory. } \\
\mathrm{C}_{14} \mathrm{H}_{6} \mathrm{O}_{4} \text {. } \\
70.59 \text { per cent. }\end{array}$ & $\begin{array}{l}\text { Found. } \\
70 \cdot 63 \text { per cent. }\end{array}$ \\
\hline & $2 \cdot 52$ & $2 \cdot 7()$ \\
\hline & $26 \cdot 89$ & $26 \cdot 67$ \\
\hline
\end{tabular}

The substance therefore has the formula $\mathrm{C}_{14} \mathrm{H}_{6} \mathrm{O}_{4}$, and a careful comparison showed that it is without doubt identical with that produced by the direct oxidation of the red substance $\mathrm{C}_{28} \mathrm{H}_{14} \mathrm{O}_{6}$ with chromic acid. Attempts to produce the substance $\mathrm{C}_{28} \mathrm{H}_{14} \mathrm{O}_{7}$ by cautious oxidation of $\mathrm{C}_{28} \mathrm{H}_{14} \mathrm{O}_{6}$ with chromic acid were unsuccessful, the reaction in all cases going as far as the formation of the compound $\mathrm{C}_{14} \mathrm{H}_{6} \mathrm{O}_{4}$.

The more soluble substance obtained in the above oxidation was found on examination to consist of unchanged substance, as the following analysis shows :-

0.1699 gram substance gave 0.0490 gram $\mathrm{H}_{2} \mathrm{O}$ and 0.4520 gram $\mathrm{CO}_{2}$.

\begin{tabular}{|c|c|c|}
\hline C....... & $\begin{array}{l}\text { Theory. } \\
\mathrm{C}_{23} \mathrm{H}_{14} \mathrm{O}_{7} \text {. } \\
72 \cdot 72 \text { per cent. }\end{array}$ & $\begin{array}{l}\text { Found. } \\
72.51 \text { per cent. }\end{array}$ \\
\hline H ...... & $3 \cdot 03$ & $3 \cdot 20$ \\
\hline $0 \ldots \ldots$ & $24 \cdot 25$ & $24 \cdot 29$ \\
\hline
\end{tabular}

Action of Hydriodic Acid on $\mathrm{C}_{28} \mathrm{H}_{14} \mathrm{O}_{6}$.

Experiments were next made with the object of removing some of the oxygen-atoms from the molecule $\mathrm{C}_{28} \mathrm{H}_{14} \mathrm{O}_{6}$ in the hope of thus 
obtaining some well-known compound, the formation of which might throw additional light on the nature of the red substance.

If $\mathrm{C}_{28} \mathrm{H}_{14} \mathrm{O}_{6}$ is suspended in hot glacial acetic acid, and fuming aqueous hydriodic acid added drop by drop, the crystals rapidly dissolve, forming a deep red solution which contains free iodine; this is filtered and poured into water, when a lemon-yellow precipitate is deposited, which is collected, vell washed, dried, and purified by recrystallisation from a mixture of hot aniline and alcohol; on cooling, it is deposited as a yellow, crystalline powder. Two different samples dried at $110^{\circ}$ were analysed with the following results :-

I. 0.1457 gram substance gave 0.0470 gram $\mathrm{H}_{2} \mathrm{O}$ and 0.4160 gram $\mathrm{CO}_{2}$.

II. 0.0659 gram substance gave 0.0212 gram $\mathrm{H}_{2} \mathrm{O}$ and 0.1880 gram $\mathrm{CO}_{2}$.

\begin{tabular}{|c|c|c|c|c|}
\hline \multirow[b]{3}{*}{ C...... } & \multicolumn{2}{|c|}{ Found. } & \multicolumn{2}{|c|}{ Theory. } \\
\hline & I. & II. & $\mathrm{C}_{28} \mathrm{H}_{14} \mathrm{O}_{5}$. & $\overline{\mathrm{C}_{28} \mathrm{H}_{16} \mathrm{O}_{5}}$ \\
\hline & $77 \cdot 86$ & $77 \cdot 80$ p. c. & $78 \cdot 14$ & $77 \cdot 77$ p. c. \\
\hline$H \ldots$. & 3.58 & 3.57 & $3 \cdot 26$ & $3 \cdot 70^{1}$ \\
\hline O...... & $18 \cdot 56$ & 18.53 & $18 \cdot 60$ & $18 \cdot 52$ \\
\hline
\end{tabular}

The numbers obtained agree, therefore, better with the formula $\mathrm{C}_{28} \mathrm{H}_{14} \mathrm{O}_{5}$ than with $\mathrm{C}_{28} \mathrm{H}_{16} \mathrm{O}_{5}$, and it is probable that this new compound is formed from the red substance simply by elimination of oxygen, thus :-

$$
\mathrm{C}_{28} \mathrm{H}_{14} \mathrm{O}_{6}+\mathrm{H}_{2}=\mathrm{C}_{28} \mathrm{H}_{14} \mathrm{O}_{5}+\mathrm{H}_{2} \mathrm{O} \text {. }
$$

This substance, $\mathrm{C}_{28} \mathrm{H}_{14} \mathrm{O}_{5}$, which is insoluble in aqueous potash, dissolves in a strong, hot solution of potash in methyl alcohol, with an intense orange-brown colour which on long boiling does not change. If the alcohol is evaporated, the colour becomes intenser as the solution becomes stronger, until at last a dark-brown residue is left; this dissolves in water with an orange-red colour, which, however, almost disappears on shaking with air.

When boiled with zinc-dust and potash, $\mathrm{C}_{28} \mathrm{H}_{14} \mathrm{O}_{5}$ gives scarcely any coloration, but it dissolves in concentrated sulphuric acid, forming an intense reddish-brown solution.

I'he ease with which the red substance $\mathrm{C}_{28} \mathrm{H}_{14} \mathrm{O}_{6}$ loses 1 atom of oxygen, and is reduced to $\mathrm{C}_{28} \mathrm{H}_{14} \mathrm{O}_{5}$, led us to think that it would be interesting to study the further action of hydriodic acid on this substance. For this purpose, a mixture of 0.5 gram of the pure red substance, 5 grams of fuming hydriodic acid, and 5 grams of glacial acetic acid were heated in a sealed tube for about half an hour to $160^{\circ}$. The dark-red liquid thus obtained was gently evaporated to get rid of most of the acetic acid, the residue freed from iodine by 
boiling with dilute aqueous sulphurous acid. The precipitate, which separated on cooling, was collected, filtered, washed with water, and dried on a porous plate. On extracting the product with alcohol, nearly the whole dissolved, leaving a small quantity of a yellow substance behind. This residue, after careful drying and sublimation in a test-tube, formed microscopic yellow needles melting at about $260-265^{\circ}$, and which on boiling with potash solntion and zinc-dust gave a red solution becoming colourless again on shaking with air. This substance was, therefore, probably anthraquinone.

The alcoholic solution on evaporation deposited a considerable quantity of a somewhat sticky yellow substance, which, especially when boiled with water, possessed in a marked degree the peculiar odour of dihydroanthracene. In order to determine whether the reduction really had gone as far as this, the crude substance was distilled in a test-tube, the solid distillate washed with a little alcohol, and then recrystallised from this solvent. In this way, small crystals were obtained which melted at $104-106^{\circ}$, and otherwise showed all the properties of dihydroanthracene.

\section{Action of Sulphuric Acid on $\mathrm{C}_{28} \mathrm{H}_{14} \mathrm{O}_{7}$.}

In studying this action, a small quantity of the substance was heated with fuming sulphuric acid at $180^{\circ}$. The reddish-brown solution first formed became gradually darker and eventnally, after 20 minutes' heating, of a bluish-red colour. At this stage, the decomposition was evidently complete, the product dissolving completely in water, showing that a sulphonic acid had been formed. On adding acetic acid to the strong hot solution, and allowing it to cool, a small quantity of a crystalline precipitate separated. It was, however, found impracticable to purify the sulphonic acid by this method, as it was too soluble in acetic acid to admit of sufficient washing. The mixture of the sulphonic acid and sulphuric acid dissolved in water forming a greenish solution, with which lead or barium carbonate yielded insoluble compounds only.

In order, if possible, to determine the nature of the sulphonic acid thus formed, resort was had to fusion with potash. The solution was mixed with a considerable excess of concentrated aqueous potash, and heated in closed iron tubes at $180^{\circ}$ for about 12 hours. The product dissolved in water, forming a red-violet solution, and on the addition of acids a brownish-yellow precipitate was thrown down, which was collected and well washed with water. This substance dyed mordanted cloth shades intermediate between those given by anthrapurpurin and flavopurpurin, and, therefore, probably consists of a mixture of these two substances. 


\section{Fusion of $\mathrm{C}_{14} \mathrm{H}_{6} \mathrm{O}_{4}$ with Potash.}

This substance, the preparation of which was given in the previous paper (loc. cit., p. 683), is formed by the oxidation of the red substance, $\mathrm{C}_{28} \mathrm{H}_{14} \mathrm{O}_{6}$, with chromic acid in acetic acid solution. Considering the interesting constitution previously assigned to this substance and its mode of formation from the red substance, it was thonght that valuable results might be obtained from the further study of its properties. The first decomposition which was studied was the action of caustic potash at high temperatures on this substance. Two grams of pure $\mathrm{C}_{14} \mathrm{H}_{6} \mathrm{O}_{7}$ were heated with concentrated potash solution for about 12 hours at $180^{\circ}$, the dark-coloured product extracted with boiling water and filtered. A small quantity of lime was then added to the bluish-violet filtrate, the lime compound thus precipitated, collected, washed with water, and decomposed with dilute hydrochloric acid. This caused the precipitation of an orange-red compound, which after collecting, washing with water, drying and recrystallising once or twice from benzene, was obtained in long red needles; it melts at about $285^{\circ}$, and possesses all the properties of alizarin.

The analysis gave the following numbers:-

\section{* 0.1175 gram substance gave 0.0391 gram $\mathrm{H}_{2} \mathrm{O}$ and 0.1175 gram $\mathrm{CO}_{2}$.}

\begin{tabular}{|c|c|c|}
\hline & $\begin{array}{l}\text { Theory. } \\
\mathbf{C}_{14} \mathrm{H}_{8} \mathrm{O}_{4} \text {. } \\
70.00 \text { per cent. }\end{array}$ & $\begin{array}{l}\text { Found. } \\
69.98 \text { per cent. }\end{array}$ \\
\hline & $3 \cdot 33$ & $3 \cdot 69$ \\
\hline & $20 \cdot 67$ & $20 \cdot 43$ \\
\hline
\end{tabular}

The portion of the product insoluble in water obtained during the fusion of $\mathrm{C}_{14} \mathrm{H}_{6} \mathrm{O}_{2}$ with potash, was extracted several times with glacial acetic acid, and boiling water added to the filtrate. On cooling, long orange-coloured needles were deposited which on analysis gave numbers corresponding with the formula $\mathrm{C}_{14} \mathrm{H}_{8} \mathrm{O}_{2}$.

I. 0.1235 gram substance gave 0.0463 gram $\mathrm{H}_{2} \mathrm{O}$ and 0.3648 gram $\mathrm{CO}_{2}$.

II. 0.1285 gram substance gave 0.0466 gram $\mathrm{H}_{2} \mathrm{O}$ and $0 \cdot 3800$ gram $\mathrm{CO}_{2}$.

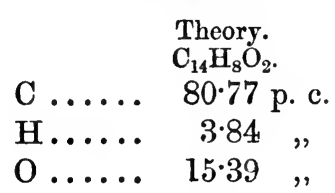

Found.

$\begin{array}{rr}\text { I. } & \text { II. } \\ 80 \cdot 60 & 80 \cdot 65 \text { p. c. } \\ 4.17 & 4.03 \quad, \\ 15.23 & 15.32 \quad,\end{array}$

This compound, obtained by the fusion of $\mathrm{C}_{14} \mathrm{H}_{6} \mathrm{O}_{4}$ with potash, did not at all resemble anthraquinone outwardly, as, however often it was 
recrystallised, it was always deposited in flat, silky needles of an orange colour. It melted, however, at $275^{\circ}$, the melting point of anthraquinone, and when heated strongly it sublimed much in the same way as ordinary anthraquinone, so that there can be no doubt as to its identity with that substance.

The action of caustic potash on $\mathrm{C}_{14} \mathrm{H}_{6} \mathrm{O}_{4}$ appears, therefore, to be entirely a reducing action, alizarin and anthraquinone being formed according to the equations-

$$
\begin{aligned}
& \text { I. } \mathrm{C}_{14} \mathrm{H}_{6} \mathrm{O}_{4}+\mathrm{H}_{2}=\mathrm{C}_{14} \mathrm{H}_{8} \mathrm{O}_{4} \\
& \text { II. } \mathrm{C}_{14} \mathrm{H}_{6} \mathrm{O}_{4}+3 \mathrm{H}_{2}=\mathrm{C}_{14} \mathrm{H}_{8} \mathrm{O}_{2}+2 \mathrm{H}_{2} \mathrm{O} .
\end{aligned}
$$

\section{Action of Sulphuric Acid on $\mathrm{C}_{14} \mathrm{H}_{6} \mathrm{O}_{4}$.}

In studying this reaction, the substance $\mathrm{C}_{14} \mathrm{H}_{6} \mathrm{O}_{4}$ was heated with three or four parts of Nordhansen sulphuric acid at $200^{\circ}$ until a sample taken out dissolved entirely in water. As soon as this was found to be the case, the product. was poured into glacial acetic acid drop by drop, the whole being constantly stirred during the operation. This caused the separation of a white substance which, when examined under the microscope, was seen to consist of a mass of small crystals. When the precipitation was complete, about twice the bulk of glacial acetic acid was added, the mixture thrown on a filter, washed with glacial acetic acid till free from sulphuric acid, and then well drained. The product was dissolved in a little water, and alcoholic soda added to the solution until the white crystalline salt thus formed had been completely precipitated. After collecting, well washing with alcohol and drying at $100^{\circ}$, this salt gave the following numbers on analysis :-

0.1963 gram substance gave 0.0333 gram $\mathrm{H}_{2} \mathrm{O}$.

0.3540 gram $\mathrm{CO}_{2}$.

\begin{tabular}{|c|c|c|c|c|}
\hline \multicolumn{3}{|c|}{$\begin{array}{c}\text { Theory. } \\
\mathrm{C}_{14} \mathrm{H}_{5} \mathrm{O}_{4} \cdot \text { SO }_{3} \mathrm{Na} \text {. }\end{array}$} & \multicolumn{2}{|c|}{$\begin{array}{l}\text { Found. } \\
49 \cdot 18 \text { per cent. }\end{array}$} \\
\hline $\mathrm{H} \ldots \ldots$ & $1 \cdot 47$ & , & $1 \cdot 88$ & , \\
\hline $\mathrm{Na} . . . .$. & $6 \cdot 76$ & $"$ & $6 \cdot 84$ & $"$ \\
\hline $0 \ldots \ldots$ & $32 \cdot 95$ & $"$ & - & \\
\hline s........ & $9 \cdot 41$ & $"$ & - & \\
\hline
\end{tabular}

0.0415 gram $\mathrm{Na}_{2} \mathrm{SO}_{4}$.

Solutions of this salt give precipitates with salts of copper, lead, barium, and other metals. On fusion with potash, this sulphonic acid yields a reddish-violet solution which, on addition of acids, deposits a yellow precipitate. This after being collected and washed 
with water, was found to dye mordanted cloths shades very closely resembling those produced by flavopurpurin. As the substance also gave the same absorption-bands as flavopurpurin there can be no doubt of its identity with this substance.

In order to examine more completely the action of sulphuric acid on $\mathrm{C}_{14} \mathrm{H}_{6} \mathrm{O}_{4}$, the acetic acid filtrate from the precipitated sulphonic acid was next experimented on, thus :-After distilling off the acetic acid, the residue was dissolved in potash, fused with an excess of strong potash solution in an iron tube, and the product isolated in the usual way. The yellowish-brown flocculent precipitate thus obtained dyed mordanted cloth with shades slightly yellower than, bat otherwise exactly similar to, those produced by anthrapurpurin, and a careful examination of the substance showed that it agreed with this compound in all its reactions. By the action of Nordhausen sulphuric acid on $\mathrm{C}_{14} \mathrm{H}_{6} \mathrm{O}_{4}$, it wonld appear, therefore, that two sulphonic acids are formed, of which one is insoluble and the other soluble in glacial acetic acid. On fusion with potash, the former yields flavopurpurin and the latter anthrapurpurin.

\section{Theoretical Remarks.}

On considering the results described in the body of this paper, it appears very doubtful whether the red substance $\mathrm{C}_{28} \mathrm{H}_{14} \mathrm{O}_{6}$ can have the formula first assigned to it (loc. cit., p. 684), namely-

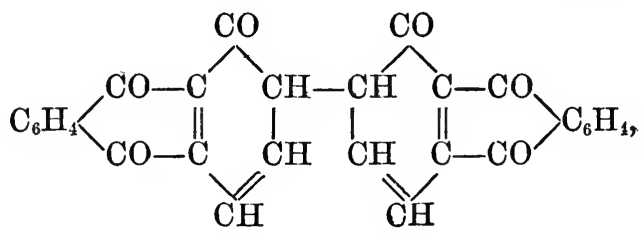

as this formula does not in any way explain the formation of the oxidation compound $\mathrm{C}_{28} \mathrm{H}_{14} \mathrm{O}_{7}$, or the decomposition into alizarin by fusion with potash. A reaction of this kind would involve the severing of the two anthraquinone molecules, which would hardly take place so easily if they were joined simply carbon to carbon, as shown above.

In order to be able to explain the formation of its various derivatives, it is necessary to suppose that the two anthraquinone molecules are linked together in a much more feeble way, as for instance with the intervention of an oxygen-atom.

The most probable formula for the red substance $\mathrm{C}_{28} \mathrm{H}_{14} \mathrm{O}_{6}$, and one which would explain all its decompositions, would be- 


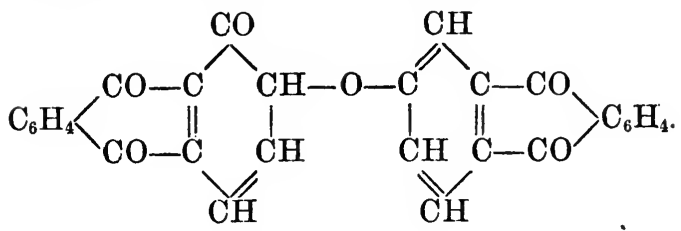

By the action of nitric acid this substance is converted into $\mathrm{C}_{28} \mathrm{H}_{14} \mathrm{O}_{7}$, which would be represented by the formula-

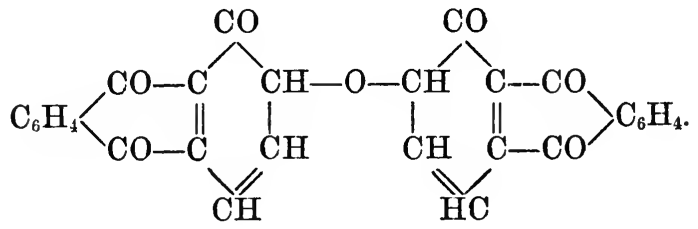

On further oxidation this substance is converted into 2 mols. of $\mathrm{C}_{14} \mathrm{H}_{6} \mathrm{O}_{4}$, a change which is easily understood if we assume that the latter substance has the constitution which was previously assigned to it; that is-

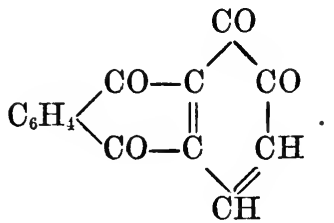

There is every reason to believe that this substance has the simple formula $\mathrm{C}_{14} \mathrm{H}_{6} \mathrm{O}_{4}$, and not the double formula $\mathrm{C}_{28} \mathrm{H}_{12} \mathrm{O}_{8}$. The comparatively low melting point of this substance (about $300^{\circ}$ ), and the fact that it sublimes easily and completely when heated, almost exclude the possibility of its having the double molecular weight $\mathrm{C}_{28} \mathrm{H}_{12} \mathrm{O}_{8}$.

The constitutional formula given above for this substance easily explains the reduction to alizarin and anthraquinone by the action of potash at a high temperature.

Lastly, the substance $\mathrm{C}_{28} \mathrm{H}_{14} \mathrm{O}_{5}$, produced by the action of hydriodic acid on $\mathrm{C}_{28} \mathrm{H}_{14} \mathrm{O}_{6}$, would be represented by the formula-

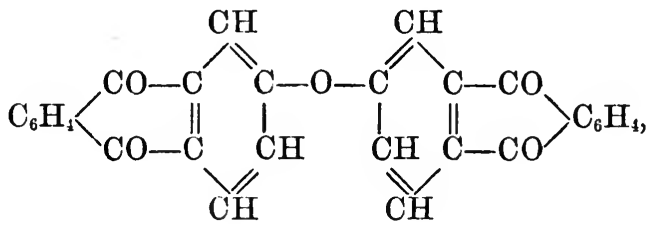


844 ON SOME DERIVATIVES OF ANTHRAQUINONE.

which in its turn would easily explain the formation of anthraquinone and dihydroanthracene by the further reducing action of hydriodic acid. 


\section{CONTRIBUTIONS FROM THE LABORATORY OF THE ROYAL COLLEGE OF CHEMISTRY.}

On the Action of Sodium on Phenylic Acetate.

By W. H. Perkin, Jun., and W. R. Hodgkinson.

IN previous communications to this Society and to the Annalen, the results of the action of metallic sodium on ethereal salts containing mixed fatty aromatic groups has been shortly described by one of us, and the results obtained led us to extend the inquiry to ethereal salts containing an aromatic, so-called, group of a simpler constitution than in those therein described.

We therefore commenced with phenylic acetate, $\mathrm{C}_{6} \mathrm{H}_{5} \mathrm{OOCCH}_{3}$, which was obtained by treating perfectly dry phenol with acetylic chloride in slight excess until hydrochloric acid gas ceased to be evolved. On distillation, a beautiful limpid liquid, boiling at $193^{\circ}$, and having a refractive power almost exactly that of crown glass, is obtained. A combustion gave-

Substance $0 \cdot 1801$.

$$
\begin{gathered}
\mathrm{CO}_{2} 0.4647=\mathrm{C} \quad 70.37 \\
\mathrm{OH}_{2} 0.0958=\mathrm{H} \quad 5.90 \\
\text { Theory for } \mathrm{C}_{8} \mathrm{H}_{3} \mathrm{O}_{2}=\mathrm{C} 70.59 \\
\mathrm{H} \quad 5.89
\end{gathered}
$$

On warming this ether with sodium to about $100^{\circ}$, most violent action takes place, the temperature rising rapidly to above the boiling point of the acetate, and in some cases causing projection of the contents of the flask in which the operation is performed.

Hydrogen is evolved during the action, and considerable quantities of a light boiling liquid was carried over as vapour, and condensed in a receiver connected with the flask in which the action took place. This liquid had a most powerful odour of ethylic acetate, and on being fractioned distilled almost entirely at $76^{\circ}$, and gave on combustion-

$0 \cdot 1534$ substance gave $\mathrm{CO}_{2} 0 \cdot 3080$ and $\mathrm{OH}_{2} 0 \cdot 1244=\mathrm{C} 54 \cdot 76$ H 9.01

Theory for acetic ether $\left(\mathrm{CH}_{3} \mathrm{COOC}_{2} \mathrm{H}_{5}\right)=\mathrm{C} 54 \cdot 54$ H $\quad 9 \cdot 09$

A portion was saponified with $\mathrm{OKH}$, the potassic salt distilled with $\mathrm{SO}_{2}(\mathrm{HO})_{2}$, and the distillate digested with argentic carbonate. The crystalline silver salt thereby obtained gave-

$$
\begin{aligned}
& 0.1030 \text { gave } 0.0665 \mathrm{Ag}=64.56 \\
& \text { Theory for }\left(\mathrm{CH}_{3} \mathrm{COOAg}\right)=64.66
\end{aligned}
$$


There is therefore no doubt that the liquid distilling over during the reaction is acetic ether.

The solid body remaining in the flask after the sodium has disappeared dissolves partially when treated with water, forming a dark coloured solution, and leaving a small quantity of a thick oil.

The watery solution, after shaking with ether to remove a little of the above oil, gives on acidifying with $\mathrm{SO}_{2}(\mathrm{HO})_{2}$ a copious precipitate of an oil, which was shaken with dilute ammonia, in which a great part remained insoluble.

This, separated from the ammoniacal solution, on fractioning distilled entirely between $180-190^{\circ}$, and solidified on cooling, forming needles characteristic of phenol, of which it gave all the reactions.

On acidifying the ammoniacal solution, after boiling with animal charcoal, a solid acid was precipitated, which on repeated crystallisation from hot water was obtained in white needles melting at $155^{\circ}$. An analysis gave-

0.2088 substance gave $\mathrm{CO}_{2} 0.4645$ and $\mathrm{OH}_{2} 0.0858=$
$\mathrm{H} \quad 40.67$

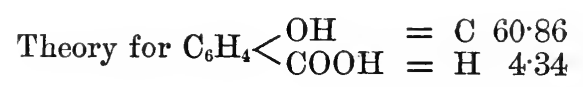

which was further confirmed by the ferric chloride reactions, \&c.

The aqueous solution, from which the phenol was precipitated by addition of sulphuric acid, was distilled with steam, and the acid distillate neutralised with baric carbonate, and the resulting barium salt converted into silver salt. An analysis of this gave the following :-

\section{$1 \cdot 1105$ substance gave Ag 0.7159 $=64.47$ per cent. Theory for $\mathrm{CH}_{3} \mathrm{COOAg}=64.66 \quad$,}

So far the products of the sodium action appear to be acetic ether, acetic acid, phenol, salicylic acid, and a thick oil. On submitting this to distillation, undecomposed acetate of phenyl first came off, then the thermometer rapidly rose to $300^{\circ}$. The remainder after distillation in a vacuum solidified on being subjected to a freezing mixture.

The crystalline mass was pressed between paper and crystallised from petroleum. As two distinct forms of crystals were observed to separate out on cooling; these were separated by repeated crystallisation from alcohol, in which one of them is almost insoluble.

The substance most soluble in alcohol, which may provisionally be called $\alpha$, forms glistening white needles melting at $48^{\circ}$. When an alcoholic solution of this substance is warmed with potash, it produces a beautiful red coloration, destroyed by water, but reappearing on 
evaporation. The specific gravity of this body is $1 \cdot 026$. On analysis it yielded the following figures:-

$\begin{array}{crc}\text { I. } & \text { II. } & \text { Theory for } \mathrm{C}_{15} \mathrm{H}_{12} \mathrm{O}_{3} . \\ \text { C } 75.13 & 74.55 & 75.00 \\ \text { H } 5.30 & 5 \cdot 4 & 5.00\end{array}$

The other substance, $\beta$, is much less soluble in alcohol, and crystallises in fine yellow needles melting at $138^{\circ}$. When warmed with alcoholic potash it forms a fine yellow crystalline compound. Its specific gravity is 1.076 . On analysis it yielded the following figures :-

$\begin{array}{ccc}\text { I. } & \text { II. } & \text { Theory for } \mathrm{C}_{18} \mathrm{H}_{14} \mathrm{O}_{4} . \\ \text { C } 73 \cdot 2 & 73.54 & 73 \cdot 4 \\ \mathrm{H} 4.6 & 4.63 & 4.7\end{array}$

From the proportions of ether and sodium employed in the reaction-

$$
2\left(\mathrm{CH}_{3} \mathrm{COOC}_{6} \mathrm{H}_{5}\right)+\mathrm{Na}_{2},
$$

it is difficult to account for all the products by an equation.

The reaction may, however, consist first in the formation of sodium phenol, and acetic anhydride. Part of the latter reunites with the sodium phenol, and forms phenyl acetate and sodic acetate, and part is immediately reduced by the nascent hydrogen from the phenol sodium undergoing at the high temperature the decomposition shown to take place by Barth and Schreder (Ber., 12, 417) by fusing phenol with sodic hydrate, when diphenol, phloroglucin, \&c., are produced.

The two crystalline substances, $\alpha$ and $\beta$, may possibly be formed from an aceto-acetic ether compound, produced in the first instance and acted upon by sodium and sodium phenol, which, however, we must reserve for experimental proof.

In order to determine whether this was a general reaction, experiments were tried with cresyl acetate, prepared by treating cresol from Kahlbaum) with acetyl chloride. Cresyl acetate is a beautiful colourless liquid, boiling at $214^{\circ}$. An analysis gave-

$\begin{array}{lc} & \text { Theory. } \\ \text { C } 72 \cdot 26 & 72 \cdot 00 \\ \text { H } 6.65 & 6 \cdot 66\end{array}$

This was treated with $\mathrm{Na}$ in the same manner as the phenyl acetate, and the low boiling liquid formed distilled off. This was evidently acetic ether. It was saponified, and the acid turned into $\mathrm{Ag}$ salt. On analysis the following numbers were obtained :-

0.1366 substance gave $0.881 \mathrm{Ag}=64.49$ per cent.

Theory for $\mathrm{CH}_{3} \mathrm{COOAg}=64.66^{\circ}$, 
4 PERKIN AND HODGKINSON ON THE ACTION OF SODIUM, ETC.

Salicylic acid was also obtained, as in the action of Na on phenyl acetate, not methyl salicylic acid, as might have been supposed. It is therefore probable that acetic ether and salicylic acid are constant products of the action of $\mathrm{Na}$ on acetyl phenols. 


$$
\text { By W. H. Perkin, Jun., Ph.D. }
$$

Condensation-products of Isobutaldehyde.

A short time ago there appeared in the Berichte $(15,2363)$ an abstract of a preliminary notice by Fossek (Monatsh. f. Chem. 3, 622 ; C. J., Abstr., 1882, 1274), on the action of an aqueous solution of caustic potash on isobutaldehyde. As I have for some time been working on the condensation-products of isobutaldehyde produced by means of alcoholic potash, in continuation of a research on the condensation of œnanthaldehyde, on which I have been engaged for the last two years, I am compelded to publish my results, although not quite completed, 
especially as they are quite different from those obtained by Fossek, alcoholic potash seeming to bring about a series of reactions differing from those obtained by the action of aqueous potash. The method employed for preparing the isobutaldeliyde was the following:100 grams isobutylalcohol and 200 grams water were put into a large flask connected with a condenser, warmed up to about $50^{\circ}$, and a concentrated solution of bichromate of potash, mixed with an equal volume of sulphuric acid slowly run in, the contents of the flask being well shaken after each addition, to facilitate the escape of the aldehyde as soon as formed. This concentrated bichromate solution was added, until the oily layer of isobutyl alcohol disappeared from the surface of the mixture in the flask, and the whole was then boiled to drive off any aldehyde still remaining in the liquid.

The oily part of the distillate was separated from water, dried as quickly as possible with calcium chloride, and fractioned. The portion which distilled over below $100^{\circ}$ was collected apart, and carefully fractioned by using a tube 3 feet high, to facilitate the separation of the aldehyde from the other products. By this means the aldehyde is easily obtained pure, boiling from $60-62^{\circ}$. The quantity was from $50-60$ per cent. of the theoretical.

The condensation with alcoholic potash was first tried in the following way:-50 grams of isobutaldehyde were dissolved in 100 grams absolute alcobol, and then a solution of 2 grams potash in 20 grams alcohol slowly added, the temperature not being allowed to rise above $30^{\circ}$. The mixture after cooling was then mixed with a second 2 grams potash in alcohol, and after standing for 12 hours, warmed up to $50^{\circ}$ for about 10 minutes. It is important not to warm too long, as otherwise the condensation is apt to go too far, and only high boiling condensation-products to be prodnced. The liquid was left to cool, and the saline products separated from the condensed oils, by adding much water and taking the oil up with ether. If the alcohol be first distilled off, the excess of potassium hydrate and saline matter act further on the aldehyde producing higher condensation-products. The aqueous saline solution from several experiments was first examined; it was thoroughly freed from oily products with ether, concentrated, and acidulated with hydrochloric acid. This caused the separation of some acids, which were extracted with ether. The ethereal solution was washed, dried over chloride of calcium, and distilled.

After the ether had distilled off, the principal part of the product came over between $145-180^{\circ}$. A considerable quantity afterwards passed over between $180^{\circ}$ and $260^{\circ}$, leaving a thick tarry residue in the retort; the portion boiling at $145-180^{\circ}$ consisted essentially of isobutyric acid. It was, however, purified, and converted into its silver salt. A silver determination gave the following numbers :- 
0.3846 gram substance gave 0.2142 gram $\mathrm{Ag}=55.69$ per cent.

Calculated for $\underset{\mathrm{CH}_{3}}{\mathrm{CH}_{3}}>\mathrm{CH} . \mathrm{COOAg}=55.38$,

The fraction boiling between $180-260^{\circ}$ was then several times carefully fractioned, when the largest part obtained boiled at $245-255^{\circ}$. This gave on analysis the following numbers:-

I. $0 \cdot 1565$ gram substance gave 0.1479 gram $\mathrm{OH}_{2}$ and 0.3839 gram $\mathrm{CO}_{2}$.

II. $0 \cdot 1676$ gram substance gave $0 \cdot 1575$ gram $\mathrm{OH}_{2}$ and 0.4111 gram $\mathrm{CO}_{2}$.

Found.

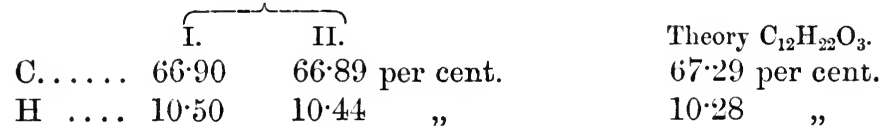

These numbers therefore agree with those required by the formula $\mathrm{C}_{12} \mathrm{H}_{22} \mathrm{O}_{3}$. The body is therefore an isomeride of octylacetoacetic acid; this acid is probably formed by the action of potash on the aldehyde $\mathrm{C}_{12} \mathrm{H}_{22} \mathrm{O}_{2}$ (which will be found described further on) according to the equation-

$$
2 \mathrm{C}_{12} \mathrm{H}_{22} \mathrm{O}_{2}+\mathrm{KOH}=\mathrm{C}_{12} \mathrm{H}_{21} \mathrm{O}_{3} \mathrm{~K}+\mathrm{C}_{12} \mathrm{H}_{24} \mathrm{O}_{2} \text {. }
$$

This acid dissolves somewhat readily in dilute potash, but after standing for some time the potassium salt separates out as a gummy layer on the surface of the liquid. Attempts were also made to prepare the potassium salt by dissolving the acid in dilute alcoholic potash, passing $\mathrm{CO}_{2}$, and filtering off the precipitated potassium carbonate, but in this case the potassium salt was left behind, on evaporating off the alcohol, as an uninviting soap. The acid itself is a light brown oil, having but little odour; it dissolves in ammonia, and precipitates silver from a solution of silver oxide in ammonia. It distils apparently without decomposition, cooled to $-10^{\circ}$. It does not solidify.

By the action of aqueous potash on isobutaldehyde, Fossek obtains an acid to which he assigns the formula $\mathrm{C}_{8} \mathrm{H}_{16} \mathrm{O}_{3}$; this requires $\left\{\begin{array}{l}\mathrm{C}=60.00 \text { per cent. } \\ \mathrm{H}=10.00 \text { per cent. }\end{array}\right.$ It melts at $75-80^{\circ}$, and is therefore evidently quite different from the one described above.

The ethereal solution of the condensed oils was well washed. It was dried with chloride of calcium, and fractioned in a stream of carbonic anhydride. The fraction below $100^{\circ}$ contained besides ether a considerable quantity of the unchanged aldehyde. That from $100-140^{\circ}$ was very small, but between $140-180^{\circ}$ a considerable quantity of a colourless oil was obtained, smelling strongly of camplor. The residue 
in the retort was very small. The oil boiling between $140-180^{\circ}$ was fractioned as rapidly as possible, when nearly the whole came over between $70-100^{\circ}$, and only a small quantity distilled over between $145-160^{\circ}$. There was also a considerable quantity of a high boiling product, which had been produced during the distillation, left behind.

This last fraction was once more rapidly fractioned, when the principal part distilled between $154-157^{\circ}$. On analysis it gave the following numbers:-

I. $0 \cdot 1156$ gram substance gave $0 \cdot 1170$ gram $\mathrm{OH}_{2}$ and $0 \cdot 3070$ gram $\mathrm{CO}_{2}$.

II. 0.1195 gram substance gave 0.1198 gram $\mathrm{OH}_{2}$ and 0.3168 gram $\mathrm{CO}_{2}$.

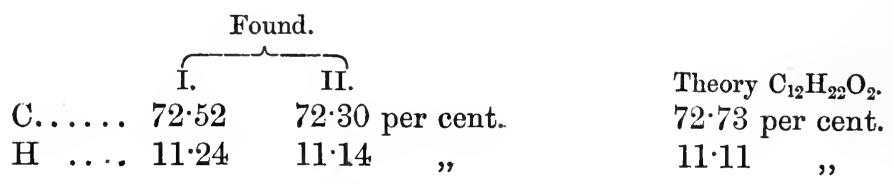

This body appears to have the formula $\mathrm{C}_{12} \mathrm{H}_{22} \mathrm{O}_{2}$, and is probably formed by the separation of a molecule of water from three molecules of isobutaldehyde, thus :-

$$
3 \mathrm{C}_{4} \mathrm{H}_{3} \mathrm{O}=\mathrm{C}_{12} \mathrm{H}_{22} \mathrm{O}_{2}+\mathrm{H}_{2} \mathrm{O} \text {. }
$$

It is a colourless oil, having a powerful ethereal smell and burning taste. It reduces an ammoniacal silver solution readily, and combines slowly with acid sulphite of sodium, forming an amorphous-looking mass, very slightly soluble in water, which under the microscope is seen to consist of small crystals displaying colours in polarised light. On the addition of an acid or of sodium carbonate this compound is decomposed, an oil separating out. It does not solidify when cooled down to $-10^{\circ}$. If dissolved in carbon disulphide, and the solution cooled in a freezing mixture, it takes up bromine. It is easily decom. posed by potash, apparently forming a considerable quantity of potassium isobutyrate. This oil is probably the same body as that which Urech obtained (Ber., 13, 590) by distilling the polymerised modification of isobutaldehyde produced by the action of potassium carbonate. He describes it as a colourless oil, possessing an ethereal smell, and boiling at $154^{\circ}$. He also notices that on distilling it decomposes, leaving a high-boiling residue in the retort.

A vapour-density which he made agreed fairly well with the formula $\mathrm{C}_{8} \mathrm{H}_{14} \mathrm{O}$, but on analysis numbers were obtained which agreed with the formula $\mathrm{C}_{12} \mathrm{H}_{22} \mathrm{O}_{2}$. As the substance decomposes on prolonged heating, a vapour-density has probably but little value. Fossek, by the action of sodium acetate on aqueous potash, obtained an 
aldehyde $\mathrm{C}_{8} \mathrm{H}_{14} \mathrm{O}$, boiling at $149-151^{\circ}$, which, however, seems to be different from the one described above, as it appears to distil without decomposition. It requires 76.19 per cent. of carbon, whereas the substance examined by me contained 72:5. By the action of $\mathrm{ZnCl}_{2}$ or $\mathrm{PCl}_{3}$ on isobutaldehyde, Fossek obtains polymerides derived from 3 mols. of isobutaldehyde.

\section{Action of Nascent Hydrogen, on the Aldehyde $\mathrm{C}_{12} \mathrm{H}_{22} \mathrm{O}_{2}$.}

This experiment was tried in order, if possible, to obtain an alcohol from the aldehyde $\mathrm{C}_{12} \mathrm{H}_{22} \mathrm{O}_{2}$, and by this means to obtain some further clue as to its constitution. About 50 grams of the aldehyde were dissolved in 200 grams of ether, mixed with a small quantity of water, placed in a large flask connected with a reversed condenser, and sodium was added at intervals and in small pieces, the mixture being kept as cool as possible, and shaken up from time to time to dissolve out any sodic hydrate suspended in the ether. The ethereal solution was then washed, dried, and fractioned. After the ether had distilled off, most of the oil came over between $260-280^{\circ}$, leaving however a small residue in the retort. This oil was slowly fractioned in a Wurtz flask, with a neck about 8 inches long, when the principal portion came over between $270-275^{\circ}$ as a colourless oil, possessing a very strong odour. On analysis it gave the following numbers :-

I. 0.1038 gram substance gave $0 \cdot 1219$ gram $\mathrm{OH}_{2}$ and 0.2721 gram $\mathrm{CO}_{2}$.

II. $0 \cdot 1160$ gram substance gave $0 \cdot 1341$ gram $\mathrm{OH}_{2}$ and $0 \cdot 3029$ gram $\mathrm{CO}_{2}$.

Found.

\begin{tabular}{|c|c|c|c|}
\hline & I. & II. & Theory $\mathrm{C}_{12} \mathrm{H}_{26} \mathrm{O}_{2}$. \\
\hline C...... & $71 \cdot 49$ & $71 \cdot 21$ per cent. & 71.28 per cent. \\
\hline $\mathrm{H} \ldots$ & $13 \cdot 05$ & $12 \cdot 85$ & $12 \cdot 87$ \\
\hline
\end{tabular}

It appears therefore that an alcohol of the formula $\mathrm{C}_{12} \mathrm{H}_{26} \mathrm{O}_{2}$, which is isomeric with ethyl propyl pinacone, had been formed, according to the equation-

$$
\mathrm{C}_{13} \mathrm{H}_{22} \mathrm{O}_{2}+2 \mathrm{H}_{2}=\mathrm{C}_{12} \mathrm{H}_{26} \mathrm{O}_{2} \text {. }
$$

This body does nct solidify at $-10^{\circ}$, neither does it combine with acid sodium sulphite. On distillation it appears to decompose slightly, forming lower and higher fractions. This makes it difficult to obtain it in the pure state. In order, if possible, to obtain an acetate from this body, it was treated with an excess of acetic auhydride at $180^{\circ}$ for four hours, and then fractioned. After the anhydride and acetic acid had 
been slowly distilled off, nearly all the residue boiled between 175 $190^{\circ}$. This, on carefully refractioning, gave quantities of distillate of about the same size, boiling at $180-185^{\circ}$ and $185-190^{\circ}$. Analysis of these gave the following numbers :-

1. $\left(180-185^{\circ}\right) 0 \cdot 1298$ gram substance gave $0 \cdot 1245$ gram $\mathrm{OH}_{2}$ and 0.3211 gram $\mathrm{CO}_{2}$.

II. $\left(185-190^{\circ}\right) 0 \cdot 1336$ gram substance gave $0 \cdot 1311$ gram $\mathrm{OH}_{2}$ and 0.3305 gram $\mathrm{CO}_{2}$.

$$
\begin{aligned}
& \text { Found. } \\
& \text { I. } \\
& \text { C..... 67 } 46 \\
& \text { H } \ldots . .10 .66 \\
& \text { II. } \\
& 67 \cdot 46 \text { per cent. } \\
& 10.90 \quad,
\end{aligned}
$$

These numbers indicate that both specimens consisted of a diacetate of the formula $\mathrm{C}_{16} \mathrm{H}_{30} \mathrm{O}_{4}$. This substance would be produced according to the equation :-

$$
\mathrm{C}_{12} \mathrm{H}_{26} \mathrm{O}_{2}+\left(\begin{array}{l}
\mathrm{CH}_{3} \mathrm{CO} \\
\mathrm{CH}_{3} \mathrm{CO}
\end{array}>\mathrm{O}\right)_{2}=\mathrm{C}_{12} \mathrm{H}_{24} \mathrm{O}_{2}\left(\mathrm{C}_{2} \mathrm{H}_{3} \mathrm{O}\right)_{2}+2 \mathrm{CH}_{3} \mathrm{COOH} .
$$

A Dumas vapour-density determination was made of this body, and gave 6.289 . The molecular weight should therefore be $6 \cdot 289 \times 28.92$ $=181.87$, whereas the theoretical molecular weight for $\mathrm{C}_{24} \mathrm{H}_{42} \mathrm{O}_{6}=$ 286 , so that apparently in this case, as with the body $\mathrm{C}_{12} \mathrm{H}_{22} \mathrm{O}_{2}$, decom. position takes place on prolonged heating of the rapour. This acetate is a very strong-smelling body, reminding one of peppermint. These results must only be looked upon as preliminary, as owing to the very small quantity of the aldehyde $\mathrm{C}_{12} \mathrm{H}_{22} \mathrm{O}_{2}$, at my command, it has been, up to the present, impossible to confirm them.

In order to study the higher condensation-products the isobutaldehyde was treated in the same manner as in the previous experiment, twice as much potash, however, being employed, and the temperature of the reactions allowed to rise to $45^{\circ}$. At the conclusion of the operation the mixture was heated on the water-bath to near its boiling point for ten minutes; it was then diluted with water, and the neutral oils were taken up with ether, separated, and treated as before. On distillation a small quantity of oil came over under $200^{\circ}$, which contained, besides the aldehyde $\mathrm{C}_{12} \mathrm{H}_{22} \mathrm{O}_{2}$, a small quantity of a body boiling between $190-200^{\circ}$; this gave on analysis, numbers approximately agreeing with the formula $\mathrm{C}_{16} \mathrm{H}_{30} \mathrm{O}_{3}$; the quantity was, however, too small to further examine. After this the thermometer rose rapidly to $215^{\circ}$, and between this and $235^{\circ}$ about 20 grams of cil distilled over, leaving in the retort a residue which was reserved for further examination (see p. 10). 
On repeatedly distilling this oil in an atmosphere of carbonic anhydride, in a long-necked W urtz flask, eventually two principal fractions were obtained, boiling between $223-224^{\circ}$ and $224-225^{\circ}$; these gave on analysis the following numbers :-

I. 0.1307 gram substance gave 0.1363 gram $\mathrm{OH}_{2}$ and $0.3381 \mathrm{CO}_{2}$. II. $0.1778, \quad, \quad 0.1833, \quad, \cdot 0.4576$,

\begin{tabular}{|c|c|c|c|}
\hline \multicolumn{3}{|c|}{ Found. } & \multirow{3}{*}{$\begin{array}{c}\text { Theory } \\
\text { for } \mathrm{C}_{20} \mathrm{H}_{38} \mathrm{O}_{4} \text {. } \\
70 \cdot 17 \text { per cent. }\end{array}$} \\
\hline & I. & II. & \\
\hline & 70.55 & $70 \cdot 19$ per cent. & \\
\hline 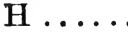 & 11.58 & $11 \cdot 45$ & $11 \cdot 11$ \\
\hline
\end{tabular}

This body appears therefore to have the formula $\mathrm{C}_{20} \mathrm{H}_{38} \mathrm{O}_{4}$. Its formation is easily understood, thus :-

$$
5 \mathrm{C}_{4} \mathrm{H}_{8} \mathrm{O}=\mathrm{C}_{20} \mathrm{H}_{38} \mathrm{O}_{4}+\mathrm{H}_{2} \mathrm{O} \text {. }
$$

It is an almost colourless oil, which smells strongly of camphor. When it was left in contact with hydrogen sodium sulphite for two or three weeks, with constant agitation, long transparent needles were formed; it is therefore apparently an aldehyde. It does not solidify in a freezing mixture, but becomes extremely viscid. It reduces ammoniacal silver solution, but does not quickly absorb oxygen from the air. When heated for some hours with 50 per cent. sulphuric acid, it first becomes black, giving off a considerable quantity of $\mathrm{CO}_{2}$, and at last it becomes nearly solid. Ether removes an oil from this product which principally distils between $200-240^{\circ}$. There was, however, also a low-boiling body present, which $I$ hope to investigate further.

This aldehyde, $\mathrm{C}_{20} \mathrm{H}_{38} \mathrm{O}_{4}$, is perhaps the same body as that which Urech obtained (Ber., 13, 593) by distillation of the polymeride of isobutaldehyde produced by the action of potassium carbonate. His product was an intensely yellow oil, boiling between $230-240^{\circ}$, and gave, on analysis, $\mathrm{C}=71.50$ per cent., $\mathrm{H}=11.80$ per cent.; considering the wideness of the range of boiling point, these agree fairly with the numbers which I obtained. He also determined the vapourdensity by Hofmann's method, and found it equal to $6 \cdot 80$, which gives a molecular weight $=196.70$ as against 342 , calculated for $\mathrm{C}_{20} \mathrm{H}_{38} \mathrm{O}_{4}$, so that it appears that this body decomposes on the prolonged heating of its vapour. In order to confirm these results, two vapour-density determinations were made, the first by the Dumas method, the second by the Victor Meyer method.

The vapour-density obtained by the Dumas method was $6 \cdot 64$, which agrees fairly with the one found by Urech. The one made by the Victor Meyer method gave 5.77.

The molecular weight was therefore $28.92 \times 5 \cdot 77=167$. 
This vapour-density was taken in a lead-bath, so that the body was probably fully decomposed, as this result is about half of the calculated density. It is worth noticing that during the determination by means of the Dumas method, most of the substance distilled off when the bath was up to $240^{\circ}$; but it was not till the bath had gone up to $252^{\circ}$ that all was vaporised. This seems to be a clear indication of its decomposing, as the substance used boiled between $223-224^{\circ}$.

By the action of aqueous potash on isobutaldehyde, Fossek obtained a body boiling at $222-223^{\circ}$, and fusing at $15.5^{\circ}$, to which he gave the formula $\mathrm{C}_{8} \mathrm{H}_{18} \mathrm{O}_{2}$. This formula requires $\mathrm{C}=65.75$ per cent.; $H=12.33$ per cent., and a molecular weight $=146$. There is no doubt it is a different body from the one described above.

\section{Action of Acetic Anhydride on $\mathrm{C}_{20} \mathrm{H}_{38} \mathrm{O}_{4}$.}

As it seemed possible that the aldehyde $\mathrm{C}_{20} \mathrm{H}_{38} \mathrm{O}_{4}$ might, by the action of acetic anhydride, form some acetyl compound which would throw light on its constitution, the following experiments were made:-A quantity of this substance was first sealed up in a tube with a slight excess of acetic anhydride (for a monoacetate), and slowly heated till the temperature reached $180^{\circ}$, and then allowed to cool down. On fractioning the product as soon as the acetic anhydride and acetic acid had distilled off, nearly all came over between $235-250^{\circ}$. This was several times carefully fractioned, when at last the largest quantity was obtained boiling at $240-242^{\circ}$. On analysis it gave the following results :-

I. 0.1345 gram substance gave 0.1263 gram $\mathrm{OH}_{2}$ and $0.3375 \mathrm{CO}_{2}$.

II. $0.1549, \quad, 0.1452, \quad, \quad$ " 0.3905 ,

Found.

$\begin{array}{cccc} & \overbrace{\text { I. }} & \text { II. } \\ \text { C } \ldots \ldots & 68 \cdot 43 & 68 \cdot 75 & \text { per cent. } \\ \text { H .... } & 10.43 & 10.42 \quad,\end{array}$

Theory for $\mathrm{C}_{22} \mathrm{H}_{40} \mathrm{O}_{5}$. 68.75 per cent.

10.42

These numbers agree with those required for a monoacetate, produced according to the following equation :-

$$
\mathrm{C}_{20} \mathrm{H}_{38} \mathrm{O}_{4}+\underset{\mathrm{CH}_{3} \mathrm{CO}}{\mathrm{CH}_{3} \mathrm{CO}}>\mathrm{O}=\mathrm{C}_{22} \mathrm{H}_{40} \mathrm{O}_{5}+\mathrm{CH}_{3} \mathrm{COOH} .
$$

This body is an almost colourless oil, having only a very faint smell. It does not solidify in a freezing mixture. Treated with potash it turns black, appearing to saponify very easily. In order to see if acetic anhydride had any further action on this body, it was heated in a sealed tube with excess of acetic anhydride to $200-220^{\circ}$ for five 
hours, and then refractioned. Nearly all of the oil came over between $240^{\circ}$ and $255^{\circ}$. This when refractioned two or three times gave, as the principal part, an oil boiling constantly from $248-252^{\circ}$, and yielding on analysis the following numbers :-

I. 0.1344 gram substance gave $0.1189 \mathrm{OH}_{2}$ and $0.3217 \mathrm{CO}_{2}$.

II. $0.1372, \quad, \quad 0.1235 \quad$, $0.3389 \quad$,

Found.

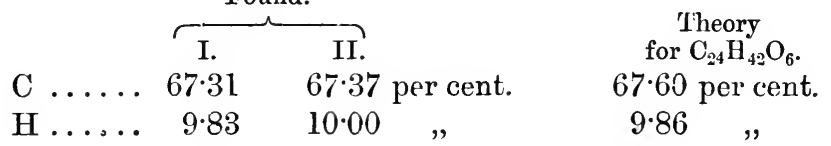

This body may therefore be regarded as a diacetate derived from $\mathrm{C}_{20} \mathrm{H}_{38} \mathrm{O}_{4}$, and produced from the monoacetate, according to the equation:-

$$
\mathrm{C}_{23} \mathrm{H}_{40} \mathrm{O}_{5}+\underset{\mathrm{CH}_{3} \mathrm{CO}}{\mathrm{CH}_{3} \mathrm{CO}}>\mathrm{O}=\mathrm{C}_{24} \mathrm{U}_{42} \mathrm{O}_{6}+\mathrm{CH}_{3} \mathrm{COOH} .
$$

This diacetate resembles the monoacetate in most respects; it is an almost colourless oil, has very little smell, and does not solidify in a freezing mixture. It distils without the least decomposition. In order to prove that this was really a diacetate, it was quantitatively saponified, and the acetic acid formed was determined. As there was not sufficient of the fraction $248-252^{\circ}$, a fraction boiling at $246-254^{\circ}$ was taken, and gave the following results:-

4.1428 gram substance was saponified with alcoholic potash, water added, and the oil extracted with ether. The potash-solution was concentrated, acidulated with sulphuric acid, and distilled into barytawater, and afterwards the excess of baryta was separated with carbonic anhydride. The weight of barium salt obtained, after evaporation, was $3 \cdot 1862$ grams. As the salt did not look pure, it was thought that it probably contained a small quantity of barium isobutyrate. A barium determination was therefore made which gave $52 \cdot 74$ per cent.

Theory for $\mathrm{Ba}\left(\mathrm{C}_{2} \mathrm{H}_{3} \mathrm{O}_{2}\right)_{2}=53 \cdot 72$. This therefore apparently consisted of-

Barium acetate $\ldots \ldots 89.86$ per cent. $=2 \cdot 863$ grams.

Barium isobutyrate... 10.14 , $=0.3231 \mathrm{gram}$.

Theory requires that from $4 \cdot 1428$ grams of the diacetate, $2 \cdot 4800$ grams of barium acetate should be formed, which agrees as well as could be expected with the quantity found. There is, therefore, no doubt that it was a diacetate. The dark-brown ethereal solution, which was separated from the potassium acetate, was well washed, dried, and fractioned. After the ether was distilled off, the thermometer rose rapidly to $200^{\circ}$, the greater quantity coming over between $215^{\circ}$ and 
$240^{\circ}$. A considerable quantity of a black tar was left behind in the retort, which was not further examined. The presence of this, as well as the apparent formation of isobutyric acid, shows that, besides saponifying, the potash had also exercised a further action on the product. The fraction $215-240^{\circ}$ was then redistilled, when the principal portion passed over at $217-223^{\circ}$, and gave on analysis the following result:--

I. $0 \cdot 1435$ gram substance gave $0 \cdot 1568 \mathrm{OH}_{2}$ and $0.3629 \mathrm{CO}_{2}$.

II. $0 \cdot 1254$

,$\quad 0 \cdot 1370$

0.3165,

Found.

$\begin{array}{llll} & \overbrace{\text { I. }} & \text { II. } \\ \text { C } \ldots \ldots & 68 \cdot 97 & 68 \cdot 83 & \text { per cent. } \\ \text { H.... } & 12 \cdot 14 & 12 \cdot 14 \quad,\end{array}$

Theory
for $\mathrm{C}_{20} \mathrm{H}_{42} \mathrm{O}_{4}$.
$69 \cdot 37$ per cent.
$12 \cdot 14 \quad$ "

This body has therefore the formula $\mathrm{C}_{20} \mathrm{H}_{42} \mathrm{O}_{4}$, or $4 \mathrm{H}$ more than the body $\mathrm{C}_{20} \mathrm{H}_{38} \mathrm{O}_{4}$, which should have been reproduced by the saponification. This new product is an almost colourless oil, having a peculiar odour, which does not resemble that of the aldehyde $\mathrm{C}_{20} \mathrm{H}_{38} \mathrm{O}_{4}$. The first of the above combustions is from a fraction boiling between $217-219^{\circ}$, and the second from a fraction $\left(219-223^{\circ}\right)$. It therefore appears that by the saponification of the diacetate, the body $\mathrm{C}_{20} \mathrm{H}_{38} \mathrm{O}_{4}$, probably first produced, is further acted on by the reducing and oxidising action of the potash, part being oxidised and part forming the body $\mathrm{C}_{20} \mathrm{H}_{42} \mathrm{O}_{4}$.

The residue of the condensed isobutaldehyde left in the retort and boiling above $235^{\circ}$ was distilled under a pressure of $100 \mathrm{~mm}$. A considerable quantity came over between $185^{\circ}$ and $200^{\circ}$; the next large fraction was between $220-235^{\circ}$. The distillation was discontinued when the temperature had reached $280^{\circ}$, and decomposition set in. No solid bodies were obtained. The portion boiling at 185$200^{\circ}$ was then fractioned under the ordinary pressure, when it nearly all distilled over between $245^{\circ}$ and $260^{\circ}$. This was then collected between every $5^{\circ}$; the principal product thus obtained boiled at 250 $255^{\circ}$.

On analysis it gave the following results :-

I. $0 \cdot 1234$ gram substance gave $0 \cdot 1231 \mathrm{OH}_{2}$ and $0.3328 \mathrm{CO}_{2}$. II. $0 \cdot 1370$

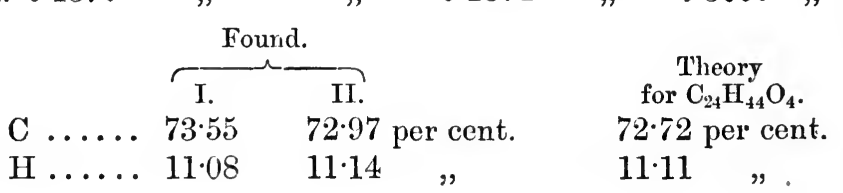


It thus appears to have the formula $\mathrm{C}_{24} \mathrm{H}_{44} \mathrm{O}_{4}$, and is probably produced according to the equation-

$$
6 \mathrm{C}_{4} \mathrm{H}_{8} \mathrm{O}=\mathrm{C}_{24} \mathrm{H}_{44} \mathrm{O}_{4}+2 \mathrm{OH}_{2} \text {. }
$$

It is a stable body, and requires long boiling with dilute sulphuric acid to decompose it. Owing to the small quantity produced in the action of potash on isobutaldehyde, it could only be very little examined. It is an almost colourless oil, and distils at ordinary pressures without decompositiou. It does not seem to combine with acid sulphite of soda. On heating with strong alcoholic potash, it turns black and is decomposed, forming a considerable quantity of a potassium salt. Two vapour-density determinations were made which gave $12 \cdot 61$ and $13 \cdot 21$.

The calculated density for $\mathrm{C}_{24} \mathrm{H}_{44} \mathrm{O}_{4}=13 \cdot 69$.

From these determinations, it appears evident that this body is much more stable tban the lower condensation-products, as the figures obtained, althougb not agreeing well with the calculated ones, yet go to show that the body is produced by the eondensation of six molecules of isobutaldehyde, the next lower one, $\mathrm{C}_{20} \mathrm{H}_{38} \mathrm{O}_{4}$, having a calculated density $=11 \cdot 82$.

The fraction obtained between $220-235^{\circ}$ under a pressure of $100 \mathrm{~mm}$. was then carefully distilled uuder the same pressure. After repeating this operation several times, a considerable quantity was obtained, boiling constantly at $227-229^{\circ}$, which gave on analysis the following numbers:-

I. $0 \cdot 1281$ gram substance gave $0 \cdot 1305 \mathrm{OH}_{2}$ and $0 \cdot 3630 \mathrm{CO}_{2}$.

II. $0.1420 \quad, \quad, 0 \cdot 1420 \quad, \quad 0 \cdot 4038 \quad$,

Found.

\begin{tabular}{|c|c|c|c|}
\hline & I. & II. & $\begin{array}{c}\text { Theory } \\
\text { for } \mathrm{C}_{28} \mathrm{H}_{48} \mathrm{O}_{3} \text {. }\end{array}$ \\
\hline$\cdots$ & $77 \cdot 28$ & 77.55 per cent. & $77 \cdot 77$ per cent. \\
\hline ... & $11 \cdot 32$ & $11 \cdot 11$ & $11 \cdot 11$ \\
\hline
\end{tabular}

This body appears therefore to have the formula $\mathrm{C}_{28} \mathrm{H}_{48} \mathrm{O}_{3}$. The formation may be expressed by assuming that four molecules of water are removed from seven molecules of isobutaldehyde according to the equation:-

$$
7 \mathrm{C}_{4} \mathrm{H}_{8} \mathrm{O}=\mathrm{C}_{28} \mathrm{H}_{48} \mathrm{O}_{3}+4 \mathrm{OH}_{2} \text {. }
$$

This body is a very thick yellowish oil, having a very faint smell. It distils in a vacuum without decomposition, but at ordinary pressures it appears to split up into lower and higher boiling bodies, a tarry residue being left behind in the retort. This decomposition was also seen when two vapour-density determinations were attempted. By the Victor Meyer process in a lead-bath, they gave 10.42 and 10.77. 
The calculated density for $\mathrm{C}_{28} \mathrm{H}_{48} \mathrm{O}_{3}$ is $14 \cdot 94$.

The still higher-boiling condensation-products would be very difficult to obtain pure, and were not analysed. There appeared, however, to be a definite body boiling at about $250^{\circ}$, under a pressure of $100 \mathrm{~mm}$. A table is appended of the bodies obtained and their boiling points.

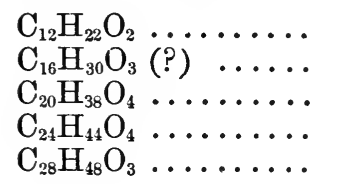

$\left.\begin{array}{l}\mathrm{C}_{12} \mathrm{H}_{26} \mathrm{O}_{2} \\ \mathrm{C}_{12} \mathrm{H}_{2 \downarrow} \mathrm{O}_{2} \\ \mathrm{C}_{2}\end{array} \ddot{\mathrm{H}}_{3} \mathrm{O}\right)_{2} \cdots$

$\mathrm{C}_{20} \mathrm{H}_{37} \mathrm{O}_{4}\left(\mathrm{C}_{2} \mathrm{H}_{3} \mathrm{O}\right)$
$\mathrm{C}_{20} \mathrm{H}_{36} \mathrm{O}_{4}\left(\mathrm{C}_{2} \mathrm{H}_{3} \mathrm{O}\right)_{2}$

$\mathrm{C}_{20} \mathrm{H}_{42} \mathrm{O}_{4} \ldots \ldots \ldots$

$\mathrm{C}_{12} \mathrm{H}_{22} \mathrm{O}_{3} \ldots \ldots \ldots$ $154-157^{\circ}$

$190-200^{\circ}$

$223-225^{\circ}$

$250-255^{\circ}$

$227-229^{\circ}$

(100 mm. pressure)

$170-175^{\circ}$

$185-190^{\circ}$

$240-242^{\circ}$

$248-252^{\circ}$

$217-223^{\circ}$

$245-255^{\circ}$
Condensation-products.

Alcohol (and acetate) produced by the action of nascent hydrogen on $\mathrm{C}_{12} \mathrm{H}_{22} \mathrm{O}_{2}$.

$\int$ Acetates produced by $\{$ the action of acetic anhydride on $\mathrm{C}_{20} \mathrm{H}_{38} \mathrm{O}_{4}$. $\{$ Produced by saponifying $\mathrm{C}_{20} \mathrm{H}_{36} \mathrm{O}_{4}\left(\mathrm{C}_{2} \mathrm{H}_{3} \mathrm{O}\right)_{2}$. $\int$ Acid produced by the action of potash on isobutaldehyde. 


\title{
ON THE CONDENSATION_PRODUCTS OF ENANTHALDEHYDE.
}

\author{
(Parts I ANd II.)
}



[Reprinted from the Journal of the Chemical Society, Februarg, 1883.]

\title{
ON THE CONDENSATION-PRODUCTS OF CENANTHALDE- HYDE. (PART I.)
}

\author{
By W. H. Perkin, Junr., Ph.D.
}

AMoNg the many researches on the condensation of the aldehydes, it may be well to mention a few on account of their special bearing on this investigation.

Urech (Ber., 13, 483, 590, and 12, 190) found that by the action of dry potassium carbonate on isobutaldehyde, a polymeride of that body is formed, to which he gives the formula $\left(\mathrm{C}_{4} \mathrm{H}_{8} \mathrm{O}\right)_{3}$. On distillation, it decomposes into isobutaldehyde, $\mathrm{C}_{12} \mathrm{H}_{22} \mathrm{O}_{2}$, and other condensationproducts, at the same time losing water.

Demtschenko (Ber., 6, 1176) obtained from isobutaldehyde by the action of sulphuric acid a polymeride which has also the formula $\left(\mathrm{C}_{4} \mathrm{H}_{8} \mathrm{O}\right)_{3}$, and which he names paraisobutaldelyde. This body is, however, not decomposed on distillation.

Borodin (Ber., 6, 982) found that by the action of caustic alkali on isovaleraldehyde at $0^{\circ}$, a polymerised modification of that body is formed, which decomposes on distillation, with separation of water, into $\mathrm{C}_{19} \mathrm{H}_{18} \mathrm{O}$, and another condensation-product, $\mathrm{C}_{20} \mathrm{H}_{38} \mathrm{O}_{3}$, both of which are oils. This last body is decomposed by heating with alkalis into isovaleric acid, isopentylalcohol, and isovaleraldehyde, which bodies are also formed by heating isovaleraldehyde alone to $240^{\circ}$, or with zinc-turnings to $180^{\circ}$.

If isovaleraldehyde is allowed to stand for some time with soda, a hydrate of the formula $\mathrm{C}_{20} \mathrm{H}_{42} \mathrm{O}_{5}=\left(\mathrm{C}_{10} \mathrm{H}_{20} \mathrm{O}_{2}\right)_{2} \mathrm{H}_{2} \mathrm{O}$ is formed, which on distillation is decomposed into isovaleraldehyde and the condensation-products $\mathrm{C}_{10} \mathrm{H}_{18} \mathrm{O}$ and $\mathrm{C}_{20} \mathrm{H}_{38} \mathrm{O}_{3}$. By the action of sodium on isovaleraldehyde, Borodin (Juhresb., 1864, 338 ; Ber., 5, 481) obtained the condensation-products $\mathrm{C}_{10} \mathrm{H}_{18} \mathrm{O}$ and $\mathrm{C}_{20} \mathrm{H}_{38} \mathrm{O}_{3}$, polyisovaleraldehyde, and the alcohol $\mathrm{C}_{10} \mathrm{H}_{23} \mathrm{O}$.

This last body gives on oxidation isocapraldehyde, the acid $\mathrm{C}_{10} \mathrm{H}_{18} \mathrm{O}_{2}$, an oil which boils at $250-290^{\circ}$, and to which Borodin ascribes the formula $\left(\mathrm{C}_{10} \mathrm{H}_{18} \mathrm{O}\right)_{n}$, also isovaleric acid and isopentylalcohol.

Grenier (Jahresb., 1866, 465) obtained the same body, $\mathrm{C}_{10} \mathrm{H}_{18} \mathrm{O}$, by the action of sodium on ethylisovalerate.

Gass and Hell found that dry potassium carbonate has, at the ordinary temperature, the same action on isovaleraldehyde as caustic potash, but that if the aldehyde is boiled with potassium carbonate, the bodies $\mathrm{C}_{10} \mathrm{H}_{18} \mathrm{O}, \mathrm{C}_{15} \mathrm{H}_{28} \mathrm{O}_{2}$, and $\mathrm{C}_{20} \mathrm{H}_{38} \mathrm{O}_{3}$, are formed. This last 
body on distillation decomposes with separation of water, and gives the aldehyde $\mathrm{C}_{10} \mathrm{H}_{18} \mathrm{O}$.

Of the researches on the condensation-products of oenanthaldehyde, those of Borodin, Bruylants, Tilley, Fittig, and Schiff may be cited.

Borodin found (Ber., 5, 481) that by the action of caustic alkalis on œnanthaldehyde at ordinary temperatures, two polymeric modifications are formed. One is a solid and the other an oil. They both give off water on distillation, with formation of $\mathrm{C}_{14} \mathrm{H}_{26} \mathrm{O}$ and $\mathrm{C}_{28} \mathrm{H}_{54} \mathrm{O}_{3}$.

By heating œnanthaldehyde at $240^{\circ}$, the same bodies are formed.

Bruylants (Ber., 8, 415) obtained by the action of potassium carbonate on œnanthaldehyde, a polymeric modification, which he purified by washing with water and repeatedly crystallising. It melts at $51-52^{\circ}$. Like the polymerides of isobutyl and isovaleryl aldehydes, it decomposes with separation of water on distillation, condensationproducts being formed; one of these boils at $160-170^{\circ}$, and appears to be an aldehyde.

Fittig (Annalen, 117, 76) found that if onanthaldehyde is left in contact with quicklime, heptoic acid, heptyl alcohol, and the hydrocarbons $\mathrm{C}_{7} \mathrm{H}_{11}, \mathrm{C}_{8} \mathrm{H}_{16}, \mathrm{C}_{9} \mathrm{H}_{18}$, and œnanthacetone, $\mathrm{C}_{13} \mathrm{H}_{26} \mathrm{O}$, are formed.

Tilley (Jaliresb., 1, 566) obtained, by warming cenanthaldehyde with caustic potash to $120^{\circ}$, an oil, $\mathrm{C}_{14} \mathrm{H}_{28} \mathrm{O}$, which boiled at $220^{\circ}$. By the action both of fused caustic potash and also of aqueous potash on conanthaldehyde, he found that heptoic acid is formed. In the latter case, an oil was separated from the potassium salt by distilling in steam, and was then found to have the boiling point $220^{\circ}$, and on analysis numbers were obtained which seemed to show it to be the same body, $\mathrm{C}_{14} \mathrm{H}_{28} \mathrm{O}$, mentioned above. By oxidation with nitric acid it gives heptoic acid, and by the action of potash heptoic acid is also produced, and a tarry body, hydrogen being evolved at the same time.

Schiff found that by saturating an alcoholic solution of cenanthaldehyde with hydrochloric acid gas, a body of the formula

$$
\mathrm{Cl}\left(\mathrm{C}_{7} \mathrm{H}_{14}\right) \mathrm{OC}_{2} \mathrm{H}_{5}
$$

is formed, which on distillation is completely decomposed into $\mathrm{C}_{14} \mathrm{H}_{26} \mathrm{O}$ and other bodies.

Rieth and Beilstein (JaTresb., 16, 478) obtained the same body, $\mathrm{C}_{14} \mathrm{H}_{25} \mathrm{O}$, by the action of zinc-ethyl on cnanthaldehyde.

As in many cases in the above-mentioned researches the formulæ only, and not the properties of the bodies obtained, are described, I undertook, at the suggestion of Professor Wislicenus, the following research, in order, if possible, to obtain some clue to the constitution of the bodies formed by the condensation of the aldehydes, and more especially of those derived from œnanthaldehyde.

The onanthaldehyde used in the following experiments was obtained 
from Kahlbaum, in Berlin. It boiled between $150^{\circ}$ and $160^{\circ}$. Pure œnanthaldehyde was found to boil at $153-154^{\circ}$ (thermometer in vapour), and has the specific gravity

$$
\begin{aligned}
& \text { at } 15^{\circ}=0.8231 \\
& \text { at } 30=0.8128 \\
& \text { at } 35=0.8099
\end{aligned}
$$

compared with water at the same temperatures.

\section{Action of Potash on Enanthaldehyde.}

The action of potash on this aldehyde is extremely violent. If cnanthaldehyde is mixed with a concentrated solution of potash in alcohol, the rise of temperature, chiefly owing to condensation, is so great as often to cause the alcohol to boil. The products of this reaction consist mainly of potassium heptoate and high condensation-products which, owing to their high boiling points, could not be isolated and examined. However, after many experiments, it was found that if a very dilute solution of alcoholic potash is used, the reaction is less complicated, the following method of procedure giving the best results :-

3 grams of potash were dissolved in about 200 grams of absolute alcohol, and then 200 grams of œnanthaldehyde slowly added, care being taken that the temperature did not rise above $30^{\circ}$, otherwise the reaction was found to go too far, large quantities of high-boiling bodies being produced. The condensation of the cenanthaldehyde takes place very rapidly, and is probably finished in half an hour. At the end of about 24 hours not a trace remains unchanged.

In order to isolate the products of the reaction, the alcohol was first distilled off and then water added, or the product was directly diluted with much water. In either case it was found necessary to shake up the product with ether to perfectly separate the oil from the alkaline solution. The ethereal solution was well washed, first with dilute hydrochloric acid, and then with water, and finally dried over calcium chloride.

The following is the examination of the aqueous alkaline solutions from a number of these operations. They were first treated two or three times with ether to remove traces of oil, saturated with carbonic anhydride to convert any caustic alkali present into carbonate, and evaporated to dryness on a water-bath. The product was then further dried and several times extracted with absolute alcohol.

After the alcohol had been distilled off from these extracts, the saline residue was dissolved in water, acidified with hydrochloric acid, and the acids thus liberated separated with ether, the ethereal solu. 
tion was then fractioned. After the ether had been distilled off, the greater part of the product came over between $180^{\circ}$ and $230^{\circ}$. A small quantity, however, referred to further on, was leit in the retort.

The portion boiling between $180-230^{\circ}$, on fractioning, came over mostly between $220-225^{\circ}$, and was, doubtless, heptoic acid. It gave on analysis the following numbers :-

0.2389 substance gare $0.2372 \mathrm{OH}_{2}$ and $0.5659 \mathrm{CO}_{2}$.

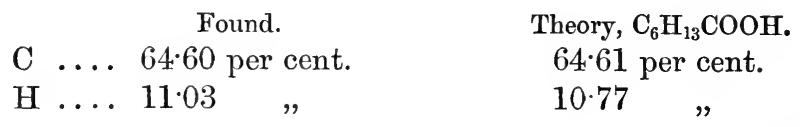

The small residue left in the retort was distilled in vacuo, and then fractioned under a pressure of $200 \mathrm{~mm}$., when it came over without the least decomposition. The best fraction which could be obtained boiled, at this pressure, between $270-290^{\circ}$, and gave the following numbers on analysis. These agree fairly well with the calculated percentages for an acid of the formula $\mathrm{C}_{14} \mathrm{H}_{26} \mathrm{O}_{2}$ :-

0.1405 substance gave $0.1445 \mathrm{OH}_{2}$ and $0.3811 \mathrm{CO}_{2}$.

Found.

C ... 73.97 per cent.

$\mathrm{H} \ldots 11.42$,
Theory, $\mathrm{C}_{14} \mathrm{H}_{26} \mathrm{O}_{2}=\mathrm{C}_{13} \mathrm{H}_{25} \mathrm{COOH}$.

74.33 per cent.

$11 \cdot 50 \quad$,

The yield of this acid is perhaps improved by adding hydrochloric acid and extracting the acids directly from the crude potash-salt, instead of saturating with carbonic anhydride, extracting with alcohol, \&c., as long boiling seems to decompose the potassium salt. The properties and salts of this acid are described further on, under the heading "Action of Potash on the Aldehyde $\mathrm{C}_{14} \mathrm{H}_{26} \mathrm{O}$."

The following is the examination of the ethereal solution of the oily condensation-products. After the ether had been distilled off the remaining oil was carefully fractioned in a stream of carbonic anhydride.* The thermometer rose rapidly to $260^{\circ}$, between which temperature and $300^{\circ}$ by far the largest quantity of the product distilled over as an almost colourless oil, leaving a black residue in the retort which is referred to further on. This distillate was then repeatedly fractioned, the greatest care being taken to prevent the product from coming in contact with the air. An oil at last was obtained boiling between $277^{\circ}$ and $279^{\circ}$.

On analysis it gave the following numbers :-

* It is very important to bear in mind that, owing to the rapid absorption of oxygen from the air by these products, all operations with them must be conducted in an atmosphere of carbonic anhydride. 


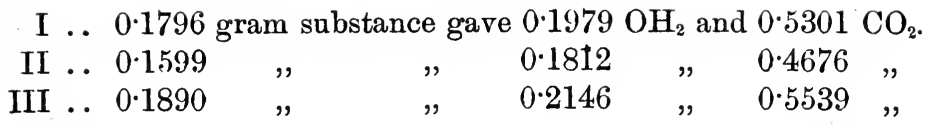

Found.

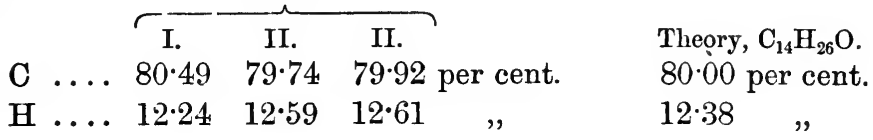

These combustions were made from three different preparations. This body has therefore the composition $\mathrm{C}_{14} \mathrm{H}_{26} \mathrm{O}$, and is apparently the same substance as that obtained by Borodin, Schiff and Rieth, and Beilstein. It is a colourless oil, having a faint smell and a burning taste. It does not solidify at $-20^{\circ}$. Its specific gravity is-

$$
\begin{aligned}
& \text { at } 15^{\circ}=0.8494 \\
& \text { at } 30=0.8416 \\
& \text { at } 35=0.8392
\end{aligned}
$$

compared with water at the same temperatures.

It absorbs oxygen very rapidly, so much so, that when placed in a tube full of air, over mercury, in a short time nothing but nitrogen is left in it. This oil reduces a solution of $\mathrm{Ag}_{2} \mathrm{O}$ in ammonia very easily.

It is an aldehyde, and combines, though very slowly, with hydrogen sodium sulphite, so that, only after keeping the two in contact for months, with frequent agitation, a definite body was obtained; it then had the form of silky crystals slightly soluble in water; it is apparently decomposed by a solution of sodium carbonate. This compound was filtered from the excess of hydrogen sodium sulphite, pressed between filter-paper, and in order to insure its freedom from inorganic matter, two or three times wetted with water and again pressed.

It was then thoroughly dried, and washed with ether to remove any uncombined oil. When pure it is a beautiful silky-looking crystalline substance. A sodium determination gave the following numbers:-

0.4622 gram substance gare 0.1105 gram $\mathrm{Na}_{2} \mathrm{SO}_{4}=7 \cdot 74$ per cent. $\mathrm{Na}$.

The formula $\mathrm{C}_{14} \mathrm{H}_{26} \mathrm{O}, \mathrm{NaHSO}_{3}$ requires $7 \cdot 32$ per cent.

This aldehyde is soluble in ether, alcohol, carbon disulphide, and glacial acetic acid, in 50 per cent. acetic acid it is only sparingly soluble. It is evidently formed by the elimination of the elements of water from 2 mols. of cenanthaldehyde, according to the equation-

$$
2 \mathrm{C}_{6} \mathrm{H}_{13} \mathrm{COH}=\mathrm{C}_{13} \mathrm{H}_{25} \mathrm{COH}+\mathrm{OH}_{2} \text {. }
$$


The condensation-products boiling above $300^{\circ}$, which were left in the retort after distilling off the aldehyde, $\mathrm{C}_{14} \mathrm{H}_{26} \mathrm{O}$, just described, were very thick; they were first distilled in a vacuum, and then fractioned under a pressure of $350 \mathrm{~mm}$. The principal portion came over between $320-350^{\circ}$, above that temperature, however, there remained a considerable quantity in the retort, which could not be purified. The product boiling between $320-350^{\circ}$ was very carefully fractioned under the same pressure, till at last the principal quantity was obtained, boiling between $330-340^{\circ}$. It is a thick light yellow oil, and has a disagreeable smell and burning taste. It does not solidify at $-20^{\circ}$. It mixes in all proportions with the usual solvents, and reduces ammoniacal silver solution.

The analyses gave the following numbers :-

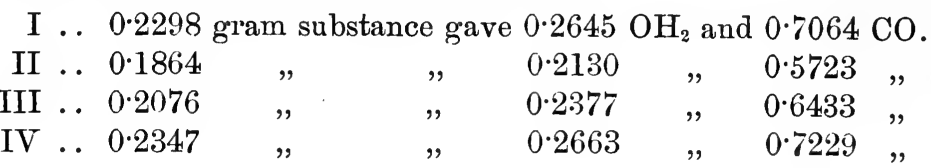

Found.

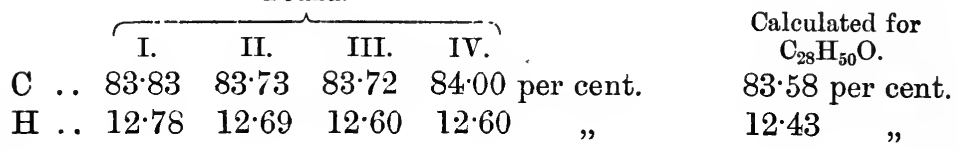

Nos. I and IV were from the first experiment, Nos. II and III from one made later on. The above results show that the body has the formula $\mathrm{C}_{28} \mathrm{H}_{50} \mathrm{O}$. Its specific gravity is-

$$
\begin{aligned}
& \text { at } 15^{\circ}=0.8831 \\
& \text { at } 30=0.8751 \\
& \text { at } 35=0.8723
\end{aligned}
$$

compared with water at the same temperatures.

It is apparently formed from 4 mols. of conanthaldehyde by the elimination of 3 mols. of water, according to the equation-

$$
4 \mathrm{C}_{7} \mathrm{H}_{14} \mathrm{O}=\mathrm{C}_{28} \mathrm{H}_{50} \mathrm{O}+3 \mathrm{OH}_{2} \text {. }
$$

The intermediate body, containing $\mathrm{C}_{21}$, and standing between this body and the aldehyde previously described, could not be obtained pure; it appears to be formed but only in very small quantities.

The principal products of the action of potash on cenanthaldehyde, so far as they have been studied, are, therefore, heptoic acid, the acid $\mathrm{C}_{14} \mathrm{H}_{26} \mathrm{O}_{2}$, and the aldehydes $\mathrm{C}_{14} \mathrm{H}_{26} \mathrm{O}$, and $\mathrm{C}_{28} \mathrm{H}_{50} \mathrm{O}$.

\section{Action of Zinc Chloride on Enanthaldehyde.}

These experiments were tried in the hope of getting a body corresponding to crotonic aldehyde, by the removal of the elements of water 
from œnanthaldehyde. The action of zinc chloride on dry œnanthaldehyde is very violent. If the two are warmed together, two layers are quickly formed; the lower one being a solution of zinc chloride, and the upper one a dark brown oil, from which nothing definite could be obtained, as it decomposed on distillation. After numerous trials it was found that by modifying the process in the following way, the reaction was less complicated: œnanthaldehyde was shaken up with water until it was thoroughly saturated, separated from the excess of water, and warmed on a water-bath with a very small quantity of zinc chloride. In about 15 hours the action was finished. The product was then dissolved in a small quantity of ether, washed with water, dried, and fractioned. After the removal of the ether, it was distilled first in vacuo, as a quantity of high-boiling products were present. It was then further fractioned at the ordinary pressure. As soon as the unchanged conanthaldehyde had distilled over, the thermometer rose rapidly to $260^{\circ}$, between which temperature and $300^{\circ}$ the principal portion distilled over.

The residue was not distilled over; it contained probably the same high-boiling condensation-products as those obtained by the action

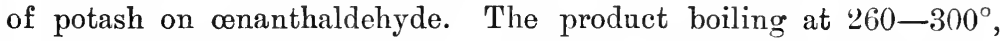
on being fractioned, passed over principally at $276-280^{\circ}$, and judging from its boiling point was apparently the aldehyde $\mathrm{C}_{14} \mathrm{H}_{26} \mathrm{O}$. This was proved to be the case by the determination of its specific gravity and analysis :-

$$
\begin{aligned}
& \text { I .. } 0.1598 \text { gram substance gave } 0.1790 \mathrm{OH}_{2} \text { and } 0.4659 \mathrm{CO}_{2} \text {. } \\
& \text { II .. } 0.1527 \quad \text { " } \quad 0.1710 \quad \text { " } 0.4479 \text {, }
\end{aligned}
$$

\begin{tabular}{|c|c|c|}
\hline \multicolumn{2}{|c|}{ Found. } & \multirow{3}{*}{$\begin{array}{l}\text { Theory, } \mathrm{C}_{14} \mathrm{H}_{26} \mathrm{O} \text {. } \\
80^{\circ} 00 \text { per cent. }\end{array}$} \\
\hline I. & II. & \\
\hline $79 \cdot 54$ & 79.97 per cent. & \\
\hline $12 \cdot 44$ & $12 \cdot 44$ & $12 \cdot 38$ \\
\hline
\end{tabular}

The zinc chloride acts therefore as a dehydrating agent on œnanthaldehyde, as in the case of ordinary aldehyde-

$$
2 \mathrm{C}_{7} \mathrm{H}_{14} \mathrm{O}=\mathrm{C}_{14} \mathrm{H}_{26} \mathrm{O}+\mathrm{OH}_{2} \text {. }
$$

The yield of this aldehyde is about 30 per cent., whereas by the potash reaction it ranges from 70 to 83 per cent. of the theoretical quantity.

Action of Acetic Anhydride on the Aldehyde, $\mathrm{C}_{14} \mathrm{H}_{26} \mathrm{O}$.

This experiment was tried in the hope of getting a diacetate from this body, as in the case of most aldehydes, according to the equation-

$$
\mathrm{C}_{13} \mathrm{H}_{25} \mathrm{COH}+\underset{\mathrm{CH}_{3} \mathrm{CO}}{\mathrm{CH}_{3} \mathrm{CO}}>\mathrm{O}=\mathrm{C}_{13} \mathrm{H}_{25} \mathrm{CH}<\mathrm{OOCCH}_{3},
$$

and of thus obtaining another proof of its being an aldehyde. 
A mixture of this aldehyde and acetic anhydride was first boiled for three days in a flask connected with a reversed condenser; as it was found, however, that little or no reaction had taken place, it was then transferred to a sealed tube, and heated to about $180^{\circ}$ for three days.

On distilling the product, and after the unchanged anhydride and acetic acid had distilled off, the thermometer rose rapidly to about $270^{\circ}$, when a small quantity of the unchanged aldehyde came over. The distillation was continued till the thermometer had reached $300^{\circ}$, and then the thick black residue was distilled under a pressure of $350 \mathrm{~mm}$., when by far the largest quantity came over between $300-350^{\circ}$.

This portion was carefully fractioned under the same pressure. After repeating this operation two or three times, the principal portion distilled over between $330-340^{\circ}$, as a viscid light yellow oil. On heating this with alcoholic potash it turned dark brown, but no trace of acetic acid could be afterwards found combined with the alkali. The oil left after this treatment decomposed on distillation. It appeared therefore that the body was not an acetate. On analysis it gave the following numbers:-

I. 0.2353 gram substance gave $0.2600 \mathrm{OH}_{2}$ and $0.7242 \mathrm{CO}_{2}$. II. $0 \cdot 1975$ $0.2219 \quad, \quad 0.6069$,

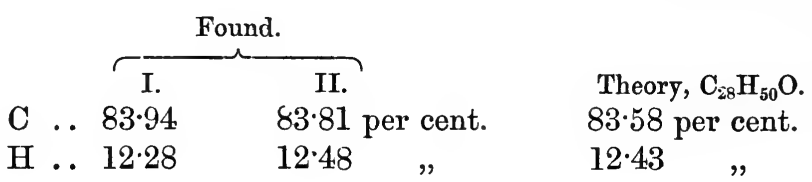

This substance has therefore the composition $\mathrm{C}_{28} \mathrm{H}_{50} \mathrm{O}$, and is doubtless identical with the one produced by the action of potash on cenanthaldehyde, as it possesses practically the same boiling point and specific gravity, and in fact resembles it in every respect. It appears therefore that the acetic anhydride acts as a dehydrating agent, extracting water from 2 mols. of $\mathrm{C}_{14} \mathrm{H}_{26} \mathrm{O}$, being itself converted into acetic acid, according to the equation-

$$
2 \mathrm{C}_{14} \mathrm{H}_{26} \mathrm{O}+\left(\mathrm{CH}_{3} \cdot \mathrm{CO}\right)_{2} \mathrm{O}=\mathrm{C}_{28} \mathrm{H}_{50} \mathrm{O}+2\left(\mathrm{CH}_{3} \cdot \mathrm{COOH}\right) .
$$

In order, if possible, to get some idea of the constitution of this body, $\mathrm{C}_{28} \mathrm{H}_{50} \mathrm{O}$, and of the nature of the groups contained in it, it was fused with excess of caustic potash in a silver crucible. In a short time the whole mass turned quite black and began to froth up and fume. After about five hours' heating it was allowed to cool, dissolved in water, and the oily matter removed with ether. The alkaline solution was then acidulated with hydrochloric acid, and the oily acid which separated taken up with ether. On distillation this acid boiled between 
$190^{\circ}$ and $230^{\circ}$, and smelt like heptoic acid. It was converted into a potassium salt and then into a silver salt.

This salt gave the following numbers on analysis :-

$0 \cdot 3307$ gram substance gave 0.1572 gram $\mathrm{Ag}=47 \cdot 53$ per cent. Calculated for $\left\{\begin{array}{l}\mathrm{C}_{5} \mathrm{H}_{11} \\ \mathrm{COOAg}\end{array}=48.43\right.$ per cent. $:\left\{\begin{array}{l}\mathrm{C}_{6} \mathrm{H}_{13} \\ \mathrm{COOAg}\end{array}=4.5 .57\right.$ percent.

It appeared therefore to be a mixture of hexoate and heptoate of silver. In order to see whether acetic acid, or any low-boiling acid soluble in water, had been formed, the original aqueous solution, which had been treated with ether, was distilled in steam. The distillate, however, was only very feebly acid, which was probably due to traces of heptoic acid. It appears, therefore, that only hexoic and heptoic acids are formed by the action of fused potash on $\mathrm{C}_{28} \mathrm{H}_{50} \mathrm{O}$, and that, therefore, probably only hexyl and heptyl-groups are contained in it, which, it will be seen, is borne out by the oxidation-products of the body $\mathrm{C}_{14} \mathrm{H}_{26} \mathrm{O}$, described further on.

It was attempted to distil in vacuo the oil which was separated from the potash salt, but it decomposed, and as only a few drops came over below $350^{\circ}$, it could not be examined.

\section{Action of Nascent Hydrogen on the Aldehyde $\mathrm{C}_{14} \mathrm{H}_{26} \mathrm{O}$.}

As the body $\mathrm{C}_{14} \mathrm{H}_{26} \mathrm{O}$ has the properties of an aldehyde, and at the same time is an unsaturated one, it was thought that it would be interesting to submit it to the action of nascent hydrogen. This was done in two ways-(I) in acetic acid solution; (II) in ethereal solution.

I. The aldehyde was dissolved in glacial acetic acid, and excess of sodium-amalgam slowly added, care being taken to keep the solution cool, in order to prevent, as far as possible, further condensation of the body $\mathrm{C}_{14} \mathrm{H}_{26} \mathrm{O}$. Owing to the addition of considerable quantities of the amalgam, the sodium acetate formed rendered the liquid thick and unmanageable. It was found advisable therefore to precipitate the oil by means of water, wash, dry, \&c., dissolve it again in acetic acid, and continue the treatment as before. When a considerable excess of amalgam had been used, the whole was diluted with water, and the oil taken up with ether. The ethereal solution, after being well washed in order to remove as much acetic acid as possible, was dried and fractioned. After the ether had distilled off, the principal quantity came over between $270-300^{\circ}$, and by repeated fractioning gave as the chief product a colourless oil boiling between $282-290^{\circ}$. An analysis of this substance gave the following numbers :- 
$0 \cdot 1906$ gram substance gave $0.2199 \mathrm{OH}_{2}$ and $0.5527 \mathrm{CO}_{2}$.

Found.

C ..... 79.08 per cent.
Theory for $\mathrm{C}_{14} \mathrm{H}_{23} \mathrm{O}$.

$79 \cdot 25$ per cent.

$13 \cdot 21 \quad$,

These numbers agree fairly well with those required by the formula $\mathrm{C}_{14} \mathrm{H}_{28} \mathrm{O}$, which represents the aldehyde as having taken on $\mathrm{H}_{2}$. As will be seen further on this product is an alcohol.

II. The treatment of the aldehyde in ethereal solution with nascent hydrogen was conducted in the following way:-

100 grams $\mathrm{C}_{14} \mathrm{H}_{26} \mathrm{O}$ were dissolved in 400 grams ether, and the mixture poured upon water contained in a large flask, connected with a reversed condenser; small pieces of sodium were then very slowly added. The reaction was at first very violent, but became more controllable as the water got saturated with sodium hydrate. Before each addition of sodium, the mixture was well shaken, in order to dissolve out the sodium hydrate formed from the ethereal solution, which would otherwise cause the further condensation of the aldehyde $\mathrm{C}_{14} \mathrm{H}_{26} \mathrm{O}$. The operation took about seven days, 50 grams of sodium being used. The ethereal solution was well washed, first with dilute hyllrochloric acid, then with water, and afterwards dried over calcium chloride, and fractioned.

The principal part distilled over between $260-310^{\circ}$, but a considerable residue was left behind, which is referred to further on. In order to separate the new alcohol which is contained in this distillate from any unchanged aldehyde, it was found best to heat the fraction $260-310^{\circ}$ with acetic anhydride in a sealed tube at $180^{\circ}$ for three days. By this means the aldehyde is condensed to $\mathrm{C}_{28} \mathrm{H}_{50} \mathrm{O}$, and the boiling point thereby considerably raised, whilst the new alcohol is at the same time converted into an acetate, which boils at about the same temperature as the alcohol itself, and is therefore very easy to obtain moderately pure by fractional distillation.

On distillation the largest portion of the product came over between $280-295^{\circ}$, and this on repeated fractioning gave as the principal product an oil boiling between $285-290^{\circ}$.

This oil, which is the acetate derived from the new alcohol, is a colourless, highly refractive liquid, having a pleasant smell. Its specific gravity is-

$$
\begin{aligned}
& \text { at } 15^{\circ}=0.8680, \\
& \text { at } 30=0.8597, \\
& \text { at } 35=0.8568,
\end{aligned}
$$

compared with water at the same temperatures.

On analysis it gave the following numbers:- 
I. 0.2367 gram substance gave $0.2600 \mathrm{OH}_{2}$ and $0.6583 \mathrm{CO}_{2}$.

II. $0.2130 \quad$ " $\quad 0.2298 \quad$ " 0.5919 ,

$\begin{array}{ccc}\text { II. } & \text { II. } & \begin{array}{c}\text { Found. } \\ \text { H } \ldots \ldots\end{array} \\ \text { for } \mathrm{C}_{14} \mathrm{H}_{27} \mathrm{OC}_{2} \mathrm{H}_{3} \mathrm{O} .\end{array}$

This acetate is readily decomposed by potash. A determination of the acetic acid produced by its saponification gave the following results :-

$2 \cdot 3442$ grams substance saponified with dilute alcoholic potash gave 0.9256 gram potassium acetate $=24 \cdot 16$ per cent. $\mathrm{C}_{2} \mathrm{H}_{4} \mathrm{O}_{2}$.

Calculated for $\mathrm{C}_{14} \mathrm{H}_{27} \mathrm{OC}_{2} \mathrm{H}_{3} \mathrm{O}=23 \cdot 62$ per cent.

To obtain the alcohol, the acetate was warmed with alcoholic potash, and completely saponified. Water was then added, and the alcohol taken up with ether. The ethereal solution after being dried was fractioned. When the ether had passed over, the thermometer rose rapidly, the largest quantity of the alcohol coming over between $278^{\circ}$ and $290^{\circ}$ as a colourless oil. On repeatedly fractioning this, it was obtained nearly pure, and boiling between $280-283^{\circ}$. It gave the following numbers on analysis, which agree satisfactorily with the formula $\mathrm{C}_{14} \mathrm{H}_{28} \mathrm{O}$ :-

I. $0 \cdot 1723$ gram substance gave $0.2021 \mathrm{OH}_{2}$ and $0.4999 \mathrm{CO}_{2}$.

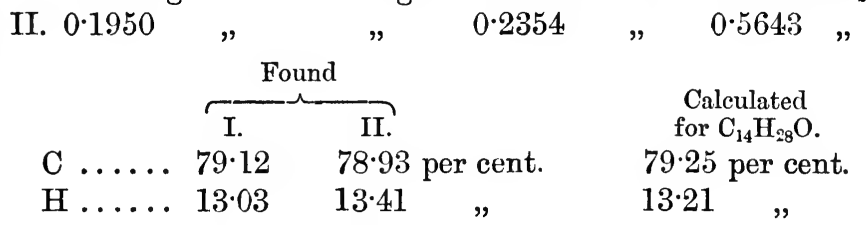

This alcohol is a colourless oil, having but little odour. It does not solidify at a temperature of $-20^{\circ}$, and is not oxidised in contact with the air. It does not combine with acid sodium sulphite, nor reduce ammoniacal silver solution. The specific gravity of this alcohol is-

$$
\begin{aligned}
& \text { at } 15^{\circ}=0.8520, \\
& \text { at } 30=0.8444, \\
& \text { at } 35=0.8418,
\end{aligned}
$$

compared with water at the same temperatures.

Its formation from the aldehyde may be represented thus :-

$$
\mathrm{C}_{13} \mathrm{H}_{25} \mathrm{COH}+\mathrm{H}_{2}=\mathrm{C}_{13} \mathrm{H}_{25} \mathrm{CH}_{2} \mathrm{OH} \text {. }
$$

The oily residue boiling above $300^{\circ}$ left in the retort (produced by the action of nascent hydrogen on $\mathrm{C}_{14} \mathrm{H}_{26} \mathrm{O}$ in ethereal solution) was 
distilled in a vacuum, and then fractioned under a pressure of $300 \mathrm{~mm}$. At first almost all the product came over between $310-350^{\circ}$, but after repeated fractioning the principal part boiled between $330-340^{\circ}$. It gave the following numbers on analysis :-

$0 \cdot 1424$ gram substance gave $0 \cdot 1634 \mathrm{OH}_{2}$ and $0.4370 \mathrm{CO}_{2}$.

\begin{tabular}{|c|c|c|}
\hline & $\begin{array}{l}\text { Found. } \\
83-69 \text { per cent. }\end{array}$ & $\begin{array}{l}\text { Theory for } \mathrm{C}_{28} \mathrm{H}_{50} \mathrm{O} \text {. } \\
83.58 \text { per cent. }\end{array}$ \\
\hline & $12 \cdot 74$ & $12 \cdot 43$ \\
\hline
\end{tabular}

These numbers agree with the formula for the body $\mathrm{C}_{28} \mathrm{H}_{60} \mathrm{O}$, which is no doubt produced from the aldehyde $\mathrm{C}_{14} \mathrm{H}_{25} \mathrm{O}$, by the condensing action of the sodium hydrate formed in the operation. Nascent hydrogen appears therefore to have no action on this body.

\section{Action of Nascent $\mathrm{H} y$ drogen on the Alcohol $\mathrm{C}_{14} \mathrm{H}_{28} \mathrm{O}$.}

In order to see whether it was possible to obtain a saturated alcohol of the formula $\mathrm{C}_{14} \mathrm{H}_{30} \mathrm{O}$ by the continued action of nascent hydrogen on the unsaturated alcohol $\mathrm{C}_{1 \S} \mathrm{H}_{28} \mathrm{O}$, the following experiments were made :-

The alcohol was dissolved in ether, and treated with water and a large excess of sodium (in the same way as in the preparation of the alcohol itself). At the end of seven days the ethereal solution was separated from the water, and treated again with nascent hydrogen, using a 50 per cent. solution of acetic acid instead of ordinary water; the ethereal solution was then separated, well washed, dried, and after the ether had been distilled off, the oil fractioned. At first the greater part came over between $260^{\circ}$ and $290^{\circ}$, but after fractioning two or three times, and last of all collecting between every $5^{\circ}$, the largest fraction obtained boiled between $270-275^{\circ}$.

On analysis this gave the following numbers:-

$0 \cdot 1612$ gram substance gave $0 \cdot 2028 \mathrm{OH}_{2}$ and $0.4669 \mathrm{CO}_{2}$.

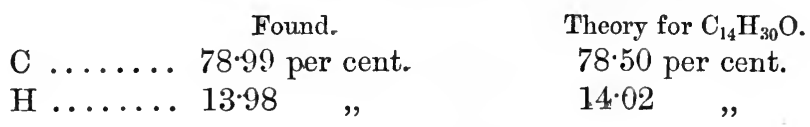

It appears therefore that the saturated alcohol, $\mathrm{C}_{14} \mathrm{H}_{30} \mathrm{O}$, is produced by the action of nascent hydrogen on $\mathrm{C}_{14} \mathrm{H}_{28} \mathrm{O}$, though with difficulty, this unsaturated alcohol, as might be expected, being very slowly acted on by nascent hydrogen. This saturated alcohol obtained was not quite free from the unsaturated one, for on dissolving it in carbon bisulphide, cooling to $-10^{\circ}$, and titrating with bromine, it took 
up a small quantity without evolution of hydrobromic acid. The formation of this alcohol is expressed according to the equation-

$$
\mathrm{C}_{13} \mathrm{H}_{25} \mathrm{CH}_{2} \mathrm{OH}+\mathrm{H}_{2}=\mathrm{C}_{13} \mathrm{H}_{27} \mathrm{CH}_{2} \mathrm{OH} \text {. }
$$

A further description of this alcohol will be found in Part II of this investigation under the heading "Action of Nascent Hydrogen on the Aldehyde $\mathrm{C}_{14} \mathrm{H}_{28} \mathrm{O}$."

In order to obtain an acetate of this alcohol, it was heated in a sealed tube with an excess of acetic anhydride for some time to 180$200^{\circ}$. On fractioning the product, almost all distilled over between $260--290^{\circ}$, and after repeating this operation two or three times, the principal portion obtained boiled at $275-280^{\circ}$. It gave the following results on analysis, agreeing with those required by the formula $\mathrm{C}_{14} \mathrm{H}_{29} \mathrm{O} . \mathrm{C}_{2} \mathrm{H}_{3} \mathrm{O}$ :- -

$0 \cdot 1320$ gram substance gave $0 \cdot 1501$ gram $\mathrm{OH}_{2}$ and $0.3643 \mathrm{CO}_{2}$.

$$
\begin{aligned}
& \text { Calculated } \\
& \text { Found. } \quad \text { for } \mathrm{C}_{14} \mathrm{H}_{29} \mathrm{OC}_{2} \mathrm{H}_{3} \mathrm{O} \text {. } \\
& \text { C . . . . 75.27 per cent. } \quad 75.00 \text { per cent. } \\
& \text { H ..... } 12.63 \text { " } 12.50 \text {, }
\end{aligned}
$$

Oxidation of $\mathrm{C}_{13} \mathrm{H}_{25} \mathrm{COH}$.

In order if possible to obtain some definite clue as to the constitution of this aldehyde, several experiments were made with different oxidising agents.

\section{(1.) Oxidation with Chromic Acid.}

50 grams of $\mathrm{C}_{14} \mathrm{H}_{26} \mathrm{O}$ were dissolved in glacial acetic acid, and treated with twice its weight of chromic acid dissolved in glacial acetic acid. The reaction was very violent, and the oxidising mixture had to be added very slowly, the product being well cooled after each aadition. Every time the solution of chromic acid was run in, the liquid frothed up, carbonic anhydride being given off in large quantities. As soon as all the chromic acid solution had been added, the product was heated on a water-bath for two hours, to ensure the oxidation being as complete as possible. A considerable quantity of water was then added, and the whole shaken up with ether three or four times. It was afterwards found best to distil off the volatile products from the green liquid by means of steam, and then to treat the distillate with ether. The ethereal solution was separated, washed several times with water, in order to remove as much acetic acid as possible, and the acids contained in it were separated from any neutral bodies formed in the reaction, by treatment with a dilute solution of potash. The potash 
solution was then separated from the ethereal, acidulated with hydrochloric acid, and the acids thus liberated taken up with ether. The ethereal solution was well washed, dried, and fractioned. After the ether had distilled off, almost all the remaining products came over between $190-230^{\circ}$, but all attempts to get substances with a constant boiling point were in vain. The whole was therefore made into a potassium compound by dissolring it in a solution of potassium carbonate and evaporating it to dryness. The saline product was then repeatedly extracted with absolute alcohol, to free it from potassium carbonate, and the solution evaporated to dryness. After repeating this purification with alcohol, the salt was dissolved in water, and a silver salt prepared from it by precipitation with nitrate of silver. The salt thus obtained, after being well washed and dried, was analysed, but no good results were obtained, the numbers always coming between those calculated for heptoate and hexoate of silver. Several attempts were made to crystallise the salt from water and alcohol, but without success.

As it appeared possible that both acids might be present, the oxidation was again repeated, with 100 grams aldehyde, as the yield of acid is very small, most of the aldehyde being oxidised to carbonic anhydride. The acids obtained in this operation were then fractioned with great care, and separated principally into two quantities, boiling at $203-207^{\circ}$ and $213-228^{\circ}$. Each of these was separately converted into potassium salts, dried, and purified as before with absolute alcohol, and after repeating the purification, dissolved in water. In order to obtain silcer salts as pure as possible, each product was fractionally precipitated with silver nitrate into five quantities. Silver determinations were then made with the 10 fractions, and these showed without doubt that the acids found consist of a mixture of hexoic and heptoic acids.

The fractions which gave the best silver determinations were completely analysed. Of those prepared from the potassinm salt of the acid (b. p. $203-207^{\circ}$ ), fraction III gave the best results; and from the potassium salt of the acid (b. p. $218-228^{\circ}$ ) fraction II. No. III $\left(203-207^{\circ}\right)$ gave the following results on analysis :-

I. 0.2098 substance gave $0.2463 \mathrm{CO}_{2}, 0.0924 \mathrm{OH}_{2}, 0.1019 \mathrm{Ag}$. II. $0.2016, \quad, 0.2366,0.0900,0.0978$,

\begin{tabular}{|c|c|c|c|c|c|}
\hline \multicolumn{4}{|c|}{ Found. } & \multirow{2}{*}{\multicolumn{2}{|c|}{$\begin{array}{c}\text { Calculated } \\
\text { for } \mathrm{C}_{5} \mathrm{H}_{11} \mathrm{COOAg} .\end{array}$}} \\
\hline & I. & II & & & \\
\hline $\mathrm{C}$ & $32 \cdot 17$ & $32 \cdot 01$ & cent. & $32 \cdot 28$ & $r$ cent. \\
\hline $\mathrm{H}$ & $4 \cdot 89$ & $4 \cdot 96$ & , & $4: 93$ & , \\
\hline$\ldots$ & $48 \cdot 57$ & $48 \cdot 51$ & , & $48 \cdot 43$ & , \\
\hline
\end{tabular}

There can therefore be no doubt it consisted of hexoate of silver. 
No. II $\left(218-228^{\circ}\right)$ fraction gave on analysis the following numbers :-

I. $0 \cdot 2200$ substance gave $0 \cdot 1102 \mathrm{OH}_{2}, 0.2855 \mathrm{CO}_{2}$, and $0.1008 \mathrm{Ag}$.

II. $0.2163 \quad, \quad$, $0.1057, \quad 0.2796 \quad$, 0.0991 ,

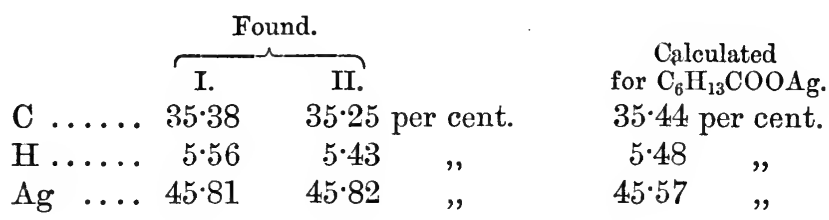

It was therefore without doubt heptoate of silver.

The aldehyde $\mathrm{C}_{13} \mathrm{H}_{25} \mathrm{COH}$ splits up therefore on oxidation into carponic anhydride, and heptoic and hexoic acids, according to the equation-

$$
\mathrm{C}_{14} \mathrm{H}_{26} \mathrm{O}+\mathrm{O}_{5}=\mathrm{C}_{6} \mathrm{H}_{13} \mathrm{COOH}+\mathrm{C}_{5} \mathrm{H}_{11} \mathrm{COOH}+\mathrm{CO}_{2} \text {. }
$$

The ethereal solution of the neutral oils from these oxidation experiments was then repeatedly fractioned, but no constant boiling point could be obtained. The oil began to boil at $250^{\circ}$, but mostly distilled above $360^{\circ}$. I hope at some future time to be able to study this subject in a more exhaustive way.

\section{(2.) Oxidation by the Air.}

As it seemed probable that by gentle oxidation, such as direct absorption of oxygen from the air, the acid of the formula $\mathrm{C}_{14} \mathrm{H}_{26} \mathrm{O}_{2}$ would be formed, identical with that obtained by the action of potash, both on cenanthaldehyde and $\mathrm{C}_{14} \mathrm{H}_{26} \mathrm{O}$, the following experiments were made:-First, a considerable quantity of the aldehyde was exposed to the air in flat dishes for three weeks. In order to hasten the oxidation, the product was gently heated by placing the dishes on a water-bath, and well stirred every day. In a few days it became very thick and slightly yellow, and appeared inclined to become solid on the surface. A second experiment was made by saturating filterpaper with the oil, and then hanging it up in the air for a week to oxidise. The oil was then extracted by digesting with ether. In both cases the oil was afterwards dissolved in ether, and the acids formed separated from the unchanged or neutral oils by shaking with dilute potash-solution. The potash-solutions were treated with ether two or three times, to ensure their freedom from oil, and after being carefully neatralised with sulphuric acid, evaporated to dryness. The whole mass was then extracted several times with absolute alcohol, and again evaporated to dryness. The organic potassium salts were acidulated. 
with dilute hydrochloric acid, and treated with ether, the ethereal solution then separated, well washed, dried, and the ether distilled off.

The quantity of acid left was very small, and smelt strongly of heptoic acid. It was carefully fractioned, when by far the largest, quantity distilled over between $200-230^{\circ}$. There was only a very small quantity of a high-boiling acid left behind, which was most probably the acid $\mathrm{C}_{14} \mathrm{H}_{26} \mathrm{O}_{2}$, but it could not be obtained pure enough for analysis. The fraction $200-230^{\circ}$ was made into the potassium compound in the usual way, and then precipitated with nitrate of silver; the silver salt gave the following numbers on analysis :-

$0 \cdot 2219$ gram substance gave 0.1072 $\mathrm{OH}_{2}, 0.2756 \mathrm{CO}, 0 \cdot 1030 \mathrm{Ag}$.

\begin{tabular}{|c|c|c|}
\hline $\begin{array}{c}\text { Found. } \\
33.87 \text { per cent. }\end{array}$ & Calculated $\left\{\begin{array}{l}\mathrm{C}_{5} \mathrm{H}_{11} \\
\mathrm{COOAg}\end{array}\right.$ & $\left\{\begin{array}{l}\mathrm{C}_{6} \mathrm{H}_{13} \\
\mathrm{COOAg}\end{array}\right.$ \\
\hline $5 \cdot 37$ & 4.93 & $5 \cdot 48$ \\
\hline
\end{tabular}

It was therefore undoubtedly a mixture of hexoate and heptoate or silver. The acid $\mathrm{C}_{14} \mathrm{H}_{26} \mathrm{O}_{2}$ may probably have been first formed, but being very unstable rapidly oxidiscd under the influence of the air and potash into heptoic and hexoic acids. The ethereal solution of the neutral oils, which was separated from the acids by shaking with potash, was examined. The oils it contained began to boil at $250^{\circ}$, but the principal part passed over above $300^{\circ}$. Several of the fractions were analysed, but no definite results were obtained; there appeared, however, to be no unchanged substance left.

\section{(3.) Oxidation with Silver Oxide.}

As it seemed possible that the acid $\mathrm{C}_{14} \mathrm{H}_{26} \mathrm{O}_{2}$ might perhaps be obtained in larger quantities by the oxidation of the aldehyde $\mathrm{C}_{14} \mathrm{H}_{26} \mathrm{O}$ with a gentle oxidising agent, such as silver oxide, several experiments were made in this direction. The following method was tried :-About 20 grams of $\mathrm{C}_{14} \mathrm{H}_{26} \mathrm{O}$ were shaken up with freshly precipitated silver oxide and water in a flask connected with a reversed condenser. The oil quickly adheres to the oxide of silver, and falls to the bottom of the flask, when the greater quantity of the water can be poured off. The mixture was then heated to boiling in a bath of a concentrated solution of carbonate of soda for about 24 hours; at the end of this time all the silver was reduced. Dilute hydrochloric acid was then added, to decompose any silver salts formed, and the whole well shaken up with ether several times, to extract the oil from the precipitated silver. The acid products were separated from the ethereal solution of the oil as before by shaking with dilute potash. The potash-solution was acidulated with dilute hydrochloric acid, treated with ether, and 
the ethereal solution separated, dried, and fractioned. As soon as the ether had distilled off, nearly all the residue boiled between $190-230^{\circ}$. The residue, consisting of high-boiling acid, was again so small that it was found impossible to purify it. The fraction $190-230^{\circ}$ was turned into the potash compounds, and then precipitated with nitrate of silver ; a silver determination of the silver salt thus made gave the following result :-

$0 \cdot 2320$ gram substance gave $0 \cdot 1087 \mathrm{Ag}=46.85$ per cent.

Calculated for $\mathrm{C}_{6} \mathrm{H}_{13} \mathrm{COOAg}=45.57 ; \mathrm{C}_{5} \mathrm{H}_{11} \mathrm{COOAg}=48.43$.

It was therefore as before a mixture of hexoic and heptoic acids. The unoxidised oil gave also the same curious results as those obtained when the other oxidising agents were used.

\section{(4.) Action of Potash on the Aldehyde $\mathrm{C}_{14} \mathrm{H}_{26} \mathrm{O}$.}

As the body $\mathrm{C}_{14} \mathrm{H}_{26} \mathrm{O}$ is an aldehyde, it was thought that it would be interesting to see what the further action of potash on it would be, more especially as it appeared probable that by this means some clue might be obtained to the constitution of the higher condensationproducts of œnanthaldehyde. Alcoholic potash acts very readily on this aldehyde, when it is gently heated with it, the whole becoming black, a considerable quantity of a potash salt being at the same time formed, but the oils in this case are very uninviting, and difficult to purify. If the potassium salts are to be examined, it is best to act on the aldehyde with moderately strong alcoholic potash, and to warm the mixture. In examining the oils, however, the following method was found to give the best results:-200 grams of the aldehyde were dissolved in 500 grams alcohol, and 20 grams of a concentrated solution of potassium hydrate in alcohol added in small quantities every day for about six weeks, the whole being well shaken after each addition. By this means the condensation and other changes take place slowly, and there are also less tarry products formed. The mixture, after standing for another two weeks, was diluted with water, and the oil taken up with ether. The aqueous potash-solution was then acidulated with dilute hydrochloric acid, and the liberated acids which it contained removed with ether. The ethereal solution was dried and fractioned. The greater part distilled over between $210-230^{\circ}$; there was a small residue left. The fraction $210-230^{\circ}$ was made into potash salt, and then into a silver salt. A silver estimation gave the following result :-

0.7520 gram substance gave 0.3433 gram $\mathrm{Ag}=45 \cdot 65$ per cent. Calculated for $\mathrm{C}_{6} \mathrm{H}_{13} \mathrm{COO} \mathrm{Ag}=45.57$ per cent.

It was therefore without doubt heptoate of silver. The residue, 
which boiled above $230^{\circ}$, was transferred to a small retort, and two or three times very carefully fractioned under a pressure of $250 \mathrm{~mm}$. The largest fraction obtained, boiled between $275-280^{\circ}$. The quantity was, however, very small-about 2 grams. An analysis of it gave the following results :-

$0 \cdot 1469$ gram substance gave $0 \cdot 1520$ gram $\mathrm{OH}_{2}$ and $0.3969 \mathrm{CO}_{2}$.

\begin{tabular}{|c|c|c|}
\hline C & $\begin{array}{l}\text { Found. } \\
74 \cdot 24 \text { per cent. }\end{array}$ & $\begin{array}{c}\text { Calculated } \mathrm{C}_{14} \mathrm{H}_{26} \mathrm{O}_{2} . \\
74.33 \text { per cent. }\end{array}$ \\
\hline 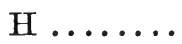 & $11 \cdot 49$ & $11 \cdot 50$ \\
\hline
\end{tabular}

This body has therefore the formula $\mathrm{C}_{14} \mathrm{H}_{26} \mathrm{O}_{2}$, and is without doubt the same acid as that which was obtained by the action of alcoholic potash on œnanthaldehyde. It is a light yellow oil, possessing but little odour, and does not solidify in a freezing mixture. It dissolves without much difficulty in dilute ammonia. On standing over sulphuric acid, the ammonia salt quickly deposits an oil which is probably the amide of the acid. A solution of the ammonia salt was prepared by dissolving the acid in dilute ammonia, nearly neutralising with dilute nitric acid, and then allowing the salt to stand for a short time over sulphuric acid. It was then filtered through a wet filterpaper, and the following salts prepared :-

The silver salt is precipitated on adding silver nitrate as a white flocculent precipitate, which is instantly decomposed on boiling with water.

The barium salt, precipitated by adding barium chloride, is a white amorphous precipitate, insoluble in water, but apparently slightly soluble in alcohol.

The calcium salt resembles in every respect the barium salt.

The copper salt is precipitated on adding sulphate of copper, as a beautiful emerald-green precipitate, which is insoluble in water and becomes black on boiling.

Unfortunately, the amount of acid procured has been so small that it has not been found possible to prepare enough of its salts for analysis. The potassium salt is difficult to prepare, a soap being formed on treating the acid with a solution of potassium carbonate.

The ethereal solution containing the condensed aldehyde and other neutral bodies was next washed, dried over calcium chloride, and fractioned. After the ether had been slowly distilled off, about 3 grams of oil came over between $100^{\circ}$ and $200^{\circ}$; the thermometer then rose rapidly to $250^{\circ}$, between which temperature and $300^{\circ}$ a considerable quantity of product distilled over. By far the largest quantity remained behind in the retort above $300^{\circ}$, and was reserved for distillation in a vacuum. The fraction $100-200^{\circ}$ was fractioned, and 
mostly came over between $170-180^{\circ}$. On analysis it gave the following results :-

$0 \cdot 1145$ gram substance gave $0 \cdot 1394$ gram $\mathrm{OH}_{2}$ and $0.3050 \mathrm{CO}_{2}$.

Found.

C $\ldots \ldots \ldots 72 \cdot 64$ per cent.

H ....... 1:35: ,
Calculated for $\mathrm{C}_{6} \mathrm{H}_{13} \mathrm{CH}_{2} \mathrm{OH}$.

$72 \cdot 41$ per cent.

$13 \cdot 79$,

It was therefore heptyl alcohol. It is formed in very small quantity, only about 1 gram boiling between $170-180^{\circ}$ being obtained.

The fraction $250-300^{\circ}$ was then heated in a sealed tube with excess of acetic anhydride at $180^{\circ}$ for three days, in order to modify any unchanged aldehyde. On fractioning, from 5-10 grams distilled over below $300^{\circ}$. In order to be certain that none of the aldehyde $\mathrm{C}_{14} \mathrm{H}_{26} \mathrm{O}$ was present, this fraction was again heated with acetic anhydride to $200^{\circ}$ for two days, and refractioned. The principal quantity distilled over between $270-295^{\circ}$, and after further fractioning, gave from $2-3$ grams of an oil which boiled between $280-290^{\circ}$. Two analyses of these gave the following results :-

I. 0.1210 gram substance gave $0.1293 \mathrm{OH}_{2}$ and $0.3386 \mathrm{CO}_{2}$. II. $0 \cdot 1114$ $0 \cdot 1164$ 0.3124,

\begin{tabular}{|c|c|c|c|}
\hline \multicolumn{3}{|c|}{ Found. } & \multirow{2}{*}{$\begin{array}{c}\text { Theory } \\
\text { for } \mathrm{C}_{14} \mathrm{H}_{27} \mathrm{OC}_{2} \mathrm{H}_{3} \mathrm{O} \text {. } \\
75.59 \text { per cent. }\end{array}$} \\
\hline & & II. & \\
\hline & $11 \cdot 87$ & $11 \cdot 61$ & $11 \cdot 81$ \\
\hline
\end{tabular}

These numbers agree fairly with the formula $\mathrm{C}_{14} \mathrm{H}_{27} \mathrm{O} . \mathrm{C}_{2} \mathrm{H}_{3} \mathrm{O}$, which is that of the acetate of the alcohol $\mathrm{C}_{14} \mathrm{H}_{28} \mathrm{O}$; but owing to the smallness of the quantity, it was found impossible to get it in a purer condition. In order, if possible, to confirm this result by preparing the alcohol, the alcoholic potash reaction was tried again, 150 grams of the aldehyde $\mathrm{C}_{14} \mathrm{H}_{26} \mathrm{O}$ being used, the mixture being warmed for two days. The oil was then fractioned as before, and the fraction $250-300^{\circ}$ treated with acetic anhydride. The acetate was then saponified with alcoholic potash, and the resulting alcohol repeatedly fractioned. In this way a considerable quantity was obtained boiling between 280$290^{\circ}$, and gave the following result on analysis :-

$0 \cdot 1088$ gram substance gave $0 \cdot 1304$ gram $\mathrm{OH}_{2}$ and $0.3176 \mathrm{CO}_{2}$.

Found.

C ...... 79.61 per cent.

H ....... 13.31
Theory for $\mathrm{C}_{14} \mathrm{H}_{28} \mathrm{O}$.

$79 \cdot 25$ per cent.

$13 \cdot 21$,

It is therefore evident that this alcohol is formed in this reaction, 
although in but small quantities. The residue of the original condensed product which boiled above $300^{\circ}$ was, without doubt, the principal product of the reaction, and as it was very black, it was first distilled under reduced pressure. A considerable quantity came over between $280-360^{\circ}$ under $250 \mathrm{~mm}$. pressure. This was several times fractioned under $350 \mathrm{~mm}$. pressure; the principal quantity obtained boiled between $335-340^{\circ}$. It was a light yellow oil, which distilled without the least decomposition in a vacuum. On analysis it gave the following numbers :-

$0 \cdot 1235$ gram substance gave $0 \cdot 1374 \mathrm{OH}_{2}$ and $0.3770 \mathrm{CO}_{2}$.

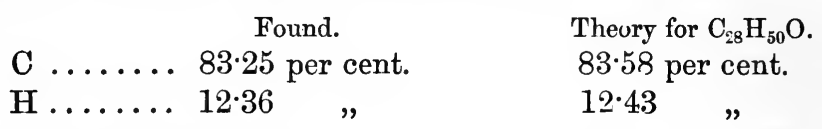

It was therefore the compound $\mathrm{C}_{28} \mathrm{H}_{50} \mathrm{O}$ previously described. The products of the action of alcoholic potash on $\mathrm{C}_{14} \mathrm{H}_{26} \mathrm{O}$ are therefore: $\mathrm{C}_{28} \mathrm{H}_{50} \mathrm{O}, \mathrm{C}_{14} \mathrm{H}_{28} \mathrm{O}, \mathrm{C}_{6} \mathrm{H}_{13} \mathrm{CH}_{2} \mathrm{OH}$, and the acids $\mathrm{C}_{6} \mathrm{H}_{13} \mathrm{CUOH}$ and $\mathrm{C}_{14} \mathrm{H}_{26} \mathrm{O}_{2}$.

The body $\mathrm{C}_{28} \mathrm{H}_{50} \mathrm{O}$ is evidently produced by the condensation of two molecules of the aldehyde $\mathrm{C}_{14} \mathrm{H}_{26} \mathrm{O}$-in exactly the same way as the aldehyde $\mathrm{C}_{14} \mathrm{H}_{26} \mathrm{O}$ itself is formed from œnanthaldehyde-according to the equation-

$$
2 \mathrm{C}_{14} \mathrm{H}_{26} \mathrm{O}=\mathrm{C}_{28} \mathrm{H}_{50} \mathrm{O}+\mathrm{OH}_{2} \text {. }
$$

Heptoic acid and heptyl alcohol are probably formed according to the equation-

$$
\mathrm{C}_{14} \mathrm{H}_{26} \mathrm{O}+\mathrm{KOH}+\mathrm{OH}_{2}=\left\{\begin{array}{l}
\mathrm{C}_{6} \mathrm{H}_{13} \\
\mathrm{COOK}
\end{array}+\left\{\begin{array}{l}
\mathrm{C}_{6} \mathrm{H}_{13} \\
\mathrm{CH}_{2} \mathrm{OH}
\end{array}\right.\right.
$$

The formation of the acid $\mathrm{C}_{14} \mathrm{H}_{26} \mathrm{O}_{2}$ and the alcohol $\mathrm{C}_{14} \mathrm{H}_{29} \mathrm{O}$ may also be represented thus:

$$
2 \mathrm{C}_{14} \mathrm{H}_{26} \mathrm{O}+\mathrm{KOH}=\mathrm{C}_{14} \mathrm{H}_{25} \mathrm{OOK}+\mathrm{C}_{14} \mathrm{H}_{28} \mathrm{O} \text {. }
$$

In these two last equations the potash evidently exerts an oxidising as well as a reducing action, analogous to the action of the same agent on the aromatic aldehydes, as for example in the case of benzaldehyde, which is converted by it into potassic benzoate and benzyl alcohol:-

$$
2 \mathrm{C}_{6} \mathrm{H}_{5} \mathrm{COH}+\mathrm{KOH}=\mathrm{C}_{6} \mathrm{H}_{5} \mathrm{COOK}+\mathrm{C}_{6} \mathrm{H}_{5} \mathrm{CH}_{2} \mathrm{OH} \text {. }
$$

In order to more thoroughly examine these secondary reactions, it would be necessary to work with very large quantities of the aldehyde $\mathrm{C}_{14} \mathrm{H}_{26} \mathrm{O}$.

Theoretical Remarks.

As the body $\mathrm{C}_{14} \mathrm{H}_{26} \mathrm{O}$ on oxidation splits up into heptoic and 
hexoic acids, and is an aldehyde, it can only have the following constitution :-

$$
\begin{aligned}
\mathrm{CH}_{3} \cdot \mathrm{CH}_{2} \cdot \mathrm{CH}_{2} \cdot \mathrm{CH}_{2} \cdot \mathrm{CH}_{2} \cdot \mathrm{CH}_{2} \cdot \mathrm{CH} \\
\mathrm{CH}_{3} \cdot \mathrm{CH}_{2} \cdot \mathrm{CH}_{2} \cdot \mathrm{CH}_{2} \cdot \mathrm{CH}_{2} \cdot \mathrm{C} \cdot \mathrm{COH} .
\end{aligned}
$$

It may be called hexyl-pentyl-acryl aldehyde, and is formed from two molecules of œnanthaldehyde, according to the equation-

$$
\begin{gathered}
\mathrm{CH}_{3} \cdot \mathrm{CH}_{2} \cdot \mathrm{CH}_{2} \cdot \mathrm{CH}_{2} \cdot \mathrm{CH}_{2} \cdot \mathrm{CH}_{2} \cdot \mathrm{CH} \mathrm{O}_{2} \\
\mathrm{CH}_{3} \cdot \mathrm{CH}_{2} \cdot \mathrm{CH}_{2} \cdot \mathrm{CH}_{2} \cdot \mathrm{CH}_{2} \cdot \mathrm{C} \cdot \mathrm{COH} \\
\mathrm{CH}_{3} \cdot \mathrm{CH}_{2} \cdot \mathrm{CH}_{2} \cdot \mathrm{CH}_{2} \cdot \mathrm{CH}_{2} \cdot \mathrm{CH}_{2} \cdot \mathrm{CH} \\
\mathrm{CH}_{3} \cdot \mathrm{CH}_{2} \cdot \mathrm{CH}_{2} \cdot \mathrm{CH}_{2} \cdot \mathrm{CH}_{2} \cdot \mathrm{C} \cdot \mathrm{COH}
\end{gathered}
$$

In order to show it was unsaturated as indicated by the equation, 51.25 grams of the aldehyde $\mathrm{C}_{14} \mathrm{H}_{26} \mathrm{O}$ were dissolved in bisulphide of carbon cooled down to $-10^{\circ}$, and titrated with dry bromine. It took up $39 \cdot 10$ grams bromine (traces of hydrobromic acid only being produced) $=76.32$ per cent. ; calculated quantity for $\mathrm{C}_{14} \mathrm{H}_{26} \mathrm{O}+\mathrm{Br}_{2}$ is $=76.66$ per cent. In this case there is no doubt that a body of the following formula is formed :-

\section{$\mathrm{CH}_{3} \cdot \mathrm{CH}_{2} \cdot \mathrm{CH}_{2} \cdot \mathrm{CH}_{2} \cdot \mathrm{CH}_{2} \cdot \mathrm{CH}_{2} \cdot \mathrm{CHBr}$ $\mathrm{CH}_{3} \cdot \mathrm{CH}_{2} \cdot \mathrm{CH}_{2} \cdot \mathrm{CH}_{2} \cdot \mathrm{CH}_{2} \cdot \mathrm{CBr} \cdot \mathrm{COH}$}

It is, however, impossible to isolate this product; for as soon as the solution in carbon bisulphide attains a temperature of $30^{\circ}$, hydrobromic acid begins to come off, and the whole turns black. The oxidation of the aldehyde $\mathrm{C}_{14} \mathrm{H}_{26} \mathrm{O}$ takes place apparently according to the following equation:-

$$
\begin{gathered}
\mathrm{CH}_{3} \cdot \mathrm{CH}_{2} \cdot \mathrm{CH}_{2} \cdot \mathrm{CH}_{2} \cdot \mathrm{CH}_{2} \cdot \mathrm{CH}_{2} \cdot \mathrm{CH}+\mathrm{O}_{5}= \\
\mathrm{CH}_{3} \cdot \mathrm{CH}_{2} \cdot \mathrm{CH}_{2} \cdot \mathrm{CH}_{2} \cdot \mathrm{CH}_{2} \cdot \mathrm{C} \cdot \mathrm{COH} \\
\mathrm{CH}_{3} \cdot \mathrm{CH}_{2} \cdot \mathrm{CH}_{2} \cdot \mathrm{CH}_{2} \mathrm{CH}_{2} \cdot \mathrm{CH}_{2} \cdot \mathrm{COOH} \\
+\mathrm{CH}_{3} \cdot \mathrm{CH}_{2} \cdot \mathrm{CH}_{2} \cdot \mathrm{CH}_{2} \cdot \mathrm{CH}_{2} \cdot \mathrm{COOH}+\mathrm{CO}_{2} \cdot
\end{gathered}
$$

As the body $\mathrm{C}_{28} \mathrm{H}_{50} \mathrm{O}$ is produced by the action of potash or acetic anhydride on $\mathrm{C}_{14} \mathrm{H}_{26} \mathrm{O}$ with separation of water, it follows that it must be formed from two molecules of $\mathrm{C}_{14} \mathrm{H}_{26} \mathrm{O}$. By melting with potash, it gives only the two acids: heptoic and hexoic acids. The following formula will therefore represent its constitution :- 


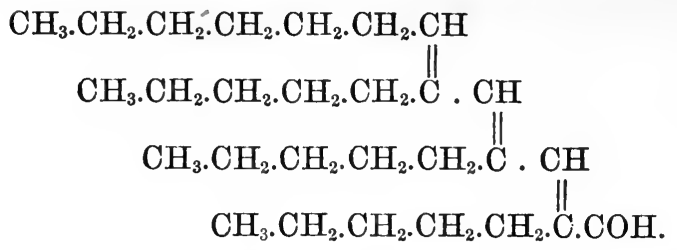

Its formation from two molecules of $\mathrm{C}_{14} \mathrm{H}_{26} \mathrm{O}$ would then take place according to the equation:-

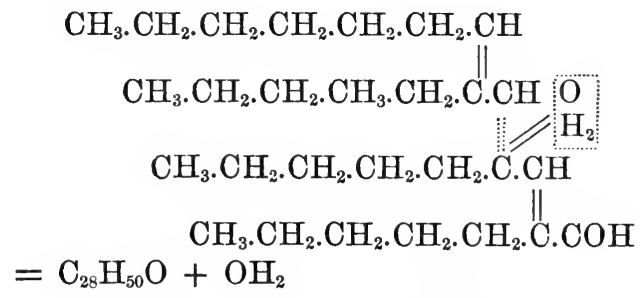

If the constitution given for the aldehyde $\mathrm{C}_{14} \mathrm{H}_{26} \mathrm{O}$ be correct, the unsaturated alcohol $\mathrm{C}_{14} \mathrm{H}_{28} \mathrm{O}$, which is formed from it by the action of nascent hydrogen, may be represented thus-

$$
\begin{aligned}
\mathrm{CH}_{3} \cdot \mathrm{CH}_{2} \cdot \mathrm{CH}_{2} \cdot \mathrm{CH}_{2} \cdot \mathrm{CH}_{2} \cdot \mathrm{CH}_{2} \cdot \mathrm{CH} \\
\mathrm{CH}_{3} \cdot \mathrm{CH}_{2} \cdot \mathrm{CH}_{2} \cdot \mathrm{CH}_{2} \cdot \mathrm{CH}_{2} \cdot \mathrm{C} \cdot \mathrm{CH}_{2} \mathrm{OH},
\end{aligned}
$$

and the saturated alcohol, being formed by the action of nascent hydrogen on the unsaturated alcohol, will therefore have the following constitation :-

$$
\begin{aligned}
& \mathrm{CH}_{3} \cdot \mathrm{CH}_{2} \cdot \mathrm{CH}_{2} \cdot \mathrm{CH}_{2} \cdot \mathrm{CH}_{2} \cdot \mathrm{CH}_{2} \cdot \mathrm{CH}_{2} \\
& \mathrm{CH}_{3} \cdot \mathrm{CH}_{2} \cdot \mathrm{CH}_{2} \cdot \mathrm{CH}_{2} \cdot \mathrm{CH}_{2} \cdot \mathrm{CH} \cdot \mathrm{CH}_{2} \mathrm{OH},
\end{aligned}
$$

and is therefore $\beta$-heptyl-heptyl alcohol, or heptyl-pentyl-ethyl alcohol. And finally the acid $\mathrm{C}_{14} \mathrm{H}_{26} \mathrm{O}_{2}$ will have the constitution

$$
\begin{aligned}
\mathrm{CH}_{3} \cdot \mathrm{CH}_{2} \cdot \mathrm{CH}_{2} \cdot \mathrm{CH}_{2} \cdot \mathrm{CH}_{2} \cdot \mathrm{CH}_{2} \cdot \mathrm{CH} \\
\mathrm{CH}_{3} \cdot \mathrm{CH}_{2} \cdot \mathrm{CH}_{2} \cdot \mathrm{CH}_{2} \cdot \mathrm{CH}_{2} \cdot \mathrm{C} . \mathrm{COOH},
\end{aligned}
$$

and is therefore hexyl-pentyl-acrylic acid. 


\title{
CONDENSATION-PRODUCTS OF CENANTHALDEHYDE
} (Part II).

\author{
By W. H. Perkin, Jun., Ph.D. \\ Action of Nascent Hydrogen on Enanthaldehyde.
}

Is the preparation of heptyl alcohol by the action of nascent hydrogen on œnanthaldehyde, there is always a considerable quantity of a highboiling bye-product formed, which often amounts to 20 per cent. of the œnanthaldehyde used. This remains in the retort, on distilling off the heptyl alcohol, as a thick brown oil, which, however, does not seem to have been investigated. As it was probable that these oils were simply condensation-products, produced by the dehydrating action of the agents used, and possibly acted on by the nascent hydrogen; it was thought that they might yield interesting results upon examination. The inquiry was divided into two parts: (1), action of nascent hydrogen on œnanthaldehyde in acid solution; (2), in ethereal solution.

\section{(1.) Action of Nascent Hydrogen on Enanthaldehyde in Acetic Acid} Solution.

The operations were conducted in the same manner as described in Part $\mathrm{I}$, in the section on the action of nascent hydrogen in acid solution on the aldehyde $\mathrm{C}_{14} \mathrm{H}_{26} \mathrm{O}$. It is practically the same as that employed by C. F. Cross (Jour. Chem. Soc., 32, 123) for the preparation of heptyl alcohol. 200 grams of œnanthaldehyde were used in each operation, and about twice as much sodium amalgam as theoretically required for the formation of heptyl alcohol.

At the end of the reaction water was added, and the oil, after being washed, was boiled with a dilute solution of potash, in order to decompose acetates, which are always formed. It was then washed, dried, and distilled under reduced pressure $(350 \mathrm{~mm}$.), until the temperature reached $300^{\circ}$. The residue was not examined. The distillate was fractioned under ordinary pressure. The principal quantity, consisting of heptyl alcohol and a small quantity of unchanged œnanthaldehyde, came over below $200^{\circ}$; the thermometer then rose rapidly to $240^{\circ}$, between which temperature and $300^{\circ}$ a large amount of product distilled over. This last quantity was repeatedly rectified, when three fractions were obtained, boiling between $270-275^{\circ}, 275-280^{\circ}$, and $280-283^{\circ}$, all of about the same size. The analyses of these gave numbers which always came between those calculated for the alde- 
hyde $\mathrm{C}_{14} \mathrm{H}_{26} \mathrm{O}$, and the alcohol $\mathrm{C}_{14} \mathrm{H}_{28} \mathrm{O}$, so that it appeared very probable that both were present. In order to separate them the fractions between $265-285^{\circ}$ were heated in sealed tubes with excess of acetic anhydride at $180-200^{\circ}$ for four days, and then distilled. After the acetic acid and excess of anhydride had passed over, a considerable quantity of oil was obtained boiling between $270-300^{\circ}$. There was, however, a dark brown residue left, which would seem to show that, as was anticipated, some aldehyde, such as $\mathrm{C}_{14} \mathrm{H}_{26} \mathrm{O}$, was previously present, and had been condensed by the action of the acetic anhydride.

The oil distilling between $270-300^{\circ}$ was then repeatedly fractioned, and at last gave a product which boiled pretty steadily between 280 $285^{\circ}$. It was a colourless, highly refracting oil, possessing an agreeable odour. The analysis gave the following numbers :-

I. $0 \cdot 1860$ gram substance gave $0 \cdot 1992$ gram $\mathrm{OH}_{2}$ and 0.5170 gram $\mathrm{CO}_{2}$.

II. 0.2167 gram substance gave 0.2302 gram $\mathrm{OH}_{2}$ and 0.6030 gram $\mathrm{CO}_{2}$.

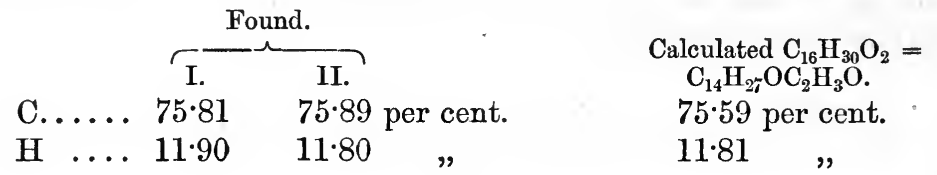

This was therefore the acetate derived from the alcohol $\mathrm{C}_{14} \mathrm{H}_{28} \mathrm{O}$. As this substance, from its composition, should be an unsaturated body, it was dissolved in carbon disulphide, cooled down to $-10^{\circ}$, and titrated with bromine. The colour of the bromine disappeared instantaneously without evolution of hydrobromic acid. A quantitative experiment gave the following numbers:-

$2 \cdot 2355$ grams substance took up, without evolution of hydrobromic acid, 1.3477 gram bromine $=60 \cdot 3$ per cent.

Calculated for $\mathrm{C}_{16} \mathrm{H}_{30} \mathrm{O}_{2}+\mathrm{Br}_{2}=62 \cdot 9$ per cent.

Attempts were made to isolate the bromine addition-product, but the body decomposes on distilling off the carbon disulphide, giving off hydrobromic acid.

This acetate was saponified by boiling with alcoholic potash. On distilling off the alcohol and adding water to the residue, the alcohol $\mathrm{C}_{14} \mathrm{H}_{28} \mathrm{O}$ separates as an oil. This was taken up with ether, and the ethereal solution distilled. After a few fractional distillations the principal quantity came over as a colourless oil boiling at $280-283^{\circ}$. Analysis gave the following result:-

$0 \cdot 1494$ gram substance gave $0 \cdot 1777 \mathrm{OH}_{2}$ and $0.4340 \mathrm{CO}_{2}$. 
Found.

$$
\text { C ..... 79.23 per cent. }
$$$$
\text { H..... 13.22 , }
$$

Calculated $\mathrm{C}_{13} \mathrm{H}_{25} \mathrm{CH}_{2} \mathrm{OH}$. $79 \cdot 25$ per cent. $13 \cdot 21 \quad$,

There can be no donbt that this alcohol and the acetate from which it was separated are the same bodies as those described in Part I, p. 13, as the properties of the two so closely agree. The formation of this alcohol from œnanthaldehyde is probably due to the dehydrating action of the glacial acetic acid and sodium acetate, formed in the reaction, first producing the aldehyde $\mathrm{C}_{14} \mathrm{H}_{26} \mathrm{O}$, a part of which is then acted on by nascent hydrogen, yielding this product. It is practically impossible to separate the aldehyde $\mathrm{C}_{14} \mathrm{H}_{26} \mathrm{O}$ from the alcohol $\mathrm{C}_{14} \mathrm{H}_{28} \mathrm{O}$ by fractional distillation, but the treatment with acetic anhydride forms an easy way of separating them, as shown in Part I.

The products of the action of nascent hydrogen in acid solution, as far as they have been examined, are therefore, heptyl alcohol, probably the aldehyde $\mathrm{C}_{14} \mathrm{H}_{26} \mathrm{O}$, and the alcohol $\mathrm{C}_{14} \mathrm{H}_{28} \mathrm{O}$.

\section{(2.) Action of Nascent Hydrogen on Enanthaldehyde in Ethereal Solution.}

This operation was conducted in the same way as that described in Part I, p. 12, where the treatment of an ethereal solution of the aldehyde, $\mathrm{C}_{11} \mathrm{H}_{26} \mathrm{O}$, is described. 450 grams of œnanthaldehyde dissolved in about $1 \frac{1}{2}$ to 2 litres of ether, and from 200 to 210 grams of sodium, were used in each experiment, which required from four to five days for completion.

The ethereal was separated from the aqueous solution, well washed, first with dilute hydrochloric acid and then with water, and dried over calcium chloride. The examination of the aqueous solution showed that it contained an oily acid boiling between $221-225^{\circ}$; it gave the following numbers on analysis :-

0.2714 gram substance gave 0.2631 gram $\mathrm{OH}_{2}$ and 0.6400 gram $\mathrm{CO}_{2}$.

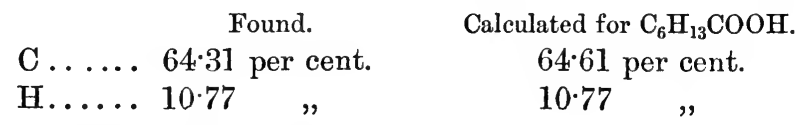

It was therefore heptoic acid.

The ethereal solution, containing the neutral oils, was next examined. After the ether had been distilled off, the oil was fractioned in a stream of carbonic anhydride.

It began to boil at $150^{\circ}$, between which temperature and $200^{\circ}$ a considerable quantity distilled over, consisting of a mixture of heptyl 
alcohol and unchanged œnanthaldehyde. The thermometer then rose rapidly to $250^{\circ}$, a large quantity coming over between $250-300^{\circ}$. The residue was reserved for distillation in vacuo, and is referred to further on. The portion boiling between $250-300^{\circ}$ was several times carefully fractioned in a stream of carbonic anhydride, as the oils oxidise in the air. It was found that when cooled down to $0^{\circ}$ all the fractions from $255-280^{\circ}$ deposited crystals, some becoming almost solid. In order to obtain this solid product in a pure state, the various fractions were cooled down to $-20^{\circ}$ in a mixture of ice and hydrochloric acid, and the oil then filtered off from the crystals as quickly as possible, by means of a vacuum pump. They were then quickly pressed between filter-paper at as low a temperature as possible, and further purified by crystallisation from ether, in which they are very soluble; on allowing the solution to evaporate spontaneously, large transparent crystals, with a beautiful lustre, were deposited. These were filtered off from the ethereal solution, and placed over sulphuric acid in an atmosphere of $\mathrm{CO}_{2}$ for some time. The following analyses were made from several preparations :-

I. 0.1693 gram substance gave $0.1977 \mathrm{OH}_{2}$ and $0.4939 \mathrm{CO}_{2}$.

II. $0.2333 \quad 0.2760 \quad 0.6755$

III. $0.2426 \quad " \quad$ " $\quad " \quad 0.2846 \quad ", 0.7015$,

IV. $0 \cdot 1543 "$

V. $0 \cdot 1565$,

VI. $0 \cdot 1985$

VII. $0 \cdot 1308$

VIII. $0 \cdot 1467$

,

,

,

"

,

,

$\begin{array}{ll}" & 0 \cdot 2846 \\ , & 0 \cdot 1818 \\ , & 0 \cdot 1872 \\ , & 0.2355 \\ , & 0 \cdot 1561 \\ , & 0 \cdot 1770\end{array}$

0.7015,

0.4474 ,

$0 \cdot 4543$

0.5741

,

,

,

, 01770

Found.

\begin{tabular}{|c|c|c|c|c|c|c|}
\hline & I. & II. & III. & IV. & V. & VI. \\
\hline 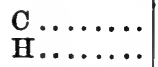 & $\begin{array}{l}79 \cdot 56 \\
12 \cdot 97\end{array}$ & $\begin{array}{l}78 \cdot 96 \\
13 \cdot 14\end{array}$ & $\begin{array}{l}78 \cdot 86 \\
13 \cdot 03\end{array}$ & $\begin{array}{l}79 \cdot 07 \\
13 \cdot 09\end{array}$ & $\begin{array}{l}79 \cdot 17 \\
13 \cdot 29\end{array}$ & $\begin{array}{l}78 \cdot 88 \\
13 \cdot 18\end{array}$ \\
\hline
\end{tabular}

Found.

\begin{tabular}{|c|c|c|c|c|}
\hline & VII. & VIII. & Mean. & Theory $\mathrm{C}_{14} \mathrm{H}_{28} \mathrm{O}$. \\
\hline $\begin{array}{l}\mathbf{c} \ldots \ldots \ldots \\
\mathbf{H} \ldots \ldots \ldots\end{array}$ & $\begin{array}{l}79 \cdot 13 \\
13 \cdot 26\end{array}$ & $\begin{array}{l}79 \cdot 01 \\
13 \cdot 40\end{array}$ & $\begin{array}{l}79 \cdot 09 \text { per cent. } \\
13 \cdot 19 \text { " }\end{array}$ & $\begin{array}{l}79 \cdot 25 \text { per cent. } \\
13 \cdot 21 \text { " }\end{array}$ \\
\hline
\end{tabular}


This substance therefore has the formula $\mathrm{C}_{14} \mathrm{H}_{28} \mathrm{O}$. It is a beautiful body, crystallising in plates which melt at $29.5^{\circ}$. It boils without the least decomposition at $266-268^{\circ}$. It is easily soluble in carbon disulphide, ether, alcohol, petroleum ether, and chloroform. The solution in carbon disulphide, if cooled down to $-10^{\circ}$, does not take up bromine, it therefore appears to be a saturated body. It combines very slowly with hydrogen sodium sulphite, so that, after 'standing for six months with a concentrated solution of the latter body, it had not quite ceased depositing crystals. The amount of the compound obtained was unfortunately too small for analysis. This body also reduces an ammoniacal silver solution, and oxidises, when left in contact with the air, but only very slowly when in the solid state. From these properties it appears to be an aldehyde. Heated with acetic anhydride for four days in a sealed tube at $180^{\circ}$, it did not form an acetate. Acetic anhydride appears to act on it very slowly, as on fractioning the product there was only a very small residue left in the retort above $280^{\circ}$. The specific gravity of this aldehyde in the fused state is-

$$
\begin{aligned}
& \text { at } 30^{\circ}=0.8274 \\
& \text { at } 35=0.8258
\end{aligned}
$$

compared with water at the same temperatures. Its formation from cnanthaldehyde may be expressed by the following equation :-

$$
2 \mathrm{C}_{7} \mathrm{H}_{14} \mathrm{O}+\mathrm{H}_{2}=\mathrm{C}_{14} \mathrm{H}_{28} \mathrm{O}+\mathrm{OH}_{2} \text {. }
$$

The residue left in the retort above $310^{\circ}$, after distilling off the aldehyde just described, was very thick and black, and was therefore first distilled in vacuo, and then fractioned under a pressure of $300 \mathrm{~mm}$. By the first distillation a considerable quantity of an almost colourless oil distilled over between $310-350^{\circ}$. On repeatedly fractioning under the same pressure, two principal products were obtained, boiling between $315-320^{\circ}$ and $320-325^{\circ}$, of which the first was the larger. Analyses of these gave the following results :-

I. $\left(320-325^{\circ}\right) \quad 0.2466$ gram substance gave $0.2882 \mathrm{OH}_{2}$ and $0.7347 \mathrm{CO}_{2}$.

II. $\left(315--320^{\circ}\right) \quad 0 \cdot 1782$ gram substance gave $0.2084 \quad \mathrm{OH}_{2}$ and $0.5332 \mathrm{CO}_{2}$.

III. $\left(315-320^{\circ}\right) \quad 0.1480$ gram substance gave $0.1734 \mathrm{OH}_{2}$ and $0.4432 \mathrm{CO}_{2}$.

IV. $\left(315-320^{\circ}\right) \quad 0.2159$ gram substance gave $0.2540 \quad \mathrm{CO}_{2}$ and $0.6466 \mathrm{CO}_{2}$. 
Found.

\begin{tabular}{|c|c|c|c|c|c|}
\hline & I. & II. & III. & IV. & Theory $\mathrm{C}_{21} \mathrm{H}_{40} \mathrm{O}$. \\
\hline & $81 \cdot 26$ & $81 \cdot 60$ & $81 \cdot 67$ & $81 \cdot 66$ per cent. & 81.82 per cent. \\
\hline $\mathrm{A}$. & $12 \cdot 99$ & $12 \cdot 99$ & $13 \cdot 02$ & $13 \cdot 07$ & $12 \cdot 99$ \\
\hline
\end{tabular}

This body has therefore the formula $\mathrm{C}_{21} \mathrm{H}_{40} \mathrm{O}$. It is a slightly yellow, very thick oil, which does not solidify at $-10^{\circ}$. It reduces ammoniacal silver solution, but does not appear to combine with hydrogen sodium sulphite. Heated with potash it becomes thick and black, a small quantity of a potash salt being formed. Boiling with dilute sulphuric acid also decomposes it. In order to see if it were unsaturated, it was dissolved in carbon disulphide, cooled down to -10 , and titrated with bromine. The colour of the bromine disappeared instantly. A quantitative experiment gave the following result :-

20.65 grams dissolved in carbon disulphide, took up 10.8 grams bromine without the evolution of hydrobromic acid $=52 \cdot 3$ per cent.

Calculated for $\mathrm{C}_{21} \mathrm{H}_{40} \mathrm{O}+\mathrm{Br}_{2}=51 \cdot 9$ per cent.

It appears therefore to be an unsaturated body. Its formation from cnanthaldehyde may be represented by the following equation :-

$$
3 \mathrm{C}_{7} \mathrm{H}_{14} \mathrm{O}+\mathrm{H}_{2}=\mathrm{C}_{21} \mathrm{H}_{40} \mathrm{O}+2 \mathrm{OH}_{2} \text {. }
$$

Its specific gravity was found to be:-

$$
\begin{aligned}
& \text { at } 15^{\circ}=0.8744 \\
& \text { at } 30=0.8665 \\
& \text { at } 35=0.8637
\end{aligned}
$$

compared with water at the same temperatures.

The yield of this body is from 5 to 10 per cent. of the cenanthaldehyde used.

The residne in the retort, boiling above $350^{\circ}$ in vacuo, became solid on cooling. On extracting with ether a white solid body remained behind, which dissolved on boiling with water. On cooling, needles of calcium heptoate crystallised out. A calcium estimation gave $13.44 \mathrm{Ca}$. Calculated for $\left(\mathrm{C}_{6} \mathrm{H}_{13} \mathrm{COO}\right)_{2} \mathrm{Ca}=13.43$ per cent. Ca. The presence of this salt is probably due to the fact that the crude oil was in the first place dried over calcium chloride, which is a little soluble in the oil, and that, at the high temperature employed in the following distillations, it was converted into calcic heptoate. The products of the action of nascent hydrogen in alkaline solution on œnanthaldehyde, as far as they have been examined, are therefore:-

Heptyl alcohol: heptoic acid: the solid aldehyde $\mathrm{C}_{14} \mathrm{H}_{28} \mathrm{O}$ : and the body $\mathrm{C}_{21} \mathrm{H}_{40} \mathrm{O}$. 


\section{Oxidation of the Aldehyde $\mathrm{C}_{14} \mathrm{H}_{28} \mathrm{O}$.}

In order to obtain some idea as to the constitution of this aldehyde, $\mathrm{C}_{14} \mathrm{H}_{28} \mathrm{O}$, and of the nature of the groups contained in it, several oxidation experiments were made, much in the same way as in the case of the aldehyde $\mathrm{C}_{14} \mathrm{H}_{26} \mathrm{O}$, described in Part $\mathrm{I}$. The aldehyde $\mathrm{C}_{14} \mathrm{H}_{28} \mathrm{O}$ was dissolved in glacial acetic acid, and one and a-half times its weight of chromic acid dissolved in glacial acetic acid slowly added, the product being well cooled after each addition. The action is very energetic, large quantities of carbonic anhydride being given off. As soon as all the chromic acid had been added, the product was heated for two hours on a water-bath to complete the oxidation. The prodnct was treated as in the analogons experiments in Part I. The acids obtained came over between $190^{\circ}$ and $230^{\circ}$, and the liquid thus obtained was separated by further distillation into two fractions boiling between $195-210^{\circ}$ and $210-230^{\circ}$. Each of these was converted into a potassium compound and then fractionally precipitated with nitrate of silver into five fractions. Silver estimations were made in each of these precipitates.

The results showed clearly that a mixture of hexoate and heptoate of silver was present. The silver salts which gave the best results were fully analysed, and gave the following numbers. Of that from the potassium salt of the acid $\left(195-210^{\circ}\right)$, fractions IV and $V$ were analysed, with the following results :-

I. 0.2202 gram substance gave $0.1000 \quad \mathrm{OH}_{2}, 0.2649 \mathrm{CO}_{2}$, and $0.1052 \mathrm{Ag}$.

II. 0.2479 gram substance gave $0.1121 \mathrm{OH}_{2}, 0.2939 \mathrm{CO}_{2}$, and $0 \cdot 1202 \mathrm{Ag}$.

III. $0 \cdot 2267$ gram substance gave $0.1040 \quad \mathrm{OH}_{2}, 0.2742 \quad \mathrm{CO}_{2}$, and $0 \cdot 1083 \mathrm{Ag}$.

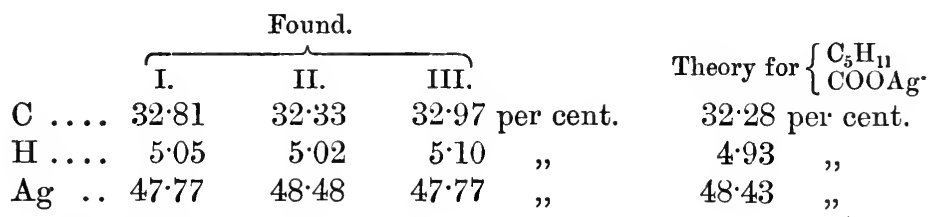

It was, therefore, without doubt, hexoate of silver.

Of that from the potassium salt of the acid $218-230^{\circ}$ fractions II and III were analysed with the following results :-

I. 0.2435 gram substance gave $0.1215 \quad \mathrm{OH}_{2}, 0.317 .4 \mathrm{CO}_{2}$, and $0 \cdot 1112 \mathrm{Ag}$. 


\section{0.1533 gram substance gave $0.0759 \mathrm{OH}_{2}, 0.1975 \mathrm{CO}_{2}$, and $0.0702 \mathrm{Ag}$. \\ III. 0.2368 gram substance gave $0.1145 \mathrm{OH}_{2}, 0.3039 \mathrm{CO}_{\varepsilon}$, and $0 \cdot 1084 \mathrm{Ag}$.}

Found.

\begin{tabular}{|c|c|c|c|c|}
\hline & I. & II. & III. & Theory for $\left\{\begin{array}{l}\mathrm{C}_{6} \mathrm{H}_{13} \\
\text { COOAg}\end{array}\right.$ \\
\hline $\mathrm{C}$ & $35 \cdot 55$ & $35 \cdot 13$ & 35.00 per cent. & 35.44 per cent. \\
\hline $\mathrm{H}$ & $5 \cdot 56$ & $5-50$ & $5 \cdot 37$ & $5 \cdot 48$ \\
\hline $\mathrm{Ag}$ & $4.5 \cdot 66$ & $45 \cdot 79$ & $45 \cdot 77$ & $45 \cdot 57$ \\
\hline
\end{tabular}

It was, therefore, heptoate of silver. The products of the oxidation of the aldehyde $\mathrm{C}_{14} \mathrm{H}_{28} \mathrm{O}$, by means of chromic acid and acetic acid, are therefore carbonic anhydride, and heptoic and hexoic acids, according to the equation-

$$
\mathrm{C}_{14} \mathrm{H}_{28} \mathrm{O}+3 \mathrm{O}_{2}=\left\{\begin{array}{l}
\mathrm{C}_{5} \mathrm{H}_{11} \\
\mathrm{COOH}
\end{array}+\left\{\begin{array}{l}
\mathrm{C}_{6} \mathrm{H}_{13} \\
\mathrm{COOH}
\end{array}+\mathrm{CO}_{2}+\mathrm{OH}_{2} .\right.\right.
$$

Oxidation with silver oxide was next tried.

This experiment was made in the same way as with the aldehyde $\mathrm{C}_{14} \mathrm{H}_{25} \mathrm{O}$, Part I. The acids obtained were carefully fractioned. By far the largest fraction obtained boiled between $195-230^{\circ}$; there was, however, a small quantity of a dark-brown residue left, which was reserved for distillation in vacuo. The distillate $195-230^{\circ}$ was converted into a potassium and then into a barium compound. This dried at $120-130^{\circ}$ gave on analysis the following results :-

I. 0.1666 gram substance gave $0 \cdot 1013 \mathrm{BaSO}_{4}=35 \cdot 79$ per cent. Ba. II. $0 \cdot 1290$ $0.0785, \quad=35 \cdot 78$, Calculated for heptoate of barium ....... 34.70 per cent. " " hexoate of barium ........ 37.33 ",

This barium compound was therefore a mixture of barium hexoate and heptoate, the two acids being formed as in the case of the oxidation by means of chromic acid.

The dark-brown residue remaining in the retort above $230^{\circ}$ was first distilled under a pressure of $300^{\circ}$, when most of it came over at about $230^{\circ}$. This was then carefully fractioned at the ordinary pressure. After a small quantity of heptoic acid had passed over, the thermometer rose rapidly to $300^{\circ}$, almost all the product (about $1 \frac{1}{2}$ grams) distilling between $300-310^{\circ}$. On analysis it gave the following numbers :-

I. 0.0967 gram substance gave $0 \cdot 1029 \mathrm{OH}_{2}$ and $0.2623 \mathrm{CO}_{2}$.
II. $0 \cdot 1341$
"
$0 \cdot 1428$
$0 \cdot 3620$, 


\begin{tabular}{|c|c|c|c|}
\hline \multicolumn{3}{|c|}{ Found. } & \multirow[b]{2}{*}{$\begin{array}{c}\text { Theory } \\
\text { for } \mathrm{C}_{14} \mathrm{H}_{28} \mathrm{O}_{2} \text {. }\end{array}$} \\
\hline & I. & $\mathrm{II}$ & \\
\hline$C \ldots \ldots$ & $73 \cdot 97$ & 73.59 per cent. & 73.60 per cent. \\
\hline$H \ldots \ldots$ & $11 \cdot 82$ & $11 \cdot 83$ & $12 \cdot 28$ \\
\hline
\end{tabular}

This acid, therefore, has the formula $\mathrm{C}_{14} \mathrm{H}_{28} \mathrm{O}_{2}$. It is an almost colourless oil, which distils at the ordinary pressure, apparently without the least decomposition. The yield from the aldehyde $\mathrm{C}_{14} \mathrm{H}_{28} \mathrm{O}$ is very small, so that it was found impossible to prepare salts pure enough for analysis.

This acid dissolves in ammonia, but the ammonium salt, on standing, quickly deposits an oil which may possibly be the amide of the acid.

The potash salt is a soap, and difficult to obtain pure. It is formed by shaking the acid with very dilute potash; on evaporating, however, it appears to decompose. The formation of this acid from the aldehyde may be expressed thus:-

$$
\mathrm{C}_{14} \mathrm{H}_{28} \mathrm{O}+\mathrm{O}=\mathrm{C}_{14} \mathrm{H}_{28} \mathrm{O}_{2} \text {. }
$$

By cooling to $-10^{\circ}$ it becomes very thick, but was not obtained in a sclid condition.

As it seemed possible that the acid just described might be obtained in larger quantity from the aldehyde by employing an alkaline solution of potassium permanganate, the following experiment was made. About 20 grams of $\mathrm{C}_{14} \mathrm{H}_{28} \mathrm{O}$ were mixed with rather more than the calculated quantity of permanganate dissolved in dilute soda, and from time to time the mixture was well shaken up. The oxidation took place rapidly at first, but afterwards proceeded very slowly. At the end of a week alcohol was added, and the soda solution separated from the precipitated hydrate of manganese by filtering through glass wool. The acids which had been formed were then liberated with hydrochloric acid, taken up with ether, and fractioned. However, almost all the acids came over between $200-230^{\circ}$, and only a trace of an acid with a high boiling point was present; this, however, appeared to boil at the same temperature as that obtained by the oxidation with silver oxide. The fraction boiling between $200-230^{\circ}$ was converted into potash salts, purified as usual with absohute aleohol, and the silver salts precipitated with nitrate of silver. Analysis gave the following result:-

0.3208 gram substance gave $0.1475 \mathrm{OH}_{2}, 0.3896 \mathrm{CO}_{2}$, and $0.1567 \mathrm{Ag}$.

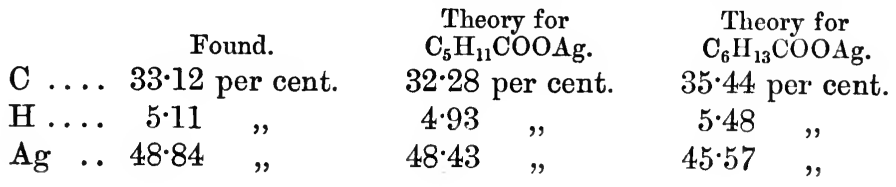


It was therefore a mixture of hexoate and heptoate of silver. The products of the oxidation of $\mathrm{C}_{14} \mathrm{H}_{28} \mathrm{O}$ are therefore carbonic anhydride, heptoic and hexoic acids, and $\mathrm{C}_{13} \mathrm{H}_{27} \mathrm{COOH}$.

Several attempts were made to purify the neutral oils which were obtained in these experiments by fractional distillation, but although several anaylses were made of different fractions, no good results could be obtained. They begin to boil at $250^{\circ}$, but by far the greater part boil above $360^{\circ}$.

\section{Action of Nascent Hydrogen on the Aldehyde $\mathrm{C}_{14} \mathrm{H}_{28} \mathrm{O}$.}

The crystalline body, $\mathrm{C}_{14} \mathrm{H}_{28} \mathrm{O}$, being an aldehyde, it was thought interesting to try the action of nascent hydrogen on it, in order, if possible, to get the corresponding alcohol $\mathrm{C}_{14} \mathrm{H}_{30} \mathrm{O}$. This would have the same formula as that previously obtained by the action of nascent hydrogen on the alcohol $\mathrm{C}_{14} \mathrm{H}_{28} \mathrm{O}$ (Part I, p. 14). The experiment was made in the following way: The aldehyde was first dissolved in glacial acetic acid, and zinc turnings added, the whole being warmed on a water-bath. It was found advantageous to cover the surface of the zinc with copper, by dipping it in a solution of sulphate of copper, the action being thus very much accelerated. From time to time small quantities of water were added; the mixture was heated at last nearly to boiling. Water was then added in excess, and after nearly neutralising the acetic acid with potash, the oil was separated and well washed. On distilling, there still appeared to be a considerable quantity of unchanged aldehyde present, the product was, therefore, again treated with nascent hydrogen. This time, however, the oil was dissolved in ether and treated with water and sodium in exactly the same way as was employed in the reduction of conanthaldehyde, as it was found to give better results than zinc and acetic acid. After a considerable excess of sodium had been added, the oil was extracted from the aqueous solution with ether, then dried and fractioned. After the ether had been distilled off, the principal quantity of the product came over between $260-290^{\circ}$, only a small residue remaining behind in the retort.

This distillate was then several times carefully fractioned, the largest part obtained boiling between $270-275^{\circ}$.

On analysis it gave the following numbers :-

$0 \cdot 1500$ gram substance gave $0 \cdot 1881 \mathrm{OH}_{2}$ and $0 \cdot 4304 \mathrm{CO}_{2}$.

Found.

C ...... 78.25 per cent.

$\mathrm{H} \ldots \ldots . .13 .93$,
Theory for $\mathrm{C}_{14} \mathrm{H}_{30} \mathrm{O}$. 78.50 per cent. 14.02 "

It therefore has the formula $\mathrm{C}_{14} \mathrm{H}_{30} \mathrm{O}$. 
It is a colourless oil, having but little odour. Cooled down to $-10^{\circ}$ it solidifies to a waxy mass, which melts again at the ordinary temperature. It does not oxidise in contact with the air. When dissolved in carbon disulphide, and cooled down to $-10^{\circ}$, it does not take up bromine. It is soluble in most of the ordinary solvents. Its specific gravity is .

$$
\begin{aligned}
& \text { at } 15^{\circ}=0.8368 \\
& \text { at } 30=0.8301 \\
& \text { at } 35=0.8279
\end{aligned}
$$

compared with water at the same temperatures. It is, without doubt, the same alcohol as that previously obtained by the action of nascent hydrogen on the unsaturated alcohol $\mathrm{C}_{14} \mathrm{H}_{28} \mathrm{O}$, as it has the same boiling point. To further establish this, however, the acetate was prepared by heating it in a sealed tube with excess of acetic anhydride at $180^{\circ}$ for two days. On the first distillation almost all the oily product distilled over between $270-285^{\circ}$, very little residue being left in the retort.

On repeating the fractioning, the principal portion came over

\begin{tabular}{|c|c|c|}
\hline & $\begin{array}{l}\text { Found. } \\
75 \cdot 14 \text { per cent. }\end{array}$ & $\begin{array}{c}\text { Theory for } \mathrm{C}_{14} \mathrm{H}_{29} \mathrm{OC}_{2} \mathrm{H}_{3} \mathrm{O} \text {. } \\
75.00 \text { per cent. }\end{array}$ \\
\hline 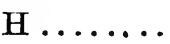 & $12 \cdot 49$ & $12 \cdot 50$ \\
\hline
\end{tabular}
between $275-280^{\circ}$. On analysis it gave the following numbers :-

$0 \cdot 1165$ gram substance gave $0.1310 \mathrm{OH}_{2}$ and $0.3210 \mathrm{CO}_{2}$.

The numbers agree therefore with those required for the formula $\mathrm{C}_{14} \mathrm{H}_{29} \mathrm{O} . \mathrm{C}_{2} \mathrm{H}_{3} \mathrm{O}$.

In order to prove that this body is an acetate, a determination of the acetic acid, formed by its saponification, was made, which gave the following numbers:-

3.5293 grams substance gave 1.3065 grams potassium acetate $=$ $22 \cdot 67$ per cent. $\mathrm{C}_{2} \mathrm{H}_{4} \mathrm{O}_{2}$. The formula $\mathrm{C}_{14} \mathrm{H}_{29} \mathrm{O} . \mathrm{C}_{2} \mathrm{H}_{3} \mathrm{O}$ requires 23.43 per cent.

This acetate is a beautiful colourless oil, possessing an aromatic odour. It boils at the same temperature as that described in Part I $\left(275-280^{\circ}\right)$. It refracts light very strongly. Cooled to $-10^{\circ}$ it does not solidify. When warmed with alcoholic potash it is very easily decomposed into potassic acetate and the alcohol $\mathrm{C}_{14} \mathrm{H}_{30} \mathrm{O}$. Its specific gravity is

$$
\begin{aligned}
& \text { at } 15^{\circ}=0.8559 \\
& \text { at } 30=0.8476 \\
& \text { at } 35=0.8448
\end{aligned}
$$

compared with water at the same temperatures. 
It is therefore seen that both the aldehyde $\mathrm{C}_{13} \mathrm{H}_{27} \mathrm{COH}$, and the alcohol $\mathrm{C}_{14} \mathrm{H}_{27} \mathrm{OH}$, when treated with nascent hydrogen, yield the same body, viz., the alcohol $\mathrm{C}_{14} \mathrm{H}_{29} \mathrm{OH}$.

\section{Theoretical Remarks.}

As the aldehyde $\mathrm{C}_{14} \mathrm{H}_{28} \mathrm{O}$ splits up on oxidation into heptoic, hexoic, and carbonic acids, according to the equation-

$$
\mathrm{C}_{14} \mathrm{H}_{28} \mathrm{O}+3 \mathrm{O}_{2}=\left\{\begin{array}{l}
\mathrm{C}_{5} \mathrm{H}_{11} \\
\mathrm{COOH}
\end{array}+\left\{\begin{array}{l}
\mathrm{C}_{6} \mathrm{H}_{13} \\
\mathrm{COOH}
\end{array}+\mathrm{CO}_{2}+\mathrm{OH}_{2},\right.\right.
$$

it must have the following constitution :--

$$
\begin{array}{r}
\mathrm{CH}_{3} \cdot \mathrm{CH}_{2} \cdot \mathrm{CH}_{2} \cdot \mathrm{CH}_{2} \cdot \mathrm{CH}_{2} \cdot \mathrm{CH}_{2} \cdot \mathrm{CH}_{2} \\
\mathrm{CH}_{3} \cdot \mathrm{CH}_{2} \cdot \mathrm{CH}_{2} \cdot \mathrm{CH}_{2} \cdot \mathrm{CH}_{2} \cdot \mathrm{CH} \cdot \mathrm{COH} .
\end{array}
$$

This shows that it is a saturated body. It is isomeric with the alcohol $\mathrm{C}_{14} \mathrm{H}_{28} \mathrm{O}$ described in Part $\mathrm{I}$, obtained by the action of nascent hydrogen on the aldehyde $\mathrm{C}_{14} \mathrm{H}_{26} \mathrm{O}$.

This aldehyde $\mathrm{C}_{14} \mathrm{H}_{28} \mathrm{O}$ contains the group $\left.\right|_{-\mathrm{CH} . \mathrm{COH}} ^{-\mathrm{CH}_{2}}$, whereas the alcohol contains the group $\|_{-\mathrm{C} . \mathrm{CH}_{2} \mathrm{OH}}^{-\mathrm{CH}}$

The production of this aldehyde is somewhat difficult to understand, as it is not formed by the action of nascent hydrogen on the aldehyde $\mathrm{C}_{14} \mathrm{H}_{26} \mathrm{O}$. It would seem to be probably formed by the action of the nascent hydrogen at the moment of the condensation of the œnanthaldehyde. A further attempt to obtain it from the aldehyde $\mathrm{C}_{14} \mathrm{H}_{26} \mathrm{O}$ was made by dissolving the latter in ether, cooling in a freezing mixture, and then adding bromine. As soon as this was no longer decolorised, water was added and then sodium, till a small quantity of the ethereal solution taken out was found no longer to contain bromine. The ether was then distilled off, and the residual oil fractioned.

A considerable quantity came over between $270-280^{\circ}$, which on combustion gave numbers agreeing fairly well with the formula $\mathrm{C}_{14} \mathrm{H}_{30} \mathrm{O}$; it was therefore apparently the same alcohol as that obtained by the action of nascent hydrogen on the aldehyde and alcohol $\mathrm{C}_{14} \mathrm{H}_{28} \mathrm{O}$. On heating with acetic anhydride in a sealed tube, it also gave an acetate. It is quite possible that some of the aldehyde $\mathrm{C}_{14} \mathrm{H}_{28} \mathrm{O}$ was formed in this experiment, but could not be separated from the alcohol $\mathrm{C}_{14} \mathrm{H}_{30} \mathrm{O}$. The aldehyde $\mathrm{C}_{14} \mathrm{H}_{28} \mathrm{O}$ is isomeric with myristic aldehyde, which F. Krafft (Ber., 13, 1415) obtained by the dry distil- 
lation of a mixture of barium myristate, barium formate, and lime. It melts at $52.5^{\circ}$, or $23^{\circ}$ higher than my product.

The constitution of the acid $\mathrm{C}_{14} \mathrm{H}_{28} \mathrm{O}_{2}$ obtained by the oxidation of the aldehyde $\mathrm{C}_{14} \mathrm{H}_{28} \mathrm{O}$, is represented thus :-

$$
\begin{aligned}
& \mathrm{CH}_{3} \cdot \mathrm{CH}_{2} \cdot \mathrm{CH}_{2} \cdot \mathrm{CH}_{2} \cdot \mathrm{CH}_{2} \cdot \mathrm{CH}_{2} \cdot \mathrm{CH}_{2} \\
& \mathrm{CH}_{3} \cdot \mathrm{CH}_{2} \cdot \mathrm{CH}_{2} \cdot \mathrm{CH}_{2} \cdot \mathrm{CH}_{2} \cdot \dot{\mathrm{C} H} . \mathrm{COOH} \text {. }
\end{aligned}
$$

It is therefore heptylpentylacetic acid. It also appears to be formed by the action of alcoholic potash on the aldehyde $\mathrm{C}_{14} \mathrm{H}_{28} \mathrm{O}$ in the same way as the corresponding acrylic acid $\mathrm{C}_{14} \mathrm{H}_{26} \mathrm{O}_{2}$ is obtained from the aldehyde $\mathrm{C}_{14} \mathrm{H}_{26} \mathrm{O}$. In the oxidation of the aldehyde $\mathrm{C}_{14} \mathrm{H}_{28} \mathrm{O}$, it is probable that this acid is first formed, and then on account of its easy oxidisability is quickly converted into hexoic and heptoic acids. This acid is isomeric with myristic acid, which Krafft (Ber., 12, 1669) obtained by saponifying nutmeg oil. He describes it as a solid body melting at $53.8^{\circ}$, but gives no particulars as to its constitution.

The body having the formula $\mathrm{C}_{21} \mathrm{H}_{40} \mathrm{O}$, which is formed by the union of 3 mols. of cenanthaldehyde, according to the following equation:-

$$
3 \mathrm{C}_{7} \mathrm{H}_{14} \mathrm{O}+\mathrm{H}_{2}=\mathrm{C}_{21} \mathrm{H}_{40} \mathrm{O}+2 \mathrm{H}_{2} \mathrm{O} \text {, }
$$

has probably the following constitution :-

$$
\begin{aligned}
& \mathrm{CH}_{3} \cdot \mathrm{CH}_{2} \cdot \mathrm{CH}_{2} \cdot \mathrm{CH}_{2} \cdot \mathrm{CH}_{2} \cdot \mathrm{CH}_{2} \cdot \mathrm{CH} \\
& \mathrm{CH}_{3} \cdot \mathrm{CH}_{2} \cdot \mathrm{CH}_{2} \cdot \mathrm{CH}_{2} \cdot \mathrm{CH}_{2} \cdot \mathrm{C} \cdot \mathrm{CH}_{2} \\
& \mathrm{CH}_{3} \cdot \mathrm{CH}_{2} \cdot \mathrm{CH}_{2} \cdot \mathrm{CH}_{2} \cdot \mathrm{CH}_{2} \cdot \mathrm{CH} \cdot \mathrm{COH} .
\end{aligned}
$$

This is borne out by the fact that when dissolved in carbon disulphide and cooled down to $-10^{\circ}$, it takes up 2 atoms of bromine, showing that it is unsaturated. The constitution of the alcohol $\mathrm{C}_{14} \mathrm{H}_{30} \mathrm{O}$, obtained by the action of nascent hydrogen on the aldehyde $\mathrm{C}_{14} \mathrm{H}_{28} \mathrm{O}$, and the alcohol isomeric with it, has already been referred to in Part I. It is isomeric with myristic alcohol, which is contained in spermaceti, together with cetyl alcohol and other bodies.

\section{Polymerisation of Enanthaldehyde.}

Bruylants (Ber., 8, 415) has shown that by the action of dry potassium carbonate on wnanthaldehyde a solid polymeride of this body is produced, and it was thought that it would be of interest to make some experiments with this compound, with a view, if possible, of getting some clue as to its molecular weight, and also of comparing it with aldol. Borodin mentions that these two bodies appear to be closely related, both of them losing water on distillation, aldol giving 
crotonic aldehyde; while the polymeride of conanthaldehyde gives the bodies $\mathrm{C}_{14} \mathrm{H}_{26} \mathrm{O}$ and $\mathrm{C}_{28} \mathrm{H}_{54} \mathrm{O}_{3}$, œnanthaldehyde being also apparently regenerated. The latter reaction seems, however, to be more complicated than that with aldol, and the formation of the body $\mathrm{C}_{28} \mathrm{H}_{54} \mathrm{O}_{3}$ would appear to indicate that the polymeride contained more than twice $\mathrm{C}_{7} \mathrm{H}_{14} \mathrm{O}$. The polymeride was prepared as follows by the method used by Bruylants :-

100 grams of $œ$ nanthaldehyde were mixed with about 20 grams of dry potassium carbonate, and allowed to stand at ordinary temperatures, the formation of the polymeride generally requiring about four days. It is, however, hastened by gently warming and shaking the mixture from time to time at about $40-45^{\circ}$; the polymerisation is then generally complete in $5-6$ hours, the aldehyde becoming quite solid. In order to ensure the polymerisation of all the œnanthaldehyde the mass was melted at $60^{\circ}$, shaken up with a small quantity of fresh potassium carbonate, and left to crystallise.

The product was carefully melted, and well washed with warm water, taking care that the temperature was not above $70^{\circ}$. As soon as all the carbonate was dissolved out the water was separated as much as possible, and the oil left to crystallise over sulphuric acid. This generally requires about two days. It was then placed in a vessel with a capillary tube at the bottom end connected with a vacuum pump. On working this, the oil was gradually drawn off, leaving the nearly pure polymeride as a white waxy crystalline mass In order to free it perfectly from oil, it was pressed between filterpaper, moistened with ether, and again pressed. Obtained in this manner, it melted at $52-53^{\circ}$, which corresponds with the melting point found by Bruylants. The oil which was drawn off from the crystals on standing for some time at $0^{\circ}$, deposits a further quantity of this body in crystals-generally as long needles. By repeating this cooling process, most of the product is eventually obtained in a solid state, only a very small quantity of oil remaining. The polymeride of œnanthaldehyde is very easily soluble in alcohol, ether, chloroform, carbon disulphide, petroleum, ether, \&c., but it could not be crystallised from these solvents. It reduces an ammoniacal solution of oxide of silver very readily. If bromine be added to a solution of this body in carbon disulphide and cooled in a freezing mixture, it is not decolorised.

It was analysed, and gave the following numbers :-

0.2255 gram substance gave $0.2500 \mathrm{OH}_{2}$ and $0.6079 \mathrm{CO}_{2}$.

Found.

C ...... 73.53 per cent.

H ...... 12.32,
Theory for $\left(\mathrm{C}_{7} \mathrm{H}_{14} \mathrm{O}\right)_{n}$.

73.68 per cent.

$12 \cdot 28$, 
As already stated, it melts at $52-53^{\circ}$, and decomposes on heating above $115^{\circ}$. The decomposition seems to begin at that temperature, water being given off, and collecting at the top of the vessel in which it is heated, as a cloud. In order to examine the decomposition-products of this body, 50 grams were distilled in a stream of carbonic anhydride. The substance first melts, and at about $120^{\circ}$ froths up a little. Between $120^{\circ}$ and $200^{\circ}$ a good deal came over, the thermometer then rose rapidly to $270^{\circ}$, and between this and $300^{\circ}$ the amount which distilled over was considerable. A small residue remained behind, which was reserved for distillation in a vacuum. It was noticed that when the substance was quickly distilled, the residue was very small, whereas if the decomposition is allowed to take place slowly, the amount of residue is increased. The oil boiling between $120-200^{\circ}$ was then several times carefully fractioned in a current of carbonic anhydride, nearly all of it at last coming over between $152-154^{\circ}$, and smelling strongly of œnanthaldehyde. Shaken up with a solution of hydrogen sodium sulphite it combined directly, and on analysis gave the following numbers:-

0.0929 gram substance gave $0.0988 \mathrm{OH}_{2}$ and $0.2507 \mathrm{CO}_{2}$.

$$
\begin{aligned}
& \text { Found. } \\
& \text { C ...... 73.59 per cent. } \\
& \text { H ...... 11.81 , } \\
& \text { Calculated for } \mathrm{C}_{6} \mathrm{H}_{13} \mathrm{COH} \text {. } \\
& 73.68 \text { per cent. } \\
& 12 \cdot 28 \text { " }
\end{aligned}
$$

It was therefore cenanthaldehyde. The fraction $270-290^{\circ}$ was then several times fractioned in an atmosphere of carbonic anhydride. Almost all came over at last between $275-280^{\circ}$, and gave the following numbers on analysis :-

0.2414 gram substance gave $0.2700 \mathrm{OH}_{2}$ and $0.7063 \mathrm{CO}_{2}$.

$$
\begin{aligned}
& \text { Found. } \\
& \text { C ...... 79.79 per cent. } \\
& \text { H ...... 12.43 ", } \\
& \text { Calculated for } \mathrm{C}_{14} \mathrm{H}_{26} \mathrm{O} \text {. } \\
& 80.00 \text { per cent. } \\
& 12 \cdot 38 \text { ", }
\end{aligned}
$$

It was therefore the aldehyde $\mathrm{C}_{14} \mathrm{H}_{26} \mathrm{O}$, and was undoubtedly identical with that obtained from œnanthaldehyde by the action of alcoholic potash, and described in Part I. Its specific gravity was practically the same, viz., 0.8504 at $15^{\circ}$, as compared with 0.8494 . Dissolved in carbon disulphide it combines with bromine without evolution of hydrobromic acid, and does not solidify at $-15^{\circ}$. It may be mentioned that by the decomposition of the polymeride of œnanthaldehyde by distillation, œnanthaldehyde and the body $\mathrm{C}_{14} \mathrm{H}_{26} \mathrm{O}$ are easily obtained in a pure state, as the decomposition takes place nearly quantitatively, only a very small residue being formed. The two bodies are very easily separated by fractional distillation, 
œnanthaldehyde boiling at $154^{\circ}$, and the aldehyde $\mathrm{C}_{14} \mathrm{H}_{26} \mathrm{O}$ at $179^{\circ}$. The residues boiling above $290^{\circ}$ (collected from a series of experiments) were first distilled in a vacuum, and then fractioned under a pressure of $250 \mathrm{~mm}$. At the first distillation nearly all came over between $310-350^{\circ}$ as a light yellow oil.

This fraction was then several times carefully fractioned under the same pressure, when most of it boiled at $330-340^{\circ}$, and gave the following numbers on analysis :-

I. $0 \cdot 1211$ gram substance gave $0 \cdot 1349 \mathrm{OH}_{2}$ and $0 \cdot 3427 \mathrm{CO}_{2}$.
II. $0 \cdot 1324$
",
$0 \cdot 1474$
0.3737,

Found.

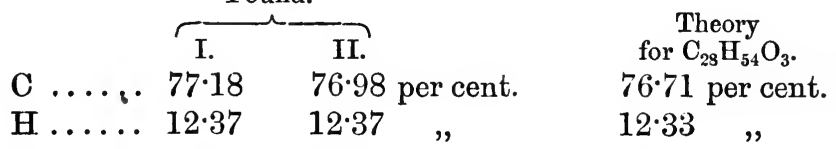

This body seems therefore to have the composition $\mathrm{C}_{28} \mathrm{H}_{54} \mathrm{O}_{3}$. It is an oil of a slightly yellow colour and disagreeable smell, which does not solidify when cooled down to $-10^{\circ}$. When dissolved in carbon disulphide and cooled down to $-10^{\circ}$, it takes up bromine without evolution of hydrobromic acid. Quantitative experiments were made with a view of determining the degree of unsaturation of the body, and gave numbers which agreed fairly well with those calculated for the formula $\mathrm{C}_{28} \mathrm{H}_{54} \mathrm{O}_{3} \mathrm{Br}_{2}$; but on titration with bromine, after a certain point is reached, the oil becomes slightly brown, and this makes it difficult to ascertain accurately when the bromine ceases to be taken up. It is apparently formed by removal of the elements of water from 4 mols. of œnanthaldehyde. Thus :-

$$
4 \mathrm{C}_{7} \mathrm{H}_{14} \mathrm{O}=\mathrm{C}_{28} \mathrm{H}_{54} \mathrm{O}_{3}+\mathrm{OH}_{22} \text {. }
$$

This body reduces ammoniacal silver solution. On heating it with strong alcoholic potash it becomes brown, apparently being converted into higher condensation-products. A small quantity of potash compound was formed at the same time, which appeared to be potassium heptoate. Boiling with dilute sulphuric acid also decomposes this oil.

The polymeride of œnanthaldehyde splits up, therefore, on distillation into water, œnanthaldehyde, the aldehyde $\mathrm{C}_{14} \mathrm{H}_{26} \mathrm{O}$, and the body $\mathrm{C}_{28} \mathrm{H}_{54} \mathrm{O}_{3}$. These results therefore agree with those obtained by Borodin.

In order, if possible, to get some idea as to the molecular weight of this polymeric modification of œnanthaldehyde, it was thought that by carefully distilling known quantities and then weighing the decomposition-products, i.e., the œnanthol $\mathrm{C}_{14} \mathrm{H}_{26} \mathrm{O}$ and the residue, some 
relation might be found which would point to a probable formula. The following results were obtained from three experiments made with the pure substance:-

I. 8.9 grams polymeride gave on distillation 4.5 grams crude œnanthaldehyde and water, $3 \cdot 7$ grams $\mathrm{C}_{14} \mathrm{H}_{26} \mathrm{O}$, and 0.6 gram residue $\left(=\mathrm{C}_{28} \mathrm{H}_{54} \mathrm{O}_{3}\right)$.

II. $33 \cdot 3$ grams polymeride gave 16.3 grams œnanthaldehyde and water, $14 \cdot 1$ grams $\mathrm{C}_{14} \mathrm{H}_{26} \mathrm{O}$, and $1 \cdot 3$ gram residue boiling above $285^{\circ}$.

III. $33 \cdot 2$ grams polymeride gave $16 \cdot 7$ grams œnanthaldehyde and water, 14:3 grams $\mathrm{C}_{14} \mathrm{H}_{26} \mathrm{O}$, and 0.9 gram residue.

The residue was all that boiled above $285^{\circ}$, and possibly still contained small quantities of $\mathrm{C}_{14} \mathrm{H}_{26} \mathrm{O}$. These three experiments were purposely made from three different preparations. The last one was made from crystals which had separated out from the oil (obtained by filtering the crude polymeride), on standing for some weeks at $0^{\circ}$. The water can be calculated on the supposition that for every molecule of $\mathrm{C}_{14} \mathrm{H}_{26} \mathrm{O}$ that is formed, one molecule of water is separated, which then gives the following results for conanthaldehyde and water :-

I. Water calculated $=0.3$ gram, therefore œnanthaldehyde $=$ $4 \cdot 2$ grams.

II. Water calculated $=1 \cdot 2$ gram, therefore enanthaldehyde $=$ $15 \cdot 1$ grams.

III. Water calculated $=1.2$ gram, therefore $œ$ nanthaldehyde $=$ $15 \cdot 5$ grams.

Calculated into percentages, the polymeride gives on distillation :-

\begin{tabular}{lrrrr} 
& \multicolumn{1}{c}{ I. } & \multicolumn{1}{c}{ II. } & \multicolumn{1}{c}{ III. } \\
Enanthaldehyde $\ldots \ldots \ldots$ & $47 \cdot 19$ & $45 \cdot 34$ & $46 \cdot 68$ \\
$\mathrm{C}_{14} \mathrm{H}_{26} \mathrm{O} \ldots \ldots \ldots \ldots \ldots$ & $41 \cdot 58$ & $42 \cdot 34$ & $43 \cdot 07$ \\
Water $\ldots \ldots \ldots \ldots \ldots \ldots$ & $3 \cdot 37$ & $3 \cdot 60$ & $3 \cdot 61$ \\
Residue $\ldots \ldots \ldots \ldots \ldots \ldots$ & $6 \cdot 74$ & 3.93 & $2 \cdot 71$
\end{tabular}

These numbers show that equal weights of œnanthaldehyde and $\mathrm{C}_{14} \mathrm{H}_{26} \mathrm{O}$ (+ water) are formed by the destructive distillation of the polymeride, $i . e$., two molecules of œenanthaldehyde and one of $\mathrm{C}_{14} \mathrm{H}_{26} \mathrm{O}$, probably according to the equation:-

$$
\left(\mathrm{C}_{7} \mathrm{H}_{14} \mathrm{O}\right)_{4}=2 \mathrm{C}_{7} \mathrm{H}_{14} \mathrm{O}+\mathrm{C}_{14} \mathrm{H}_{26} \mathrm{O}+\mathrm{OH}_{2} \text {. }
$$

It appears therefore likely that the polymeride is formed froni fonr molecules of onanthaldehyde, and has the following constitution :- 


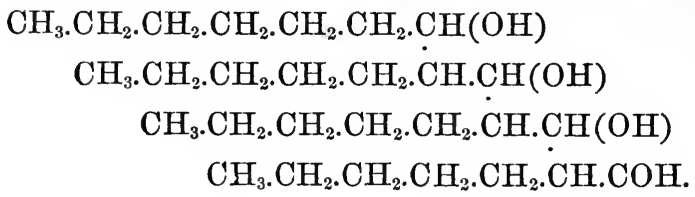

That it is a saturated body is proved by the fact, that this substance when dissolved in bisulphide of carbon, does not take up bromine without evolution of hydrobromic acid, and, if it is an aldehyde, this must also be the case from its formula. The different groups in this formula are linked together much in the same way as those in aldol, which splits up into water and crotonic aldehyde according to the equation :-

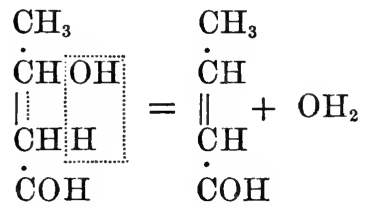

It is also very easy to understand the splitting up of the polymeride of œnanthaldehyde, in a similar way, into water, the aldehyde $\mathrm{C}_{14} \mathrm{H}_{26} \mathrm{O}$, and two molecules of onanthaldehyde; thus :-

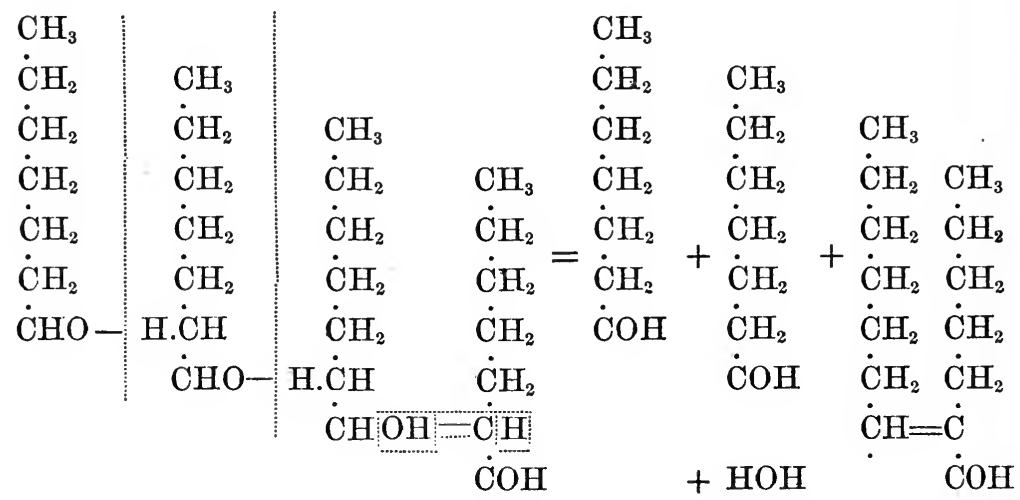

The amount of the body $\mathrm{C}_{25} \mathrm{H}_{54} \mathrm{O}_{3}$ formed is dependent, as before mentioned, on the rapidity of the distillation. In Experiment I, for example, the polymeride was slowly distilled, and 6.74 per cent. of residue was obtained; whereas in Experiment III the retort was heated with an open flame and the distillation conducted very rapidly when only $2 \cdot 71$ per cent. of residue was obtained. The amount obtained has rarely reached more than 10 per cent. The formation of this substance from the polymeride of œnanthaldehyde is in itself in 
favour of this latter substance being built up from four molecules of œnanthaldehyde, as the formation of the former is then very easy of explanation; thus:-

$$
\mathrm{C}_{28} \mathrm{H}_{56} \mathrm{O}_{4}=\mathrm{C}_{28} \mathrm{H}_{54} \mathrm{O}_{3}+\mathrm{OH}_{2} \text {. }
$$

It would be difficult to understand how such a body could be produced by the simple distillation of a mixture of conanthaldehyde and the aldehyde $\mathrm{C}_{14} \mathrm{H}_{26} \mathrm{O}$. Its constitution may probably be represented thus:-

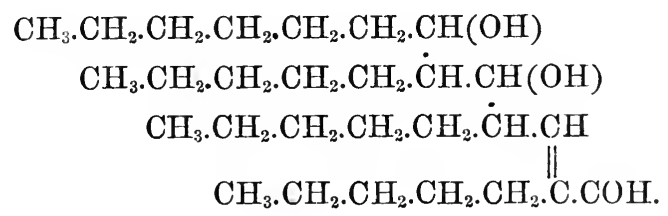

This shows it to be an unsaturated body, which is borne out by its combining directly with bromine. By the action of solid potash at ordinary temperatures on cenanthaldehyde, Borodin states that he obtained two different polymerides; the one solid and the other liquid. In order to see if this were the casc when potassium carbonate was used, the oil which was filtered off from the solid polymerides was allowed to stand at $0^{\circ}$ till it deposited no more crystals, and then completely filtered off. Only a small quantity of oil was obtained, having the appearance of glycerol, and smelling strongly of cenanthaldehyde, whereas the solid product has very little smell. It was then left for some time over sulphuric acid. On heating, the oil began to decompose at $120^{\circ}$, giving off water; at a temperature of $130-140^{\circ}$ the decomposition was very rapid. In order to see whether it were a different polymeride or only the solid one mixed with a little impurity which prevented its crystallising, two quantitative experiments were made and the distillation-products weighed as before. They gave the following results :-

I. $21 \cdot 6$ grams of the oil gave $10 \cdot 3$ grams crude œnanthaldehyde and water, $8 \cdot 1$ grams $\mathrm{C}_{14} \mathrm{H}_{26} \mathrm{O}$, and $2 \cdot 4$ grams residue.

II. $12 \cdot 2$ grams gave $5 \cdot 7$ grams onanthaldehyde and water, $4 \cdot 6$ grams $\mathrm{C}_{14} \mathrm{H}_{26} \mathrm{O}$, and $1 \cdot 0$ gram residue.

If the water be calculated as before, the following amounts of conanthaldehyde and water are obtained:-

I. Water calculated $=0 \cdot 7$ gram. Enanthaldehyde $=9 \cdot 6$ grams. II. $\quad=0.4$ gram. $\quad=5.3 \%$

Calculated into percentages these give the following numbers, as the relative amounts of onanthaldehyde and $\mathrm{C}_{14} \mathrm{H}_{26} \mathrm{O}$ formed :- 


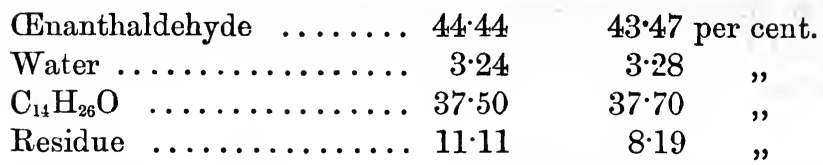

These two experiments were made from two different preparations. From these results, it appears that the oil is most probably only a mixture of the solid polymeride and a little unchanged cenanthaldehyde. By the action of potassium carbonate on œnanthaldebyde, it seems therefore that only one polymeride is formed, which is solid and melts at $52-53^{\circ}$. By boiling cenanthaldehyde with potassium carbonate, the same condensation-products are produced as by the distillation of the polymeride, together with potassium heptoate. It is probable that the polymeric modification of isobutaldehyde, which Urech (Ber., 12, $190 ; 13,483$ and 590) obtained by the action of potassium carbonate on that aldehyde at ordinary temperatures, is derived from at least four molecules of isobutaldehyde. Urech considers that it has the constitution $\left(\mathrm{C}_{4} \mathrm{H}_{8} \mathrm{O}\right)_{3}$, and describes it as a very thick oil, which can be distilled in steam. When distilled, alone, however, it decomposes into water, isobutaldehyde and condensation-products. From these he isolated a body, $\mathrm{C}_{12} \mathrm{H}_{22} \mathrm{O}_{2}=$ $3 \mathrm{C}_{4} \mathrm{H}_{8} \mathrm{O}-\mathrm{OH}_{2}$.

Demtschenko (Ber., 6, 1176) also obtained a polymeride by the action of, sulphuric acid on isobutaldehyde, which has the formula $\left(\mathrm{C}_{4} \mathrm{H}_{8} \mathrm{O}\right)_{3}$, and which he called "paraisobutaldehyde." It distils without decomposition, and he was thus able to determine its molecular weight by taking its vapour-density; this agreed with the formula $\left(\mathrm{C}_{4} \mathrm{H}_{8} \mathrm{O}\right)_{3}$. It therefore appears that the polymeride obtained by Urech is quite a different body, possibly containing at least $4\left(\mathrm{C}_{4} \mathrm{H}_{8} \mathrm{O}\right)$.

The same remark applies to the polymeride obtained by Borodin (Ber., 6, 982) from isovaleraldehyde, which, on distillation, splits up into water, isovaleraldehyde, $\mathrm{C}_{10} \mathrm{H}_{18} \mathrm{O}$, and $\mathrm{C}_{20} \mathrm{H}_{38} \mathrm{O}_{2}$, and the hydrate of which is produced by the action of soda solutions on this aldehyde at ordinary temperatures. This latter body has the formula $\left(\mathrm{C}_{10} \mathrm{H}_{20} \mathrm{O}_{2}\right)_{2} \mathrm{H}_{2} \mathrm{O}=\left(\mathrm{C}_{5} \mathrm{H}_{10} \mathrm{O}\right)_{4} \mathrm{H}_{2} \mathrm{O}$.

By distillation, this hydrate also splits up into water, isovaleraldehyde, $\mathrm{C}_{10} \mathrm{H}_{18} \mathrm{O}$, and $\mathrm{C}_{20} \mathrm{H}_{38} \mathrm{O}_{2}$.

\section{Action of Nascent Hydrogen on Polymerised Ginanthaldehyde.}

This experiment was made in the hope of obtaining from the polymerised cenanthaldehyde, a glycol of the formula $\mathrm{C}_{28} \mathrm{H}_{58} \mathrm{O}_{4}$, by the reduction of the $\mathrm{COH}$-group to $\mathrm{CH}_{2} \mathrm{OH}$, and of thus confirming the supposition that it is derived from 4 mols. of cenanthaldehyde. 
Thirty grams of the polymeride were dissolved in ether, and then treated with 50 per cent. acetic acid, and a large excess of sodium in a flask connected with a reversed condenser. The sodium was added very slowly, and now and then a little acetic acid was poured in, to insure the contents having an acid reaction, as it appeared probable that the polymeride would be easily decomposed by caustic alkali. At the end of the reaction the ethereal solution was 'well washed to remore sodium acetate and acetic acid, dried, and the ether distilled off. The residual oil was then carefully fractioned. The first fraction obtained was from $100-210^{\circ}$, and weighed about 5 grams; the thermometer then rose rapidly to $260^{\circ}$, and between this and $300^{\circ}$ about 5 grams more came over. The greater part, however, boiled above $310^{\circ}$, and was left behind in the retort. The product which came over between $100-210^{\circ}$ was then repeatedly fractioned, when at last most of it came over between $174-176^{\circ}$, and gave the following numbers on analysis :-

0.1535 gram substance gave $0.1857 \mathrm{OH}_{2}$ and $0.4121 \mathrm{CO}_{2}$.

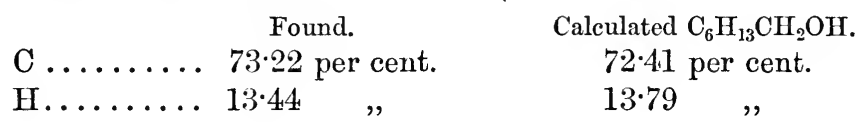

It is therefore undoubtedly heptyl alcohol. On shaking it with acid sodium sulphite, a small quantity of a compound was formed, indicating that a trace of onanthaldehyde was also present. This would account for the hydrogen being low and the carbon high in the above analysis.

The oil boiling between $250-310^{\circ}$ was then repeatedly fractioned; a product was thus obtained boiling at $297-300^{\circ}$. The quantity was, however, very small, so that it was very difficult to obtain pure. It gave on analysis the following numbers:-

I. 0.2563 gram substance gave $0.3133 \mathrm{OH}_{2}$ and $0.7268 \mathrm{CO}_{2}$.

II. $0.1746 \quad " \quad$ " $0.2133 \quad$, 0.4936 ,

Found.

\begin{tabular}{|c|c|c|}
\hline I. & II. & Theory $\mathrm{C}_{21} \mathrm{H}_{44} \mathrm{O}_{2}$. \\
\hline$\cdots \quad 1704$ & 13.57 per cent. & 13.41 per cent. \\
\hline
\end{tabular}

This substance therefore appears to have the formula $\mathrm{C}_{21} \mathrm{H}_{44} \mathrm{O}_{2}$. Its formation, together with that of heptyl alcohol by the action of nascent hydrogen on the polymeride of œnanthaldehyde, may be represented thus :-

$$
\mathrm{C}_{23} \mathrm{H}_{56} \mathrm{O}_{4}+3 \mathrm{H}_{2}=\left\{\begin{array}{l}
\mathrm{C}_{6} \mathrm{H}_{13} \\
\mathrm{CH}_{2} \mathrm{OH}
\end{array}+\mathrm{C}_{21} \mathrm{H}_{44} \mathrm{O}_{2} .\right.
$$


This reaction can be better understood if the equation is written out as follows; thus:-

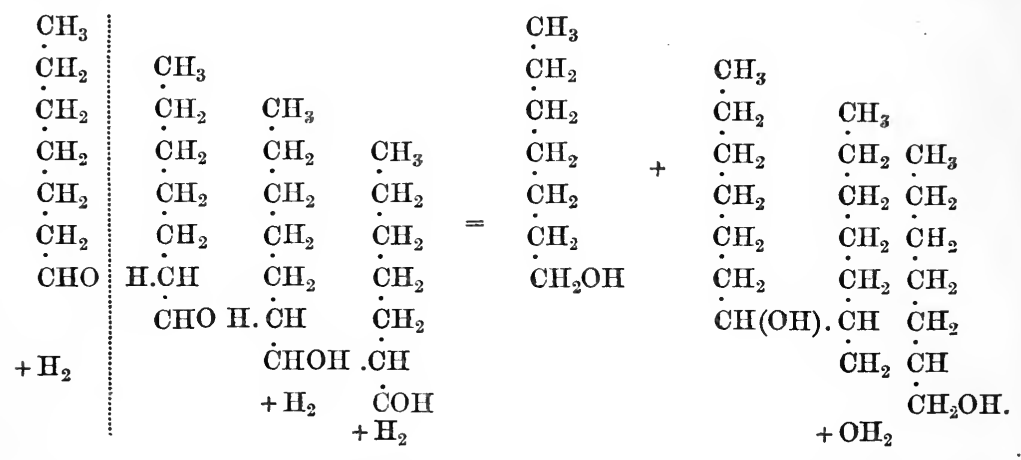

This substance is a yellowish oil, which does not solidify at $-20^{\circ}$. If dissolved in carbon disulphide and cooled down to $-10^{\circ}$, it does not appear to take up bromine. In order to confirm its molecular weight several attempts were made to determine its vapour-density, but as it decomposes a few degrees above its boiling point, no useful results were obtained.

I hope to have another opportunity of more thoroughly studying this interesting reaction, and more especially of examining the products boiling above $310^{\circ}$, which, as yet, I have not been able to obtain pure.

Action of Alcoholic Potash on Acetic Aldehyde.

A few experiments were made on the action of very dilate alcoholic potash on ordinary aldehyde, in order to see if it were possible to obtain as good a yield of crotonic aldehyde by this reaction, as of the corresponding aldehyde, $\mathrm{C}_{14} \mathrm{H}_{26} \mathrm{O}$, from œnanthaldehyde, more especially as the preparation of the former by the zinc chloride reaction is unsatisfactory, the yield being very small.

The aldehyde employed in these experiments was the so-called " concentrated solution" from Kahlbaum, which is a mixture of water, aldehyde, and a trace of alcohol. The aldebyde was mixed with a little alcohol, and then treated every day with small quantities of alcoholic potash, cooling the mixture well after each addition. After standing for some time, a considerable quantity of metaldehyde separated out in large crystals, which were filtered off. From the solution an oil was obtained, which, after washing with a solution of calcium chloride and drying, was fractioned. At first a considerable quantity of unchanged aldehyde came over, then from $90-135^{\circ}$ 
a large fraction was obtained; above $130^{\circ}$ a dark brown residue was left, which probably contained aldol, as on further heating it decomposed, giving off water and a small quantity of an oil smelling very like crotonic aldehyde.

The product boiling between $90-135^{\circ}$ was carefully fractioned in carbonic anhydride. Two fractions were obtained between $100-110^{\circ}$ and $115-130^{\circ}$. The latter was by far the larger: On repeatedly fractioning the first of these, nearly all of it boiled between $102-106^{\circ}$, and gave the following numbers on analysis :-

0.2093 gram substance gave $0.1636 \mathrm{OH}_{2}$ and $0.5229 \mathrm{CO}_{2}$.

Found.

C......6 68.14 per cent.

H...... 8.68 ,
Calculated $\mathrm{CH}_{3} \cdot \mathrm{CH}=\mathrm{CH} . \mathrm{COH}$. 68.57 per cent.

$8.57 \quad$,

It was therefore crotonic aldehyde. On standing for some few days in the air, it deposited crystals of crotonic acid, melting at $70^{\circ}$, whereas the pure acid melts at $72^{\circ}$. The secund fraction, on being redistilled, eventually boiled mostly between $123-125^{\circ}$, and gave the following result on analysis :-

$0 \cdot 1264$ gram substance gave $0 \cdot 1014 \mathrm{OH}_{2}$ and $0 \cdot 2509 \mathrm{CO}_{2}$.

\begin{tabular}{|c|c|c|}
\hline & $\begin{array}{l}\text { Found. } \\
54 \cdot 13 \text { per cent. }\end{array}$ & $\begin{array}{c}\text { Calculated }\left(\mathrm{CH}_{3} \mathrm{COH}\right)_{3} \text {. } \\
54.54 \text { per cent. }\end{array}$ \\
\hline $\mathrm{H}$ & $8 \cdot 91$ & 9.09 \\
\hline
\end{tabular}

It was therefore paraldehyde. The yield of crotonic aldehyde was unfortunately small, but it appears probable that, by modifying the conditions, it might be improved, and this process found to be the easiest method of preparing it. Paraldehyde was the chief product of the reaction.

Finally, I append a table of the substances obtained from œnanthaldehyde, with their boiling points and specific gravities. 


\begin{tabular}{|c|c|c|c|c|}
\hline \multirow{2}{*}{ Name of substance. } & \multirow{2}{*}{ Boiling point. } & \multicolumn{3}{|c|}{$\begin{array}{l}\text { Specific gravity compared with } \\
\text { water at the same temperature. }\end{array}$} \\
\hline & & $15^{\circ}$ & $30^{\circ}$ & $35^{\circ}$. \\
\hline Enanthaldehyde ...... & $153-154^{\circ}$ & $0 \cdot 8231$ & $0 \cdot 8128$ & $0 \cdot 8099$ \\
\hline $\mathrm{C}_{14} \mathrm{H}_{26} \mathrm{O}=\left\{\begin{array}{l}\mathrm{C}_{13} \mathrm{H}_{25} \\
\mathrm{COH}\end{array}\right\}$. & $277-279^{\circ}$ & $0 \cdot 8494$ & $0 \cdot 8416$ & $0 \cdot 8392$ \\
\hline $\mathrm{C}_{13} \mathrm{H}_{27} \mathrm{COH} \ldots \ldots \ldots \ldots$ & $\begin{array}{c}266-268^{\circ} \\
\left(\mathrm{m} \cdot \mathrm{p} .-29^{\circ} \mathrm{o}^{\circ}\right)\end{array}$ & - & $0 \cdot 8274$ & $0 \cdot 8258$ \\
\hline $\mathrm{C}_{13} \mathrm{H}_{25} \mathrm{CH}_{2} \mathrm{OH} \ldots \ldots \ldots$ & $280-283^{\circ}$ & $0 \cdot 8520$ & $0 \cdot 8444$ & $0 \cdot 8418$ \\
\hline $\mathrm{C}_{13} \mathrm{H}_{27} \mathrm{CH}_{2} \mathrm{OH} \ldots \ldots$ & $270-275^{\circ}$ & $0 \cdot 8368$ & $0 \cdot 8301$ & $0 \cdot 82.79$ \\
\hline $\mathrm{C}_{13} \mathrm{H}_{25} \mathrm{CH}_{2} \mathrm{OC}_{2} \mathrm{H}_{3} \mathrm{O} \ldots \ldots$ & $285-290^{\circ}$ & $0 \cdot 8680$ & $0 \cdot 8597$ & $0 \cdot 8568$ \\
\hline $\mathrm{C}_{13} \mathrm{H}_{27} \mathrm{CH}_{2} \mathrm{OC}_{2} \mathrm{H}_{3} \mathrm{O} \ldots \ldots$ & $275-280^{\circ}$ & $0 \cdot 8559$ & $0 \cdot 8476$ & $0 \cdot 8448$ \\
\hline $\mathrm{C}_{13} \mathrm{H}_{25} \mathrm{COOH} \ldots \ldots \ldots$ & $\begin{array}{l}275-285^{\circ} \\
\text { (at } 250 \mathrm{~mm} \text {.) }\end{array}$ & - & - & \\
\hline $\mathrm{C}_{13} \mathrm{H}_{27} \mathrm{COOH} \ldots \ldots \ldots$ & $300-310^{\circ}$ & - & - & $\overline{-}$ \\
\hline $\mathrm{C}_{21} \mathrm{H}_{40} \mathrm{O} \ldots \ldots \ldots \ldots$ & $\begin{array}{l}310-315^{\circ} \\
\text { (at } 300 \mathrm{~mm} .)\end{array}$ & $0 \cdot 8744$ & $0 \cdot 8665$ & $0 \cdot 8637$ \\
\hline $\mathrm{C}_{28} \mathrm{H}_{50} \mathrm{O} \ldots \ldots \ldots \ldots \ldots$ & $\begin{array}{l}330-340^{\circ} \\
(\text { at } 200 \mathrm{~mm} \text {. }\end{array}$ & $0 \cdot 8831$ & $0 \cdot 8751$ & $0 \cdot 8723$ \\
\hline $\mathrm{C}_{28} \mathrm{H}_{54} \mathrm{O}_{3} \ldots \ldots \ldots \ldots$ & $\begin{array}{l}330-340^{\circ} \\
\text { (at } 200 \mathrm{~mm} .)\end{array}$ & - & - & - \\
\hline $\mathrm{C}_{28} \mathrm{H}_{56} \mathrm{O}_{4} \ldots \ldots \ldots \ldots$ & melts at $52-53^{\circ}$ & 一 & - & - \\
\hline $\mathrm{C}_{21} \mathrm{H}_{44} \mathrm{O}_{2} \ldots \ldots \ldots \ldots$ & $297-300^{\circ}$ & - & - & 一 \\
\hline
\end{tabular}

I cannot conclude this paper without expressing my sincere thanks to Prof. Wislicenus, in whose laboratory this research was carried out, for his kind help and advice during its progress. 


\section{ON BERBERINE. (PART I.)}

By W. H. Perkin, Jun., Ph.D.

Berberine, the yellow alkaloïd of Berberis.vulgaris, was discovered in the year 1826 by Chevallier and Pelletan (Journ. de Chim. Médicale, 2, 314) in Xanthoxylum clava Herculis, and described by them under the name of xanthopicrit.

Subsequent researches have shown that this alkaloid is a constituent of a large number of plants, in some of which it is present in considerable quantities.

It has been found in Cocculus palmatus, or Columbo root (Boedecker, Annalen, 66, 384 ; 69, 40); in Berberis vulgaris (Buchner, Ann., 24, 228); in Calceline polycarpa (Stenhouse, Ann., 95, 108; 105, $360)$; in the wood of Coscinium fenestratum from Ceylon, and in Xanthorrisa apii folia, a North American plant of the Ranunculacea order (Perrins, Ann., 83, 276); in Hydrastis canadensis (Mahla, Sill. Am. J. [2], 33, 43 (1862)); another North American plant of the Ranunculacea order, which, as it contains about 4 per cent. of crude alkaloid, and is readily obtainable in England, can be used as a source of the alkaloid (Perrins, Ann., Suppl. 2, 173).

Berberine has also been found in Woodumpar, a yellow wood from Upper Assam, in the root of Coptis tecta or Mahmira-a plant growing in Hindoostan and China, and which is much valued in India on account of its tonic properties. Perrins (loc. cit., p. 174), who examined this source, obtained as much as $8 \frac{1}{2}$ per cent. berberine from the root-a far larger quantity than has up to the present been found in any plant.

The French chemists Chevallier and Pelletan, the discoverers of berberine, although they carefully and accurately described some of its properties, do not appear to have made any analyses of their 
product, and it was not till 1835 that any attempts were made to ascertain the composition of this interesting substance. In this year, Buchner and Herberger (Annalen, 24, 228) published the results of an important series of experiments on this alkaloïd, together with a number of analyses of the base itself, and also of its compound with oxide of silver. These experiments led them to the supposition that berberine was represented by the formula $\mathrm{C}_{33} \mathrm{H}_{36} \mathrm{~N}_{2} \mathrm{O}_{12}(\mathrm{C}=6: \mathrm{O}=8)$.

At a later date, Fleitmann made a careful examination of berberine, and showed that instead of being a weak acid, as Buchner had suggested, it was in reality a strong base, forming with acids stable salts, and in all respects showing the properties of a well-defined alkaloïd.

Fleitmann analysed not only berberine itself but also several of its salts, and the results of his experiments led him to the conclusion that the formula proposed by Buchner was erroneous, and that the composition of this base was $\mathrm{C}_{42} \mathrm{H}_{18} \mathrm{NO}_{9}(\mathrm{C}=6: \mathrm{O}=8)$.

In the year 1861, J. Dyson Perrins (Annalen, Suppl. 2, 176) published the most accurate investigation of berberine which had up to that time been made, in the course of which he analysed a large number of its salts, and showed, in a most conclusive manner, that Fleitmann's formula was inaccurate, and that the true formula was $\mathrm{C}_{20} \mathrm{H}_{17} \mathrm{NO}_{4}(\mathrm{C}=12: \mathrm{O}=16)$.

Although the analytical results obtained by Perrins agree so closely with this formula, I still thought it necessary, before starting an investigation on the constitution of this interesting alkaloid, to repeat some of these analyses with specially purified substances, so as to leave no doubt as to the relation of the carbon, hydrogen, nitrogen, and oxygen-atoms in it. The berberine used in these experiments was purified in the following manner :-

Crude berberine hydrochloride (from Merck in Darmstadt) was recrystallised twice from water acidified with hydrochloric acid, the beautiful yellow, silky needles dissolved in a little boiling water, and the solution rendered alkaline by the addition of an excess of carbonate of soda solution.

On cooling, the liquid became filled with brownish, silky crystals. These were filtered from the dark brownish-red mother-liquor, washed with water till the washings were only slightly yellowish, and dried on a porous plate. The beautiful silky mass thus obtained was next dissolved in boiling 80 per cent. alcohol (carefully purified methylated spirits may also be used) from which, on slowly cooling, long, yellowish-brown needles separated. After repeated crystallisaticn from dilute alcohol, these crystals entirely lose their original brownish colour, and are at length deposited in the form of long, silky needles of an intensely yellow colour. This substance was repeatedly analysed under varying conditions, but in spite of this no 
satisfactory results could be obtained. One reason for this is that the substance contains several molecules of water of crystallisation, part of which is given off slowly at ordinary temperatures and part at $100^{\circ}$.

The substance dried at $100^{\circ}$ is, however, never free from water of crystallisation, and if attempts are made to drive this off at a higher temperature, slight decomposition invariably takes place sufficient to spoil the analytical results. Another reason for the difficulty experienced in obtaining concordant numbers with berberine prepared in this way, is that the substance almost invariably contains traces of chlorine.

Experience showed that the nitrate, hydriodide, and platinochloride of berberine were far more suitable for analytical examination than the base itself.

Berberine Nitrate, $\mathrm{C}_{20} \mathrm{H}_{17} \mathrm{NO}_{4}, \mathrm{HNO}_{3}$. - This salt has been described by both Fleitmann and Perrins. It is readily prepared by adding an excess of dilute nitric acid to a warm, concentrated solution of the base. The nitrate is thus precipitated as a yellow crystalline mass, which is collected, well washed with water, and repeatedly recrystallised from this solvent. In this way beautiful yellow needles were obtained which, after drying first over sulphuric acid in a vacuum and then at $100^{\circ}$, gave the following results on analysis:-

I. 0.1724 gram substance gave 0.0722 gram $\mathrm{H}_{2} \mathrm{O}$ and 0.3808 gram $\mathrm{CO}_{2}$.

II. 0.1638 gram substance gave 0.0679 gram $\mathrm{H}_{2} \mathrm{O}$ and 0.3621 gram $\mathrm{CO}_{2}$.

III. 0.2056 gram substance gave 12.5 c.c. $\mathrm{N}$. Bar $=760 \mathrm{~mm} . t=$ $15^{\circ}$.

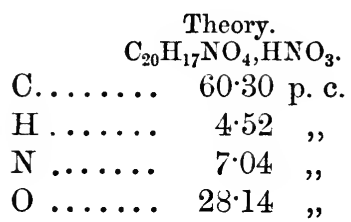

Found.

\begin{tabular}{|c|c|c|}
\hline I. & II. & III. \\
\hline $60 \cdot 24$ & $60 \cdot 27$ & - \\
\hline $4 \cdot 65$ & $4 \cdot 52$ & - \\
\hline- & - & $7 \cdot 16^{\circ}$ \\
\hline - & 一 & - \\
\hline
\end{tabular}

These numbers agree well with those previously found by Fleitmann and Perrins, but not so well with those given by Henry.

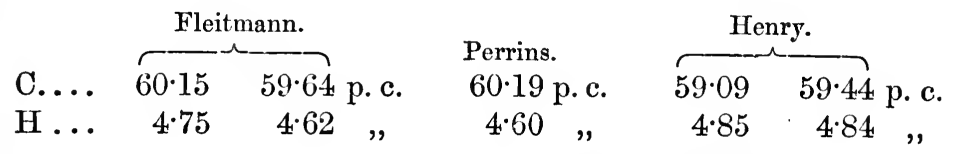

This salt is readily soluble in hot water, sparingly in cold, and almost insoluble in dilute nitric acid. 
If the hot solution of berberine nitrate be mixed with a small quantity of ammonia, on cooling a remarkable compound separates in sparingly soluble golden plates. I have not as yet been able to determine the nature of this substance, but from the analyses already carried out it appears to have the formula $\mathrm{C}_{20} \mathrm{H}_{20} \mathrm{~N}_{2} \mathrm{O}_{9}$.

(Found $\mathrm{C}=55 \cdot 2 . \quad \mathrm{H}=4 \cdot 8 . \quad \mathrm{N}=6 \cdot 80$. Theory requires $\mathrm{C}=$ $55 \cdot 55 . \mathrm{H}=4 \cdot 63 . \quad \mathrm{N}=6 \cdot 48$.)

I am at present engaged in the investigation of this substance.

Berberine Hydriodide, $\mathrm{C}_{20} \mathrm{H}_{17} \mathrm{NO}_{4}, \mathrm{HI}$. - This very insoluble salt is obtained as a yellow precipitate when an aqueous solution of berberine is acidified with a few drops of aqueous hydrogen iodide-or when potassic iodide is added to a solution of nitrate of berberine. The salt was collected, well washed with water, dried on a porous plate, and recrystallised from a large quantity of boiling 80 per cent. alcohol.

In this way, beautiful satiny needles were obtained which on analysis gave the following numbers :-

I. 0.1460 gram substance gave 0.0523 gram $\mathrm{H}_{2} \mathrm{O}$ and 0.2767 gram $\mathrm{CO}_{2}$.

II. 0.3561 gram substance gave 0.1795 gram AgI.

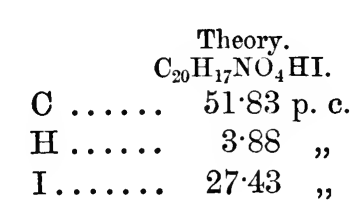

Found.

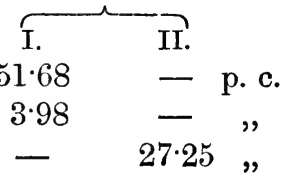

Perrins and Henry, who also analysed this salt, obtained numbers agreeing closely with the above.

\begin{tabular}{|c|c|c|c|}
\hline \multirow[b]{2}{*}{ C ...... } & \multicolumn{2}{|c|}{ Perrins. } & \multirow{2}{*}{$\begin{array}{l}\text { Henry. } \\
51.59 \text { p. c. }\end{array}$} \\
\hline & $51 \cdot 72$ & 51.77 p. c. & \\
\hline & $3 \cdot 92$ & $3 \cdot 96$ & $4 \cdot 23$ \\
\hline & $27 \cdot 02$ & $27 \cdot 12$ & $25 \cdot 7 \mathrm{~L}$ \\
\hline
\end{tabular}

Berberine Platinochloride, $\left(\mathrm{C}_{20} \mathrm{H}_{17} \mathrm{NO}_{4}\right)_{2}, \mathrm{H}_{2} \mathrm{PtCl}_{6}$. - This double salt has, on account of its insolubility and suitability for determining the molecular weight of berberine, been repeatedly examined. It is readily prepared by adding platinum chloride in excess to a slightly acid solution of berberine hydrochloride. The yellow precipitate thus thrown down was collected, well washed with water, dried on a porous plate over sulphuric acid, and then at $100^{\circ}$, and analysed with the following results :- 
I. 0.2251 gram substance gave 0.0708 gram $\mathrm{H}_{2} \mathrm{O}, 0.3657$ gram $\mathrm{CO}_{2}$, and 0.0406 gram Pt.

II. 0.6807 gram substance gave 0.1228 gram Pt.

III. 0.2780 gram substance gave 0.2192 gram $\mathrm{AgCl}$.
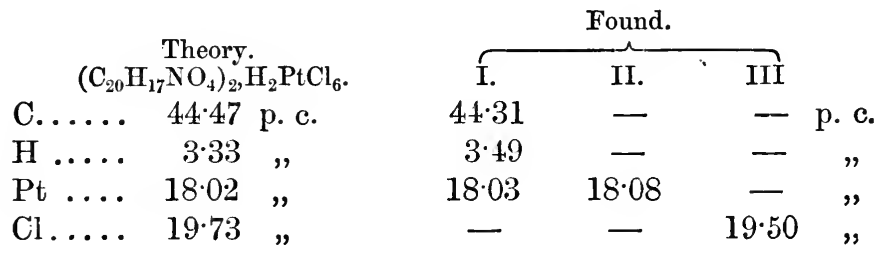

Previous analyses of this salt by Fleitmann, Perrins, and Henry gave the following results, which are in each case the mean of a number of experiments :-

\begin{tabular}{|c|c|c|c|}
\hline $\begin{array}{l}\mathrm{C} \ldots \\
\mathrm{H} \ldots\end{array}$ & $\begin{array}{l}\text { Fleitmann. } \\
44.40 \text { p. c. } \\
3.50 \text {, }\end{array}$ & $\begin{array}{l}\text { Henry. } \\
44.38 \text { p. c. } \\
3.57 \text {, }\end{array}$ & $\begin{array}{l}\text { Perrins. } \\
44.33 \text { p. c. } \\
3.411\end{array}$ \\
\hline$\ldots \ldots$ & $18 \cdot 11$, & $17 \cdot 90 \quad$ & $18 \cdot 21$ \\
\hline
\end{tabular}

This salt is almost insoluble in all the ordinary solvents. It crystallises from its solution in boiling amyl alcohol, on cooling in small, yellow needles, but it is extremely insoluble in this solvent even on boiling.

The analyses of these three salts show conclusively that the correct formula for berberine is $\mathrm{C}_{20} \mathrm{H}_{17} \mathrm{NO}_{4}$. Fior a complete list of the various berberine salts which have from time to time been analysed by different investigator's, I must refer to a paper by Hlasiwetz and Gilm (Annalen, Suppl. 2, 197-201); and also to a recent paper by Ernst Schmidt (Mittheilungen aus pharm. chem. Institut, Marburg), which I have only lately had the opportunity of consulting.

In his researches on berberine (loc. cit.), Fleitmann states that it crystallises from water with $4 \frac{1}{2}$ mols. $\mathrm{H}_{2} \mathrm{O}$. Several analyses which I made with different samples agree rather with the formula $\mathrm{C}_{20} \mathrm{H}_{17} \mathrm{NO}_{4}+5 \frac{1}{2} \mathrm{H}_{2} \mathrm{O}$. In these experiments the berberine used was prepared by Fleitmann's method, very carefully purified by repeated recrystallisation from water, and then dried in the air on a porous plate at the ordinary temperature. Determinations of the amount of water of crystallisation given off at $100^{\circ}$ gave the following results :-

I. $1 \cdot 5241$ gram substance heated to $100^{\circ}$ till coustant lost $0 \cdot 1944$ gram. 
II. 0.5883 gram substance heated to $100^{\circ}$ till constant lost 0.0702 gram.

III. $0 \cdot 6444$ gram substance heated to $100^{\circ}$ till constant lost 0.0758 gram.

IV. $0 \cdot 7017$ gram substance heated to $100^{\circ}$ till constant lost 0.0851 gram.

$\begin{array}{lcccc}\text { Found. } & \text { I. } & \text { II. } & \text { III. } & \text { IV. } \\ \mathrm{H}_{2} \mathrm{O} \ldots \ldots & 12 \cdot 7 & 11 \cdot 9 & 11 \cdot 9 & 12 \cdot 1 \text { p. c. }\end{array}$

Complete analyses of different preparations of berberine dried at $100^{\circ}$ till constant gave the following numbers :-

I. 0.2385 gram substance gave 0.1239 gram $\mathrm{H}_{2} \mathrm{O}$ and 0.5550 grám $\mathrm{CO}_{2}$.

II. 0.2095 gram substance gave 0.1041 gram $\mathrm{H}_{2} \mathrm{O}$ and 0.4877 gram $\mathrm{CO}_{2}$.

III. 0.1316 gram substance gave 0.0666 gram $\mathrm{H}_{2} \mathrm{O}$ and 0.3032 gram $\mathrm{CO}_{2}$.

IV. 0.3430 gram substance gare $11 \cdot 4$ c.c. $\mathrm{N} . \quad t=16 \cdot 5^{\circ} . \quad$ Bar. $=$ $736 \mathrm{~mm}$.

\begin{tabular}{|c|c|c|c|c|c|c|}
\hline \multirow{2}{*}{\multicolumn{3}{|c|}{$\begin{array}{l}\text { Theory. } \\
\mathrm{C}_{20} \mathrm{H}_{17} \mathrm{NO}_{4}+2 \frac{1}{\mathrm{H}_{2}} \mathrm{H}_{2} \mathrm{O} \text {. }\end{array}$}} & \multicolumn{4}{|c|}{ Found. } \\
\hline & & & I. & II. & III. & IV. \\
\hline$\ldots$ & $63 \cdot 16$ & o. c. & $63 \cdot 08$ & $63 \cdot 49$ & $63 \cdot 8.3$ & - p.c. \\
\hline $\mathrm{H}$ & $5 \cdot 78$ & " & $5 \cdot 77$ & $5 \cdot 52$ & $5 \cdot 66$ & 一 \\
\hline$N \ldots$ & $3 \cdot 68$ & , & - & - & - & $3 \cdot 72$ \\
\hline
\end{tabular}

From these analyses, it seems probable that berberine dried at $100^{\circ}$ still contains $2 \frac{1}{2}$ mols. $\mathrm{H}_{2} \mathrm{O}$, and that the air-dried substance originally contained $5 \frac{1}{2}$ mols. $\mathrm{H}_{2} \mathrm{O}, 3$ of which are driven off at $100^{\circ}$. This would require a loss of 12.4 per cent., which agrees approximately with the numbers given above.

Hermann Schreiber (Dissertation Marburg, 1888) is of opinion that berberine crystallises with 6 mols. $\mathrm{H}_{2} \mathrm{O}$, all of which is expelled at $100^{\circ}$ in a stream of hydrogen.

\section{Properties of Berberine.}

Berberine crystallises from water in brilliant, yellow needles, which when dried in the air have a fine silky lustre. The base itself and also its salts are remarkable for their excessively bitter taste; when warmed, they emit a faint but peculiar odour strongly resembling that of ordinary quinone.

When heated to $100^{\circ}$, the yellow berberine crystals gradually lose their lustre, and become yellowish-brown, at $110^{\circ}$ the change is very 
rapid, and at temperatures above $160^{\circ}$ total decomposition soon sets in with formation of a brownish-black mass, from which $I$ have not as yet been able to obtain anything of a crystalline nature.

On account of this behaviour of berberine when heated, I have not made any attempts to determine its melting point. Fleitmann (loc. cit.) gives as the melting point of berberine $120^{\circ}$, but this is certainly too low.

If bromine-water be added to even very dilute solutions of berberine, a yellow precipitate is produced, which on standing rapidly becomes darker. If the product is collected and washed with dilute sulphurons acid, a yellowish-orange mass is obtained, crystallising from large quantities of boiling water in needles, which appear to consist of the hydrobromide of a new base. Schreiber (loc. cit., p. 38) considers this substance to be simply berberine hydrobromide.

Berberine not only combines with acids to form salts, but it also gives peculiar compounds with alkalis. The addition of methyl alcoholic potash to a strong solution of berberine in alcoholic potash produces a pinkish-white, amorphous precipitate, which contains considerable quantities of inorganic matter. The analyses of this compound have not as yet given satisfactory results.

Berberine does not appear to combine either with hydroxylamine or phenylhydrazine, and although the alkaloïd dissolves readily in a mixture of oxychloride and pentachloride of phosphorus, no change seems to take place even when the mixture is allowed to remain for a long time at ordinary temperatures.

Before publishing the details of the experiments which have been made in this direction, I propose to confirm the results already obtained, by using, instead of the alkaloïd itself, sorne salt, such as the nitrate, which contains no water of crystallisation.

In its behaviour towards reagents, berberine shows the following characteristic reactions :-

It dissolves in concentrated sulphuric acid, forming a yellow solution, which on warming turns olive-green.

Very dilute solutions of berberine give with chlorine-water a decided brownish-red coloration, whilst strong solutions are coloured intensely by this reagent. Reducing agents, such as tin and hydrochloric acid, destroy this colour, which, however, reappears on the further addition of chlorine-water.

Concentrated nitric acid dissolves berberine, forming a dark, reddish-brown solution, from which water precipitates a yellow, flocculent mass, partly soluble in ammonia. If the brown-red solution in nitric acid be warmed, oxidation rapidly sets in with evolution of abundance of red fumes, and formation of a yellow solution which contains ber- 
beronic acid, oxalic acid, and other products. Potassium ferricyanide in alkaline solution gives with berberine a yellow precipitate, being itself for the most part reduced to ferrocyanide.

If a trace of berberine be boiled for a short time with concentrated aqueous hydrogen iodide, the product diluted with water and rendered slightly alkaline by the addition of ammonia, an intense blackishviolet coloration is produced.

Berberine is also characterised by the insolability of some of its compounds, as, for instance, its hydriodide, platinochloride, aurochloride, chromate, \&c., whilst a number of soluble salts, such as the nitrate, are rendered almost insoluble by the addition of a mineral acid.

A solution of iodine in potassic iodide gives with extremely small quantities of berberine salts a brownish precipitate of the periodide, $\mathrm{C}_{20} \mathrm{H}_{17} \mathrm{NO}_{4}, \mathrm{HI}, \mathrm{I}_{2}$. If the precipitation be carefully conducted in warm alcoholic solution, beautiful, shining, green plates separate.

Berberine Hydrochloride, $\mathrm{C}_{20} \mathrm{H}_{17} \mathrm{NO}_{4}, \mathrm{HCl}+2 \mathrm{H}_{2} \mathrm{O}$.- This beautiful salt crystallises from water in long, yellow needles. As mentioned at the commencement of the paper, this salt, in spite of its beautiful appearance, does not give very satisfactory results on analysis.

If attempts are made to dry it at $100^{\circ}$, in a short time the yellow colour of the crystals gradually changes to brownish-yellow, and then to brownish-red, small quantities of hydrochloric acid being invariably lost during the operation. Analysis of the pure substance dried over sulphuric acid gave numbers agreeing fairly well with those required by the formula $\mathrm{C}_{20} \mathrm{H}_{17} \mathrm{NO}_{4}, \mathrm{HCl}+2 \mathrm{H}_{2} \mathrm{O}$.

I. 0.2075 gram substance gave $0 \cdot 1057$ gram $\mathrm{H}_{2} \mathrm{O}$ and $0 \cdot 4461$ gram $\mathrm{CO}_{2}$.

II. 0.1557 gram substance gave 0.0792 gram $\mathrm{H}_{2} \mathrm{O}$ and 0.3383 gram $\mathrm{CO}_{2}$.

III. 0.5091 gram substance gave 16.5 c.c. $\mathrm{N}$. Temp. $=17^{\circ}$. Bar. $727 \mathrm{~mm}$.

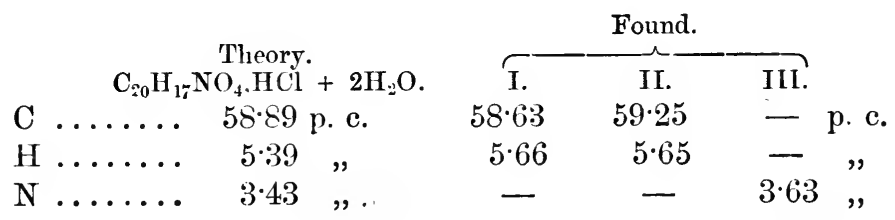

The difficulty in obtaining good results in the analysis of this substance is partly accounted for by the fact that the salt dried as above is so excessively hygroscopic as to render it very difficult to weigh it out with the necessary degree of accuracy.

As the result of a number of careful analyses, E. Schmidt (loc. cit., 
p. 54) has shown that berberine hydrochloride crystallises from dilute alcohol with 4 mols. $\mathrm{H}_{2} \mathrm{O}$.

\section{Oxidation of Berberine with Potassium Permanganate.}

In most of the investigations on the constitution of the alkaloids which have up to the present time been carried out, it has been found that the careful examination of the behaviour of this class of substances towards oxidising agents has almost invariably given interesting results, and in some cases very important clues as to their structure.

The molecule of the alkaloïd is usually split up, yielding acids of much simpler constitution, such as hemipinic acid (from narcotine), carboxylic acids of pyridine, quinoline, and their derivatives (in the case of quinine, cinchonine, \&c.), \&c., although in some cases acids containing nearly the same number of carbon-atoms as the alkaloïd employed are obtained.

One of the most remarkable examples of the value of careful oxidation in investigating an alkaloï is that of papaverine, from which by the use of potassium permanganate under various conditions Goldschmiedt was able to obtain among other substances papaverinic, veratric, hemipinic, pyridinetricarboxylic, and dimethyloxycinchoninic acids and papaveraldine.

Experiments on the oxidation of berberine with potassic permanganate have already been made by E. Schmidt and C. Schilbach (Arch. Pharm. [3], 25, 164-170; Abstr., 1887, 604), who obtained thus considerable quantities of hemipinic acid.

In some preliminary experiments on berberine made early in 1887, I also obtained hemipinic acid in considerable quantities by the oxidation of berberine with alkaline permanganate, and lately, in view of the remarkable results obtained by Goldschmiedt in his examination of hemipinic and isohemipinic acids (Monats., 1888, 861), I have again very carefully studied this decomposition, partly with the object of determining whether the acid obtained is simply hemipinic acid or a mixture of this acid with metahemipinic acid, and partly from a desire to obtain some substance intermediate between berberine and this acid, which might afford some clue to the constitution of the alkaloïd.

The first experiments were carried out almost exactly in the way Goldschmieât recommends in his first paper on papaverine (Monats., 1885, 1112).

30 grams of pure berberine hydrochloride were dissolved in 1 litre of boiling water, the salt decomposed by the addition of 6 grams of potassic carbonate, and then oxidised by slowly running 
in 150 grams of potassic permanganate dissolved in 3 litres of hot water. At first the oxidation is very rapid, but as soon as about two-thirds of the permanganate have been added, decolorisation takes place much more slowly, continued boiling being necessary to remove the last traces of the oxidiser.

At the end of this operation, the product was saturated with carbonic acid, filtered through a cloth filter, and the manganese precipitate well pressed; this was then carefully extracted by repeated boiling with water, and pressing in a vice. The filtrate and washings were evaporated to dryness, the residue pulverised and repeatedly exhausted with boiling absolute alcohol (or purified methylated spirits), the alcoholic solution distilled, and the residue, after freeing it from the last traces of alcohol by gently warming on a water-bath, was dissolved in a little water and filtered. On acidifying with dilute sulphuric acid, the brown aqueous solution deposits a small quantity of a resinous precipitate, from which, however, nothing crystalline could be obtained. It was removed by filtration, the clear filtrate extracted 20 times with pure ether, the ethereal solution dried over calcic chloride and evaporated, and the brownish residue thus obtained was allowed to remain for some days over sulphuric acid in a vacuum. The crude semi-solid mass was then roughly purified by spreading it on a porous plate, and afterwards repeatedly recrystallised from boiling water. In this way beautiful transparent crystals were obtained, which on analysis gave numbers agreeing with those required for hemipinic acid :-

0.1827 gram substance gave 0.0720 gram $\mathrm{H}_{2} \mathrm{O}$ and 0.3550 gram $\mathrm{CO}_{2}$.

\begin{tabular}{|c|c|c|}
\hline & $\begin{array}{l}\text { Theory. } \\
\mathrm{C}_{10} \mathrm{H}_{10} \mathrm{O}_{6} \text {. } \\
53 \cdot 10 \text { per cent. }\end{array}$ & $\begin{array}{l}\text { Found. } \\
52 \cdot 99 \text { per cent. }\end{array}$ \\
\hline & $4 \cdot 42$ & $4 \cdot 38$ \\
\hline & $42 \cdot 48$ & $4 \cdot 2 \cdot 73$ \\
\hline
\end{tabular}

Determination of water of crystallisation :-

0.8878 gram of substance heated to $100^{\circ}$ till constant lost 0.1236 gram $=13.92$ per cent. Theory for $\mathrm{C}_{10} \mathrm{H}_{10} \mathrm{O}_{6}+\mathrm{H}_{2} \mathrm{O}=13 \cdot 74$ per cent.

Considerable difference of opinion exists as to the melting point of hemipinic acid. Wegscheider (Monats., 3, 351) gives $175-179^{\circ}$ for the acid from narcotine; Liebermann (Ber., 19, 2279), 180-181 , and Grüne (Ber., 19, 230) $180^{\circ}$, whereas E. Schmidt and Schilbach (Arch. Pharm., 225, 164) found for the hemipinic acid prepared by them from berberine, and also for the acid from narcotine, the low melting point $160-161^{\circ}$. Goldschmiedt, who has lately most 
carefully re-examined the hemipinic acid from narcotine, found that although when rapidly heated it melted at $172-175^{\circ}$ with decomposition into anhydride and water, yet when slowly beated it melted at $160-161^{\circ}$. I have repeated these experiments with the hemipinic acid from berberine, and although I can corroborate the experience of Goldschmiedt that the melting point varies considerably according to the rapidity of the heating, still I have never been able to observe a melting point lower than $170^{\circ}$. When heated moderately rapidly, the acid obtained as above melts regularly at $177-178^{\circ}$ with decomposition.

In studying the action of small quantities of permanganate on berberine, I subsequently obtained an acid, $\mathrm{C}_{10} \mathrm{H}_{10} \mathrm{O}_{6}$, of totally different appearance, which melted at $159-160^{\circ}$ with decomposition, and thus showed all the properties of hemipinic acid from narcotine.

As I was, therefore, not quite satisfied that the acid obtained as described above, melting at $177-178^{\circ}$, was hemipinic acid and not metahemipinic acid, I carefully examined my product in the way suggested by Goldschmiedt (Monats., 1888, 870).

In his researches on papaverine, Goldschmiedt obtained by the oxidation with alkaline permanganate an acid of the formula $\mathrm{C}_{10} \mathrm{H}_{10} \mathrm{O}_{6}$, which from its properties (m. p. 174-175 , water of crystallisation, \&c.) he at first supposed to be identical with the hemipinic acid from narcotine. The further study of the constitution of papaverine, however, showed that these two acids could not be identical, but must be isomeric merely, a conclusion which was borne out by the further examination of their properties. As both these acids on fusion with potash give protocatechuic acid, and both are orthocarboxylic acids, their constitution must be represented by the formulæ-

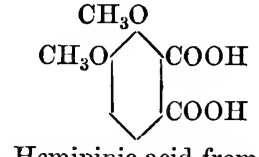

Hemipinic acid from narcotine.

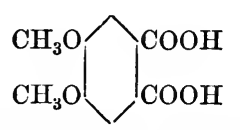

Metahemipinic acid from papaverine.

In examining the hemipinic acid from berberine, I first made a determination of the methoxyl-groups according to Zeisel's method (decomposition with hydrogen iodide and determination of the amount of methyl iodide formed by leading into an alcoholic solution of silver nitrate, and estimating the silver iodide precipitated (Monats., 1883, 704), with the following results :-

0.2740 gram substance dried at $100^{\circ}$ gave 0.7350 gram $\mathrm{AgI}$. 


\section{Found. \\ Theory for \\ $\mathrm{OCH}_{3} \ldots \quad 27 \cdot 74$ per cent. \\ $\mathrm{C}_{10} \mathrm{H}_{10} \mathrm{O}_{6}$. \\ $27 \cdot 43$ per cent.}

In order, then, to prove that the acid from berberine was a derivative of pyrocatechol, a small quantity was decomposed by fusion with potash. 2 grams of the pure acid was dissolved in a little potash, the solution evaporated to dryness, and the residue fused in a silver dish with 50 grams of pure caustic potash and a little water for about half an hour. The resulting almost colourless melt was dissolved in water and acidified with dilute sulphuric acid, during which operation the solution became first blue, then violet, and lastly reddish, but remained clear. It was extracted several times with ether, the ethereal solution dried over calcic chloride, and the ether distilled off; in this way, a crystalline residue was obtained which, after recrystallising once or twice from a little water, gave the following results on analysis :-

0.1217 gram substance dried at $100^{\circ}$ gave 0.0442 gram $\mathrm{H}_{2} \mathrm{O}$ and $0 \cdot 2436$ gram $\mathrm{CO}_{2}$.

\begin{tabular}{|c|c|c|}
\hline & $\begin{array}{l}\text { Theory. } \\
\mathrm{C}_{7} \mathrm{H}_{6} \mathrm{O}_{4} \text {. } \\
54.55 \text { per cent. }\end{array}$ & $\begin{array}{l}\text { Found. } \\
54.59 \text { per cent. }\end{array}$ \\
\hline & $3 \cdot 89$ & 4.03 \\
\hline & $4 \cdot 56$ & $4 \cdot 48$ \\
\hline
\end{tabular}

A determination of the water of crystallisation gave the following results :-

0.3326 gram of air-dry substance dried at $100^{\circ}$ lost 0.0347 gram.

$$
\begin{array}{cl}
\multicolumn{2}{c}{\text { Theory. }} \\
\mathrm{C}_{7} \mathrm{H}_{6} \mathrm{O}_{4}+\mathrm{H}_{2} \mathrm{O} . & \text { Found. } \\
\mathrm{H}_{2} \mathrm{O} \ldots \ldots \ldots \quad 10 \cdot 46 \text { per cent. } & 10 \cdot 43 \text { per cent. }
\end{array}
$$

This acid has, therefore, the composition of protocatechuic acid, with which it agrees in all its properties.

It crystallised from hot water in needles melting at $195-196^{\circ}$. The aqueous solution gives with ferric chloride a deep, emerald-green coloration, which, on the careful addition of sodic carbonate solution, becomes first blue, then violet, and at last red. It reduces an ammoniacal silver solution at once, gives with lead acetate a white precipitate soluble in acetic acid, and on heating is decomposed into carbonic anhydride and a white crystalline sublimate which shows all the reactions of pyrocatechol. These reactions, however, do not determine whether the acid oltained from berberine is hemipinic or metahemipinic acid, as both these acids contain two methoxyl-groups, and 
both on fusion with potash yield protocatechuic acid. These two acids can, however, be readily distinguished by conversion into their ethylimides; the ethylimide of hemipinic acid melting at $96^{\circ}$, whilst that of metahemipinic acid melts at $226-227^{\circ}$.

In order to prepare the ethylimide of the acid from berberine, the pure substance was dissolved in an aqueous solution of ethylamine, the solution evaporated to dryness on a water-batb; and the residue distilled from a small retort. The solid distillate was dissolved in a little boiling methyl alcohol and allowed to stand, when beautiful needle-shaped crystals were obtained, which on analysis gave the following numbers :-

$0 \cdot 1753$ gram substance gave $9 \cdot 7$ c.c. N. Bar. $=760 \mathrm{~mm} . \quad t=$ $14.5^{\circ}$.

$$
\begin{aligned}
& \text { Theory. } \\
& \mathrm{C}_{12} \mathrm{H}_{13} \mathrm{NO}_{4} \text {. Found. } \\
& \text { N. ....... } \quad 5.96 \text { per cent. } \quad 6.09 \text { per cent. }
\end{aligned}
$$

This ethylimide melts at $94-95^{\circ}$, but when recrystallised from water, at $96^{\circ}$, and shows all the properties of ethylhemipinimide, prepared by Liebermann by the action of ethyl iodide on the potassiumderivative of hemipinimide. It is therefore proved beyond doubt that hemipinic acid from berberine is identical with that obtained from narcotine.*

Besides hemipinic acid, there are small quantities of at least two other acids formed when berberine is oxidised with permanganate in the proportions given above, both of which are present in the potassic carbonate residues, from which the potash salt of hemipinic acid has been extracted with alcohol. These residues were dissolved in a small quantity of water, neutralised with acetic acid, and treated with acetate of copper. On long standing in a warm place, the green solntion thus produced deposited a small quantity of a light bluish-green precipitate. This was collected, well washed with water, and decomposed by passing sulphuretted hydrogen through the salt suspended in boiling water; the filtrate, on evaporation, gave a small quantity of a crystalline residue which on examination was found to contain nitrogen.

On recrystallising from water, small nodular crystals were obtained, melting at $238-242^{\circ}$. These gave on analysis the following numbers:0.2629 gram substance gave 14.5 c.c. $\mathrm{N} . \quad \mathrm{Bar}=759 . \quad t=14^{\circ}$.

$$
\begin{aligned}
& \text { Theory. } \\
& \mathrm{C}_{8} \mathrm{H}_{5} \mathrm{NU}_{6}+\mathrm{H}_{2} \mathrm{O} \text {. Found. } \\
& \mathrm{N} \text {....... } 6 \cdot 11 \text { per cent. } \quad 6.48 \text { per cent. }
\end{aligned}
$$

* This confirms the results obtained by E. Schmidt (loc. cit., 63-73). 
Unfortunately the amount of this acid obtained by this reaction was too small to allow of further experiments being made to determine its composition, but from its melting point and analysis it would appear to be either carbocinchomeronic acid or berberonic acid. This is the more probable, as berberine when oxidised by nitric acid yields berberonic acid. It is interesting to notice that $\mathrm{E}$. Smith and C. Schilbach (Arch. Pharm. [3], 25, 164-170) in their experiments on the oxidation of berberine with permanganate, also noticed the formation of a nitrogenous acid, crystallising in nodular crystals, and melting at $218-220^{\circ}$. The silver salt, however, gave numbers showing this acid to be a pyridinemonocarboxylic acid (nicotinic acid?).

The mother-liquor from the copper salt was treated with sulphuretted hydrogen, filtered from the precipitated sulphide of copper, and subacetate of lead added so long as a precipitate was produced. The lead salt was collected, well washed, suspended in water, decomposed with sulphuretted hydrogen, and the filtered solution evaporated to dryness.

The residue, when dissolved in a small quantity of boiling water, and the solution allowed to cool slowly, deposited, after some time, a quantity of crystals ; these, on examination, were found to consist of two distinct substances, the one crystallising in thick, brownish prisms, and the other in long, colourless needles. The brownish prisms were mechanically separated, recrystallised several times from water, and the colourless crystalline mass thus obtained dried at $100^{\circ}$, and analysed with the following result :-

0.1932 gram substance gave 0.0777 gram $\mathrm{H}_{2} \mathrm{O}$ and 0.3763 gram $\mathrm{CO}_{2}$.

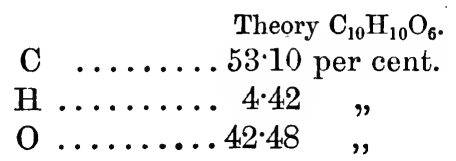

Theory $\mathrm{C}_{10} \mathrm{H}_{10} \mathrm{O}_{6}$. $53 \cdot 10$ per cent.

$42 \cdot 48$
Found.

$53 \cdot 12$ per cent.

As this substance melted at $177-178^{\circ}$, there can be no doubt that it was simply hemipinic acid, with which it agreed in all its reactions.

The needle-shaped crystals obtained from the lead salt together with the hemipinic acid, after recrystallisation from water, were found to consist of pure oxalic acid. It is very remarkable that in the oxidation of berberine with alkaline permanganate so little oxalic should be formed. The amount actually obtained from 60 grams of berberine was but little more than 3 grams.

From the above it will be seen that berberine, when oxidised with an excess of potassium permanganate, yields principally hemipinic 
acid, the other oxidation products isolated being formed only in very small quantity. In this respect, berberine differs very widely from papaverine, which, under similar conditions, yields a number of important decomposition products, such as papaverinic acid, $\mathrm{C}_{16} \mathrm{H}_{13} \mathrm{NO}_{7}$, veratrinic acid, $\mathrm{C}_{9} \mathrm{H}_{10} \mathrm{O}_{4}$, metahemipinic acid, $\mathrm{C}_{10} \mathrm{H}_{10} \mathrm{O}_{6}$, $\alpha$-pyridinetricarboxylic acid, $\mathrm{C}_{8} \mathrm{H}_{5} \mathrm{NO}_{6}$, \&c., all of which are found in considerable quantity in the product of the oxidation. The reason for this is that berberine and its derivatives are so very readily oxidised, that unless a limited amount of permanganate is used, and the oxidation very carefully carried out, the whole molecule is split up, yielding principally hemipinic acid. As the result of a very large number of experiments carried out under a great variety of conditions, it has, however, been found possible to arrest the oxidation before it has gone as far as hemipinic acid, and in this way several new substances have been isolated, the study of which it is hoped will furnish some important clue as to the constitution of this interesting alkaloïd.

In experimenting on the formation of these new derivatives I have found it necessary to work with small quantities at a time (never more than 7 grams of berberine in one operation), as when large quantities are used, even under precisely similar conditions of temperature and amount of oxidiser, very small yields are obtained.

The best results have been obtained with the following quantities :-

5 grams berberine (dried at the ordinary temperature).

9 " potassic permanganate.

$1 \frac{1}{2}, " \quad, \quad$ carbonate.

The potassic permanganate and carbonate are dissolved in half a litre of water of $90^{\circ}$, and the solution very slowly run through a - separating funnel or burette, into a hot solution $\left(90^{\circ}\right)$ of the berberine (in a quarter of a litre of water), the whole being well stirred during the operation. As soon as all the permanganate has been added (the oxidation requires about $10-15$ minutes), the product is transferred to a flask, cooled well under the tap, and the manganese precipitate brought into solution by passing a slow stream of sulphurous acid into the mixture. In this way a slightly yellowish liquid is obtained, in which are suspended small, yellow, flocculent particles. These are collected, well washed with water, and freed from the last traces of mother-liquor by spreading them on a porous plate. The purification of the resulting amorphous yellow mass which contains at least three new substances, is a very difficult operation, and has, up to the present, only been successfully carried out when the following conditions have been carefully observed.

The well washed residue is suspended in water and well agitated 
at a temperature of about $40^{\circ}$, with a slight excess of sodic carbonate, by which treatment the crude product is separated into two portions, the one soluble, the other insoluble in carbonate of soda; these are separated by filtration.

The new acid contained in the brownish-coloured filtrate is deposited on the addition of an acid in the form of an amorphous yellow precipitate, which, on account of the presence of a quantity of a resinous substance, can only with difficulty be obtained in a crystalline condition. The method of purification which has given the best results is the following:-The solution in sodic carbonate is nearly neutralised with dilute sulphuric acid, and the new acid fractionally precipitated in three fractions by running into the well-stirred solution a fine stream of very dilute sulphuric acid. The first fraction should be about one-fourth, the second one-half, the third one-fourth of the whole. The second fraction is carefully washed, dried on a porous plate, dissolved in a little methyl alcohol, and the solution transferred to a flat dish, and allowed to evaporate completely at the ordinary temperature. During the evaporation, the new acid separates in yellow nodular masses, covered with a transparent resin, which is invariably deposited along with the crystals. The resulting mixture of acid and resin is scraped off the sides of the dish and well kneaded between the fingers with a small quantity of methyl alcohol. In this way the resin is removed, leaving the warty masses almost entirely undissolved. The purified product is now dissolved in a small quantity of boiling methyl alcohol, from which it is deposited on cooling in almost colourless nodular masses, which, after repeated recrystallisation, gave the following results on analysis :-

I. 0.1511 gram substance gave 0.0602 gram $\mathrm{H}_{2} \mathrm{O}$ and 0.3192 gram $\mathrm{CO}_{2}$.

II. 0.1421 gram substance gave 0.0552 gram $\mathrm{H}_{2} \mathrm{O}$ and 0.3000 gram $\mathrm{CO}_{2}$.

III. 0.3633 gram substance gave 10.4 c.c. N. $t=11^{\circ}$. Bar. $=$ $727 \mathrm{~mm}$.

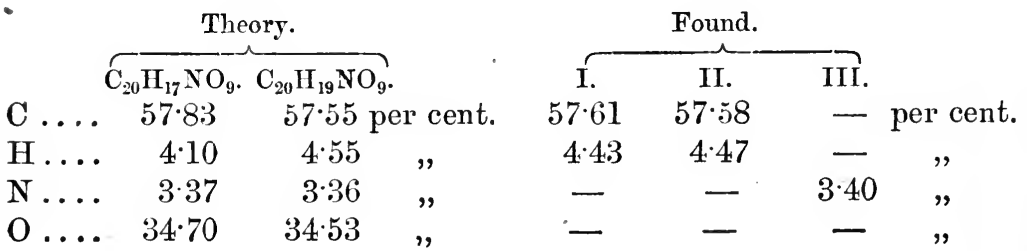

It is as yet very difficult to decide whether this new substance has the formula $\mathrm{C}_{20} \mathrm{H}_{17} \mathrm{NO}_{9}$ or $\mathrm{C}_{20} \mathrm{H}_{19} \mathrm{NO}_{9}$, but for various reasons $\mathrm{I}$ am 
inclined, in the meantime, to adopt the latter formula, leaving the definite settlement of this point to further experimental proof.

This substance is readily soluble in ethyl and methyl alcohol and in acetic acid, but only sparingly in benzene, toluene, and ether, and almost insoluble in light petroleum, chloroform, ethyl acetate, and cold water. It is, however, best purified by recrystallisation from methyl alcohol, from which it is deposited, on long standing, either in warty masses or in small plates.

Considerable difficulty was experienced in determining the melting point of this new substance. When rapidly heated, it softens at about $138-140^{\circ}$, and then melts completely at $141-142^{\circ}$ with evolution of gas. At $150-155^{\circ}$ it again becomes solid, and on further heating melts again between $195^{\circ}$ and $200^{\circ}$.

The pure compound, $\mathrm{C}_{20} \mathrm{H}_{19} \mathrm{NO}_{9}$, dissolves readily in alkalis and in alkaline carbonates.

The barium salt was prepared by dissolving the acid in barytawater, removing the excess of the latter by passing carbonic anhydride through the boiling solution, filtering, and evaporating on a waterbath. In this way a quantity of an almost colourless crystalline barium salt was obtained which, after washing with a little water and drying first over sulphuric acid in a vacuum and then at $100^{\circ}$, gave the following results on analysis :-

$0 \cdot 4598$ gram substance gave $0 \cdot 1926$ gram $\mathrm{BaSO}_{4}$.

$$
\begin{aligned}
& \text { Theory. } \\
& \mathrm{C}_{20} \mathrm{H}_{17} \mathrm{NO}_{9} \mathrm{Ba} \text {. Found. } \\
& \mathrm{Ba} \text {.. 24.82 per cent. } \quad \mathrm{Ba} \ldots 24.63 \text { per cent. }
\end{aligned}
$$

The neutral solution of the ammonium salt of this acid gives with nitrate of silver a white, gelatinous precipitate, soluble in a large quantity of boiling water: with cupric acetate a beantiful bluishgreen precipitate, and, with acetate of lead, a white, gelatinous precipitate. Fused with a small quantity of pure caustic potash, this substance is readily decomposed, yie!ding an almost colourless melt which, when dissolved in water, acidified with sulphuric acid, and extracted with ether, gives a crystalline acid readily soluble in water. The solution of this acid gives with ferric chloride a deep-green coloration, changing to violet and then to deep red on the addition of sodic carbonate. As it also gives with acetate of lead a white precipitate, and readily reduces ammoniacal silver solution, it is probable that this substance is either berberinic acid or protocatechuic acid.

The residual product of the oxidation of berberine, insoluble in sodic carbonate, obtained as described above, contains, besides a quantity of a resinous substance, two new compounds which are separated in the following way :- 
The crude, brownish product is well washed, first with a dilute solution of sodic carbonate, then several times with water, dried on a porous plate, and dissolved in a small quantity of boiling 90 per cent. acetic acid ( 5 grams of substance require about 20 grams of acetic acid). This solution on cooling gradually deposits a considerable quantity of a crystalline substance. At the end of 48 hours, this is collected, washed with a little glacial acetic acid, and purified by repeated recrystallisation from this solvent.* It is thus obtained in flat, glittering plates, with a remarkable silky lustre: these after drying, first on a porous plate and then at $100^{\circ}$, gave the following results on analysis :-

I. 0.2001 gram substance gave 0.0800 gram $\mathrm{H}_{2} \mathrm{O}$ and 0.4387 gram $\mathrm{CO}_{2}$.

II. 0.1395 gram substance gave 0.0589 gram $\mathrm{H}_{2} \mathrm{O}$ and 0.3053 gram $\mathrm{CO}_{2}$.

III. 0.1354 gram substance gave 0.0550 gram $\mathrm{H}_{2} \mathrm{O}$ and 0.2971 gram $\mathrm{CO}_{2}$.

IV. $0 \cdot 1560$ gram substance gave 0.0620 gram $\mathrm{H}_{2} \mathrm{O}$ and 0.3433 gram $\mathrm{CO}_{2}$.

V. 0.3773 gram substance gave 11.2 c.c. $\mathrm{N}\left(t=13^{\circ}\right.$. Bar. $=$ $760 \mathrm{~mm}$.).

VI. 0.3372 gram substance gave 10.6 c.c. $\mathrm{N}\left(t=13.5^{\circ}\right.$. Bar. $=$ $737 \mathrm{~mm}$.$) .$

Found.

Theory. $\mathrm{C}_{20} \mathrm{H}_{17} \mathrm{NO}_{8}$.

C.... $60 \cdot 15$ p. c.

H... 4.26,

$\mathrm{N} \ldots . .3 \cdot 5 \mathrm{l}$,

O ... 32.08,
Found.

$\begin{array}{ccccccc}\text { I. } & \text { II. } & \text { III. } & \text { IV. } & \text { V. } & \text { VI. } \\ 59.87 & 59.69 & 59 \cdot 77 & 60.02 & - & - & \text { p. c. } \\ 4.44 & 4.53 & 4.51 & 4.41 & - & - & " \\ - & - & - & - & 3.50 & 3.58 & " \\ - & - & - & - & - & - & "\end{array}$

This substance melts at $236-237^{\circ}$. It is readily soluble in hot acetic acid, from which it crystallises on cooling in beautiful glittering plates, but is only sparingly soluble in ethyl and methyl alcohol, benzene, toluene, light petroleum, and acetone, even on boiling. Although insoluble in alkaline carbonates, this substance dissolves readily in warm solutions of ammonia, caustic potash, \&c., forming well-defined salts. If the solution in ammonia be allowed to evaporate over sulphuric acid, a very curious jelly-like ammonium salt is deposited, which gradually dries up to a mass resembling dried albumin. This dissolves readily in water, and the solution gives with reagents some very characteristic salts.

* The mother-liquors contain considerable quantities of a second neutral substance, which will be described further on. 
Nitrate of silver gives a white, gelatinous precipitate which is readily soluble in hot water, but only sparingly in cold; it could not be recrystallised, as it somewhat readily decomposes on warming with water. A small quantity of this salt was prepared, well washed with cold water, dried on a piece of porous plate over sulphuric acid in a vacuum, and analysed with the following results :-

I. 0.1996 gram substance gave 0.0560 gram $\mathrm{H}_{2} \mathrm{O}, 0.2547$ gram $\mathrm{CO}_{2}$, and 0.0623 gram $\mathrm{Ag}$.

II. 0.3286 gram substance gave 0.1023 gram $\mathrm{Ag}$.

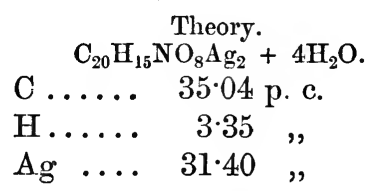

Found.

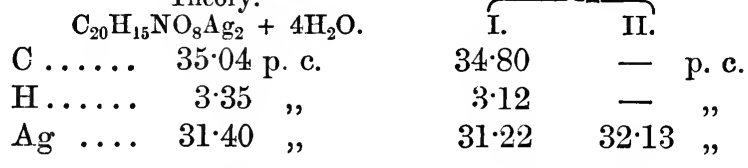

From this analysis it would appear as though the neutral substance, $\mathrm{C}_{20} \mathrm{H}_{17} \mathrm{NO}_{8}$, when dissolved in alkalis took up 4 mols. $\mathrm{H}_{2} \mathrm{O}$, forming a bibasic acid of the formula $\mathrm{C}_{20} \mathrm{H}_{25} \mathrm{NO}_{12}$. It is, however, much more probable that. the silver salt when prepared as above contains water of crystallisation, as is the case, for example, with the silver salt of

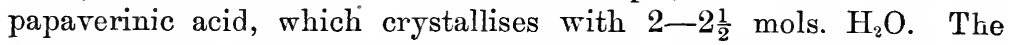
formula of the acid formed by dissolving the substance $\mathrm{C}_{20} \mathrm{H}_{17} \mathrm{NO}_{8}$ in alkalis, can therefore only be accurately determined by the analysis of some other of its salts, with the investigation of which I am at present engaged.

The solution of the ammonium salt of this acid gives with acetate of copper a splendid, light bluish-green precipitate, and with acetate of lead a white, gelatinous precipitate which dissolves somewhat sparingly in boiling water. The chlorides of barium and calcium give no precipitate in the cold; on boiling, white, amorplious salts are precipitated.

The mother-liquors from the precipitation of the substance $\mathrm{C}_{20} \mathrm{H}_{17} \mathrm{NO}_{8}$ contain, besides small traces of this sparingly soluble substance, a considerable quantity of a second neutral substance which is much more readily soluble. To isolate this, the dark-brown acetic acid solution is heated to boiling, diluted with one-fourth of its bulk of boiling water, and allowed to stand for 24 hours. The crystals which separate are collected, the mother-liquor again treated as above, and the operation repeated until no more crystals separate. In this way nearly the whole of the new substance crystallises out in yellowish plates, whereas if too much water be added at first the crystals are invariably mixed with a black, tarry substance which hinders their subsequent purification. The crude substance thus obtained can now 
easily be purified by recrystallisation, once from dilute acetic acid and twice from pure alcohol. From the hot, alcoholic solution it is deposited on cooling in beautiful colourless plates with a pearly lustre. For analysis the substance was dried at $100^{\circ}$.

I. 0.1372 gram substance gave 0.0540 gram $\mathrm{H}_{2} \mathrm{O}$ and 0.3155 gram $\mathrm{CO}_{2}$.

II. 0.1792 gram substance gave 0.0741 gram $\mathrm{H}_{2} \mathrm{O}$ and 0.4093 gram $\mathrm{CO}_{2}$.

III. $0 \cdot 1395$ gram substance gave 0.0584 gram $\mathrm{H}_{2} \mathrm{O}$ and 0.3213 gram $\mathrm{CO}_{2}$.

IV. $0 \cdot 1680$ gram substance gave 0.0720 gram $\mathrm{H}_{2} \mathrm{O}$ and 0.3852 gram $\mathrm{CO}_{2}$.

V. $0 \cdot 3070$ gram substance gave $10 \cdot 1$ c.c. $\mathrm{N}\left(t=15^{\circ}\right.$. Bar. $=$ $742 \mathrm{~mm}$.$) .$

VI. 0.2493 gram substance gave $8 \cdot 2$ c.c. $\mathrm{N}\left(t=18 \cdot 5^{\circ}\right.$. Bar. $=$ $759 \mathrm{~mm}$.$) .$

Found.

\begin{tabular}{|c|c|c|c|c|c|c|c|c|c|}
\hline \multirow{2}{*}{\multicolumn{3}{|c|}{$\begin{array}{c}\text { Theory. } \\
\mathrm{C}_{20} \mathrm{H}_{1} \mathrm{NO}_{7} \text {. }\end{array}$}} & \\
\hline & & & . & 11. & III. & ( & $\mathrm{V}$. & VI. & \\
\hline & $62 \cdot 66$ & o. c. & $62 \cdot 71$ & $62 \cdot 61$ & $62 \cdot 81$ & 62 & - & - & p. c. \\
\hline & $4: 43$ & $"$ & $4: 38$ & $4 \cdot 70$ & $4 \cdot 65$ & 4.76 & - & - & ", \\
\hline & $3 \cdot 66$ & $"$ & - & - & - & - & $3 \cdot 75$ & $3 \cdot 80$ & " \\
\hline & $29 \cdot 24$ & , & - & - & - & - & - & - & \\
\hline
\end{tabular}

This new compound, which from the analysis appears to have the formula $\mathrm{C}_{20} \mathrm{H}_{17} \mathrm{NO}_{7}$, melts at $150^{\circ}$. It is readily soluble in hot methyl and ethyl alcohol, but only sparingly soluble in these liquids in the cold. It dissolves with difficulty in boiling water, and the solution on slowly cooling deposits the substance in beautiful, glittering plates, which resemble anthracene in appearance; if rapidly cooled, however, the aqueous solution becomes milky before crystallising. It is also readily soluble in hot, but almost insoluble in cold benzene and toluene. If this substance is not quite pure, its solutions fluoresce in a most beautiful way, reminding one strongly of a dilute solution of fluoresceïn.

The substance $\mathrm{C}_{20} \mathrm{H}_{17} \mathrm{NO}_{7}$ does not dissolve in alkalis in the cold, and only very slowly on boiling. The solution in hot, strong caustic potash on cooling deposits what appears to be the unchanged substance, but as the substance is more soluble in caustic potash than in water, it is possible that it forms with alkalis very unstable salts. Fused with a little potash, the substance $\mathrm{C}_{20} \mathrm{H}_{17} \mathrm{NO}_{7}$ yields what appears to be protocatechuic acid (? berberinic acid). In studying this decomposition, about $\frac{1}{4}$ of a gram of substance was gently fused with 2 grams of potash and a little water in a thick test-tube. The crystals dissolved at once with evolution of quantities of a pungent 
gas, which resembled methylamine or ethylamine rather than ammonia. The colourless residue dissolved in water with a reddish colour, and on acidifying and extracting with ether, a colourless crystalline substance was obtained, which showed all the properties of protocatechuic acid. The aqueous solution gave with ferric chloride a deep-green coloration, which on the addition of sodic carbonate became first blue, then violet, and at last red. On the addition of acetate of lead to the aqueous solution, a white precipitate was obtained, soluble in acetic acid.

The substance $\mathrm{C}_{20} \mathrm{H}_{17} \mathrm{NO}_{7}$ dissolves in strong nitric acid with a deep orange colour, and in warm sulphuric acid forming a deep violet-black solution. The addition of strong hydrochloric acid causes the crystals to turn yellow without alissolving them, and if a drop of hydrochloric acid be added to the alcoholic solution of the substance the solution is coloured slightly yellow, and acquires a bluish-green fluorescence.

In order to further control the formula of the substance $\mathrm{C}_{20} \mathrm{H}_{17} \mathrm{NO}_{7}$, its molecular weight was determined by Raoult's method, with the following result :-

\begin{tabular}{|c|c|}
\hline Substance taken & 0.7558 gram. \\
\hline Acetic acid ..... & $42 \cdot 1296$ \\
\hline Meiting point of acetic acid. & $16 \cdot 447^{\circ}$ \\
\hline of mixture... & $16 \cdot 263$ \\
\hline Depression of melting point. & $0 \cdot 184$ \\
\hline
\end{tabular}

Molecular weight of substance found from the above data 380 Theory for $\mathrm{C}_{20} \mathrm{H}_{17} \mathrm{NO}_{7} \ldots \ldots \ldots \ldots \ldots \ldots \ldots \ldots . \ldots . \ldots 383$

The yield of crude, dry, yellow substance obtained by the oxidation of 200 grams of berberine as described above is about 70 grams, of which 12 grams are insoluble, and 55 grams soluble in a solution of sodic carbonate. These numbers show clearly that a large portion of the products of the oxidation must be contained in the filtrate from the yellow substance. In order to test this, the yellow solution was evaporated over a free flame, with occasional addition of a little aqueous sulphurous acid, until the inorganic salts commenced to separate as a crust on the surface. The liquid was then allowed to cool, filtered from the crystalline matter, and again evaporated, the operation being repeated until the solution from the oxidation of 200 grams of berberine was reduced in bulk to 1 litre. The brownish product was then extracted 20 times with ether, the ethereal solution dried over calcic chloride, and the ether distilled off until about 150 c.c. remained.

During this operation a quantity of a white, crystalline substance separates, which after collecting, washing with a little ether, and 
drying on a porous plate, presented the appearance of hard, sandy crystals. Subsequent examination showed that this product was a mixture of two substances, which are readily separated from each other by recrystaWisation from water. The solution in a small quantity of boiling water deposits on cooling first of all a quantity of glittering plates, which are rapidly filtered off. On standing, the second substance separates from the mother-liquor in hard prisms.

The more insoluble substance, which is formed only in very small quantity, was purified by recrystallisation from water; the crystals, dried first over sulphuric acid in a vacuum and then at $100^{\circ}$, gave the following numbers on analysis :-

I. $0 \cdot 1493$ gram substance gave 0.0618 gram $\mathrm{H}_{2} \mathrm{O}$.

II. $0.2907 \quad " \quad$ " 8.8 c.c. $\mathrm{N} ; t=17^{\circ}$. Bár. $=$ $757 \mathrm{~mm}$.

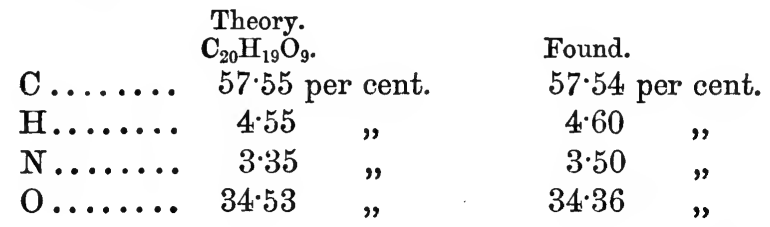

This interesting substance, which from the above analysis appears to have the constitution $\mathrm{C}_{20} \mathrm{H}_{19} \mathrm{NO}_{9}$, melts at $178-179^{\circ}$ with decomposition, due probably to loss of water. It is sparingly soluble in alcohol and ether, fairly soluble in boiling water, from which it crystallises on cooling, either in small plates, or in very small, warty masses.

At present only sufficient of this substance has been obtained for the above analyses, and subsequent investigation must show whether the constitution assigned to it is correct.

This acid has the same formula as the acid obtained from the yellow precipitate (p. 78), but as it melts $38^{\circ}$ higher it must be isomeric, and not identical with this substance.

The crude crystals of the more soluble substance obtained, as described above, were separated from the substance $\mathrm{C}_{20} \mathrm{H}_{17} \mathrm{NO}_{9}$ by repeated recrystallisation from water, from which they were invariably deposited as a hard, crystalline crust on the bottom of the beaker. The air-dried crystals, when heated on a water-bath, lost scarcely any weight, showing the absence of water of crystallisation.

On analysis the following nambers were obtained :-

I. 0.1518 gram substance gave 0.0608 gram $\mathrm{H}_{2} \mathrm{O}$ and 0.2970 gram $\mathrm{CO}_{2}$.

II. 0.1720 gram substance gave 0.0701 gram $\mathrm{H}_{2} \mathrm{O}$ and 0.3361 gram $\mathrm{CO}_{2}$. 


\begin{tabular}{|c|c|c|c|}
\hline \multirow{2}{*}{\multicolumn{2}{|c|}{$\begin{array}{l}\text { Theory. } \\
\mathrm{C}_{10} \mathrm{H}_{10} \mathrm{O}_{6} \text {. }\end{array}$}} & \multicolumn{2}{|c|}{ Found. } \\
\hline & & I. & II. \\
\hline & $53 \cdot 10$ p. c. & $53 \cdot 36$ & $53 \cdot 28$ p. c. \\
\hline & 4.42, & $4 \cdot 45$ & 4.52 \\
\hline $0 \ldots$. & $42 \cdot 48 \quad$ & $42 \cdot 19$ & $42 \cdot 20$ \\
\hline
\end{tabular}

This substance melts at $159-160^{\circ}$ with decomposition, and as it gives protocatechuic acid on fusion with potash, there can scarcely be a doubt that it is hemipinic acid. It is, however, in appearance totally different from the hemipinic acid previously obtained (see p. 73). The crystals, which contain no water of crystallisation, are very soluble in hot water, and separate from the solution very slowly indeed, the deposition often being incomplete after standing for 24 hours.

The hemipinic acid obtained as described at the commencement of this chapter, and also a sample of the same substance prepared from narcotine (for which I am indebted to Dr. Goldschmiedt), crystallise rapidlý with 2 mols. $\mathrm{H}_{2} \mathrm{O}$ of crystallisation, when the hot aqueous solution is allowed to cool moderately slowly. The crystals dried at $100^{\circ}$, and heated under the same conditions as the above, melt at $177-178^{\circ}$ with decomposition.

The great difference in the properties of the two preparations lead me to doubt their identity, and in order to establish this the silver salt of the acid, melting at $159-160^{\circ}$, was prepared and examined. This salt is thrown down on the addition of silver nitrate to the ammonium salt as a heavy, white precipitate. It was well washed with water, dried over sulphuric acid in a vacuum, and then analysed with the following result:-

I. 0.2928 gram substance gave 0.1430 gram Ag.

II. $0 \cdot 2505$

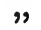

$"$

$0 \cdot 1222$

"

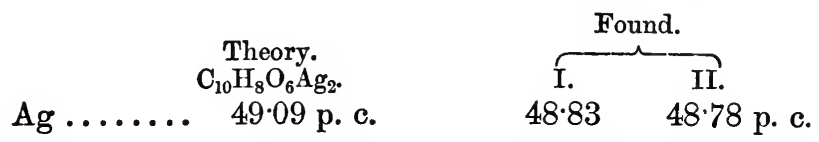

This salt gives therefore the same numbers as those required for the silver salt of hemipinic acid.

Subsequent experiments on these two preparations of hemipinic acid make it appear probable that this substance exists in two distinct forms, the one meliing at $159-160^{\circ}$, and the other at $177-178^{\circ}$. If a crystal of the latter acid be added to a warm saturated solution of the former, in some cases at least, the one modification is completely converted into the other, but this change appears only to take place under certain conditions of concentration, \&c. 
I hope to be able to investigate this point more completely at an early date.

\section{Action of Hydrogen Iodide on Berberine.}

When heated with fuming aqueous hydrogen iodide, berberine is readily decomposed with evolution of methyl iodide, and formation of a new substance, which dissolves in alkalis with a blackish-violet coloration. This preliminary experiment led me to suppose that berberine is decomposed by hydrogen iodide in a way similar to papaverine, which under this treatment is split up into papaveroline, $\mathrm{C}_{16} \mathrm{H}_{13} \mathrm{NO}_{4}$, and 4 mols. of methyl iodide. In determining the constitution of berberine, it was a matter of great importance to discover the number of $\mathrm{OCH}_{3}$ groups contained in the formula $\mathrm{C}_{20} \mathrm{H}_{17} \mathrm{NO}_{4}$, and this is readily done by Zeisel's method (Monats., 6, 995), which consists in decomposing the alkaloïd with fuming hydrogen iodide, and determining the amount of methyl iodide formed by means of alcoholic silver nitrate. The first experiments in this direction were made with berberine itself, with the following results :-

I. 0.2930 gram of berberine (dried at $100-110^{\circ}$ ) gave 0.3990 gram AgI.

II. 0.3315 gram of berberine (dried at $100-110^{\circ}$ ) gave 0.4440 gram AgI.

III. 0.3290 gram of berberine (dried at $100-110^{\circ}$ ) gave 0.4430 gram AgI.

Theory for $2\left(\mathrm{OCH}_{3}\right)$ groups in $\left(\mathrm{OCH}_{3}\right) \ldots .17 \cdot 56$ p. c.

Found.

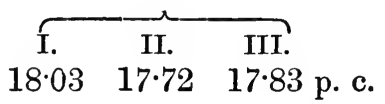

These experiments show that berberine contains only two $\mathrm{OCH}_{3}$ groups in its molecule, these two being present in the portion which on oxidation yields hemipinic acid. Owing, however, to the difficulty in determining accurately the amount of water of crystallisation present in the samples of berberine used, these results were not considered perfectly trustworthy, and in order to be quite certain similar experiments were instituted with the nitrate and hydriodide of the base, these salts being so readily obtained pure and free from water of crystallisation.

I. 0.3903 gram berberine nitrate, dried at $100^{\circ}$, gave 0.4669 gram $\mathrm{AgI}$.

Theory for $2\left(\mathrm{OCH}_{3}\right)$ groups in

$\left(\mathrm{OCH}_{3}\right) \ldots \quad 15 \cdot 59$ per cent.

Found.

$15 \cdot 74$ per cent. 
II. 0.4401 gram berberine hydriodide gave 0.4520 gram AgI.
Theory for $2\left(\mathrm{OCH}_{3}\right)$ groups in

$$
\mathrm{C}_{20} \mathrm{H}_{17} \mathrm{NO}_{4}, \mathrm{HI} \text {. }
$$
$\left(\mathrm{OCH}_{3}\right) \ldots \quad 13 \cdot 39$ per cent.
Found.
$13 \cdot 61$ per cent.

In order, if possible, to isolate the phenolic substance formed during this reaction, the experiment was carried. out with larger quantities. 25 grams of pure berberine were boiled with a considerable excess of fuming aqueous hydrogen iodide in a flask connected with a reflux condenser, until evolution of methyl iodide had ceased.

The dark-coloured solution on cooling deposited a small quantity of the new substance in the form of blackish-coloured nodules. These were collected, the solution distilled from a small retort until half had passed over and again allowed to cool, when a further quantity of solid was obtained. The crude product was well washed with water till free from hydrogen iodide, and ground to a paste in a mortar with a weak solution of sulphurous acid, to remove a small quantity of iodine. The yellowish-brown emulsion was now collected on a filterpump, well washed with water, and extracted with boiling water containing a little sulphurous and sulpharic acids. The hot solution, after filtering from a quantity of a brown insoluble residue, deposited the new substance on cooling in beautiful orange-coloured, amorphous flocks, which entirely filled the liquid. These were collected, reprecipitated twice from hot water containing sulphurous acid, dried over sulphuric acid in a vacuum, and analysed, with the following result :-

I. 0.1478 gram substance gave 0.0582 gram $\mathrm{H}_{2} \mathrm{O}$ and 0.3097 gram $\mathrm{CO}_{2}$.

II. $0 \cdot 1546$ gram substance gave 0.0631 gram $\mathrm{H}_{2} \mathrm{O}$ and 0.3244 gram $\mathrm{CO}_{2}$.

III. 0.4354 gram substance gave $15 \cdot 2$ c.c. $\mathrm{N} ; t=0.12$. Bar. $=$ $740 \mathrm{~mm}$.

IV. 0.4086 gram, decomposed by nitric acid according to Carius' method, gave $0 \cdot 1314$ gram $\mathrm{BaSO}_{4}$.

V. 0.4401 gram, decomposed by nitric acid according to Carins' method, gave $0 \cdot 1412$ gram $\mathrm{BaSO}_{4}$.

Ia. 0.2784 gram substance, heated to $100^{\circ}$ till constant, lost 0.0123 gram.

II $a .0 \cdot 2502$ gram substance, heated to $100^{\circ}$ till constant, lost 0.0112 gram. 
W. H. PERKIN, JUN., ON BERBERINE.

Found.

\begin{tabular}{|c|c|c|c|c|c|c|c|c|}
\hline & & & & & & & & \\
\hline$\left(\mathrm{C}_{18} \mathrm{H}_{13} \mathrm{~N}\right.$ & $\left.{ }_{4}\right)_{2}, \mathrm{H}_{2} \mathrm{SO}$ & $+2]$ & . I. & II. & III. & IV. & $\vec{v}$. & \\
\hline$C \ldots \ldots$ & $57 \cdot 75$ & c. & $57 \cdot 12$ & $57 \cdot 22$ & - & - & - & \\
\hline $\mathrm{H}$. & $4 \cdot 28$ & " & $4 \cdot 37$ & $4 \cdot 60$ & - & - & - & \\
\hline $\mathrm{N}$. & $3 \cdot 74$ & $"$ & - & - & $4 \cdot 01$ & - & 一 & \\
\hline $\mathrm{H}_{2} \mathrm{SO}_{4} \ldots$ & $13 \cdot 10$ & 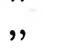 & - & - & - & $13 \cdot 52$ & $13 \cdot 45$ & \\
\hline $\mathrm{H}_{2} \mathrm{O} \ldots$ & $4: 81$ & ," & $4 \cdot 43$ & $4 \cdot 47$ & - & - & - & \\
\hline
\end{tabular}

This substance is therefore the sulphate of a base of the formula $\mathrm{C}_{18} \mathrm{H}_{13} \mathrm{NO}_{4}$, for which I propose the name berberoline.

This base is formed from berberine simply by substituting hydrogen for methyl in each of the two methoxy-groups; thus :-

$$
\begin{array}{cc}
\mathrm{C}_{18} \mathrm{H}_{11} \mathrm{NO}_{2}\left(\mathrm{OCH}_{3}\right)_{2} & \mathrm{C}_{18} \mathrm{H}_{11} \mathrm{NO}_{2}(\mathrm{OH})_{2} . \\
\text { Berberine. } & \text { Berberoline. }
\end{array}
$$

The sulphate of this base is stable, and does not appear to undergo decomposition even on long standing. In the presence of a trace of alkali, decomposition sets in at once owing no doubt to oxidation-a dark, blackish-violet solution being formed. An aqueous solution of the sulphate shows the following reactions :-

Chlorine-water gives a dark-violet, flocculent precipitate. Nitric acid gives a magnificent violet coloration which on standing-and particularly on warming-becomes a deep, reddish-brown. Berberoline solutions decompose solutions of platinic chloride, auric chloride, and ammoniacal nitrate of silver with separation of the metal.

\section{Fusion of Berberine with Potash.}

Hlasiwetz and Gilm (Jahresbericht, 1864, 407), who were the first to study the action of melted potash on berberine, obtained by this decomposition two new substances, that is, berberinic acid, $\mathrm{C}_{8} \mathrm{H}_{8} \mathrm{O}_{4}+$ $\mathrm{H}_{2} \mathrm{O}$, and an acid of the formula $\mathrm{C}_{9} \mathrm{H}_{8} \mathrm{O}_{5}$. With the object of determining the constitution of these two acids, I have again taken up the study of this important decomposition, the experiment being conducted as follows:-

100 grams of caustic potash and 5 c.c. of water were heated in a silver dish to about $180^{\circ}$ and then 60 grams of berberine slowly added in small portions at a time. The berberine on coming in contact with the melted potash was rapidly decomposed-little black balls being formed at first, which swam about on the surface of the liquid, but on stirring, as the temperature gradually rose (to $240^{\circ}$ ), these dissolved, forming a dark-brown liquid. During the operation-which lasts about an hour-a quantity of thick vapours, smelling strongly of ammonia and guaiacol, were evolved. The pro- 
duct was dissolved in water, acidified with dilute sulphuric acid, filtered from a quantity of brownish flocks which separated, evaporated to a syrupy consistence, and well agitated with about twice its volume of strong alcohol. The potassic sulphate thus precipitated was filtered off, washed with a little alcohol, the combined alcoholic solutions gently evaporated on a water-bath, and the residue, dissolved in a little water, was repeatedly extracted with ether. The ethereal extract after carefully drying over calcic chloride and evaporating deposited 5 grams of a dark-brownish, crystalline mass consisting of impure berberinic acid. This was purified first by spreading it on a porous plate and then by repeated recrystallisation from water. The beantiful white crystals thus obtained gave on analysis the following numbers :-

I. 0.1506 gram substance dried at $80^{\circ}$ gave 0.0667 gram $\mathrm{H}_{2} \mathrm{O}$ and 0.3160 gram $\mathrm{CO}_{2}$.

II. $0 \cdot 1683$ gram substance dried at $80^{\circ}$ gave 0.0735 gram $\mathrm{H}_{2} \mathrm{O}$ and 0.3521 gram $\mathrm{CO}_{2}$.

\begin{tabular}{|c|c|c|c|}
\hline \multirow[b]{3}{*}{ C $\ldots . . .}$. & \multirow{2}{*}{$\begin{array}{l}\text { Theory. } \\
\mathrm{C}_{8} \mathrm{H}_{8} \mathrm{O}_{4} \text {. }\end{array}$} & \multicolumn{2}{|c|}{ Found. } \\
\hline & & I. & II. \\
\hline & $57 \cdot 15$ p. c. & $57 \cdot 22$ & 57.05 р. с \\
\hline H. & $4 \cdot 76 \quad$, & $4 \cdot 92$ & $4: 84$ \\
\hline $0 \ldots \ldots$ & $38 \cdot 04 \quad$, & $37 \cdot 86$ & $38 \cdot 11$ \\
\hline
\end{tabular}

Berberinic acid appears to crystallise with 1 mol. $\mathrm{H}_{2} \mathrm{O}$, which, however, is gradually given off at the ordinary temperatures if the crystals are placed over sulphuric acid in a vacuum. The melting point of this acid, which is not given by Hlasiwetz and Gilm, I found to be about $165^{\circ}$. No accurate melting point can be given, as when heated the substance softens at $145^{\circ}$, and then melts with evolution of carbonic anhydride at about $165^{\circ}$; this temperature may therefore be looked upon as its decomposing point rather than its melting point.

The other properties of this acid agree in all respects with those given by Hlasiwetz and Gilm.

If a drop of ferric chloride is added to an aqueous solution of berberinic acid, a splendid dark-green coloration is produced which, on the addition of sodic carbonate solution, becomes first violet, then violet-red, and at last deep-red coloured. This reaction, and the fact that this acid reduces Fehling's solution and an ammoniacal solution of silver nitrate, and gives with acetate of lead a white precipitate soluble in acetic acid, shows at once that berberinic acid must be very closely related to protocatechuic acid.

As protocatechuic acid on distillation is split up into pyrocatechol and carbonic acid, it was thought that, under similar treatment, 
berberinic acid might give some decomposition-product which would afford some clue as to its constitution. In order to test this, 2 grams of the pure dry acid were heated in a small retort until the evolution of carbonic anhydride had ceased, and the residue then rapidly distilled. In this way an almost colourless syrup was obtained, which on analysis gave the following numbers:-

0.1460 gram substance gave 0.0842 gram $\mathrm{H}_{2} \mathrm{O}$ and 0.3605 gram $\mathrm{CO}_{2}$.

\begin{tabular}{|c|c|c|}
\hline & $\begin{array}{l}\text { Theory. } \\
\mathrm{C}_{7} \mathrm{H}_{8} \mathrm{O}_{2} \text {. } \\
67 \cdot 74 \text {. per cent. }\end{array}$ & $\begin{array}{l}\text { Found. } \\
67 \cdot 37 \text { oer cent. }\end{array}$ \\
\hline & $6 \cdot 46$ & $6 \cdot 4 \mathrm{~L}$ \\
\hline 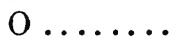 & $25 \cdot 80$ & $26 \cdot 22$ \\
\hline
\end{tabular}

This substance has, therefore, the same formula as homopyrocatechol, with which it agrees in all its reactions. It is readily soluble in water; the solution reduces Fehling's solution and ammoniacal nitrate of silver. Ferric chloride produces a deep-green coloration which, on the addition of sodic carbonate, becomes reddish-violet. There can, therefore, be no doubt that berberinic acid on distillation is split up into homopyrocatechuic and carbonic acid, thus :-

$$
\mathrm{C}_{8} \mathrm{H}_{8} \mathrm{O}_{4}=\mathrm{C}_{7} \mathrm{H}_{8} \mathrm{O}_{4}+\mathrm{CO}_{2} \text {, }
$$

but it is at present difficult to decide the positions of the carboxylgroup with reference to the $\mathrm{OH}$ and $\mathrm{CH}_{3}$-groups.

I am at present engaged on the further investigation of oxidising agents on berberine and on the alkyl addition-products of tetrahydroberberine, and also on a series of experiments on the constitution of berberonic acid.

The completion of these experiments will be somewhat delayed owing to an unfortunate fire in the laboratories of the Heriot Watt College having consumed a number of new products which had already been prepared. For this reason also I have not been able to complete the examination of the substances described in this paper so thoroughly as I could have wished.

I hope, however, at an early date to be able to communicate to the Society a further paper on this interesting alkaloïd.

Heriot Watt College,

Chemical Laboratory, Edinburgh. 






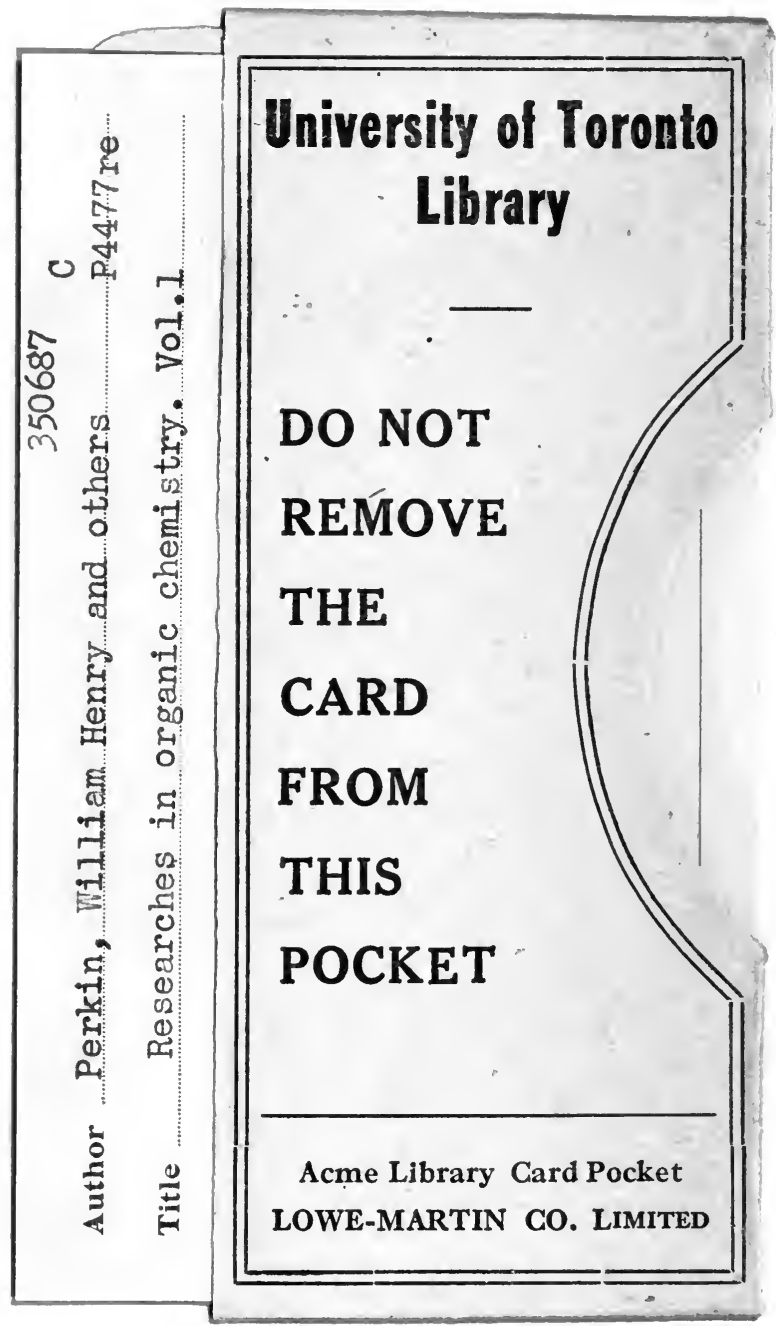


